\title{
Update on the Scientific Status of the Plasma Focus
}

\author{
Sunil Auluck 1,2,*(1), Pavel Kubes 1,3, Marian Paduch ${ }^{4}$, Marek J. Sadowski ${ }^{1,5}$, Vyacheslav I. Krauz ${ }^{1,6}$, Sing Lee ${ }^{7,8}$, \\ Leopoldo Soto 1,9,10, Marek Scholz ${ }^{1,11}{ }^{10}$, Ryszard Miklaszewski ${ }^{4}$, Hellmut Schmidt ${ }^{1}$, Alexander Blagoev ${ }^{1,12}$, \\ Maurizio Samuelli ${ }^{1}$, Yeow Sing Seng ${ }^{13}$, Stuart Victor Springham ${ }^{14} \mathbb{D}^{\mathbb{B}}$, Alireza Talebitaher ${ }^{15}$, Cristian Pavez ${ }^{9,10}$, \\ Mohammad Akel 1,16, Seong Ling Yap ${ }^{1,17} \mathbb{1}$, Rishi Verma ${ }^{18,19}$, Karel Kolacek ${ }^{1,20} \mathbb{D}_{\text {, Paul Lee Choon Keat }}^{1,14}$, \\ Rajdeep S. Rawat ${ }^{1,14}$, Ali Abdou ${ }^{1,21}$, Guixin Zhang ${ }^{1,22}$ and Tõnu Laas ${ }^{1,23}$
}

1 International Scientific Committee on Dense Magnetized Plasmas, IPPLM, HERY 23, P.O. Box 49, 00-908 Warsaw, Poland; kubes@fel.cvut.cz (P.K.); marek.sadowski@ncbj.gov.pl (M.J.S.); krauz_vi@nrcki.ru (V.I.K.); leopoldo.soto@cchen.cl (L.S.); marek.scholz@ifj.edu.pl (M.S.); mail@hellmutschmidt.de (H.S.); blagoev@phys.uni-sofia.bg (A.B.); maurizio.samuelli@tiscali.it (M.S.); makel@aec.org.sy (M.A.); yapsl@um.edu.my (S.L.Y.); kolacek@ipp.cas.cz (K.K.); paul.lee@nie.edu.sg (P.L.C.K.); rajdeep.rawat@nie.edu.sg (R.S.R.); aabdou@zewailcity.edu.eg (A.A.); guixin@mail.tsinghua.edu.cn (G.Z.); tony@tlu.ee (T.L.)

2 HiQ TechKnowWorks Pvt Ltd., Navi Mumbai 400706, India

3 Faculty of Electrical Engineering, Czech Technical University in Prague, 16627 Prague, Czech Republic

4 International Center for Dense Magnetized Plasmas, 00-908 Warsaw, Poland; marian.paduch@ifpilm.pl (M.P.); ryszard.miklaszewski@ifpilm.pl (R.M.)

5 National Centre for Nuclear Research (NCBJ), A. Soltana 7, 05-400 Otwock-Swierk, Poland

6 National Research Center Kurchatov Institute, 123182 Moscow, Russia

check for updates

Citation: Auluck, S.; Kubes, P.; Paduch, M.; Sadowski, M.J.; Krauz, V.I.; Lee, S.; Soto, L.; Scholz, M.; Miklaszewski, R.; Schmidt, H.; et al. Update on the Scientific Status of the Plasma Focus. Plasma 2021, 4 , 450-669. https://doi.org/10.3390/ plasma4030033

Academic Editor: Andrey Starikovskiy

\section{Received: 28 April 2021}

Accepted: 1 July 2021

Published: 7 September 2021

Publisher's Note: MDPI stays neutral with regard to jurisdictional claims in published maps and institutional affiliations.

Copyright: (c) 2021 by the authors. Licensee MDPI, Basel, Switzerland. This article is an open access article distributed under the terms and conditions of the Creative Commons Attribution (CC BY) license (https:/ / creativecommons.org/licenses/by/ $4.0 /)$.
Institute for Plasma Focus Studies, Melbourne 3148, Australia; leesing@optusnet.com.au

8 Physics Department, University of Malaya, Kuala Lumpur 50603, Malaysia

9 Center for Research on the Intersection of Plasma Physics, Matter and Complexity, $\mathrm{P}^{2} \mathrm{mc}$ Comisión Chilena de Energía Nuclear, Av. Nueva Bilbao 12.501, Las Condes, Santiago 7600713, Chile; c.pavez.morales@gmail.com

10 Departamento de Física, Facultad de Ciencias Exactas, Universidad Andres Bello, Sazié 2212, Piso 7, Santiago 8370136, Chile

11 The Henryk Niewodniczański Institute of Nuclear Physics, Polish Academy of Sciences, 01-224 Krakow, Poland

12 Faculty of Physics, Sofia University, 5 J. Bourchier Blv., 1164 Sofia, Bulgaria

13 Emerging Nanoscience Research Institute, Nanyang Technological University, Singapore 639798, Singapore; ysseng@ntu.edu.sg

14 Natural Sciences \& Science Education (NSSE), National Institute of Education, Nanyang Technological University, Singapore 639798, Singapore; stuart.springham@nie.edu.sg

15 Department of Physics, University of Regina, Regina, SK S4S 0A2, Canada; Alireza.Talebitaher@uregina.ca

16 Department of Physics, Atomic Energy Commission, Damascus P.O. Box 6091, Syria

17 Plasma Technology Research Centre, Department of Physics, Faculty of Science, University of Malaya, Kuala Lumpur 50603, Malaysia

18 Pulsed Power \& Electromagnetics Division, Bhabha Atomic Research Centre, Visakhapatnam 531011, India; rishiv@barc.gov.in

19 Homi Bhabha National Institute, Anushaktinagar, Mumbai 400094, India

20 Department of Pulse Plasma Systems, Institute of Plasma Physics, Czech Academy of Sciences, 18200 Prague, Czech Republic

21 Technical Advisor, Halliburton, Houston, TX 77032, USA

22 Lab of Gas Discharge and Plasma, Dept of Electrical Engineering, Tsinghua Univeisity, Beijing 100084, China

23 School of Natural Sciences and Health, Tallinn University, 25 Narva Road, 10120 Tallinn, Estonia

* Correspondence: skhauluck@gmail.com

Abstract: This paper is a sequel to the 1998 review paper "Scientific status of the Dense Plasma Focus" with 16 authors belonging to 16 nations, whose initiative led to the establishment of the International Center for Dense Magnetized Plasmas (ICDMP) in the year 2000. Its focus is on understanding the principal defining characteristic features of the plasma focus in the light of the developments that have taken place in the last 20 years, in terms of new facilities, diagnostics, models, and insights. Although it is too soon to proclaim with certainty what the plasma focus phenomenon is, the results available to date conclusively indicate what it is demonstrably not. The review looks at the experimental data, cross-correlated across multiple diagnostics and multiple devices, to delineate the contours of an 
emerging narrative that is fascinatingly different from the standard narrative, which has guided the consensus in the plasma focus community for several decades, without invalidating it. It raises a question mark over the Fundamental Premise of Controlled Fusion Research, namely, that any fusion reaction having the character of a beam-target process must necessarily be more inefficient than a thermonuclear process with a confined thermal plasma at a suitably high temperature. Open questions that need attention of researchers are highlighted. A future course of action is suggested that individual plasma focus laboratories could adopt in order to positively influence the future growth of research in this field, to the general benefit of not only the controlled fusion research community but also the world at large.

Keywords: plasma focus; fusion energy; neutron emission; neutron spectrum; beam-target mechanism; ion acceleration; ion spectroscopy; ion imaging; coded aperture imaging; soft x-ray imaging; magnetic reconnection; plasma modeling; Monte-Carlo method; magnetic probes

\section{Introduction}

This article is a sequel to the 78-page review article "Scientific Status of the Dense Plasma Focus" [1] published in 1998 with 16 authors belonging to 16 nations. That review paper expressed its purpose clearly in its Introduction: it was expected to serve as "the foundation lying at the base of the International Center for Dense Magnetized Plasmas created under the auspices of UNESCO". This center came into existence as an initiative of the International Plasma Focus Community with the following important milestones:

- The idea of the International Center for Dense Magnetized Plasmas (ICDMP) appeared in 1990 (Appendix B shows group photographs of the first meeting at Moscow).

- $\quad$ The ICDMP Project (including ICDMP basic concept, organization structure, financial requirements, and scientific program) was elaborated by the working group in the period 1991-1995.

- $\quad$ Proposals for locating the Center in various countries were considered: Italy (Ferrara University), Poland (INS and IPPLM), China, Romania, Argentina.

- $\quad$ The 28th UNESCO General Conference (on 7 November 1995) took note of the Draft Resolution 28 C/DR 291 on the creation of ICDMP, drafted by the ICDMP Steering Committee, which consisted of 16 members including authorized representatives of 8 governments (Argentina, Czech Republic, Singapore, South Korea, South Africa, Russia, Poland, and Spain).

- In 1997, members of the steering committee decided that the best place for locating ICDMP was IPPLM in Warsaw, because of the availability of the large PF-1000 device and modern diagnostic apparatus along with buildings, infrastructure and manpower.

- UNESCO Director General, Dr. Federico Mayor, visited plasma laboratories at IPPLM in Warsaw in 1998 and accepted the proposal of locating the ICDMP at IPPLM.

- On 24 August 1999, an official agreement was signed in Warsaw between the UNESCO Director General and President of the Polish Atomic Energy Agency. This document was the starting point for ICDMP activities at IPPLM in Warsaw. Director of IPPLM organized a separate group of workers assigned to the ICDMP (ICDMP research team) and according to the UNESCO recommendations created a foundation for financial support of the ICDMP, together with its council and board.

- $\quad$ The International Steering Committee, recognizing that it had successfully fulfilled its major task of selecting a site for the ICDMP, decided to end its mission as of 19 November 1999. The International Steering Committee also decided to form a new International Scientific Committee for Dense Magnetized Plasmas (ISC DMP), constituted by the former members of the International Steering Committee.

- The actual establishment of the International Center for Dense Magnetized Plasmas was formalized in the AGREEMENT between the International Scientific Committee for Dense Magnetized Plasmas and the Institute of Plasma Physics and Laser Micro- 
fusion "On the Establishment and Operation of the International Center for Dense Magnetised Plasmas" that was signed on October 17, 2000, by the Chairman of the ISCDMP and the Director of IPPLM.

- $\quad$ The stated aims of the ICDMP are:

- To carry out advanced fundamental investigations in the domain of a dense magnetized plasma.

- To realize research programs aimed at application of plasma technologies and utilization of soft- and hard X-ray, ions and neutrons emitted by a plasma in industry, medicine, biology, etc.

- To teach and train young scientific workers in performing experimental investigations with the use of modern and complex apparatus under the supervision of experienced scientists from various countries.

The 'pandemic year' 2020 marks the 20th anniversary of the agreement that finally established the ICDMP and is, thus, a proper occasion to consider an update on the scientific status of the plasma focus. A brief summary of activities of ICDMP over its 20 year span is presented in the Appendix A. Photographic remembrance of the meeting in Moscow in 1990, where the idea of ICDMP was first discussed, is presented in Appendix B.

There are several developments in these 20 years that necessitate such update.

- New facilities have come up.

- New diagnostics have been deployed in a novel manner.

- New experimental results have been reported.

- New models have been developed.

- New insights have been revealed.

- New directions have been proposed in the utilization of plasma focus.

A unified overview of all these should, therefore, be useful for the plasma focus community to chalk its path going forward.

This review chooses to focus its attention on understanding the principal defining characteristic features of the plasma focus. In order to sharpen this focus, some aspects of current research dealing with plasma focus have been left out of its scope. These deal with the applications of plasma focus and post-pinch-phase phenomenology, which deserve their own dedicated reviews.

There have been many review articles [2-13] on the plasma focus. This review does not aim to duplicate their effort. Nor does it aspire to be a comprehensive bibliographic reference source. Rather, it aims to identify an outline of an emerging narrative regarding the complex physics of the plasma focus, that is yet to become 'established science' but can no longer be ignored as mere conjecture. Along the way, it demonstrates that certain commonly held beliefs about the plasma focus phenomenon are not supported by experimental data. It makes a distinction between reporting experimental observations and conjectures, postulates, or scenarios which are advanced to "explain data". It attempts to bring out deeper meaning inherent in already published data that can be discerned using insights from later publications and from informal discussions in the scientific community. For this purpose, this review has chosen to modify some already published figures from the works of its coauthors to bring out that meaning using various annotations and digital enhancement. In this way, while the original figures published in the cited references maintain their data fidelity, the annotations enable commentary in the text that aims to bring out the deeper meaning. The fluid state of this emerging narrative is expected to provide opportunities and impetus for creative experimental and theoretical investigations. That is the hope and motivation behind the present review.

\section{Summary of Scientific Status of PF as of $\mathbf{1 9 9 4}$}

The purpose of this section is to provide a context for this review of PF research over the last 20 years. Although the scientific details of PF research in the period 1961-1994 have been extensively covered in the previous review [1], and much information about various 
facilities and their contributions is available in the cited review papers [2-13], this section looks at some of its historical aspects with the benefit of hindsight. It is especially important for the scientific community to appreciate why PF research evolved along the trajectory it actually followed. This, in turn, is essential to appreciate how the future trajectory of $\mathrm{PF}$ research could be affected by the new narrative emerging from ongoing research and certain proactive initiatves by the plasma focus community.

It is also necessary to appreciate the nature and scope of the prevailing consensus in the field of plasma focus in order to comprehend the novelty and promise of the emerging narrative. To this end, this section also outlines a "Standard Narrative" regarding plasma focus, together with known deviations. This is to be understood primarily as context for discussion of the significance of ongoing research.

\subsection{Summary of Major Trends of Research 1961-1994}

This period should be logically divided into three eras: the first one is of general optimism that begins with the discovery of the plasma focus (PF) effect by Filippov [14] in 1961 and Mather [15] in 1964, and ends with the discontinuation of funding for plasma focus program at Limeil in 1981 and of Frascati 1 MJ focus in 1982. This period is marked by rapid emergence of government funded large ( $>100 \mathrm{~kJ}$ stored energy) and medium sized (10-100 kJ stored energy) PF facilities and grand plans $[5,16]$ about scaling it up to reactor size. This is followed by the second era of gradual decline in interest, that saw the plasma focus research slowly going out of fashion and the end of experiments at POSEIDON in early 1990s. The third era began around 1990 and marks the emergence of smaller facilities $(<10 \mathrm{~kJ})$. A histogram of the number of year-wise paper publications of DPF shown in Figure 1 of [11] clearly illustrates this trend: a low level of publications from 1961-1970, a strong steady trend from 1970-1980, a steady decline during 1980-90, and a steady growth from 1990 onwards. The causes for the early optimism, later decline and resurgence deserve a close scrutiny and have an important bearing on the conclusions of this review paper. These have their origin in the scientific climate in which the plasma focus effect was discovered.

The history of the early days of controlled fusion research (CFR) has been covered in the classic review paper by R.F. Post [17]. The history of the research program into harnessing the pinch effect for CFR has been discussed in the review paper by Bostick [18]. The following aspects of this historical reconstruction are of special significance:

- At the beginning of CFR, three methods of generating fusion reactions were known: (1) bombardment of a target with a beam of accelerated ions, as in a CockcroftWalton machine. (2) generation and confinement of a hot, fusion-reactive plasma, as in the stars. (3) explosive release of fusion energy as in a hydrogen bomb. The first was dismissed with the following argument: an accelerated linear beam of ions bombarding a solid target would spend most of its energy in heating the target and too few fusion reactions would occur before eventual disassembly of the target, resulting in a net loss rather than gain of energy. Later in this review (see Section 8.5), this shall be referred to as the Fundamental Premise of Controlled Fusion Research and shall have significant implications. The second appeared to be promising provided the role of gravity in confining the hot, fusion-reactive plasma of the star could be played by magnetic field. The third could be considered as a viable CFR option only after the advent of high power lasers and particle beams and sophisticated computational resources for radiation hydrodynamics.

- The idea of confinement of a stream of ions and electrons by their own magnetic field was suggested by Bennett in 1934 [19] (the word "plasma", although introduced by Langmuir in 1927, was not in common scientific use at that time). This was the only conceptual candidate for confining a hot plasma available to the scientific community at the beginning of CFR research in early 1950s. The only theoretical tools to deal with this concept were based on the theory of magneto hydro dynamics (MHD), developed primarily in the context of astrophysics $(17,18)$. This theory dealt with charged particles 
moving in adiabatic orbits in the magnetic field generated by their own motion. MHD analysis revealed that the CFR goals of heating and confinement faced fundamental obstacles in the form of poor stability of the pressure equilibrium between the magnetic field and the plasma and loss of plasma through open ends [17,18]. MHD analysis also later guided the CFR community towards inventing workarounds in the form of complex magnetic field and plasma configurations with toroidal topology-an effort that continues to this date. The goal of this MHD-inspired-and-guided effort remains confinement of a thermal, sufficiently hot, fusion-reactive plasma for a sufficiently long time to achieve net energy gain.

- The observation of high fusion neutron yields $\sim 10^{10}$ neutrons/pulse in the Filippov and Mather type devices and the poor neutron yield from linear z-pinches and its MHD-inspired derivatives were the main cause for the surge of interest in the PF in the 1960s and 1970s. Discovery of scaling of neutron yield, with the fourth power of current already mentioned by Mather in his much cited first review paper [2], was a major driver of this optimism. This interest was dampened by the conclusion that much of the neutron yield appeared to be produced by a linear beam along the axis, which went against the then prevailing consensus that only a confined thermal plasma could achieve breakeven. Coupled with the fact that the plasma focus effect could not be adequately explained in terms of a theory, the plasma focus lost its place in the list of viable candidates for CFR and got labeled as "a high intensity neutron source" [16].

- The only point still in favor of plasma focus was the scaling of neutron yield as 4th power of current over three decades of current that saw a record yield of more than $10^{12}$ neutrons per shot in 1973 [20]. When the Frascati 1 MJ focus failed to scale up, it was game over. This facility lost funding, its leadership migrated to the emerging consensus in favor of tokamak research and the facility was eventually dismantled, making way for another experiment, ending the era of optimism.

- Theoretical work, beginning in 1972, suggesting the possibility of miniaturizing the hydrogen bomb using temporally profiled shock waves [21,22] served as a major attraction to the fusion community. Theoretical work related to understanding magnetic confinement devices became a major undertaking in the 1970s and 1980s in contrast to plasma focus research, which had a bewildering variety of experimental results, none of which fitted any theoretical narrative that could be used to scale it up to a fusion reactor. This absence of a theoretical basis for PF physics, in contrast to a huge army of theoreticians working on inertial and magnetic confinement schemes, had a severe negative impact on the prospects of subsequent funding for large PF projects.

- The invention of lasers in the 1960s complemented efforts to understand the real phenomenology of plasma focus with some hopes of augmenting the plasma focus reaction rate by laser heating. It inspired Sylwester Kaliski, a prominent Polish scientist, member of Polish Academy of Sciences, of the military General Staff, and the Council of Ministers, to establish a scientific institute and a facility, known today as the Institute for Plasma Physics and Laser Microfusion (IPPLM) and PF-1000, respectively, where he hoped to enhance the fusion reaction rate of the plasma focus by injecting a powerful laser pulse. It also led to the deployment of advanced time-resolved imaging diagnostics (Schlieren, shadography, and interferometry), Faraday rotation diagnostics, and Thomson scattering. Development of gated micro-channel plate detectors also allowed for time resolved images using soft X-ray pinhole cameras. Significant insights into the complex phenomenology of PF were obtained by correlating these with neutron spectroscopy and ion measurements. However, constraints arising from the pressure of reporting progress to funding agencies probably prevented sustained follow up investigations. Instead of leading to well-researched scientific conclusions, this resulted in a few conference papers that merely reported 'first impressions' which were not followed up with detailed investigations and did not have much impact on later research. This will be further commented upon again in a later section (see Section 9.3). 
- Smaller plasma focus groups concentrated efforts on developing skills in diagnostics and fundamental investigations, rather than building large facilities. Notable examples are:

$\bigcirc \quad$ The group at Hoboken, USA, which did significant work on high spatial resolution fast image convertor photography and X-ray imaging, as well as reaction product analysis.

$\bigcirc \quad$ Groups in Argentina and Germany, which did fundamental work on sheath formation, structure, and propagation.

$\bigcirc \quad$ The group in Heidelberg, Germany, which carried out neutron pinhole imaging and light scattering experiments on multiple DPF installations.

- Large and small PF groups reacted to the decline in the popularity of PF research by suggesting and investigating probable causes of failure of neutron yield scaling and demonstrating applications of $\mathrm{PF}$ in various areas.

- The resurgence of interest in plasma focus after the 1990s can be seen as the direct result of the initiative by Sing Lee to design a simple low cost plasma fusion facility that could be operated by a small university group on a low budget and the financial support for this initiative from the International Center for Theoretical Physics, Trieste. This is described in detail in Section 3.3.1.1.

- There was a notable absence of any research program that was mandated to answer the fundamental questions related to plasma focus physics, in particular the question: why was the neutron yield 2-3 orders of magnitude more than the thermonuclear yield corresponding to experimentally determined plasma parameters?

\subsection{The "Standard Narrative" about the Dense Plasma Focus}

It is necessary to comprehend the nature and scope of the prevailing consensus in the field of plasma focus in order to appreciate the novelty and promise of the emerging narrative. This is described in terms of a 'Standard Narrative'. The term "narrative" is deliberately used to emphasize that the present state of knowledge is inbetween a scientifically well-founded theory and conjectures partially rooted in emprical evidence. It will be seen later (see Section 8.3) that the emerging narrative is fascinatingly different from the standard narrative, without invalidating it.

The standard narrative regarding the dense plasma focus emerged from Mather's review article [2] (circa 1971) and has largely been accepted till now, with several known phenomenological deviations and additions, but no major conceptual departure other than emphasizing the "beam-target process" as the cause of bulk of the neutron production. This is briefly summarized below and shall be referred as "Standard Narrative" which is applicable to "well-optimized" PF devices:

1. A plasma sheath is initially formed as a surface discharge over the insulator.

2. The initial plasma sheath gets detached from the insulator and gets accelerated by magnetic pressure, turns around the anode corner and moves towards the axis in a characteristic curved shape.

3. The current flows behind the dense plasma sheath until it approaches the axis. The moving current layer acts as a time-varying inductance that increases as the plasma approaches the axis. A characteristic sharp dip in the current derivative signal and a spike in the voltage across the electrodes occur as the plasma reaches the axis.

4. The sheath stagnates at the axis in a funnel-shaped formation whose stem gets compressed and heated.

5. The compressed and heated plasma is a z-pinch equilibrium where the thermal pressure of the plasma trying to push it away from the axis is resisted by the magnetic pressure because of the plasma current flowing axially through the plasma stem that tends to push it towards the axis.

6. The z-pinch equilibrium undergoes instability that suddenly decreases the plasma current which creates intense electric fields which accelerate ions which produce neutrons by a beam-target interaction. 
7. Until the stagnation, the radial and axial movement of the plasma has good azimuthal symmetry: a given $(\mathrm{r}, \mathrm{z})$ coordinate is reached by the plasma at the same time, irrespective of the associated azimuthal coordinate $\theta$.

This narrative still serves as the often invisible backdrop for most publications on plasma focus today.

Known phenomenological deviations from (or additions to) the standard narrative include observations of filaments, hot spots, turbulent density fluctuations, anomalous resistivity, localized sources of fast deuterons, and local sub-millimeter-sized super-dense plasma domains. They do not affect the prevailing consensus except in terms of offering multiple postulates for interpreting data but do not offer experimental proof compelling enough to modify the standard narrative. Some of these are discussed in detail in Section 2.3.

This standard narrative has guided a significant portion of the research in DPF physics. Unfortunately, it also has caused many researchers to ignore or downplay their own experimental findings which did not fit this narrative. This is discussed in Section 2.4.

\subsection{Known Phenomenological Deviations from (or Additions to) the Standard Narrative}

This section describes some aspects of plasma focus research which have been studied from the very beginning in a more or less continuous stream, with similar techniques, reaching more or less similar conclusions across multiple devices. They reveal inadequacy of the standard narrative in explaining their findings and provide some insight into a more complex phenomenology. They suggest certain postulates which can be used to interpret their observations. Direct experimental proof compelling enough to cause a major revision of the standard narrative is, however, not yet in sight. These are presented below as minireviews to convey the scale of efforts and achievements in their areas of specialization. A selection from these observations is discussed in more detail later.

\subsubsection{Ion Emission from the Plasma Focus}

Transfer of energy from the magnetized plasma to ions and electrons is a process not fully understood even today. The study of ion emission from the plasma focus, therefore, continues to be at the forefront of plasma focus research. This section provides a historic background of the relevant work and its continuation to the present in the form of a mini-review.

From the very beginning of research on plasma-focus discharges, which were performed with deuterium (either pure or with some admixtures) it was known that the main fusion reactions occur between accelerated primary deuterons. It was evident that the accelerated deuterons have a wide energy spectrum, and those not-lost in the fusion reactions can be emitted from PF discharges, e.g., in the form of high-energy deuteron beams [23-29]. In fact, such deuteron beams have been observed in many PF experiments [30-43]. There were also recorded high-energy ions of working-gas impurities and admixtures [30,34].

Research on the ion emission from PF discharges was of primary importance not only because it provided information about plasma-focus phenomena, but also enabled some technological applications to be realized. A concise description of the ion emission studies performed until 1998 was presented in a comprehensive review paper [1].

\section{Investigation of Fast Ions from PF discharges until 1998}

The most important results of ion studies in various PF facilities, which were carried out before 1998, can be summarized as follows:

1. Many experiments proved that PF facilities are powerful generators of pulsed ion streams. To estimate the total deuteron emission from PF discharges performed with a pure deuterium filling different diagnostic techniques were applied. Using deuteron reactions with a lithium target and a time-of-flight (ToF) method in a hybrid Filippov-Mather experiment, it was estimated that the total yield of fast deuterons with an average energy of about $1 \mathrm{MeV}$ was about $10^{15}$ [23]. Using nuclear emulsions it was found that in the Filippov experiment [24] the yield was about $10^{13}$ deuterons 
per shot. In order to analyze the fast deuterons $(>100 \mathrm{keV})$ the use was made of a magnetic analyzer and ToF method. It was shown that the energy spectrum of the emitted deuterons extended from about $70 \mathrm{keV}$ to above $400 \mathrm{keV}$, and it could be described by an exponential function $\exp \left(-\mathrm{kE}_{\mathrm{d}}\right)$. The total deuteron yield from a small $1-\mathrm{kJ}$ PF device was estimated to reach even $10^{14}$. Using activation measurements of carbon foils it was found that a 76-kJ discharge [26] produced about $10^{15}$ deuterons of energy $>330 \mathrm{keV}$. Based on the foil-stack technique, it was shown that a larger 250-kJ PF device [28] was able to emit the fast deuterons of energy ranging above $6 \mathrm{MeV}$.

2. Numerous PF experiments showed that the highest ion emission was recorded at pressures lower than that corresponding to the maximum fusion-neutron yield. This ion emission was usually peaked at the $z$-axis, but in many cases it showed a strong asymmetry $[1,30]$. The total deuteron yield depended on experimental conditions, i.e., energy delivered from the condenser bank, the initial pressure, etc. [25,36,43]. It was deduced that the fast neutrons from $\mathrm{d}-\mathrm{d}$ fusion reactions in PF discharges were produced mainly by deuterons of energy (20-100) keV [32,42].

3. Measurements performed with pinhole cameras equipped with nuclear track detectors proved that the fast deuterons are emitted in the form of numerous micro-beams originated from many micro-sources (probably of sub-millimeter dimensions) located inside and at the surface of the pinch column [30,31,36]. Ion pinhole images showed, usually, the central spot (containing numerous beam tracks) and a few ringshaped images produced by many micro-beams $[1,31]$. The central spot was evidently formed by the deuterons emitted along the $z$-axis, while the ring-shaped images could be produced by the deuteron beams emitted at different angles to the $z$-axis and deflected by local magnetic fields. It was observed that the deuteron beams had durations of (2-8) ns [29,31] and could form intense bunches of the total power ranging from $10^{11}$ to $10^{12} \mathrm{~W}[1,29]$. Some examples of the ion pinhole images and angular distributions of the fast deuterons are shown in Figure 1.

4. It was also proved experimentally that heavy impurity and admixture ions could be accelerated to higher energies ranging between 10 and $15 \mathrm{MeV}$ [34,36]. Such ions were recorded with pinhole cameras and filtered track detectors, as well as with a Thomsontype analyzer [34]. For highly ionized $(Z>4)$ impurity-ions the maximum $E_{i} / Z$ ratio was about $1.8 \mathrm{MeV}$, while for lower-ionized $(Z<4)$ species this ratio amounted to (2-2.8) MeV. Mechanisms of the ion acceleration were not identified, but taking into account that the considered ions were emitted from sub-millimeter sources, it was estimated that they were accelerated by strong local electrical fields ranging even up to $50 \mathrm{MV} / \mathrm{cm}$.

5. In Filippov geometry, it was shown that evaporated anode material could be transported to the acceleration zone of the pinch and subjected to an intense electric field. Thus, with an aluminium insert at the center of the anode, $\mathrm{Al}$ ions with ionization multiplicity 5 to 9 and energy of several $\mathrm{MeV}$ were detected with an ion time-of-flight method [44]. With tantalum, multicharged Ta ions accelerated to $100 \mathrm{MeV}$ were detected [45]. 


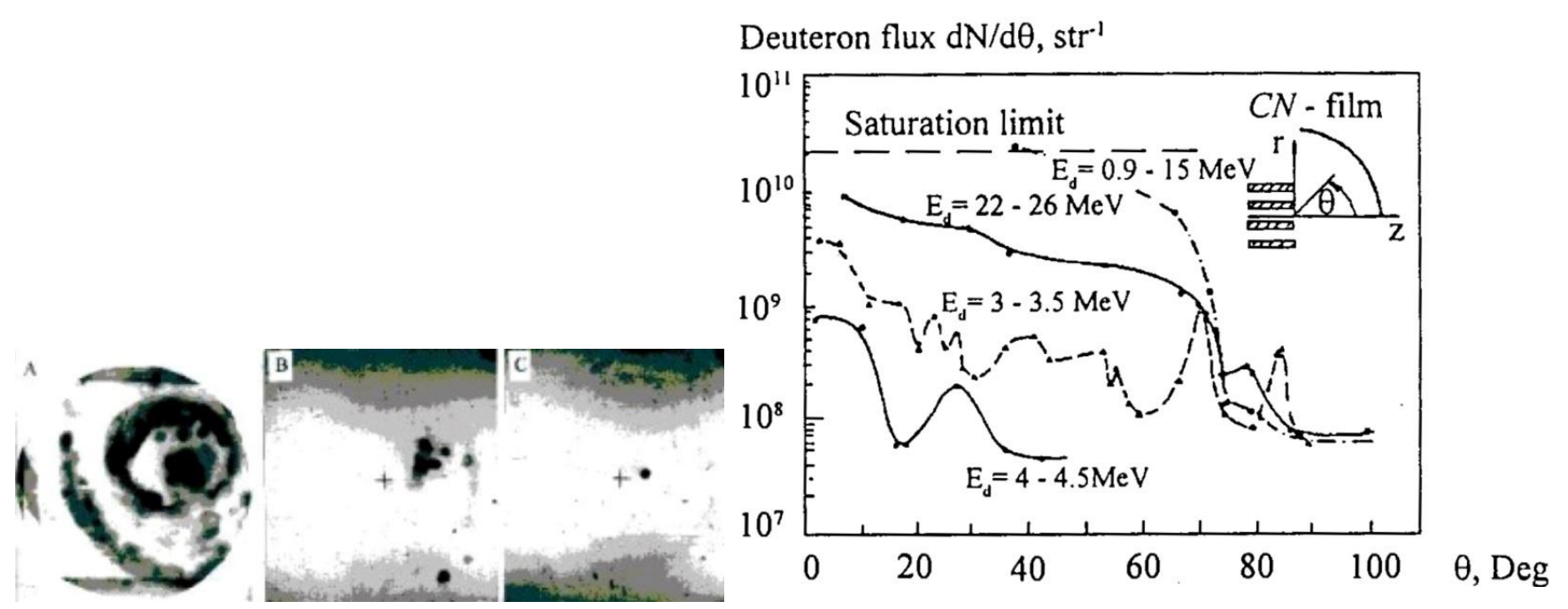

Figure 1. Typical images of the fast ion beams. Ions emitted from a 20-kJ PF device, as recorded with an axial pinhole camera and filtered track detectors: (A) 1.5- $\mu \mathrm{m} \mathrm{Al-filter,}>220 \mathrm{keV},($ B) $6-\mu \mathrm{m} \mathrm{Al-filter,}>700 \mathrm{keV}$, (C) $30 \mu \mathrm{m} \mathrm{Al-filter,}>2.1 \mathrm{MeV}$, and the angular distribution of the fast deuterons emitted from a 40-kJ NESSI experiment. Adapted from original data reported in $[1,30]$ (reprinted with permission of IOP).

\section{Studies of Fast Ions from PF Discharges Since 1998}

Taking into consideration importance of the ion studies for basic plasma physics and for different technological applications, research on the fast ions emitted from PF facilities was continued in many laboratories all over the world, e.g., an analysis of nitrogen ion beams (generated in a low-energy plasma focus device) was performed in Argentina [46]. Extensive theoretical and experimental studies were also continued in Poland [47-52]. Theoretical analysis of ion trajectories was carried out taking into account different filamentary structures of a pinch column [47]. There were also performed experimental studies of the current-sheath dynamics and charged particle emission from a PF-1000 facility [48] and PF-360 device [49]. Using nuclear track detectors placed upon a semicircular support (in front of the electrode outlet) it was shown that the angular distributions of the emitted deuterons had a local minimum at the $z$-axis and two side-on wings ranging to above $60^{\circ}$ to the $z$-axis (with an evident asymmetry). The theoretical studies of the ion motions were continued [50], and a more detailed theoretical analysis, based on the known gyrating particle model (GPM) and assumptions about the filamentary structure of the pinch column, made it possible to explain the angular distribution of the fast deuterons observed in several PF experiments. Particular attention was, however, focused on experimental studies in the large PF-1000 facility [51,52], as well as in small PF devices, e.g., [53,54]. In the first case it was confirmed that the angular distributions of fast ions (both primary protons and deuterons) showed two distinct peaks and a lower emission along the discharge axis. In the small PF device, for shots with a lower neutron yield the distinct and well-collimated deuteron beams were recorded, while for shots producing higher neutron yields the ion pinhole images showed numerous deuteron micro-beams. It concerned the accelerated primary deuterons of energy above $100 \mathrm{keV}$, because lower-energy deuterons were mostly lost in nuclear fusion reactions or stopped within the dense plasma column.

Many efforts were devoted to the development of different diagnostic techniques for the ion (and electron) studies [55-57]. Using nuclear track detectors shielded with different absorption foils it was possible to perform a rough energy analysis of the recorded ions, e.g., using an 80- $\mu \mathrm{m}$-thick Al-foil and CR-39 plastic there were recorded fusionproduced protons of energy $>2.9 \mathrm{MeV}$ and very fast deuterons of energy $>3.9 \mathrm{MeV}$. From $15-\mathrm{kJ} \mathrm{PF}$ discharges, at a distance of $34 \mathrm{~cm}$ from the focus region there were obtained about $4.5 \times 10^{3}$ protons $/ \mathrm{cm}^{2}$.

It should be noted that simultaneously with experimental studies of the ion emission from different large $[51,52]$ and small $[53,54,58]$ facilities there were continued theoretical 
analyses of ion motions $[59,60]$. In particular, some efforts were undertaken to explain an influence of temporal changes of the current filaments on trajectories of the emitted ions. Characteristics of the pulsed ion beams emitted from different small PF devices were compared [61] and measurements of charged-particle beams (i.e., ions and electrons) from PF discharges were carried out [62]. There were observed correlations of the ion beams (emitted mainly along the $z$-axis) and fast electron beams (emitted in the upstream direction through an axial channel in the anode). Some examples are presented in Figure 2.

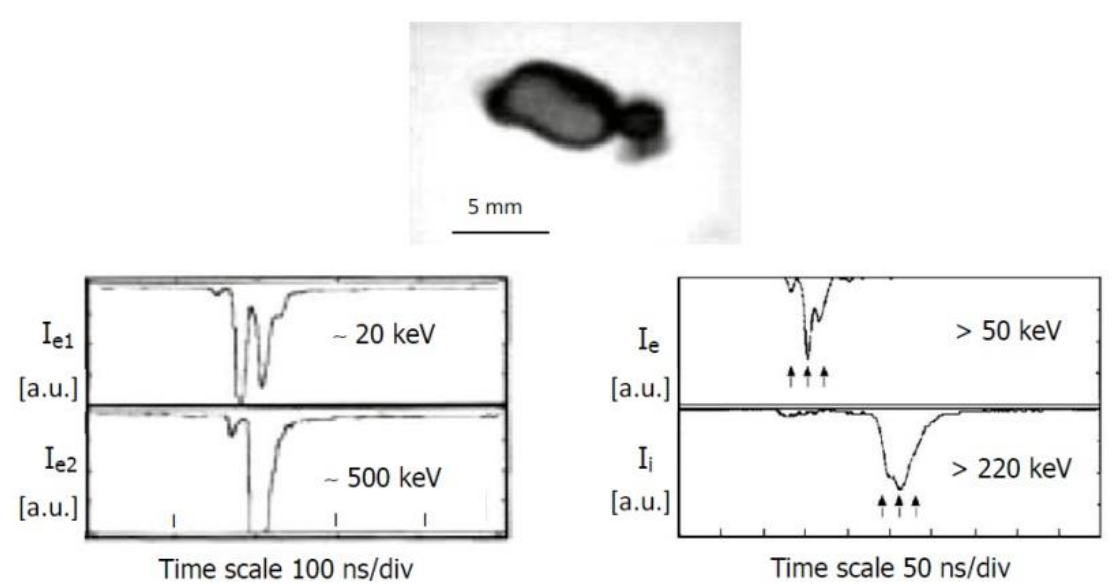

Figure 2. (Top) Enlarged image of distinct spots formed by numerous tracks (microcraters) produced by very fast $(>1.3 \mathrm{MeV}$ ) deuteron beams emitted from a single 44-kJ discharge in the MAJA-PF facility, as recorded upon a shielded $\mathrm{CN}$-foil placed perpendicular to the $z$-axis, inside a pinhole camera, which was oriented along the discharge axis and provided 1.63-magnification: (Bottom left) Timeresolved signals of fast electron beams (Ie1 and Ie2) emitted from the same shot in the upstream direction, and recorded with miniature filtered-scintillators located $70 \mathrm{~cm}$ behind a pinhole in the front plate of the anode. (Bottom right) Data from another 44-kJ shot: Time-resolved signals of electrons (emitted in the upstream direction and measured with a Cherenkov-type detector) and fast deuterons (Id) recorded with a filtered scintillation detector placed at the $z$-axis, at a distance of $70 \mathrm{~cm}$ from the anode end. The vertical arrows indicate parts of the pulses which were correlated with 3 hot-spots observed in this discharge. Selected from the original data presented in [62] (Nucl. Fusion 2001, 41, 755-760) and reproduced with permission of IOP.

In the same PF experiment [62] it was determined that the FWHM value of a single ion pulse was about $20 \mathrm{~ns}$, and the energy spectrum of the recorded deuterons with the maximum at (400-500) $\mathrm{keV}$ was extended up to above $1 \mathrm{MeV}$.

The reported experimental studies were supported by numerical modeling of deuteron motions in a filamentary PF pinch column [63]. Since earlier studies showed that current filaments could exist inside two (or three) cylindrical plasma layers, as well as at the $z$-axis, computations were carried out for different numbers and spatial distributions of the filaments, as well as for different initial energies of the deuterons. It was shown that the formation of the central axial filament changed the angular distribution of the emitted deuterons very strongly. In such a case this distribution was peaked at the $z$-axis, while in the case without the central filament the deuteron distribution had a ring-like shape, as shown in Figure 3. 

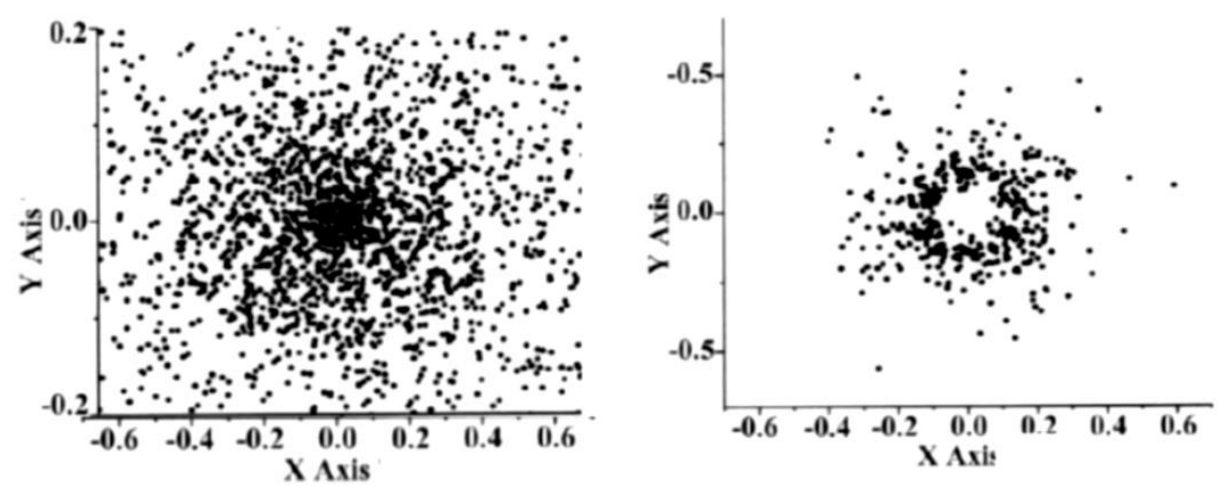

Figure 3. Tracks of the fast deuterons on the plane perpendicular to the $z$-axis, which were computed for two pinch configurations: at the presence of the central axial filament (left) and for the case without the central filament (right). Adapted from original data reported in Figure 4 of [63] (Nukleonika 2001, 46, S29-S32).

Successive experiments and studies of the ion emission were performed in the PF-1000 facility equipped with new larger electrodes (the outer squirrel-cage electrode of $400 \mathrm{~mm}$ in diameter, inner cylindrical one $230 \mathrm{~mm}$ in diameter, both of $600 \mathrm{~mm}$ in length) operated at initial pressures of 1-5 Torr and supply energy of 500-1100 kJ [64]. The intense ion beams emitted along the $z$-axis were observed mostly at lower filling pressures, and the ion pinhole camera images confirmed a complex structure of the recorded ions. At the same time there was investigated a spatial structure and energy spectrum of ion beams within a small 2-kJ PF device in Argentina [65]. In that case, an influence of the initial gas conditions was studied, and it was found that at the gas-puffing the yield of the fast ( $>220 \mathrm{keV}$ ) deuterons was about one order of magnitude lower, and the energy spectrum extended to lower values, as shown in Figure 4.

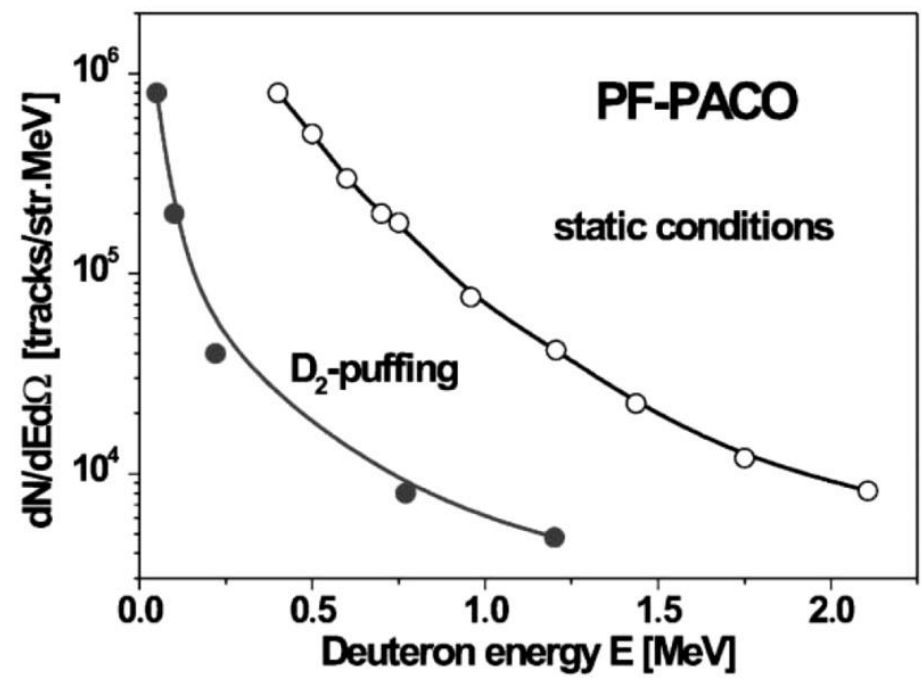

Figure 4. Energy distributions of the fast deuterons emitted from the small (2-kJ) PF device operated at the static initial gas conditions and at the $\mathrm{D}_{2}$-puffing. Reproduced with permission from [65] (Radiat. Meas. 2001, 34, 315-318). CElsevier (2001).

In another small-scale (45 kJ) PF experiment [66] particular attention was focused on the formation of so-called "hot spots" which emitted intense X-ray pulses. It was shown that such "hot spots" are also sources of energetic electron beams (emitted in the upstream direction) and fast ion beams (emitted mainly along the z-axis). Some progress was also achieved in 3D modeling of ion kinetics in PF discharges [67]. The computations were performed for various numbers and distributions of the current filaments, and for different starting points and energies of ions (both protons and deuterons). It was shown that the 3D 
model, which took into account ion motions and collisions, described behavior of different ions and their angular distributions.

The plasma focus group at the Chilean Nuclear Energy Commission performed measurements of ions in a plasma focus of a few hundred joules, PF-400 J, charging at $27 \mathrm{kV}$, operating at 5 to $12 \mathrm{mbar}$ in $\left(\mathrm{H}_{2}\right)$ and at 1 to 9 mbar in a mixture $\left(\mathrm{H}_{2}+5 \% \mathrm{Ar}\right)$ as filling gas, using Faraday cups, time of flight technique (TOF), and electromagnetic deflector with CR39 track detector [68]. It was found that in the case of hydrogen discharges, the ion energy range was from $0.5 \mathrm{keV}$ to $50 \mathrm{keV}$, and in discharges using gas mixtures $\left(\mathrm{H}_{2}+5 \% \mathrm{Ar}\right)$ the ion energy range increased from $0.5 \mathrm{keV}$ to $270 \mathrm{keV}$. Additionally, in some cases, copper (from anode) and aluminum (from insulator) ions with energies lower than $1 \mathrm{keV}$ were observed. In other experiments in a plasma focus of $2 \mathrm{~kJ}$ using Faraday cups, TOF technique and visible spectroscopy [69], the experiments were operated at $20 \mathrm{kV}, 1.6 \mathrm{~kJ}$ stored energy in $\mathrm{H}_{2}, \mathrm{D}_{2}$ and a mixture of $98 \% \mathrm{H}_{2}+2 \% \mathrm{Ne}$ at 9 to 10 mbar. Results obtained with TOF using Faraday cup in hydrogen $\left(\mathrm{H}_{2}\right)$ at 9 mbar showed, that the ion energies were distributed from $40 \mathrm{kV}$ to $370 \mathrm{kV}$ in two regions. The region of higher energy corresponded to the ion species with the highest ionization stages, $\mathrm{Al}^{+5}, \mathrm{Al}^{+6}$ (from the alumina insulator), and $\mathrm{O}^{+5}$. On the other hand, the region of lower energy corresponds to the ion species with lower ionization stages, $\mathrm{H}^{+}, \mathrm{Al}^{+}$and $\mathrm{Al}^{+2}, \mathrm{~N}^{+}, \mathrm{Cu}^{+}$and $\mathrm{Cu}^{+2}$ (from the anode), and $\mathrm{O}^{+2}$. Complementary visible spectral measurements were done to identify the elements present and their ionization degree. Only some ionic species were detected, as $\mathrm{Al}^{+}$and $\mathrm{Al}^{+2}, \mathrm{Cu}^{+2}$ and $\mathrm{Cu}^{+3}$, and $\mathrm{O}^{+}$.

The Chilean group also reported experimental evidence of more than one ion acceleration mechanisms in plasma focus device [70] in the PF-2 kJ operating at $17 \mathrm{kV}, 1.15 \mathrm{~kJ}$ stored energy, at pressure range of 3 to 11 mbar of hydrogen gas. One Faraday cup was mounted in the axial direction, and two Faraday cups were mounted in radial directions (at $180^{\circ}$ to each other). The ion signals were converted into ion number densities at each pressure. The maximum ion number densities were found at $3 \mathrm{mbar}$ in both directions. The variation in the number density with pressure suggests the existence of more than one ion acceleration mechanism. Moreover, at the lower pressures ( 3 mbar), ion emissions are observed before the dip in the current derivative, which suggests that there are acceleration mechanisms acting not only in the pinch stage but also during the radial compression. It is conjectured that Fermi acceleration could be a suitable mechanism for the charge particle acceleration that was measured before the dip in the $\mathrm{dI} / \mathrm{dt}$ signal in the radial direction.

An interesting result of the ion studies was obtained in the large PF-1000 facility, where PM-351 nuclear track detectors, shielded with appropriate absorption filters (e.g., 80- $\mu \mathrm{m}$-thick Al-foils), were used to record fusion-produced protons of energy above 3.1 MeV [71]. Later on, similar detectors were used for more accurate measurements of angular distributions of the fast deuterons emitted from 1-MJ PF discharges [72]. In this case, the results were consistent with earlier measurements described above.

An important direction of the ion studies was research on interactions of intense ion-plasma streams with different targets irradiated in the PF-1000 facility [73]. The chosen nuclear track detectors, which were covered with some filters and placed upon a semicircular support (in front of the electrode outlets), made it possible to determine the angular distribution and energies of the recorded ions (mainly protons or deuterons). Advantages of the use of nuclear track detectors were also demonstrated in measurements of fusion-produced protons emitted from the PF-1000 facility [74]. In that case, samples of the PM-351 detector were covered with 80- or 100- $\mu$ m-thick Al-foils (which protected those detectors from direct plasma interactions and stopped almost all fast deuterons) and placed at different angles to the $z$-axis. The microscope analysis of the etched tracks made it possible to determine numbers of proton tracks of different diameters, as shown in Figure 5, and to estimate the numbers of the recorded protons. 

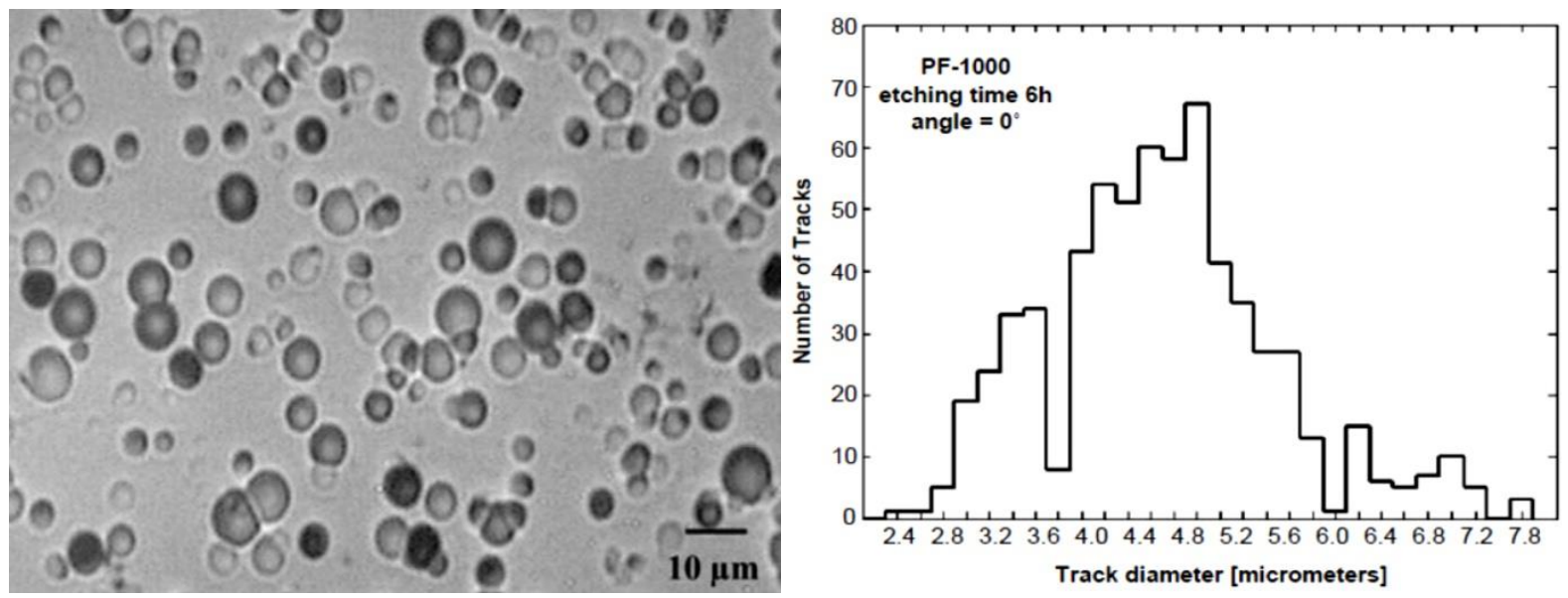

Figure 5. Image of the tracks produced by fusion-protons in the PF-1000 facility (left) and histogram of the measured track diameters (right), which were recorded at the $z$-axis. Reproduced with permission from [74] (Radiat. Meas. 2005, 40, 479-482). (OElsevier (2005).

Further ion studies were continued in a frame of research on correlation of neutron emission with other corpuscular and X-ray pulses in different PF experiments [75]. In the PF-360 device it was observed that the deuterons of energy $>1.3 \mathrm{MeV}$ were emitted about $10 \mathrm{~ns}$ before the $\mathrm{dI} / \mathrm{dt}$ dip, while $600-\mathrm{keV}$ deuterons were emitted about $40 \mathrm{~ns}$ after that, what was explained by a stochastic character of the formation of ion sources.

Very important information was obtained from time-integrated measurements of fusion-produced protons emitted from PF-360 and PF-1000 facilities [76]. On the basis of the pinhole images recorded at different angles, it was shown that the fusion-protons were emitted from small (sub-millimeter) regions localized mostly around the electrode axis, at a distance of a few centimeters from the anode surface, as shown in Figure 6.
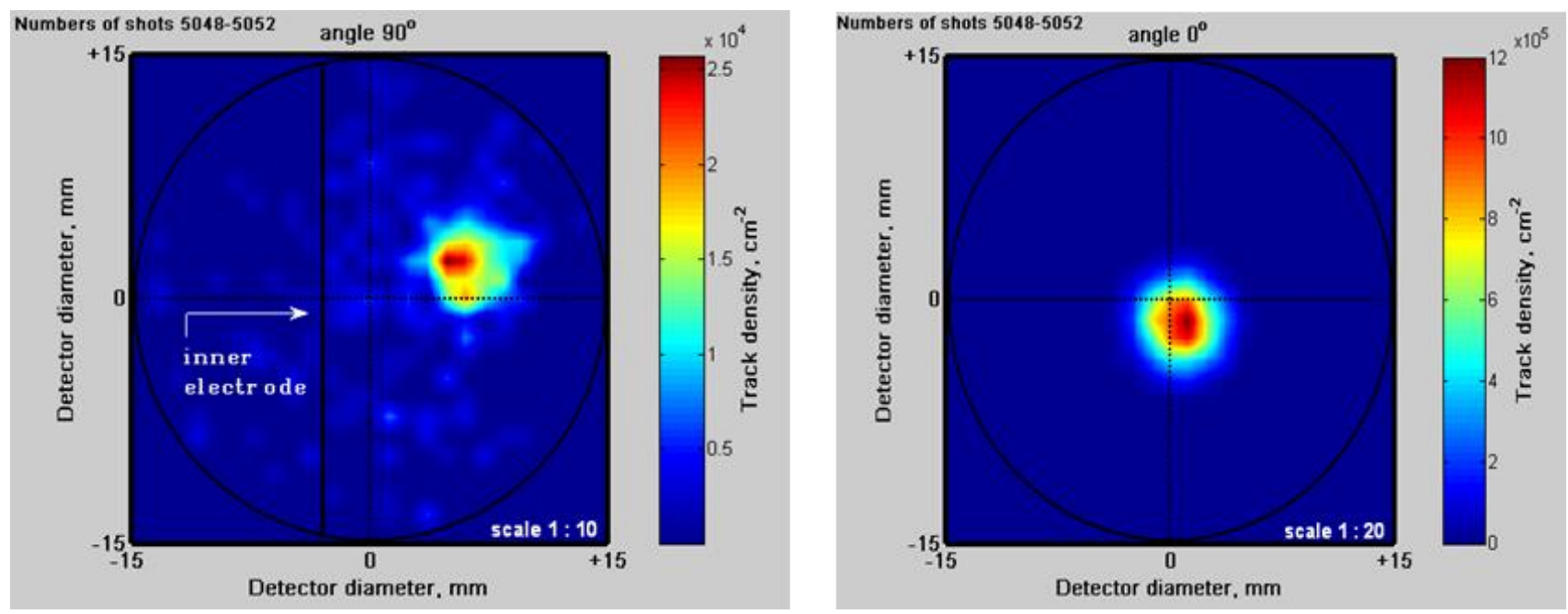

Figure 6. Images of the sources of the fast fusion-produced protons emitted from five successive shots in the PF-1000 experiment, as recorded with two ion pinhole cameras (and NTDs) oriented perpendicular and along the $\mathrm{z}$-axis. The cameras were irradiated by 5 successive shots (at $\mathrm{p}_{0}=3$ torr $\mathrm{D}_{2}$ and $\mathrm{U}_{0}=31 \mathrm{kV}$ ). Adapted from original data presented at the ICDMP Workshop 2008.

Detailed information about the spatial structure of the ion micro-beams emitted from PF discharges was obtained from measurements performed in the PF-1000 facility [77]. It was observed that the accelerated primary deuterons (not-lost in the fusion reactions), some impurity ions, as well as fusion-produced protons, showed a distinct anisotropy which was explained by a stochastic influence of local magnetic fields. Characteristics of 
the ion beams in the PF-1000 experiment were investigated also during the operation at the full energy [78], taking into consideration their possible technological applications.

Later on, particular attention was focused on detailed studies of the fusion-produced protons [79]. Using several pinhole cameras, equipped with filtered nuclear track detectors and fixed upon a semi-circular support (at a radial distance of $44 \mathrm{~cm}$ from the electrode outlet center), it was possible to determine the angular distribution of the fusion-protons, and (from an analysis of the track diameters) to estimate the proton energy spectrum. It was found that the angular distribution was peaked at the $z$-axis, and the energy spectrum extended from about 3.2 MeV to about 4.5 MeV. Those high-energy protons were evidently produced in the $\mathrm{d}-\mathrm{d}$ fusion reactions, probably by a beam-target mechanism.

Further studies concerned applications of the intense plasma-ion streams from PFdischarges for material engineering [80]. The use was made of the fast primary protons accelerated during PF-discharges with the hydrogen filling. Measurements with nuclear track detectors showed that the ion flux amounted to $(0.6-2.0) \times 10^{5}$ ions $/ \mathrm{cm}^{2}$, and the observed irradiation damages in different austenite-steel samples corresponded to protons of energy $>100 \mathrm{keV}$. Some information about the ion emission was also gained during studies of the pinch dynamics and fusion-products emission in the PF-1000 facility [81], and during research on correlations of X-ray pulses with the electron-, ion-, and neutronemission from PF-discharges in a 50-kJ MAJA-PF device, as well as in the PF-360 and PF-1000 facilities [82]. The main conclusion was that the characteristics of the emitted ions depended on the operational conditions, and the emission was relatively reproducible in the macroscopic scale, but it was irreproducible in the microscopic scale.

Detailed studies of the plasma dynamics and investigation of the characteristics of the fast ions (and electrons) emission, as a function of the neutron emission parameters, were performed in the PF-1000 facility operated at 480-850 kJ [10]. Pinhole images of the ions (mainly deuterons) emitted along the $z$-axis were recorded and the angular distribution of the fast deuterons was measured. The emission of the fast ions (and electrons) was correlated with a release of energy stored in the magnetic field surrounding the pinch column. To explain the acceleration of charged particles, a model of local plasma-diodes was proposed which could be formed during the breaking of the pinch column and current filaments, as shown in Figure 7. It was concluded that the neutron yield depended on the time of the magnetic energy release, the confinement of a large population of the fast deuterons with energies ranging from $10 \mathrm{keV}$ to $100 \mathrm{keV}$, and the confinement time of the dense pinch column (constituting the hot plasma target).
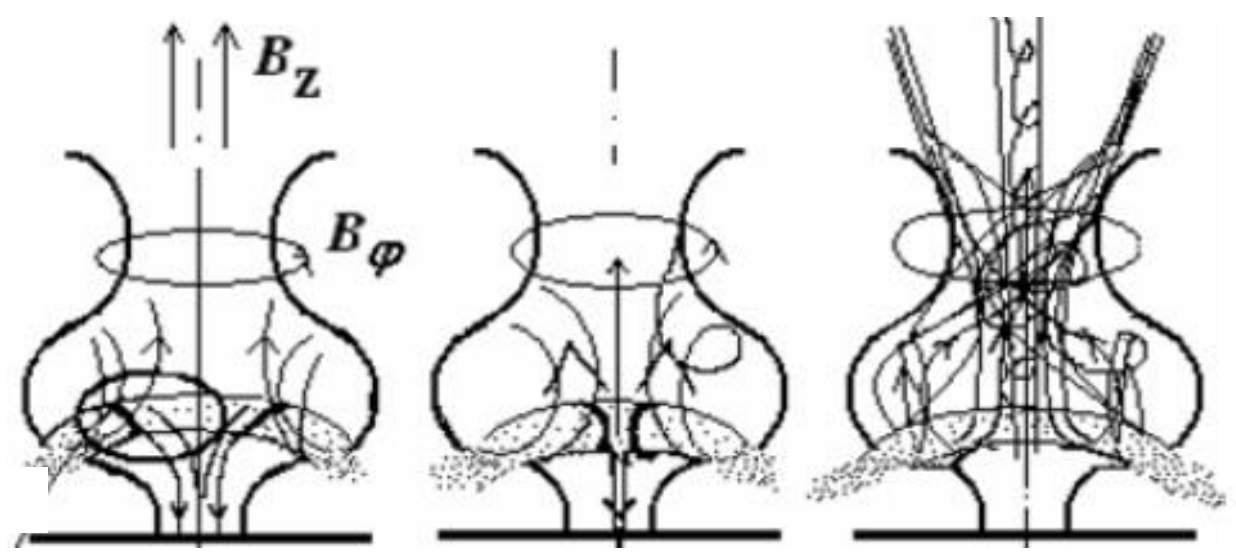

Figure 7. Successive phases of the evolution of a plasma-diode in the PF pinch column. Adapted from original data published in Figure 14, p. 53 of the preprint IC/2006/127 [4].

In the PF-1000 facility, there were also continued studies of pulsed plasma-ion streams during their free propagation, and during their interactions with carbon or tungsten targets, which were placed at a distance of $15 \mathrm{~cm}$ from the electrode outlets [83]. Optical emission spectra were recorded with a Mechelle ${ }^{\circledR} 900$ spectrometer operated with a chosen exposure 
(from $100 \mathrm{~ns}$ to $100 \mu \mathrm{s}$ ), at some delay in relation to the $\mathrm{dI} / \mathrm{dt}$ minimum. It made possible to estimate parameters of a free plasma-ion stream and those of plasma produced at the target surface.

Particular attention was, however, focused on the continuation of experimental studies of the fast fusion-produced protons in the PF-360 device and PF-1000 facility $[84,85]$. It was observed that in both the experiments, the fusion-proton emission was strongly peaked at the $z$-axis, but the azimuthal distribution (around the $z$-axis) had a distinct anisotropy, as shown in Figure 8.
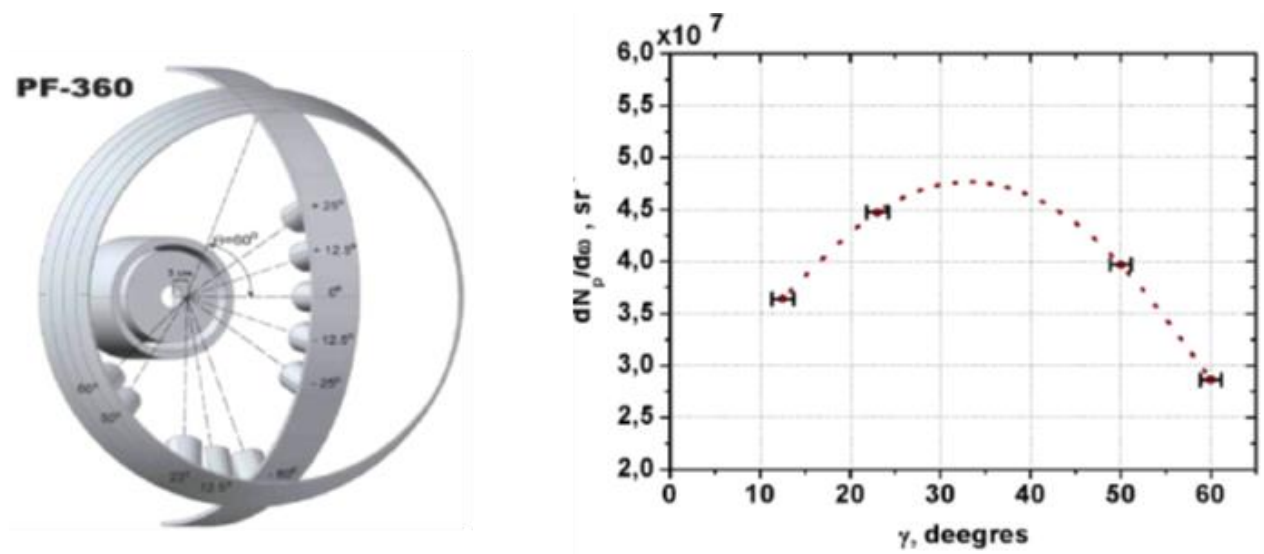

Figure 8. Arrangement of pinhole cameras used for fusion-proton studies in the PF-360 device, and a distribution of the measured protons as a function of the azimuthal angle $\gamma$ around the pinch axis. Reproduced from Figures 1 and 4b of [84], (AIP Conf. Proc. 2008, 993, 354-356) with the permission of AIP Publishing.

Simultaneously with the fusion-proton studies described above, detailed analysis was also performed of the neutron measurements in order to determine an energy spectrum of the fast deuterons involved in the fusion reactions [86]. In that study an energy spectrum of fusion-neutrons was determined from their ToF measurements (at different distances and angles). On the basis of the neutron energy-distribution functions it was possible to compute axial and radial components of the velocity of the fast deuterons responsible for the neutron production, as well as to estimate the total deuteron energy distribution, shown in Figure 9.

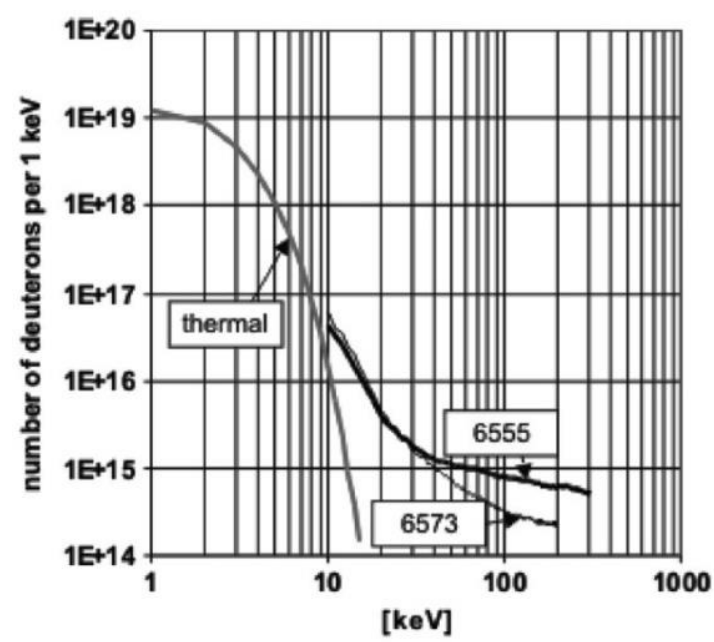

Figure 9. Energy distribution of the fast deuterons in the pinch column, as computed from neutron signals for two shots: \#6555 $\left(\mathrm{Y}_{\mathrm{n}}=2 \times 10^{11}\right)$ and \#6573 $\left(\mathrm{Y}_{\mathrm{n}}=4 \times 10^{10}\right)$, and the distribution of low-energy deuterons (calculated for an assumed $\mathrm{T}_{\mathrm{d}}=1 \mathrm{keV}$ ). Adapted from original data published in [86]. (C) 2021 IEEE. Reprinted, with permission, from IEEE Trans. Plasma Sci. 2008, 37, 83-87. 
Some progress was also achieved in the analysis of the motion of fusion-protons in filamentary PF discharges [87]. Different configurations of current filaments (central homogenous filament, parallel and funnel-like filaments) were considered and it was computed that in the most realistic funnel-like configuration some deuterons were accelerated not only in the downstream direction, but also in the upstream one. The modeling made it also possible to compute an azimuthal distribution of the fusion-protons, as shown in Figure 10.
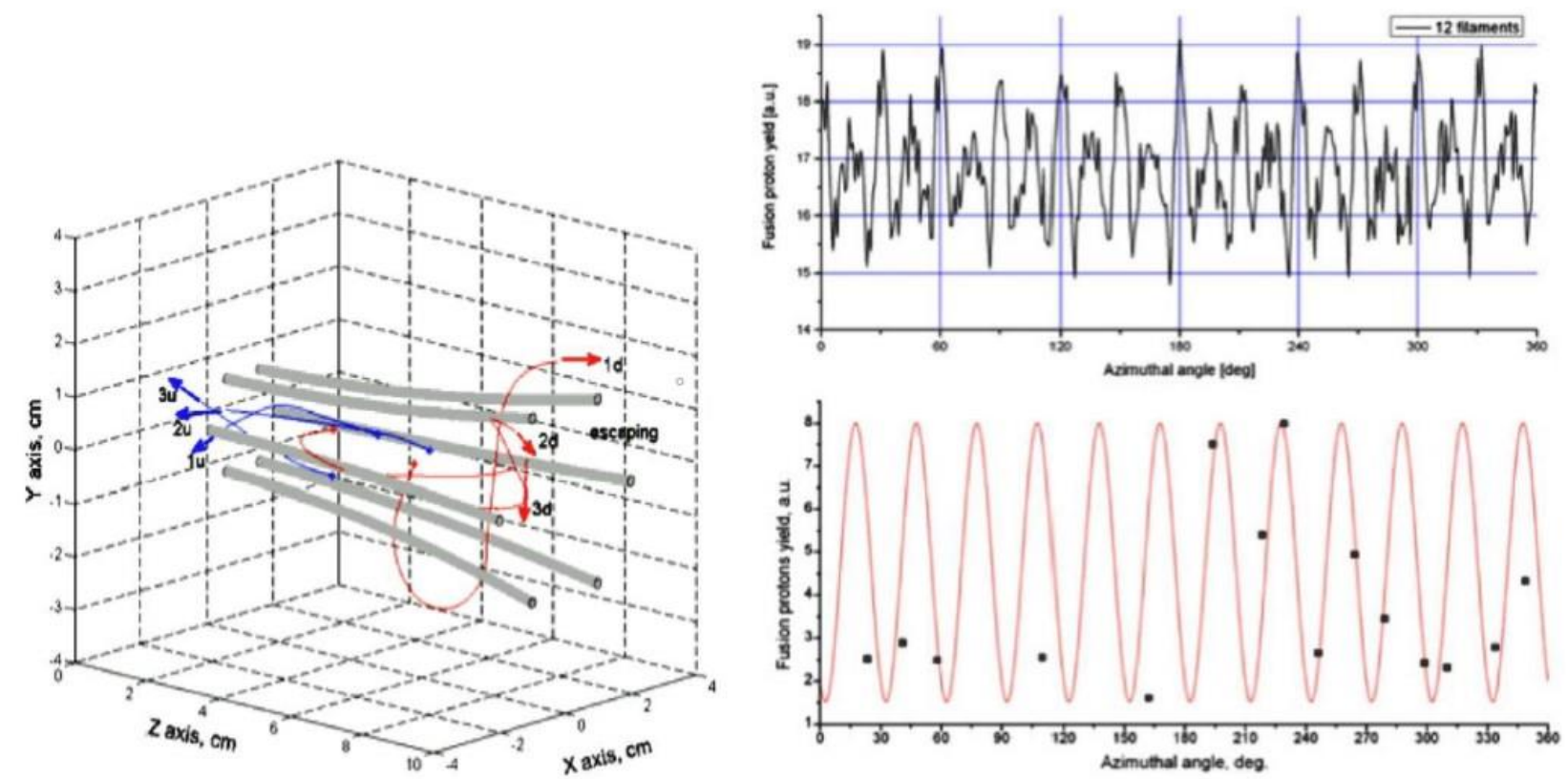

Figure 10. (Left diagram): Examples of trajectories of 3-MeV fusion-protons which were emitted from different points in a pinch column (containing 6 funnel-like filaments) and could escape in the down-stream direction (red lines) and in the up-stream direction (blue lines). Right diagram: (top) The azimuthal distribution of the fusion-protons emitted perpendicularly to the $z$-axis, which was computed for a pinch column containing 12 parallel filaments). (bottom) The PF-1000 experimental data (points) fitted by the periodical function (red sinusoidal line), which shows that in this case the data could be interpreted by an influence of 12 plasma-current filaments. Adapted from the original data reported in [87].

More detailed measurements of the fusion-protons in the PF-360 and PF-1000 facilities, which were performed by means of calibrated nuclear track detectors [88], confirmed that in both the experiments the fusion-protons were emitted mostly along the discharge axis, but considerable populations of them were also recorded at different angles. It was also confirmed that the fusion-protons had energies ranging from $3.1 \mathrm{MeV}$ to $4.5 \mathrm{MeV}$, which supported the hypothesis about the main role of the beam-target mechanism.

Studies of the fusion-produced protons were also performed at the Nanyang Technological University in Singapore, using a small 3-kJ PF device operated with ${ }^{3} \mathrm{He}-\mathrm{D}_{2}$ or ${ }^{4} \mathrm{He}-\mathrm{D}_{2}$ gas mixtures [89]. The fast protons from $\mathrm{D}\left({ }^{3} \mathrm{He}, \mathrm{p}\right)^{4} \mathrm{He}$ and $\mathrm{D}(\mathrm{d}, \mathrm{p})^{3} \mathrm{H}$ reactions were recorded with an axial pinhole camera. When the ${ }^{3} \mathrm{He}-\mathrm{D}_{2}$ gas mixture was applied there were recorded two groups of the fast protons of energies equal to about $3 \mathrm{MeV}$ and $15 \mathrm{MeV}$, simultaneously. It was observed that the proton yields from the considered reactions were similar, but their angular distributions were different. Monte Carlo computations showed that in both cases the fusion yields were produced by the beam-target mechanism.

To perform more accurate mass- and energy-analysis of the emitted ions it was necessary to improve diagnostic techniques. For this purpose a miniature Thomson-type spectrometer was constructed, and calibrations of new nuclear track detectors were performed [90]. Using the mastered diagnostics there were performed: more detailed studies of the ion emission from the PF-1000 facility [91], measurements of the ion (and electron) beams emitted in the downstream and upstream directions [92], measurements of the 
spatial- and energetic-characteristics of the ion beams in the PF-360 device [93], as well as time- and space-resolved measurements of the high-energy ion beams from different PF discharges [94]. The main results were more accurate data about the spatial- and energy-characteristics of the emitted ions, as shown in Figure 11.
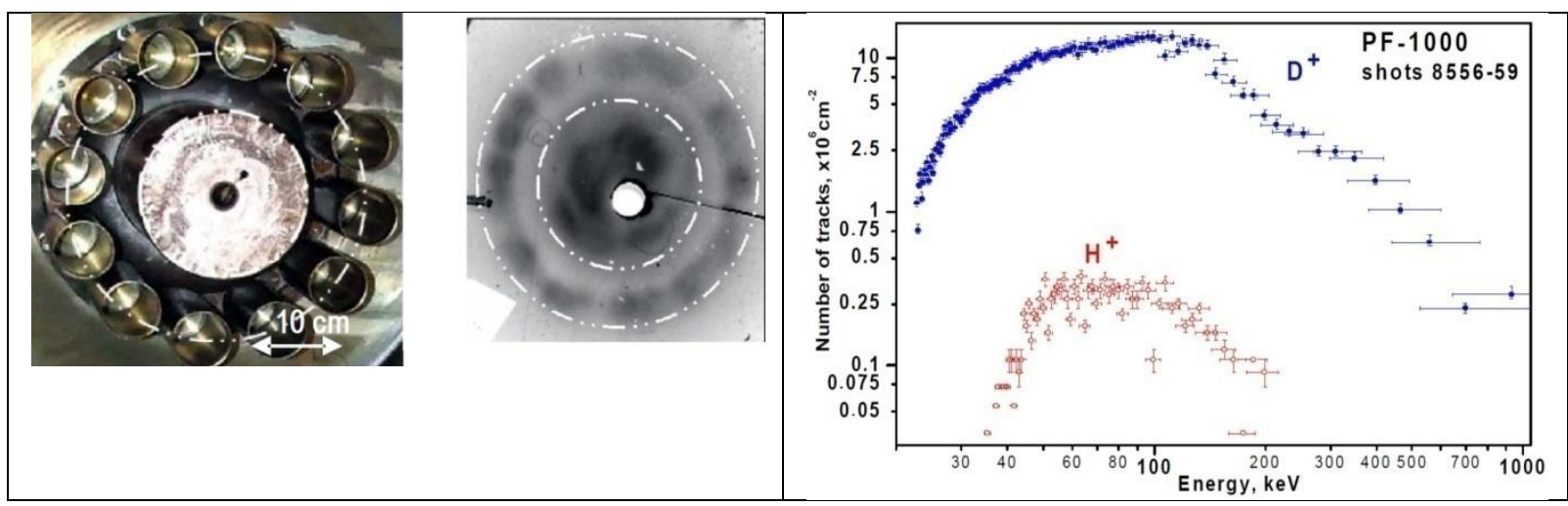

Figure 11. End-view of the modified PF-1000 electrodes, an ion pinhole image recorded for a 290-kJ shot, at a distance of $160 \mathrm{~cm}$ (with marked projections of the electrodes, and a hole used to observe ion micro-beams behind this detector), and energy distributions of the accelerated primary deuterons and protons. Adapted from original data reported in [91,93].

Another important result was the confirmation that at the modified PF electrodes, the emitted ion beams had also a complex spatial structure, composed of many micro-beams of energy exceeding even $700 \mathrm{keV}$. It was also confirmed experimentally that some ion beams were emitted in the upstream direction, but in that case the ion fluxes were about two orders of magnitude smaller. Similar features of the ion emission were also observed in the PF-360 device.

Some researchers suspected that the fast ions could be trapped inside internal plasma structures for a longer time, before being involved in the fusion reactions. It was estimated that in 1.2 MA PF discharges within the FF-1 facility some deuterons, which were trapped in plasmoids of 300-500 $\mu \mathrm{m}$ diameter and plasma density of about $3 \times 10^{19} \mathrm{~cm}^{-3}$, had a mean energy ranging up to $150 \mathrm{keV}$ [95]. This estimation was based on ICCD images of the plasmoids (recorded with a $30 \mu \mathrm{m}$ resolution) and the observed isotropy of the fusion-neutron emission.

New ion measurements in the modified PF-1000 facility were undertaken about three years later [96]. There were also performed new energy- and time-resolved measurements of the ion beams emitted from different PF discharges [97,98]. The use was made of a Thomson-type spectrometer, equipped with miniature scintillation detectors placed in chosen locations on the deuteron parabola. The most important result was the determination of time-intervals when the fast ions (recorded in different energy channels) were emitted, (Figure 12). 

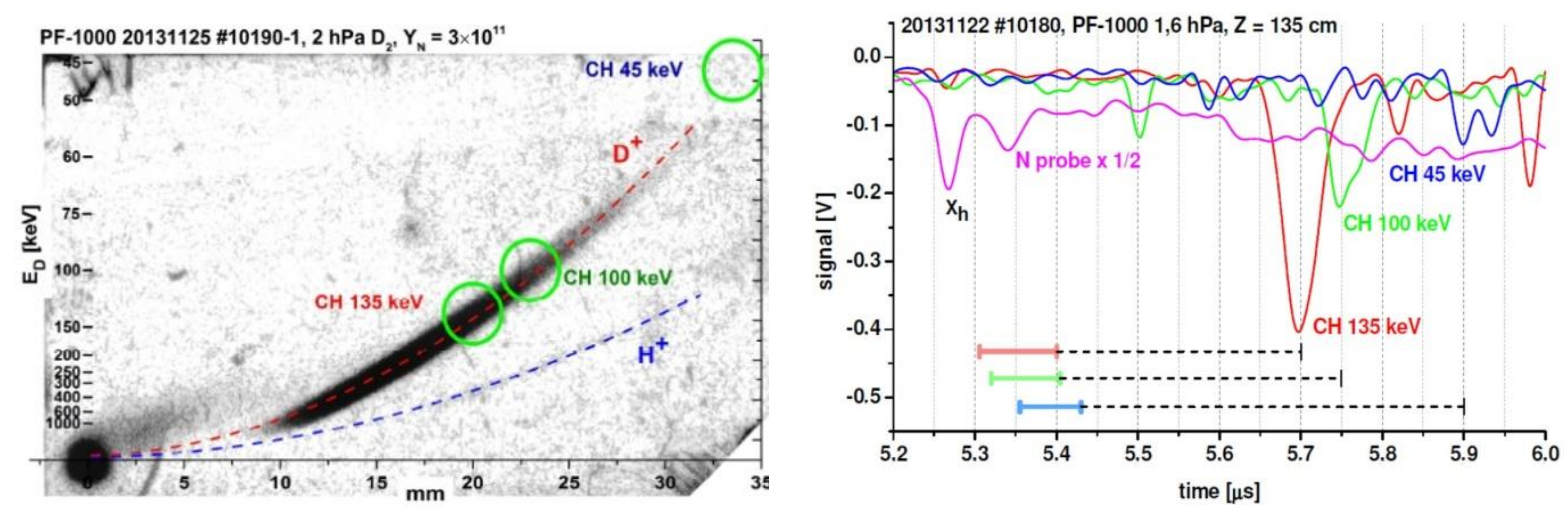

Figure 12. (Left diagram) Time-integrated Thomson parabolas of the accelerated primary deuterons and protons, which were recorded for a 335-kJ shot in the PF-1000 facility by means of a Thomson mass-spectrometer with an input diaphragm located at the $z$-axis, at a distance of $125 \mathrm{~cm}$ from the electrode ends. The green circles marked the positions of miniature scintillation detectors located in the image plane $(10 \mathrm{~cm}$ behind the input diaphragm), which were used for time-resolved measurements. (Right diagram) Time-resolved signals for such a shot: characteristic $X_{h}$ and neutron pulses with remarkable time shifts in relation to the discharge current beginning (violet line) and distinct deuteron pulses recorded in different energy channels (red-about $135 \mathrm{keV}$, green—about $100 \mathrm{keV}$, blue—about $45 \mathrm{keV}$ ). The thick color lines at the bottom show possible time intervals of the observed ion emission. Adapted from original data reported in [97].

At the same time there were carried out new studies of anisotropy of fusion-produced protons (and neutrons) emitted from PF discharges [99]. The use was made of a new "sandwich" detector consisting of an Al-absorber and two PM-351 foils, separated by a polyethylene-plate which converted the fast neutrons into recoil-protons. That detector made it possible to record the fusion-protons in the first PM-351 foil and the fusion-neutrons (through the recoil protons) in the second PM-351 foil. Using several "sandwich" detectors, it was possible to investigate the anisotropy of the fusion-products emission.

An important issue of research on the ion emission from PF discharges was the question concerning a correlation of that emission with filamentation phenomena. Detailed studies of the formation of plasma-current filaments in the column were performed within the PF-1000 facility [100]. In addition to parallel and/or funnel filaments (described above), in shots with an additional gas (deuterium or hydrogen) puffing there were observed many short filaments oriented in the radial or quasi-radial directions. It should be mentioned that the tiny plasma-current filaments were not visible on the laser-interferometer frames, but they were very distinct on the pinhole XUV-frames recorded with a 4-frame microchannel plate, which detected photons of energy $>30 \mathrm{eV}$ without any filter. It was deduced that magneto-hydro-dynamic instabilities of individual filaments could lead to the formation of plasma micro-diodes accelerating some ions (and electrons in different directions), while local magnetic fields generated around other current filaments could deflect trajectories of the acceleratedcharged particles. This effect was particularly connected with the main quasi-axial filaments, and could be neglected for many tiny filaments.

Successive studies concerned the characterization of fast deuterons involved in the production of fusion neutrons within the PF-1000 facility operated at 250-kJ level [101]. In that experiment the use was made of the laser interferometer and a set of ion pinhole cameras, equipped with filtered nuclear track detectors, and oriented at different angles to the $z$-axis. The ion pinhole images recorded along and at small angles to the $z$-axis (similar to those presented in Figure 1) showed a central spot of tracks and characteristic ring-shaped track regions, but the images obtained at larger angles did not reveal such ring-shaped forms, as shown in Figure 13. 


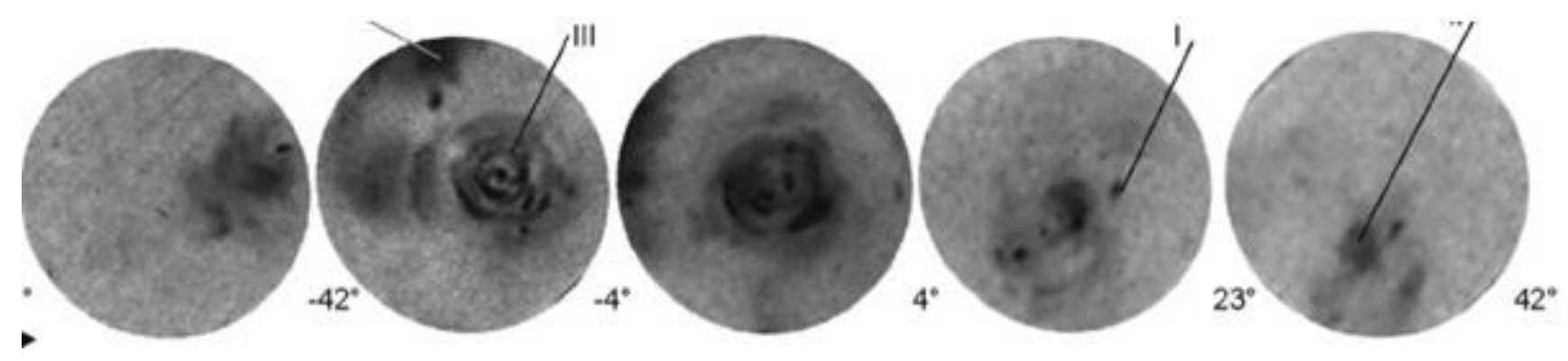

Figure 13. Ion pinhole images recorded at different angles in the recent PF-1000 experiments. Adapted from original data reported in [101] (Phys. Plasmas 2018, 25, 012712), with the permission of AIP Publishing.

From an analysis of the recorded ion images and the interferometer frames (described elsewhere) it was deduced that the fast deuteron beams $(>220 \mathrm{keV})$ were emitted from two regions. The central part of the pinch column (probably the inner plasmoids) emitted the fast deuterons at different angles (up to about $60^{\circ}$ ) and produced the distinct track spots. The external plasma layer (which contained some lobules) emitted the fast deuterons in a form of a quasi-cylindrical stream and produced non-uniform ring-shaped images.

Different PF-1000 experiments were reviewed, and an influence of plasma-current filaments on the emission of the fast ion beams was discussed in an invited paper [102]. It was confirmed that the fast deuteron beams were generated in different local sources located inside and in close surrounding of the pinch column. The main current filaments, which generated strong local magnetic fields, could strongly change the deuteron trajectories.

The evolution of a pinch column during the acceleration of deuterons (and electrons) in PF discharges was also subject of another study [103]. Using different diagnostic techniques in the PF-1000 facility, it was demonstrated that inside the pinch column there were formed different ordered plasma structures which contained local currents with poloidal and toroidal components and associated magnetic fields. The filamentary structure of current flows was taken into consideration to explain fast transformations of the magnetic energy in kinetic energies of the accelerated charged particles.

An evident progress was achieved in theoretical modeling of ion motions, and in particular the characteristics of ion beams generated in a small 2-kJ PF device, which was operated with the neon and argon filling, were investigated [104]. It was shown that the results of computations performed on the basis of a Lee model code were very consistent with the measured values of ion speed and energy, ion flux, and ion fluence.

Features of the fast deuterons emitted from PF discharges were considered in another study [105]. In that case comparison of the collected ion pinhole images with the corresponding interferometric frames was supported by a detailed microscope analysis of the recorded deuteron tracks. This analysis also concerned a hypothetical distribution of the local current flows inside and outside the dense pinch column. It was confirmed that the fast deuterons producing the central spot were evidently emitted from the internal plasma structures (e.g., plasmoids), while the deuterons producing ring-shaped images were probably produced in separate local sources (e.g., plasma lobules) formed in the external plasma layer. This study was completed by estimations of the azimuthal magnetic fields in the chosen cross-section of the pinch column.

In order to explain what the mechanisms of the energy transfer and acceleration of the charged particles during a PF discharge were, numerous interferometric images of pinch dynamics were analyzed, and a scenario of a magnetic dynamo and magnetic reconnections was considered in details [106]. It was deduced that energy stored in ordered plasma structures, which were formed in the pinch during several hundred nanoseconds, could finally be released during the fast decay of these structures (in the form of the kinetic energy of ion and electron beams).

The recent studies, connected with the problem of the fast ion (and electron) emission, concerned characteristics of some closed currents and magnetic fields formed outside the dense pinch column [107]. A detailed analysis of the recorded interferometric images and 
XUV frames, which was supported by estimations of pressure values in different plasma regions, confirmed the role of closed currents and the appearance of local sources in the near surrounding of the pinch column. The recent achievements of plasma studies within the PF-1000 facility were also reviewed in an invited talk given at the international conference PLASMA-2019 and in a paper [108]. In particular there were discussed characteristics of fast deuteron emission their relation with the operational conditions, as well as the evolution of the pinch column during the emission of charged particle beams. The characteristics of the fast deuteron sources have recently been described in a new paper [109].

Summary and Conclusions of Research on the Fast Ion Emission

The most important results of research on the ion emission from PF discharges, which have been obtained during recent 20 years, can be summarized as follows:

1. Many new data about the emission of fast ions from PF discharges have been collected in different experiments of energy ranging up to $1 \mathrm{MJ}$, and some characteristics concerning this emission have been verified, e.g., information about ion micro-beams and their bunches, information about a dependence of ion energy spectra on the operational conditions, the range of ion energies, etc.

2. Contrary to statements in some early papers, it has been shown that plasma-current filaments (and their magnetic fields), which probably exist in each PF discharge, can strongly influence trajectories of the emitted ions (and electrons).

3. Completely new results have been obtained from measurements of fast fusionproduced protons. It has been shown that such protons are emitted not only along the $z$-axis, but also at different angles, and their azimuthal distribution has usually a periodical character which can be explained by an influence of magnetic fields generated by the main current filaments. Energies of these protons, which extend from $3.2 \mathrm{MeV}$ to above $4.0 \mathrm{MeV}$, have confirmed that the $\mathrm{d}-\mathrm{d}$ fusion reactions are produced by energetic deuteron beams.

4. Valuable results have also been obtained from studies of interactions of pulsed plasmaion streams with different solid targets, e.g., materials of interest for fusion technology. An erosion caused by energetic ion beams and intense plasma streams has been investigated in various PF facilities, and at different conditions. In such studies, the chosen fast ions can reach the target first, and the main portion of energy is usually delivered later by a plasma stream, which does not penetrate the irradiated material very deeply $(<10 \mathrm{~nm})$, but can produce strong thermal effects (melting, evaporation) and form a shock wave penetrating through the sample volume.

5. Time-resolved measurements of the ion emission have shown that the most energetic ions (e.g., deuterons of energy $>1.3 \mathrm{MeV}$ ) can be emitted even above $10 \mathrm{~ns}$ before the current peculiarity (dI/dt dip), while lower energy ions (e.g., deuterons of energy about $600 \mathrm{keV}$ ) can be emitted even above $40 \mathrm{~ns}$ after that instant.

6. Ion measurements performed behind the main collector plate of the PF-1000 facility (through an axial channel in the anode) have provided the first experimental evidence that some ion beams can be emitted also in the upstream direction (although their population is about two orders of magnitude smaller).

7. A microscopic irreproducibility of the ion emission, as observed in many PF experiments, has confirmed a stochastic character of the formation of the fast ion (and electron) sources. It has also been confirmed that the most energetic ions (e.g., deuterons of energy exceeding $700 \mathrm{keV}$ ) are emitted from the pinch column (probably from individual plasmoids) and on the pinhole images they produce the central spot of tracks, while lower-energy ions are emitted from many local sources (probably from plasma lobules) formed in a plasma layer surrounding the pinch, and produce ring-shaped regions of tracks

In conclusion, it can be stated that the performed ion studies have already delivered valuable information about physical processes occurring in the PF discharges. The measurements of the fast ions escaping from PF discharges also shed some light on mechanisms of the acceleration of deuterons engaged in the fusion reactions. It can help in research on the 
optimization of fusion neutron yields. The detailed characteristics of the ion emission from a given PF facility are also of importance for its technological applications, e.g., in material engineering. Therefore, the ion studies should be continued, e.g., in order to collect more accurate data about correlations of the investigated ions emission with other phenomena in PF discharges.

\subsubsection{Filaments and Hot Spots in the Plasma Focus}

Bostick and coworkers [18,110] (and references therein) took the lead in advocating the idea that the fine structure of the plasma focus current sheath, consisting of filaments that form during its axial phase and hot spots in the pinch phase, plays a significant role in plasma focus physics. This has been a popular theme in plasma focus literature from its early days till today (see Section 2.3.1). This section looks at the experimental basis of this idea, theoretical models proposed and at various conjectures, hypotheses, analogies, or postulates that revolve around this experimental basis and are proposed to explain the observed features in ion emission studies reviewed in Section 2.3.1.

\section{(1) Existence and role of filaments}

Impressive image convertor photography, with a few thousand photographs, submillimeter spatial resolution, and less than 5 nanosecond exposure times revealed iconic pictures (see Figure 1 from [110]) showing bright filamentary structures distributed uniformly in the azimuthal direction in the radially imploding sheath (seen axially and obliquely), also in the pinch phase. The photographs in Figures 14 and 15 [111,112] illustrate the main points of Bostick's ideas summarized below:

(a) Filaments are stable structures embedded within the plasma current sheath, running mostly perpendicular to the azimuthal background magnetic field, but sometimes also in a spider-web fashion with azimuthal ribs connecting the radial spokes.

(b) They have a component of magnetic field along their axis, which has been detected [113] using magnetic probes in the axial phase. Neighboring filaments have opposite polarity of this magnetic field [113] showing that they occur in pairs, as shown in Figure 14 below.

(c) They have been referred to as vortex filaments [110,111]. However, literature describing experimental evidence showing helical topology of magnetic field, or mass flow inside filaments in plasma focus devices, has not been found.

(d) Based on the supposition that they are vortex structures containing mass flow and magnetic flux in opposite directions, their mutual annihilation is postulated to be responsible for the generation of micro-beams of energetic ions, which are recorded as clusters of tracks on solid-state nuclear track detectors (see Section 2.3.1). The main supporting evidences for this postulate are optical image convertor photographs (see Figures 14 and 15), and their correlation with the fusion neutron emission. 


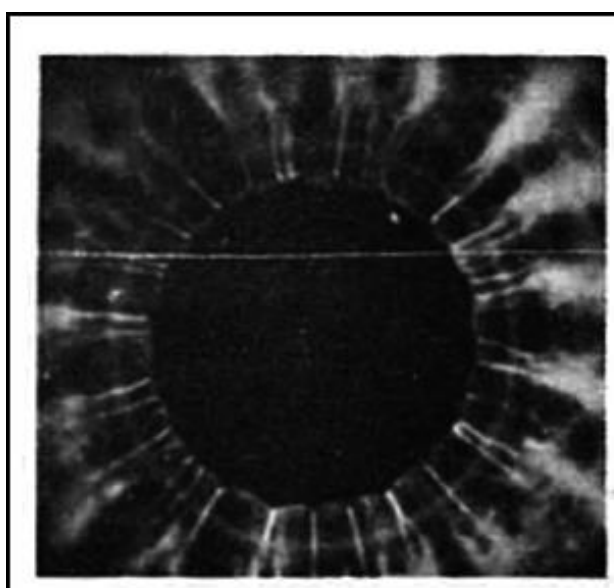

Fig. 1. $t=-250 \pm 20$ nsec

Current sheath is already orossing face

of center conductor. Note vortex pair combining at 5 o'clock.

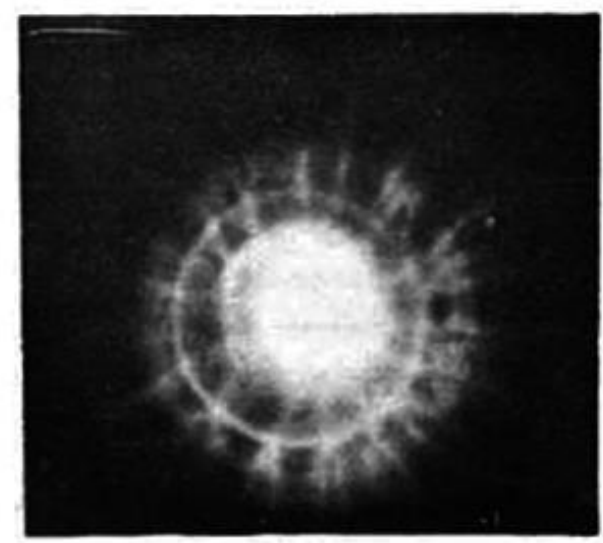

Fig. 3. $t=230 \pm 20$ nsec

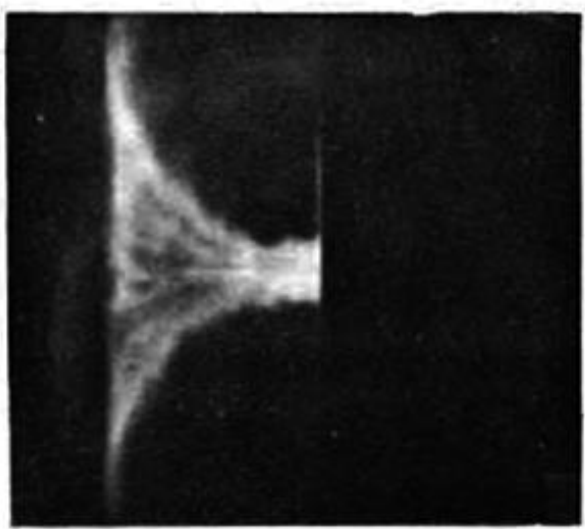

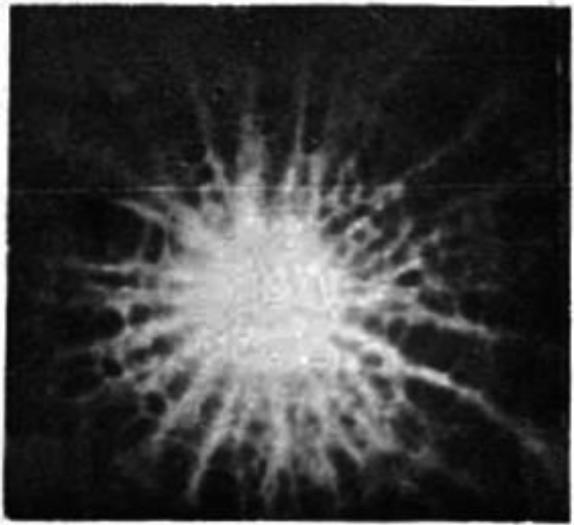

Fig. 2. $t=20 \pm 20$ nBec

"Plasna focus" has occured.

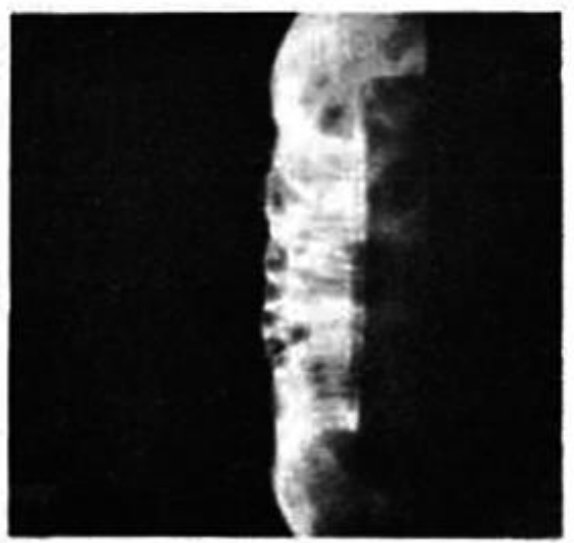

Fig. 4. $t=-250 \pm 20$ nsec

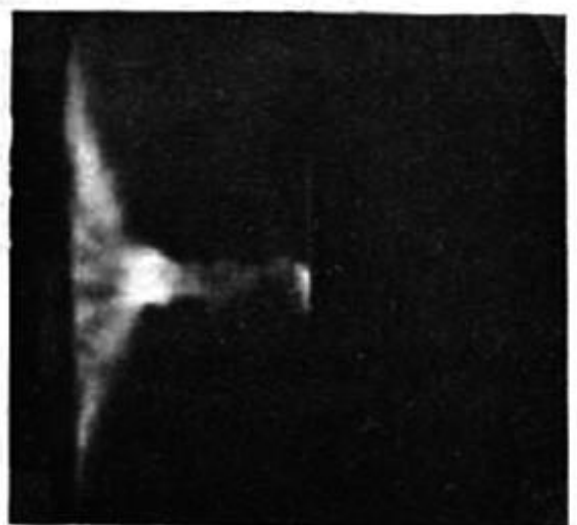

Figure 14. Supporting evidence for the ideas of vortex filaments occurringin pairs. They appear to lie in azimuthal corrugations of the plasma sheath. They are visible during the pinch formation and disruption [111]. The first figure caption mentions "Note vortex pair combining at 5 o'clock". 


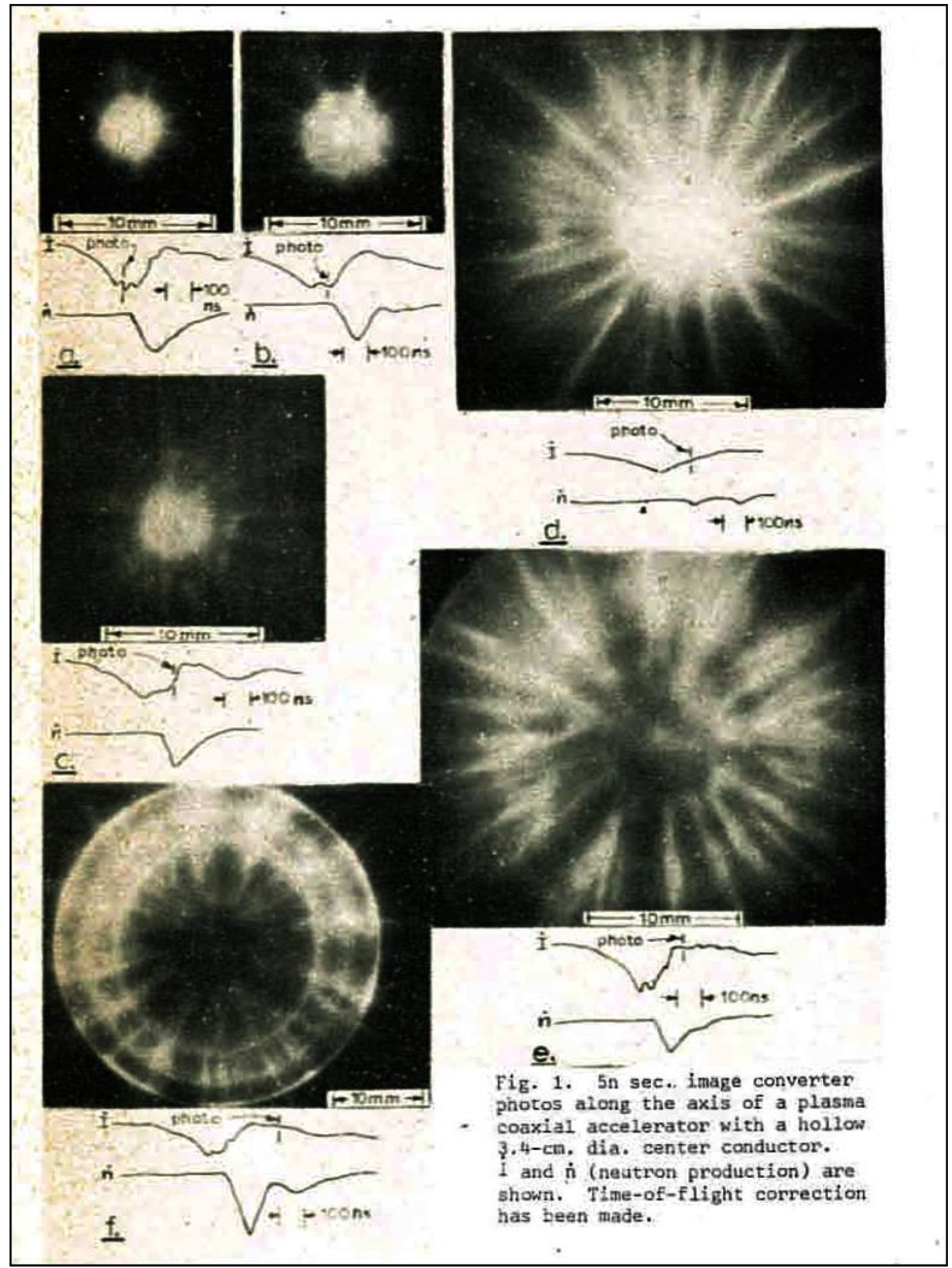

Figure 15. Evidence supporting the idea of annihilation of vortex filaments [112] and their relation with neutron production.

Revisiting their work, particularly related to observation of filaments by shadowgraphy and Schlieren techniques, detection of helical magnetic fields and mass flow in filaments is difficult due to the fact that a significant portion of their cited work is not available in an electronic form. 
Contrary to Bostick's hypothesis about the annihilation of filaments during the compression phase, numerous plasma focus experiments performed at the Stuttgart University $[114,115]$ proved that some filaments exist also during the maximum compression phase, as one can see in pictures obtained by means of a Schlieren technique and X-ray cameras (presented in Figure 16).

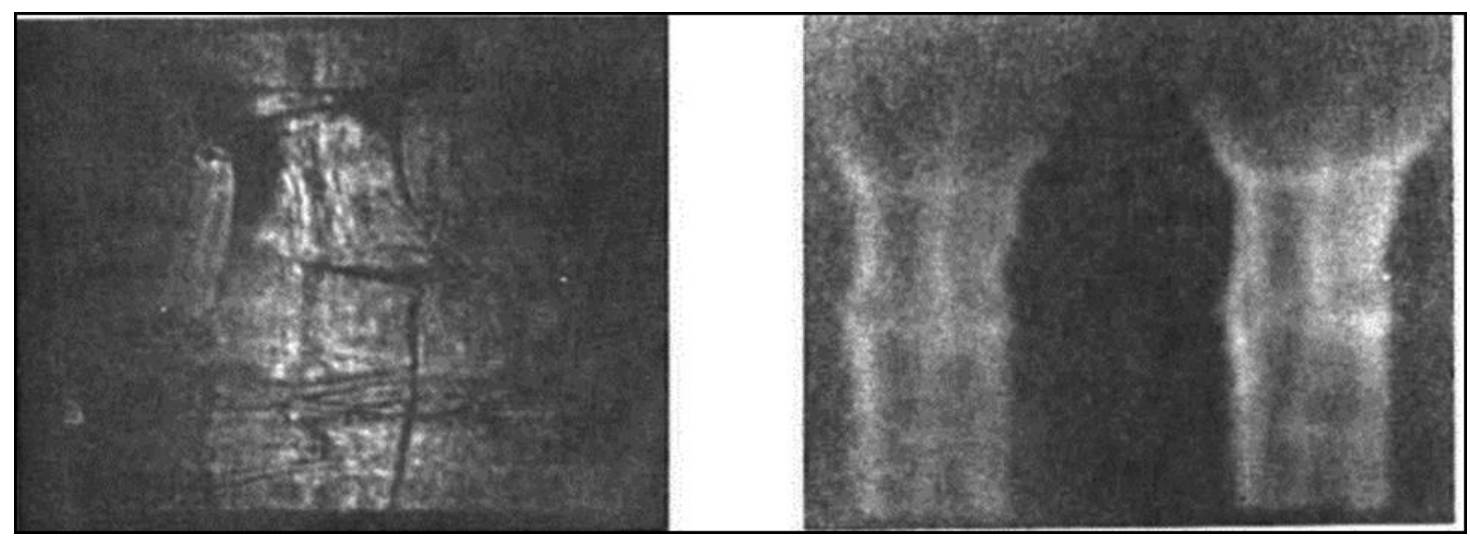

Figure 16. Example of a Schlieren picture taken in the POSEIDON facility at $t=+40 \mathrm{~ns}$ (left) and X-ray pictures recorded behind $25-\mu \mathrm{m}$ and 10- $\mu \mathrm{m}$ Be-filters at the maximum compression (right). Both the diagnostic techniques showed the central filaments in the pinch column. Reproduced from [114].

The existence of some central filaments in the pinch column was confirmed both by time-resolved and time-integrated pictures of soft $X$-rays, as one can see from a comparison of such measurements, presented in Figure 17.

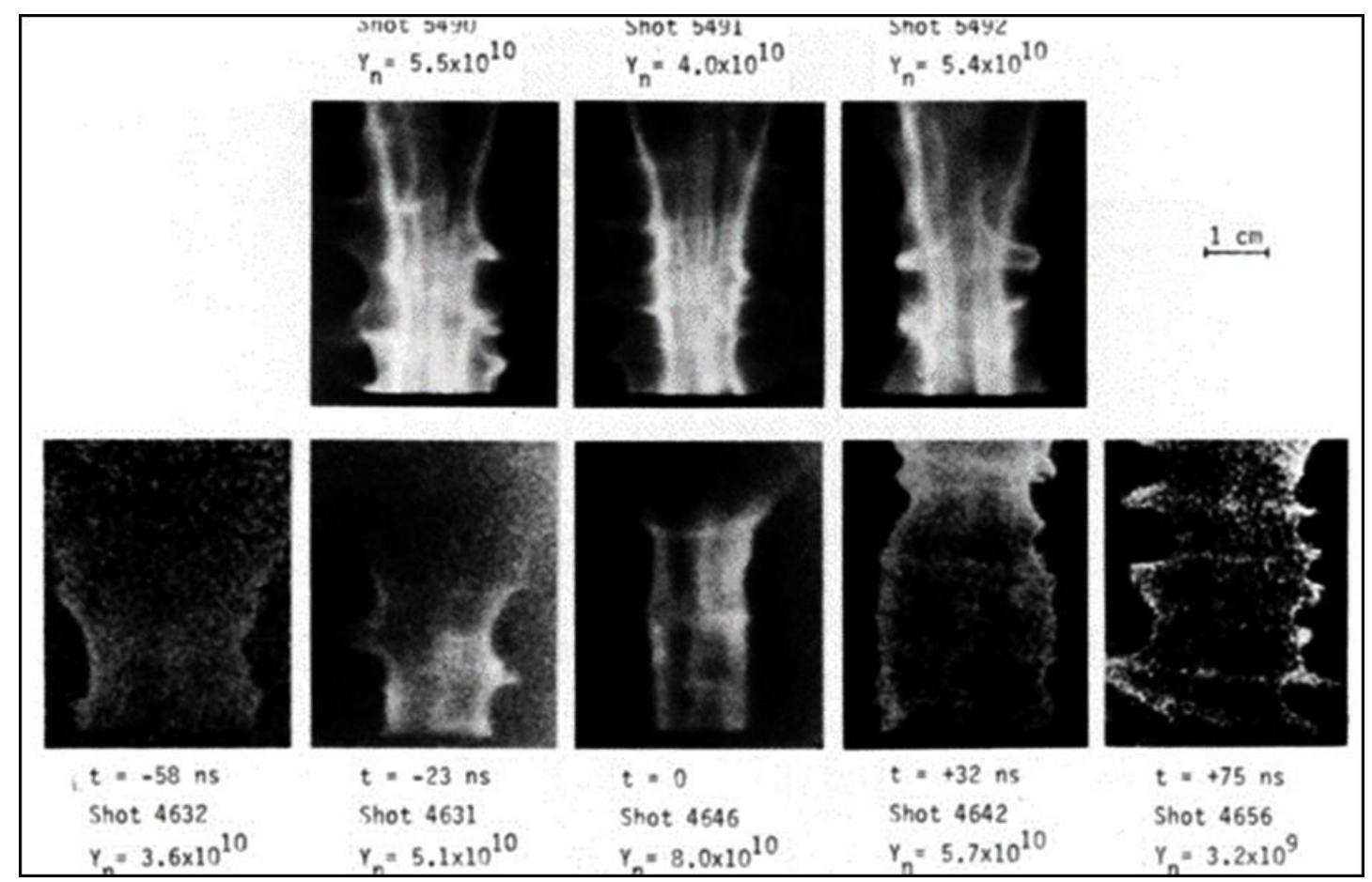

Figure 17. Comparison of time-integrated x-ray pinhole pictures (upper row) with time-resolved X-ray pictures recorded in the POSEIDON facility with gated intensifier images during different shots (lower row). The time-integrated pictures were probably a superposition of two x-ray flashes (emitted during the maximum compression and expansion phases). Reproduced with permission from Figure 3 of [115] (Phys. Lett. 1984, 105A, 117-123) OElsevier (1984). 
Filaments were detected in the Frascati 1 MJ plasma focus using a 3-frame shadowgraphy system by V. Ya. Nikulin of Lebedev Institute in 1980 based on a $100 \mathrm{~mJ}, 2$ ns ruby laser, with $18 \mathrm{~ns}$ inter-frame separation [116]. They became visible in the return current path above a critical pressure $\sim 12 \mathrm{mbar}$, well beyond the neutron optimized regime (see Figure 18). Digitally or photographically enhanced versions of individual frames from this image have been reported elsewhere (frame b in [117] and frame $c$ in [118]).

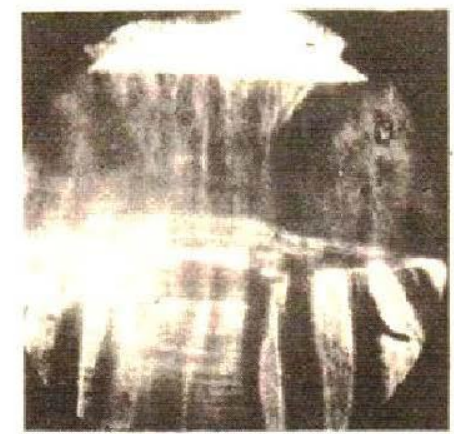

a
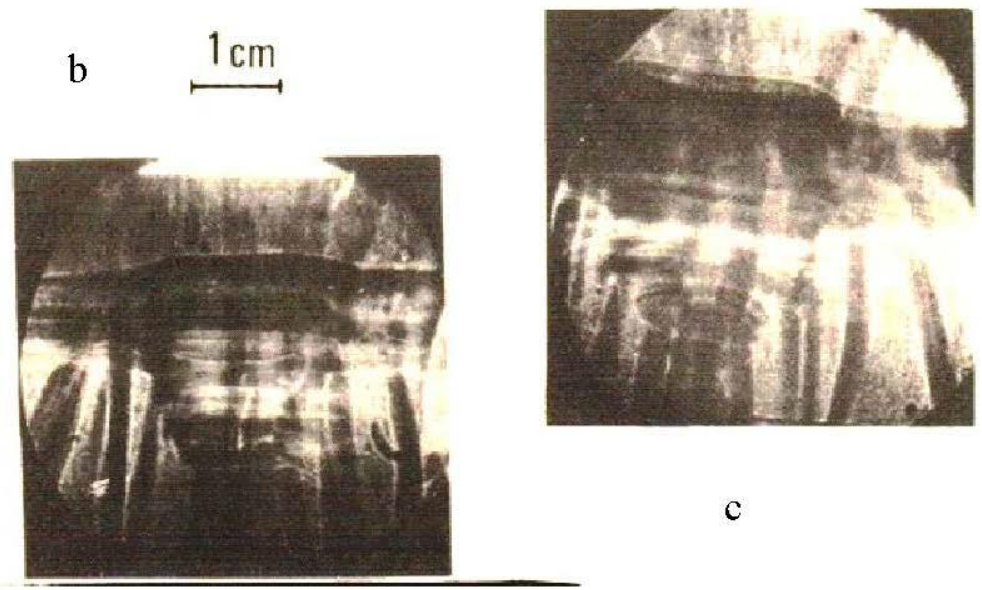

c

Figure 18. Sequence of 3 shadow frames showing filaments [116]. Image kind courtesy Dr. V. Ya Nikulin. (b) Reported in [117] (c) Reported in [118].

The Düsseldorf group [119] has published X-ray pinhole photographs that show filamentary structures similar to those seen in POSEIDON (Figure 17) but has not claimed them to be filaments.

There are many reports [114,120-123] on filamentary structures persisting in the radial phase of a plasma focus, as shown in visible radiation short-exposure photographs, besides those reported by the Bostick's group.

Filaments recorded in a deuterium plasma focus by means of laser interferometry were reported by two laboratories only $[120,124]$. The Chilean group at the Chilean Nuclear Energy Commission has reported [120] filaments seen in visible framing images, schlieren and interferometry in the small plasma focus PF-400J operating at $350 \mathrm{~J}$.

Interferometric evaluation [120] of the electron density of isolated filaments in the radial phase of a small plasma focus showed a peak density of about $1 \times 10^{25} \mathrm{~m}^{-3}$, varying on a spatial scale that matched the value of $\mathrm{c} / \omega_{\mathrm{pi}}=0.1 \mathrm{~mm}$.

Indirect evidence of the existence of filaments was found from azimuthally distributed discrete erosion marks left upon the anode surface after many plasma-focus shots [125]. Anisotropy of ion pinhole images has been interpreted by deflections produced by local magnetic field gradients associated with different filaments (see Section 2.3.1)

There is categorical mention of absence of filaments in interferograms obtained in the POSEIDON [42] and PF-1000 [126] facilities.

This is almost all of the direct experimental results on filaments observed during the normal operation of plasma focus and available in accessible literature till now.

Recently, filaments have been observed in XUV images recorded during a modified plasma focus operation on the PF-1000 facility $[100,127]$, when the plasma current sheath imploded on to a gas-puff injected axially and containing admixtures (in order to help visualization of the filaments). Some examples of XUV and interferometric images recorded at the PF-1000 facility with the deuterium filling and puffing are shown in Figure 19. 

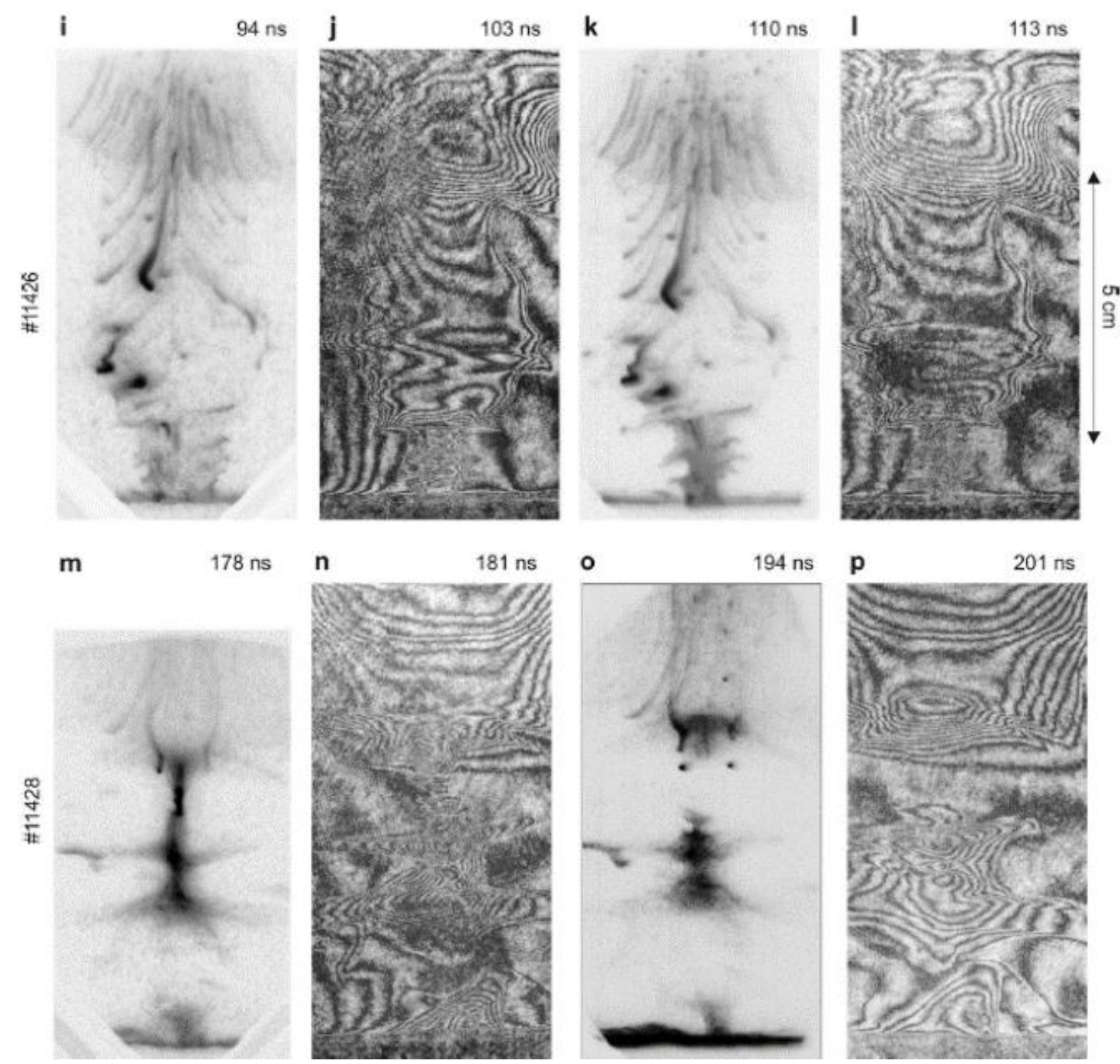

Figure 19. Comparison of XUV and interferometric pictures recorded between the first X-ray and neutron pulses (i-1) and during the second X-ray and neutron pulses $(\mathbf{m}-\mathbf{p})$ in the PF-1000 experiment in a gas puff mode of operation. Reproduced from Figure 4 of [100] (Phys. Plasmas 2017, 24, 032706) with the permission of AIP Publishing.

It should however be noted that it is not clear whether this mode of the operation can be directly compared with the normal plasma focus operation, since there were no reports of the same diagnostics exhibiting similar filaments during the normal PF-1000 operation [126] (see Section 5). Additionally, these filaments do not appear to be aligned with the current flow like the Bostick filaments as in the cases discussed above. So these filaments appear to be a different phenomenon than the other cases discussed above.

(2) Theoretical models of filaments

Similarity between filaments observed in the plasma focus and astrophysics [128,129] and interest in the emergence of the self-organizing structures in complex systems [130] have led to efforts in the understanding their physics from a theoretical perspective. This section reviews the theoretical work on filaments in the context of a plasma focus.

(a) H. Alfven [131] assumed a force-free magnetic field and then concluded that it had to be confined to a finite radius. In this model the total current carried by a filament varies as cube-root of the axial electric field.

(b) Furth, Killeen, and Rosenbluth [132] showed that in magnetized plasma regions where magnetic field changes direction ("current sheets with magnetic shear"), finite resistivity can lead to "bunching" of a current element leading to current filamentation. 
(c) V. Nardi [133] constructed a solution of the Vlasov equation, treating energy and components of the canonical momentum along the filament as constants of motion, and got explicit expressions for the electric and magnetic potentials. He also estimated a magnetic field along the filament axis and a flow approximately collinear with the magnetic field lines.

(d) G. Marklund [134] described a model for filamentary plasma structures associated with force-free magnetic fields. In this model, a homogenous electric field parallel to the symmetry axis of the magnetic field creates a radially directed electric drift velocity $\overrightarrow{\mathrm{E}} \times \overrightarrow{\mathrm{B}} / \mathrm{B}^{2}$. Recombination loss prevents accumulation of plasma in the central region, with the resulting neutrals streaming out across the magnetic field lines.

(e) Auluck [135] suggested a model of the filament formation specifically for plasma focus discharges. In this model, a plasma sheath undergoes gravitational instability in the rundown phase with a wave-vector parallel to the ambient azimuthal magnetic field, creating an azimuthal modulation of density (visible in Figure 14). Efficient electron thermal conduction parallel to the azimuthal magnetic field would ensure that azimuthal gradients of the temperature would be smoothed out. The pressure gradients along the direction of propagation of a sheath and density modulation in the azimuthal direction create the source term $\vec{\nabla} \mathrm{p}_{\mathrm{e}} \times \vec{\nabla} \mathrm{n} / \mathrm{en}^{2}$ for a magnetic field, as well as a fluid vorticity with alternating signs in the azimuthal direction.

(f) Ferro-Fontan and Sicardi-Schifino [136] demonstrated the generation of kilogauss radial magnetic fields using the $\vec{\nabla} \mathrm{p}_{\mathrm{e}} \times \vec{\nabla} \mathrm{n} / \mathrm{en}^{2}$ source term, but invoked an electrothermal instability that creates azimuthal modulation of temperature, but not of density.

(g) Haines and Marsh [137] showed that the Ohmic heating of electrons by a current parallel to a magnetic field leads to spatial oscillations in the electron temperature, number density, and current, with a scale length of $\sim \mathrm{c} / \omega_{\text {pe }}$ (microscopic filamentation).

(h) Trubnikov [138] described the formation of current filaments of an electron beam within plasma, and also discussed other mechanisms.

(i) Di Vita [139] formulated a variational principle, which generalized several wellknown results, e.g., Ginzburg-Landau superconductor theory, as well as Taylor's and Turner's plasma relaxation theories. He derived qualitative conclusions concerning filaments based on conjectures concerning the radiative collapse.

(j) E.J. Lerner [140] suggested the minimum energy dissipation principle to explain filamentation phenomena. According to his opinion "A heated plasma moves into a cool plasma or neutral gas in a way that minimizes the total dissipation of energy. The two principal ways how energy is dissipated from the current sheath in a plasma focus device are through electrical resistance of the current moving through the sheath, and through hydrodynamic friction of the sheath moving through the background medium. Electrical resistance is minimized by an even distribution of current, but hydrodynamic friction is minimized by filamentation-the balance between the two processes determines if filamentation will occur.".

(k) Role of ionization processes in formation of filaments has been suggested in [123].

(l) A model based on London current of superconductivity has been proposed in [141].

The present status of filaments in plasma focus can be summarized as follows:

The reported experimental observations on normally operating plasma-focus devices:

- demonstrate the existence of filaments in the axial, radial, and pinch phases using luminosity of the visible radiation and soft $\mathrm{X}$-rays (many reports).

- show that the filaments are associated with density modulations recorded with Schlieren and shadowgraphy techniques and laser interferometry (4 reports).

- demonstrate that there exists a magnetic field component along the filament axis (1 report).

- $\quad$ show that their radial size is of the order of $c / \omega_{\mathrm{pi}}$ (1 report). 
- demonstrate that they are spatially located within azimuthal corrugations of the plasma sheath (1 report).

- $\quad$ show some time correlation between neutron emission signals and image convertor pictures of the explosive destruction of filaments (1 report).

Theoretical studies deal with various ways a filamentary structure can be conceptualized within certain plasma models, which can be quite dissimilar not only in their physical origin but also their scale length and appearance. Establishing a correspondence between these models and experimental results is still an open question.

The following conjectures, hypotheses, analogies, assumptions, or assertions sometimes made in literature are not supported by any complete experimental report, but they have been proposed to explain some features of data on the ion emission (see Section 2.3.1) or hot spots.

- Filaments in plasma focus are local concentrations of currents. Azimuthally distributed discrete erosion marks upon the anode faces [125] strongly suggest the formation of local current concentrations, but are not a direct evidence of current filaments (for example, they could be formed by arcing in the plasma afterglow).

- Filaments in plasma focus are local z-pinch Bennett equilibria. This apparently has its origin in a Russian language theory paper published in 1965 [142].

- Filaments in plasma focus undergo MHD instability and form virtual plasma diodes.

(3) Existence and properties of hot-spots observed in soft X-ray images

Soft X-ray pinhole photographs reported by Mather in his early paper [143] showed also sub-millimeter $(\sim 0.2 \mathrm{~mm})$ sized intense spots (hot-spots) embedded within a larger plasma region of 1.5-2.5 mm dimensions, as shown in Figure 20.

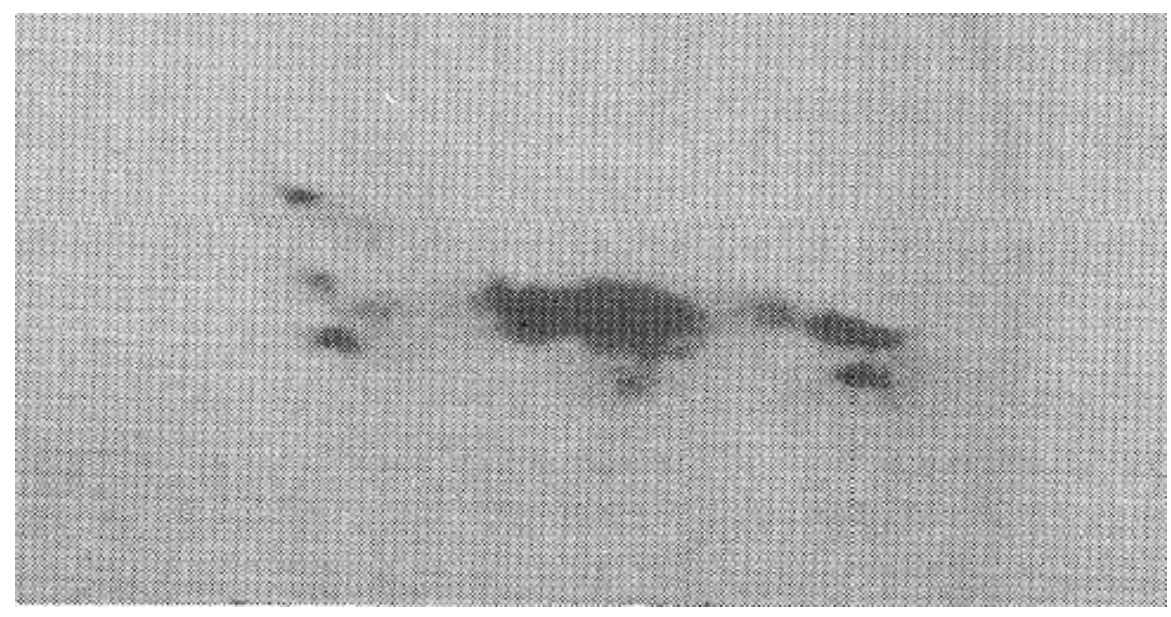

Figure 20. X-ray pinhole picture taken for a single PF shot at the (D2 $+1 \%$ Ar) filling, by means of a 0.16 -mm-diameter pinhole and 50- $\mu \mathrm{m}$-thick Be-filter. Micro-regions of an increased X-ray emission (hot-spots) are visible at and near the z-axis. Reproduced from Figure 14 of [18].

This section reviews subsequent investigations into their origin, nature, and properties. The central concern of these investigations is the relation of these structures, if any, with the $\mathrm{m}=0$ instability and filaments. A common feature of these investigations is a deliberate addition of some dopants to deuterium gas in order to enhance the $\mathrm{X}$-ray emission under the assumption that they do not affect the unknown mechanisms which might be responsible for the appearance of these features. However, there are also reports of such structures from Filippov type devices (see (d) below) under certain conditions, where these structures were formed in pure deuterium shots and the radiation from these structures was identified as line radiation from $\mathrm{Cu} X X$ lines from impurities introduced into the pinch from the ablated copper anode. Such investigations have been continued from the earliest days 
of PF research till now, adding much data and conjecture but arriving at no conclusive statements about their role in the performance of PF operated with pure deuterium.

(a) Mather [143] suggested that the multiple hot-spots observed in filtered time-integrated X-ray pinhole pictures "resemble the $\mathrm{m}=0$ sausage-type instability of a pinched discharge".

(b) Bostick and coworkers $[110,144]$ reported the following observations:

- The neutron yield is not changed by argon doping of deuterium less than $3 \%$ (by pressure). The size and sharpness of the boundary of sub-millimeter features, as well as the texture of the softer $\mathrm{X}$-ray emitting region surrounding them, is not changed by the addition of $0.5 \% \mathrm{Ar}$, but it increases the $\mathrm{X}$-ray intensity by a factor $\sim 3$. This observation is interpreted as an indicator that the mechanism responsible for the generation of the hot spots remains the same with and without Ar doping [144].

- Fast X-ray scintillation detectors recorded as many peaks (of a temporal width $\sim 10 \mathrm{~ns})$ as the number of hot-spots [110].

- X-ray pinhole pictures showing a double column of hot-spots and multiple offaxis hot-spots at the same axial position are not consistent with the adiabatic compression and re-pinching scenario. This is taken to indicate that a singlechannel description of the pinch column may not be a correct one, and that the plasma column may consist of multiple parallel filamentary channels of current flow [144].

- Increasing the thickness of beryllium filters on pinholes decreased the linear dimensions of the recorded hot-spots, but their shapes remained substantially unchanged [110].

- With pinhole diameters equal to (13-25) $\mu \mathrm{m}$, the spatial fine structure of the recorded hot spots reached (50-100) $\mu \mathrm{m}$ (i.e., 20 times the grain size of the applied emulsion).

(c) K.H. Schönbach and his co-workers from Aachen [145] demonstrated some correlation between time-integrated pinhole pictures and signals from plastic scintillation detectors (both having identical filters). Since the number of spots and number of pulses is identical, and the width of the X-ray pulse is 5-10 ns (as expected for the lifetime of the $\mathrm{m}=0$ neck), they assert that "The observed spot structures are $\mathrm{m}=0$ instabilities..."

(d) A research report from Russia [146] mentions "sufficiently reproducible" observation of micropinches with pure deuterium filling (6 torr) with a special geometry where the insulator diameter was $55 \mathrm{~mm}$ and the anode diameter was $110 \mathrm{~mm}$ (see Section 7 (5)). This geometry produced "a number of successive pinches at intervals of fractions of microseconds". The authors explain, "At the time of the first pinching, as a result of contact with the hot and dense plasma, the central part of the anode surface explodes and the partially ionized copper plasma spreads along the pinch axis with a velocity of $2-3 \mathrm{~cm}-\mu \mathrm{s}^{-1}$. During the second (and subsequent) pinching a part of the discharge current flows through the region occupied by the metal plasma, which undergoes compression under radiative cooling conditions." The smallest dimension of the dark spots on the film was determined to be $\sim 10 \mu \mathrm{m}$. Spectral measurements with a mica spectrograph showed that the radiation from the recorded structures corresponded to $\mathrm{Cu}$ XX lines.

(e) Koshelev et al. [147,148] were the first to report deliberate addition of heavy impurities in a plasma focus for producing few micron sized micropinches. The best results were obtained with a $2 \%$ addition of xenon to deuterium. Using a pinhole camera, a sequence of bright "hot spots" (up to 10 or more) was detected, grouped mainly along the axis of the system. A $0.5 \mathrm{~mm}$, Be filter which cuts off the relatively soft component of radiation, showed that the "points" had a very sharp border and, most importantly, the size of all the "points" was the same and equal to twice the image of the pinhole aperture $(0.6 \mathrm{~mm})$. This indicated that the size of the radiating points was 
less than the pinhole. Since production of micron-sized holes with smooth edges is a rather complex technological procedure, and small diaphragms can also lead to a strong attenuation of the radiation flux, they designed a new kind of imaging camera with square pinholes having polished edges of size $0.5 \mathrm{~mm} \times 0.5 \mathrm{~mm}$ in Ta plates of $0.1 \mathrm{~mm}$ thickness. Using the penumbral imaging method that measures the blurring of the image of a sharp edge, they could determine micropinch sizes down to $10 \mu \mathrm{m}$. Figure 21 shows an example.

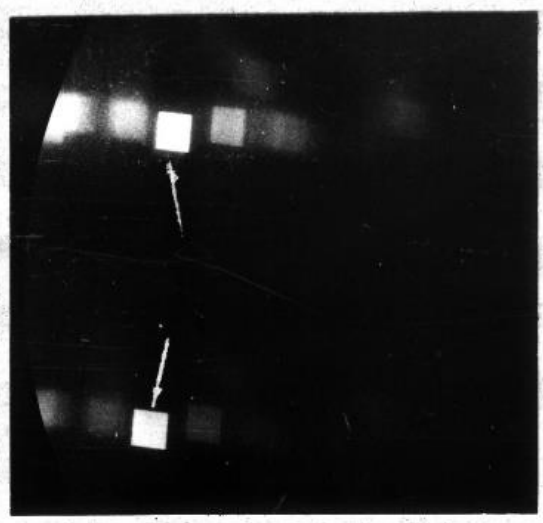

a)

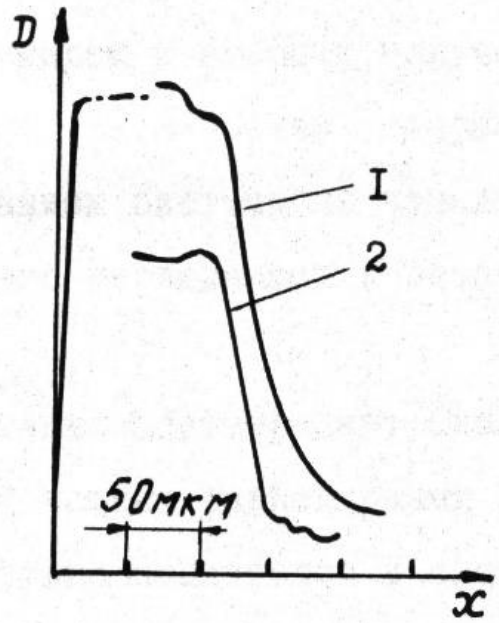

б)

Figure 21. (a) Pinhole images of micropinches obtained in a deuterium discharge with $2 \%$ xenon addition using a two-hole pinhole camera with square diaphragms and (b) a microphotogram of the image edge shown by the arrow. 1-0.5 mm Be filter, 2-1 mm Be filter. (from [149] in Russian) (OV I Krauz).

In some shots, both individual "spots" and their entire branches located at a considerable distance from the axis were observed, which indicated a complex distribution of the current in the pinch region (Figure 22). The typical diameter of a micropinch was 30-50 microns. In some cases, the diameter was close to the limit of the spatial resolution of the method (10 microns). In the longitudinal direction, the micropinches were more blurred, indicating either their axial movement or the existence of an aspect ratio of $\sim 3$.

a)

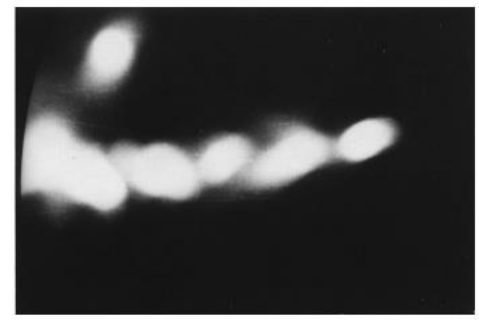

c)

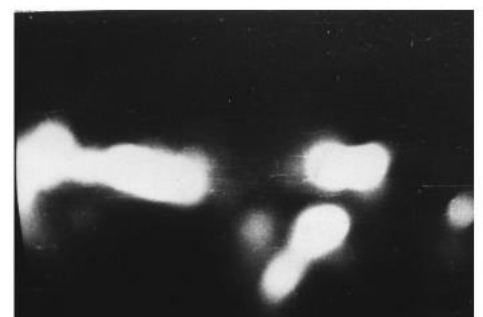

b)

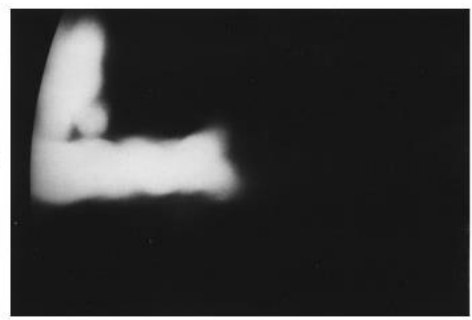

d)

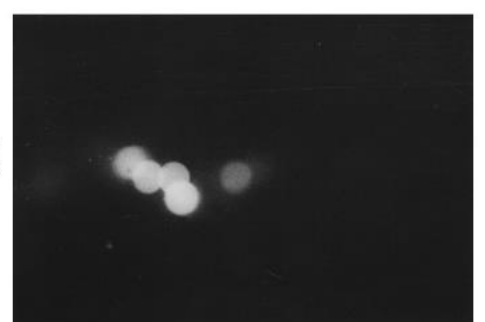

Figure 22. Pinhole images of micropinches in discharges with $2 \%$ xenon addition: (a)-(c) - Al filter 18 microns, (d)—Be filter $0.5 \mathrm{~mm}$. (from [149] in Russian (OV I Krauz). 
(f) Antsiferov et al. [150] determined density of micropinches from $\mathrm{Ne}-$ like ArXVII dielectronic satellites working with a mixture of $\mathrm{D}_{2}, \mathrm{Ar}$ and Xe. Electron density values for a 50 -micron micropinch were estimated as $\mathrm{n}_{\mathrm{e}} \sim 6 \times 10^{22} \mathrm{~cm}^{-3}$, corresponding to the ion density $\mathrm{n}_{\mathrm{i}} \sim 3 \times 10^{21} \mathrm{~cm}^{-3}$ along with electron temperature of $0.7-1 \mathrm{keV}$. Assuming the average ion mass of the plasma to be close to deuterium, the scale length $\mathrm{c} / \omega_{\mathrm{pi}} \sim 6 \mu \mathrm{m}$.

(g) P Antsiferov and the Stuttgart team [151] have also investigated the formation of hot-spots and emission characteristics of plasma focus discharges at various gas conditions.

(h) P. Choi and colleagues [152] reported studies on the dynamics of the hot spot formation in a $28 \mathrm{~kJ}, 60 \mathrm{kV}$, DPF78 plasma focus device at the Stuttgart University. The highlights of their report are as follows:

- They cited references indicating that reproducibility of hot-spots is improved by doping the $\mathrm{H}_{2}$ or $\mathrm{D}_{2}$ working gas with high- $\mathrm{Z}$ noble gases, but not with low- $\mathrm{Z}$ gases $\left(\mathrm{N}_{2}\right.$ or $\left.\mathrm{Ne}\right)$.

- The following diagnostic techniques were applied:

- Single- and double-frame bright-field Schlieren photography with a nitrogen laser pumped Rhodamine 6G dye laser, which generated about 1-ns pulses with 8-ns delay between the two frames.

- Silicon PIN diodes with collimators and a selection of X-ray absorption filters.

- The axial and radial optical streak photography to look at the implosion dynamics.

- A set of nine scintillator-photomultiplier detectors to observe the neutron emission.

- A soft X-ray streak camera, using two pinholes covered with different spectral filters and forming images on an NE102A thin plastic scintillator, which was viewed by an optical streak camera equipped with an image intensifier.

- An axially displaced radially resolved multi-pinhole (ADRRM) streak technique, which gave 2D image data with a continuous time evolution.

- A large slit axially displaced radially resolved multi-pinhole (LSADRRM) streak technique, which provided 2D image data with a continuous time evolution (with added redundancy for the detection of hot-spots).

- A simultaneous introduction of controlled amounts of $\mathrm{Ne}, \mathrm{Ar}$, and $\mathrm{Kr}$, to study hot-spot evolution in different spectral bands, using composite multimaterial X-ray filters.

- All diagnostics had time markers providing a few ns accuracy in time-correlations.

- Their findings related to hotspots were as follows:

- The first hot-spot occurs usually within $5 \mathrm{~ns}$ after the maximum compression and is followed by many others with few-ns intervals and at different axial locations.

- All hot-spots are embedded within a 3-mm-diameter grossly stable pinch column that lasts usually for $30 \mathrm{~ns}$.

- The positions of the observed hot-spots are determined with $\pm 1 \mathrm{~mm}$ accuracy, and their lifetimes are below $1 \mathrm{~ns}$.

- With the Ar doping, the recorded hotspots are about $1.5 \mathrm{~mm}$ in diameter and several $\mathrm{mm}$ in length.

- The size and shape of a hot-spot depends almost entirely on the spectral region, in which it is observed.

- Hot-spots are rarely recorded in Ne-doped discharges, although some Schlieren photographs show the necking and other local instabilities.

- The number of observable hot-spots usually increases slightly with $\mathrm{Z}$ and with the impurity fraction.

- There are no correlations between the number of hot-spots and the neutron yields. 
(i) H. Schmidt and co-workers [153] used two curved crystal spectrometers positioned side-on and end-on to study space-resolved X-ray spectra of hydrogen- and heliumlike argon plasma from micro-pinches in the DPF78 device (28 kJ, $60 \mathrm{kV}, 0.9 \mathrm{MA})$. Supporting diagnostics included a time-integrated filtered pinhole camera with an X-ray film, and a time-resolving filtered pinhole camera with a 10-channel PIN-diode array in its detection plane. A penumbral imaging technique was used to obtain the spatial dimensions of the hot-spots. Their main findings were as follows:

- Micro-pinches occur earlier near the anode, than at more distant positions.

- Usually two to four micro-pinches appear in a discharge during a time up to $50 \mathrm{~ns}$.

- Hot-spot durations of 200-400 ps were measured using an X-ray streak camera.

- A comparison of the observed and simulated spectral line profiles indicated a good fit for a temperature of $\sim 900 \mathrm{eV}$ and density $\sim 9 \times 10^{21} \mathrm{~cm}^{-3}$.

- A penumbral imaging method applied to space-resolved spectra indicated hotspots dimensions ranging from 150 to $370 \mu \mathrm{m}$.

- The measured Doppler shifts between lines of the adjacent micro-pinches ranged up to $8 \mathrm{~mA}$ and corresponded to axial velocities of about $6 \times 10^{5} \mathrm{~m} / \mathrm{s}$.

- The Doppler shifts (up to $4 \mathrm{~m} \AA$ ) from the radially observed spectra were always in a direction which corresponded to radially-outward moving micro-pinches.

(j) The Düsseldorf group $[119,154,155]$ investigated spatial-, temporal-, and spectralfeatures of micro-pinches in the SPEED-II facility with a 4-frame gated MCP camera and curved crystal spectrometer with a deuterium plasma focus collapsing on an argon cloud. They reported:

- “...necking is a necessary condition for micropinches to be actuated, but not a sufficient one for them to occur", "...only the brightest neckings result in micropinches, whereas diffuse ones often fail to produce them" [119].

- "There is no correlation between electron, ion (neutron) and X-ray production" [119].

- A comparison between the measured and simulated spectra indicated in the micro-pinches a density of $\sim 10^{29} \mathrm{~m}^{-3}$ and a temperature of about 1-1.5 keV [154].

(k) Hotspot formation was studied in a small $3.8 \mathrm{~kJ}$ plasma focus device operating in hydrogen-argon mixtures $(5 \%, 10 \%, 20 \% \mathrm{Ar})$, at pressures from below 0.2 torr upward at the Pontifical Catholic University of Chile [156]. A combination of multi-pinhole and slit-wire $\mathrm{X}$-ray photography was used to measure the size and temperature of the hotspots, over a range of pressure and gas mixing ratios. Filtered p-i-n diodes and a scintillator detector were used to investigate the time evolution of the hotspots. Typical size for the hottest emitting region, at temperatures between 200 and $400 \mathrm{eV}$, was found to be around $150 \mu \mathrm{m}$, with a typical duration of the order of $10 \mathrm{~ns}$. The temperature in the final phase of the time evolution of the hotspots reached values nearly twice those of the plasma column where they were formed. Calculations using three different complementary codes (XRAYFIL, RATION, and FLY) to analyze the X-ray emission, assuming full ionization of a $20 \%$ argon content plasma, indicated that a significant fraction of the radiation is emitted in the $3.2 \mathrm{keV}$ to $3.88 \mathrm{keV}$ region, corresponding to $\mathrm{K} \alpha$ emission from highly ionized argon [157]. In addition, the particle inventory in a column of the same diameter as the anode results in a maximum plasma line density of $9.5 \times 10^{16} \mathrm{~cm}^{-1}$. This value further supports the estimation of a plasma line density at maximum compression of around $10^{16} \mathrm{~cm}^{-1}$, i.e., of the order of one-tenth of the maximum attainable from the initial particle inventory in the cylindrical volume above the anode. With the lower estimated value of the plasma line density, the mean electron density in a $115 \mu \mathrm{m}$ diameter hotspot is estimated at $\sim 1.3 \times 10^{20} \mathrm{~cm}^{-3}$, if the dominant ionization state for the argon ions is taken to be $\mathrm{H}$ like, as suggested by a calculation with FLY, for a $300 \mathrm{eV}, 10^{20} \mathrm{~cm}^{-3}$ argon-hydrogen plasma [157]. 
(4) Existence and properties of super-dense plasma domains

Filaments, hotspots, and ion micro-beam sources discussed in Section 2.3.1 are examples of plasma micro-structures that reveal intrinsic azimuthal asymmetry in the plasma focus phenomenology. An additional aspect is revealed by investigations of the azimuthal asymmetry in nuclear reaction rates. These rates are interpreted to reveal the existence of "localized plasma domains of enhanced nuclear reactivity (PDER)" [158]. This section reviews reports that study these aspects.

(a) In a $7 \mathrm{~kJ}, 17 \mathrm{kV}$ plasma focus at Stevens Institute of Technology, Hoboken, USA, 15 thin walled Geiger-Muller (GM) counters were arranged inside the vacuum chamber in the space above the anode [158]. Their output was recorded with a computer. The radioactive decay curves were fitted to identify various half-lives, and hence the radioactive nuclides. The counters were covered with a metal foil ( $\mathrm{Al}$ of $0.254 \mathrm{~mm}$ in thickness or $\mathrm{Cu}$ of $0.127 \mathrm{~mm}$ in thickness). The plasma focus was operated with a pure $\mathrm{D}_{2}$ and a mixture $\mathrm{D}_{2}(90 \%)+\mathrm{O}_{2}(10 \%)$ or $\mathrm{D}_{2}(50 \%)+{ }^{3} \mathrm{He}(50 \%)$. The following nuclear reactions produced radioactivity:

- $\quad{ }^{27} \mathrm{Al}(\mathrm{d}, \mathrm{p})^{28} \mathrm{Al}\left(\mathrm{E}_{\text {thresh }} \sim 1.8 \mathrm{MeV}\right), \mathrm{T}_{1 / 2} \sim 2.4 \mathrm{~min}$ (by $\mathrm{D}^{+}$beams from plasma with $\mathrm{E}>\mathrm{E}_{\text {thresh}}$ ).

- ${ }^{65} \mathrm{Cu}(\mathrm{d}, \mathrm{p})^{66} \mathrm{Cu}\left(\mathrm{E}_{\text {thresh }} \sim 2 \mathrm{MeV}\right), \mathrm{T}_{1 / 2} \sim 5.1 \mathrm{~min}$ (by $\mathrm{D}^{+}$beams from plasma with $\left.\mathrm{E}>\mathrm{E}_{\text {thresh }}\right)$.

- ${ }^{16} \mathrm{O}(\mathrm{d}, \mathrm{n})^{17} \mathrm{~F}\left(\mathrm{E}_{\text {thresh }} \sim 2 \mathrm{MeV}\right), \mathrm{T}_{1 / 2} \sim 1.1 \mathrm{~min}$ (when ${ }^{17} \mathrm{~F}$ is implanted in a $\mathrm{Cu}$ foil).

- $\quad{ }^{63} \mathrm{Cu}(\mathrm{p}, \mathrm{n})^{63} \mathrm{Zn}\left(\mathrm{E}_{\text {thresh }} \sim 5 \mathrm{MeV}\right), \mathrm{T}_{1 / 2} \sim 38.1 \mathrm{~min}$. This reaction would be caused by $14.7 \mathrm{MeV}$ protons from the ${ }^{3} \mathrm{He}(\mathrm{d}, \mathrm{p})^{4} \mathrm{He}$ fusion reaction with a ${ }^{3} \mathrm{He}$ admixture.

- The observed azimuthal asymmetry in the production of radioactivity was consistent with localized PDERs displaced by $(3-5) \mathrm{cm}$ from the axis of symmetry.

- A comparison of radioactivity produced by $\mathrm{D}^{+}$beams in ${ }^{16} \mathrm{O}(\mathrm{d}, \mathrm{n}){ }^{17} \mathrm{~F}$ reactions to the total radioactivity from the same PDER enabled estimation of a lower bound on the density of the PDER, which was $\sim 7 \times 10^{21} \mathrm{~cm}^{-3}$.

(b) A re-analysis of the neutron activation and nuclear emulsion data from Frascati [159] has led to an estimation of the triton burnup fraction $\mathrm{R}$ from pure deuterium discharges. This fraction was defined as the ratio of space and time integrated yields of 14.1-MeV and 2.45-MeV neutrons. The $14-\mathrm{MeV}$ neutrons arise from $\mathrm{D}(\mathrm{t}, \mathrm{n})^{4} \mathrm{He}$ fusion reactions between 1-MeV tritons produced in the $\mathrm{D}(\mathrm{d}, \mathrm{p}) \mathrm{T}$ reaction and deuterium plasma. The cross-section for the $\mathrm{D}(\mathrm{t}, \mathrm{n})^{4} \mathrm{He}$ fusion reaction has a resonance peak at $160 \mathrm{keV}$, which is 13 times higher than that at the initial energy $(1.1 \mathrm{MeV})$ of the triton. The plasma density and temperature in the immediate vicinity of the point of origin of the triton play a crucial role in defining the slowing down environment of the triton and directly affect the triton burnup ratio $\mathrm{R}$. A detailed description of the definition, measurement, error analysis, computer simulation, and theoretical modeling of the triton burnup ratio led to the following conclusions:

- For the Frascati Plasma Focus working at $500 \mathrm{~kJ}$ the space and time integrated neutron yield ratio $\mathrm{R}$ for $14.1-$ and $2.45 \mathrm{-MeV}$ neutrons was in the range $1 \times 10^{-3}<\mathrm{R}<4 \times 10^{-3}$.

- This value of $\mathrm{R}$ could consistently be explained only if the tritons were generated and trapped within plasma domains of a high density in the range of $10^{21}$ to $10^{22} \mathrm{~cm}^{-3}$, a temperature in the range of $1 \mathrm{keV}$ to $5 \mathrm{keV}$, and trapping time amounting to $5-20 \mathrm{~ns}$.

Detailed studies of the formation of hot-spots and their correlation with other phenomena in PF-type discharges were carried out by a team at the Soltan Institute for Nuclear Studies in Swierk, Poland [160]. It was proved experimentally that the appearance of the observed hot-spots correlates well with the emission of electron and ion beams (in opposite directions).

Recently, experiments performed with the PF-1000 facility in Warsaw, Poland, have also confirmed the formation of super-dense plasma micro-regions in discharges performed 
with a high- $Z$ gas admixture. In some cases PF discharges carried out with the $\mathrm{D}_{2}$ filling and puffing have also shown distinct hot-spots [161], as shown in Figure 23.

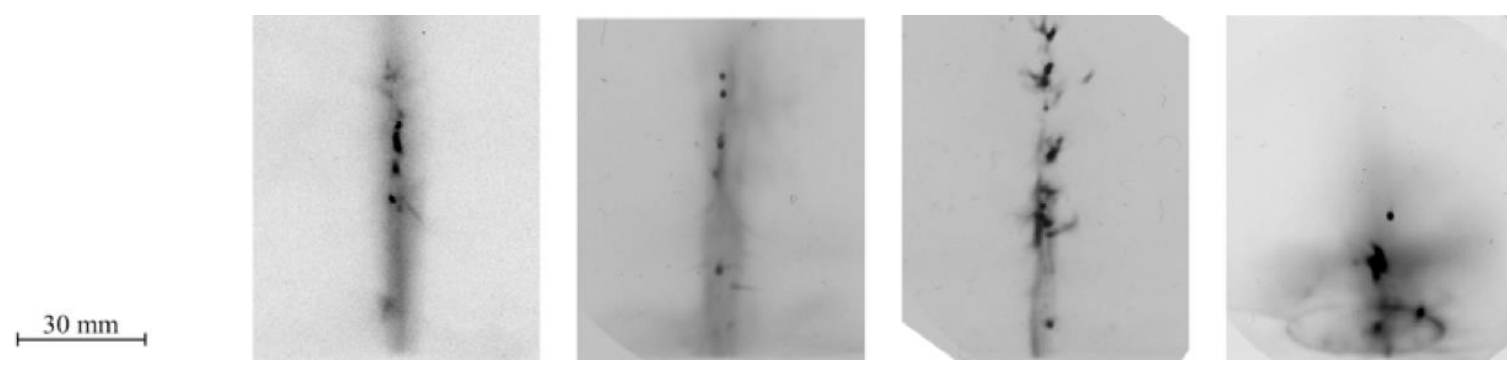

Figure 23. Soft $\mathrm{X}$-ray pinhole pictures of PF-1000 discharges performed with the $\mathrm{D}_{2}$ filling and puffing, which show the appearance of different hot-spots. Reproduced from Figure 6 of [161].

Detailed studies of PF-1000 discharges, as performed with pinhole cameras and filtered PIN-diodes [162], showed that a local electron temperature can reach even $7.5 \mathrm{keV}$, as shown in Figure 24.
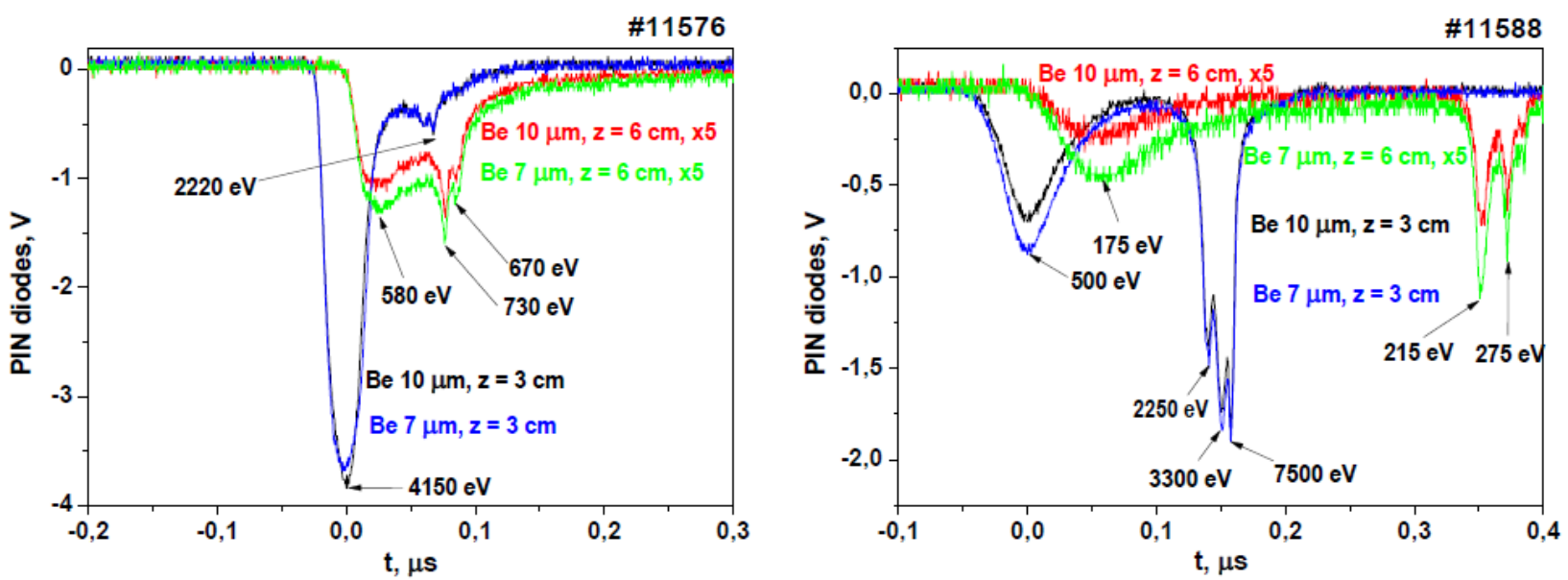

Figure 24. Soft $\mathrm{X}$-ray signals from different observation regions of two PF-1000 discharges: (left) at $1.2 \mathrm{hPa}\left(90 \% \mathrm{D}_{2}+10 \% \mathrm{Ne}\right)$, and (right) with additional puffing of a $\left(75 \% \mathrm{D}_{2}+25 \% \mathrm{Ne}\right)$ mixture. The sharp peaks and high $\mathrm{T}_{\mathrm{e}}$ corresponded probably to individual hot-spots [162] (Figure 6).

\section{(5) Other miscellaneous indications of plasma micro-structures}

Attributes of plasma microstructure other than observations of filaments, ion microbeams, hot-spots, and super-dense plasma domains of an enhanced reactivity were found in other experiments. Neil and Post [163] used a $20 \mathrm{MW}, 100 \mathrm{~ns}$ FWHM, TEA $\mathrm{CO}_{2}$ laser to observe over-dense scattering from plasma focus. The critical density for the $\mathrm{CO}_{2}$ laser is $\sim 10^{19} \mathrm{~cm}^{-3}$. When plasma density exceeds this value even in a small volume, the overdense scattering occurs. This study used a 45-kJ plasma focus operated with deuterium doped with $4 \%$ of $\mathrm{Ar}$, at the initial pressure 1.4 torr. The highlight was an observation of the over-dense scattering signal after the pinch break-up, in a few pulses of FWHM less than $20 \mathrm{~ns}$, in about $50 \%$ of shots, which also showed hot-spots in several soft X-ray pinhole pictures. The scattered power was interpreted to infer the scattering volume of about $40 \mu \mathrm{m}$ dimensions.

(6) Significance of plasma filaments and micro-structures

The phenomena discussed in this section indicate that the common view of plasma current being carried by streams of free electrons may be an over-simplification. They also point towards the existence of energy concentration mechanisms of different kinds. A widely cited theory for one of such mechanisms is Shearer's radiative collapse model [164]. 
A z-pinch equilibrium state between magnetic and thermal pressures is expected to decrease its equilibrium radius indefinitely above a critical current, because radiated power can exceed the energy coupled to the plasma through the resistive heating, and this deficit can only be covered by the work done by the magnetic field in compressing the plasma. The collapse is predicted to proceed until the plasma reaches a Fermi degenerate state. A comprehensive theory of this effect is presented by Meierovich [165]. The triton burnup analysis [159] showed that such a mechanism must be operating during the neutron production in pure deuterium discharges. As the over-dense scattering experiment [163] showed, at least one such mechanism must operate after the pinch. The contribution of such mechanisms to the overall fusion yield from plasma focus devices during their normal operation is an open question at the moment (see Section 8.4.1.5).

\subsection{Experimental Findings That Were Ignored but Are Found Relevant in Hindsight}

The initial surge of funding and official interest allowed deployment of advanced experimental and diagnostics equipment leading to significant findings. However, while most of these findings were consistent with the standard narrative described above, some did not fit into this narrative and were downplayed and never pursued further by their authors. Their significance has been rediscovered in the light of a new narrative regarding $\mathrm{PF}$ that is still evolving as a result of recent and ongoing research work that is discussed later. This section aims to put on record these old findings so that their authors are duly (although belatedly) acknowledged and their modern significance can be appreciated in an appropriate manner later in this article.

\subsubsection{Existence of a Spontaneous Axial Magnetic Field Component}

Early research indicated [2] that application of an external axial magnetic field decreased the neutron emission and increased the stability of the plasma column, which was one of the arguments in favor of the hypothesis of instability-induced acceleration of ions leading to neutron emission. Axial component of Earth's magnetic field was considered to have negligible effect on the plasma dynamics. Neglect of axial magnetic field and assumption of strict azimuthal symmetry have always been standard assumptions in theoretical discussions of DPF Physics $[8,166,167]$ and even now, there is no perceived need to specially justify these assumptions. The topological role of a non-zero axial component of magnetic field in generating flux surfaces of a spontaneously self-organized relaxed state was not widely appreciated, probably because the idea of magnetic reconnection was then in its infancy, and is even now a work in progress [168].

In 1979, Gribkov et al. [169] inferred the existence of an axial component of magnetic field associated with the implosion phase of the plasma focus. A laser pulse ablated the center of the copper anode of the Filippov type DPF at the Lebedev Physical Institute in Moscow much before the plasma focus sheath collapsed to form a pinch column. The resulting copper plasma plume was observed to emerge with negligible radial expansion, using time integrated soft $X$-ray pinhole photography and 5 frame pulsed ruby laser interferometry. In a subsequent experiment [170], the plume was generated in an off-axis location and was seen to be pushed towards the axis and then to expand axially with negligible radial expansion (see Figure 1 of [171]). Since the DPF plasma sheath and its associated azimuthal magnetic field had not yet reached the vicinity of the copper plume, the authors concluded that this behavior could be caused only by an axial magnetic field filling the space between the still-imploding plasma focus sheath and the plasma plume.

This matter was not investigated further at the Lebedev Institute. It has been confirmed recently at the PF-1000 facility (Section 4.3.1.1 A(4)c).

In 1980, multi-frame schlieren pictures revealed [172] a helical perturbation of the plasma in Frascati 1 MJ experiment leading to the suspicion that a spontaneously generated axial magnetic field may exist. Its relation to neutron emission was suspected: "...one explanation for the long neutron production time may be the creation of magnetic structures involving the generation of a $B_{Z}$ magnetic field component, which would eventually trap 
fast particles" [117]. A Faraday rotation experiment was carried out by Gerardo Fischfeld from Rosario University, Argentina, on the Frascati $1 \mathrm{MJ}$ experiment to look for an axial component of magnetic field. Its conclusion was mentioned in a conference paper in 1981 [172] and no further investigations of these findings were reported.

Signature of existence of axial magnetic field was found in 1984 [42,173] at POSEIDON in Stuttgart in the tilt of the reaction proton image with respect to the neutron image in about $50 \%$ of analyzed shots. Both Frascati [172] and Stuttgart [42,173] reports mention that the polarity of the axial magnetic field / azimuthal current does not change from shot to shot. This was also confirmed on PF-1000 (see Section 4.2.2 (4)d).

Magnetic probes were deployed through the anode at POSEIDON plasma focus at Stuttgart in 1989 to look at radial, azimuthal, and axial components of magnetic field. These probes had transverse component rejection ratios better than 30:1. The radial, azimuthal and axial probe signals were found [174] to have distinct temporal features showing that the signals represented independent phenomena. This work could not be followed up with detailed investigations because funding for POSEIDON came to an end.

The scientific reason for the general neglect of these indications of axial magnetic field in PF research is that there is no place for an axial component of magnetic field in the standard narrative (see Section 8.2.1). This is because of the excellent azimuthal symmetry of the spatial propagation of the plasma-there is no inherent choice between $+\hat{\theta}$ and $-\hat{\theta}$ directions for the azimuthal current required to create an axial magnetic field with a given orientation. There are exactly four physical phenomena that can break the symmetry between $+\hat{z}$ and $-\hat{z}$ directions: (1) component of Earth's magnetic field along the device axis, (2) component of Earth's angular momentum along device axis, (3) direction of current flow, and (4) direction of plasma propagation. It is difficult to conceive of any mechanism other than the convection or compression of Earth's magnetic field that can create an axial component of magnetic field in the plasma focus device. Since externally applied axial magnetic field was already shown to have a negative influence on the PF neutron yield, investigating axial magnetic field found no interested researchers. However, the possibility that geomagnetic field may have an influence on plasma focus phenomena was considered by Mather and Ahluwalia in 1988 [175]. Existence of paramagnetic drift of charged particles placed in an arbitrarily weak axial magnetic field under the action of an implosive force directed towards the axis was shown in 1984 [176] to create an azimuthal current density that strengthens the weak initial axial magnetic field (see Section 8.2.1).

Existence of axial magnetic field was finally confirmed in PF-1000 in 2011 using magnetic probes, (Section 4.3.1.1 A(4)) and led to significant new insights into PF physics as discussed later in this paper (Section 4.3.1.3).

\subsubsection{Aspects of Neutron Emission Not Conforming to the Standard Narrative}

The standard narrative ascribed bulk of the neutron emission to ions accelerated along the axis by an intense electric field generated by the sudden decrease in current because of plasma instability. The evidence in favor of this supposition was very clear: (1) The neutron spectrum measured along the axis was shifted to higher energy. (2) The anisotropy (ratio of end-on to side-on neutron emission) was greater than unity. (3) When the instability was suppressed by application of an external axial magnetic field, the neutron emission was decreased by an order of magnitude. However, more detailed investigations revealed many features of neutron emission that do not conform to this narrative.

- In POSEIDON plasma focus, the neutron emission routinely began $\sim 80$ ns before maximum compression. A similar phenomenon was observed in SPEED-II plasma focus at Düsseldorf [177] with neutron emission beginning $30 \mathrm{~ns}$ before maximum compression. Figure 9 of [118] shows that, even in Frascati, the neutron emission began before the maximum compression.

- $\quad$ Bernstein and Comisar [178] mention having measured neutrons having energy as high as $3 \mathrm{MeV}$ in the backward direction in 1967. This was a clear hint that the insta- 
bility induced axial ion acceleration idea was flawed. However, this was confirmed by other authors only after the turn of the century, as discussed later.

- Neutron time-of-flight spectra were measured in 1975 in the Limeil plasma focus [179] using a flight path of $120 \mathrm{~m}$ and large volume plastic scintillator detectors were used at $0^{\circ}$ and $90^{\circ}$, simultaneously, to attain better than $0.1 \mathrm{MeV}$ energy resolution. It was shown that the side-on neutron spectrum had much higher width than the end-on spectrum and both were centered at $2.45 \mathrm{MeV}$. This indicated that most of the neutron emission was by deuterons streaming perpendicular to the axis.

- Side-on neutron time-of-flight measurements in 1978 by Milanese and Pouzo on the 1 MJ Frascati facility with $128 \mathrm{~m}$ flight path, revealed [180] two lateral sub-peaks on either side of a central peak in the neutron energy spectrum which could only be explained in terms of loops of $100-\mathrm{keV}$ deuterons in the plane determined by the device axis and the direction of observation.

- Frascati team inferred $[172,181]$ the existence of a $\sim 100 \mathrm{keV}$ deuteron beam circulating around the device axis from time-integrated single-shot neutron spectra measured through four collimators looking to the left and right of the axis from the front and back.

- Evidence of azimuthal motion of deuterons responsible for fusion reactions was also reported in a Ph.D. thesis from POSEIDON [171,182] and was never followed up.

- $\quad$ Time resolved neutron emission anisotropy measurements on POSEIDON in 1983 showed [183] that anisotropy of neutron emission was less than and greater than 1 before and after the instability, respectively, particularly in high neutron yield shots.

- Time-integrated images of protons emitted from the reaction $D(d, p) T$ in POSEIDON were larger in size than the time-integrated X-ray pinhole images from the same shot $[171,182,183]$.

These findings had no influence on the developments on PF research for two obvious reasons. One was that their authors did not follow them up (probably for reasons related to funding) with detailed confirmatory experiments, which could address issues related with detection statistics, effectively abandoning their results. The second was that they contradicted the accepted narrative and did not fit into any theoretically justifiable alternative narrative.

\subsubsection{Aspects of Plasma Dynamics Not Conforming to the Standard Narrative}

Three-frame Schlieren diagnostics at Frascati 1 MJ facility [116,117] revealed in 1980 that at the time of maximum neutron production, the plasma column undergoes instability with a wavelength comparable with pinch radius leading to emission of "plasma tongues" which reconnect to form "long-lasting plasma blobs whose decay time (100-150 ns) corresponds to that of the local neutron emission" [114,184]. Quasi-spherical structures of $\sim 3-6 \mathrm{~mm}$ size were sometimes observed using soft X-ray pinhole diagnostics in the Nessi plasma focus [116]. These findings found no mention in subsequent PF research because their significance could not be related to the standard narrative. These features were confirmed on the PF-1000 facility quite recently (Section 4.3.1).

\section{Major Developments around the World between 1994 and 2020}

This section gives an overview of major developments around the world in terms of new research facilities, diagnostic techniques, simple models, numerical simulations, and empirical and analytical insights, and discusses their contribution to overall progress.

\subsection{Facilities}

A recent review paper [13] gives a good overview of research in the laboratories of Asia, Africa, and Latin America including information about their facilities. A new trend is seen that emphasizes design and construction of inexpensive, fast, compact, and very low energy (under kJ) PF devices reaching down to $0.1 \mathrm{~J}$ stored energy. They have made a valuable contribution to basic research and the extension of scaling laws towards lower 
end of the energy scale. However, to avoid duplication, this section gives only a brief introduction to a few of the new plasma focus facilities with one exception. The UNU ICTP plasma fusion facility is covered in detail with emphasis on its historical role in promoting plasma focus research.

The PF-3 facility, although not a new facility, is described because it has not been described in any other review paper and has a historical significance. Only the standard Mather and Filippov configurations are covered here. Non-conventional electrode profiles are covered in Section 7.

\subsubsection{The UNU ICTP Plasma Fusion Facility (PFF)}

\section{Brief Time Line and Motivation}

The University of Malaya Plasma Research Laboratory had built a 40 kV, 50 kJ capacitor bank in 1972 with a measured current of 1.92 MA and a short-circuited inductance of $23.7 \mathrm{nH}$ [185]. A plasma focus was constructed which produced $10^{8}$ neutrons at $20 \mathrm{kV}$, $700 \mathrm{kA}$ peak current in mid-1973. The neutrons were measured by time-of flight method to have energies of $2.5 \mathrm{MeV}$ in the forward axial direction and 2.2 MeV rearwards [186]. This was likely the first measurement of fusion neutrons from an electric plasma in geographical Asia. It was with this background that the UNU ICTP PFF was developed. The United Nations University/International Center for Theoretical Physics Plasma Fusion Facility is a $3 \mathrm{~kJ}$ plasma focus system powered by a single $15 \mathrm{kV}$ capacitor switched by a triggered parallel-plate spark-gap. It was designed, assembled, and tested in late 1985 at the University of Malaya, Kuala Lumpur in the course of a UNU Training Programme attended by eight UNU Fellows from Egypt, India, Indonesia, Nigeria, Pakistan, and Sierra Leone [187]. The objective of that project, initiated in 1983 by an informal group at the ICTP Spring College on Plasma Physics, was to provide a facility to study plasma fusion that could be put together and maintained by small research groups in developing countries. Hence a requisite guiding principle in the design was that each part of the facility could be built and maintained by a small university laboratory. Every part-electrodes, insulators, collectors, vacuum chamber, spark gap, high voltage charger, triggering and synchronising electronics, diagnostics including Rogowski coils, voltage probes, magnetic probes, neutron counter and a nanosecond shadowgraphy system using a home-made nitrogen laser-was designed and manufactured during the training programme from components that were typically available in a university laboratory or workshop setting. Only the capacitor needed to be specially procured. Accompanying the hardware was a microcomputer code [188] to compute the electro-dynamics of the plasma focus. The plan was for the Fellows to build the plasma focus when they return to their respective institutions.

This plan was upgraded when, in January 1986, Professor Abdus Salam visited the training programme [187] during his Malaysian tour and on the spot provided funding for follow-up hardware for the fellows. Six devices were manufactured with the resources of the workshop. Each device was assembled and tested by its owner using the only available capacitor and rotary pump. In April 1986, the six plasma focus machines were packed and shipped to the laboratories of the fellows.

\section{What Was Physically Achieved}

The design of the UNU ICTP PFF was broken down into systems [189] and described in a 1988 paper [190] in the American Journal of Physics. Data collected during the testing of the original six devices were included in the paper detailing its best performance regime in various gases, including the production of $10^{8}$ neutrons per shot in each device. Today, UNU ICTP PFF and variants based on the original design are operational in Egypt, India, Indonesia, Iran, Malaysia, Nigeria, Pakistan, Singapore, Syria, Thailand, USA, and Zimbabwe. At the last informal count in 2006, more than 20 Ph.D.s, 40 master's degrees, and 200 refereed research papers have been produced using the network machines, contributing significantly to the understanding of dense plasmas. About half of these research documents were already listed in a 1998 review document [191]. At the December 1990 meeting convened in Moscow to discuss the formation of the International Center 
for Dense Magnetised Plasmas (see Appendix B), it was mentioned that the idea of the ICDMP was triggered by the collaborative success of the AAAPT on the UNU ICTP PFF mentioned by Ashok Gholap at the IV Latin American Workshop on Plasma Physics in Buenos Aires, Argentina in July 1990. The proposal document of the International Center for Dense Magnetized Plasmas [192] states that the training, education, and scientific exchange component of the proposal was based on the experience of the AAAPT (using the UNU ICTP PFF).

\section{What Innovations Were Developed, What New Insights Were Generated}

A standardized, easily affordable plasma focus was developed that could be manufactured, assembled, and maintained in any small laboratory. Moreover the design could be modified to suit the availability of the capacitor, with the help of the code which was integrated as part of the package. The system was used in Ph.D. studies in neutrons, ion beams, REB, soft and hard X-rays [187,191], in simulations of astrophysical plasmas (also see Section 3.3.1.1) and in a series of experiments at the ICTP Trieste to demonstrate nonlinear deterministic mechanics $[187,191]$. Improved neutron scaling using a shaped anode for speed-enhancement of yield was demonstrated. That research also yielded insight into the scaling of dimensions and lifetimes of plasmas with anode radius, and the constancy of the speed factor $\mathrm{S}=\mathrm{I} / \mathrm{a} \sqrt{\mathrm{p}}$ for optimized neutron yields across the range of plasma focus devices from sub $\mathrm{kJ}$ to MJ. Pulsed neutron activation was used to determine the half-lives of indium-116 and rhodium-104 and metastable sodium-24 and to study radiation safety. In another notable study neutron measurements, with copper and deuterated obstacles placed at various positions downstream from the anode face, showed that $85 \%$ of the fusion neutrons were from the deuterium ion beam bombardment of the deuterium gas in the region 20-60 $\mathrm{mm}$ from the end of the anode [193]. The plasma focus system was used to pioneer plasma processing of thin film materials including superconducting and fullerene films on silicon. That research in Delhi then progressed to diamond-like carbon and carbon nanostructured materials [191]. The code developed within the UNU ICTP PFF has now expanded in concept to become a universal plasma focus tool described later (see Section 3.3.1.1).

\section{Conclusions}

The UNU ICTP PFF is now an established research device operating in 12 countries. Its simple design and flexible operation in various gases enables it to have become a most effective plasma device for international cooperation at the same time producing exceptional numbers of Ph.D. theses and research papers

\subsubsection{PF-1000}

The PF-1000 Mather type facility was commissioned in the year 1999 [194]. Its salient features are summarized below:

Capacitor bank parameters

- $\quad$ The charging voltage $-\mathrm{U}_{0}=20-40 \mathrm{kV}$.

- The condenser bank capacitance- $\mathrm{C}_{0}=1.332 \mathrm{mF}$ (12 modules each with 24 units of $4.625 \mu \mathrm{F})$.

- $\quad$ The stored capacitor bank energy- $\mathrm{E}_{0}=266-1064 \mathrm{~kJ}$.

- The nominal inductance- $\mathrm{L}_{0}=8.9 \mathrm{nH}$.

- $\quad$ The quarter discharge time $-\mathrm{T}_{1 / 4}=5.4 \mu \mathrm{s}$.

- $\quad$ The short-circuit current- $\mathrm{I}_{\mathrm{SC}}=15 \mathrm{MA}$.

- $\quad$ The characteristic impedance- $Z_{0}=2.6 \mathrm{~m} \Omega$.

At present, the plasma focus configuration is as follows:

- Anode: copper tube of $230 \mathrm{~mm}$ diameter.

- Cathode: 12 stainless-steel tubes, each $82 \mathrm{~mm}$ in diameter, on $400 \mathrm{~mm}$ diameter.

- Insulator: Alumina.

- $\quad$ Stainless steel vacuum chamber $1.4 \mathrm{~m}$ diameter $\times 2.5 \mathrm{~m}$ length with neoprene seals.

- Oil diffusion vacuum pump with anti-creep liquid $\mathrm{N}_{2}$ traps. 
Some novel features of this facility are listed below:

- This is the only mega Joule level plasma focus facility with a horizontal axis with a $85 \mathrm{~m}$ clear distance behind the anode (where Sylwester Kaliski wanted to place his laser to interact with the plasma focus). This allowed high resolution measurements of neutron time of flight spectra in upstream $(\sim 84 \mathrm{~m})$, downstream $(\sim 84 \mathrm{~m})$, and radial $(\sim 7 \mathrm{~m})$ directions [86].

- Large dimensions of coaxial electrodes ensure the formation of a big plasma column about $10 \mathrm{~cm}$ in length and $1 \mathrm{~cm}$ diameter. Electron density $\sim 10^{24}-10^{25} \mathrm{~m}^{-3}$, transparent for interferometry in the visible radiation and plasma characteristic velocity $\sim(1-2) \times 10^{5} \mathrm{~m} / \mathrm{s}$, facilitate refractivity diagnostic measurements with ns-temporal and $\mathrm{mm}$-spatial resolutions.

- In the year 2000, this facility was placed at the disposal of the International Center for Dense Magnetized Plasma, with an explicit mandate "to carry out the advanced fundamental investigations in the domain of a dense magnetized plasma" making it the only MJ level plasma focus facility in the world to be explicitly linked with open-ended fundamental investigations.

\subsubsection{PF-24}

PF-24 is a Mather type plasma focus based on a $116 \mu \mathrm{F}, 100 \mathrm{~kJ}$ capacitor bank working at $40 \mathrm{kV}$. Its front view is shown in Figure 25 below.

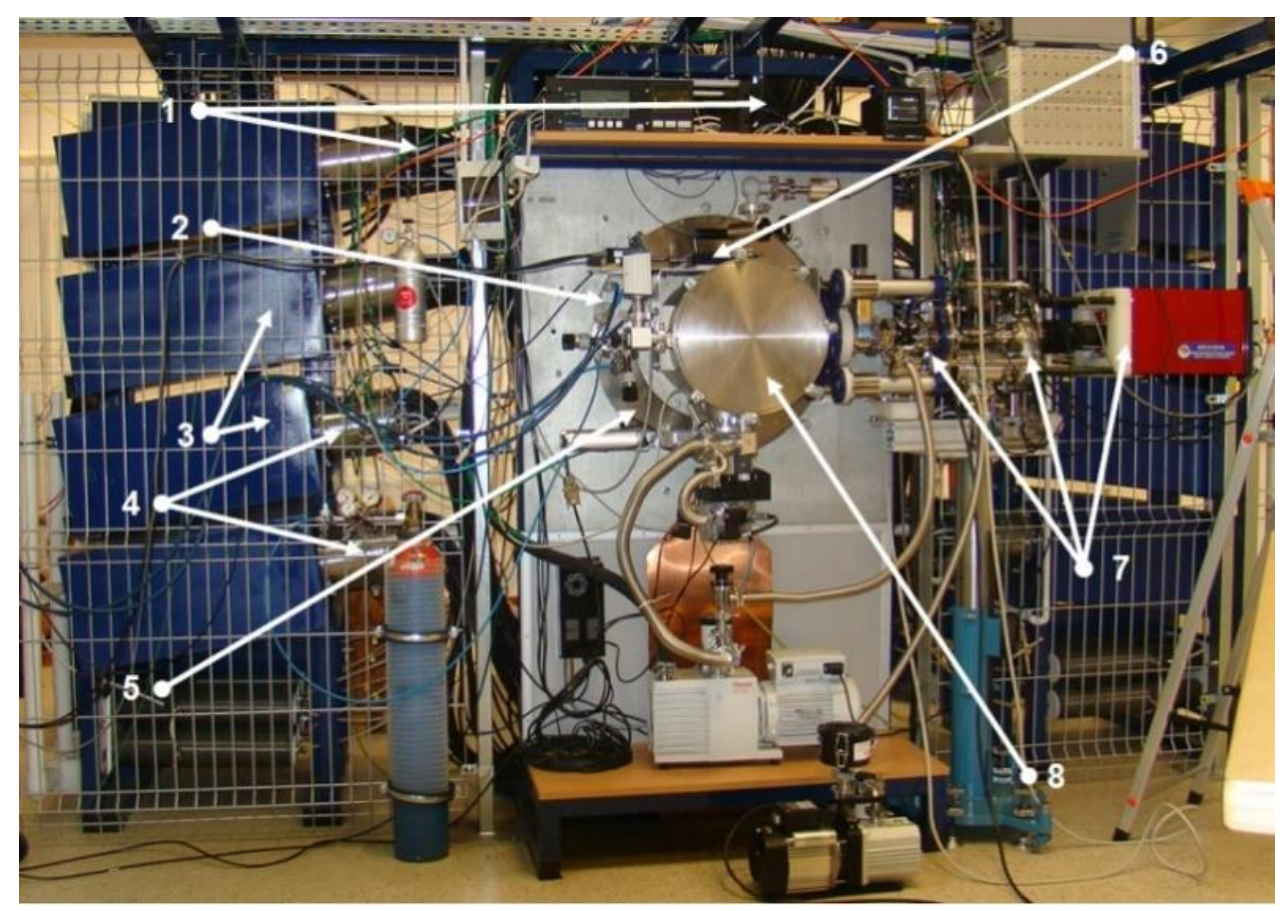

Figure 25. PF-24 $100 \mathrm{~kJ}$ plasma focus at IPJ, Krakow, Poland. 1. Transmission line. 2. Electrical diagnostics. 3. Capacitors. 4. Spark gaps. 5. Collector. 6. Neutron counter. 7. Plasma imaging system. 8. Experimental chamber.

The capacitor bank consists of 24 capacitors each with an integrated, pressurized air, field distortion spark gap with $50 \mathrm{~ns}$ jitter. Each capacitor is connected to the collector plate with 18 RG213/U cables of $3.5 \mathrm{~m}$ length. The estimated parallel inductance of capacitors, spark gaps, cables and collector plate is $\sim 7.5 \mathrm{nH}$. The PF anode made of anaerobic copper has $31 \mathrm{~mm}$ radius and $170 \mathrm{~mm}$ length inside the vacuum chamber. The squirrel cage cathode consists of 16 rods of $12 \mathrm{~mm}$ diameter and has inner radius of $49 \mathrm{~mm}$. The insulator made of $\mathrm{Al}_{2} \mathrm{O}_{3}$ has an outer diameter of $60 \mathrm{~mm}$ and $4 \mathrm{~mm}$ wall thickness. The vacuum chamber has $400 \mathrm{~mm}$ length and $320 \mathrm{~mm}$ diameter. It is operated with a base vacuum of $10^{-6}$ mbar and has facility to inlet multiple gases. Short-circuit tests carried out for $16 \mathrm{kV}$ 
of charging voltage and deuterium pressure of 20 mbar and 25 mbar were evaluated to determine the short circuit inductance $\mathrm{L}_{0}=8.5( \pm 3.1) \mathrm{nH}$ and the total, nominal resistance $\mathrm{R}_{0}=3.1( \pm 2.3) \mathrm{m} \Omega$.

The facility has standard current diagnostic with a Rogowski coil, voltage diagnostic with a capacitive voltage divider built into the collector plate, current derivative diagnostic with a magnetic probe built into the collector plate. Other diagnostics include a beryllium activation neutron yield monitor, a neutron pinhole camera with $5 \mathrm{~mm}$ spatial and $5 \mathrm{~ns}$ temporal resolution, a 4 frame MCP based Soft X-ray/vacuum ultraviolet camera with 1.2 ns exposure time and $4.7 \mathrm{~ns}$ inter-frame delay.

\subsubsection{Gemini and other NSTec PF Facilities}

National Security Technologies (also known as NSTec LLC) developed and operated plasma focus facilities at the Nevada National Security Site (NNSS) from 2006 to 2017 to support programs of many US government agencies related to defense experimentation and stockpile stewardship, homeland security and defense applications, and environmental management [195]. The main facilities at NSTec Nevada are described in Table 1 below.

Table 1. Facilities at NSTec Nevada.

\begin{tabular}{cccc}
\hline & OneSys & TallBoy & Gemini \\
\hline Type & & Two stage Marx & Two Stage Marx \\
Energy $(\mathrm{kJ})$ & 133 & 515 & 1058 \\
Capacitance $(\mu \mathrm{F})$ & 216 & 210 (effective) & 432 (effective) \\
Max Voltage $(\mathrm{kV})$ & 35 & 70 (effective) & 70 (effective) \\
Anode radius & $2^{\prime \prime}$ & $2^{\prime \prime}$ & $3^{\prime \prime}$ \\
Anode length & $15.5^{\prime \prime}$ & $27.5^{\prime \prime}$ & $23.5^{\prime \prime}$ \\
Insulator length & $4.25^{\prime \prime}$ & $10^{\prime \prime}$ & $5^{\prime \prime}$ \\
Cathode radius & $3^{\prime \prime}$ & $3^{\prime \prime}$ & $4^{\prime \prime}$ \\
Fill pressure $\left(\mathrm{D}_{2}\right)$ torr & 5.23 & 6.46 & 5.21 \\
\hline
\end{tabular}

The emphasis of the program has been on providing neutron source and diagnostic services to defense related programs of United States Government Agencies and no significant reports dedicated to elucidation of DPF physics are available in public domain [196-199]. Their operations are now carried out by Mission Support And Test Services LLC founded in 2015.

\subsubsection{Programs and Facilities at Lawrenceville Plasma Physics Inc.}

Lawrenceville Plasma Physics Inc., located at Middlesex, NJ, USA, has embarked on a program to demonstrate a net energy generating technology based on the dense plasma focus device and hydrogen-boron fuel. Their plasma focus facility named Focus Fusion 1 [200] has the following parameters, $113 \mu \mathrm{F}, 45 \mathrm{kV}$ capacitor bank, anode radius $28 \mathrm{~mm}$, cathode radius $50 \mathrm{~mm}$, anode length $140 \mathrm{~mm}$, insulator length $27 \mathrm{~mm}$.

This program is aimed at producing sub-millimeter size super-dense plasma formations, referred to as plasmoids, in which the magnetic field is claimed to be high enough to prevent energy transfer from ions to electrons by the "Quantum Magnetic Field effect" [200]. Gated ICCD camera photographs in visible and near UV, with $0.2 \mathrm{~ns}$ exposure time are claimed [95] to depict a bright filament $60 \mu \mathrm{m}$ in diameter and wrapped in a coil that is $400 \mu \mathrm{m}$ in diameter and $1.5 \mathrm{~mm}$ long. 


\subsubsection{PF-3 Facility}

The PF-3 facility, commissioned in 1982, (Figures 26 and 27) is a PF device with so-called plane geometry of electrodes (Filippov-type, or non-cylindrical z-pinch configuration). The capacitor bank is assembled from IK-12-25 capacitors. The total capacitance of the power source is $9.2 \mathrm{mF}$, the maximum charging voltage is $25 \mathrm{kV}$, the maximum stored energy is $2.8 \mathrm{MJ}$, and the minimum external inductance is $15 \mathrm{nH}$. An additional matching inductance enabling variations in the initial inductance $L_{0}$ of the circuit from 15 to $40 \mathrm{nH}$ can also be installed. The low pressure spark gap switch is traditionally used as a switch for the facilities of such type. The spark gap switch is a multi-sectional (24 sections) toroid with a $12 \mathrm{~m}$ major diameter and with a cross-section diameter of $0.08 \mathrm{~m}$. The spark gap switch is evacuated to the pressure of $\sim 10^{-2}$ Torr.

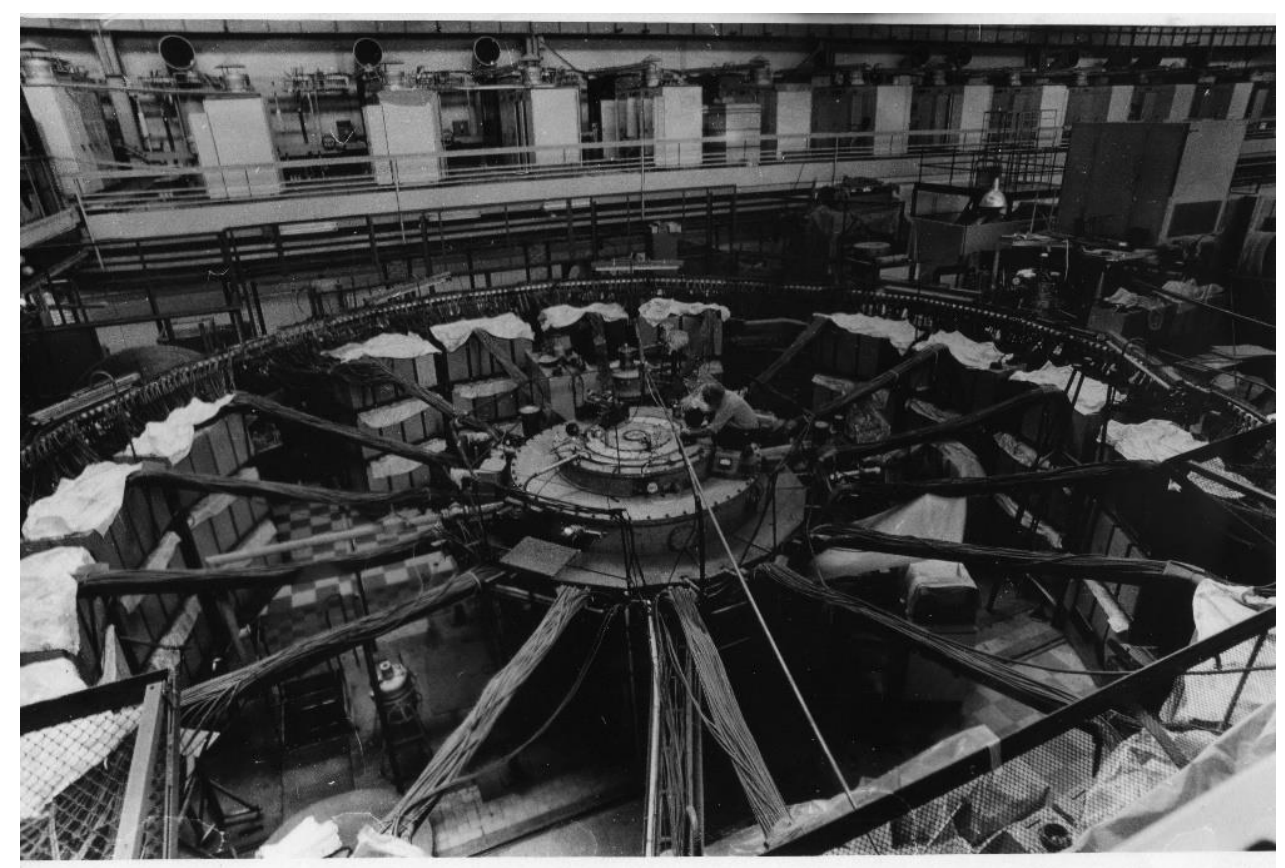

Figure 26. Nikolay Filippov and PF-3 Facility.

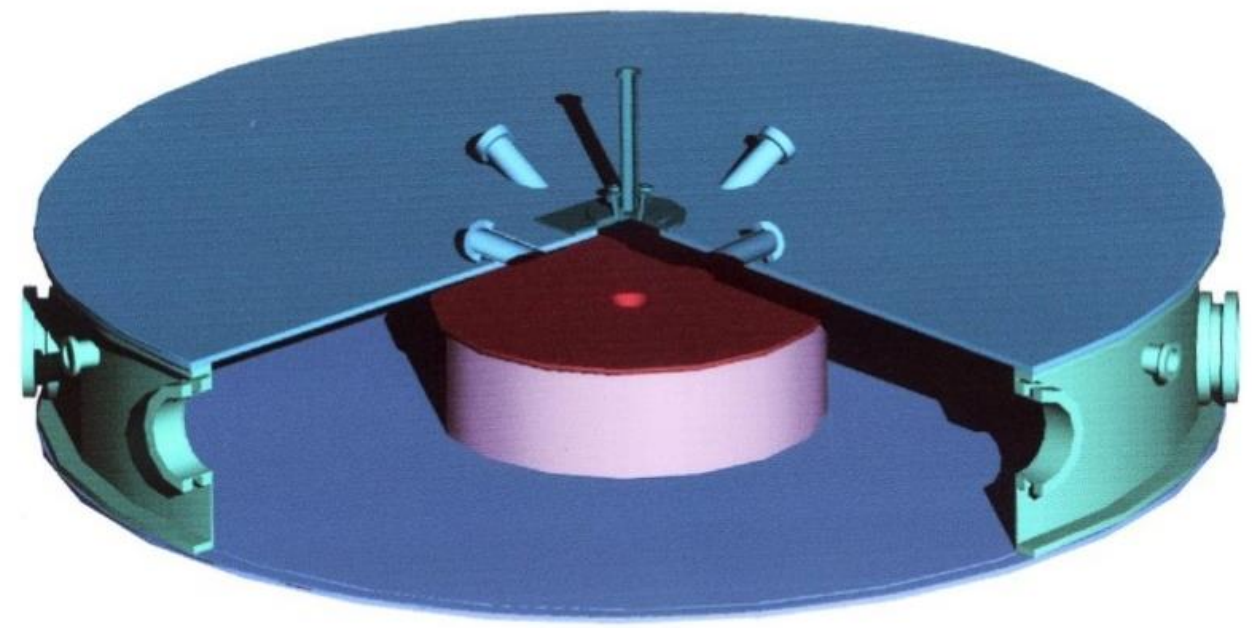

Figure 27. Schematic view of the PF-3 chamber. 
The calculated value of the short-circuit current for the PF-3-facility, at the maximum charging voltage of $25 \mathrm{kV}$ and at the minimum external inductance of $15 \mathrm{nH}$, is $20 \mathrm{MA}$. The main working gases are neon, argon, or deuterium with an admixture of krypton or xenon at a total pressure of 1-10 Torr. The insulator is $92 \mathrm{~cm}$ in diameter and $25 \mathrm{~cm}$ in height and is made of pyroceramic. In fact, the dimensions of the available insulator determine the anode diameter. The anode is a sectioned copper disc with a diameter of $92 \mathrm{~cm}$ and thickness of $2.5 \mathrm{~cm}$. In the central part of the anode, there is a replaceable insert that allows one to increase the anode lifetime and control the profile of the imploding PCS by varying the shape of this insert. In most of the experiments, an insert with a cone-shaped cavity was used, which had a base diameter of $10 \mathrm{~cm}$ and depth of $7 \mathrm{~cm}$.

In the initial stage of research, a carbon-steel vacuum vessel, $260 \mathrm{~cm}$ in diameter and $45 \mathrm{~cm}$ in height, also served as the cathode. Such cathode dimensions allow the CS to evolve freely during the discharge. For the facility parameters $\left(\mathrm{C}=9 \times 10^{-3} \mathrm{~F}, \mathrm{~L}_{\min }=15 \mathrm{nH}\right)$ the short circuit current rise time of the capacitor bank is $18 \mu \mathrm{s}$. However, under experimental conditions the current rises only for 5-6 $\mu$ s, evidently not reaching the maximum-possible value. One of the main reasons resulting in an early current "decay" — as it was shown by the numerical calculation with a "snow-plough"-model-is a practically unlimited PCS-expansion that results in an undesirable increase in the discharge loop inductance. In that case, the current drops almost to half its maximum value at the moment of the maximum compression at the axis. For improving the matching of the facility parameters with plasma dynamics, 48 rods are installed at the diameter of $115 \mathrm{~cm}$ as a "squirrel cage", playing the role of a side wall for the reverse current conductor (Figure 28). Installing a current conductor of such type allows one to avoid the reflected plasma flows, which at relatively long discharge duration could reach the insulator and lead to a secondary break-down. In order to reduce the discharge loop inductance, the inter-electrode gap between the anode and the chamber upper cover and the working height of the insulator have been also reduced.

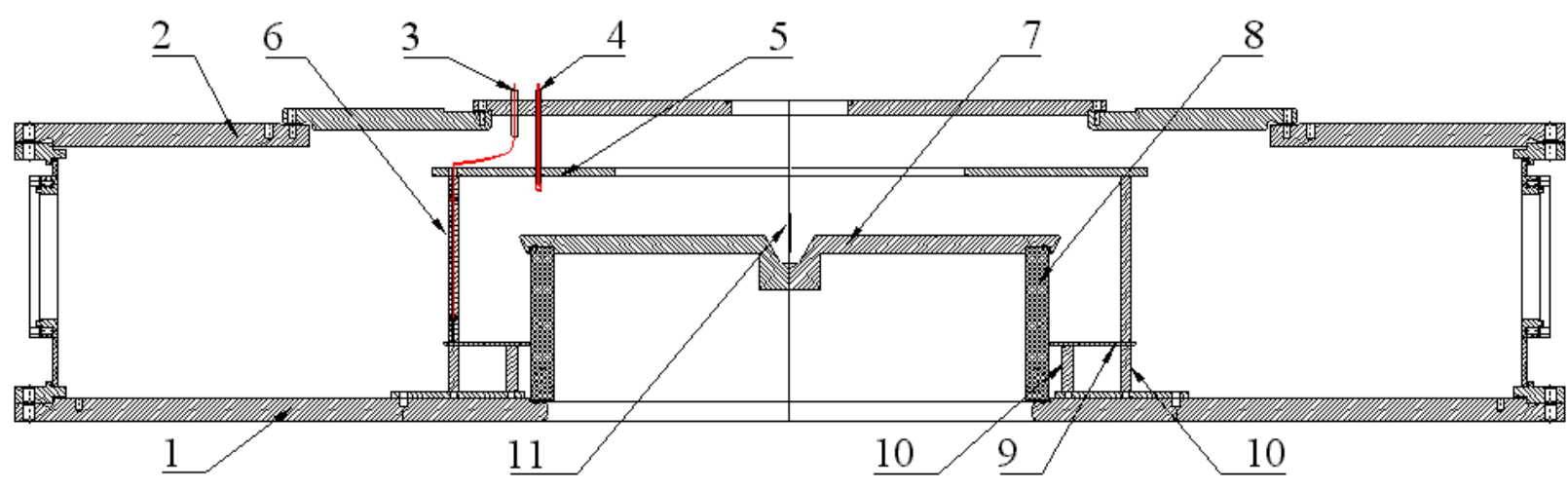

Figure 28. Design of the modernized chamber: 1-chamber floor (cathode). 2-chamber upper cover. 3-Rogowski coils. 4-magnetic probe. 5-upper flange of reverse current conductor (RCC). 6-RCC rods. 7-anode. 8-insulator. 9-false-cathode. 10-false-cathode support rods. 11-pinch. 
The study of the current dependence upon voltage in the modernized system has shown (Figure 29) that the increase in the maximum value of the discharge current has not exceeded $10 \%$ (that corresponds to the results of the numerical calculations). At the same time the value of the current at the time of "peculiarity" has essentially grown (more than 2 times) and has achieved $2 \div 3 \mathrm{MA}$ at $10-14 \mathrm{kV}$.

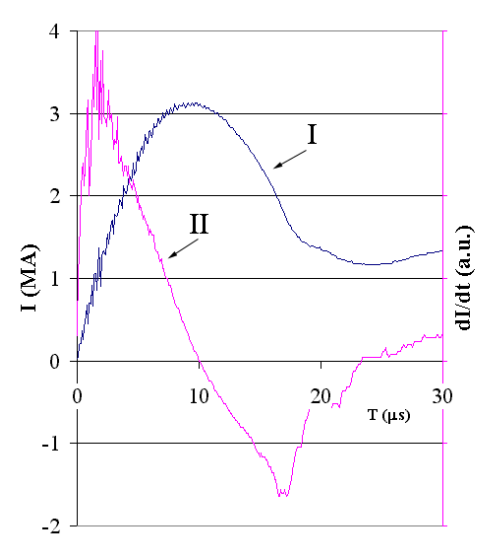

(a)

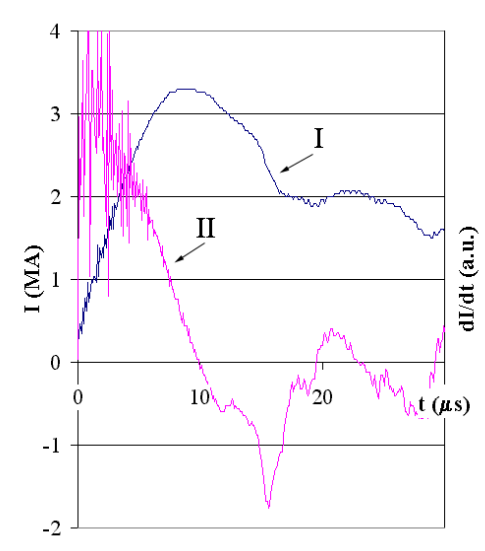

(b)

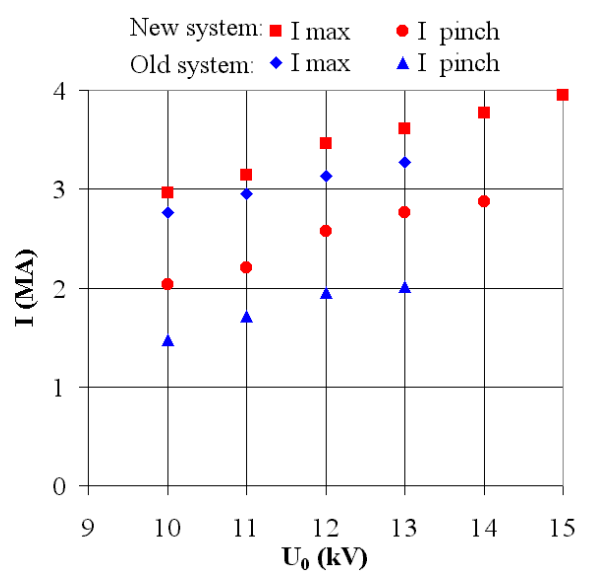

(c)

Figure 29. Typical waveforms of discharge current (I) and current derivative (II) for non-modernized (a) and modernized (b) discharge systems: $\mathrm{U}=12 \mathrm{kV}, \mathrm{P}=4$ Torr neon and current dependence on voltage (c) at facility minimum inductance, $\mathrm{L}_{0}=15 \mathrm{nH}, \mathrm{P}=4$ Torr neon. Adapted from authors' original data also presented in [201].

The PF-3 installation was created as a powerful source of neutron and X-ray radiation. Moreover, it was the prototype of the installation for the large MINIK project $[5,202]$, which was supposed to create a PF with a power source of $100 \mathrm{MJ}$ (based on the tokamak T-14 power supply). This installation was the first to demonstrate the possibility of compressing a current of $\sim 3 \mathrm{MA}$ to the axis of the system [202].

However, the first experiments showed that the existing electrode system is not well matched with the power supply at high charging voltages when operating with deuterium. At the same time, very promising results were obtained when working with heavy gases. In particular, it was reported in [203] that about $100 \mathrm{~kJ}$ was obtained in soft X-ray radiation at power supply energy of $\sim 1 \mathrm{MJ}$ when working with neon as a working gas.

Subsequently the facility was operated mainly with heavy gases: neon and argon.

FIELDS OF ACTIVITY

$\checkmark \quad$ Dynamics of plasma focus discharge in heavy gases at high level of power input.

$\checkmark \quad$ X-ray radiation of high-current neon pinch and its interaction with matter.

$\checkmark$ Acceleration of heavy ions.

$\checkmark \quad$ Axial plasma jet studies. Experimental simulation of collisionless shock waves.

$\checkmark \quad$ Usage of PF as a driver for magnetic compression of liners.

$\checkmark \quad$ Experiments with dust plasma.

$\checkmark \quad$ New approaches to nanofilm production.

$\checkmark \quad$ Experimental simulation of astrophysical processes.

\subsection{Diagnostics}

This section reviews salient features of new diagnostic techniques fielded since the last review.

\subsubsection{6-Frame Interferometry}

A 16 frame Mach-Zehnder interferometry system [204] has been operating at the PF-1000 facility since 2007. It uses a Nd:YLF laser operating at a second harmonic wavelength of $527 \mathrm{~nm}$ with $500 \mathrm{~mJ}$ pulse energy, energy stability of $7 \%$, time duration less than 
$1 \mathrm{~ns}$, timing jitter $\pm 1 \mathrm{~ns}$ and beam diameter of $12 \mathrm{~mm}$. The beam diameter is increased up to $60 \mathrm{~mm}$, minimizing beam divergence using an optical telescope. An optical delay line produces 16 light beams in the form of a $4 \times 4$ matrix around the optical axis of the system with a variable total delay of $0-220 \mathrm{~ns}$ with identical linear sizes and beam quality. A beam expander increases the beam size to $150 \mathrm{~mm}$ and directs it into the mirror system of Mach-Zehnder interferometer giving sufficiently large field of view to observe the entire plasma volume of interest.

A specially designed 16-prism assembly distributes the 16 time-delayed beams onto four photographic plates of four images each. Optical output of each of the 16 prisms passes through narrow band optical interference filters reducing the huge plasma irradiance background in visible spectrum. Four $130 \times 180 \mathrm{~mm}$ photographic cassettes are mounted to capture the 16 interferograms, which are opened just before the shot to minimize effect of ambient stray illumination. A special type of film is selected for optimal contrast and resolution of the registered images.

\subsubsection{Magnetic and Magneto-Optical Probes}

The magnetic probe diagnostics has the advantage of directly calibrated measurement of local magnetic field as a function of time. Its main disadvantage lies in the uncertainty regarding its influence on the plasma behavior and errors of magnetic field measurements caused by plasma perturbations and electrostatic coupling with the plasma. Presence of magnetic probes in the pinch formation region may also affect the pinching process. Destruction of the probe by intense plasma and radiation fluxes during a single discharge creates operational problems for experiments. Evaporation of the probe case under the action of radiation and particle fluxes, screening of the probe by the plasma and plasma perturbations arising due to plasma flow around the probe are also potential problems. These factors were taken into account when designing and manufacturing absolutely calibrated magnetic probes for experiments at the z-pinch Angara 5-1 installation and then adapted for the plasma conditions of the plasma focus experiment on the three facilities PF-3, KPF-4 Phoenix, and PF-1000 [205].

To minimize the degree of plasma perturbation introduced, the probe size should be less than the thickness of the skin layer. This requirement is difficult to meet for the classical design of a cylindrical magnetic probe. This factor is particularly important when measuring in the near-axis region.

To overcome this problem, a probe design with planar profile of the sensitive element with a thickness of 0.7-0.8 mm was developed (Figure 30) [205-207]. It was placed with the narrow dimension facing the plasma flow in order to minimize perturbation of plasma flow around the probe and reduce the influence of the probe on the processes under study. 

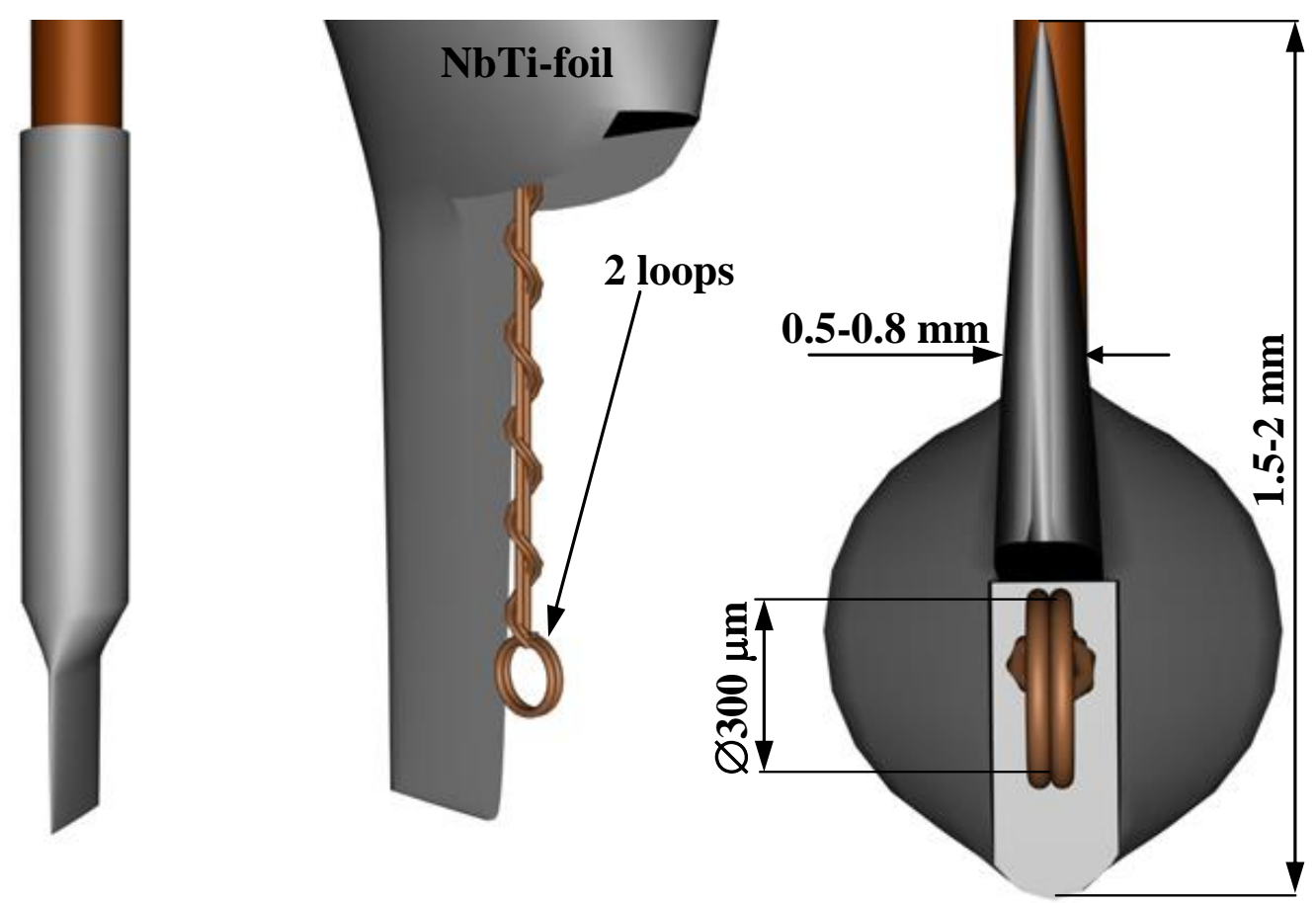

Figure 30. Design of planar magnetic probe. Reproduced with permission from Figure 1a of [206]. Plasma Phys. Rep. 2010, 36, 937-952. (C) 2010. Pleiades Publishing, Ltd.

The probe was physically protected and electrostatically shielded by niobium-titanium foil of thickness 10-15 $\mu \mathrm{m}$. Diffusion time for electromagnetic field through the thickness of the probe case was estimated to be $\sim 1.5 \mathrm{~ns}$ for a plasma flow with density $\sim 5 \times 10^{17}-10^{19} \mathrm{~cm}^{-3}$, velocity $\sim 10^{6}-5 \times 10^{7} \mathrm{~cm} / \mathrm{s}$, magnetic field $0-100 \mathrm{kG}$, temperature $1-30 \mathrm{eV}$. The sensing element consisted of two $\sim 300 \mu \mathrm{m}$-diameter coils wound in clockwise and counterclockwise directions. Two symmetric signals of opposite polarity from the two coils allow one to be sure of their magnetic origin and discriminate against spurious effects. This symmetry was lost at the instant of probe destruction confirming the adequacy of the electrostatic shielding prior to probe destruction. Numerical simulation of probe response to plasma flow with frozen-in magnetic field showed that perturbation of magnetic field by planar probes was $\sim 7 \%$ while cylindrical probes caused an error $\sim 25 \%$ [205].

Planar probes were introduced in experiments on the PF-3 facility [206,208] and then studied in detail on the PF-1000U facility [207,209] and KPF-4 Phoenix facility [210]. Figure 31 compares measurements of the magnetic field during compression of deuterium PCS in two PF-1000U shots with a cylindrical probe (curve 4) and a probe with a planar shape (curve 2) located $40 \mathrm{~mm}$ from the facility chamber axis. It should be noted that in this series of experiments, under optimally selected initial conditions, the repeatability from shot to shot was high. For example, the total currents differed by less than $10 \%$ (see curves 1 and 3), and the neutron yields Yn differed by no more than 1.5 times. 


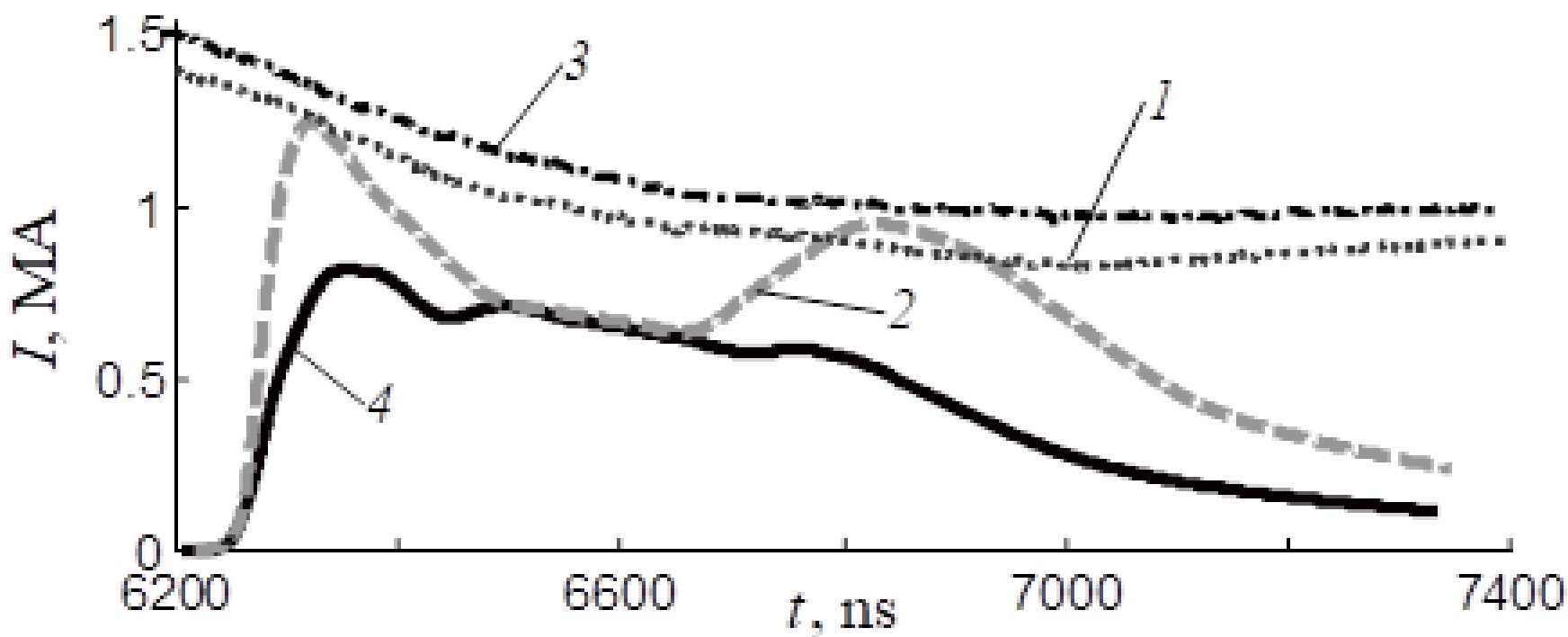

Figure 31. The comparison of the results of measuring the current in the near-axis region of the PF-1000 facility $(\mathrm{r}=40 \mathrm{~mm})$ using magnetic probes with different shapes. Gaseous $\mathrm{D}_{2}, \mathrm{P}_{0}=1.8$ Torr, $\mathrm{U}_{0}=24 \mathrm{kV}, \mathrm{W}_{0}=384 \mathrm{~kJ}:(1,2)$ the total current and the current measured with the planar probe (mica $+\mathrm{NbTi})$, respectively, $\left(\mathrm{Yn}=6.9 \times 10^{10}\right.$ neutrons $/$ pulse $)$. $(3,4)$ the total current and the current measured with a cylindrical probe (ceramic tube with $2.5 \mathrm{~mm}$ in diameter) $\left(\mathrm{Yn}=9.8 \times 10^{10}\right.$ neutrons $\left./ \mathrm{pulse}\right)$. Reproduced with permission from Figure 8 of [205] Instruments and Experimental Techniques. Volume 61. pp. 239-259. (C) 2018. Pleiades Publishing, Ltd.

Figure 31 (curves 2 and 4) shows that the planar probe registers a current 1.6 times larger in amplitude than the cylindrical probe. The signal profiles are similar. Beyond $150 \mathrm{~ns}$ after the maximum, the signals from the probes coincide for $200 \mathrm{~ns}$. Further signal divergence occurs after plasma compression and may be associated with different scenarios for the development of pinch current shunting both near the installation axis and in the installation insulator area for these shots. For the total number of shots $(\sim 20)$, the ratio of the amplitudes of the measured currents in this series was about 1.8 . This is confirmed by the experimental data of testing cylindrical and planar probes conducted at the Angara-5-1 installation [205].

The lowering of the signal amplitude from the cylindrical probe occurs due to a larger perturbation that occurs when the plasma flows around the cylindrical probe than when it flows around the planar probe. Further coincidence of the signals, both in amplitude and shape, indicates the attenuation of the plasma perturbation introduced by the sensor. The smaller the size of the probe or the smaller its cross-section to the incoming plasma flow, the smaller the plasma perturbation that occurs, and it decays faster with time. This is also qualitatively confirmed by the results of laser interferometry.

Figures 32 and 33 show interferograms of plasma flow around probes of various configurations installed at radii of $40 \mathrm{~mm}$ and $13 \mathrm{~mm}$. It is clearly seen that the introduction of probes even at the minimum radius does not disrupt the pinching process and does not lead to a decrease in the neutron yield. It also follows from the above interferograms that the level of disturbance introduced by the planar probe is about half an order of magnitude less than in the case of a cylindrical probe. Moreover, in the case of the planar probe, these disturbances fade faster.

A magneto-optical probe (Figure 34) was developed to study the change in the structure of the PCS (the "shock wave-magnetic piston" structure) during its convergence to the axis. The optical signal was associated with the passage of the shock wave over the probe position, and the electrical signal from the magnetic channel was associated with the passage of the magnetic piston. 


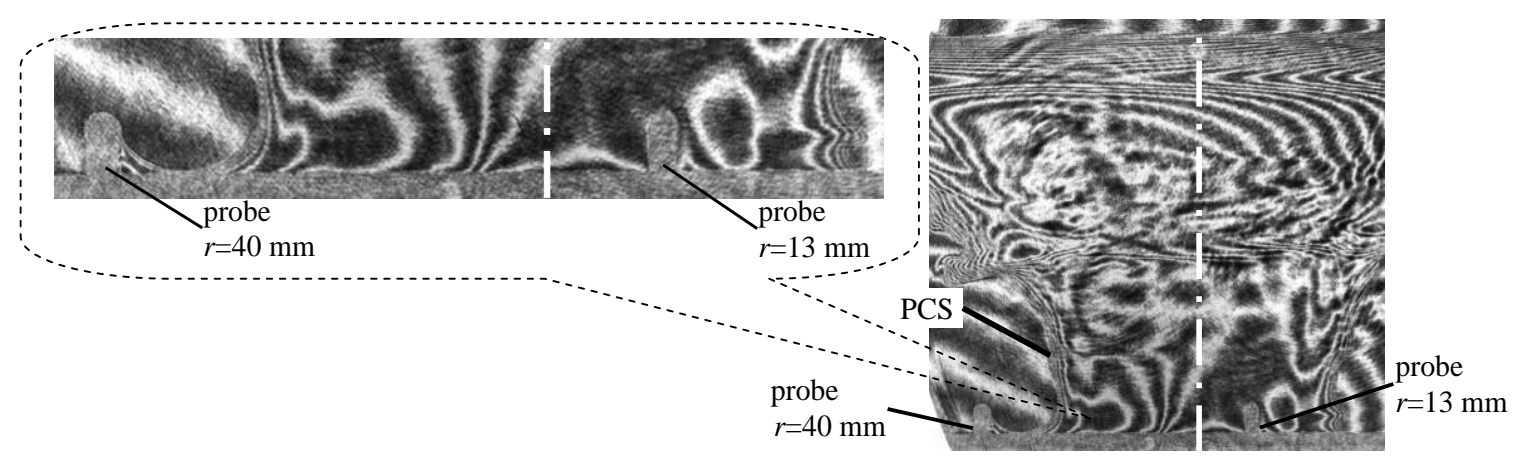

(a)

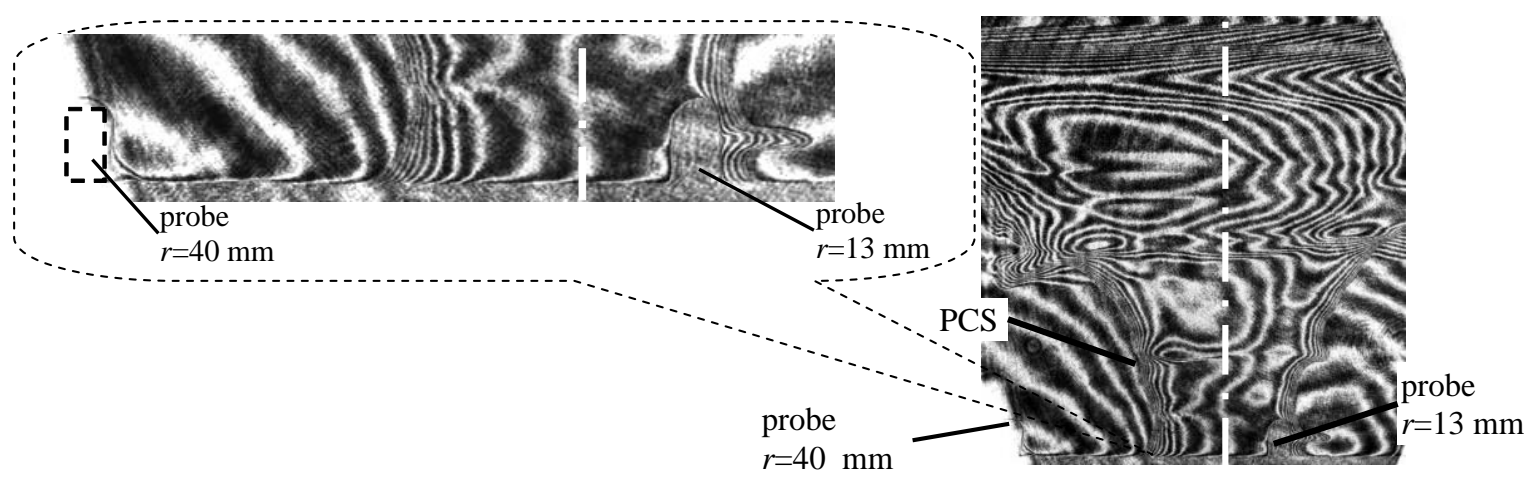

(b)

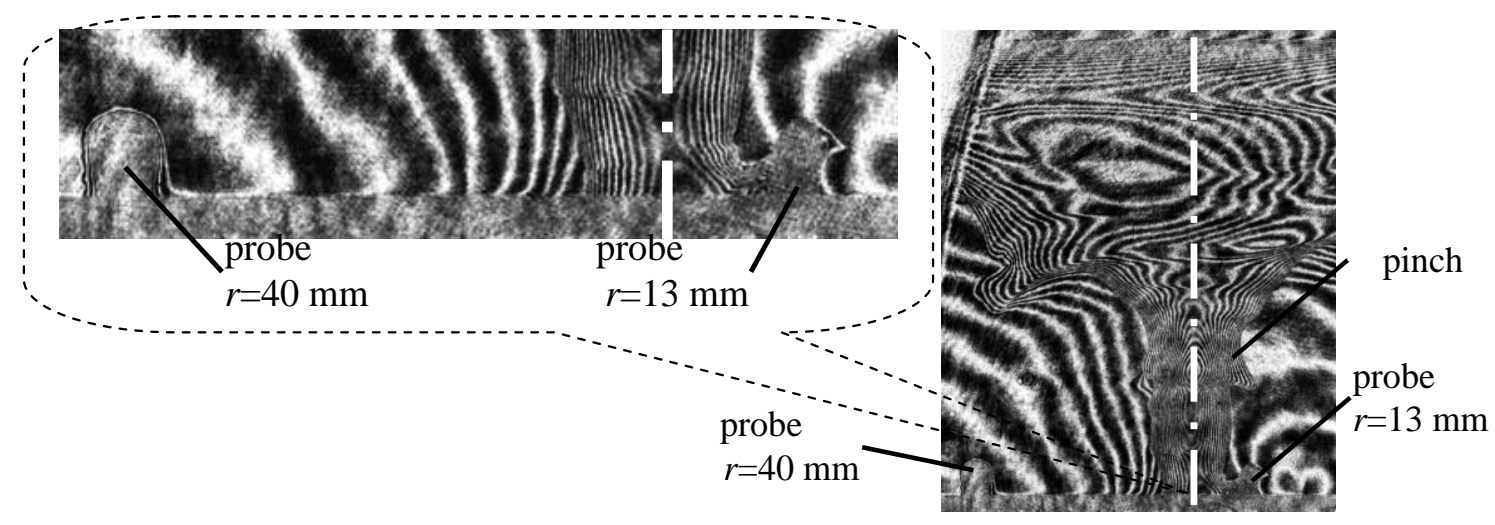

(c)

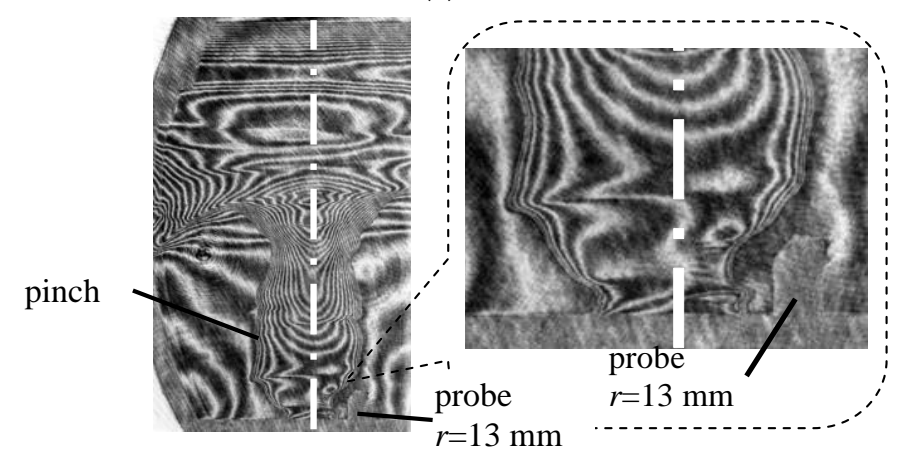

(d)

Figure 32. The interferograms in the near-axis region at different instants of time from the discharge beginning: (a) $5781 \mathrm{ns,}$ (b) $5841 \mathrm{~ns}$, (c) $5901 \mathrm{~ns}$, and (d) $5931 \mathrm{~ns}$. Plasma flow around the probe with a 2.5-mm-diameter cylindrical ceramic tube. Gaseous $\mathrm{D}_{2}, \mathrm{P}_{0}=1.2$ Torr, $\mathrm{U}_{0}=24 \mathrm{kV}, \mathrm{W}_{0}=384 \mathrm{~kJ}, \mathrm{Y}_{\mathrm{n}}=3.2 \times 10^{10}$ neutrons/pulse. Compiled with permission from Figure 10 of [205] (Instrum. Exp. Tech. 2018, 61, pp. 239-259, (c) 2018. Pleiades Publishing, Ltd.) and Figure 5 of [209] (Plasma Phys. Rep. 2014, 40, 623-639 (c) 2014. Pleiades Publishing, Ltd.). 


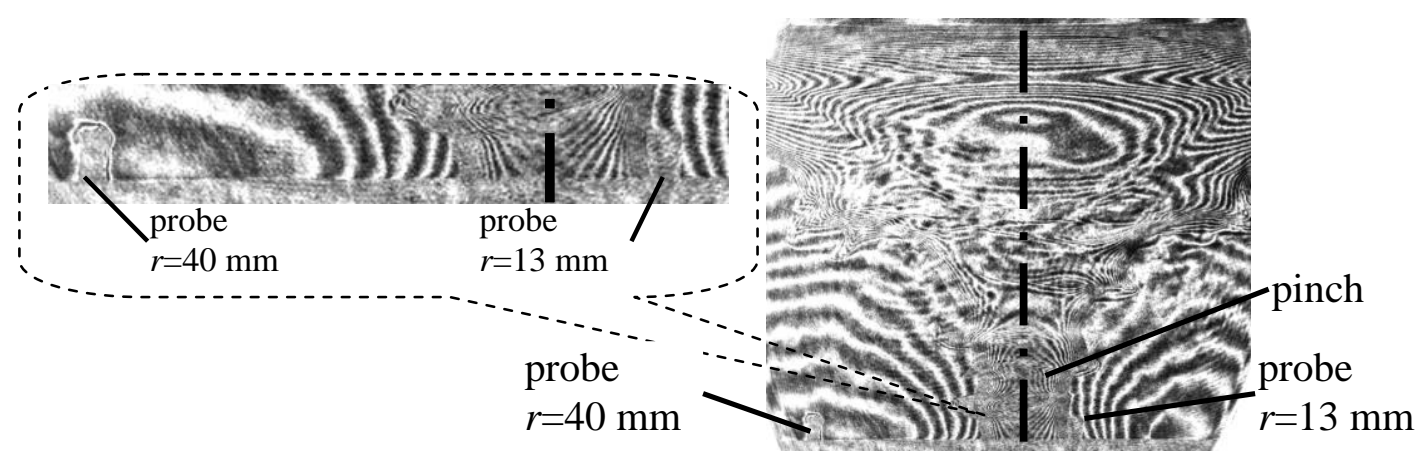

(a)

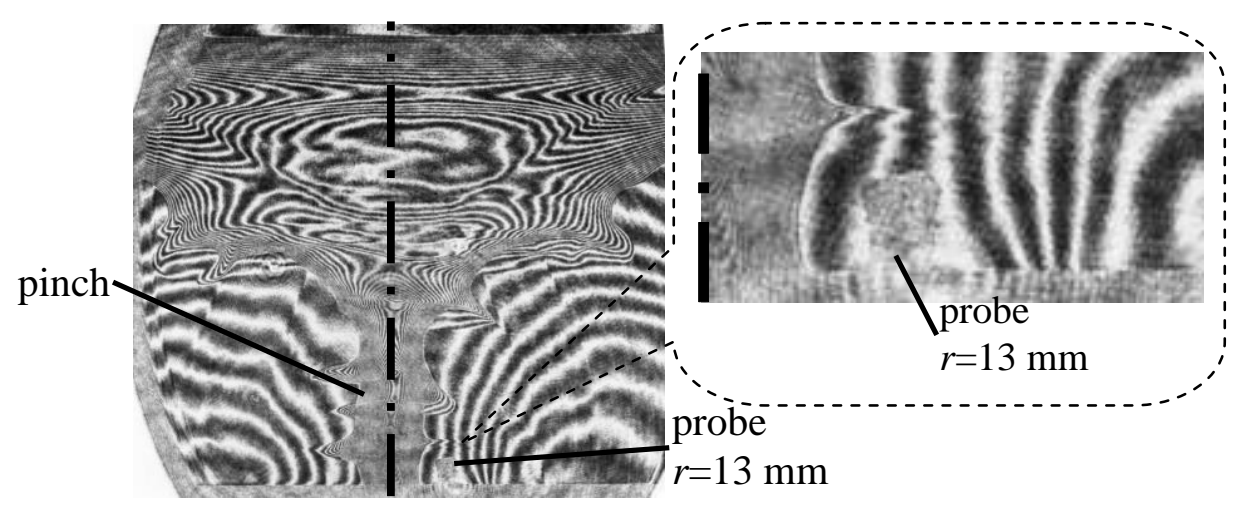

(b)

Figure 33. Interferograms of the PCS in the near-axis region at different instants of time from the discharge beginning: (a) $6348 \mathrm{~ns}$ and (b) $6378 \mathrm{~ns}$. Plasma flow around the probe with a flat mica case coated with a 15-20- $\mu$ m-thick NbTi foil. Gaseous $\mathrm{D}_{2}, \mathrm{P}_{0}=1.8$ Torr, $\mathrm{U}_{0}=24 \mathrm{kV}, \mathrm{W}_{0}=384 \mathrm{~kJ}$, and $\mathrm{Y}_{\mathrm{n}}=8.6 \times 10^{10}$ neutrons/pulse. Reproduced with permission from Figure 14 of [205] (Instruments and Experimental Techniques. 2018. Vol. 61. pp. 239-259, (c 2018. Pleiades Publishing, Ltd.).

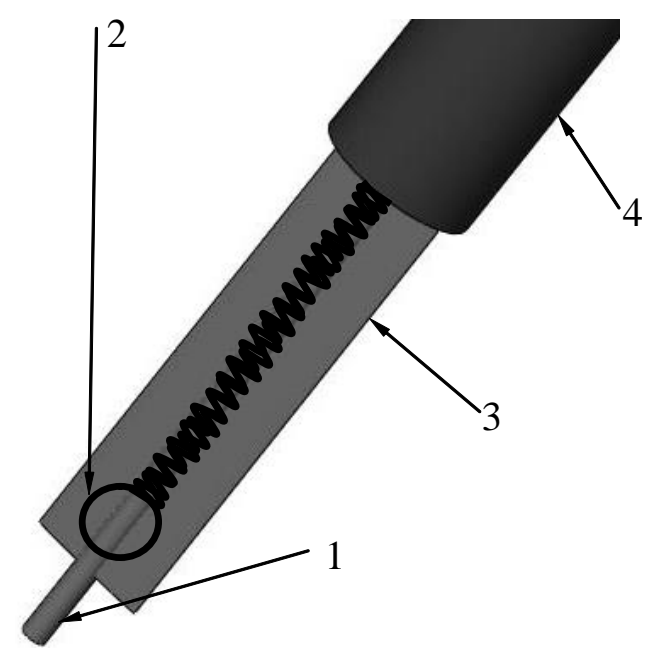

Figure 34. Design of the magneto-optical probe: (1) 0.38-mm-diameter optical fiber, (2) two coils for measuring the magnetic field, (3) 2-mm-diameter ceramic tube, and (4) 2.5-mm diameter copper tube. Reproduced with permission from Figure 2 of [211], (Plasma Physics Reports, 2011, 37, pp. 742-754). (C) 2011. Pleiades Publishing, Ltd.

The magneto-optical probe included a 0.38 core diameter optical fiber in addition to the two $0.3 \mathrm{~mm}$ diameter coils oriented for measuring azimuthal magnetic field. The optical fiber and coils were protected by a $\sim 2 \mathrm{~mm}$ diameter ceramic tube (see Figure 2 of [210], Figure $1 \mathrm{~b}$ of [207]). The optical fiber protruded outside the ceramic tube by $\sim \mathrm{mm}$ into 
the plasma. The optical glow of plasma passing over the optical fiber was detected by a photomultiplier tube whose signal was recorded on the same oscilloscope as the magnetic probe signal to generate data on the relative position of the plasma and the magnetic piston. The spectral sensitivity of the photomultiplier cathode determines the spectral range of the recorded optical radiation of 300 to $600 \mathrm{~nm}$. When processing the signals, the delay times of the cables, the transit time of photons through the optical fiber, and the duration of the photomultiplier dark phase are taken into accoumt [211].

A combination magneto-optical probe was designed [212] to measure both azimuthal $\left(\mathrm{B}_{\varphi}\right)$ and axial $\left(\mathrm{B}_{\mathrm{z}}\right)$ components of magnetic field at the same location in relation to the optical signal from the plasma (see Figure 1 of [212]). The probe had a 0.38 diameter optical fiber in the center that axially protruded $1 \mathrm{~mm}$ outside the body of the probe. The $\mathrm{B}_{\varphi}$ probe was a $0.9 \mathrm{~mm}$ diameter single turn coil. The $B_{z}$ probe used $4-10$ turns of a $0.9 \mathrm{~mm}$ diameter coil. The main physical protection of the probe was a $2 \mathrm{~mm}$ diameter ceramic tube. Additional electrical shielding of the $B_{z}$ probe consisted of two cylindrical copper shields, each of which had an axial slit but the two slits were on opposite sides. This provided a partial shielding to the $B_{z}$ probe from the $B_{\varphi}$ field of the plasma, as well as provided electrostatic shielding. Axial separation of $5-8 \mathrm{~mm}$ was provided between the $B_{z}$ probe and the $\mathrm{B}_{\varphi}$ probe to reduce capacitive cross-talk between the channels. Absolute calibration of the $\mathrm{B}_{z}$ and $\mathrm{B}_{\varphi}$ probes was performed using the homogeneous field of a Helmholtz coil with a magnetic field oscillating at $\sim 7 \mathrm{MHz}$. This set up was also used to measure the sensitivity of $B_{z}$ channel to $B_{\varphi}$ and $B_{\varphi}$ channel to $B_{z}$ so that the parasitic contribution of the unwanted field to the desired field could be quantified.

\subsubsection{Dynamic Inductance Measurement}

A technique for the determination of the inductance evolution in coaxial discharges based on measurements of the voltage between the electrodes and of the discharge current time derivative, previously proposed by Mather and Bottoms in 1967 [213] has been developed further and widely used by Bruzzone et al. [214]. A Rogowski coil is used for the measurement of the current derivative and a resistive voltage divider is used for voltage measurement. The salient features of the method are described with reference to Figure 35 .

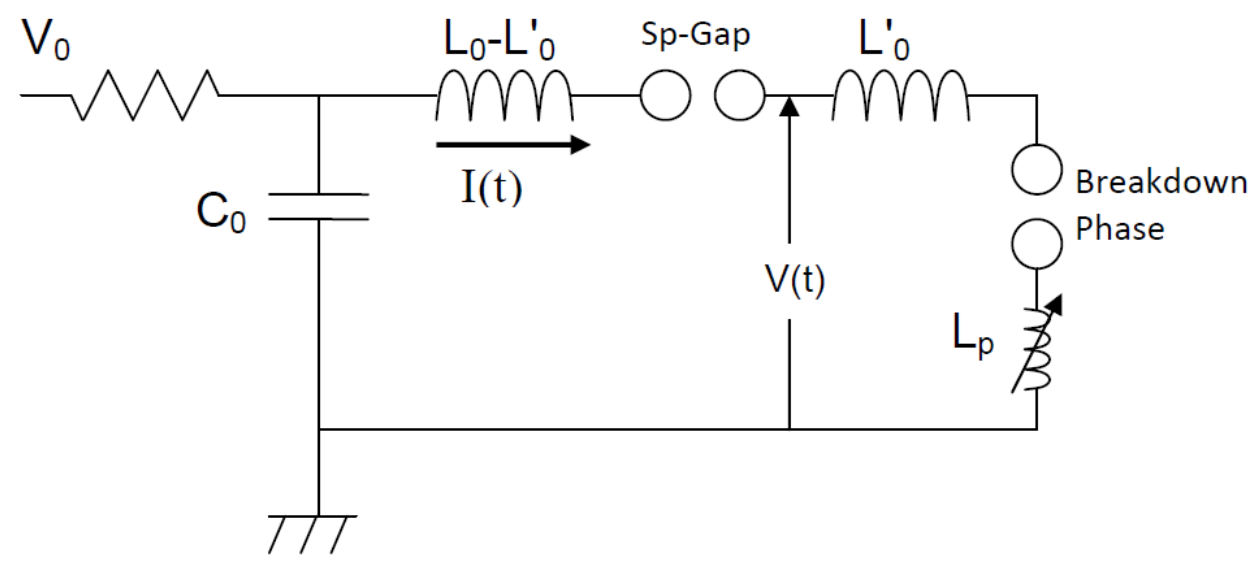

Figure 35. Circuit schematic used in [214]. $\mathrm{L}_{\mathrm{p}}$ is the gun inductance.

The key step in this procedure is the determination of the inductance of connections $\mathrm{L}_{0}{ }^{\prime}$ between the voltage divider and the discharge. This is done by making several short circuit shots (with constant inductance) and determining the proportionality constant between the calibrated voltage and current derivative signals by regression. This method makes use of the fact that after the initiation of the discharge, the circuit resistance is much 
less than the circuit impedance so that the measured voltage can be taken to be inductive, that is,

$$
\mathrm{V}(\mathrm{t})=\frac{\mathrm{d}}{\mathrm{dt}}\left(\left(\mathrm{L}_{0}^{\prime}+\mathrm{L}_{\mathrm{p}}(\mathrm{t})\right) \mathrm{I}(\mathrm{t})\right)
$$

This is inverted to get

$$
L_{p}(t)=\frac{\int_{t_{9}}^{t} V(t) d t+\left(L_{p}\left(t_{0}\right)+L_{0}{ }^{\prime}\right) I\left(t_{0}\right)}{I(t)}-L_{0}{ }^{\prime}
$$

where $t_{0}$ is the time of the first peak of the current derivative signal. This method has been applied also for PF-1000 device which is discussed in a separate section (Section 4.2.3).

The inductance measurement allows estimation of the plasma voltage at pinch time (see Section 4.2.3). The mechanical energy of the plasma at different stages of discharge [215] can be estimated from the relation

$$
E_{m}(t)=\int_{0}^{t} \frac{1}{2} \dot{L}_{p}(t) I^{2}(t) d t
$$

Correlation between neutron yield per particle-pair and mechanical energy per particle at the pinch time has been studied [215]. The shape of the inductance evolution curve allows identification of the beginning of the plasma lift-off from the insulator and radial implosion phases from which axial and radial velocities can be estimated [216].

\subsubsection{Double Wollaston Prism Laser Differential Interferometer}

M. F. Lu has developed [217] a double-Wollaston-prism Laser Differential Interferometer for plasma focus diagnostics which produces fringe shifts proportional to the gradient of plasma density. It uses a linearly polarized Nd-YAG laser pulse of FWHM 10 ns and $15 \mathrm{~mJ}$ energy in the second harmonic at $533.2 \mathrm{~nm}$ as light source and ISO 400 high speed panchromatic film for recording the interferogram. For a typical sheath thickness of $1.6 \mathrm{~mm}$ and a minimum detectable fringe shift of $5 \%$, the sensitivity of the interferometer for plasma density gradient $\mathrm{n}_{\mathrm{e}}^{\prime}$ is about $(2.58 \pm 0.46) \times 10^{25} \mathrm{~m}^{-4}$ which is equivalent to a lower limit of detectable plasma density $n_{e}$ of about $(3.25 \pm 0.59) \times 10^{22} \mathrm{~m}^{-3}$. Because of the rather large pulse duration, the fringes are significantly blurred. This hampers a quantitative evaluation of the plasma density gradients.

\subsubsection{Coded Aperture Imaging of Fusion Protons}

Single pinhole proton imaging of the plasma focus $\mathrm{d}-\mathrm{d}$ fusion reaction zone for individual shots is feasible only for large PF devices with their associated high proton yields, which leads to a large number of protons passing through the single pinhole and forming an image with good definition. For example, Jäger et al. [218] reported experimental results of single pinhole proton imaging for the POSEIDON PF, with stored energy of $280 \mathrm{~kJ}$ and an average proton (and neutron) yield of $\sim 10^{11}$ per shot. However, for moderate energy PF devices with a proton yield of $\sim 10^{8}$ per shot, a single pinhole produces very noisy images due to the low throughput of protons. Using a larger area pinhole increases the number of collected protons, but at the cost of sacrificing image resolution. To overcome these limitations, the coded aperture imaging (CAI) technique was implemented $[219,220]$ to image the fusion reaction zone for the $1.6 \mathrm{~kJ}$ NX2 PF operated in pure $\mathrm{D}_{2}$ gas, or $\mathrm{D}_{2}-\mathrm{Kr}$ gas admixture [221,222], as discussed further in Section 4.3.2.

CAI enables both goals of high proton throughput and good image resolution to be attained simultaneously by using many pinholes (of equal size) arranged in a specific mathematical pattern known as a cyclic difference set (CDS). The number of protons forming the image increases in proportion to the number of mask holes (hence improving the signal to noise ratio, SNR), while the spatial resolution remains equal to that obtained 
for a single pinhole. The CAI technique is especially suitable for imaging 'difficult-to-focus radiation', e.g., gamma-rays and protons. Image formation of a radiating object is achieved via a two-step process: incoming rays are encoded by a mask before they reach the detector plane, then the spatial distribution of detected radiation is decoded computationally to reconstruct an image of the source [223]. In experimental terms, the coded aperture mask represents the physical embodiment of the CDS pattern: either transmitting, or stopping completely, each impinging proton. The coded mask patterns used in [219-222] are based on Singer CDS. Pulsed-laser machining was used to fabricate the coded masks in metal foil of sufficient thickness to stop $\sim 3 \mathrm{MeV}$ DD fusion protons. Among the different CDS employed for CAI, Singer CDS offer the highest SNR by allowing optimization of the openarea fraction to suit the number of object pixels (i.e., size of the object). Shutler et al. [224] studied the relationship between SNR and mask open-area fraction. It was found that, in the presence of background noise, Singer masks with just three different open-area fractions can achieve $95 \%$ of the optimal SNR, as compared to $70 \%$ for half-open masks (e.g., MURA masks).

For initial experiments [219], the coded Mask-400 was fabricated in $50 \mu \mathrm{m}$ thick Havar alloy with $20 \times 20$ pixels with 57 square holes of $400 \mu \mathrm{m}$ side $\left(9.12 \mathrm{~mm}^{2}\right.$ total open area) using a Singer-CDS pattern. These experiments are described in greater detail elsewhere [220]. Upgraded experiments followed [221,222], which used two CAI cameras positioned at $60^{\circ}$ to one another (in adjacent planes of a hexagonal holder) to capture sideon views of the fusion source simultaneously for the same PF shot. In order to validate the deconvolution procedures, the two cameras employed different Singer-CDS mask patterns, denoted Mask-1365 and Mask-4680. Figure 6.36 from Reference [222] shows details of the masks in their holder, and their position with respect to the PF anode. Mask-1365 has overall dimensions of 27.3 by $25.5 \mathrm{~mm}$ with space-factor of 6 comprising $91 \times 15$ pixels (300 $\mu \mathrm{m}$ side) and 341 holes (30.69 $\mathrm{mm}^{2}$ open area). Mask-4680 has overall dimensions of 28.1 by $24.6 \mathrm{~mm}$ and space-factor of 2 comprising $104 \times 45$ pixels $(270 \mu \mathrm{m}$ side) and 585 holes (42.65 mm² open area). Both masks were fabricated in $50 \mu \mathrm{m}$ thick stainless steel. In their experimental positions, Mask-1365 and Mask-4680 present open solid angles of $1.32 \times 10^{-2}$ and $2.15 \times 10^{-2} \mathrm{sr}$, respectively, for source points near the axis of the plasma pinch. These solid angles are a factor of $\sim 100$ greater than that for the single pinhole POSEIDON experiments [218], which had open solid angle of $1.46 \times 10^{-4} \mathrm{sr}$.

CR-39 polymer nuclear track detectors of size of $67 \mathrm{~mm} \times 77 \mathrm{~mm}$ were placed in the detector plane. The source-mask and mask-detector distances were $44 \mathrm{~mm}$ and $40 \mathrm{~mm}$, respectively, giving a field of view (FOV) in the (plasma) source plane of $28 \mathrm{~mm} \times 17 \mathrm{~mm}$. The CR-39 was covered by $75 \mu \mathrm{m}$ thick polyamide film to stop all energetic charged particles (mostly deuterons) other than the $\sim 3 \mathrm{MeV}$ DD fusion protons. Optimized etching protocols were developed to obtain etched proton tracks that were large enough (20 to $30 \mu \mathrm{m}$ diameter) and dark enough for easy recognition by optical microscope, and easily distinguishable from flaws on the CR-39 surface. The CCD sensor attached to an Olympus BX51 microscope with MPlan $\times 20$ objective lens, had a $528 \times 378 \mu \mathrm{m}$ field of view of the CR-39 surface. A motorized microscope stage with laser auto-focusing and image processing software was used to capture more than 25,000 microscope images for each CR-39 detector. A track recognition algorithm based on correlations between five measured parameters including area, average brightness, roundness, were used to discriminate between genuine proton tracks and spurious features, and output an $(x, y)$ coordinate list for recognized proton tracks. A de-convolution algorithm was then applied to this $(x, y)$ list of to obtain the fusion source image. Results are discussed in Section 4.3.2.

\subsubsection{Quasi-Average Neutron Energy Measurement Using Zirconium and Beryllium Pair} Activation Detectors

Neutron time-of-flight (n-TOF) measurements performed on various PF devices are described in several sections of this review. Such measurements aim to obtain neutron energy spectra for many individual PF shots in one (or more) directions relative to the $\mathrm{PF}$ anode-typically, the axial and radial directions $\left(0^{\circ}\right.$ and $\left.90^{\circ}\right)$. These energy spectra 
can reveal information about the energy and direction of the deuterons involved in the $\mathrm{d}-\mathrm{d}$ fusion reaction. Neutron energy measurements represent some of the most important experiments performed in the early days of PF research, revealing that fusion within the $\mathrm{PF}$ is not thermonuclear in origin. Bernard et al. [179] used a flight path of $120 \mathrm{~m}$ and large volume plastic scintillator detectors at $0^{\circ}$ and $90^{\circ}$, simultaneously, to attain $\Delta E_{\mathrm{n}}<0.1 \mathrm{MeV}$ energy resolution. A long flight path at $0^{\circ}$ usually requires that the PF axis be horizontal. e.g., for the 1 MJ Frascati PF [180], n-TOF was performed with an $128 \mathrm{~m}$ path in the $90^{\circ}$ direction only, as the PF axis was vertical. However, clearly, such long flight paths are only possible for large experimental facilities, especially so if $0^{\circ}$ and $90^{\circ} \mathrm{n}$-TOF are to be performed simultaneously.

Springham et al. [225] have introduced a new technique which enables an effective neutron energy $E_{\mathrm{n}}^{\text {eff }}$ to be measured at $0^{\circ}$ and $90^{\circ}$ simultaneously. This is done using pairs of zirconium and beryllium fast neutron activation (FNA) detectors. $E_{n}^{\text {eff }}$ can be regarded as a quasi-average neutron energy for the burst of $d-d$ neutrons. This new technique relies on the different trends with energy of the nuclear activation cross-sections $\sigma_{\mathrm{Zr}}^{\mathrm{a}}\left(E_{\mathrm{n}}\right)$ and $\sigma_{\mathrm{Be}}^{\mathrm{a}}\left(E_{\mathrm{n}}\right)$. This enables $E_{\mathrm{n}}^{\text {eff }}$ to be inferred from the $\mathrm{Zr} /$ Be detector count ratio $\left(C_{\mathrm{nZr}}^{\theta} / C_{\mathrm{nBe}}^{\theta}\right)$ for each PF shot. Operation of the zirconium and beryllium activation detectors are fully described in papers $[225,226]$. The $\mathrm{Zr} /$ Be detector Pairs (ZBP) are sufficiently compact that two of them can be used simultaneously at $\theta=0^{\circ}$ and $90^{\circ}$. Figure 36 shows a schematic of the experimental setup drawn to scale.

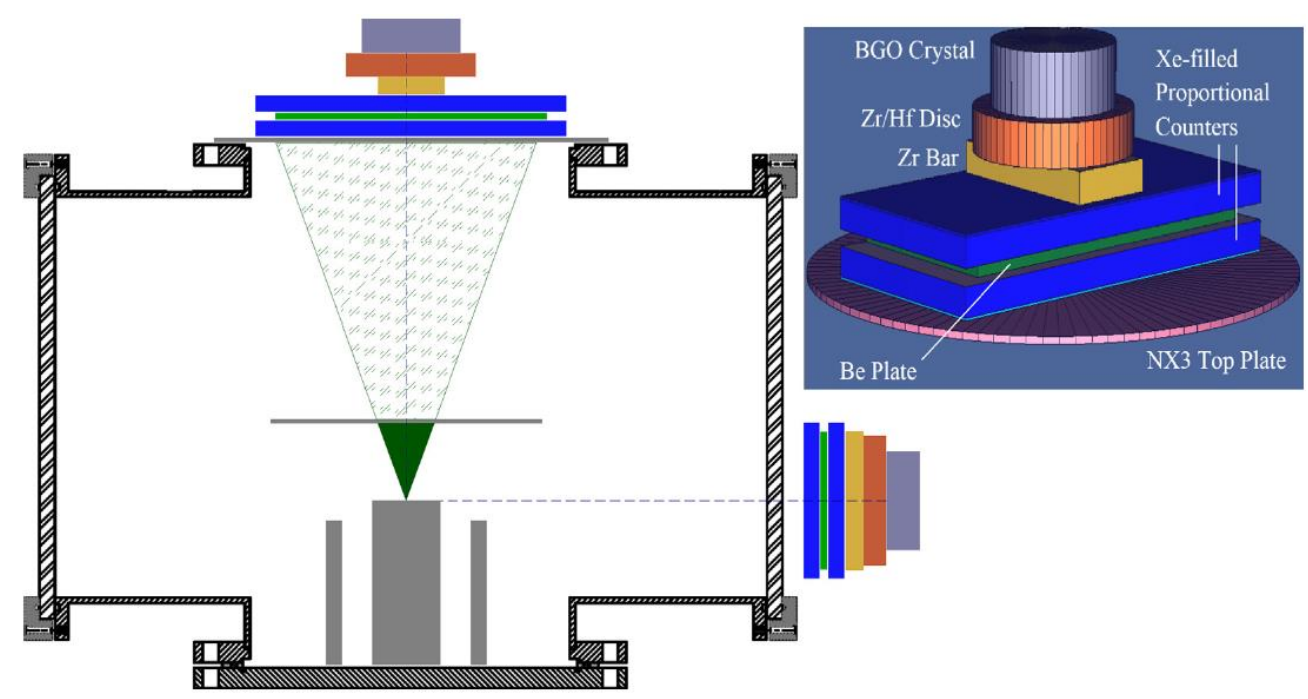

Figure 36. This shows a schematic diagram of the NX3 vacuum chamber with Zr and Be FNA detector-pairs (drawn to scale) positioned immediately adjacent to it, at $0^{\circ}$ and $90^{\circ}$ orientations. As an example, a $20^{\circ}$ half-angle deuteron beam emitted from the pinch is shown with the obstacle plate (OP) $6 \mathrm{~cm}$ from the anode top. Reprinted from Figure 2 of [225] (Springham et al. NIMA 2021988 164830). () (2021) with permission from Elsevier.

Neutron activation of beryllium occurs via the ${ }^{9} \mathrm{Be}(\mathrm{n}, \alpha)^{6} \mathrm{He}$ reactions in a Be metal plate, producing a population of ${ }^{6} \mathrm{He}$ nuclei which undergo beta-decay with $0.807 \mathrm{~s}$ halflife. Neutron activation of zirconium occurs via inelastic neutron scattering producing a population of metastable ${ }^{90 \mathrm{~m}} \mathrm{Zr}$ nuclei with $0.809 \mathrm{~s}$ half-life. Such similar $(<1 \%$ difference) half-lives for the two activation products $\left({ }^{6} \mathrm{He}\right.$ and ${ }^{90 \mathrm{~m}} \mathrm{Zr}$ ) makes it possible to use the same 3.0 s counting-interval, in the pulse processing electronics, to accumulate the gross count for each of the four detectors ( 2 zirconium and 2 beryllium). The most probable gamma-ray emissions from ${ }^{90 \mathrm{~m}} \mathrm{Zr}$ is $2.319 \mathrm{MeV}(82 \%)$. ${ }^{90 \mathrm{~m}} \mathrm{Zr}$ de-excitation gamma-rays are detected by a BGO scintillator in close proximity to the zirconium target. The activation secondary radiations of Be and $\mathrm{Zr}$ are easily discriminated from each other and measured separately using pulse height analysis counting methods with standard nuclear electronics. 
The authors discuss the possibility of 'cross-talk' between the detectors and show that its influence is negligible.

The $\mathrm{Zr}$ and Be detector response functions were characterized using MCNP5 simulations over the neutron energy range of 2.3 to $3.6 \mathrm{MeV}$ at $0.1 \mathrm{MeV}$ intervals. In these simulations, an isotropic point mono-energetic neutron source was positioned at the center of the anode tip. The simulation included neutron energy, geometry of source, chamber, and detector, material compositions and masses, $\gamma$-ray energies and branching ratios, ${ }^{6} \mathrm{He}$ $\beta$-particle energy spectrum, and Gaussian energy broadening (simulating the BGO energy resolution). Simulations were also performed to determine the spatial distributions of the active nuclides ${ }^{6} \mathrm{He}$ and ${ }^{90 \mathrm{~m}} \mathrm{Zr}$ within the respective metal targets and to determine what fraction of: (i) ${ }^{6} \mathrm{He}$ beta-decays are detected by proportional counters. (ii) ${ }^{90 \mathrm{~m}} \mathrm{Zr} \gamma$-ray de-excitations produce BGO pulses within the energy window. Figure 37 shows the results of the simulations.

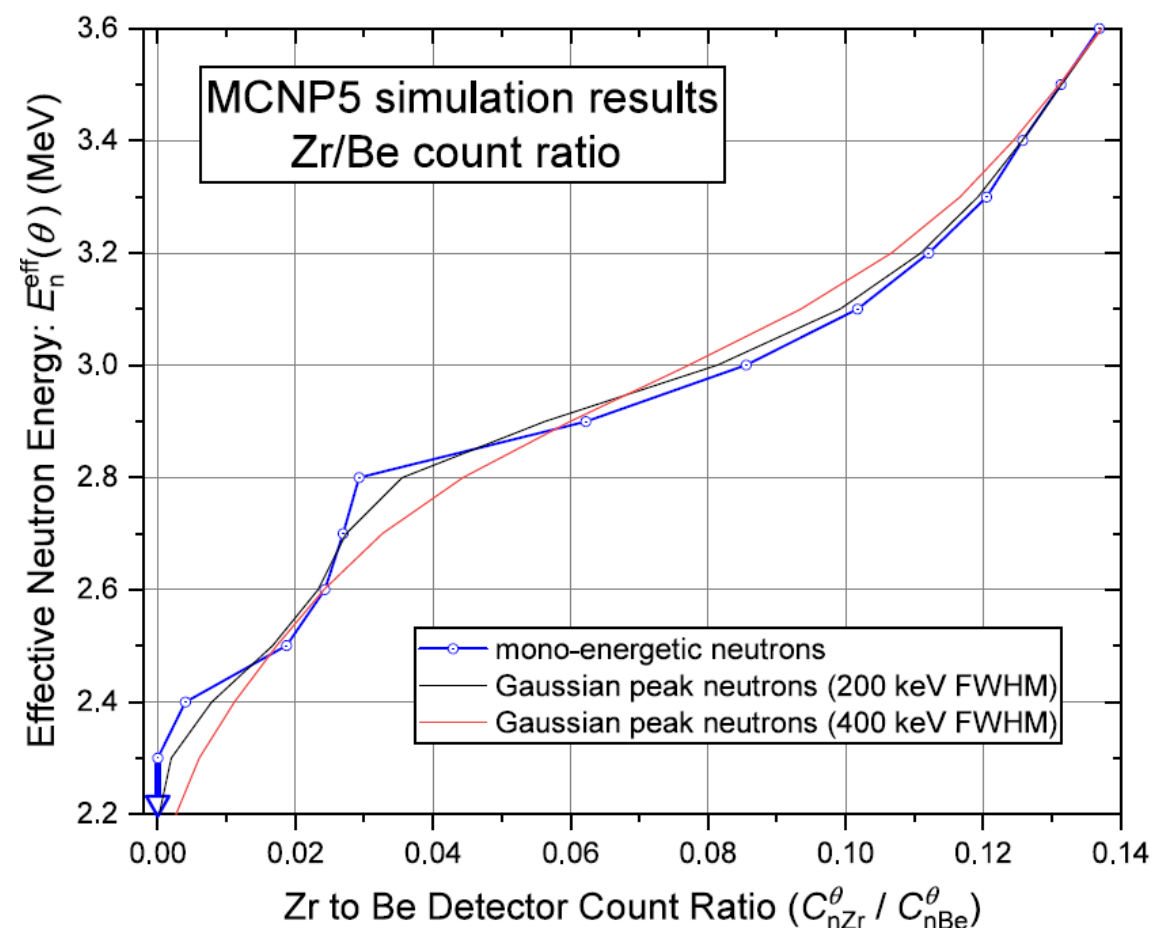

Figure 37. Graph of MCNP5 simulation results for effective neutron energy versus $\mathrm{Zr}$ to Be detector count ratio. The neutron energies are obtained in each shot from count ratios obtained for $0^{\circ}$ and $90^{\circ}$ by interpolation on this graph. Reprinted from Figure 5 of [225] (Springham et al. NIMA 988 (2021) 164830). (C) (2021) with permission from Elsevier.

In order to minimize effect of the discharge-produced transient RF noise, the pulse processing electronics and computer were located inside a large screen-cage. Pulses corresponding to BGO gamma-ray deposited energy in the range 0.40 to $2.60 \mathrm{MeV}$ were counted by a scaler. The scaler was triggered by a gate pulse delayed by $10 \mathrm{~ms}$ with respect to the trigger output of an oscilloscope used to record the current derivative pulse of the discharge. This delay allowed " ... various forms of interference (hard x-rays, discharge EM noise, and prompt $\gamma$-rays from neutron captures) to die away before the [start of the] $=3.0 \mathrm{~s}$ counting interval".

The results from this diagnostics are discussed in Section 4.3.2 (8).

\subsubsection{Digital Interferometry}

Digital interferometry is a new refractive optical technique applied to plasmas produced by PF discharges. This technique consists of digital recording of three microinterferometric patterns of parallel fringes of high spatial frequency (15-20 lines/mm 
or higher, depending on the CCD resolution), one with a phase object information and two reference records [227]. One of the reference micro-interferograms is constructed with the same carrier frequency as the micro-interferogram with the phase object information. The second reference is constructed with a slightly higher carrier frequency, controlled by the optical arrangement (see Figure 38). The digital records are then numerically processed. The image (the pixel matrix) of the micro-interferogram with the plasma is subtracted point by point with one of the images of the reference micro-interferograms. The resulting image contains interference fringes of high frequency modulated by a fringe pattern which are related to the variation of the optical path length, which is, in turn, related to the refractive index of the plasma. The high-frequency fringes are easily removed using a digital low-pass filter [227]. This process constructs interference patterns with fringes of finite and infinite width. This is similar to an optical holographic interferometry technique [228] for viewing small-sized inhomogeneities in a much larger phase object, but instead of each exposure occurring on the same holographic plate, the recording is done in consecutive captures of a digital acquisition system (such as a commercial digital SLR camera). This technique, therefore, contains many of the attributes of optical holographic interferometry, with the additional versatility of digital processing tools (e.g., spatial filtering). For example, a homogeneous interferometric fringe patterns of parallel fringes can be formed over a wide region of observation, which facilitates the subsequent interferometric analysis.

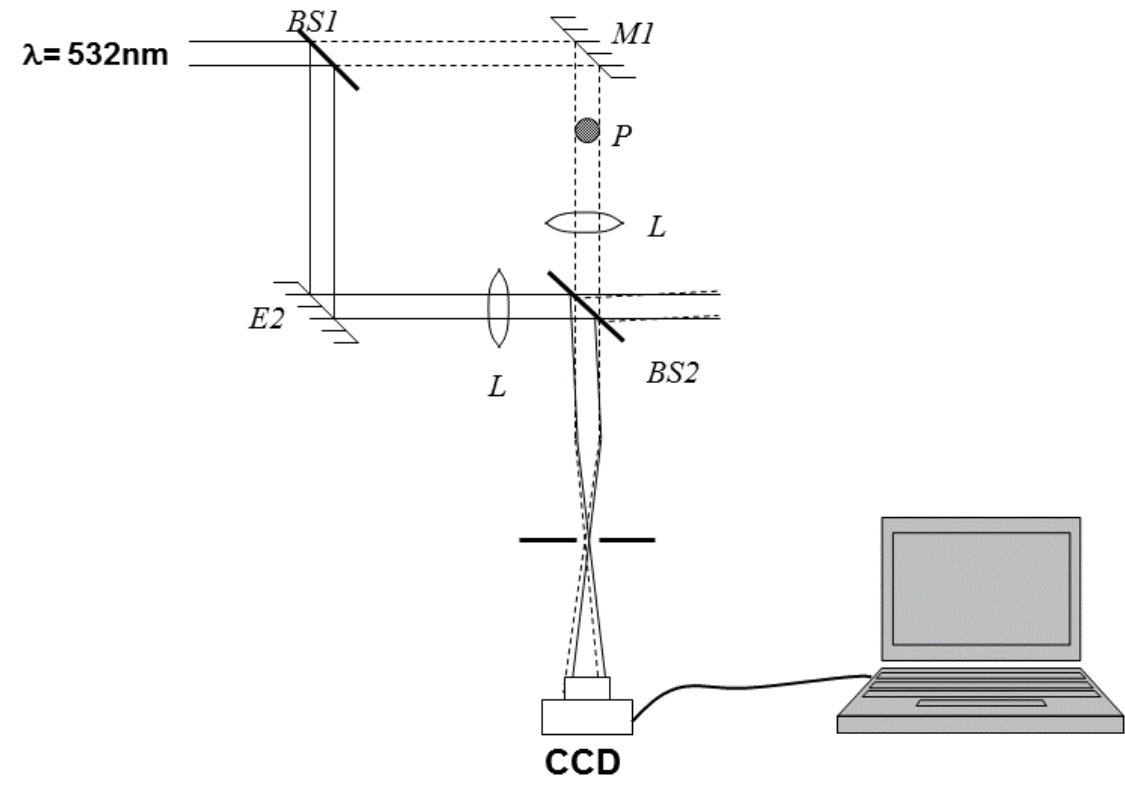

Figure 38. Optical setup for recording micro-interferograms for digital interferometry.

The optical setup is schematically shown in Figure 38. This is based on a MachZehnder interferometer, which uses two lenses of equal focal length $\mathrm{L}$, one in each arm of the interferometer. One of them forms the image of the plasma on the recording system (CCD or CMOS) and the other one produces the same curvature of the wavefront of reference beam at the recording surface. Both lenses are at the same distance from the recording system. This lens arrangement allows better control of the parallelism, frequency, and orientation of fringes at the focal plane.

An application of digital interferometry to study a plasma jet generated in a small plasma focus discharge, in the post-disruption phase of the column is shown in Figure 39. For this purpose, the PF-50J generator, synchronized with a pulsed Nd:YAG laser $(\lambda=532 \mathrm{~nm}, 6 \mathrm{~ns}$ FWHM) and a Canon digital SLR camera, model Rebel Xsi, with a CMOS size of $14.8 \mathrm{~mm} \times 22.2 \mathrm{~mm}(5.2 \mu \mathrm{m}$ pixel size) was used [229] as a recording system with the shutter open for a few seconds. This kind of device has been found to have an electronically robust performance under intense electromagnetic noise, such as those produced in facilities with plasma pulsed generators. 


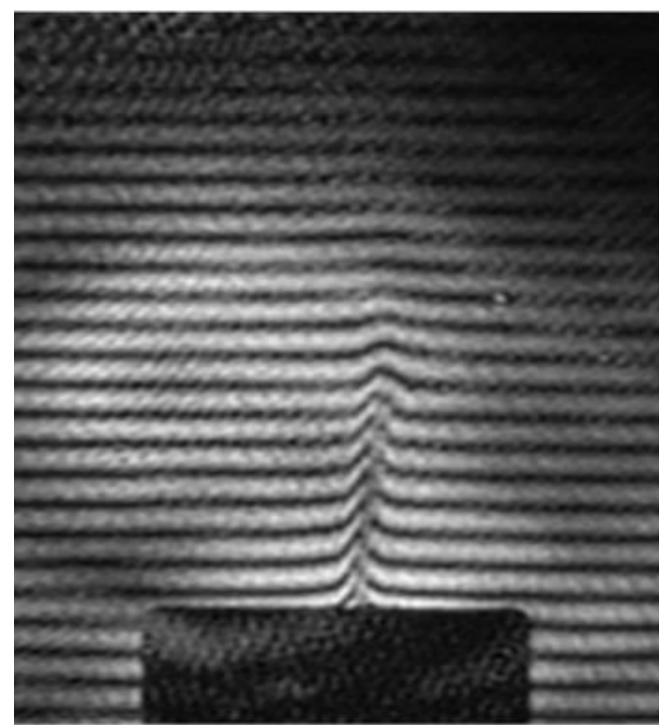

(a)

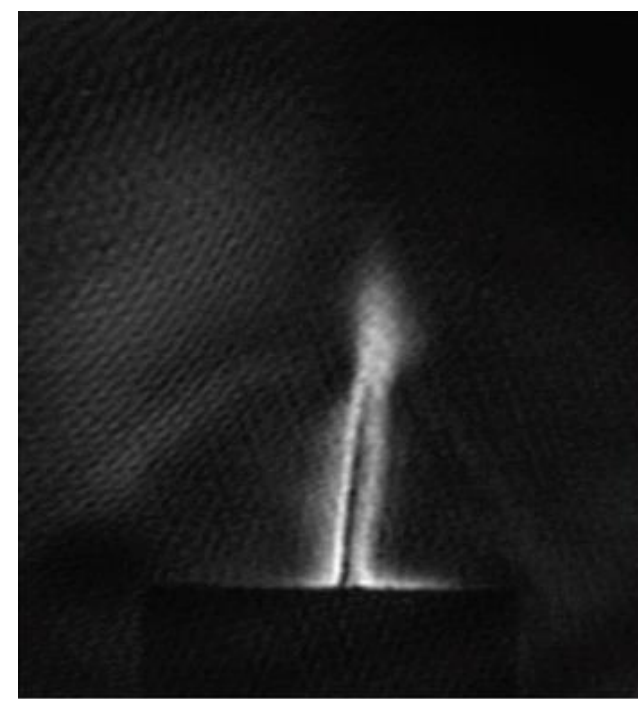

(b)

Figure 39. Digital interferograms of a plasma jet generated at the small plasma focus PF-50J. (a) Interferograms in fringes of finite width, (b) interferograms in fringes of infinite width [70]. The anode top is visible in (a) with a diameter of $6 \mathrm{~mm}$ which provides the spatial scale. Adapted from Figure 4 of [229] (Phys. Plasmas 2015, 22, 040705) with the permission of AIP publishing.

Figure 39a shows an interferogram in fringes of finite width and (b) shows an interferogram in fringes of infinite width for the same event [229]. In Figure 39b the phase-shift mapping is clearly distinguishable. From the analysis of the consecutive temporal interferograms it was possible to estimate average axial velocities of $(4.2 \pm 0.9) \times 10^{4} \mathrm{~m} / \mathrm{s}$ and areal densities ranging $(4-6) \times 10^{17} \mathrm{~cm}^{-2}$. Additionally, the electron density for the jets was measured to be in the range of $10^{24}$ to $10^{25} \mathrm{~m}^{-5}$ [229].

This technique has been extended [230] to digital reconstruction of an interferogram, a schlieren image, or a shadowgram from a single digital holographic recording using Fresnel-Kirchhoff transform.

\subsubsection{Low Neutron Yield Detection}

Miniaturized plasma foci require neutron detection technique capable of detecting pulses with less than $10^{5}$ neutrons per pulse. For neutron yields less than $10^{6}$ neutron/pulse, the well known techniques (activation counter, bubble counter system, etc.) are not effective. A conventional neutron detection technique was adapted to measure low neutron yields from d-d fusion pulses [231]. This method uses a ${ }^{3} \mathrm{He}$ proportional counter surrounded by a paraffin or polyethylene moderator. Electric signals generated in the ${ }^{3} \mathrm{He}$ tube are fed into a preamplifier. The output of the preamplifier is directly connected to a digital oscilloscope. The time-integrated signals represent the charge generated in the ${ }^{3} \mathrm{He}$ tube which is proportional to the total neutron yield. Integration time is determined by the preamplifier and moderator characteristics within some hundreds of microseconds. No meaningful neutron background counts are detected during this time window [231]. The system, previously calibrated, was used to measure the neutron yield ( $<10^{6}$ neutron/pulse) generated in a fast and very small plasma focus device designed to operate with energies of tens of joules (PF-50J) [232,233] and in a ultra-miniaturized plasma focus operating at only 0.1 joules [234]. Neutron yields as low as $10^{2}$ neutrons per pulse were measured. Details of the system and calibration were published in references [231,235]. 


\subsubsection{Plasma Sheath Dynamics Using Non-Invasive Optical Sensors}

This method is based on a lens that collects the light emitted by the plasma sheath which is detected by fast photodiodes. Two different circuit connections for the photodiodes are presented using either several or a single scope channels to record the photodiode signals [236]. The technique is applied to a hundred joules plasma focus, PF-440J, with a spatial resolution of $\sim 0.25 \mathrm{~mm}$ measuring its average propagation speeds (axial and radial), as well as its detachment time and an estimated thickness [236,237]. The setup required in this technique is easy to implement and cost-effective, and it can be applied in any plasma focus, regardless of its stored energy or operational gas.

\subsubsection{UHF Radiation}

Remote and non-invasive diagnostic of the plasma focus using antennas was developed in references [238-240]. Three different kinds of antennas tuned in the ultra high frequency range are used: monopole, vivaldi, and helical. The high frequency transients detected with the antennas were time correlated to the known inductive measurement features. The initial dielectric breakdown, later plasma pinch, and subsequent disruption are identified to be the principal phenomena detected. Signal parameter correlations between the inductive sensor and the antennas showed that the pinch phenomena can be correlated with the antenna signals. Additionally, correlation with hard X-rays emission is obtained, and deep learning and neural networks are used to improve the analysis [238,240].

\subsubsection{Hard X-ray Characterization for Radiographic Applications}

One of the applications of hard X-rays produced by plasma focus devices is flash radiography. In these applications the $\mathrm{X}$-rays outside of the discharge chamber are used. Hard X-ray emission has been studied in $\mathrm{kJ}$ and hundred joules PF devices using commercial radiographic recording system. To estimate an average energy of the $\mathrm{X}$-ray emitted by the PF devices, a mono-energetic radiation is assumed. When mono-energetic radiation interacts with an element, the classical exponential radiation decay relation through the matter is, $\mathrm{I}(\mathrm{x}) / \mathrm{I}_{0}=\exp (-\mathrm{K} \cdot \mathrm{x})$, where $\mathrm{I}(\mathrm{x}) / \mathrm{I}_{0}$ is the normalized radiation intensity after travelling a distance $x$ inside the material characterized by a linear attenuation coefficient $K$. From this relation, it is possible to obtain an effective linear attenuation coefficient $\mathrm{K}$ when different grey shades of the digitized images are linked to the $\mathrm{I}(\mathrm{x}) / \mathrm{I}_{0}$ ratio. This method provides a correlation between $\mathrm{K}$ and the $\mathrm{x}$-ray energy [241]. Using filters with different materials and $\mathrm{mm}$ thickness the method has been used to obtain an effective mean energy $\sim 70 \pm 10 \mathrm{keV}$ [242] in a kJ device. In the PF-400J device it was found to be $\sim 90 \pm 5 \mathrm{keV}$ [243], using the Curix ST-G2 AGFA system as recording media. The same method was used to characterize the $\mathrm{X}$-ray emission inside the discharge chamber from the Nanofocus device operating at $0.1 \mathrm{~J}$. Radiographs of an array of aluminum filters of 30, 45, and $60 \mu \mathrm{m}$ on HP5 Ilford film were obtained integrating 1200 shots on the film. In this case, Nanofocus, an effective mean energy of $4.3 \pm 0.3 \mathrm{keV}$ was obtained [244].

\subsection{Simple Models, Numerical Simulations, and Empirical and Analytical Insights}

Simple models make simplifying assumptions, which are á priori known to be not true, and yet produce results that mimic trends in experimental data to an astonishing degree. Their value lies in their substantial validation base which allows a reasonable expectation of experimental results to be obtained quickly with near zero cost. Thus, the Lee model code described below has been used for designing plasma focus facilities for many decades and has had a significant impact on the direction of PF research in many countries.

Simple models also imply that the experimental numbers that are being reproduced do not depend sensitively on the details of the physical phenomena involved. This contrasts with sophisticated numerical simulations which take into account many finer details of the physics and incur substantial manpower and computational cost. In many cases, they still give no better agreement with experimental numbers than simple models. 
Yet, numerical computations serve an important purpose in cross-checking the role of known physical phenomena in explaining the observed plasma focus phenomenology. In some cases, the relative importance of several processes is not known and even standard simplifications, such as representing the plasma as a fluid, are not feasible. Such numerical simulation codes serve as virtual laboratories which help understand and visualize the influence of various physical processes in a graphic manner when their results are compared with a real physical experiment.

Empirical and analytical insights serve a radically different purpose. While simple models and numerical simulations deal with numbers, empirical and analytical insights operate at the level of concepts. Such insights help bring out hidden facets of observed phenomenology or of common assumptions regarding 'what might be happening'.

This section gives an overview of significant developments in simple models, numerical simulations and empirical and analytical insights. The seemingly uneven emphasis on various sub-topics is explained as follows. Simple models are reviewed in some detail because of their evident usefulness and because of the historical role of the Lee model. Fluid simulations, which are based on the premise that the velocity distribution of various plasma species is Maxwellian with a species-specific temperature, are not covered in similar detail because they are a mature, well-known technique, widely used in plasma physics, and only prominent conclusions in the case of plasma focus simulations are mentioned. Kinetic simulations, which look at evolution of velocity distribution of plasma species in time and space, are a relatively new technique, not widely available and practiced, whose utility for understanding plasma focus phenomenology revealed by experiments (see Section 4) is not quite clearly established. Particle-in-cell simulations, coupled with Monte Carlo techniques, have been used recently at least in three laboratories for understanding aspects of the plasma focus breakdown phase, that defy the simplifications of fluid and kinetic approaches. It clearly has a huge untapped potential in understanding the physics involved in the transition from a fully neutral gas to a fully ionized plasma within the space of a few millimeters during sheath propagation where most of the simplifications made in traditional approaches to plasma physics fail (see Section 8.2.1). For this reason they are covered in greater detail. Modeling of ion acceleration requires techniques to follow ion trajectories as they make excursions over distances comparable with plasma dimensions and, therefore, require a departure from fluid and kinetic approaches. These need to be reviewed separately. Empirical and analytical insights are discoveries of small but important relationships that have considerable significance for the development of plasma focus physics. They are covered in a summary form.

Many researchers are tempted to look upon simple models and numerical simulations as a substitute for doing experiments. One of the objectives of this review paper is to demonstrate that real world experiments show facets of plasma focus physics that are not contained in any simple model, sophisticated numerical code or theory at present.

\subsubsection{Simple Models}

\subsubsection{Lee Model}

\section{Brief Time Line and Motivation}

A simple 2-phase code [188] was written to guide the design of the UNU ICTP PFF package (see Section 3.1.1) and for the understanding of the dynamics and electrical characteristics of the plasma focus in its axial and radial phase. As the usage of the UNU ICTP PFF expanded, the code and model were continuously developed until 5 phases were included and each updated code version was made available as widely as possible through the ICTP network at the annual Plasma Physics Colleges. In June 2005, a paper was published by Siahpoush et al. in Plasma Physics and Controlled Fusion titled "Adaptation of Sing Lee's model to the Filippov type plasma focus geometry" [245]. Thereafter Iranian researchers published several papers with reference to the modified Lee (ML) model, thus naming the continuously developing code as the Lee model. It was not until the 13th version of the code was available on the website of the Institute of Plasma Focus 
Studies [246] that the Lee model was deemed sufficiently developed to be published formally in 2014 as the Lee model code $[247,248]$.

\section{What Was Physically Achieved}

Importantly, the physics of the model is clear so that what may be computed is clear, and what may not be computed is also clear. In the axial phase, the code couples the voltage equation with an equation of motion. In the radial phase, the voltage equation is coupled with a shock wave equation and an adiabatic equation allowing the piston to separate from the shock wave and an equation to model the elongation of the collapsing structure additionally, critically incorporating finite small disturbance speed in the radial phase [249]. A reflected shock phase follows leading to the final slow compression or pinch phase which is modeled using a radiation-coupled equation of motion.

Non-dimensionalization of the governing equations of the axial and radial phases produces intrinsic scaling parameters, including alpha, a parameter matching the characteristic transit time to the current rise time and beta, a parameter matching the current sheath inductance to the static inductance. The analytical treatment also yields the scaling of speed with $\mathrm{S}=\mathrm{I} / \mathrm{a} \sqrt{\mathrm{p}}$, where $\mathrm{a}$ is anode radius and $\mathrm{p}$ is pressure. The observation from published data that $S$ is practically constant across the range of existing neutron-optimized plasma focus machines, small and big, confirms the constancy of speed, energy density, and temperature across the range of plasma focus machines [250]. A further observation is that the density of operation of optimized plasma focus machines remains within an order of magnitude over six orders of magnitude in storage energy. These two observations mean that all plasma focus machines have essentially the same plasma conditions in terms of density and temperature. The model further shows that the lifetimes and dimensions of the plasma focus pinch scale as the anode radius ' $a$ ', which scales as the discharge current ' $\mathrm{I}$ '. These properties of the plasma focus machines are in agreement with all neutron-optimized plasma focus machines, thus confirming that the basic physics of the model are realistic and sound. For plasma focus operating in gases other than deuterium, these properties are also inherent, modified by the requirements of the application, for example, in neon for characteristic neon SXR production, the requirement to operate in a specific temperature window in order to have the requisite levels of ionization for the emission of the required SXR lines. Further modifications to the dimensions and lifetime scaling in the case of heavy gases such as argon, krypton, and xenon due to radiation cooling and collapse are also computed by the model [246-248].

\section{What Innovations Were Developed}

A major innovation of the Lee code is the use of two model parameters for each phase, the mass swept up factor and the plasma current factor to include the effects of physical mechanisms not specifically modeled [246-248]. To use the code, a set of trial model parameters are used to generate the discharge current, which is then compared to the time profile of a measured current. The model parameters are then adjusted until the computed current profile matches that of the measured current. The fitted model parameters then represent the effects of all the mechanisms which operated in that particular shot which are not included specifically in the model. This use of fitting these pairs of model parameters gives the code modeling capacities far beyond the mechanisms specifically modeled in the equations of each phase. The way the code is formulated makes it mass, energy, and charge consistent. Because the computation is charge conservative there is the added bonus that once the computed current trace is fitted to the measured current trace in all temporal features, the values of the computed current are intrinsically correct, so that the measured current may be calibrated from the computed current [251]. This major innovation could be beneficial to other models which could use the same model parameters or alternatives which have the equivalent effect, such as, for example, adjusting the operating pressure [252] instead of using the mass swept up factor and using the inductance as a fitting parameter [252] instead of the more logical plasma current fraction. Another technical innovation of the Lee code is the use of a time shift to compensate for 
starting effects including non-ideal switching, lift-off times, and ill-defined run-down lengths of the axial phase.

Besides the dynamics and the current, the code computes the tube voltage, various components and distributions of plasma and inductive energies, the neutron yield (when operated in deuterium and D-T), including beam-target and thermonuclear components, the bremsstrahlung, line and recombination radiation, soft $\mathrm{X}$-ray yield (for various gases), beam ion and REB yields and characteristics, pinch dimensions and lifetimes, and postpinch plasma flow characteristics. The computations of neutron yields (D-D and D-T), soft x-ray yields (in Ne), Ar all-line yields, beam ion yield, and characteristics (in D, Ne and Ar) pinch dimensions and lifetimes and post-pinch fast plasma flow characteristics have been compared in general agreement with many experiments. The code predicts and quantifies radiative cooling in gases heavier than nitrogen and radiative collapse in gases heavier than argon. The radiative collapse in krypton has been verified by current fitting. Studies of radiative collapse on Ar and D doped with Ar have been made.

\section{What New Insights Were Generated}

The code is used to produce reference numbers for experiments for example axial and radial speeds, energy distributions, radiation yields (neutrons, beam ions, REB, soft X-rays), pinch dimensions and lifetimes, and fast plasma stream characteristics [246-248]. Systematic numerical experiments have produced insights into a pinch current limiting effect as static inductance is reduced towards very small values below $20 \mathrm{nH}[253,254]$ and corresponding radiation yield limitation $[255,256]$. Scaling laws for neutrons and soft X-rays, all-line yields, ion beams, and REB and fast plasma streams have been generated [246-248,257-268] and a deterioration in scaling of yields as capacitor energy goes towards and beyond MJ is attributed to the dynamic resistance of current sheet motion [269]. Radiation-coupled dynamics of the plasma focus pinch delineates the regimes of radiation cooling and collapse of various gases operated in various conditions [246-248,270-273]. The model finds that beyond the need to reach the PeaseBraginskii current (or modified, reduced P-B current for gases heavier than D) a longenough pinch time in comparison with an energy-depletion time [272] is a further requirement to observe radiative cooling and collapse.

The philosophy, the phases, the outputs and applications of the Lee model code may be summarized in the following sketch (Figure 40) [248].

Experimental based; Energy Mass \& Charge consistent; Connected to reality:

Utility prioritised: Cover whole process: birth to streaming death.

Universal: all gases and all plasma focus from smallest to largest and beyond

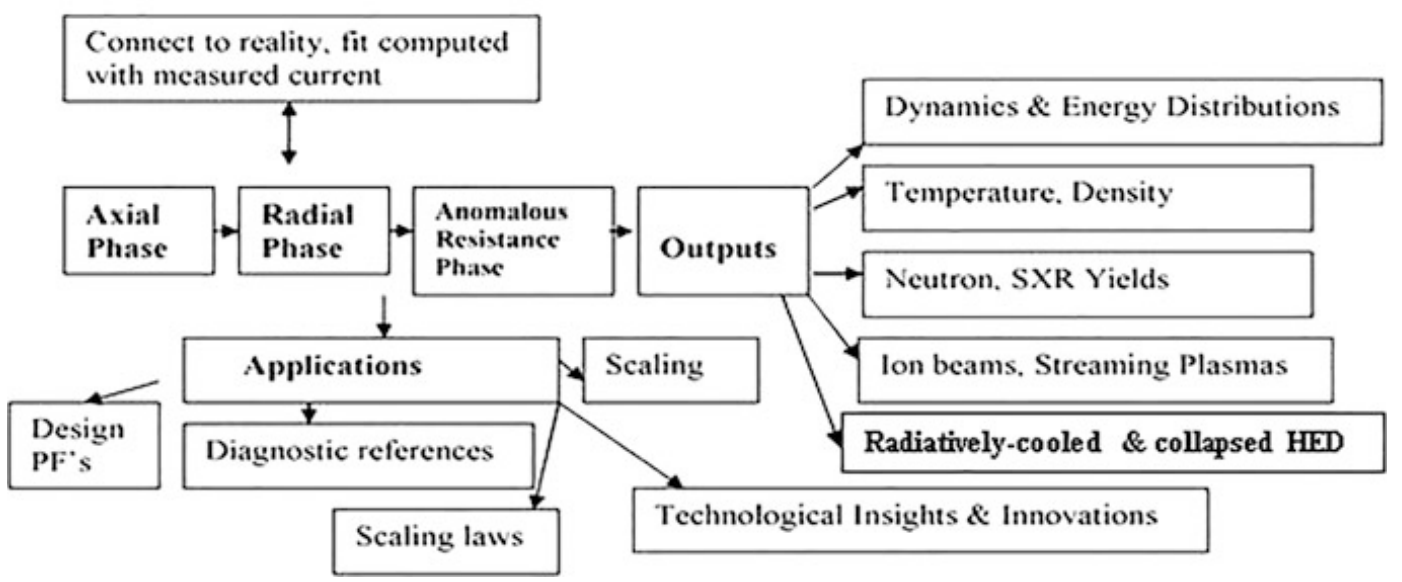

Figure 40. Schematic of the Lee model code. Reprinted by permission from Chapter 3: "The Plasma Focus-Numerical Experiments, Insights and Applications". In Plasma Science and Technology for Emerging Economies Ed. Rawat, R.S (C) 2021, Springer Nature, Singapore. 


\section{Experimental Verification of the Code}

Importantly, verification of the code has been achieved rather extensively by comparison of the code with experimental results for dynamics, neutrons, and soft X-rays, and to some extent with experiments in ion beams and post shock fast plasma streams and also to several experiments in radiative cooling and collapse.

\section{Dynamics and Time Scales}

The results of the code in terms of dynamics, dimensions and lifetime are generally in agreement with experiments. For example the scaling may be illustrated in the following comparison of the $3 \mathrm{~kJ}$ UNU ICTP PFF and the MJ PF1000 [10,248] (Figure 41):
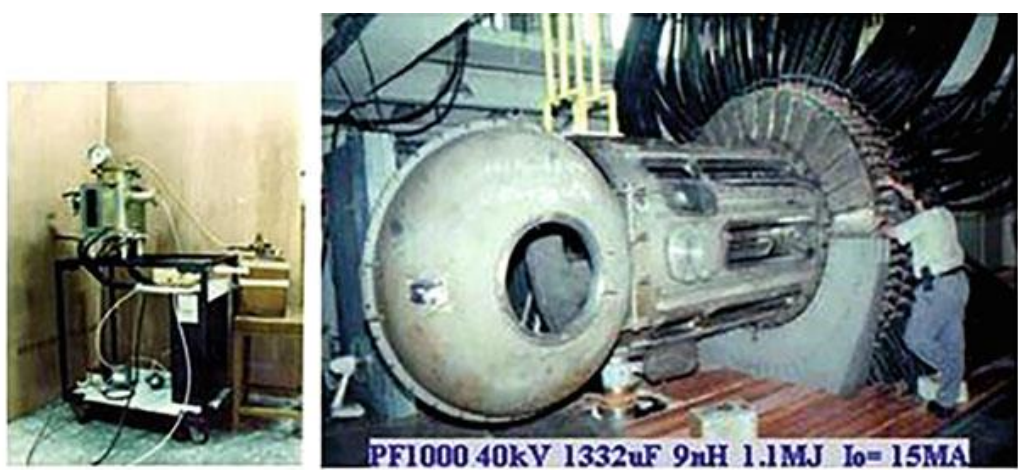

Above: A small PF (3kJUNU ICTP PFF) on same scale for comparisonwith a large PF (1 MJPF1000) Below: Shadowgraphs of the pinches of the two machines placed on the same scale.

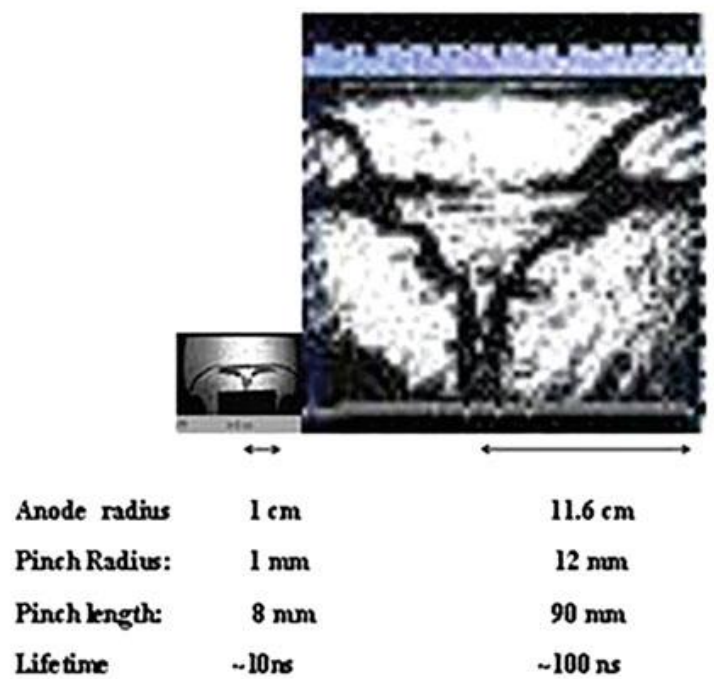

Figure 41. Scaling of plasma focus, dimensions and lifetimes. Reprinted by permission from Chapter 3: "The Plasma Focus-Numerical Experiments, Insights and Applications". In Plasma Science and Technology for Emerging Economies Ed. Rawat, R.S (c) 2021, Springer Nature, Singapore.

It was found that high performance machines (static inductance less than $50 \mathrm{nH}$ ) could be well fitted whilst low performance machines (static inductance more than $100 \mathrm{nH}$ ) always produce a bigger dip in the measured current than could be fitted to the computed current. To fit low performance machines (such as the UNU ICTP PFF) the standard 5 -phase code needed an extension of a 6th phase which included anomalous resistivity terms [246,274].

The dynamics of the axial phase in numerous machines, more than 40 as tabulated by A Singh [275] has been computed and found to compare well with measured dynamics in terms of speeds and speed profiles. Direct radial phase comparison is less common because of the dearth of experimental data. Figure 42 shows an outstanding comparison 
of computed radial trajectories with measured data from streak photograph [276]. Other more complex comparisons include combination of measured SXR profiles compared with radial dynamics $[277,278]$.

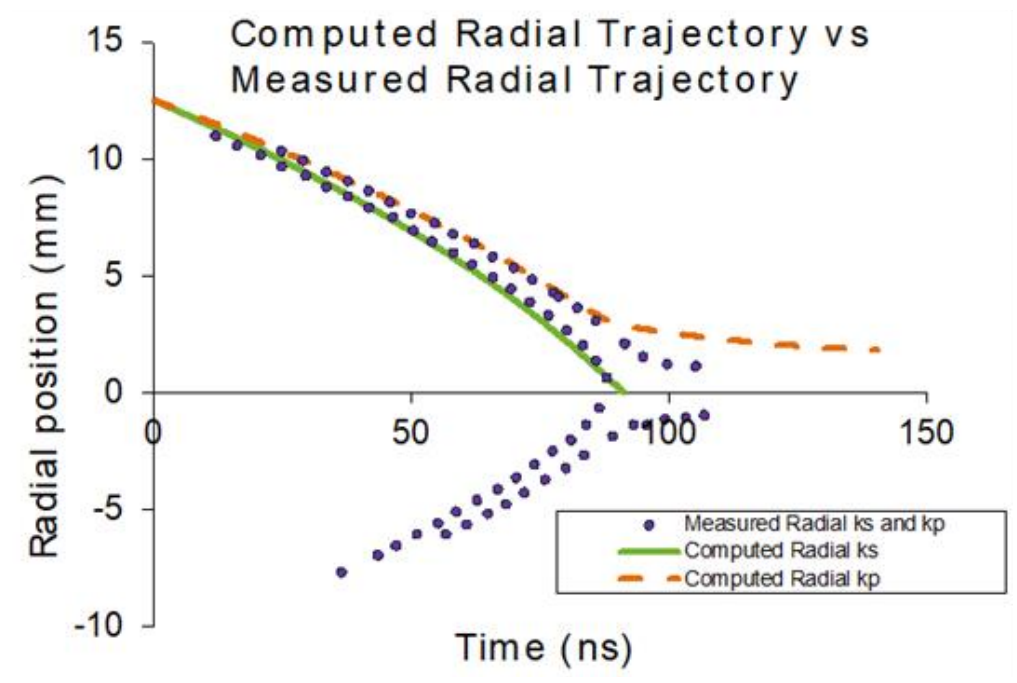

Figure 42. Comparing computed and measured radial inward shock trajectory, ks, and piston trajectory, kp. The measured trajectory (digitized from a streak photograph) shows reasonable symmetry of top half and bottom half indicating good axisymmetry for the measured radial compression. So only the top half is compared. The upper dots show the trajectory of the magnetic piston (i.e., rear of the slug), the lower dots show the trajectory of the radial inward shock (i.e., front of slug). Reproduced from [276] (Phys. Plasmas 2015, 22, 092702) with the permission of AIP Publishing.

\section{Neutron Yields}

The Lee Code computes both the thermonuclear, as well as the beam-target component of neutron yield. Neutron yields produced by the code configured to specific machines have shown reasonable agreement for the following machines:

PF1000 [10], PF-400J [279], NX2 [253,280-282], Mexico FNII [280,283], FMPF-3, NX3 [282],GN1 [284], ICPF [285], BARCPF2 [286], PF50 [275], Tsinghua Uni DPF2.2 [275], Texas 126 kJ TAMU [275], POSEIDON [275], UMDPF1 [275], UMDPF2 [275], Assam DPF [275], DPF78 [275], BORA [287] and PF24 [288], and in the KPU200 [247,289,290] at the level of $10^{13} \mathrm{D}-\mathrm{T}$ neutrons per shot. A comparison of computed vs. measured neutron yield is shown in Figure 43. As in typical cases, the peak value of computed neutron yield at optimum pressure is within experimental error of the measured, but there is a shift in the computed optimum pressure from the measured optimum pressure. 


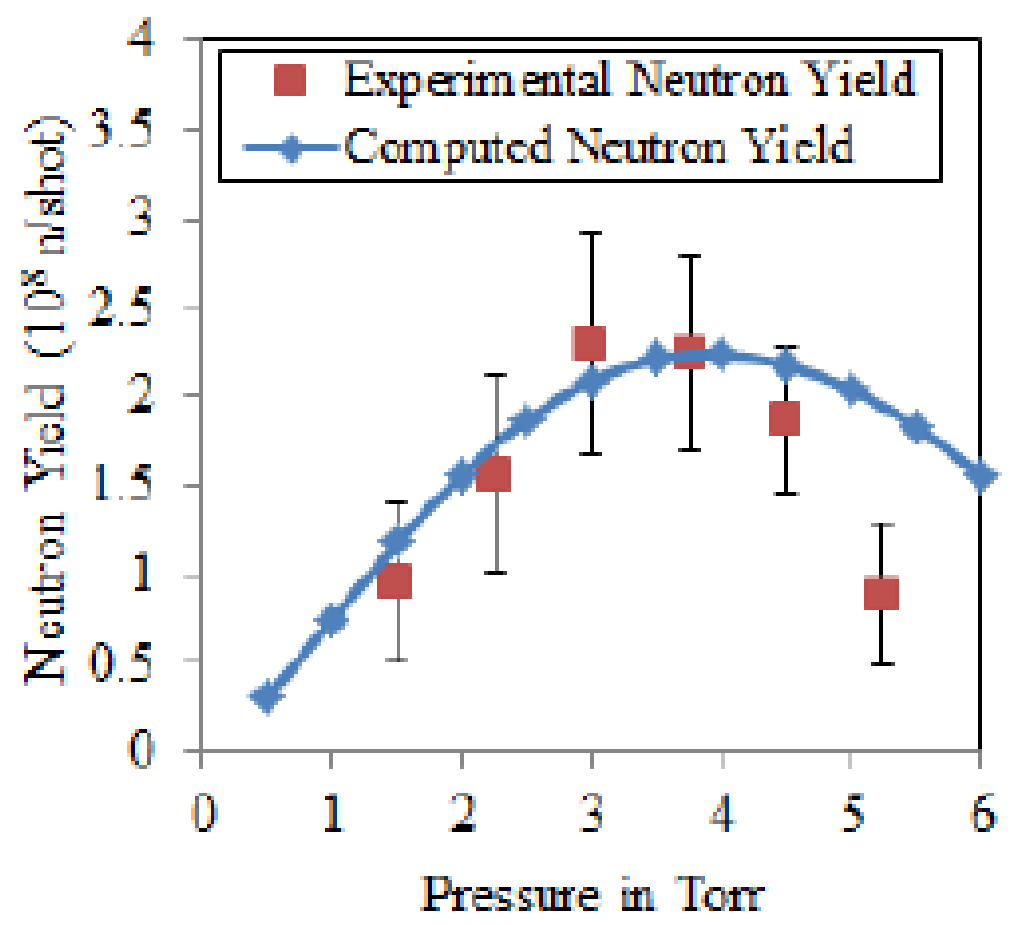

Figure 43. Argentina GN1: Computed neutron yield versus pressure compared to experimental neutron yield versus pressure from 0.5 Torr and 6 Torr working at $30 \mathrm{kV}$. The neutron yield scale is in multiples of $10^{8}$ neutrons per shot. Reproduced from Figure 3 of [284] (Int. J Mod. Phys: Conf. Series 2014, 32, 1460325).

\section{Neon SXR}

Neon soft X-rays, in particular, have been extensively studied for their potential applications in micro-lithography. Comparison between computed results and measurements have been done in the following machines: NX1, NX2 [291-295], UNU ICTP PFF [277,278,296-299]. A typical comparison is shown in Figure 44. The computed profile for the soft $\mathrm{X}$-ray yield versus pressure fits the measured profile well, including peak yield value and profile spread. However there is a shift in the optimum pressure.

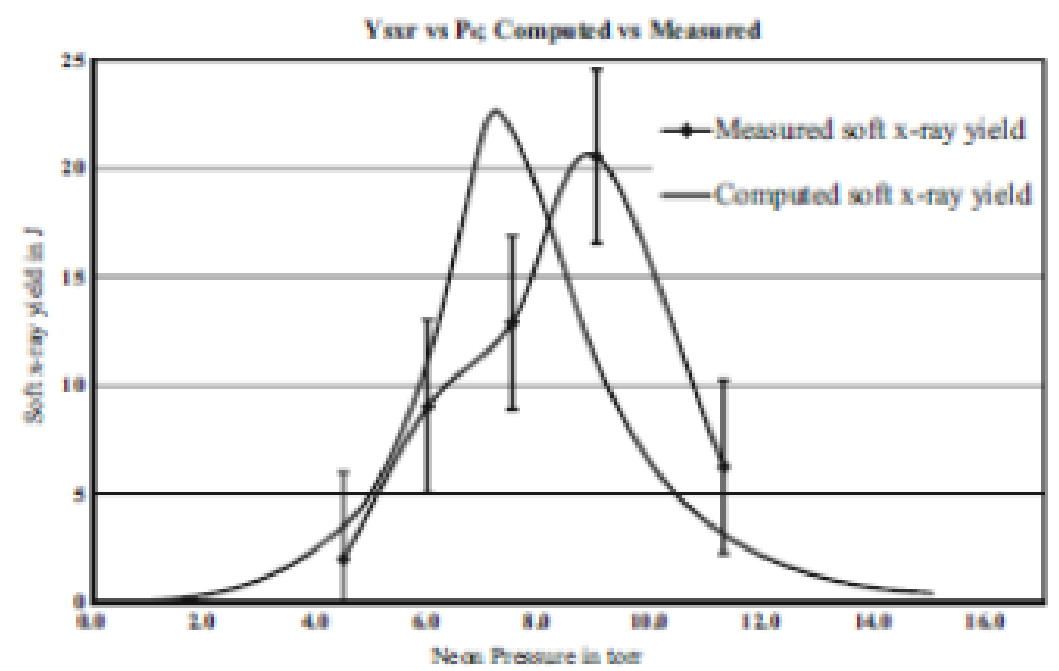

Figure 44. Computed and the measured soft X-ray yield per shot for NX1 at operating voltage 10 kV [295]. Reprinted with permission from Figure 4 of [294] (J. Fusion Energy 2015, 34, 686-693), (C) 2021 Springer Nature. 


\section{Ion Beams and Fast Plasma Streams}

In the original paper on extending the model to fast beam ions, there was found to be reasonable agreement between the computed beam ion numbers and energy in the case of PF2 [247,264] and PF1000 [10,264]. Akel et al. [300] extended the comparison with Faraday Cup data from the Assam PF operated at $25 \mathrm{kV}$ [301] and BPX PIN diode data from QAU PF operated at $2.3 \mathrm{~kJ}$ for nitriding applications [302]. The measured ion current density, ion number density, and ion energy and energy flux are found to compare reasonably well with the values computed by the code. Akel et al. [303] compared published data using time resolved Schlieren imaging in the PF-400J [304] with code results for post-focus pinch fast plasma stream speeds and stream energy, power flow density, and damage factor. Reasonable agreement was found for all the measured quantities. Vahid et al. $[104,305]$ carried out extensive and systematic measurements using Faraday Cups, PIN diode detectors, and photomultiplier-scintillator measurements to study ion beams from PFs operated in deuterium, neon, and argon correlate the results with the Lee code, thus providing conclusive experimental validation of the ion beam computations of the Lee code.

\section{Radiative Cooling and Collapse}

Experimental verification of code computation of radiative collapse in neon was presented in 2016 using time-resolved multi-frame interferometric imaging of the PF1000 pinch [306] and in krypton [307] using data inferred from measured current traces. Ł. Marciniak carried out an exhaustive and systematic study in PF-24 in deuterium and argon and in argon-doped deuterium producing experimental results [308,309] to correlate with results from the Lee code, modified for gas mixtures. D Piriaei et al. [310] correlated measurements of current and hard X-rays in the $25 \mathrm{~kJ}$ SABALAN2 with the 6-phase Lee code in order to obtain information on anomalous resistances and radiation-enhanced compressions in nitrogen and argon. Reviewing experimental data in view of the many gases involved and the required time resolution, it seems that the conclusive validation of the Lee model, in respect to its computations on radiation collapse in various gases, requires further research data which should include imaging with sufficient time resolution.

\section{Conclusions}

The Lee model is based on simple, clearly explained physics and is extensive in its coverage of dynamics, energetics, radiation yields and its range of gases and size of machines. It has been experimentally verified to various degrees in its results on dynamics, neutron and SXR yields, ion beams, and post focus fast plasma streams and radiative cooling and collapse. Its connection to the reality of any machine through a measured current time profile and thereupon to produce experimentally verified results in so many aspects of plasma focus behavior and properties makes it a useful tool which is productively used by many groups internationally.

\subsubsection{Resistive Gratton-Vargas (RGV) Model}

This is an adaptation [311-313] of the 2D electro-mechanical snowplow model of plasma focus [314] developed in late 1970s by F. Gratton and J. M. Vargas (GV). This model is based on the assumption of equality between the magnetic pressure driving the plasma focus sheath and density of fill gas times square of the normal velocity of the sheath, which is also sometimes known as the snowplow hypothesis. The basic idea behind this assumption is as follows. The sheath moving with normal velocity $v_{n}$ into the neutral gas of density $\rho_{0}$ experiences a "wind pressure" because of ingestion of a flux of momentum density $\left(-\rho_{0} v_{n}\right)\left(-v_{n}\right)$ into this region. This is balanced by the magnetic pressure driving the sheath. The "snowplow" effect is implicit in the suggested expression for wind pressure which is valid only when the gas "sticks" to the sheath during its propagation and does not bounce away or slide off. This equality is used by the model to define a dimensionless time-like independent variable $\tau$ related to the charge that has flown in time $t$ normalized to a 'mechanical equivalent of charge'. The equality also leads to a partial differential 
equation (the GV equation) for the shape of an imaginary 3D surface of rotation co-located with the sheath (called the GV surface) as a function of time.

Note that the assumed equality between the "wind pressure" and the magnetic field cannot be rigorously derived from jump conditions for planar, steady, strong, MHD shock waves because of the inherently non-planar, non-steady nature of the plasma flow. Nonplanarity involves curvature effects on momentum flow. Non-steady flow involves complications from staggered merging of sequentially launched shock wavelets. Presence of magnetic field and curvature together leads to coupled transverse flow of both plasma and magnetic field. Therefore, this equality needs to be treated as an independent assumption.

This assumption can be heuristically looked upon as the limit of the strong planar steady hydrodynamic shock relation $\mathrm{p}_{1}=2 \rho_{0} \mathrm{v}^{2} /(\gamma+1)$ for $\gamma \rightarrow 1$ with the pressure being equal to the magnetic pressure of the piston. The limit $\gamma \rightarrow 1$ implies an infinite number of degrees of freedom, specific volume tending to zero and shock velocity equal to piston velocity which is an alternate statement of the snowplow hypothesis.

This equality happens to be validated by both experiments [315] and 2D MHD codes (see Section 3.3.2.1 (C))

The importance of the GV model lies in an analytical solution of this equation that describes the GV surface algebraically and bears a striking resemblance to the experimentally determined shape of the sheath [315] at any value of $\tau$. From this, the dynamic inductance of the plasma focus sheath can be determined as a function of $\tau$ and geometric parameters of the device. The circuit equation can then be solved for the current as a function of $\tau$. This is then used to calculate the real time $t$ corresponding to $\tau$ by a quadrature of the reciprocal of calculated current as the last procedure of the model. The original GV model was not able to take into account the circuit resistance. The inclusion of resistance [311] allowed the revised resistive Gratton Vargas (RGV) model to fit experimental current waveforms by treating circuit inductance, resistance, and fill gas pressure as adjustable parameters [252], just as the Lee model fits experimental current waveforms by choosing values of certain adjustable model parameters by trial.

The significance of the RGV model lies in its analytical approach. It allows an algebraic representation of the shape of the sheath, described in terms of an imaginary 3D surface of rotation, as a function of the dimensionless time-equivalent parameter $\tau$. It also allows an algebraic treatment of the energy transfer efficiency from the capacitor bank into magnetic energy and electromagnetic work done.

It is important to emphasize that the RGV model contains no plasma and, hence, has no capability to address the pinch phase phenomena as the Lee model does. Its domain of utility lies in addressing (1) issues related to the curvature of the plasma, (2) issues related to energy transfer from the capacitor bank to the plasma, which is an attribute of the axial phase.

The 3D surface calculated in the RGV model can be used as a skeleton on which a plasma model can be constructed as an add-on. The algebraic definition of the 3D GV surface can be used $[171,316]$ to define a set of local curvilinear coordinate systems at every point on the GV surface having axes along its local normal, local tangent and along the azimuth. Physical laws expressed in these coordinates explicitly take into account all effects of plasma curvature. As an example, conservation laws of mass, momentum, and energy have been written in such local coordinate system and the theory of hyperbolic conservation laws has been used to draw useful insights $[171,316]$. This is discussed in a separate section on empirical and analytical insights. The analytical treatment of energy transfer in the RGV model has been used to construct device optimization algorithms [317,318].

It is interesting to note that the Lee model and the RGV model can provide equally good fit to experimental waveforms during the axial phase and much of the radial implosion phase using suitable adjustable parameters (see Figure 45). This is their advantage over MHD codes (see Section 3.3.2.1) which do not provide a comparable fit to the current waveforms. Transfer of energy from the capacitor bank to the plasma takes place during this period. 


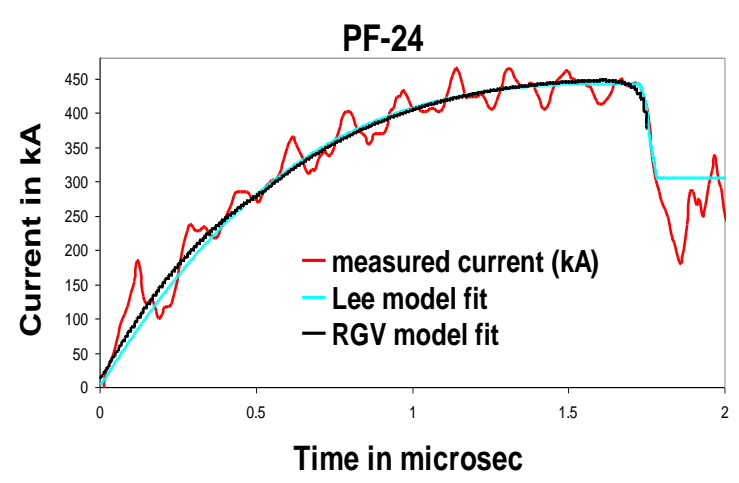

(a)

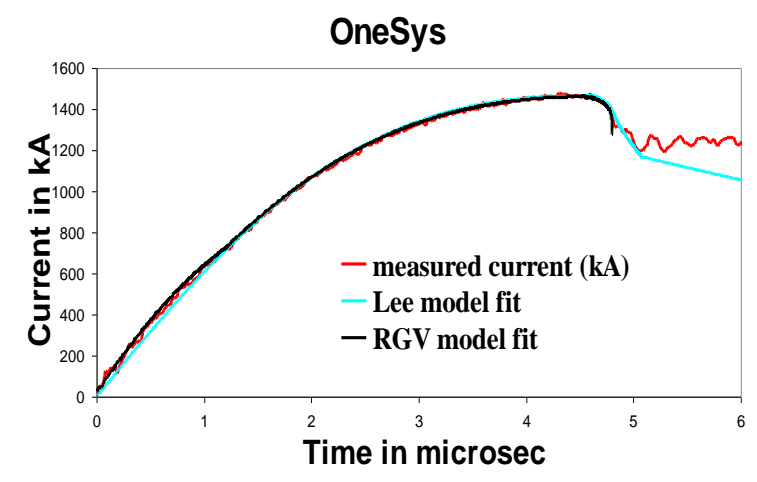

(c)

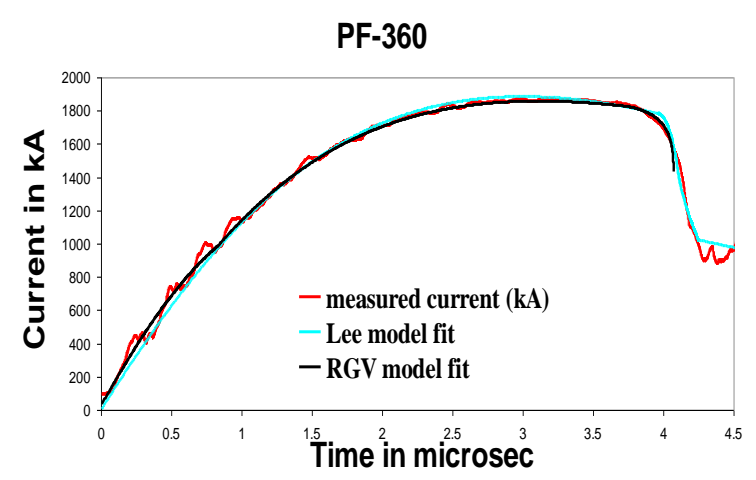

(b)

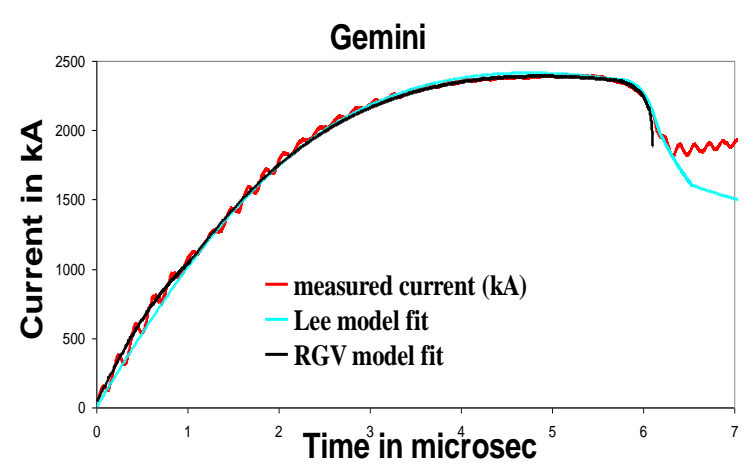

(d)

Figure 45. Comparison of Lee model and RGV model with measured current waveforms: (a) PF24 Common parameters: $\mathrm{C}_{0}=112.5 \mu \mathrm{F}, \mathrm{V}_{0}=16 \mathrm{kV}$, cathode radius $\mathrm{b}=49 \mathrm{~mm}$, anode radius $\mathrm{a}=31 \mathrm{~mm}$, anode length $\mathrm{z}_{\mathrm{A}}=172 \mathrm{~mm}$, insulator length $\mathrm{z}_{\mathrm{I}}=40 \mathrm{~mm}$, Insulator radius $\mathrm{r}_{\mathrm{i}}=31.5 \mathrm{~mm}$ Parameters used in Lee model fitting: inductance $\mathrm{L}_{0}=22 \mathrm{nH}$, resistance $\mathrm{R}_{0}=18 \mathrm{mOhm}$, pressure $\mathrm{p}=1.5$ torr, effective anode length $\mathrm{z} 0=152 \mathrm{~mm}$, mass fraction $\mathrm{f}_{\mathrm{m}}=0.157$, current fraction $\mathrm{f}_{\mathrm{c}}=0.7$, radial mass fraction $\mathrm{f}_{\mathrm{mr}}=0.02$, radial current fraction $\mathrm{f}_{\mathrm{cr}}=0.8$. RGV model parameters: $\mathrm{L}_{0}=20 \mathrm{nH}, \mathrm{R}_{0}=14 \mathrm{mOhm}$, $\mathrm{p}=1.1$ torr. Experimental value of pressure 1.5 torr. (b) PF360 Common parameters: $\mathrm{C}_{0}=262.60 \mu \mathrm{F}, \mathrm{V}_{0}=31 \mathrm{kV}, \mathrm{b}=75 \mathrm{~mm}$, $\mathrm{a}=50 \mathrm{~mm}, \mathrm{z}_{\mathrm{A}}=304 \mathrm{~mm}, \mathrm{z}_{\mathrm{I}}=75 \mathrm{~mm}, \mathrm{r}_{\mathrm{i}}=60.1 \mathrm{~mm}$. Parameters used in Lee model fitting: $\mathrm{L}_{0}=23 \mathrm{nH}, \mathrm{R}_{0}=5.7 \mathrm{mOhm}$, $\mathrm{p}=4.9$ torr, $\mathrm{z} 0=26.65 \mathrm{~mm}, \mathrm{f}_{\mathrm{m}}=0.56, \mathrm{f}_{\mathrm{c}}=0.7, \mathrm{f}_{\mathrm{mr}}=0.1, \mathrm{f}_{\mathrm{cr}}=0.7$. RGV model parameters: $\mathrm{L}_{0}=21 \mathrm{nH}, \mathrm{R}_{0}=1.7 \mathrm{mOhm}$, $\mathrm{p}=14.5$ torr. Experimental value of pressure 4.9 torr. (c) OneSys common parameters: $\mathrm{C}_{0}=216 \mu \mathrm{F}, \mathrm{V}_{0}=35 \mathrm{kV}, \mathrm{b}=76.2 \mathrm{~mm}$, $\mathrm{a}=60 \mathrm{~mm}, \mathrm{z}_{\mathrm{A}}=393.7 \mathrm{~mm}, \mathrm{z}_{\mathrm{I}}=108 \mathrm{~mm}, \mathrm{r}_{\mathrm{i}}=50.9 \mathrm{~mm}$. Parameters used in Lee model fitting: $\mathrm{L}_{0}=54 \mathrm{nH}, \mathrm{R}_{0}=3.3 \mathrm{mOhm}$, pressure $\mathrm{p}=5.23$ torr, effective anode length $\mathrm{z} 0=339.7 \mathrm{~mm}, \mathrm{f}_{\mathrm{m}}=0.221, \mathrm{f}_{\mathrm{c}}=0.7, \mathrm{f}_{\mathrm{mr}}=0.6, \mathrm{f}_{\mathrm{cr}}=0.7$. RGV model parameters: $\mathrm{L}_{0}=46 \mathrm{nH}, \mathrm{R}_{0}=2 \mathrm{mOhm}, \mathrm{p}=6.8$ torr. Experimental value of pressure 5.23 torr. (d) Gemini common parameters: $\mathrm{C}_{0}=432 \mu \mathrm{F}, \mathrm{V}_{0}=40.13 \mathrm{kV}, \mathrm{b}=101.6 \mathrm{~mm}, \mathrm{a}=76.2 \mathrm{~mm}, \mathrm{z}_{\mathrm{A}}=596.9 \mathrm{~mm}, \mathrm{z}_{\mathrm{I}}=127 \mathrm{~mm}, \mathrm{r}_{\mathrm{i}}=77 \mathrm{~mm}$. Parameters used in Lee model fitting: inductance $\mathrm{L}_{0}=36.5 \mathrm{nH}, \mathrm{R}_{0}=3.7 \mathrm{mOhm}, \mathrm{p}=5.21$ torr, $\mathrm{z} 0=533.4 \mathrm{~mm}, \mathrm{f}_{\mathrm{m}}=0.246, \mathrm{f}_{\mathrm{c}}=0.7, \mathrm{f}_{\mathrm{mr}}=0.6, \mathrm{f}_{\mathrm{cr}}=0.7$. RGV model parameters: $\mathrm{L}_{0}=29.7 \mathrm{nH}, \mathrm{R}_{0}=2.5 \mathrm{mOhm}, \mathrm{p}=5.7$ torr. Experimental value of pressure 5.21 Torr.

Note that the Lee model and the RGV model provide an equally good fit to the axial phase and the part of the radial phase before the stagnation, which most MHD codes do not provide. The Lee model provides a fit beyond the pinch phase which is outside the domain of RGV model.

The Lee model and the RGV model superficially appear to be based on widely different assumptions and inputs, physics, and philosophy, as well as mathematical tools. However, their internal physics has a common core over their domain of overlap. The discussion below looks at the apparent differences and the common physics core

\section{Apparent Differences between the Lee model and the RGV model}

- Assumptions and inputs:

- Plasma shape: The Lee model assumes the plasma to be a thin annular disc in the axial phase and a straight circular cylinder in the radial phase, although, 
the curved shape of the sheath is well known. The RGV model calculates an imaginary 3D surface of rotation that bears a resemblance to the curved shape of the sheath in both the axial and radial phases [315].

- Plasma thickness: The Lee model utilizes the slug model of Potter [249] to calculate the thickness of the plasma cylinder in the radial phase as a function of time. The RGV model deals with a 3D surface of revolution co-located with the plasma sheath and has no plasma (so there is no question of a 'thickness'). Device geometry: The Lee model does not include the insulator length and takes into account an effective length of the anode. In the RGV model, the insulator length, insulator outer radius, and anode length (all normalized to the anode radius) enter the formula for the dimensionless dynamic inductance explicitly. Operating parameters: The Lee model works with the experimental values of gas fill pressure (along with atomic number and atomic weight of the gaseous species) and "measured" static inductance and resistance, usually determined from the current waveform of a high-fill-pressure discharge, where the plasma is expected to remain at the insulator for the duration of the discharge. These values may however be tweaked slightly in practice on account of possible errors of measurement. The RGV model treats the operating pressure, static inductance and resistance as adjustable parameters on the ground that (1) the actual mass density of plasma during discharge may be different from that corresponding to the fill gas pressure because of release of surface-adsorbed gas during the discharge, and (2) the definitions of static inductance and resistance used in the model correspond to that calculated from a discharge in which a metallic sleeve snug-fitted over the insulator shorts the anode and the cathode, which is never actually performed.

- Snowplow hypothesis: The Lee model invokes the snowplow hypothesis that the sheath carries the mass of gas encountered during propagation but modifies it by introducing 'mass fraction' and 'current fraction' that participate in the snowplow process that are adjustable parameters chosen by an iterative procedure. These fractions are different for the axial and radial phases. The RGV model formulates the snowplow hypothesis as an equality between magnetic pressure and "wind pressure" of the gas that sticks to the sheath without bouncing away or sliding off.

- Physics and Philosophy:

The Lee model invokes physical laws, phenomena and models such as Newton's second law, first law of thermodynamics, shock dynamics, slug model [249], pinch effect, thermodynamics including radiation loss, anomalous resistivity, and circuit theory to set up a system of coupled ordinary differential equations. It thus has a reasonable expectation that its results should correspond to the experimental reality. The RGV model has no in-built physics other than an assumption of equality between the magnetic pressure of azimuthal magnetic field driving the plasma and the "wind pressure" resisting its motion that operates at an imaginary surface that plays a role similar to the imaginary center of mass in mechanics. This assumption superficially looks like the planar steady strong shock jump condition with an infinite number of degrees of freedom but must be treated as an independent assumption in view of the fact that it is being applied to a non-planar, non-steady plasma flow for which curvature effects on momentum transport and shock wavelet merging effects are known to play a significant role. It must, therefore, be treated as a postulate. It also uses the circuit equation. There is no á priori basis to expect the results of RGV model to agree with experiments.

- The Lee model uses the hypothesis of a virtual-plasma-diode beam-target mechanism [10] to calculate the neutron yield and calibrates the calculated numbers against experimental data. In a similar manner, it calculates soft X-ray, ion, and 
electron emission from devices operating with various gases. The RGV model, on the other hand, strongly emphasizes absence of any physics other than the snowplow hypothesis. Its ability to fit experimental current is conjectured to imply insensitivity of current waveforms to details of plasma phenomena, such as atomic physics of ionization and recombination, anisotropic transport of heat and electricity, interaction with electrodes, etc., which are well-represented in MHD codes (see Section 3.3.2.1). It specifically refuses to predict any plasma properties because its structure does not include a plasma. It can, however, be used to provide a mathematical framework for constructing a first-principles theory of plasma focus sheath propagation based on conservation of mass, momentum, and energy, and perhaps other physical principles [171,316]. It can also be used for construction of algorithms that optimize energy transfer efficiency as a function of device parameters [317,318].

- Mathematical tools:

The Lee model is formulated in terms of a system of ordinary differential equations that couple the circuit equation and the equation of motion, whose solution is obtained as a table of numbers. The inductance of the moving plasma is calculated at each step during the calculation, which uses geometrical as well as operational parameters. The RGV model is formulated as a twodimensional partial differential equation of Jacobi type describing the shape and position of a 3D surface of rotation co-located with the plasma sheath (the GV surface) as a function of $\tau$. Its solution, obtained in an algebraic form, depends only on the scaled geometry of the device and has no relation with operational parameters, such as voltage, inductance, capacitance, resistance, gas fill pressure, and gas species or of the geometrical scale represented by the anode radius. The dimensionless inductanceas a function of $\tau$ is calculated from a formula and is the same for all devices that have the same scaled geometry. The current waveform can be obtained as a closed-form algebraic expression to the zeroth order of a small parameter representing ratio of resistance to impedance of the circuit. Fractions of energy remaining in the capacitor and converted into magnetic energy can also be expressed as algebraic expressions.

Decoupling between "plasma" propagation on the one hand (represented by motion of the GV surface) and device operating parameters and the circuit on the other hand is a unique property of the GV approach, not shared by any other theory or model.

In spite of these rather spectacular differences, both models are able to provide equally good fit to the experimentally measured waveforms (as seen in Figure 45) in the axial and radial implosion phases before stagnation. This points to existence of a common physics core for the domain of overlap between the two models, which is explored below separately for the axial and radial phases.

\section{Common Physics Core of the Lee Model and the RGV Model Axial Phase:}

Both the codes use a momentum based snow-plow equation coupled to a circuit equation to compute the axial phase. The Lee model equates the rate of change of momentum to the driving force on a thin layer. The RGV code is effectively based on a strong, planar, steady shock equation with infinite (or a very large number of) degrees of freedom (for which the adiabatic index $\gamma$ approaches unity). The strong shock equation is derived from conservation of momentum. Hence, the physics of the two models are exactly equivalent for the axial phase. Any difference in proportionality constants is removed in the fitting process in which the Lee model multiplies the filling density with a swept up ratio $f_{m}$ whilst the RGV model changes the filling density. Therefore, the two codes are exactly the same in underlying physics and in fitting procedures. The two codes produce current waveforms which are fitted with the same degree of accuracy to the measured currents. Once 
properly fitted, the two codes are expected to give identical results in terms of dynamics and energetics. The above remarks apply only to the axial phase.

\section{Radial Phase:}

In the radial phase, the Lee model adopts the strong shock equation with proper thermodynamics and a finite small disturbance speed, thus allowing a plasma layer to be computed with a shock front driven by a magnetic piston at the rear of the layer. The plasma layer or slug has density and temperature defined by the speed of the shock and the thermodynamic properties of the gas. As the layer moves radially inwards, the slug elongates. The shock front is driven radially inwards until it coalesces on axis whereupon a reflected shock forms and moves radially outwards towards the incoming piston, with a growing column of stagnated doubly shocked plasma behind the reflected shock. When the reflected shock hits the piston the radiation-coupled pinch phase begins. The circuit equation continues to be coupled to the system of equations.

On the other hand, the RGV model again uses only the strong shock equation to model the radial phase. The driving magnetic field is at the same radial position as the moving front. Such a zero thickness structure will have an increasing speed as it approaches the axis with the driving magnetic field tending towards infinity at the axis, which is then a singularity point. Hence the computation will have to be terminated at some arbitrary point before the axis. There is no possibility for such a momentum based snowplow model to have a pinch phase. The RGV model, therefore, claims to represent a mathematical surface co-located with the plasma rather than a physical plasma.

Technically, both codes use non-dimensionalization to analytically derive characteristic quantities and scaling parameters. In the numerical solution of the coupled equations both codes compute in non-dimensionalized quantities for the axial and radial inward shock phases. To be practical, the inputs and outputs of both code are in numerical tabular forms and the outputs also incorporate graphs. However, RGV model has the possibility of algebraically representing the curved shape of the plasma in the radial collapse phase much before the plasma reaches the axis.

\section{Fitting the Radial Phase:}

The axial phase has a duration of several microseconds whereas the radial phase lasts only tens of nanoseconds. Therefore, a look at the fitting curves may indicate good agreement with the measured current curves for both the computed curves, since the radial phase occupies such a small region of the curves. However it is these tens of nanoseconds that really matter beyond the excellent fit of the microsecond axial phase.

The importance of the plasma focus lies more in the end of the radial phase than its axial and early radial phase. Hence the fitting of the radial phase especially towards the end of the radial phase needs to be examined carefully. The example of OneSys in Figure 45 above is discussed here. The section covering the end of axial phase to end of pinch is extracted and expanded for a closer look. This is shown in Figure 46.

It is seen that both codes compute current waveforms that are reasonably well fitted to the measured current from the axial phase through the start of the radial phase until point $\mathrm{D}$ (half way between points 1 and 2), at which point the RGV curve becomes exponentially steeper towards the vertical, deviating from the Lee code curve, which continues to follow the average curve of the measured current trace. The asymptotic behavior of RGV curve tending towards a vertical slope indicates asymptotic infinite speed, which indicates the limit of its validity. In comparison, the current waveform of the Lee code fits better to the average slope of the measured current waveform, indicating a realistic speed when compared to the actual event taking place. Whereas the RGV code, by fundamental definition has no pinch phase, the Lee code computes the pinch phase and enables various radiation yields to be computed and even enables the mechanisms of radiative cooling and collapse to be investigated using the code. Similarly the differences in the fitting of the two codes is clearly seen in all four cases of Figure 45. On the time scale of c 45, the difference in the fitted curves at the end of the radial phase appears to be small. However, for any 
research concerned with the pinch phase of the plasma focus, it is this short period of tens of nanoseconds that is more important than the microseconds of the axial phase.

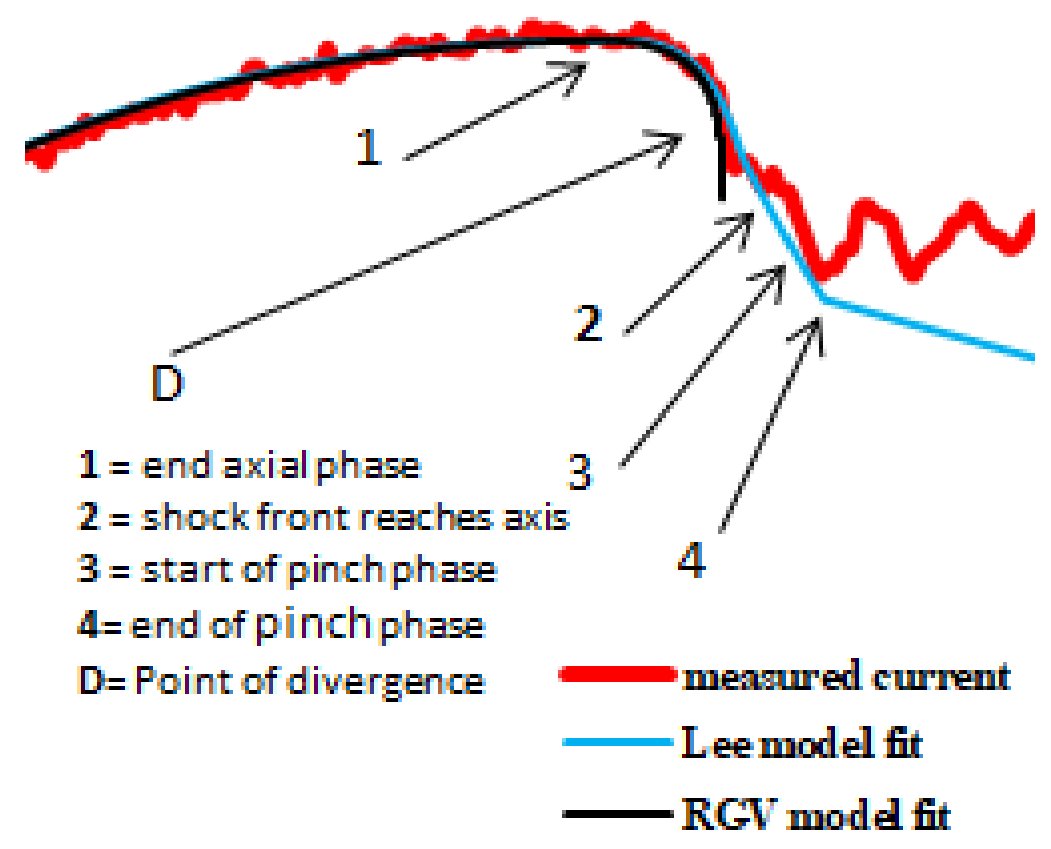

Figure 46. Fitting the radial phase. The red line is the measured current trace to which the RGV computed current (black line) and the Lee code (light blue line) are fitted.

On the other hand, considerations of device optimization are almost exclusively dependent on matching the arrival time of the plasma at the axis with the characteristic time constant of the circuit, in which, the pinch phase physics plays no part. In this respect, the RGV model provides closed-form algebraic formulation of energy partition between various components. It also provides a means to describe the shape of the plasma in both axial and radial phases which can serve as a base for formulating a first-principles theory of plasma focus sheath structure, which would be a pre-requisite of a first-principles theory of the pinch phase.

In summary, for the axial phase, the underlying physics of both codes are exactly the same, coupling one snowplow equation with the circuit equation and compensating for all other modeling deficiencies with two fitting parameters. The RGV model defines the end radial phase as a singularity, hence has no pinch phase. The Lee model computes a pinch phase.

The limitations of the Lee code are also apparent in the radial phase current fitting. Typically, the bottom of the current dip, as fitted by the Lee code, differs significantly from that of the measured current dip. This indicates that the code does not include all important mechanisms. This is not unexpected since indeed the Lee model does not take into account even the simplest mechanisms such as magnetic field diffusion as the piston slows down, or any instability mechanisms which are known to occur. Nevertheless, the Lee code has a computed pinch phase and seems to be able to produce various yields which are comparable to experimentally observed yields within typical shot-to-shot variations.

The ability of both models to fit the current waveform within their domains of applicability is accompanied by three caveats. (1) For small devices, the switch closure time may comprise a significant fraction of the circuit quarter cycle time and may cause deviation from a good overall fit in the early part of the waveform. (2) For large devices, there may be a significant jitter in switches and oscillations caused by multiple parasitic LC circuits causing the waveform to deviate from a good overall fit. (3) The Lee model calculates the waveform up to the pinch formation and re-expansion while the RGV model calculates 
the waveform only till the stagnation and have no applicability in the pinch formation and associated phenomena.

There are, thus, clearly discernible limits of what both these models can and cannot do. Within these limits, they have their unique capabilities which can be explored and leveraged to address many issues in plasma focus research. Extrapolations using both these models must be treated with due caution particularly because the fitting procedures may not lead to a unique set of fitting parameters and the conclusions derived from such extrapolations need to be taken as 'highly likely indications' but not 'absolute certainties'.

\subsubsection{PLADEMA Model}

Researchers associated with the Inter-institutional Program of Dense Magnetized Plasmas, National Research Network PLADEMA, Argentina, developed a simple model for basic engineering criteria for conceptual design of plasma focus devices [319]. This model assumes the neutron emission to be from thermonuclear reactions and the plasma configuration to be close to a Bennett equilibrium and governed by an isentropic oscillator equation obtained by linearization of the zero-dimensional radial equation of motion of a pinch. It derives an expression for the neutron yield that scales as the 4th power of the pinch current. The axial phase is represented by a snowplow equation. Its results that are benchmarked against experiments are: (1) dependence of focus timing on filling pressure, (2) variation of average neutron yield with pressure for 3 small PF devices, (3) variation of optimum neutron yield with anode length, and (4) variation of optimum pressure for various anode lengths.

\subsubsection{Finite-Elements Numerical Model of the Current-Sheet Movement}

This is a two-dimensional numerical model of the movement and shaping of the current sheath in coaxial plasma guns, based on a finite-element representation [320]. Each element moves normal to its surface accelerated by the Lorentz force. During its displacement, each element accumulates the gas mass swept by its motion. Distortions of the elements are corrected by reshaping them after each step in order to avoid geometric instabilities. The usual electrical circuit equation is used where the inductance of each element is calculated assuming a coaxial geometry of variable radius. The code is benchmarked against experimental results of sheath arrival time at various axial distances determined using axially inserted probes in the rundown phase. In addition, the model reproduces a toroidal singularity observed in the plasma sheath in the PF-50J [321].

\subsubsection{Numerical Simulations}

This subsection presents an overview of the numerical simulation efforts and their relevant conclusions. The influence of the standard narrative is easily discerned and their inherent inability to explain experimentally well-established phenomena, such as large width of side-on neutron spectra reported from multiple laboratories becomes evident.

\subsubsection{Fluid Simulations}

Many MHD simulations are reported in the literature [322-330]. This section gives a brief report on their main features and conclusions. It is seen that there are major problems in getting an agreement between such calculations and experiments. In many cases, the values of series inductance and resistance are "appropriately chosen", just as in the case of simple models discussed above. Nevertheless, there has been a steady progress in computational sophistications and significant insights have been generated concerning importance of certain phenomena. The overall impression of this body of work, however, highlights the cost-benefit advantage of simple models, particularly, the Lee model, over such sophisticated and costly computations at present.

1. Dychenko and Imshennik (1969) [322] considered only the hydrodynamic aspect of the two-dimensional flow of plasma in the radial phase by assuming infinite conductivity which excludes magnetic field from the plasma. The dimensionless current is fitted 
to the experimental waveform in the radial phase as a linear expression in time. Although the numerical simulation is inconclusive with respect to neutron emission, it emphasizes the role of axial mass outflow in heating the plasma.

2. Potter (1971) [323] formulated the first self-consistent 2D MHD code based on the explicit time step Lax-Wendroff method for the plasma focus including the Hall effect, finite ion Larmor radius effects, thermal and electrical conductivity, that coupled the plasma propagation with an external LC circuit. The characteristic shape of the plasma during the axial and radial phases was reproduced along with its density and temperature structure. Agreement between calculated sheath velocity with the expression for sheath velocity given by the snowplow model of Rosenbluth and Garwin [2] proportional to square root of applied electric field and inversely proportional to fourth root of mass density of gas was used as the test of correctness of the numerical procedure. The calculation finds that in the pinch zone ion-ion collision times $\sim 20 \mathrm{~ns}$ are of the same order as the hydrodynamic time scale and ion Larmor radius is of the same order as the plasma scale length $\sim 1 \mathrm{~mm}$ so that applicability of the fluid model is doubtful.

3. Maxon and Eddleman (1977) [324] also report on 2D MHD calculations based on Eulerian mesh. They have run Potter's problem and find significantly different results. In particular, they find that the sheath velocity agrees very well with the snowplow hypothesis that equates the wind pressure with the magnetic pressure (see Section 3.3.1.2). Current waveform is underestimated by about $10 \%$. They also note non-applicability of the fluid model at the rear of the plasma sheath where gradients are large, mean free paths are large and magnetic field varies rapidly.

4. Behler and Bruhns (1987) [325] report on a variation of Potter's 2D MHD code by including the neutral gas as the third species. Joule heating, energy exchange between ions, electrons and neutrals, losses by bremsstrahlung, ionization and recombination kinetics, heating by viscosity of individual species are taken into account. Molecular and atomic physics processes are restricted to impact ionization from the ground state, radiative recombination, three-body Auger recombination, and charge exchange. The main focus of their investigation is the residual neutral gas remaining near the insulator in the inverse pinch and lift-off stages.

5. Stepniewski (2004) [326] reports a gridless, fully dissipative MHD code with Braginskii transport coefficients, that accounts for kinetics of ionization. Only qualitative agreement with experiments is reported.

6. Garanin and Mamyshev (2008) [327] report a 2D MHD code with implicit difference scheme. In this scheme, “...the ion-velocity components are calculated using a scheme that is implicit with respect to terms comprising the magnetic field. Such a scheme makes it possible to eliminate the constraint imposed by the magnetic sound speed on the computational time step and to significantly reduce the effect of the fictitious "vacuum" density on the plasma dynamics". This allows the code "... to perform simulations at plasma densities of about six orders of magnitude lower than the initial density, while keeping the computational time step at a reasonable level". Their code calculates (1) standard hydrodynamic quantities (ion velocity, ion density, magnetic field, ion and electron energies), and the degrees of ionization, (2) electron and ion thermal conductivities, energy exchange between electrons and ions, heat transport, (3) Hall effect, (4) neutron generation rate, and (5) radiation losses. They demonstrate good agreement between calculated and measured current derivative and voltage waveforms for one Filippov type PF, one Mather type PF, and one spherical PF on both sides of the dip for "... some operating regimes". They also present a novel ion acceleration model that they use to compute neutron emission rate, which is within a factor 2 of the measured neutron yield.

7. Kueny, Flicker, and Rose (2009) [328] present simulations of 3 PF devices using the ALEGRA-HEDP code. The following quote from this report describes the capabilities "ALEGRA-HEDP is a 2D/3D ALE (Arbitrary Eulerian-Lagrangian) code featuring 
coupled, operator-split models for hydrodynamics, magnetics, thermal conduction, and radiation physics. multi-material, multiphase equation-of-state (EOS) models. material strength and fracture models. MHD including advanced thermal/electrical conductivity and lumped circuit models. two-temperature physics (separate ion and electron temperatures). emission, multi-group diffusion and implicit Monte-Carlo for radiation modeling. neutron yield and time-of-flight diagnostics. and a very full complement of output diagnostics. The ALEGRA code family runs both in serial and massively parallel mode on a wide variety of platforms, and has been successfully used to model a wide variety of experiments with high fidelity in both two and three dimensions". They report current waveforms and neutron emission until the code stops because of convergence failure. The calculated peak currents were in good agreement with experiments. However, the circuit inductance and resistance values were conveniently chosen, not measured.

8. Meehan and Niederhaus (2014) [329] report on "Fully Three-dimensional Simulation and Modeling of a Dense Plasma Focus". The report compares 2D and 3D calculations of current waveforms with experimental waveforms and finds that they do not match with respect to the rundown time and the peak current. Tweaking the 2D calculation by increasing the series inductance from $25 \mathrm{nH}$ to $28.2 \mathrm{nH}$ gave a better match with rundown time but not with peak current.

9. Liu et al. (2016) describe plasma focus simulation with a particle-in-cell code LSP, where "[t]he discharge is simulated in two phases: an MHD fluid phase followed by a fully kinetic phase". "The plasma is initiated as a MHD fluid to reduce computational time. When the plasma has reached sufficient proximity to the axis, then the code is switched into a fully kinetic simulation to capture the relevant physics during the pinch, including beam formation". The code is used to look at the effect of the radius of a cavity in the anode on the development of the $\mathrm{m}=0$ instability. The motivation is to deliberately induce the instability in the hope that it would enhance the beam-target neutron yield. The report compares the LSP calculations with a snowplow model: "The trajectories of the slugs predicted by the snowplow model match well with those of the LSP model, differing in their times to reach the axis only by several ns or less. Additionally, the temporal evolution of the sheath densities is well-recreated by the snowplow model...". The calculations reveal the existence of an optimized value of the radius of the inner cavity for enhancing the neutron yield caused by a linear ion beam formed during the $\mathrm{m}=0$ instability.

10. Li et al. (2017) [331] from Los Alamos National Laboratory report on "high-fidelity 2D and 3D numerical magnetohydrodynamic (MHD) simulations using the LACOMPASS code to study the pinch formation dynamics in a DPF and the associated instabilities and neutron production". Kelvin-Helmholtz instability is observed in the radial phase, as well as in the pinch phase. Although 2D calculations show $\mathrm{m}=0$ instability only, 3D calculations show $\mathrm{m}=1$ kink instability after imposing an azimuthal perturbation. They observe: "instabilities affect the magnitude and time and energy shape of the neutron pulses at about the $20 \%$ level". They have calculated and measured neutron spectra along the axial direction. No information is given about neutron spectrum along the radial direction. Investigation of the effect of an axially placed obstacle (called "re-entrant tube") on the neutron emission leads to the conclusion that multiple neutron pulses are emitted in the absence of the obstacle from pinches that occur at different heights, while placing it at an optimum distance produces just one neutron pulse of a smaller duration.

\subsubsection{Kinetic Simulations}

The problems with fluid simulations discovered so far and discussed in the previous subsection have prompted efforts at kinetic simulations, with hopes of mitigating some of these. Typically, these computations begin with an initial plasma placed at the end of the 
rundown phase and only treat the radial phase with the kinetic method. This subsection reviews some of these efforts.

(a) Schmidt, Tang, and Welch (2012) [332] report "Fully Kinetic Simulations of Dense Plasma Focus Z-Pinch Devices". Their 2D calculation in $(\mathrm{r}, \mathrm{z})$ coordinates is initialized at the end of the rundown phase with a $1 \mathrm{~mm}$ width plasma sheath of uniform mass density $\rho_{0}$ travelling at the speed $\mathrm{B} / \sqrt{2 \mu_{0} \rho_{0}}$ (governed by the equality between the wind pressure and magnetic pressure). Some of the important new results from fully kinetic computations are: (1) impedance approaching $1 \Omega$ in the pinch phase. (2) localized sub-millimeter size regions of high $(\sim 5 \mathrm{GV} / \mathrm{cm})$ axial electric field which are positive in some places and negative in other places, with positive regions dominating in intensity and number. (3) High frequency oscillations in axial electric field with a frequency $\sim 4 \mathrm{GHz}$. (4) High energy ions up to several MeV. From the text, it is not clear whether the localized domains are snapshots of either propagating or stationary high frequency waves.

(b) A follow-up paper from the same group [333] compares fully kinetic simulations with experimental measurements. Notable experimental results are: (1) High frequency electromagnetic fields in the $3-4 \mathrm{GHz}$ range are more intense in good quality discharges than in poor quality discharges. (2) Ion spectrum measured by ion time of flight is well reproduced by computations. (3) Neutron yields are within a factor 2 of the experimental yields.

(c) Fully kinetic simulations of a Mega Joule class plasma focus similar to Gemini [334] in cylindrical $(\mathrm{r}, \mathrm{z})$ geometry with cell sizes $0.1 \mathrm{~mm}$ radially and $0.2 \mathrm{~mm}$ axially show beam-target fusion occurring within the $2.5 \mathrm{~mm}$ pinch radius with energies from 1.8 to $5.5 \mathrm{MeV}$ with a peak at $2.45 \mathrm{MeV}$ and thermonuclear neutrons outside the pinch with $11 \mathrm{keV}$ spectral width. This apparently refers to axially observed neutron spectrum, although the text does not explicitly mention this.

\subsubsection{Particle in Cell Simulations of the Initial Phase}

This section reviews application of particle-in-cell (PIC) simulations to the initial phase of plasma focus in some detail. This phase deals with the transition from a "no-plasma" state to a weakly ionized plasma state. The number of charged particles involved in this transition varies by orders of magnitude within a short time. Initially, this number is not large enough to permit a meaningful definition of a phase space distribution function, let alone its derived quantities like density, temperature, pressure, current density, and velocity. Therefore, the resources and techniques of fluid and kinetic simulations discussed above are found to be completely inadequate.

Early simulations of the breakdown phase $[323,335,336]$ used the fluid approach which implicitly assumes a Maxwellian velocity distribution, which is certainly not a valid assumption in the first 15-20 ns of the discharge [337]. A statistical approach based on the Monte Carlo (MC) methods has been used with some success [338-343]. This approach typically calculates trajectories of charged test particles using Newtonian mechanics and classical electromagnetic fields and uses atomic physics to calculate probabilities of various excitation and ionization processes during collision of the test particle with the background neutral particles. The Monte Carlo method calculates the free path, change in the velocity vector and change in the state (excitation or ionization) of the particles involved in the collision event. The calculation of fields requires the spatial distribution of all charged particle ensembles. This uses the particle-in-cell (PIC) method. Then, the next movement of the particle take place. The combination of the MC and PIC methods allows determination of the distributions of the particle ensembles at each temporal step of the discharge evolution. Figure 47 below shows the well-known MC-PIC scheme comprising four modules: 'particle movement', 'collisions', 'weighting', and 'field', which produce all these actions. 


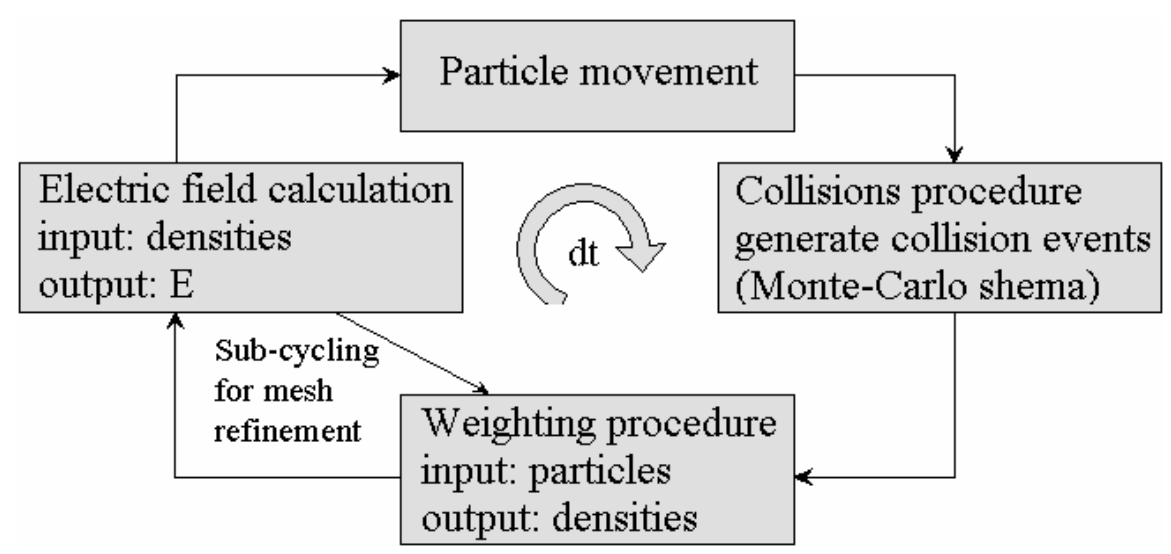

Figure 47. Monte Carlo computing cycle. Reproduced from Figure 2 of [338].

1. In the "Particle Movement" module equations of motion of charged particles are solved. If a particle leaves the volume, a collision with a boundary (either a dielectric or a metallic cathode) is generated. The dielectric absorbs the incident electrons and forms a surface charge. The electrons are reflected by the cathode while the ions hitting the cathode eject electrons with a ratio $\gamma \sim 0.15$.

2. The "Collisions procedure" generates collision events by Monte Carlo method using the cross sections of each scattering process, included in the code.

3. The densities of the charged particles are obtained by the PIC scheme in the "Weighting" module. It is optimized for faster weighting while sub-cycling for mesh refinement [338].

4. The "Field" module calculates the electric field by solving the Poisson equation. More accurate treatment [342] would be to solve Maxwell equations and to take into account induced magnetic field also. In fact, the induced magnetic field is not negligible, because of the very fast rising electric field (anode potential increases linearly with a rate of $2.10^{11} \mathrm{~V} / \mathrm{s}$ ). Probably the effect of magnetic field would be a decrease in the mobility of the charged particles and a corresponding decrease in the breakdown pressure.

Investigations using this basic scheme [338-343] use similar initial conditions and arrive at more or less similar conclusions regarding the growth of an ionization wave. The basic elements of this approach are illustrated using [338] as an example. The geometry of the problem is illustrated in Figure 48.

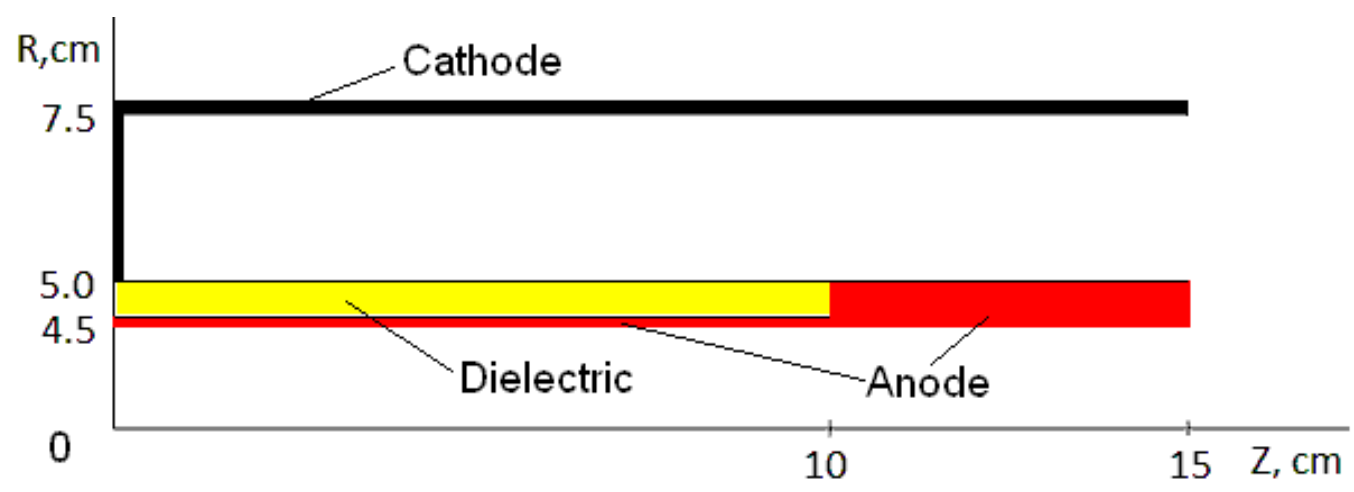

Figure 48. Simplified 2D geometry of the cross-section of a part of Mather type PF device (see Figure 1 of [338]). The picture additionally shows the geometry parameters used in the simulation. Similar geometry was used in [342]. Adapted from author's original data.

The gas was hydrogen, and a volume of $1.5 \mathrm{~L}$ that was relevant for the breakdown was considered. The initial number of charged particles was chosen to be 2000. This corresponds 
roughly to density equal to $1.4 \times 10^{6} \mathrm{~m}^{-3}$. The dielectric constant of the insulator was chosen to be $\varepsilon=10\left(\mathrm{Al}_{2} \mathrm{O}_{3}\right)$. An external potential increasing at a rate of $2 \times 10^{11} \mathrm{Vs}^{-1}$ was applied. Processes listed in Table 2 were taken into account for MC simulation of the collision events. The creation of photoelectrons from the cathode was considered in the following way [338]. It is known that in the transitions $\mathrm{H}_{2}\left(\mathrm{~B}^{1} \Sigma^{+} \mathrm{g}\right) \rightarrow \mathrm{H}_{2}\left(\mathrm{X}^{1} \Sigma^{+} \mathrm{g}\right)$ and $\mathrm{H}_{2}\left(\mathrm{C}^{1} \Pi_{\mathrm{u}}\right) \rightarrow \mathrm{H}_{2}\left(\mathrm{X}^{1} \Sigma^{+} \mathrm{g}\right)$, photons with energies of $11.3 \mathrm{eV}$ and $12.4 \mathrm{eV}$, respectively, are emitted. These photons are capable of ejecting photoelectrons from the cathode [344]. The lifetimes of the states $\mathrm{H}_{2}\left(\mathrm{~B}^{1} \Sigma^{+} \mathrm{g}\right)$ and $\mathrm{H}_{2}\left(\mathrm{C}^{1} \prod_{\mathrm{u}}\right)$ are quite small-below $1 \mathrm{~ns}$. Therefore, it was assumed that when an electron excites a molecule, it emits immediately.

Table 2. List of processes taken into account into the model. The set of cross-sections for all these processes are taken from $[345,346]$.

\begin{tabular}{cc}
\hline Elastic electron collision & $e+H_{2} \rightarrow e+H_{2}$ \\
\hline Elastic ion collision & $p+H_{2} \rightarrow p+H_{2}$ \\
\hline Ionization & $e+H_{2} \rightarrow 2 e+H_{2}^{+}$ \\
\hline Rotational excitation & $e+H_{2}\left(X^{1} \Sigma_{g}^{+}, v=0, J=0\right) \rightarrow e+H_{2}\left(X^{1} \Sigma_{g}^{+}, v=0, J=2\right)$ \\
\cline { 2 - 2 } & $e+H_{2}\left(X^{1} \Sigma_{g}^{+}, v=0, J=1\right) \rightarrow e+H_{2}\left(X^{1} \Sigma_{g}^{+}, v=0, J=3\right)$ \\
\hline Vibration excitation & $e+H_{2}\left(X^{1} \Sigma_{g}^{+}, v=0\right) \rightarrow e+H_{2}\left(X^{1} \Sigma_{g}^{+}, v=1,2,3\right)$ \\
\cline { 2 - 2 } Electronic excitation & $e+H_{2}\left(X^{1} \Sigma_{g}^{+}\right) \rightarrow e+H_{2}\left(b^{3} \Sigma_{g}^{+}\right)$ \\
\cline { 2 - 2 } & $e+H_{2}\left(X^{1} \Sigma_{g}^{+}\right) \rightarrow e+H_{2}\left(a^{3} \Sigma_{g}^{+}\right)$ \\
\hline Rydberg Excitations levels & $e+H_{2}\left(X^{1} \Sigma_{g}^{+}\right) \rightarrow e+H_{2}\left(d^{3} \Pi_{u}\right)$ \\
\hline Dissociation & $e+H_{2}\left(X^{1} \Sigma_{g}^{+}\right) \rightarrow e+H_{2}\left(B^{1} \Sigma_{u}^{+}\right)$ \\
\hline & $e+H_{2}\left(X^{1} \Sigma_{g}^{+}\right) \rightarrow e+H_{2}\left(c^{3} \Pi_{u}\right)$ \\
\hline & $\left.e+H_{2}\left(X^{1} \Sigma_{g}^{+}\right) \rightarrow e+H^{1}+H_{u}\right)$ \\
\hline & $e+H_{2}\left(X^{1} \Sigma_{g}^{+}\right) \rightarrow e+H_{2}(R y d b e r g)$ \\
\hline
\end{tabular}

When a photon is created, a ray originating from the location of the excited molecule is generated. This ray has a random spatial direction. The ray crosses the cathode at a point where a new photo-electron is created with probability $\gamma$ (the quantum yield for the given cathode material), which is assumed to have a constant value in the model. Photon reabsorption was neglected, because the pressure is quite low and the distance that a photon traverses is small. The VUV radiation played a significant role for sustaining the initial phase of PF discharge by the photo effect, considering that there are 93 optical transitions in the $\mathrm{H}_{2}$ Lyman band, $\lambda=(128.0 \div 164.6 \mathrm{~nm}), \lambda_{\max }=161.0 \mathrm{~nm}$, and 36 transitions in the Werner band, $\lambda=(109.8 \div 127.0 \mathrm{~nm}), \lambda_{\max }=116.0 \mathrm{~nm}$. It was presumed that by accounting for all of them, the re-absorption of the VUV quanta could be incorporated in the model. If the photo-effect is neglected at 3 Torr (the typical operational pressure of the device) the electrons disappear from the volume and go to the vessel walls after $22 \mathrm{~ns}$. This can be seen in Figure 49 on the curve for $\gamma=0$. 


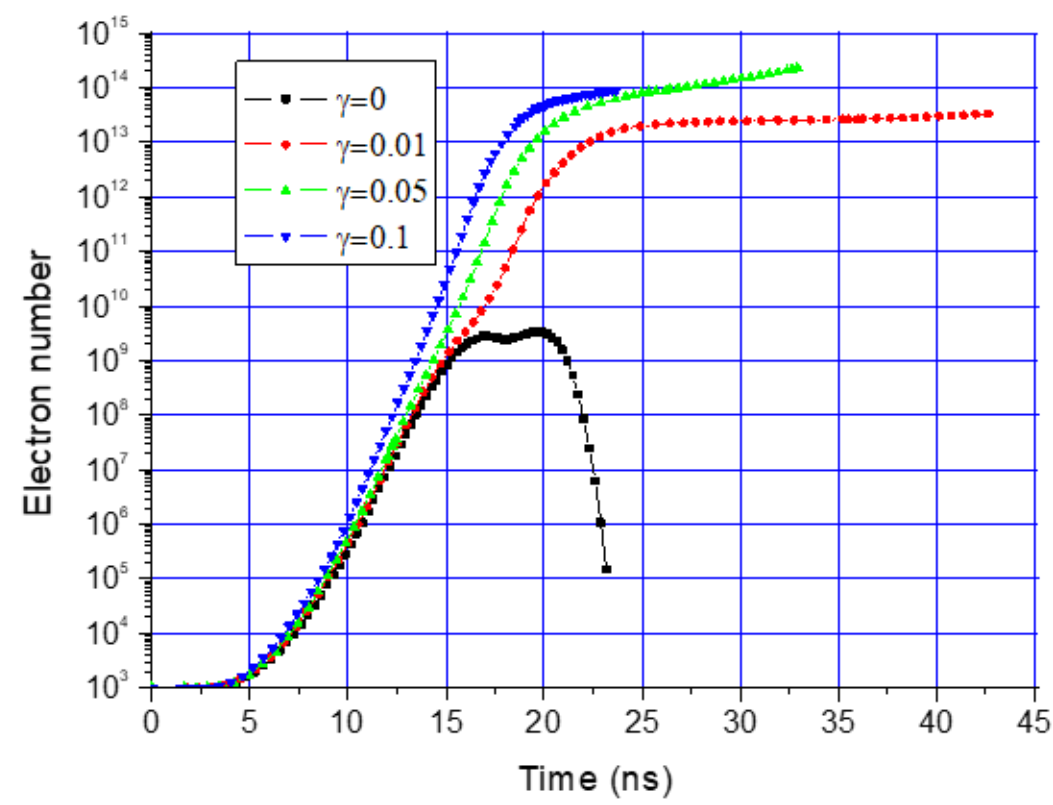

Figure 49. Number of electrons in the volume. (see Figure 2 of [343]). Reproduced with permission from Blagoev, A. Yordanov V. A modeling of the breakdown phase in the plasma focus discharge, Plasma Phys. Contr. Fusion 2009, 51, 124022. CIOP Publishing. All rights reserved.

Figures 49 and 50 show that for all non-zero values of the quantum yield, the charged particle density continues to rise even after the abrupt fall of the average electron energy.

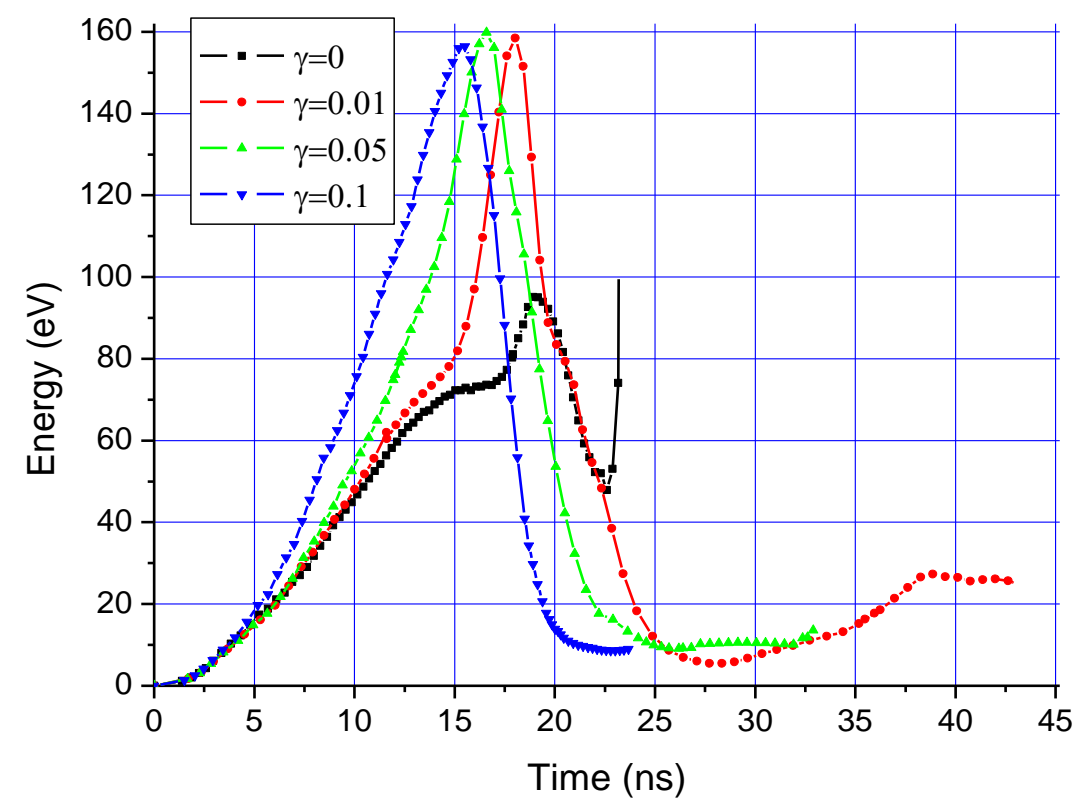

Figure 50. Average energy of the electrons (see Figure 3 of [343]). Reproduced with permission from Blagoev, A. Yordanov V. A modeling of the breakdown phase in the plasma focus discharge. Plasma Phys. Contr. Fusion 2009, 51, 124022. CIOP Publishing. All rights reserved.

Figure 51 shows the most important results of the calculations. It shows the distributions of the charges and the potential at the bottom space of the Mather type PF device at different moments of the breakdown process. The dominantly parallel equipotential lines are drawn on the right side of the picture with densely bunched curved lines in the vicinity of the cathode bottom showing the large electric field there. 

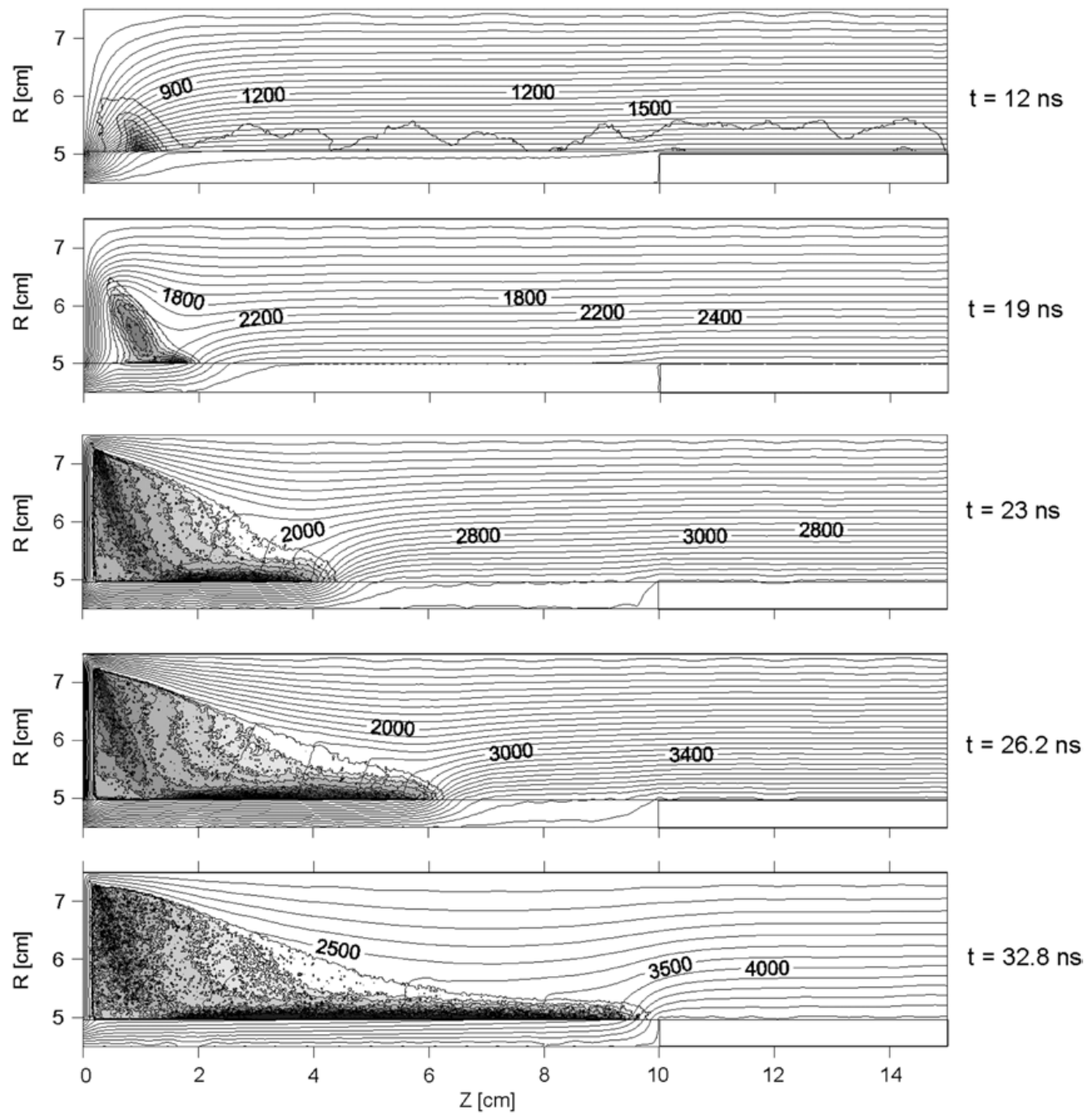

Figure 51. Spatial distribution of charged particles and electrostatic potential. The figure shows the electrons starting at the left side of the figures near $Z \sim 0 \mathrm{~cm}$ at early times, propagating towards the right with time and concentrated on the surface of the insulator sleeve at $R=5 \mathrm{~cm}$. The ionization front arrives at $\mathrm{Z} \sim 10 \mathrm{~cm}$ at time $\mathrm{t} \sim 32.8 \mathrm{~ns}$. The contour lines corresponding to the electric equi-potential lines are the smooth lines which extend all the way to the right and are labeled with values from $900 \mathrm{~V}$ to $4000 \mathrm{~V}$. These distributions were taken for $\gamma=0.05$, but similar results were observed for all other investigated non-zero values of $\gamma$. Intensification of electric field at the head of the ionization front is clearly seen. (see Figure 4 of [340]). Reproduced with permission from Yordanov, V. Ivanova-Stanik, I. Blagoev, A. Development of the ionization wave in the breakdown of the plasma focus device. J. Phys. D: Appl. Phys. 2007, 40, 2522-2525. CIOP Publishing. All rights reserved.

At $12 \mathrm{~ns}$, there is a considerable number of charged particles (shown by the closed lines of equal electron density) in the area between the bottom of the cathode and the dielectric. However, this density is still not high enough to disturb the external field. The time when a significant modification of the electric field arises can be determined from Figure 49 . For the duration of $7 \mathrm{~ns}$, between the 12th and the 19th ns, the density of charged particles increases by 5 orders of magnitude sustained by the high average electron energy. Then at the 19th ns the potential distribution exhibits a low electric field zone (Figure 51). In the same spatial region, the density of the potential lines, representing the levels of equal charged-particle concentration, is high. They form a compact structure, as can be seen at the time frames for the 12th and the 19th ns. It is seen from the next frames that this structure begins to move towards the anode and slide over the insulator surface in a zone where the field is low. Such effects are not observed for zero value of $\gamma$. The photo-electrons sustain the bulk of electrons causing the latter to grow close to the cathode bottom, thus drastically 
increasing the role of the photo-effect. There is a strong electric field at the head of this surface ionization wave moving towards the anode. In the 32.8th ns frame the head of the ionization wave is seen to come quite close to the left part of the anode indicating imminent closure of the anode-cathode gap. For experimental validation of this model, a mock-up device with flat parallel electrodes was created (see Figure 52). The cathode was L-shaped, so the short arm corresponds to the bottom part of the PF electrode's compartment. The electrodes of the discharge gap were aluminium plates separated with a $5 \mathrm{~mm}$ thick flat acryl insulator (see Figure 7 of [343]). The anode plate behind the insulator was completely insulated in order to avoid transverse discharges and direct ohmic current that could cause a strong return stroke. The 2D sectional view of the model device is similar to the bottom part of the PF. In 2D geometry the potential lines between the dielectric and the cathode resemble those in the PF device.
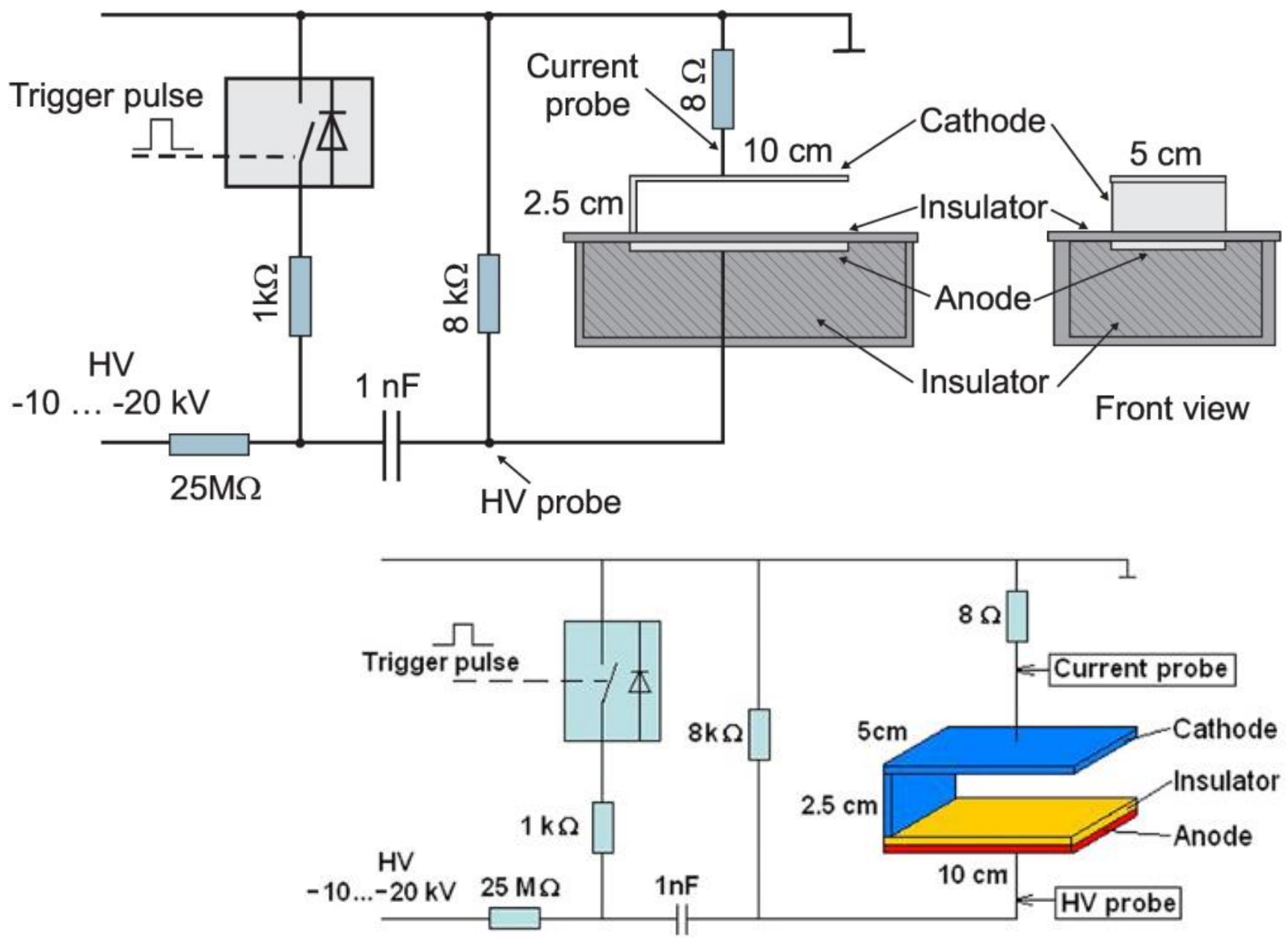

Figure 52. PF-like model device (geometry and electric circuit) (see Figure 1 of [340], Figure 7 of [343]). Reproduced with permission from Yordanov, V. Ivanova-Stanik, I. Blagoev, A. Development of the ionization wave in the breakdown of the plasma focus device.J. Phys. D: Appl. Phys. 2007, 40, 2522-2525 and. Blagoev, A. Yordanov V. A modeling of the breakdown phase in the plasma focus discharge. Plasma Phys. Contr. Fusion 2009, 51, 124022. (CIOP Publishing. All rights reserved.

The model device was placed in a vacuum chamber which permitted evacuation, control of the filling gas (Ar) pressure and optical observation. The optical images were recorded by a camera type 4 Quick Edig at 2 ns exposure time using the maximal sensitivity. The direction of observation was normal to the plane of the L shaped cathode.

The frame separation was chosen suitably using camera controls. The $1 \mathrm{nF}$ storage capacitor was charged up to $20 \mathrm{kV}$. Figure 53 compares the optical images and results from the model using the MC-PIC code. The respective changes were done in the model to 
conform to the geometry, filling gas (Ar) and external applied voltage on the anode. The list of processes involved are given in Table 3.
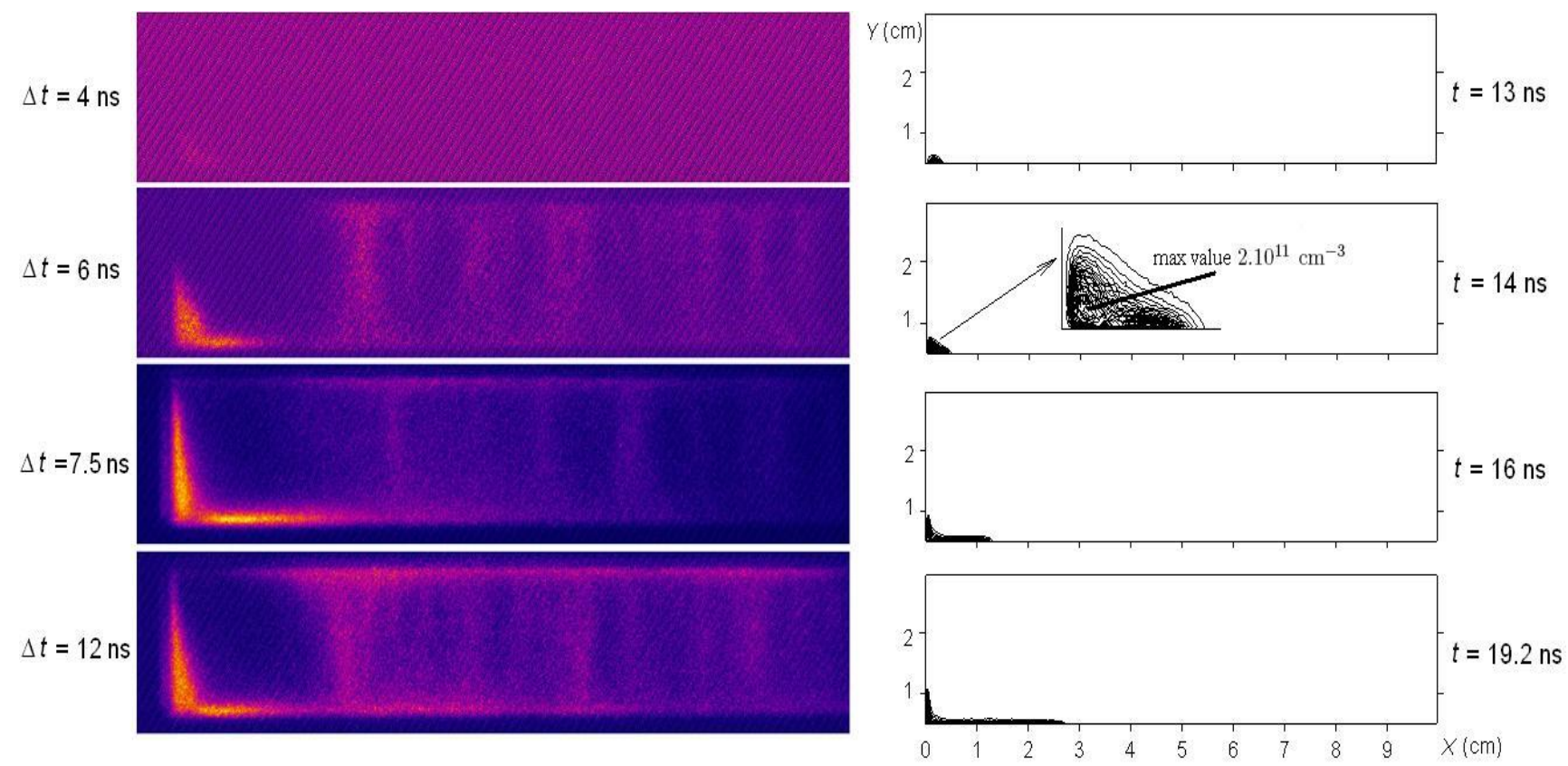

Figure 53. Comparison of the experimental data with the model (see Figure 8 of [343]). Reproduced with permission from Blagoev, A. Yordanov V. A modeling of the breakdown phase in the plasma focus discharge. Plasma Phys. Contr. Fusion 2009, 51, 124022. CIOP Publishing. All rights reserved.

Table 3. List of processes taken into account in the model. The set of cross-sections for all these processes are taken from $[345,346]$.

\begin{tabular}{cc}
\hline Elastic electron collision & $A r+e \rightarrow A r+e$ \\
\hline \multirow{2}{*}{ Electronic excitations } & $A r+e \rightarrow A r\left(4 s^{1} P_{1}\right)+e$ \\
\cline { 2 - 2 } & $A r+e \rightarrow A r\left(4 s^{3} P_{0}\right)+e$ \\
\cline { 2 - 2 } & $A r+e \rightarrow A r\left(4 s^{3} P_{1}\right)+e$ \\
\hline Ionization & $A r+e \rightarrow A r\left(4 s^{3} P_{2}\right)+e$ \\
\hline Penning ionizations & $A r+e \rightarrow A r(4 p)+e$ \\
\hline C & $A r\left(4 s^{3} P_{2}\right)+e \rightarrow A r\left(4 s^{3} P_{2}\right) \rightarrow A r^{+}+A r+e$ \\
\hline
\end{tabular}

Prompt de-excitation of the Ar resonance levels $\left(4 s^{1} P_{1}\right.$ or $\left.4 s^{3} P_{1}\right)$ was assumed as in the case of $\mathrm{H}_{2}$. The corresponding VUV spectral lines ArI 1048.2 and ArI 1066.7 are emitted with photon energies $11.83 \mathrm{eV}$ and $11.61 \mathrm{eV}$, respectively. At these energies, the photons are able to eject photoelectrons from the $\mathrm{Al}$ surface. Under the conditions shown in Figure 53 , besides the plasma layer on the horizontal insulator surface, there is also a plasma layer in front of the vertical portion of the cathode. At higher pressures this layer disappears. This layer is presumably due to the photo-effect from the cathode. This is confirmed by the next model results, shown in Figure 54. The figure presents the evolution of the chosen initial number of electrons (100) in the geometry of Figure 52. The charged particles born near the metal wall through acts of ionization multiplication are shown with arrows. The processes of CS formation to the point of voltage breakdown can be identified as volume ionization, plasma localization and gliding discharge. All of these steps, except the last one, can be observed in the models derived by the electrostatic (ES) approximation $[338,343]$. 
Figure 54 shows the volume ionization and plasma localization in the range 0 to $12 \mathrm{~ns}$. The arrows on these three time frames, $(a-c)$ denote the photo electrons created near the cathode. These photo electrons play essential role in the ionization growth in subsequent period.
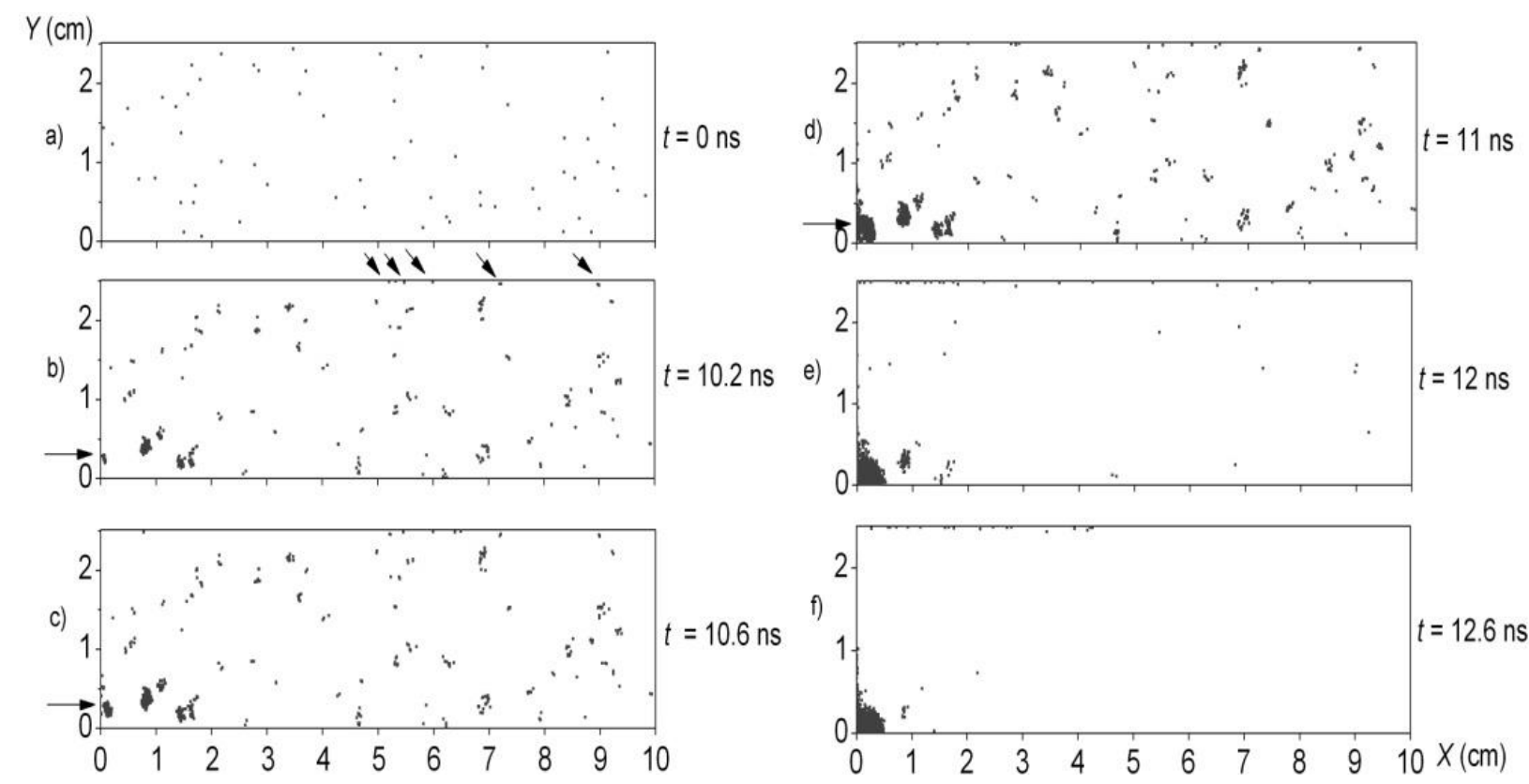

Figure 54. Initial phase of the ionization wave development (see Figure 9 of [343]). The volume ionization and plasma localization is seen in the range 0 to $12 \mathrm{~ns}$. The arrows on these three time frames, $(a-c)$ denote the photo electrons created near the cathode. Reproduced with permission from Blagoev, A. Yordanov V. A modeling of the breakdown phase in the plasma focus discharge. Plasma Phys. Contr. Fusion 2009, 51, 124022. CIOP Publishing. All rights reserved.

In the ES approximation, the magnetic field due to the current sheath is assumed to be negligible. This is true during the sliding discharge phase when the CS is forming. When the sliding discharge closes the anode-cathode gap, the formation of the CS is complete. This is followed by a rapid rise in the current, at which point magnetic effects grow in importance and the ES approximation fails. The electromagnetic PIC (EMPIC) approach [342] overcomes the limitation of the ES method by solving the full set of Maxwell's equations with the magnetic field consistently built in from the very beginning of the simulation.

Using the EMPIC framework on the configuration used in [338], the entire breakdown phase was simulated near to the point of dielectric breakdown in the filling gas [342]. It produced a physical picture in line with the ES simulations, with an identical ionization profile, generation of the sliding discharge, and plasma density with order of magnitude similar to the ES approach [338]. This work [342] further demonstrates low magnetic field strength during the sliding discharge phase, but a substantial increase in the magnetic field following an appreciable rise in the CS current near the end of this phase (Figure 55). 


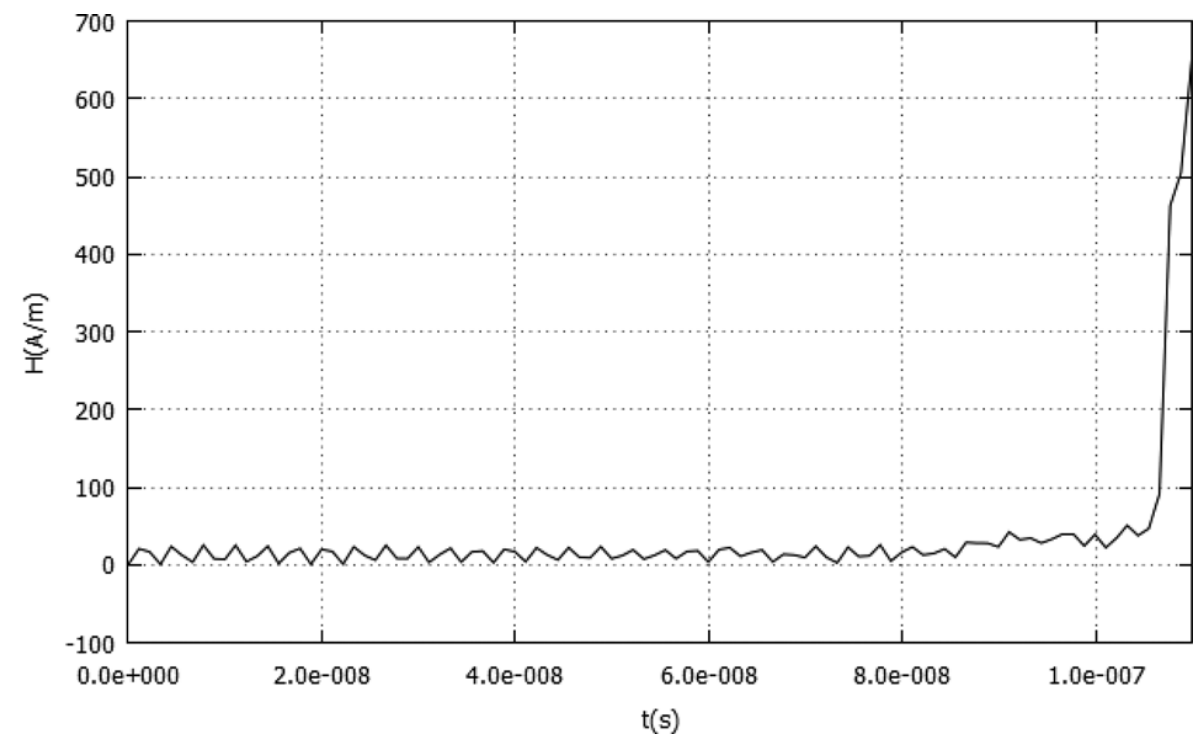

Figure 55. Time development of the magnetic field H Reproduced from Figure 5 of [342], (Phys. Plasmas 2014, 21, 023509) with the permission of AIP Publishing.

Accompanying the rise in the magnetic field strength with the closure of the anodecathode (AK) gap is a surge in the plasma density (Figure 56) and the (temporal) lifting off the electron layer near the vertical cathode plate (Figure 57).

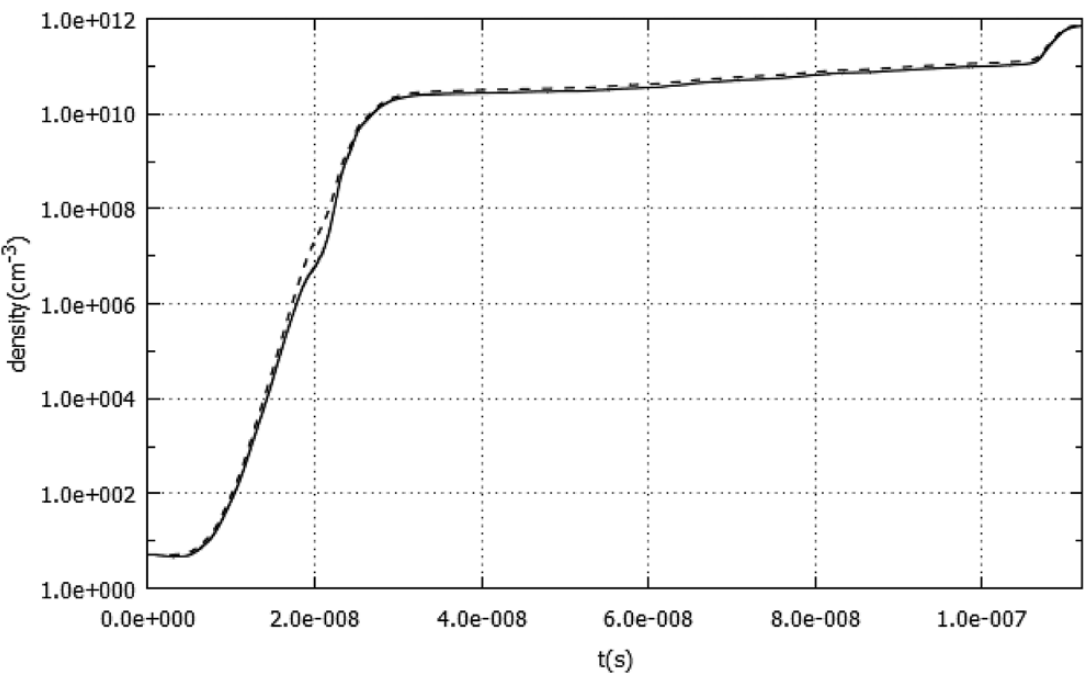

Figure 56. Averaged charge density plots (solid line for electrons, dashed line for ions). Reproduced from Figure 2 of [342], [Phys. Plasmas 2014, 21, 023509] with the permission of AIP Publishing. 


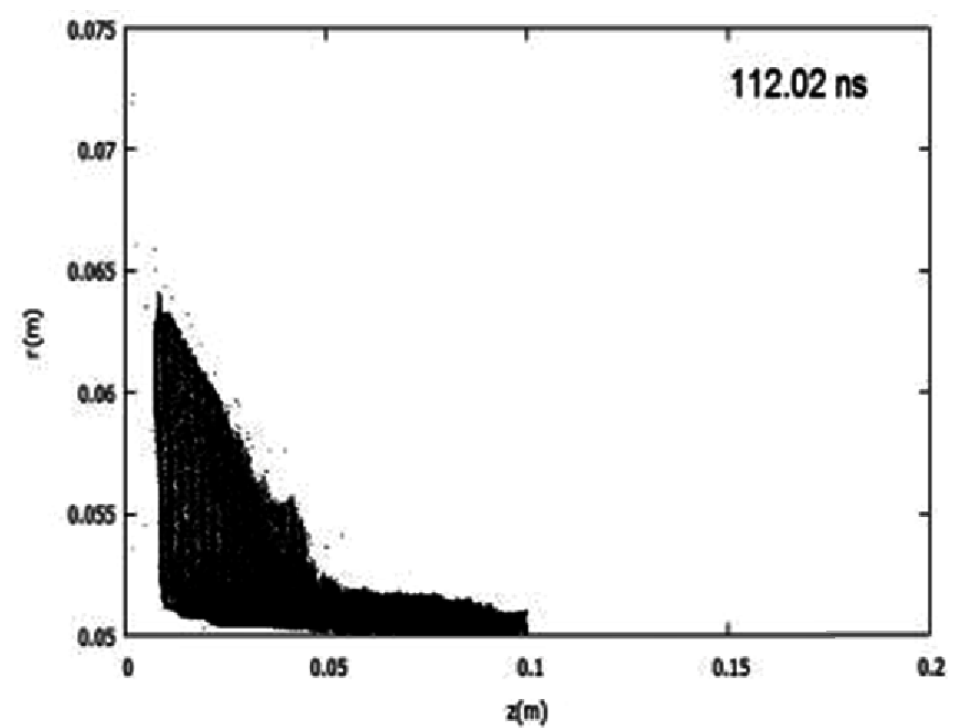

Figure 57. Lift-off of the electron layer near the vertical cathode plate at $112 \mathrm{~ns}$ [342]. The electron layer, shown by dark shaded region, separates from the insulator surface located at coordinates $(\mathrm{r}, \mathrm{z}):(0.05,0)$ to $(0.05,0.1)$ at $112 \mathrm{~ns}$ because of the Lorentz force. This is indicated by the clear unshaded space between the shaded electron cloud and the position of the insulator surface. Reproduced from Figure 3 of [342], (Phys. Plasmas 2014, 21, 023509) with the permission of AIP Publishing.

The increase in the local magnetic field strength enhances the electric field in the CS leading to further ionization. Inside the current sheath region near the vertical cathode plate, the increased ionization rate due to the surge in the local magnetic field caused a two-orders increase in the electron density, from $10^{12} \mathrm{~cm}^{-3}$ to $10^{14} \mathrm{~cm}^{-3}$. The magnetic field strength was also the highest in this region, which resulted in a Lorentz force strong enough to cause a sudden, temporal lifting of the local electron layer (Figure 57).

Following this preliminary work [342], simulations based on similar MC-EMPIC framework were performed on the UNU-ICTP PF device with a protruded anode configuration for different insulator lengths [347]. Physical processes similar to those observed in [338,342] were reproduced despite the configurational difference. In line with [342], a surge in the CS current alongside the rise in the magnetic field strength was observed near the end of the sliding discharge, indicating the beginning of voltage breakdown. Performing the simulations over different insulator lengths, the EMPIC results showed that the time to voltage breakdown scales linearly with the insulator length (Figure 58). The voltage breakdown time was taken as the time at which the current experienced a rapid surge in value

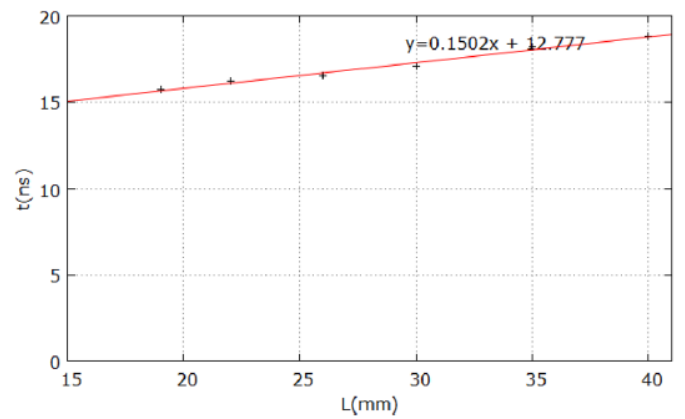

Figure 58. Simulated time for voltage breakdown as a function of insulator length from MC-EMPIC model (see text for details). Reproduced from Figure 7 of [347], (Phys. Plasmas 2014, 21, 113508) with the permission of AIP Publishing. 
Fast ionization waves, with nearly constant propagation velocities, that are responsible for generating the sliding discharge, were found for all the insulator lengths. The magnitude of these velocities, with weak dependence on the insulator length, is of the order of the ES simulation result.

Figure 59 shows the velocity variation with insulator length. It increases with insulator length, reaches a maximum, and subsequently declines. Physical details of the sliding discharge were obtained in [348], where the UNU-ICTP PF device with a $2.6 \mathrm{~cm}$ insulator was simulated. Initiation of the sliding discharge occurred near the insulator surface $5 \mathrm{~mm}$ from the vertical cathode wall.

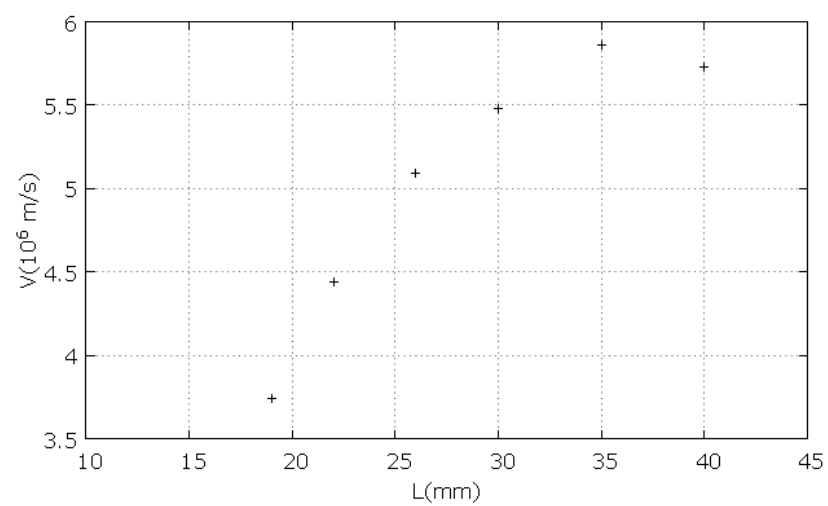

Figure 59. Velocity of the fast ionization wave for different insulator lengths Reproduced from Figure 5 of [347], (Phys. Plasmas 2014, 21, 113508) with the permission of AIP Publishing.

This was followed by the breakdown of the local electric field $(\sim 0.5 \mathrm{MV} / \mathrm{m})$ at about $11 \mathrm{~ns}$ after the application of the input voltage pulse (Figure 60).

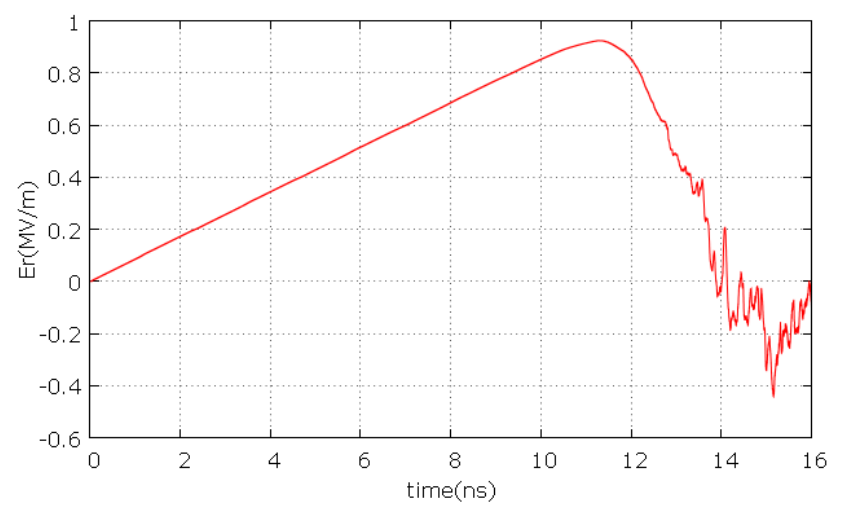

Figure 60. Simulated local (radial) electric field breakdown at the axial location $\mathrm{z}=5.2 \mathrm{~mm}$ just above the insulator surface [348]. (C) 2021 IEEE. Reprinted from Figure 3 of [348] with permission, from IEEE Trans. Plasma Sci. 2019, 47, 4883.

The high local ionization rate led to large amount of space charges from volume ionization, which contributed to the electric field enhancement. Consequently, an ionization wave driven by the high negative electric field at its tip propagated down the tube, creating new ionizations along the way. At the ionization front, the electron density reached $10^{13} \mathrm{~cm}^{-3}$. The sliding discharge continued to propagate down the vertical end of the insulator to short the AK gap after it passed the end of the top insulator surface. Figure 61 presents a snapshot of the sliding discharge at various times. 


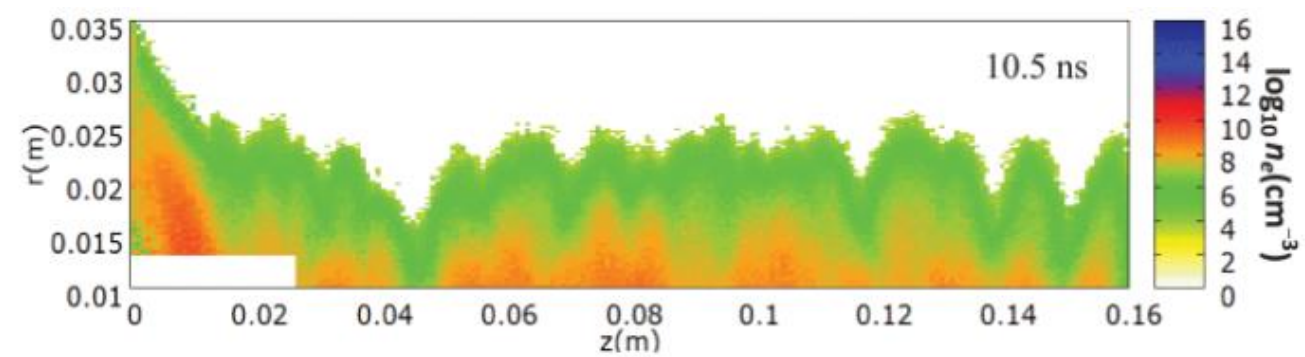

(a)

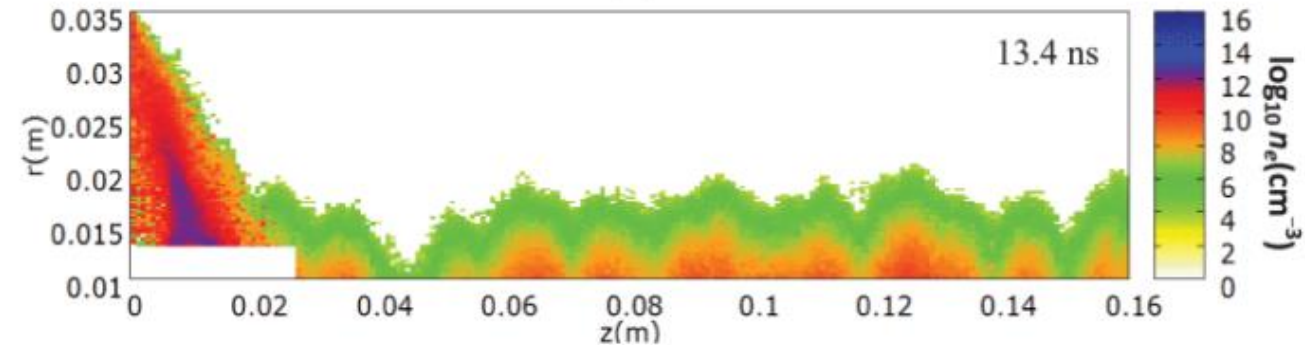

(b)

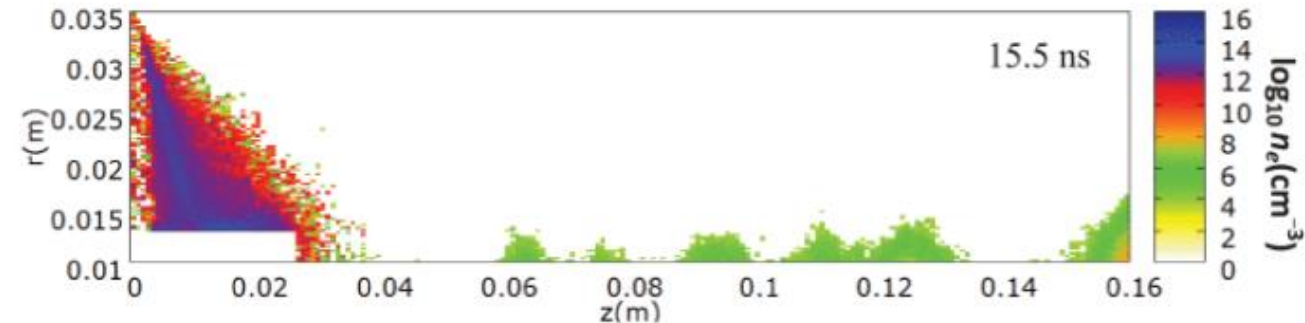

(c)

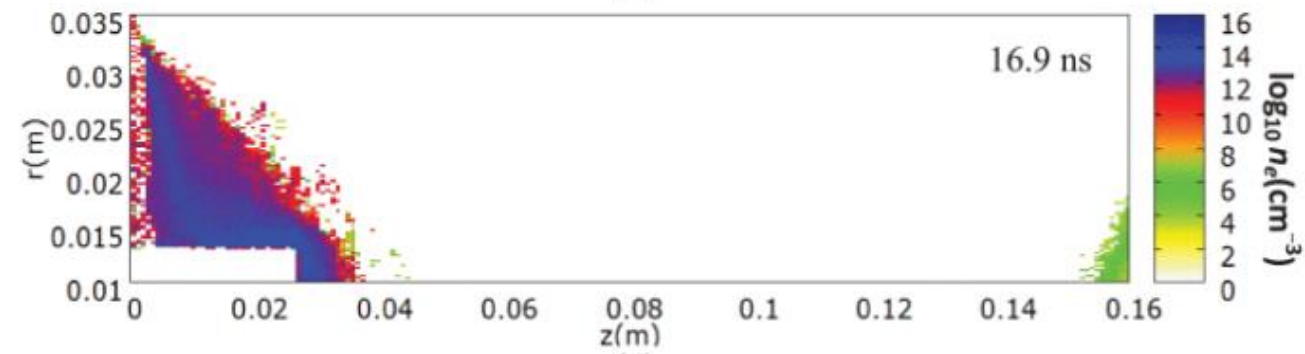

(d)

Figure 61. The sliding discharge of the UNU-ICTP PF device [348]. The figure shows snapshots of electron density distribution in (r,z) space at 4 times: $10.5 \mathrm{~ns}, 13.4 \mathrm{~ns}, 15.5 \mathrm{~ns}$, and $16.9 \mathrm{~ns}$. Electron density is depicted on a logarithmic color scale, with blue being the top of the scale. The front of the high density region coded blue can be seen sliding from $\mathrm{z}=0.01 \mathrm{~m}$ at $\mathrm{t}=13.4 \mathrm{~ns}$ to $\mathrm{z}=0.02 \mathrm{~m}$ at $\mathrm{t}=15.5 \mathrm{~ns}$ before completely enveloping the whole insulator sleeve at $\mathrm{t}=16.9 \mathrm{~ns}$. (C) 2021 IEEE. Reprinted, with permission, from Figure 4 of [348] (IEEE Trans. Plasma Sci. 2019, 47, 4883-4890).

Results from the computation of the surface charges accumulated over time are displayed in Figure 62 at various times. It shows that the charges on the insulator surface glide along with the sliding discharge. The value of the surface charge density increases with time and saturates at about $7.5 \times 10^{-5} \mathrm{~cm}^{-2}$. Note that the surface charge density is relatively uniform across the main discharge column. From the computed electric field of the simulated surface charge density, it was determined that the local electric field distribution was dominated by accumulated surface charges resulting from plasma electrons in the discharge volume striking the surface. Thus, the surface charges on the insulator play a primary role in shaping the sliding discharge characteristics. 


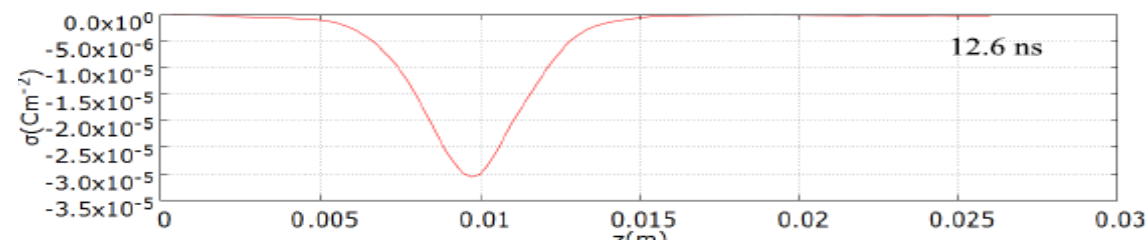

(a)

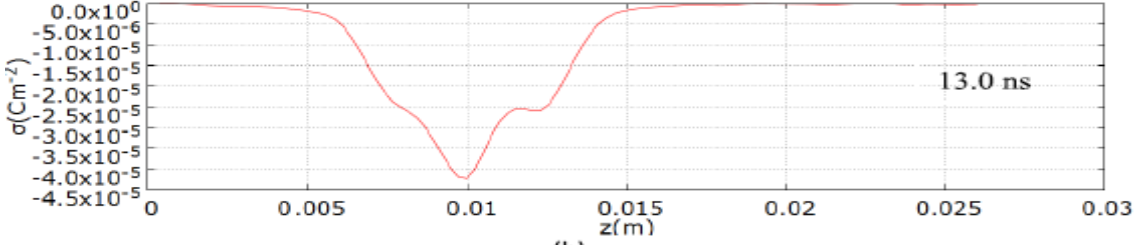

(b)

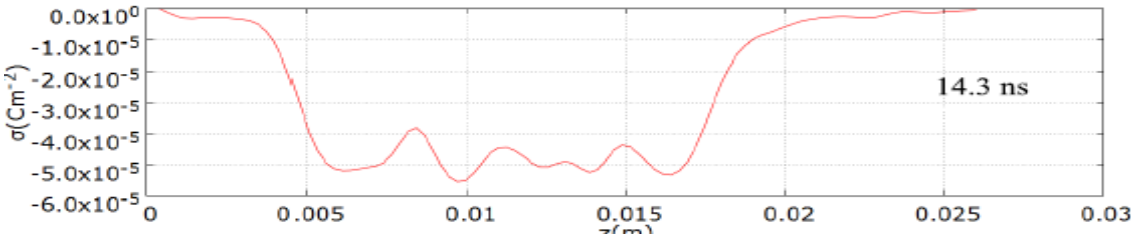

(c)

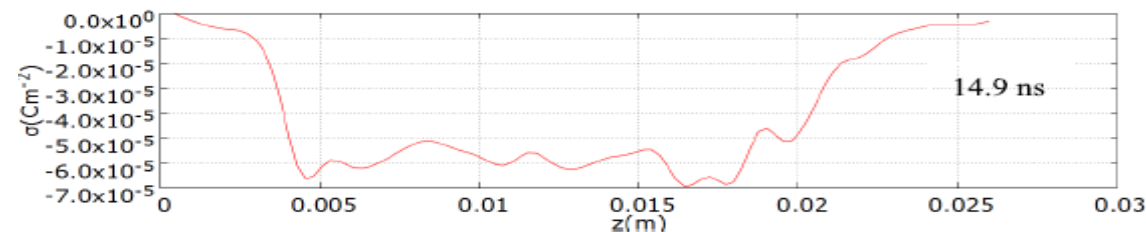

(d)

Figure 62. Spatial profile of the accumulated plasma charges on the insulator (C) 2021 IEEE. Reprinted, with permission, from Figure 7 of [348]. (IEEE Trans. Plasma Sci. 2019, 47, 4883-4890).

Many simulations [342,347,348] confirmed the exponential increase in the magnetic field strength near the end of the sliding discharge phase, thus demonstrating unequivocally the significant role of the magnetic field beyond this phase. However, the simulations could not carry on further without consistently simulating the voltage breakdown itself, since the computations so far were carried out under the assumption of an imposed constantly increasing voltage profile. To further improve the simulation and to model the voltage breakdown itself requires replacing the independent voltage source with an external circuit that couples self-consistently with the plasma dynamics. Such an external capacitive driving circuit was created and numerically implemented $[349,350]$ on the UNU-ICTP device. Incorporating the external circuit into the plasma dynamics allows one to carry the simulation from the breakdown to the axial acceleration phase, where the full lifting of the CS from the insulator surface occurs. With this modification, electrical breakdown of the filling gas was simulated successfully $[349,350]$, following a drop in the applied voltage across the device and a concurrent substantial rise in the circuit current. Consequently, the plasma became increasingly magnetized while the growing magnetic field over time led to the gradual lifting off of the well-formed CS into the axial acceleration phase. Figure 63 shows the simulated voltage-time profile which clearly shows voltage breakdown taking place at $15.8 \mathrm{~ns}$, after which the voltage drops and oscillates around $3 \mathrm{kV}$. This waveform exhibits an oscillatory behavior and voltage breakdown strength similar to those obtained experimentally (Figure 64). The difference between the simulated and experimental profiles is that the frequency of the oscillating voltage is lower in experiment than in the simulation. This difference is due to the fact that the stray capacitance used in the simulation was much smaller than the expected value inherent in the experiments. Note that the stray capacitance controls the rise time of the voltage pulse and the oscillation frequency but does not otherwise affect the essential shapes of the breakdown voltage and current profile. 


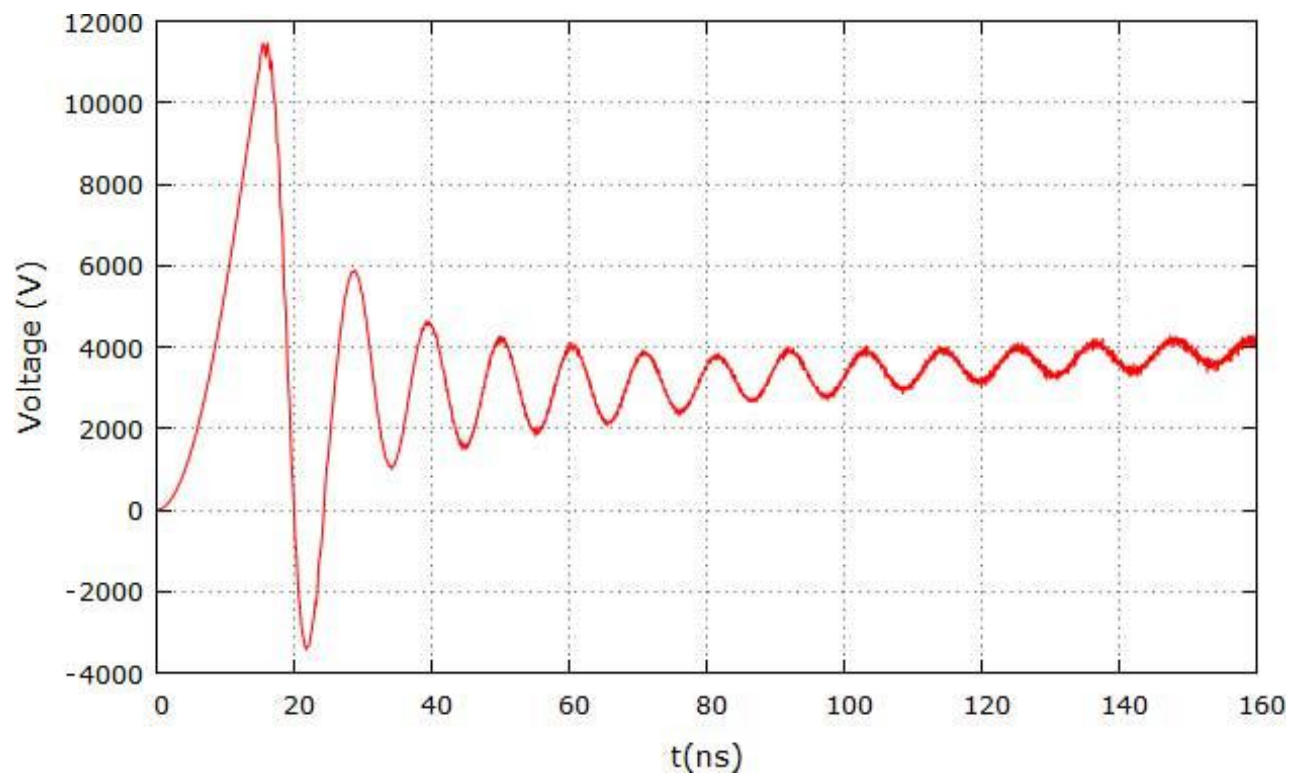

Figure 63. Simulated voltage time profile from the circuit-PF coupled simulation. @ 2021 IEEE. Reprinted, with permission, from Figure 3 of [351], (IEEE Trans. Plasma Sci. 2018, 46, 943-946).

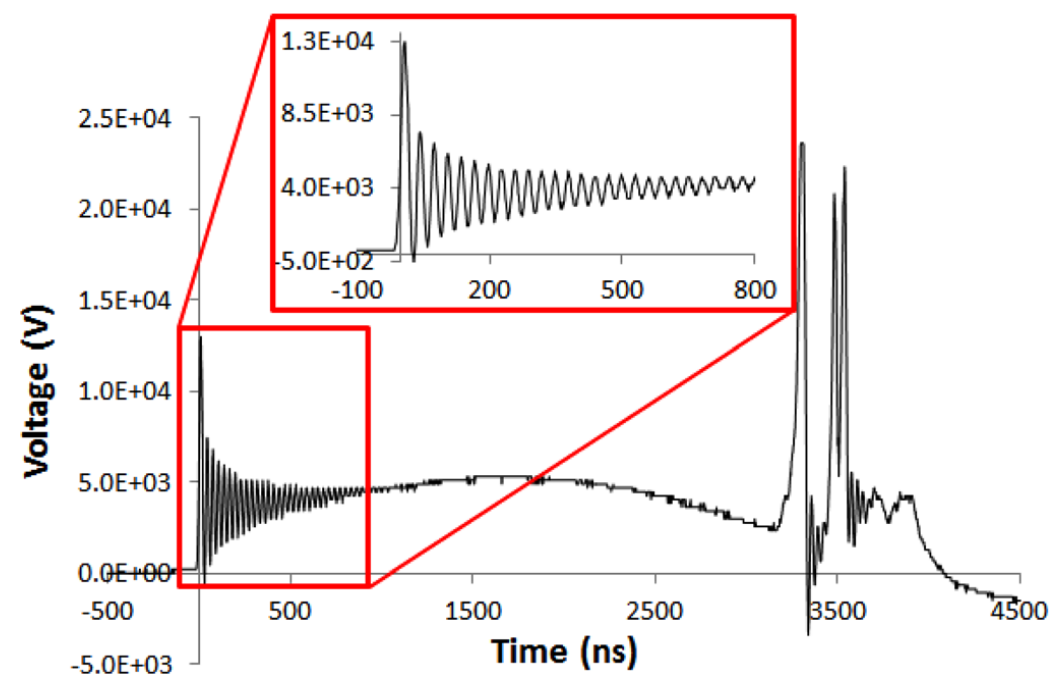

Figure 64. Typical experimental voltage waveform from the UNU-ICTP device. Reproduced from Figure 6 of [350], (Phys. Plasmas 2015, 22, 033524) with the permission of AIP Publishing.

In the simulations, the $1 \mathrm{nF}$ stray capacitance was built into the external circuit. Choosing a $1 \mathrm{nF}$ stray capacitance value allowed PF breakdown simulation to be carried out up to the early axial acceleration phase within a reasonable amount of physical simulation time. In Figure 63, although the voltage is oscillating at a regular frequency, its average value nevertheless grows slowly with time although the circuit current continues to rise (Figure 65). In Figure 65, voltage breakdown occurred at 16 ns, signified by the onset of increased current. 


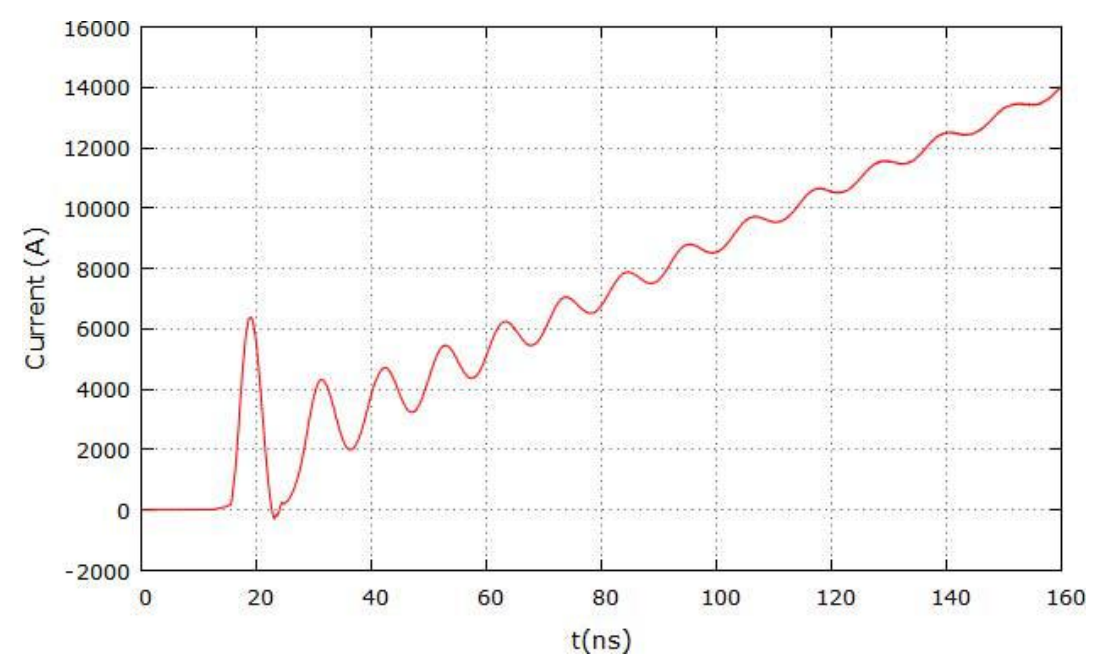

Figure 65. Current flowing through the PF walls as a function of time. Reproduced from Figure 11 of [350], (Phys. Plasmas 2015, 22, 033524) with the permission of AIP Publishing.

After voltage breakdown, the current continued to increase into the $\mathrm{kA}$ level, along with the growth in the magnetic field strength. As a result, the Lorentz force from the magnetic field acting on the plasma charges in the CS increased. Over time, the Lorentz force became strong enough to lift the CS off the insulator surface. Both ends of the CS moved vertically and horizontally along the walls of the cathode and anode, respectively, (Figure 66) during the axial acceleration phase. Between the time prior to breakdown to $177 \mathrm{~ns}$, the magnetic field strength was found to increase by 3 orders of magnitude-from 100 to $100,000 \mathrm{~A} / \mathrm{m}$.

By assembling together the physical picture from the pre-breakdown to the breakdown and finally to the axial acceleration phase, it is clear that the PF continues to undergo dynamic transformation in the physical parameters. This is also especially true in the EM fields, beginning from a purely electrostatic field to one where the magnetic field grows and increasingly becomes dominant. All these hint to a physical state of thermodynamic nonequilibrium in the CS governing its dynamics. Figure 67 shows that this is indeed the case. In the figure, the number of electrons in the discharge volume were grouped according to their energies. ES simulations had already predicted a non-Maxwellian electron energy distribution function EEDF during the pre-breakdown phase. Even when the PF was well into early the axial phase, at 39 ns say, the EMPIC simulations showed that the EEDF did not equilibrate into a Maxwellian distribution. Figure 67 shows that if the system were to reach thermal equilibrium, the average electron temperature would have been $1.9 \mathrm{eV}$. 


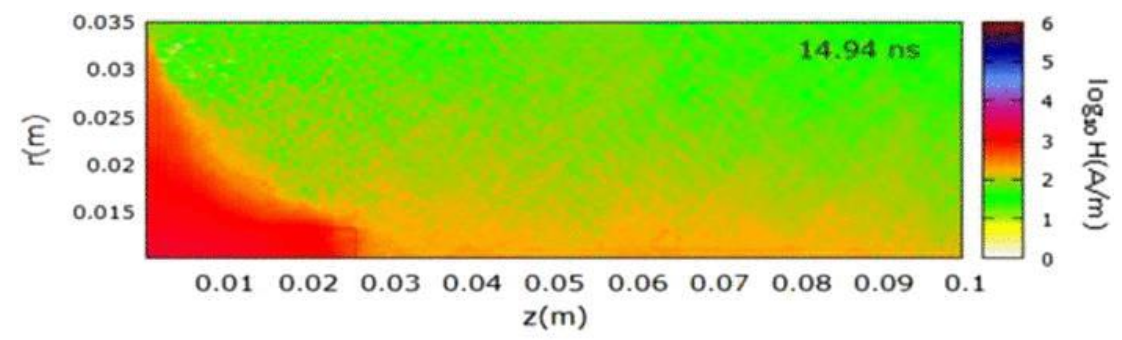

(a)

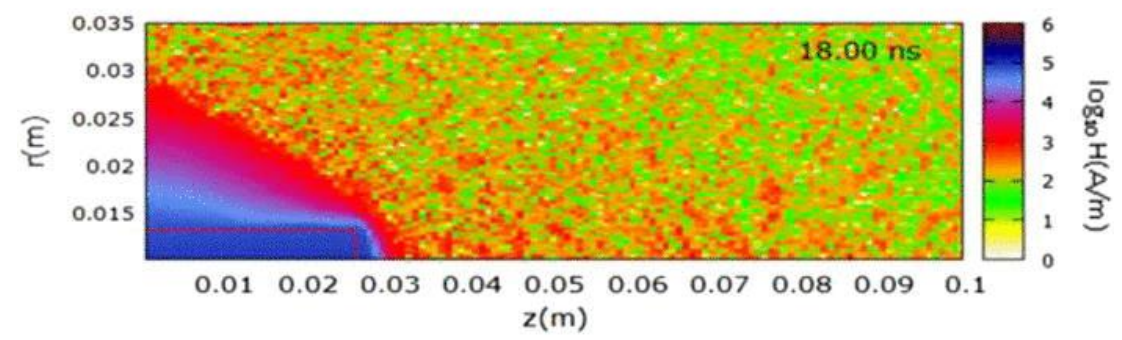

(b)

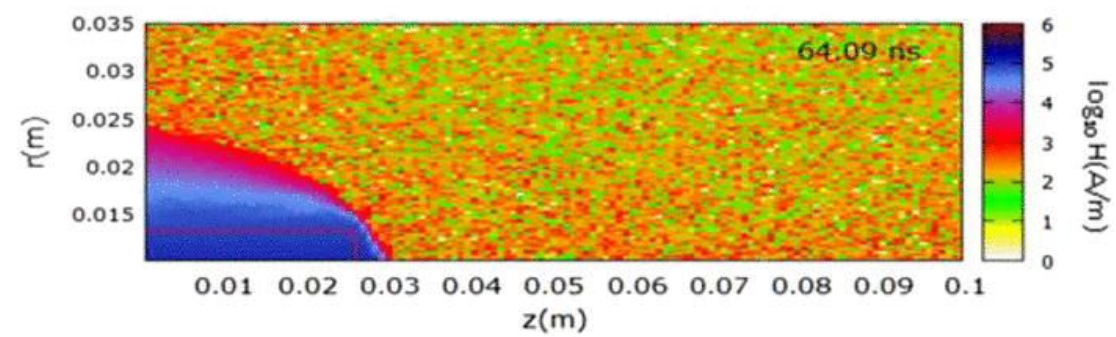

(c)

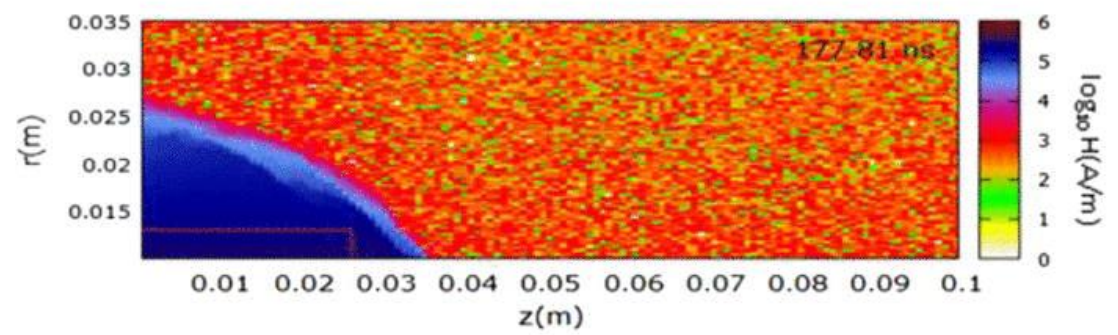

(d)

Figure 66. Development of the self generated, highly localized intense magnetic field lifting up and driving the CS in the axial acceleration phase [350]. Blue indicates the region of high magnetic field. (a) Building up of the magnetic field intensity around the insulator region is evident at $14.94 \mathrm{~ns}$. (b) At $18 \mathrm{~ns}$, the discharge has progressed sufficiently and the current through the anode has become high enough so that the entire insulator sleeve is now seen as blue. The return path of the current density is still distributed quite near to the surface of the insulator. (c)The current and magnetic field distribution expands but can be seen to mostly follow the "rectangular" shape of the insulator sleeve up to $\mathrm{t}=64 \mathrm{~ns}$. (d) By $\mathrm{t}=178 \mathrm{~ns}$ the plasma current distribution has formed into the typical curve of the current sheath as shown by the curved shape of the blue region of high magnetic field. Reproduced from [350], (Phys. Plasmas 2015, 22, 033524) with the permission of AIP Publishing. 


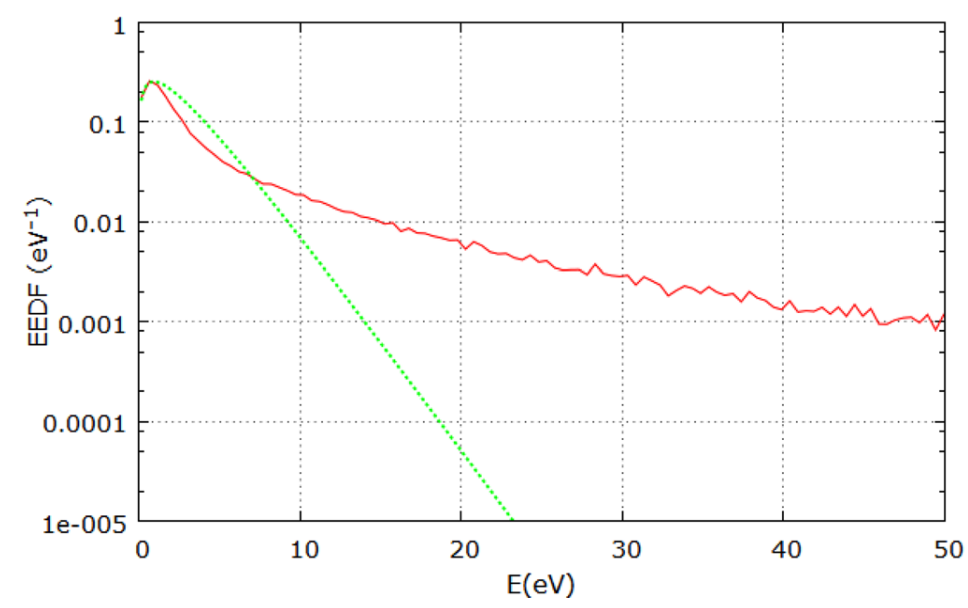

Figure 67. Simulated electron energy distribution function (EEDF) at $39 \mathrm{~ns}$ (in red), with a Maxwellian fit of temperature $1.9 \mathrm{eV}$ (in green) Reproduced from Figure 10 of [350], (Phys. Plasmas 2015, 22, 033524) with the permission of AIP Publishing.

However, lots of electrons were still having energies greater than $10 \mathrm{eV}$. The result is that up to the axial accleration phase, the electrons cannot be assumed to follow a Maxwellian distribution. This conclusion poses a fundamental difficulty to fluid-based simulation models which to a large extent rely on assumption of Maxwellian equilibrium.

Last but not the least, the oscillations in the voltage and current pulse that were experienced in the simulations $[349,350]$ and experiments were attributed to the plasma inductance, whose value was calculated from circuit analysis and agreed reasonably well with the computed experimental value [350]. In [350], an RLC circuit model comprising a plasma inductance was constructed to model the pre- and post-breakdown phases. Numerical solutions of the circuit equations produced voltage and current waveforms with a structure that matched well with the EMPIC results. As a result, the plasma inductance was identified as repsonsible for the oscillations.

To summarize, the particle-in-cell (PIC) simulation efforts achieved the following:

- Modeling in $\mathrm{H}_{2}$ showed development of the cylindrical ionization wave sliding along the insulator in the PF as observed in the real device at a pressure of 3 Torr. The model results in Ar could be compared with experiments. The development of the ionization wave was observed both in the model and in the experiment. The experiment showed that breakdown starts later in time than in the model. To have quantitative agreement for the time lag between the experiment and the simulation, a correct model for transport of radiation must be taken into account.

- EMPIC simulations carry the PF modeling further into the breakdown and the axial acceleration phase. They help uncover many interesting physics and phenomena during the early PF development and allow one to characterize the PF physical parameters. These localized physical phenomena happen on short timescales and pose serious diagnostic challenges to experiments. Without the proper diagnostic tool to carry out measurements, simulations using the coupled Monte Carlo PIC present the most viable computation tools to study the rich plasma phenomena manifesting in the breakdown and the early axial acceleration phases. Since the formation and propagation of the CS is determined in this early phase, the performance of a PF device may eventually be traced to the kinetics and structural formation and evolution of the CS in this development stage.

\subsubsection{Numerical Simulation of Ion Acceleration}

With the realization that the fusion neutrons emitted in a plasma focus had energy different from the energy in the center-of-mass frame of reference, efforts were made to understand the processes which could alter the velocity of the center-of-mass of reacting 
deuterons. These are often classified into a high laboratory kinetic energy species referred to as 'fast ions' or an 'ion beam' that interact with a relatively lower laboratory kinetic energy species called 'target ions'. Measurements carried on the high energy ions (see Section 2.3.1) also revealed the complex nature of the ion acceleration phenomenon.

The numerical simulation of ion acceleration needs to be considered as an activity separate from the simulation of the plasma itself on account of three circumstances inferred from experimental data. The first is that the number of accelerated ions is a small fraction of the total number of plasma particles present in a given volume element. The second is that the majority of the plasma particles in a small volume element can be considered to be moving together so their motion is adequately described by fluid models while the fast ions make excursions over distances comparable with plasma dimensions, so they cannot be treated as a fluid. Thirdly, the total kinetic energy of all the fast ions can be a significant fraction of the total energy content in the plasma. So the evolution of the state of the majority of the plasma particles must be determined as a first step by neglecting the presence of fast ions (see Sections 3.3.2.1 and 3.3.2.2). The acceleration of ions is then studied under the conditions determined by the resulting computed plasma environment.

Such theoretical analyses of the ion acceleration processes were performed with the use of two conceptual approaches: (1) kinetic theory implemented with particle-incell (PIC) techniques and (2) classical MHD. Capabilities of numerical codes based on both approaches were significantly enhanced during the last 10 years due to progress in numerical methods and rapid increase in computational power of contemporary multiprocessor clusters.

The simulation results are mainly seen to replicate basic concepts known from earlier efforts (see Section 2.3.1) although refined and supported by sophisticated computational infrastructure and manpower.

1. Ion acceleration takes place because of rapid increase in resistivity (anomalous) caused by development of kinetic instabilities [352,353].

2. Although the electric field in the frame moving with a current sheath is practically zero (as the plasma conductivity is very high), it can have significant value in the laboratory frame. Some ions can be accelerated in the electric field, extracted from the collisional plasma and start to behave as a collisionless beam. [178,354].

3. There is one new idea namely acceleration of ions during coalescence of magnetic loops in current carrying plasma by the so called "surfatron mechanism" proposed in the context of astrophysics.

\section{Particle-in-Cell Simulation of Ion Acceleration}

The particle-in-cell code developed by the LLNL team has been used to perform extensive simulations of the plasma focus phenomena and among other effects the ion acceleration has been investigated. The simulated energy spectrum for $\mathrm{D}_{+}$ions in $0^{\circ}$-to- $10^{\circ}$ forward-directed cone, $5 \mathrm{~ns}$ after the start of the pinch has the following characteristics [334]: the bulk of the ions have energies below $100 \mathrm{eV}$, with a high-energy tail extending to $1 \mathrm{MeV}$, in agreement with measurements reported by many laboratories.

A synthetic (artificial) Ez probe was placed in the simulated plasma, $0.5 \mathrm{~cm}$ from the axis to monitor the axial electric field. It was observed that the Ez field exhibits broadband fluctuations after the plasma starts pinching, increasing in magnitude and spectral content as the pinch lengthens. With highly non-uniform and temporally evolving magnetic fields in the simulation, the broadband oscillations observed are consistent with oscillations in the LH range of frequencies. Both simulated and measured oscillations show activity in the 3 to $4 \mathrm{GHz}$ range [333]. Measurements show this activity only in high quality (high neutron yield) pinches [333]. These results suggest that the long-suspected lower hybrid drift instability may be present $[352,353]$.

It was demonstrated that the reported kinetic simulations (done using PIC method) exhibit anomalously high plasma resistivity during the pinch. In the Mega-Joule-scale simulation [355], the plasma resistance rises to approximately $0.7 \Omega$ at peak neutron production, a value $\sim 1000$ times more than that predicted using classical resistivity calculation based 
on local density and temperature. More detailed analysis of the ion beam characteristics as simulated using PIC method can be found in [355].

\section{Ion Acceleration by Electric Field Accompanying Movement of the Current Carrying Plasma (Current Sheath)}

The high-fidelity 2D and 3D numerical magnetohydrodynamic (MHD) simulations were used [331] to study the pinch formation dynamics in a DPF, the associated instabilities, ion acceleration, and neutron production.

A special post-processor was developed that accelerates and transports the D or T ions and produces neutrons through ion-ion beam-target interactions.

As mentioned above, the electric field is practically zero in the frame moving with a current sheath, but at the same time in the laboratory frame $\vec{E} \sim \vec{V} \times \vec{B}$ where $\vec{V}$ is the velocity of the plasma and $\vec{B}$ local magnetic field. At energies near the thermal energy, random up-scattering events allow some small fraction of ions to be accelerated by local EMFs, moving them away from the thermal peak. When their energy becomes large enough, the Coulomb collision rate decreases. The streaming process then gradually becomes entirely collisionless and the particles form a runaway beam, moving in the direction of the electric field.

The energy of particles in the accelerated ion beam is determined by the integral of $\mathrm{E}$ along an ion trajectory. Such ion trajectories are dependent on a detailed solution of the MHD equations. After the energy distribution of ions is determined, it is straightforward to compute the beam-target interaction rate, which determines the neutron production rate.

The authors [331] have not shown any ion spectrum received in their simulations but the energy distributions of neutrons demonstrate proper shift to higher energies as observed in many experiments. Since the calculations assume the presence of only azimuthal magnetic field components and ignore significant poloidal component of magnetic field detected in many large plasma focus devices (see Section 2.4.1), the 3-dimensional character of simulated ion trajectories is possibly different from what is expected in real large plasma focus devices.

\section{Surfatron Acceleration Mechanism}

The surfatron acceleration mechanism refers to the resonant trapping and acceleration of ions by a large amplitude magnetosonic pulse propagating in a direction perpendicular to the magnetic field. The ions are accelerated parallel to the wavefront and are de-trapped when their velocities become of the order of the sum of the $\mathrm{E} \times \mathrm{B}$ drift velocity and the wave phase velocity, where $\mathrm{E}$ is the electric field in the direction of the wave propagation [351]. The pulse develops into a quasi-shock-wave in a collisionless plasma because of dissipation caused by the resonant ion acceleration [351].

This idea has been adapted by Saito and Sakai [356] in a two-dimensional (2D) electromagnetic and relativistic particle-in-cell (PIC) numerical study of electron and ion acceleration during the coalescence of two adjacent magnetic loops with co-helicity or counter-helicity magnetic reconnection. They found that, during such processes, at least two types of ion acceleration mechanisms are present: (1) Ions trapped in the front of fast magnetosonic shock waves generated during the coalescence process are promptly accelerated by the surfatron acceleration mechanism. (2) During the coalescence process through counter-helicity magnetic reconnection, ions outside the current sheet are accelerated by ExB drift. Yousefi et al. [357] suggest that the above mechanisms are responsible for ion acceleration in the plasma focus and can explain ring-shaped ion structures observed in ion pinhole images taken in several PF experiments (Section 2.3.1).

\subsubsection{Empirical and Analytical Insights}

This subsection deals with contributions that cannot be put in standard categories such as 'models', 'theories', 'simulations', 'data analysis', but nevertheless have a unique place in the development of plasma focus research. 


\subsubsection{Lee and Serban}

Lee and Serban [250] reported an important observation in 1996. They showed that the quantity $\mathrm{I}_{\mathrm{p}} / \mathrm{ap}^{1 / 2}$ (designated as the drive parameter), where $\mathrm{I}_{\mathrm{p}}$ is the peak current in $\mathrm{kA}$, a is anode radius in $\mathrm{cm}$ and $\mathrm{p}$ is the pressure of deuterium in torr has a nearly constant value of $(89 \pm 8) \mathrm{kA} / \mathrm{cm} \cdot$ torr $^{1 / 2}$ for neutron optimized plasma focus devices from many laboratories ranging from 3 to $280 \mathrm{~kJ}$ in energy. The drive parameter was shown to be related to the plasma speed. Scaling models showed that the minimum pinch radius $r_{\min }$, height $z_{p}$, compression time $t_{\text {comp }}$ (time in nanoseconds required for the radial shock wave to traverse the anode face), and lifetime $t_{\mathrm{pf}}$ (time between maximum plasma compression and instability) are all proportional to the anode radius a in $\mathrm{mm}: \mathrm{r}_{\min } \approx 0.12 \mathrm{a}, \mathrm{z}_{\mathrm{p}} \approx 0.8 \mathrm{a}$, $\mathrm{t}_{\text {comp }}(\mathrm{ns}) \approx 4.5 \mathrm{a}(\mathrm{mm}), \mathrm{t}_{\mathrm{pf}}(\mathrm{ns}) \approx 2 \mathrm{a}(\mathrm{mm})$.

\subsubsection{Soto}

Soto [6] extended the constancy of drive parameter to sub-kJ devices in 2005. Soto and coworkers reported [358] in 2010 that for experimentally optimized PF devices spanning 8 decades of stored energy range from $0.1 \mathrm{~J}$ to $1 \mathrm{MJ}$, the drive parameter varies over $68-95 \mathrm{kA} \mathrm{cm}^{-1} \mathrm{mbar}^{-1 / 2}$. The pinch radius and height scaling observed by Lee and Serban remains valid. The ratio of stored energy $E$ to nominal pinch volume $V_{p}=\pi r_{\min }^{2} z_{p} \approx 0.036 a^{3}$ (termed as energy density parameter) is observed to vary by a factor $\sim 10$. The energy per unit mass factor defined as $\mathrm{E} /\left(\mathrm{a}^{3} \mathrm{p}\right)$ varies by a factor 23 . Their tabulated data of $14 \mathrm{PF}$ devices shows the pressure to vary over the range 1.5 mbar to 16 mbar. Soto et al. [358] list the following inferences concerning optimized PF devices spanning 8 decades in stored energy:

(1) The magnetic field at pinch radius is nearly the same $\sim 30-40 \mathrm{~T}$.

(2) Ion density of the pinch is nearly the same $\sim 5 \times 10^{24} \mathrm{~m}^{-3}$.

(3) Mean Alfven speed is nearly the same $\sim 1-2 \times 10^{5} \mathrm{~m} / \mathrm{sec}$.

(4) The Bennett temperature varies as the square of the drive parameter and thus should be nearly the same over 8 decades in stored energy.

\subsubsection{Klir and Soto}

Klir and Soto [359] reviewed arguments related to the constancy of the drive parameter and derived an expression for the optimized drive parameter from a variant of the snowplow model. Their predicted drive parameter was shown to have a linear correlation with the empirical drive parameter for many devices. In particular, they argue that their model explains why a high voltage device like SPEED 2 has a higher drive parameter.

\subsubsection{Turner Relaxation Model for Current Sheath}

A model for current sheath of the plasma focus based on Turner's relaxation theory [360] was proposed [361] that was in conformity with the existence of axial magnetic field in the radial phase of the plasma focus (see Section 2.4.1, Section 4.2.2 (3)) and inference of $100 \mathrm{keV}$ deuterons moving azimuthally around the device axis from space resolved fusion product spectra (see Section 2.4.2).

\subsubsection{Turner Relaxation Model for Ion Trapping Structures}

Turner's relaxation theory was evaluated [362] as a model for long-lived ion-trapping structures in plasma focus and z-pinches. The relaxed state turns out to be a ChandrasekharKendall function (eigenfunction of curl) that has a toroidal form. This paper pointed out that interferograms recorded on PF-1000 facility (see Section 4.3.1.1 A(2)) had features of a toroidal structure. It also showed that this model reproduces the experimental observations $[171,172,181]$ of asymmetry in space-resolved fusion product spectra from laterally mirror symmetric points. 


\subsubsection{Upper and Lower Bounds on Snowplow Velocity}

Auluck [363] used conservation of mass, momentum, and energy including contributions from electromagnetic field to show that the snowplow velocity has both lower and upper bounds related to material properties of the gas. The lower bound is related to the requirement that the temperature behind the shock must be high enough to make the plasma highly conducting to enable the driving current to flow through it. This bound is, therefore, related to the specific energy per unit mass $\varepsilon_{\text {eff }} \approx 7.45 \times 10^{8} \mathrm{~J} / \mathrm{kg}$ required to fully dissociate and ionize deuterium gas. The moving magnetized plasma has an electric field proportional to the product of velocity and magnetic field that is parallel to the sheath. This is continuous across the boundary between the highly conducting plasma and the neutral gas ahead of the shock. This field must be less than the spontaneous electric breakdown strength $E_{b}$ of the neutral gas to prevent an ionization wave from detaching and moving away from the shock front.

A simple estimate of $E_{b}$ is obtained as follows. The probability that a photoelectron generated in the neutral gas of molecular number density $n_{0}$ causes impact ionization while moving a distance $d x$ in the electric field is given by $d P_{i m}=\sigma_{i m}(w) n_{0} d x$, where $\sigma_{i m}(w)$ is the electron impact ionization cross section as a function of electron energy $w$ (in $\mathrm{eV}$ ), which is related to the electric field by $d w=E_{b} d x-w n_{0} \sigma_{c} d x, \sigma_{c}$ being the collision cross-section in the neutral gas. Electron multiplication is guaranteed when the integrated probability equals unity. This condition can be written as

$$
\int d P_{i m}=\int \frac{\sigma_{i m}(w) d w}{\left(E_{b} / n_{0}-w \sigma_{c}(w)\right)}=1
$$

This shows that $E_{b} / n_{0}$ is a material property of the gas. Using electron impact ionization cross-section and total electron collision cross-section in hydrogen data from literature this is found to be $\kappa \equiv E_{b} / n_{0} \approx 8.21 \times 10^{-18} \cdot$ Volt- $^{2}$ for hydrogen. At a pressure of 1 mbar at NTP, the molecular number density of hydrogen is $2.9 \times 10^{22} \mathrm{~m}^{-3}$ giving a spontaneous electric breakdown field $\sim 2.4 \times 10^{5}$ volts $/ \mathrm{m}$ for hydrogen.

The instantaneous local snowplow velocity $\mathrm{v}_{\text {sp }} \equiv \mu_{0} \mathrm{I}(\mathrm{t}) /\left(2 \pi \mathrm{r} \sqrt{2 \mu_{0} \rho_{0}}\right)$ at time $\mathrm{t}$ and radius $r$ in a deuterium fill density $\rho_{0}$ is then bounded by

$$
1.08 \times 10^{5} \cdot \sqrt[4]{\mathrm{p}_{0}(\mathrm{mbar})}>\mathrm{v}_{\mathrm{sp}}>7.9 \times 10^{4} \mathrm{~m} / \mathrm{s}
$$

Note that (5) implies a maximum current for every plasma focus beyond which the snowplow sheath propagation fails as the ionization wave detaches from the piston-driven pressure wave and races ahead and, therefore, fails to form a pinch (see Section 8.4.1.3). This maximum current limit increases with the initial fill gas pressure.

\subsubsection{Plasma Focus as a Circuit Element}

The plasma focus is usually treated as a time-varying inductance in a circuit representation. Certain observations (discussed in Sections 4.2.3 and 5.1.1 (d)) are difficult to reconcile with this interpretation. The representation of a plasma focus as a circuit element has been examined from first principles [364] using Poynting's theorem. It is seen that representation of the plasma focus as a circuit element is not exactly congruent with the more accurate description in terms of fields (see Section 8.2.4). This discrepancy must be rectified by introducing an ad hoc correction in the circuit equation as an anomalous impedance. It is also shown that poloidal magnetic field associated with post-pinch dynamo activity can contribute to the plasma inductance. This is a likely explanation of monotonic increase in inductance after the current derivative singularity observed in many devices (Section 4.2.3). In the light of this development, the validity of the circuit diagram of Figure 35 is called into question. 


\section{Experimental Results: Standard PF Operation with Deuterium}

The purpose of this section is to summarize the research work related to standard operation of PF with deuterium without admixtures. Results on operation of PF with other gases, admixtures, and gas puffing are discussed in a separate section. The presence of high atomic number species in the plasma could be expected to give rise to many new effects related to enhanced resistivity causing faster magnetic diffusion or resistive instabilities [132], enhanced radiation rate causing radiative collapse scenarios [164], mass separation during sheath propagation [365], etc. Gas puffing is likely to introduce turbulent eddies and condensates of residual moisture into the initial state of the plasma. This could also lead to gradients of various scale lengths in the ambient gas which could nucleate mixing instabilities that might be manifested through new phenomenology. Using observations from non-standard operating regimes to interpret measurements on pure deuterium discharges could, therefore, result in misleading conclusions. This is avoided by devoting a separate section to such studies. An exception is made in respect to the discussion of the initial phase, where the state of experimental information is at a rather preliminary level and the anxiety for misinterpretation is far outweighed by the scarcity of data.

A side effect of this consideration is that research on Filippov type PF devices such as PF-3 needs to be discussed in a separate section as well. This is because their standard mode of operation is not with pure deuterium but with deuterium plus a heavy gas admixture.

This section is divided into subsections dealing with prominent physical aspects. There is a much larger volume of correlated experimental results on the pinch phase from PF-1000, as compared with any other facility, which needs to be interpreted coherently. Therefore, the subsection dealing with the pinch phase is divided into three parts: the results on PF-1000 subcategorized by diagnostic techniques are discussed together in the first part. Interpretation of results from PF-1000 is separately discussed making a distinction between standard evaluation methods (such as neutron spectra from time of flight and electron density from interferometry), which are discussed along with experimental data and special methods proposed by authors which may use additional assumptions, which are mentioned separately.

The credibility of interpretations of PF-1000 results suffers from an inherent defect that is not related to the quality of experiments or of their evaluation. Rather, it refers to the doubt that these results may be inherent to the device or to its regime of operating parameters and not indicative of a general phenomenon that occurs in all plasma focus devices. To mitigate this, a selection of results from other devices is discussed in Section 4.3.2 and the convergence between these and the PF-1000 interpreted results is discussed in Section 4.3.3.

\subsection{Initial Phase: Sheath Formation}

This section discusses the research related to the initial or sheath formation stage in terms of some pertinent questions, without making the distinction between discharges with deuterium and other gases.

(1) Is there an optimum length for the insulator or initial plasma?

a. The Singapore group [366] finds "that for a neon filled plasma focus device the change in insulator sleeve length changes the current sheath curvature angle and thus the length of the focused plasma column". "The pinhole images and laser shadowgraphy are used to explain the observed variation in the average soft $X$-ray yield (measured using a diode $X$-ray spectrometer) with variation of the insulator sleeve length". They conclude that X-ray yield is maximum for an optimized insulator length.

b. The QAU group from Pakistan [367] finds an optimum insulator length that maximizes neutron yield. "When sleeve length deviates from the optimum value, spokes are observed on the sleeve surface". The spokes are more prominent on the surface of shorter length.

c. Iranian group at Amir Kabir University [368] finds smaller pinch radius for an optimum insulator length. 
d. Darmstadt group in Germany [369] finds that “... the discharge tends to shorten its path with increasing pressure. Hence by increasing or decreasing the insulator length, the pressure range of the gliding discharge is shifted towards lower or higher pressure values, respectively".

e. According to the RGV model [311], variation of insulator length of a given device, keeping all other parameters constant, is also expected to vary the fraction of energy converted into magnetic energy, work done, remaining in the capacitor and dissipated resistively. Decrease in X-ray and neutron yield could be explained on the basis of less energy coupled to the pinch phase.

f. The breakdown electric field in deuterium is proportional to the fill gas density [363], so that if voltage is kept constant, a longer insulator will create uniform discharge at lower pressure. The plasma will then move faster, so the pinch occurs at a time before the quarter cycle period of the capacitor bank so that a significant amount of energy remains outside the device.

The experiments that report optimization of insulator length need to assess the amount of energy remaining in the capacitor at pinch for various insulator lengths for the sake of completeness.

(2) Is there a preferred material for the insulator? Which property of the material makes it the preferred material?

a. The occurrence of an intense sliding discharge for PTFE, $\mathrm{PVC}, \mathrm{Al}_{2} \mathrm{O}_{3}$, Pyrex has been studied [369] and it is found that the corresponding pressure range is material dependent.

b. It is found [370] that " ... considerable increase in neutron yield has been achieved in POSEIDON by replacing the Pyrex insulator by a ceramic tube".

c. Beg et al. [371] find that a higher dielectric constant insulator sleeve material produces better neutron yield.

d. Hussain [372] et al. have studied electron temperature from a device operated with argon-hydrogen mixture with Pyrex glass, fused silica, alumina, nylon, Perspex, and Teflon, and find that Pyrex and fused silica produce highest electron temperature and speculate that high surface resistivity of these materials is probably involved.

(3) What is the role of the polarity in sheath formation and pinch formation?

a. Decker and coworkers [373] explain that with positive polarity, the radial electric field attracts to the insulator free electrons from the gas and those created by field emission from the sharp edges of anode or knife edge structures nearby. This helps in rapid buildup of surface charge density on the insulator leading to breakdown. When the polarity of applied voltage is negative, electrons are repelled from the insulator and the buildup of surface charge density is retarded.

b. Mathuthu, et al. [374] find that for negative polarity, the optimum anode length is shorter than for positive polarity.

c. The above two observations appear to be mutually supporting in the following manner: With negative polarity, plasma formation is delayed by electron repulsion, so less portion of quarter cycle time is available for plasma to reach axis, hence shorter anode works better.

d. Pinch formation in the negative quarter cycle, with positive charging voltage, has been demonstrated in multiple experiments [375-377] showing that the polarity of the current has no influence on pinch formation.

e. This question can potentially be addressed by simulations reported in Section 3.3.2.3 but does not appear to have been attempted so far.

(4) Can the initial plasma be formed by any means other than surface electrical discharge on insulator? 
a. Comment by H. Bruzzone: "It has been shown [378] that the initial plasma does not always form on the insulator. A single CS is formed on the insulator just in a restricted range of pressure values, which must be determined for any device. No rationale has been found for this fact.".

b. At high pressures, a filamentary sheath is formed at the end of the insulator [378].

c. There are patents [379-381] on creation of the initial plasma using laser illumination.

d. Plasma focus action indicated by current derivatve singularity has been demonstrated using an inside-out washer gun configuration [382] (see Section 9.2.3).

\subsection{Sheath Structure and Propagation}

\subsubsection{Electron Density Profile}

One of the basic questions related to the physics of sheath structure and propagation is the role of the theory of strong shocks driven by a piston. The well-known result that the density ratio across a single, strong, planar, steady shock driven by a piston is $(\gamma+1) /(\gamma-1)$ is not expected to be applicable because of non-planarity of the sheath (sheath curvature both in azimuthal and tangential directions), converging motion of the sheath and because of the non-steady nature of the magnetic piston related to a time-dependent current. Another factor that negatively affects applicability of RankineHugoniot jump conditions is the absence of a constant-density "flat top" region, where gradients can be neglected. Experiments that measure the density behind the shock wave are summarized below.

(1) Bruzzone and Fischfeld [383] have evaluated the density profile of the sheath in the Frascati plasma focus at $250 \mathrm{~kJ}, 3$ torr $\mathrm{D}_{2}$. The following Figure 68 shows the radial density profile at various $\mathrm{z}$ coordinates. The peak density calculated by them is $(1.6-1.8) \times 10^{18}$ electrons $/ \mathrm{cm}^{3}$ while the atomic filling density corresponding to 3 torr of deuterium is $2.1 \times 10^{17} \mathrm{~cm}^{-3}$-a ratio of 8.57 . Some other features of the density profile commented by them are:

a. In the leading portion of the sheath, the electron density starts rising to a value of $1-2 \times 10^{17} \mathrm{~cm}^{-3}$ (similar to filling density) within about $3 \mathrm{~mm}$, after which it rises rapidly to $1.6-1.8 \times 10^{18} \mathrm{~cm}^{-3}$ in next 3-4 mm.

b. The decrease in density from peak to below detection limit towards the rear side of the sheath occurs over a scale of less than $1 \mathrm{~mm}$, which is well resolved.

c. The scale length of density rise agrees well with the slowing down distance of deuterons as they enter the moving sheath.

d. The scale of the slower initial rise of density agrees with atomic photoionization mean free path.

(2) Electron density profiles for the radial phase have also been published for the PF-1000 device (see Section 4.3.1.1 A(4)). However, a detailed analysis of density profile similar to that of [383] is not available as yet. 


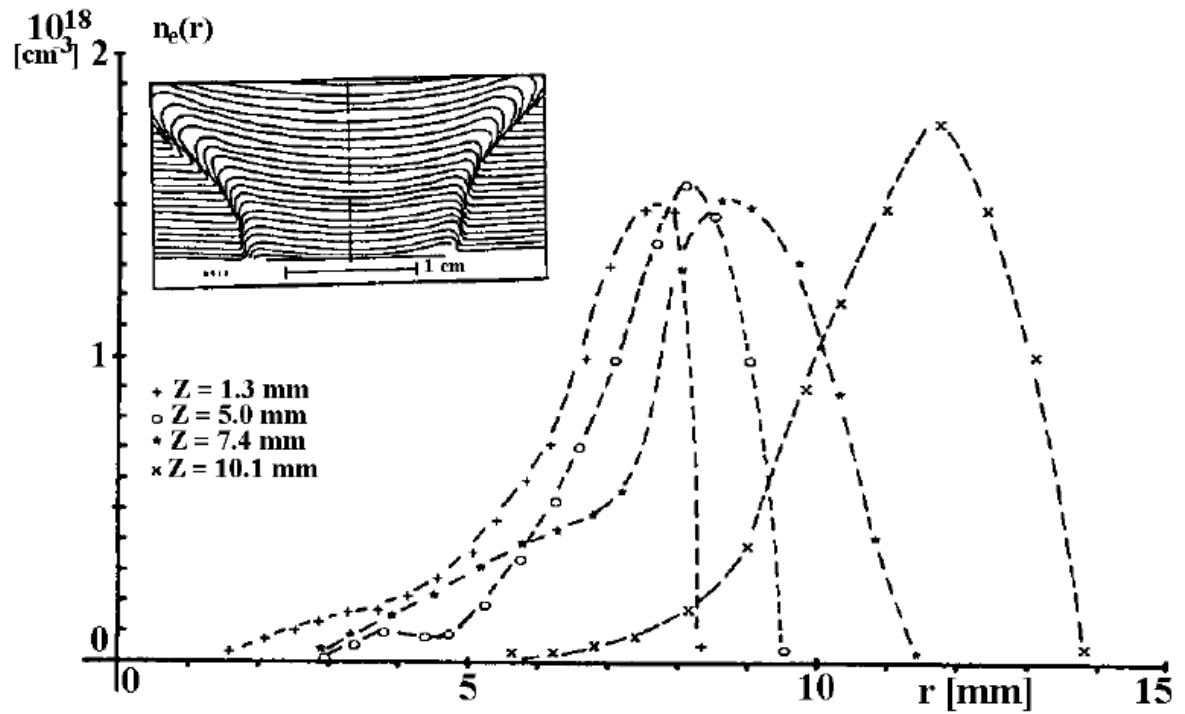

Figure 68. Reproduced from [384] with the kind permission of Prof. Bruzzone. Interferogram obtained at the Frascati 1 MJ plasma focus using a Mach-Zehnder Interferometer with a frequency doubled Nd-YAG laser.

\subsubsection{Current Density Profile}

Considerable information on current sheath structure in radial phase has become available using magnetic and magneto-optical probes described in Section 3.2.2. An important aspect of this is the efficiency of current transport from the initial phase to the stagnation phase, which is discussed in detail in Section 5.2. This is supplemented on PF-1000 with interferometry described in Section 3.2.1. This work is reviewed below.

(1) Magnetic probes (of a different design from that described in Section 3.2.2) were used at the Lebedev Institute [385] in 2010 on a Filippov type plasma focus operated with pure deuterium (see Figure 69).

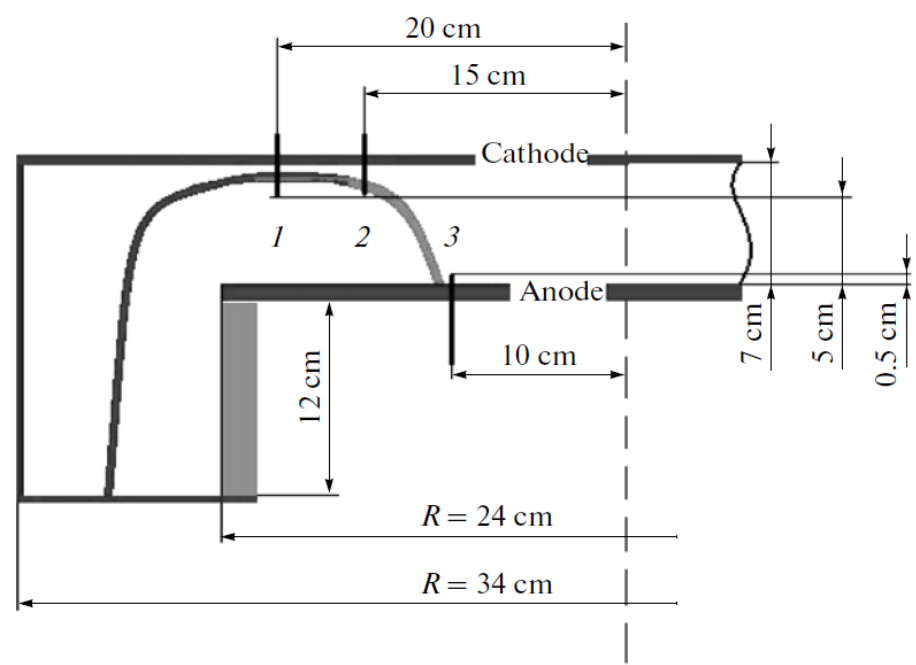

Figure 69. Schematic of the experiment. The experiment attempted to demonstrate that the lower than expected neutron yield was because of parasitic currents that did not flow through the pinch. Numerals 1, 2, and 3 show the positions of the magnetic probes. Reproduced with permission from Figure 1 of [385] (Plasma Physics Reports, 2010, 36, pp. 1013-1022) @ 2010, Pleiades Publishing, Ltd.

The plasma focus anode and cathode diameters were 47.4 and $69 \mathrm{~cm}$, respectively, and the insulator height was $12 \mathrm{~cm}$. The capacitor bank of $120-160 \mu \mathrm{F}$ was charged to $27-33 \mathrm{kV}$, and the maximum current was 0.9-1.1 MA with a current rise time of 4.2-5.6 $\mu \mathrm{s}$. 
The device was operated with pure deuterium $0.1-0.3$ torr and the neutron yield was $10^{7}-10^{9}$ per shot. "The magnetic measurements were performed using a set of calibrated magnetic probes, each of which was a $2.1 \mathrm{~mm}$ diameter 15-turn coil incased in a $5 \mathrm{~mm}$ diameter quartz tube with a sealed end". The probes were connected to the oscilloscope using $75 \mathrm{Ohm}$ cables. The authors quote the time resolution of their probes as $3 \mathrm{~L} / \mathrm{R} \approx 10 \mathrm{~ns}$ (where $\mathrm{L}$ is the coil inductance) of $0.2 \mu \mathrm{s}$. "The current distribution in the chamber can be found by numerically integrating the probe signals with allowance for their sensitivities, the drift of the zero level of the recording system, and the probe positions with respect to the discharge axis under the assumption that the discharge is axisymmetric". Major findings include the following:

a. The current derivative singularity occurs after the current maximum showing that the PF discharge is not properly matched with the capacitor bank.

b. The probe signals have multiple peaks which are interpreted as multiple sheaths formed by secondary breakdowns in the residual gas behind an initial sheath that does not efficiently sweep all the gas. The neutron signals also show similar double peaks (see Figure 70).

c. The probe measurements indicate that only about $50 \%$ of the current entering the device is transported up to the axis.

(a)

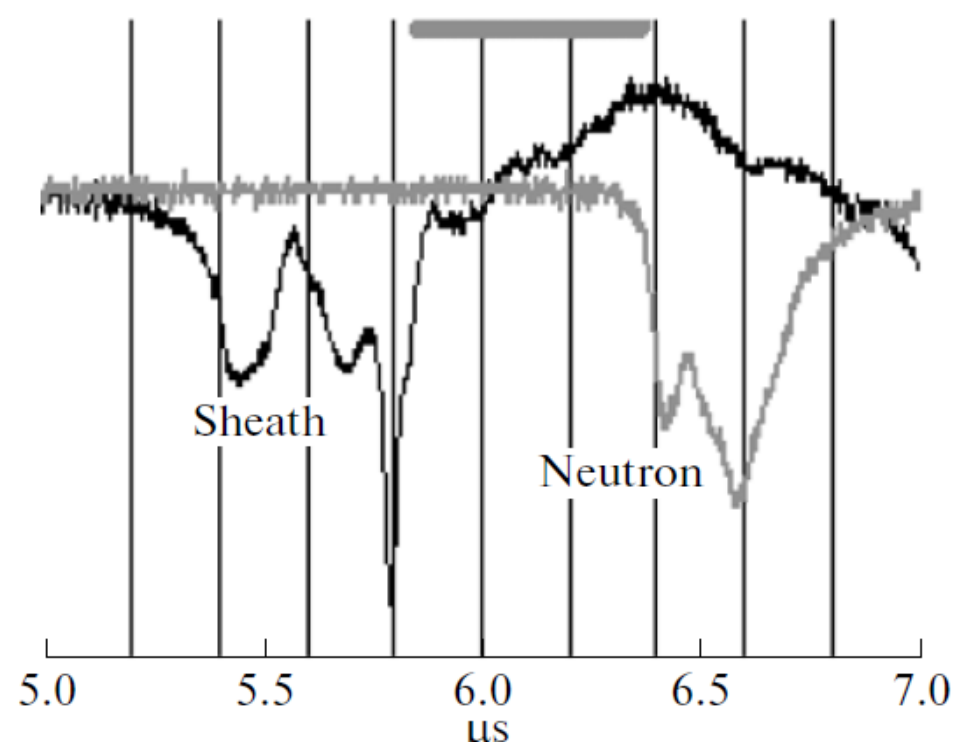

Figure 70. Reproduced with permission from Figure 14a of [385]. The black curve shows the probe signal from the passage of the sheath over the magnetic probe. The grey signal is from the neutron detector. The grey bar over the signals shows the time when the sheath is passing over the probe placed at $15 \mathrm{~cm}$ radius. (Plasma Physics Reports, 2010, Vol. 36, pp. 1013-1022) (C 2010, Pleiades Publishing, Ltd.

(2) Magnetic and magneto-optical probes of the type described in Section 3.2.2 were deployed on KPF-4 Phoenix facility [210] through the cathode, as shown in Figure 71. The results for operation between 18-24 kV with deuterium at 6 to 8 mbar are summarized below:

a. Relative position between the luminous shock front and the current carrying magnetic piston layer was studied from the optical and magnetic channels of magneto-optical probes (see Figure 72) at a radius of $2.2 \mathrm{~cm}$ and height $3 \mathrm{~cm}$ above the anode. The shaded area in Figure 72 indicates the portion of the current carrying layer that overlapped with the dense plasma layer, showing current exclusion from the dense plasma layer. 
b. However, in an uncontrolled spontaneous firing of the capacitor bank, the optical signal and the magnetic probe signal were detected with no delay between them, showing that current had penetrated fully into the luminous dense plasma sheath (see Figure 73).

c. Most of the current was seen to flow in the magnetic piston layer in good shots, which was $65 \%$ of the total discharge current. However in the spontaneous self-fired shot referred above, this portion was $42 \%$. Although the operating voltage, pressure and total current were nearly the same, the neutron yield was one order less.

d. The probes were introduced from the top and placed at a height of $3 \mathrm{~cm}$ above the anode. Because of sheath curvature, the sheath reached the axis on the anode surface before it encountered the magnetic probe at $22 \mathrm{~mm}$ radius.

e. The average radial speed of the sheath was estimated from the time difference between peak of the magnetic probe signal and current derivative minimum to be $\sim 2 \times 10^{7} \mathrm{~cm} / \mathrm{sec}$. This velocity estimate was used to construct a spatial profile of the optical and magnetic probe signals. The thickness of the luminous dense plasma layer was found to be $\sim 6 \mathrm{~mm}$ (see Figure 74). The width of the current layer is $\sim 0.9 \mathrm{~cm}$ at $5.6 \mathrm{~cm}$ radius. At $2.2 \mathrm{~cm}$ radius, the current distribution is seen to have two peaks.

f. Assuming the thickness of the current layer to be given by the skin depth at the characteristic frequency corresponding to the current rise time and the resistivity to be given by the Spitzer formula, its temperature is estimated to be $\sim 20 \mathrm{eV}$.

g. The neutron yield scaled with measured pinch current $\mathrm{I}_{\mathrm{p}}(\mathrm{MA})$ as $\mathrm{Y}_{\mathrm{n}} \sim 1-3 \times 10^{10} \mathrm{I}_{\mathrm{p}}{ }^{4}$.

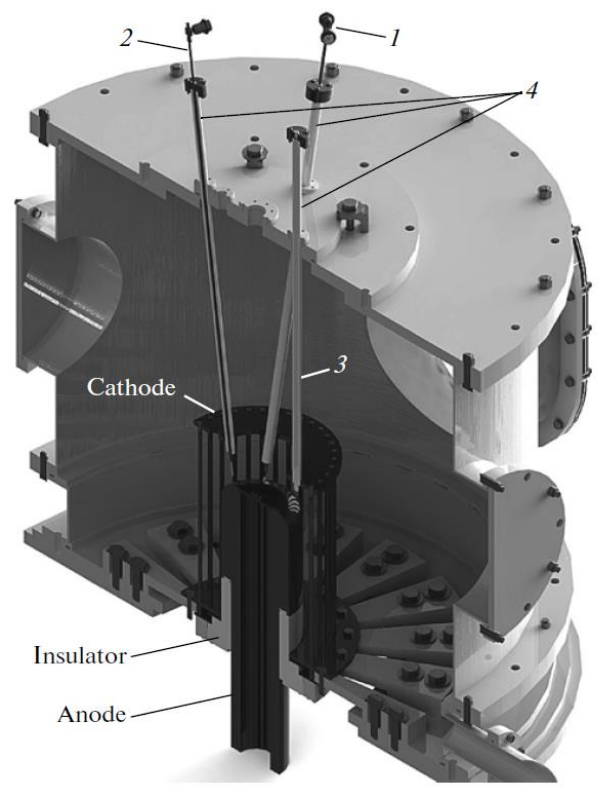

Figure 71. Arrangement of magnetic and magneto-optical probes in KPF-4 Phoenix. (diametric cross-section): (1-3) magnetic probes installed at the radii of 2.2, 5.6 and $11 \mathrm{~cm}$, respectively, and (4) vacuum inlets for the magnetic probes. Reproduced with permission from Figure 3 of [210] (Plasma Physics Reports, 2013, 39, pp. 888-899) (C 2013, Pleiades Publishing, Ltd. 


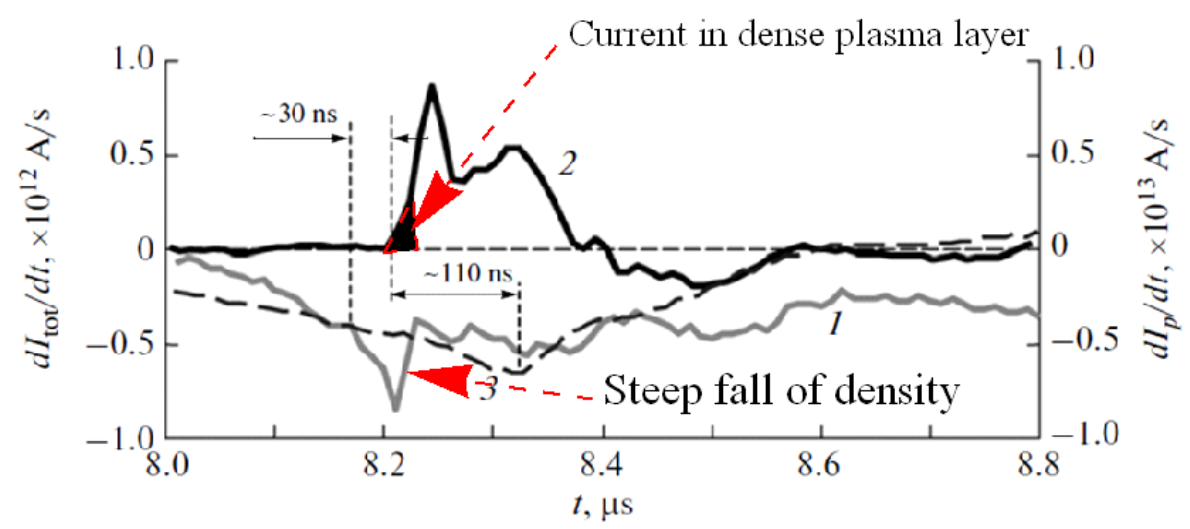

Figure 72. Adapted with permission from Figure 5 of [210] (Plasma Physics Reports, 2013, 39, pp. 888-899) (c) 2013, Pleiades Publishing, Ltd. Grey curve is the optical signal, black is the timederivative of current detected by magnetic probe, dashed line is the time-derivative of the total current. Shot no. 4908, deuterium, $\mathrm{P}_{0}=6$ Torr, $\mathrm{U}_{0}=22 \mathrm{kV}, \mathrm{W}_{0}=340 \mathrm{~kJ}, \mathrm{Y}_{\mathrm{n}} \sim 4.7 \times 10^{9}$ neutrons/shot). Note the steep fall of optical signal towards the rear side of the dense plasma shock layer, indicating a sharp decrease in density also noted in [383] and shown in Figure 68. Shaded portion at the beginning of the black solid curve 2 pointed at by the upper arrow represents the current flowing in the dense sheath.

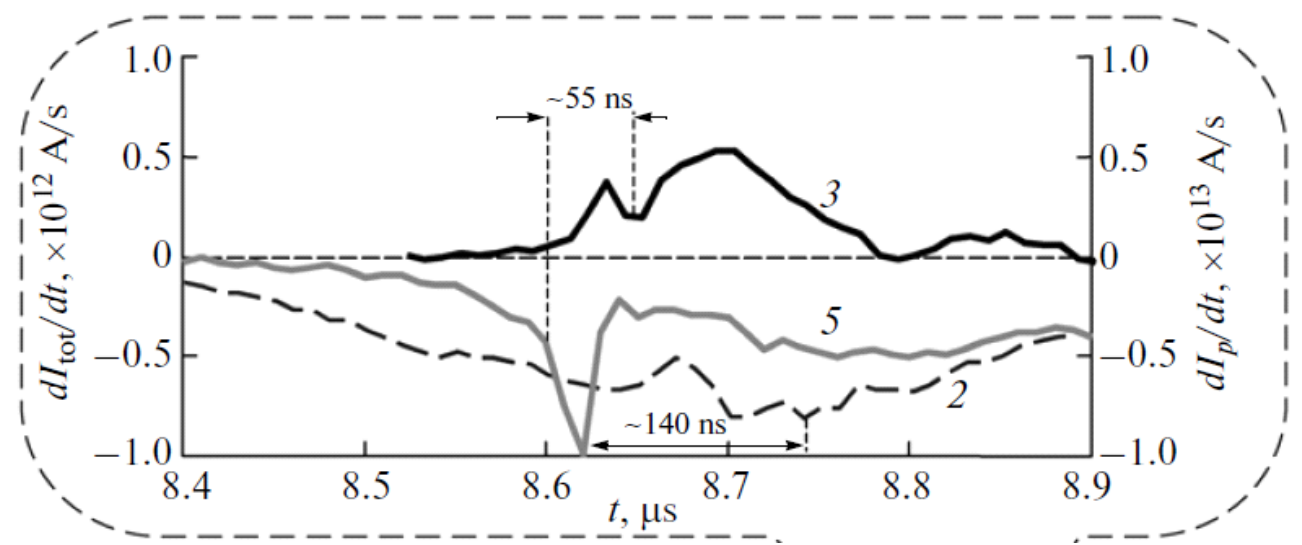

Figure 73. Reproduced with permission from Figure 6 of [210] (Plasma Physics Reports, 2013, 39, pp. 888-899) (c) 2013, Pleiades Publishing, Ltd. Grey curve is the optical signal, black is the timederivative of current detected by magnetic probe, dashed line is the time-derivative of the total current. Shot no. 4909 (deuterium, $\mathrm{P}_{0}=6$ Torr, $\mathrm{U}_{0}=23 \mathrm{kV}, \mathrm{W}_{0}=370 \mathrm{~kJ}, \mathrm{Y}_{\mathrm{n}} \sim 5.7 \times 10^{8}$. Self-fired shot. Note that the magnetic probe signal begins at the same time as the optical signal. 
$J_{1}(X), \mathrm{MA} / \mathrm{cm} ; \operatorname{Opt}(x)$, arb. units
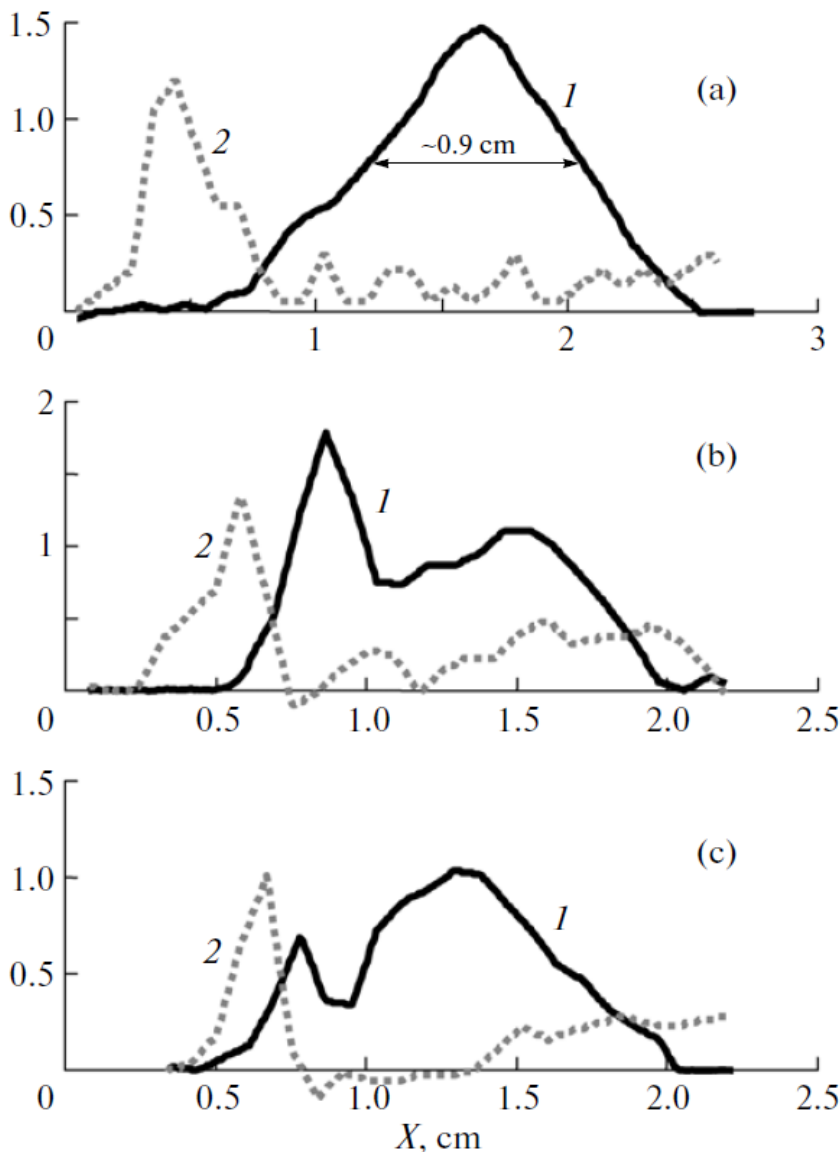

Figure 74. Reproduced with permission from Figure 10 of [210] (Plasma Physics Reports, 2013, 39, pp. 888-899) (C) 2013, Pleiades Publishing, Ltd. Dotted gray line 2 represents the optical signal, black line 1 represents the magnetic probe signal divided by velocity. The $X$ axis is sheath velocity multiplied by time axis of probe signal. (a) Probe at $5.6 \mathrm{~cm}$ radius, good shot (b) Probe at $2.2 \mathrm{~cm}$, good shot, (c) Probe at $2.2 \mathrm{~cm}$, bad shot.

(3) Magnetic probe measurements on PF-1000 [207] were performed with the probes placed through the anode and the measurement system floated to the anode voltage (See Figure 75).

a. The first series of experiments was performed in a well-reproducible mode, with deuterium pressure 3 torr, operating voltage $27 \mathrm{kV}$, stored energy $485 \mathrm{~kJ}$. Before each shot with magnetic probe, a series of training discharges under standard conditions were performed until the neutron yield became more than $10^{10}$. The working gas was refreshed before each shot because the probes got damaged in almost every shot. Major findings of the study are summarized below:

i. High neutron yield $\sim 1.24 \times 10^{11}$ showed that presence of the probe at $40 \mathrm{~mm}$ radius and $10 \mathrm{~mm}$ above anode surface did not adversely affect the normal operation of plasma focus.

ii. The probe signal typically had two peaks with FWHM 35-40 ns and a precursor pulse of smaller amplitude and opposite polarity to the main pulse. The precursor carried $5 \%-7 \%$ of the total current of the sheath. Its origin and role, if any, is unclear. See Figure 76 below.

b. Total current inferred from the integration of magnetic probe signal over its $\sim 100$ ns duration assuming azimuthal symmetry is $\sim 1.7 \mathrm{MA}$, which equals the total device current measured from the Rogowski coil or the current derivative monitor within experimental errors. The discharge current is transported almost 
completely past the $40 \mathrm{~mm}$ radius on the anode surface without suffering any leakage as found on KPF-4 Phoenix.

c. With two magnetic probes at $40 \mathrm{~mm}$ and $12 \mathrm{~mm}$, the average radial velocity was found to be $2.1 \times 10^{7} \mathrm{~cm} / \mathrm{sec}$.

d. Using magneto-optical probes, the arrival of a luminous plasma front $\sim 30 \mathrm{~ns}$ before the current front was clearly seen (Figure 77).

e. The average radial velocity in the final stage of implosion was estimated from the delay between the peak of the probe signal at $40 \mathrm{~mm}$ radius and the minimum of current derivative signal. This was found to be in the interval $(1.5-2.2) \times 10^{7} \mathrm{~cm} / \mathrm{sec}$.

f. Since the variation of total current over the duration of the probe signal was negligible, the form of the probe signal was determined mainly by the spatial distribution of linear current density. The full spatial width of the current distribution was $\sim 1 \mathrm{~cm}$ at 1.8 mbar pressure and nearly double that at $2.1 \mathrm{mbar}$ and 2.8 mbar, while the total current was nearly the same and there was no significant change in the neutron yield.

g. Neutron yield showed a good correlation with the scaling law $\mathrm{Y}_{\mathrm{n}} \sim 1.5 \times 10^{10} \mathrm{I}^{4}$ when I was taken to be the current estimated from integration of probe signal at $40 \mathrm{~mm}$ radius, but not when it was the total device current measured at the collector. The efficiency of current transport up to the axis varied from shot to shot and was a significant factor in the variation of the neutron yield.

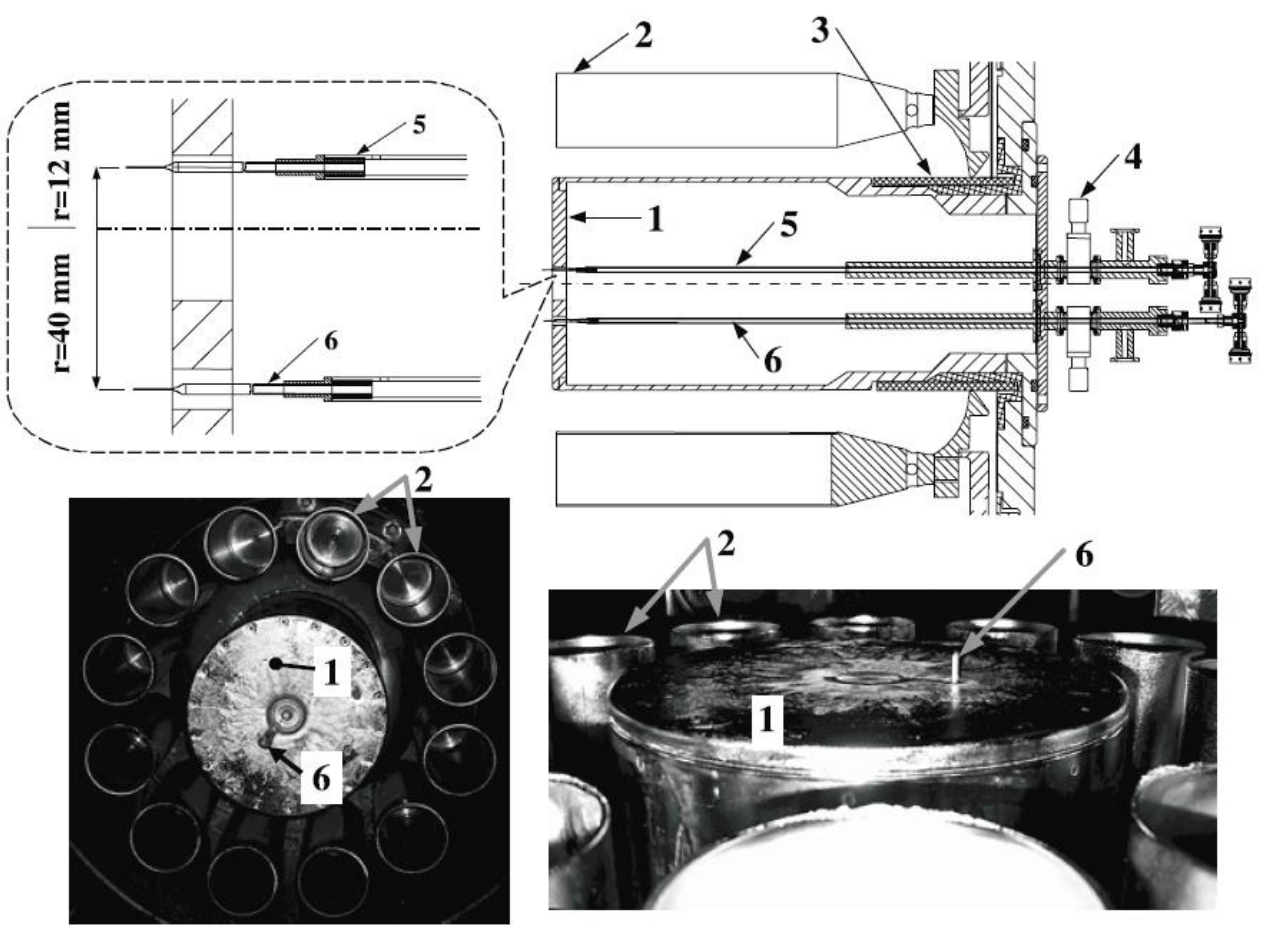

Figure 75. Geometry of the electrodes of the PF-1000 facility and arrangement of the magnetic probes: (1) anode, (2) cathode (12 rods), (3) insulator, (4) vacuum lock through which the probe is introduced, and $(5,6)$ magnetic probes measuring the azimuthal magnetic field at the radii of $12 \mathrm{~mm}$ and $40 \mathrm{~mm}$, respectively. Reproduced with permission from Figure 3 of [207] (Plasma Phys. Control. Fusion 2012, 54, 025010) @ (2012) IOP Publishing. 


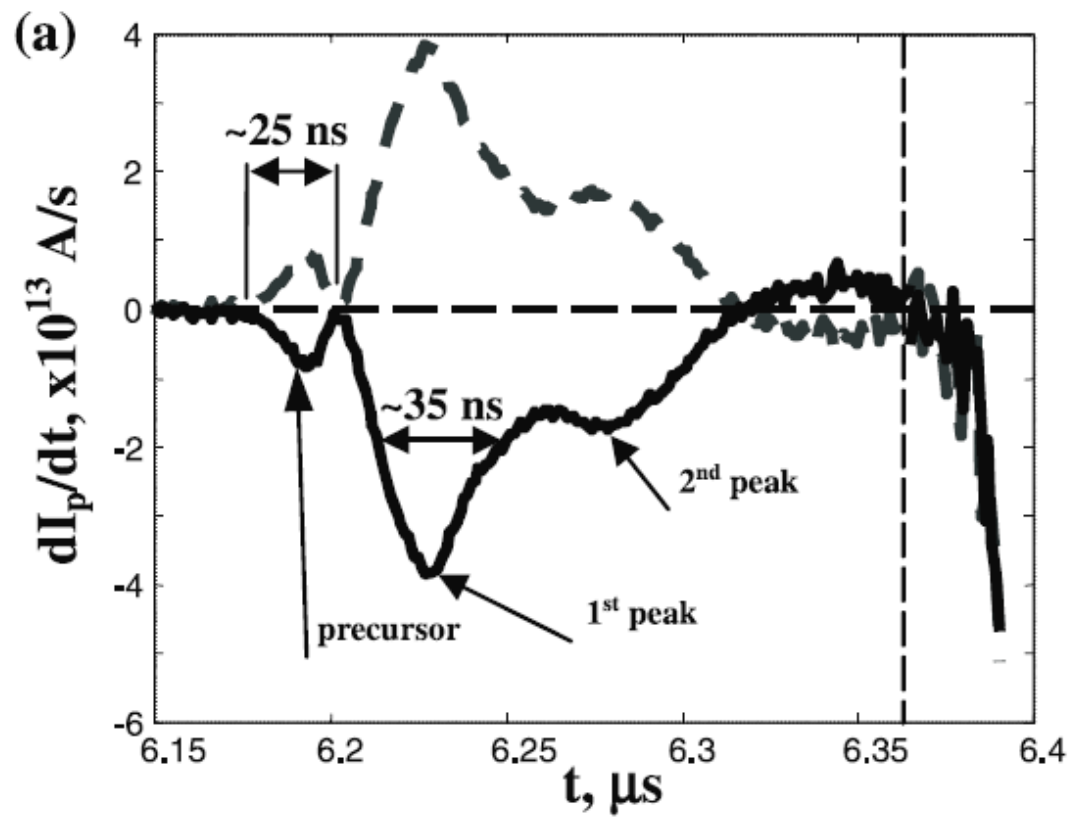

Figure 76. The two curves are outputs from two probe coils wound in opposite sense. Their mirror symmetry is good until the probes are destroyed at the dashed vertical line near $6.35 \mu$ s. Reproduced with permission from Figure 8a of [207] (Plasma Phys. Control. Fusion 54 (2012) 025010) ( ) (2012) IOP Publishing.

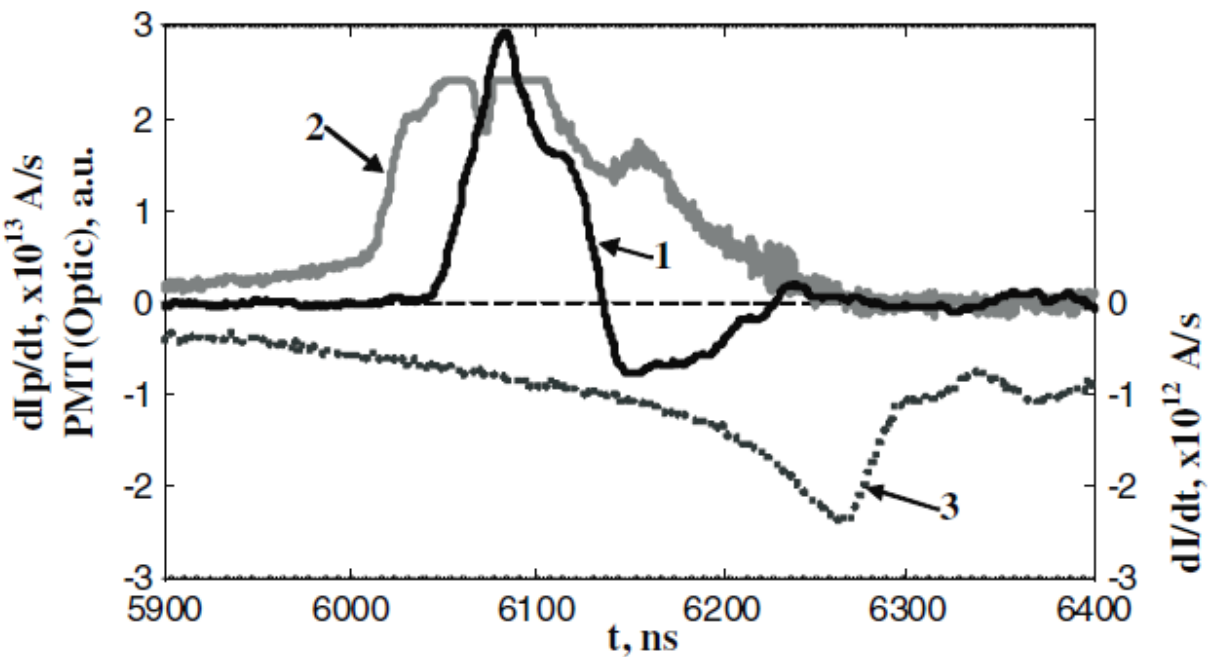

Figure 77. Reproduced with permission from Figure 12 of [207] (Plasma Phys. Control. Fusion 2012, 54,025010 ) (C (2012) IOP Publishing (shot\# 9143, D2, $\mathrm{P}_{0}=2.1$ Torr, $\mathrm{U}_{0}=24 \mathrm{kV}$, and $\mathrm{W}_{0}=384 \mathrm{~kJ}$ ). (1) Current time derivative measured by the magnetic probe installed at the radius of $40 \mathrm{~mm}$, (2) PMT signal, (3) time derivative of the total current measured by the loop detector at the collector. The neutron yield is $\mathrm{Yn} \sim 8.6 \times 10^{10}$ neutrons/shot.

(4) Existence of an axial magnetic field associated with the radial phase was demonstrated in 2012 [212], confirming the observation of Gribkov et al. [169] from 1979. The main points of investigations at operating parameters 1.8 torr $\mathrm{D}_{2}, 24 \mathrm{kV}, 384 \mathrm{~kJ}$ are summarized below:

a. Signals from consecutive shots 9347 and 9348 with neutron yields $1.24 \times 10^{11} \mathrm{n} /$ shot and $9.3 \times 10^{10} \mathrm{n} /$ shot are shown in Figure 78 and their integrations are shown in Figure 79, panels (a) and (b), respectively, with the black arrow marking the minimum of current derivative. 
b. The signal from the $B_{z}$ channels appears first, followed by the signal from the optical channel and then followed by the signal from the $\mathrm{B}_{\varphi}$ channel.

c. The $B_{z}$ probe used in panels (b) in Figures 78 and 79 had a relatively low sensitivity to the $B_{\varphi}$ channel and is, thus, expected to yield the more credible data. However, after applying corrections for the influence of the $\mathrm{B}_{\varphi}$ channel, the reconstructed $B_{Z}$ signals from both panels (a) and (b) in Figure 79 have a similar behavior which is typical for all shots with probes at $40 \mathrm{~mm}$ radius.

d. $\quad B_{Z}$ field appears $\sim 60-65 \mathrm{~ns}$ before the arrival of the PCS and increases to $\sim 0.4 \mathrm{kG}$. According to the calibration results, the $B_{z}$ field in front of the PCS is directed away from the anode. After the arrival of the PCS, the $B_{z}$ field changes its sign and reaches a value of $\sim 4 \mathrm{kGs}$. The polarity of the $\mathrm{B}_{\mathrm{z}}$ field remains unchanged from shot to shot.

e. The integration of $B_{z}$ signals implicitly assumes that the value of $B_{z}$ at the probe position was zero before the arrival of the sheath to the probe. This is an arbitrary assumption. The authors observe "If we assume that the initial $B_{z}$ field is nonzero and is directed positively (from the anode), then the negative signal from the $B_{z}$ channel can be caused by expulsion of the magnetic field by the PCS plasma". Turner Relaxed State model [362] has been suggested as a possible source of such initial axial magnetic field.

a)

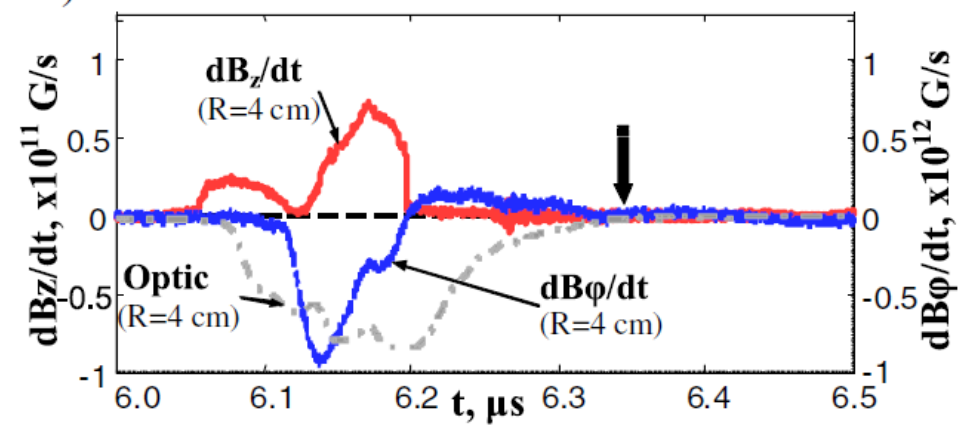

b)

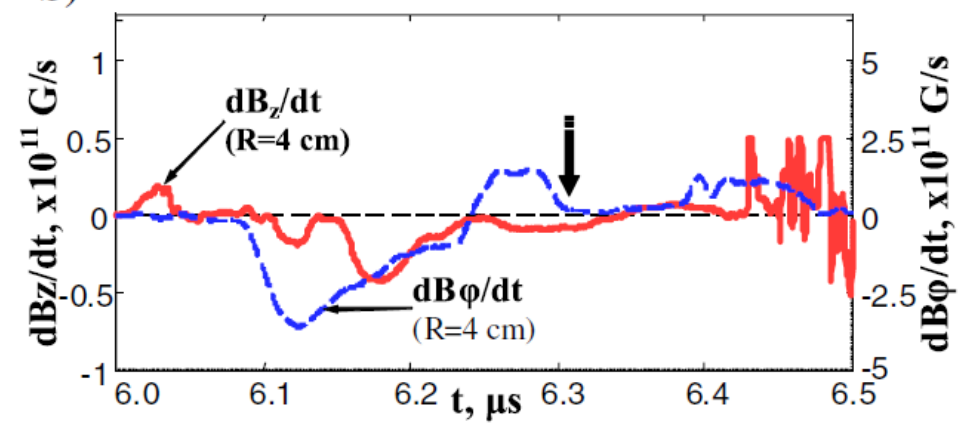

Figure 78. Figure 3 of [212]. Reproduced with permission from "Experimental Evidence of Existence of the Axial Magnetic Field in a Plasma Focus. Europhys. Lett. 2012, 98, 45001". Signals from the $B_{Z}$ and $B_{\phi}$ channels of the probes having different relative sensitivities of the $B_{Z}$ channel: $\mathrm{K}_{\mathrm{z}}\left(\mathrm{B}_{\phi}\right) / \mathrm{K}_{\mathrm{z}}\left(\mathrm{B}_{\mathrm{z}}\right)=(\mathbf{a}) 0.231$ (shot no. 9347) and (b) 0.055 (shot no. 9348). Here, "Optic" in panel (a) stands for the signal from the optical channel of the probe (in arb. units). The arrows show the instant of the dip in the current derivative. $R_{\text {probe }}=40 \mathrm{~mm} . Z_{\text {probe }}=10 \mathrm{~mm} . Y_{\mathrm{n}}=1.24 \times 10^{11}$ and $9.3 \times 10^{10} \mathrm{n} /$ shot for shot nos. 9347 and 9348 , respectively. 
a)

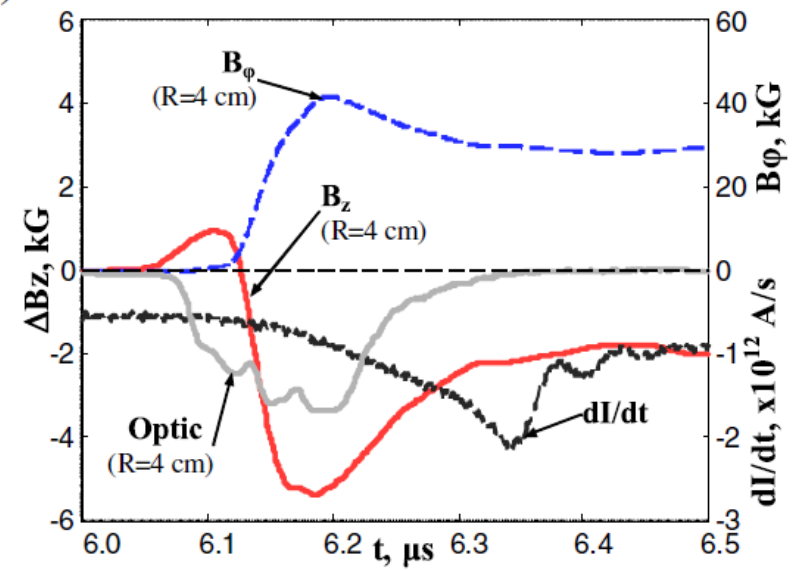

b)

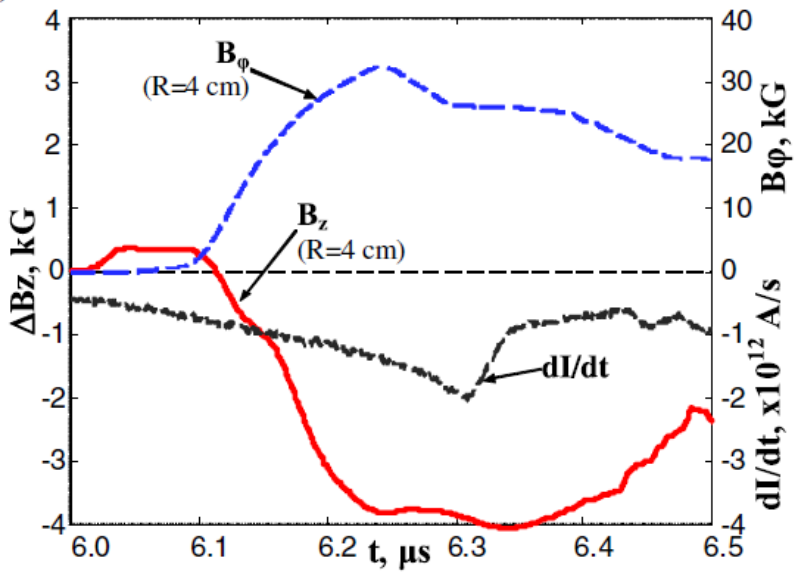

Figure 79. Figure 4 of [212]. Reproduced with permission from "Experimental Evidence of Existence of the Axial Magnetic Field in a Plasma Focus. Europhys. Lett. 2012, 98, 45001". Time dependences of the $B_{Z}$ and $B_{\phi}$ fields at the radius of $40 \mathrm{~mm}$, calculated from the probes signals shown in Figure 78 for $\mathrm{K}_{\mathrm{z}}\left(\mathrm{B}_{\phi}\right) / \mathrm{K}_{\mathrm{z}}\left(\mathrm{B}_{\mathrm{z}}\right)=(\mathbf{a}) 0.231$ (shot no. 9347) and (b) 0.055 (shot no. 9348). Here, "Optic" in panel (a) stands for the signal from the optical channel of the probe (in arb. units).

\subsubsection{Inductance of the Moving Sheath}

The method of inferring time varying inductance of the plasma discharge discussed in Section 3.2.3 was applied to PF-1000 plasma focus [386]. The voltage probe was a hybrid capacitive-resistive divider and the current derivative was measured by a Rogowski coil, both built into the current collector assembly. Figure 80 shows typical voltage and current derivative signals. Note that the sharp extrema of voltage and current derivative signals do not coincide in time. 


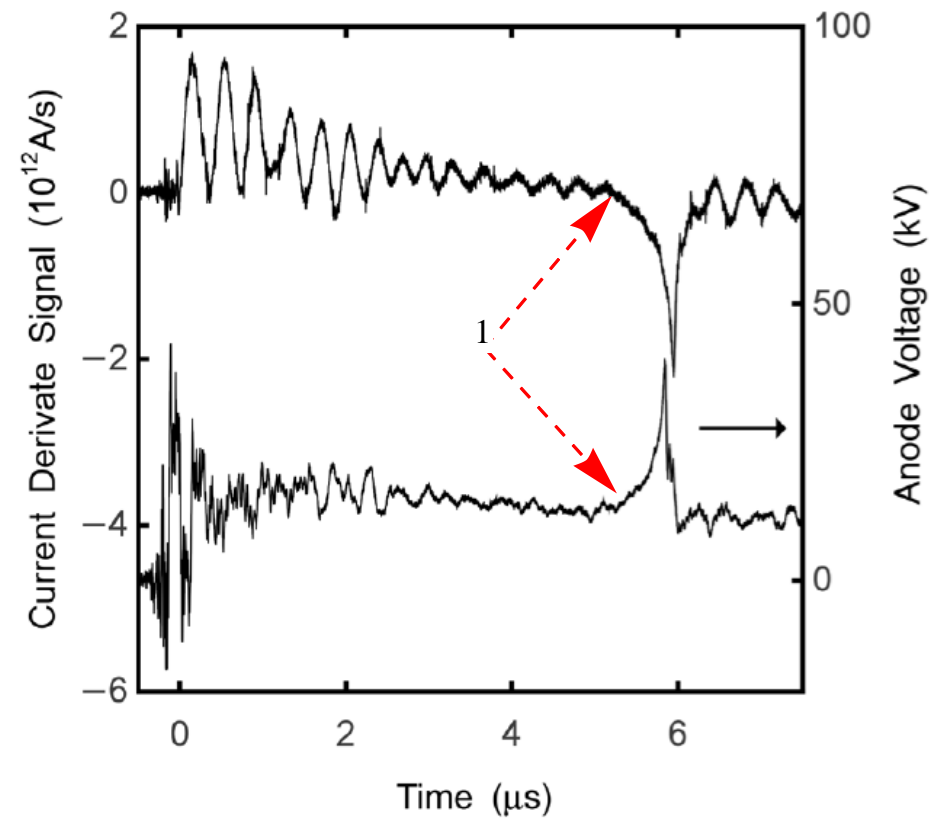

Figure 80. The red arrows mark the beginning of the radial phase. Adapted from Figure 2 of [386]. (IEEE Trans. Plasma Sci. 2016, 44, 968-972). With permission, from IEEE. (C) 2021 IEEE.

Typical results of inductance calculations are shown in Figure 81, which also provide a consistency check on the method with the following observations.

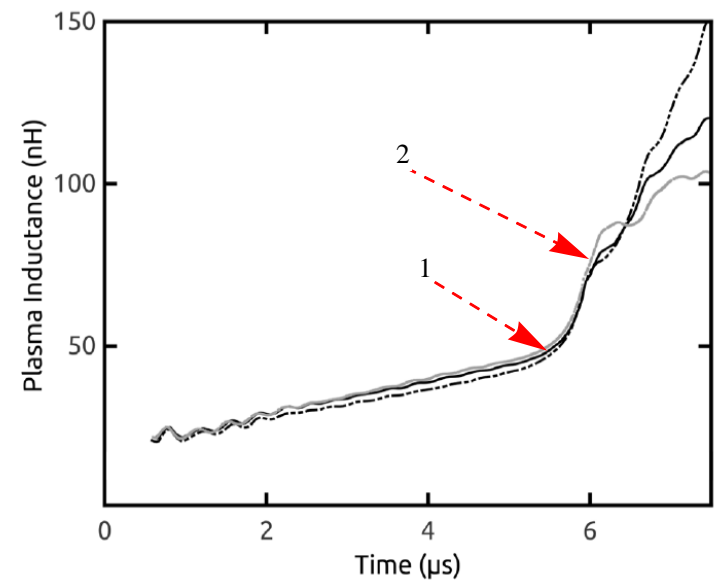

Figure 81. Inductance variation for 3 discharges at $24 \mathrm{kV}, 2.4$ mbar of $\mathrm{D}_{2}$. Red arrow labeled 1 marks the beginning of the radial phase and that labeled 2 marks the beginning of stagnation. Adapted from Figure 5 of [386] (IEEE Trans. Plasma Sci. 2016, 44, 968-972) with permission. (C 2021 IEEE.

- The initial values are approximately constant and practically coincident.

- The first change of the growth rate also occurs approximately at the same time and at the same inductance level in all of the 23 discharges studied.

- The initial value of plasma inductance is in agreement with the geometrical estimation of the fixed inductance due to connections at the base of the gun.

- The change in inductance until the first change of slope is about $30 \mathrm{nH}$, which is consistent with the total inductance of the gun.

The following major conclusions are reported:

- "The timing of the inductance-growth-rate changes and the magnitude of the inductance jumps fluctuate from shot to shot.". 
- The voltage drop across the plasma column, formed at time $t_{c}$, is estimated by the formula

$$
\mathrm{V}_{\mathrm{p}}\left(\mathrm{t}_{\mathrm{c}}\right)=\mathrm{V}\left(\mathrm{t}_{\mathrm{c}}\right)-\mathrm{L}_{\mathrm{p}}\left(\mathrm{t}_{\mathrm{c}}\right) \frac{\mathrm{dI}}{\mathrm{dt}}
$$

This is shown in Figure 82. Note the two peaks. The first peak appears in all the shots at roughly the same time and can be related with the radial convergence of the current sheath. The second peak is always the larger one, reaching hundreds of kilovolts, and has significant shot-to-shot variation. From Figure 80 and Equation (6), it is clear that the second peak corresponds to the minimum of the current derivative, which is taken as the origin of time for many experiments discussed in Section 4.3.1.

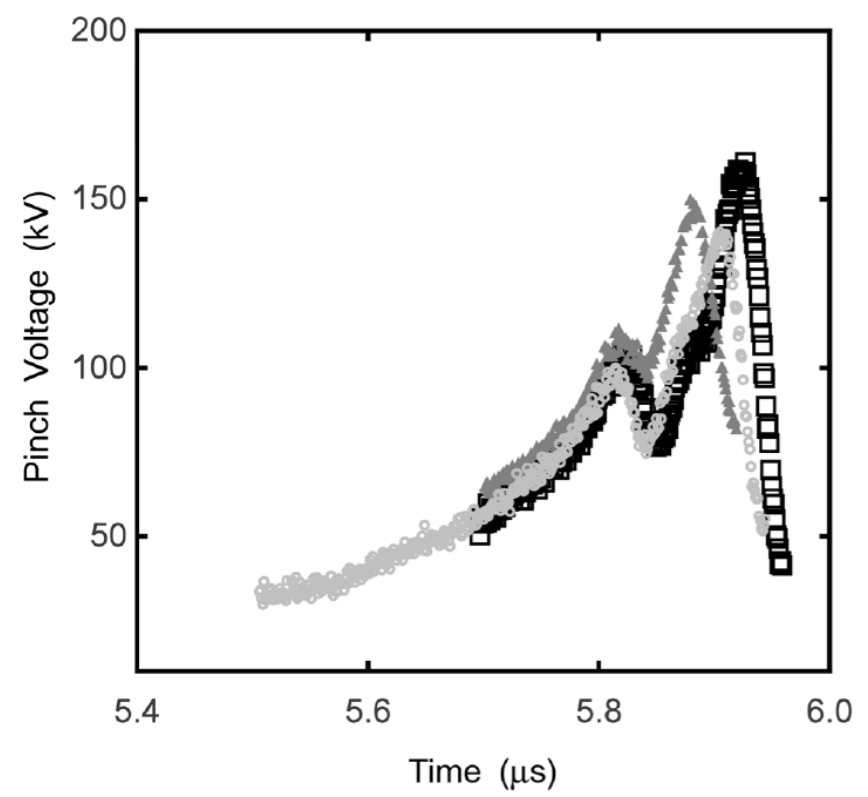

Figure 82. Voltage across the pinch vs. time. (C) 2021 IEEE. Adapted from Figure 6, [386] (IEEE Trans. Plasma Sci. 2016, 44, 968-972). With permission.

The decrease in voltage between the two peaks can be interpreted in terms of a short circuit occurring across the plasma column before the current derivative minimum (see Section 4.3.1.3 A which discusses a likely visual evidence of such short circuit).

The monotonic increase in calculated inductance particularly after the current derivative minimum in Figure 81 has not been interpreted. It is interesting to note that this feature has been observed in other studies as well, including on PF-3 [387], PACO [388] and a $400 \mathrm{~J} \mathrm{PF}$ in Chile [216]. The conjecture that the inductance should vary as logarithm of the pinch radius $[1,2,387]$ should imply that the inductance should reach a maximum at the minimum radius and then decrease. The results of inductance measurements seem to contradict this. This issue has been discussed in Sections 3.2.7 and 8.2.4.

\subsubsection{Instability of the Imploding Sheath}

M. F. Lu has reported $[389,390]$ that the radial implosion of the sheath has a stable mode and an unstable mode which "occur randomly from shot to shot in consecutive discharges and their probabilities are much influenced by the anode end structures".

Figure 83 illustrates the difference. The portion of the sheath in contact with the anode moves at a much higher radial velocity in the unstable mode than in the stable mode, resulting in a flatter sheath profile. The pinch column has a reduced height as a result of the instability in the radial implosion phase. Reduction in pinch height with pure neon operation of PF-1000 (but not with pure deuterium operation) is reported (see Section 5.1.1 (1)). 

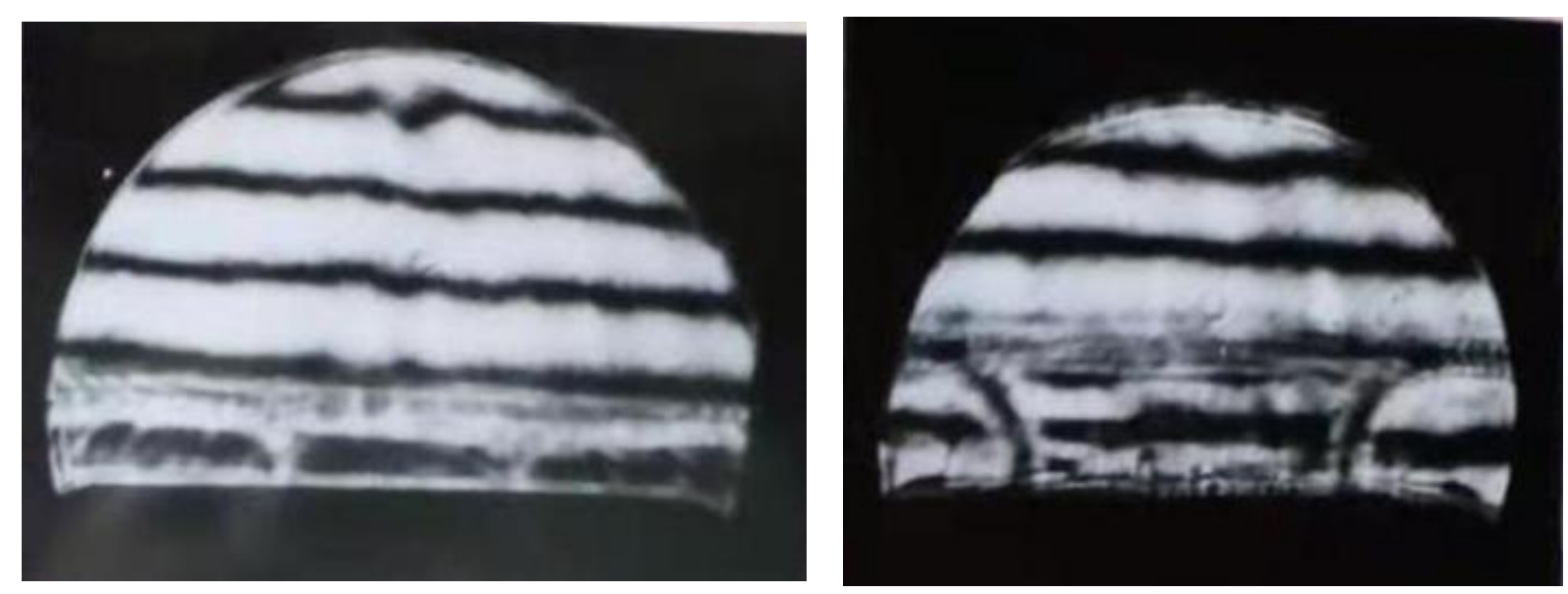

Figure 83. Unpublished images kindly provided by Prof. Ming Fang Lu. (CProf. Ming Fang Lu). They illustrate the same features as Figure 3d1,d2) of [389]. The left hand image illustrates the unstable mode and the right hand one the stable mode. The sheath appears to make a much shallower angle with the anode face in the unstable mode than in the stable mode. This is because its portion in contact with the anode moves much faster than the part not in contact with the anode.

\subsection{Plasma Dynamics Related to Stagnation, Pinch, and Instability Phases 4.3.1. PF-1000}

The investigations reported below were performed with the PF-1000 device described in Section 3.1.2. For many experiments, the operating parameters were chosen for considerations other than maximum neutron yield in order to provide slower plasma dynamics or higher plasma density in order to obtain conditions more favorable to the diagnostic technique. Some experiments were also performed in a modified regime where a gas puff injected on the axis was compressed by plasma focus sheath launched in ambient gas. These are discussed in a separate section (Section 5).

\subsubsection{Optical Diagnostics}

A. Interferometry

The interferometric system described in Section 3.2.1 enabled visualization of internal structure of the plasma from radial implosion phase until plasma decay in a sequence of 15 frames recorded in a single shot. Results of such investigations are described in a series of publications [103,105-107,126,391-397], including an overview paper [103]. Measurements of neutron, soft X-ray (SXR) and hard X-ray (HXR) emissions provided context for the interferometric observations. Magnetic probe measurements $[205,207,210,393]$ and vacuum ultraviolet emission spectroscopy of hydrogen and helium like ions of impurities $(\mathrm{O}, \mathrm{N}$, and C) provided additional information for interpretation of the observations. Comparison of XUV imaging with interferograms [107] is discussed in Section 4.3.1.1 B on XUV imaging. Salient common features of these reports are summarized below.

- In early reports [126,391-394], the interferometer was set up to cover the field of view with uniformly spaced sharp fringes in the absence of plasma whose deviation in a plasma shot was related to plasma density. Subsequently, the interferometer was set up [395] with parallel mirrors which gave widely spaced fringes in the absence of plasma. The fringes obtained in the presence of plasma were, therefore, contours of equal areal electron density (integral of electron density along the path of the collimated laser beam). Closed contours indicated presence of 3D bounded plasma structures. Densely grouped fringes indicated high plasma density gradient and widely spaced fringes indicated low density region. The uncertainty in the estimation of electron density from wide-spaced fringes outside the dense plasma was estimated [107] as $\sim 1 \times 10^{23} \mathrm{~m}^{-3}$.

- Three types of structures were observed: plasmoidal, toroidal, and lobule-like. Helical perturbations of the plasma boundary were also observed. Plasmoidal structures 
are marked by a single set of nested fringes indicating that the density peaks at the innermost region of a topological sphere. Toroidal structures are marked with a double set of nested fringes enclosed within another system of nested fringes that indicate density peaking in the innermost region of a topological torus. Lobules were marked by a sharp radial deviation of the boundary of the fringe pattern in a narrow axial region either to one side or to both sides of the plasma boundary region in the interferogram.

- Although the fringe patterns were generally not symmetric about the axis, Abel inversion could be used across those cross-sections where the asymmetry was minimal. Although not strictly accurate, this gave useful average estimates of radial electron density distribution.

- Movement of the fringe pattern in successive frames gave an estimate of plasma motion. The growth of lobules indicated outflow of plasma from the dense pinch into surrounding low density plasma. X-regions were formed at the separatrix of neighboring systems of closed fringes. Their displacement in successive frames indicated presence of axial flow into these structures from these $\mathrm{X}$-regions.

- In all investigations, time $t=0$ is assigned to the minimum of the current derivative, except where explicitly mentioned. This requires some discussion. On the one hand, this is a signal that is measured in all the shots and can thus be used as a cross-reference across many series of experiments which may not field some of the diagnostics. It is difficult to conceive of an alternative time reference on this point alone. On the other hand, there are certain problems with this time reference. In a large plasma focus like the PF-1000, the current derivative is measured at a distance of $2 \mathrm{~m}$ from the pinch. Interpretation of the instant of the current derivative minimum in terms of physical phenomena occurring at the anode surface near the axis implicitly assumes that there is no signal delay across this $2 \mathrm{~m}$ distance. In fact, Mather does mention such delay [2] (p.214) that corresponds to a wave speed of $10^{9} \mathrm{~cm} / \mathrm{sec}$ and uses it to estimate the residual density left behind the snowplow (see Section 8.4.1.4). The minimum of the current derivative is not sharply defined-it has a flat region of a few ns width, whose center may be taken as the time mark. In many shots, the current derivative has multiple minima of which the deepest one is used as a time mark. Reflections (oscillations related to impedance mismatch in the current collector assembly following current derivative singularity) can affect the amplitude of the minimum and can cause errors in locating the minimum. The multiple turns of the Rogowski coil can cause a delay between the true current derivative and the detected signal. A constant error due to propagation delay or the identification of minimum would not affect interpretations within a single shot based on time differences. However, as a matter of abundant caution, the time reference information needs to be associated with an error estimate $\sim 10 \mathrm{~ns}$ at the time of interpretation of results across multiple shots.

A brief summary of experimental results in these reports is given below.

(1) First interferometry experiments [126] were deliberately carried out in a non-optimized parameter regime: charging voltage of $20 \mathrm{kV}$ (120 kJ energy) and initial deuterium pressure of $200 \mathrm{~Pa}$ as compared to $30 \mathrm{kV}$ and $400 \mathrm{~Pa}$ standard operating regime. The neutron yield was $\sim 10^{9}$ at a current of $\sim 1 \mathrm{MA}$. In this parameter regime, the plasma compression was weaker, the plasma column had a larger diameter, with worse cylindrical symmetry and lower symmetry of stagnation. However, the advantage was a high contrast fringe pattern with distinct fringes that facilitated its first ever evaluation. This advantage was not available at the standard operating conditions.

a. The study looked at the stagnation phase from $-100 \mathrm{~ns}$ to $+120 \mathrm{~ns}$ in $10 \mathrm{~ns}$ intervals.

b. Linear electron density as a function of $\mathrm{z}$ coordinate for different interferograms revealed a dense plasma structure that moves along the axis beginning at the anode. 
c. During the implosion of the current sheath, a dense structure with a radius of $\sim 2 \mathrm{~cm}$ is formed with central peak density $\sim 2-4 \times 10^{18} \mathrm{~cm}^{-3}$ which decreases with time.

d. No evidence of filaments or sub-millimeter structures was found.

(2) The second report of interferometric investigations [391] dealt with interferometric investigations during neutron emission in the parameter regime $20 \mathrm{kV}$ and $100 \mathrm{~Pa}$ deuterium pressure producing $\sim 7 \times 10^{10}$ neutrons at $\sim 1.2 \mathrm{MA}$ current. A region of decreased density was formed on the axis at the time of maximum neutron production both during the first pulse before column disruption and the second pulse following column disruption. In the latter case, the low density structure was below the detection threshold. The computed contours of plasma density show signatures of toroidal structures. They were not identified as such in that paper but were pointed out later [362,392] (see Section 3.3.3.5).

a. The paper mentions dominant deuteron energy estimate $\sim 100-350 \mathrm{keV}$.

(3) The third report [392], operating at 1.6-1.9 MA and 250-300 $\mathrm{Pa} \mathrm{D}_{2}$, explicitly identifies toroidal, helical, and lobular structures within the plasma in the implosion phase (before stagnation) because of their higher density than surrounding plasma. The lobules get absorbed in the sheath within few tens of nanoseconds. A low density plasma region of 1-2 cm thickness is seen in front of the well-marked dense imploding sheath.

a. The period of first neutron and HXR emission usually begins at $-20 \mathrm{~ns}$, reaches a maximum at $15-20 \mathrm{~ns}$ and ends around $40-50 \mathrm{~ns}$. This period coincides with the formation of the first plasmoid embedded within a less dense plasma column of about $12-14 \mathrm{~mm}$ diameter and $30-50 \mathrm{~mm}$ length. The plasmoid moves axially starting from the anode during the radial collapse of the sheath with a velocity of 2-4 $\times 10^{7} \mathrm{~cm} / \mathrm{sec}$. During the declining portion of the HXR pulse, the diameter of the plasma stem between the anode and the moving plasmoid increases and its density decreases (see Figure 86). The radial and axial density profiles of the plasmoid have a Gaussian shape.

b. The period of second neutron and HXR emission usually coincides with the continuation of the stagnation process. In the example presented, it began at $49 \mathrm{~ns}$ and ended at $69 \mathrm{~ns}$. It coincided with the growth of a low density region between the anode and the plasmoid. During this period, the dense plasmoid moved away from the anode, interacted with the upper lobule and the column surface expanded in a quasi-spherical shape.

c. Third HXR pulse began at $106 \mathrm{~ns}$. Before this, sudden constriction of plasma column occurred near the anode at $96 \mathrm{~ns}$. The interferogram at this time indicated that about half the plasma in the constricted zone was injected into the non-collapsed part of the plasma column, where a dense plasmoid was formed. At $116 \mathrm{~ns}$, which marked the peak of HXR, the constricted region underwent a sudden increase in diameter accompanied with reduction in density, similar to an explosion. At $126 \mathrm{~ns}$, there was a rapid decrease in density. Multiple plasmoids of diameter less than $5 \mathrm{~mm}$ and peak density $\sim 3 \times 10^{18} \mathrm{~cm}^{-3}$ were observed in the constricted region.

d. The paper reports a delay of 10-30 ns between the maximum of neutron signal with respect to HXR signal.

(4) The 4th report [393] explores correlation between magnetic probe and neutron signals and interferometry in an operating regime of $24 \mathrm{kV}$ (384 kJ energy) and $240 \mathrm{~Pa}$ deuterium pressure producing about $10^{11}$ neutrons. Magnetic probes looking at time derivative of azimuthal magnetic field (designated $\mathrm{dB}_{\varphi}$ ) were fielded at $4,1.3$, and $0 \mathrm{~cm}$ from the axis on a line perpendicular to the path of laser beam. Their integration was related to current flowing in the sheath under the assumption of symmetric flow of current. In later campaigns, probes looking at time derivative of axial magnetic field 
(designated $\mathrm{dB}_{\mathrm{z}}$ ) were fielded at 4 and $0 \mathrm{~cm}$. The details of the probe construction, calibration, etc., are discussed in Section 3.2.2.

a. In a series of 8 shots looking at $\mathrm{dB}_{\varphi}$ at $4 \mathrm{~cm}$ radius, the signal started at $-200 \mathrm{~ns}$, reached its peak at $-160 \mathrm{~ns}$ and reached its zero (maximum of $\mathrm{B}_{\varphi}$ ) at $-110 \mathrm{~ns}$ with a standard deviation of just $10 \mathrm{~ns}$ shot to shot. Up to $(80 \pm 20) \%$ of discharge current flowed past the $4 \mathrm{~cm}$ radius.

b. The current carrying layer had a full width of $1.6-2.6 \mathrm{~cm}$ and was located behind the dense plasma layer (see Figure 3 of [393]).

c. In a series of 3 shots, combined $\mathrm{dB} \varphi$ and $\mathrm{dB}_{z}$ probes placed at $4 \mathrm{~cm}$ position revealed a small Bz prepulse preceding the current sheath by $70-90 \mathrm{~ns}$ and oriented away from the anode (see Section 4.2.2 (3)). The sign of the main $B_{z}$ pulse was opposite to that of the prepulse, i.e., directed towards the anode and was 2-8 times smaller in magnitude as compared with the $\mathrm{B}_{\varphi}$ field. The prepulse corresponded with a $5 \mathrm{~mm}$ wide low-density (less than $10^{24} \mathrm{~m}^{-3}$ ) region moving ahead of the dense plasma layer in which toroidal and helical structures were observed to form (see Figure 84 for illustration).

d. In a series of 5 shots with combined magnetic probes placed at $4 \mathrm{~cm}$ and $1.3 \mathrm{~cm}$, the $\mathrm{dB}_{\varphi}$ signal at $1.3 \mathrm{~cm}$ started at $-55 \mathrm{~ns}$ and reached its maximum at $16 \mathrm{~ns}$. The shot to shot fluctuation was $5 \mathrm{~ns}$. The current sheath took $90 \mathrm{~ns}$ to cross the probe at $4 \mathrm{~cm}$ and $70 \mathrm{~ns}$ for the probe at $1.3 \mathrm{~cm}$. Three interferograms at $-68 \mathrm{~ns}$, -38 ns, and 22 ns (Figure 6 of [393], adapted as Figures 84-86 below) show the position of the plasma at the start, maximum, and zero of the $\mathrm{dB}_{\varphi}$ signal. The leading edge of current density front at $-68 \mathrm{~ns}$ lies ahead of the leading edge of the dense plasma. The plasma re-expansion had already started at $22 \mathrm{~ns}$. This indicated that the current layer continued to move towards axis even after the plasma stopped imploding. Completion of current penetration into the dense plasma and end of the first neutron pulse coincided with the start of plasma column re-expansion. The value of $\mathrm{B}_{\varphi}$ at the plasma boundary at $22 \mathrm{~ns}$ was $21 \pm 4 \mathrm{~T}$. The $\mathrm{B}_{\mathrm{z}}$ channel was not used.

e. In a series of 4 shots with combined magnetic probes placed at $r=0$, the $\mathrm{dB}_{\varphi}$ signal was smaller than $\mathrm{dB}_{\mathrm{Z}}$ signal, as it registered azimuthal and radial components of magnetic field produced by asymmetry in current distribution. The $\mathrm{dB}_{\mathrm{z}}$ signal varied considerably from shot to shot. Interestingly, one of the discussed shots had a good azimuthal symmetry of the plasma enabling Abel inversion of the interferogram and also had a strong $B_{z}$ signal $(\sim 6 \mathrm{~T})$ in the radial collapse phase detected at $r=0$. This core of the investigations has been described in considerable de $e_{t}$ ail in the paper [393] and a condensed summary would not do proper justice.

f. The investigation demonstrates that the neutron pulses are correlated in time with evolution and decay of plasmoidal and toroidal structures which probably are locations of azimuthal currents that create the axial magnetic field. 


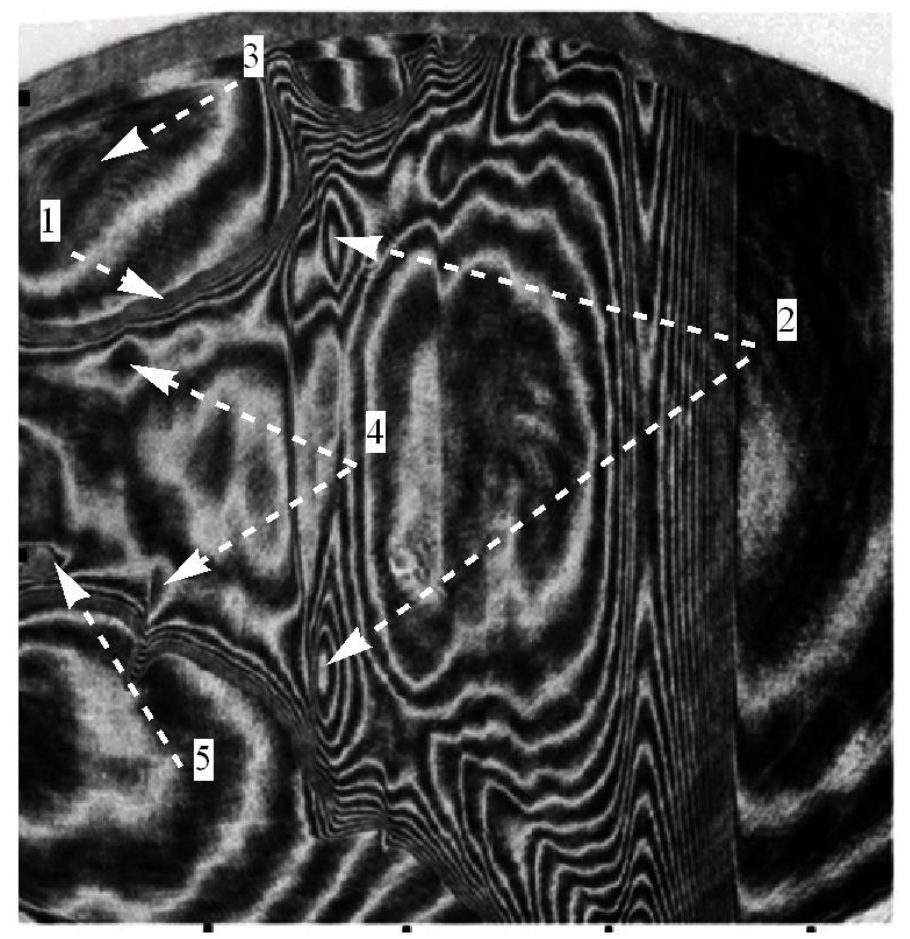

Figure 84. Interferogram at $-68 \mathrm{~ns}$ Shot \#9367 (adapted from Figure 6a of [393] (Plasma Phys. Control. Fusion 2012, 54, 105023). 1. Dense plasma sheath, 2. Density peaks on toroidal axes. 3. Low density plasma region where azimuthal current is located according to the magnetic probe measurements. 4 . Low density structures correlated with the $B_{z}$ prepulse where toroidal currents are suspected to reside (see Section 4.3.1.3 A) 5. Probe at $r=1.3 \mathrm{~cm}$. At $-68 \mathrm{~ns}$, the dense sheath had not crossed the probe at $1.3 \mathrm{~cm}$ but the probe signal had already begun indicating that the current front had now moved ahead of the density front. Reproduced with permission. OIOP Publishing. All rights reserved.

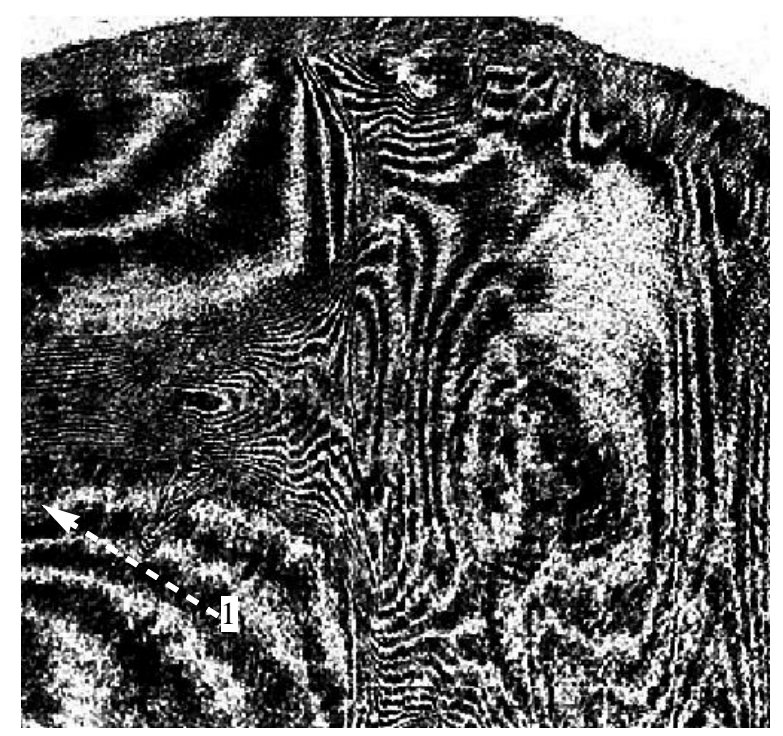

Figure 85. Interferogram at -38 ns Shot \#9367 (adapted from Figure 6b of [393] (Plasma Phys. Control. Fusion 2012, 54, 105023). 1. Probe at $r=1.3 \mathrm{~cm}$. At this time, the dense front had fully passed the probe position and reached the axis forming a dense column. The signal at this probe had reached a maximum indicating that the maximum of current density is located at the probe position marked by the white arrow. The current derivative signal had not reached its minimum as yet-that was $38 \mathrm{~ns}$ away. Reproduced with permission. (OIOP Publishing. All rights reserved. 


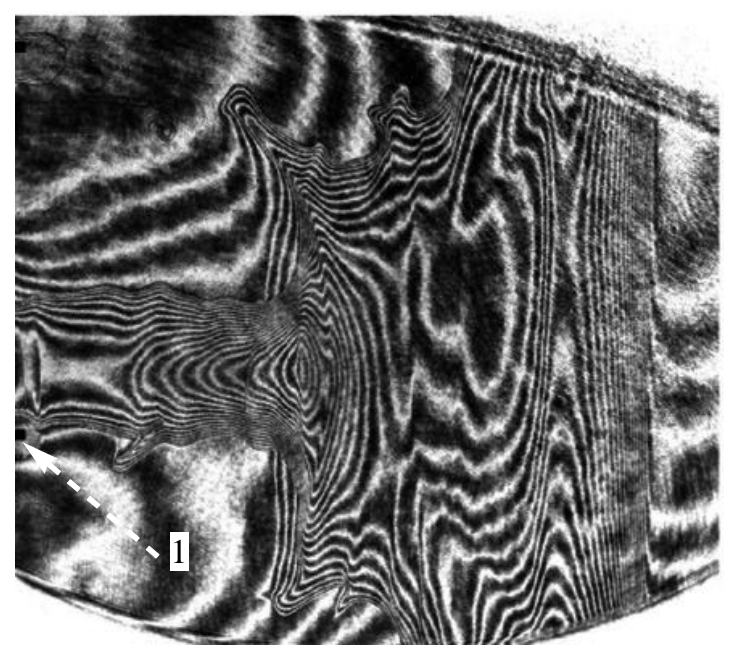

Figure 86. Interferogram at $22 \mathrm{~ns}$ Shot \#9367 (adapted from Figure 6c of [393] (Plasma Phys. Control. Fusion 2012, 54, 105023). 1. Probe at $\mathrm{r}=1.3 \mathrm{~cm}$. This time coincided with the zero of the probe signal indicating that the maximum of $\mathrm{B}_{\varphi}$ was located at the probe position marked by the white arrow. At this time, the plasma column had already started expanding as seen by comparing the dense plasma column at $-38 \mathrm{~ns}$. According to Section 4.3.1.1 A(3)a, this time marks the beginning of the declining portion of the HXR pulse. Reproduced with permission. CIOP Publishing. All rights reserved.

(5) The 5th report [394] extracts a phenomenological narrative from correlated experimental results on interferometry, magnetic probe and neutron measurements. The main departure from the standard narrative takes into account the helical nature of magnetic field in view of simultaneous presence of azimuthal and axial components of magnetic field and their source currents in the current sheath.

a. Toroidal structures spontaneously occur ahead of the dense plasma layer, as indicated by the $B_{z}$ prepulse, at various axial positions well before stagnation (see Figure 84 for illustration). They offer more resistance to the implosive force than the neighboring plasma. Therefore, the implosion slows down at their axial locations but proceeds unimpeded in the gap between them. This leads to a plasma structure with alternating large and small diameter regions. The higher pinching force on the smaller diameter regions forces the plasma to move axially into the toroids at the core of the large diameter regions, evolving them into plasmoidal structures. The peak of neutron and HXR emission coincides with the maximum of plasmoid density and of the $B_{Z}$ signal from the probe at $\mathrm{r}=0$.

b. The minimum of current derivative $20 \mathrm{~ns}$ after the peak of SXR emission corresponds to the penetration of discharge current into the plasma column and peak value of discharge inductance.

c. Axially approximately uniform density of the plasma column indicated by axially oriented open fringes before the $\mathrm{m}=0$ instability becomes non-uniform by axial flow. The regions of decreasing density implode and form necks or constrictions. In the toroidal structures between necks, plasma density increases and forms toroidal discs in a manner similar to the implosion phase.

d. The plasmoid formed at the time of the first neutron pulse persists and continues to evolve during the process of constriction. It decays during the breakup of the plasma. Formation and destruction of other plasmoids during the constriction process is tracked and found to correlate with neutron emission.

e. The boundary of constricted zone near the anode shows modulation of radius with sub-millimeter wavelength. These are correlated with formation of small and dense plasmoids. 
f. In some regions of the constricted zone of plasma, the fringe pattern becomes blurred while it continues to show high contrast fringes in neighboring areas. This indicates localized turbulent fluctuations of density on a time scale shorter than the pulse width of the laser.

g. The dominant neutron pulse is correlated with decrease in magnetic field (both $B_{Z}$ and $B_{\phi}$ components) leading to induced electric field that accelerates ions.

(6) Interferometric images of lobules [395] were interpreted in terms of existence of poloidal current loops and return path for the radially escaping plasma. These conclusions have been further elaborated in a later paper (see (9) below).

(7) Evolution of the plasmoidal structure was more closely studied [396] in 2016. However, this was not in a normal PF-1000 operation regime. Instead, annular nozzle of diameter $2.4 \mathrm{~cm}$ and $3.6 \mathrm{~cm}$ placed $2.3 \mathrm{~cm}$ inside the anode were used to inject a gas puff on the anode axis while a plasma focus sheath launched at $23 \mathrm{kV}$ charging voltage (350 kJ bank energy) and 200 Pa pressure of deuterium in chamber compressed it. Conclusions of this report are, therefore, summarized in a different section (see Section 5.1).

(8) Important differences in the evolution of internal structures were reported [397] between the first and subsequent neutron pulses in an operational regime with $16 \mathrm{kV}$ charging voltage (300 kJ energy) and $120 \mathrm{~Pa}$ deuterium pressure. The paper also reports observations on plasma sheath launched in hydrogen gas filling collapsing on a deuterium gas puff. The discussion is intimately related to evolution of features of the fringe pattern and is difficult to summarize. Interested reader needs to refer to the original paper. Interpretation of results depends on XUV spectroscopy and neutron time-of-flight and HXR diagnostics and is discussed separately.

(9) Results of comparison between XUV images and interferometry from a series of 29 shots were reported [107] for a higher than optimal pressure of $170 \mathrm{~Pa}$. This regime had a higher radiation in extreme ultraviolet but had a decreased neutron yield of $\sim 10^{9}$. It also had a slower evolution of plasma allowing assumption of quasi-stationary conditions which played an important role in interpretation. The uncertainty in the calculation of plasma density in the low density plasma region outside the dense plasma column was estimated to be less than $1 \times 10^{23} \mathrm{~m}^{-3}$. For comparison, the molecular number density of an ideal gas at $1 \mathrm{hPa}$ and $273 \mathrm{~K}$ is $2.7 \times 10^{22} \mathrm{~m}^{-3}$. The significant new results were detailed studies of the lobule structure on the outer boundary of the dense plasma.

a. The evolution of the plasma column from $-40 \mathrm{~ns}$ to $+50 \mathrm{~ns}$ was recorded by both XUV frames and interferometry, which covers the period with formation of the first plasmoid and the first neutron and SXR pulses. The images show development of a lobule structure at the junction of the umbrella-like top and the approximately cylindrical plasma column that extends towards the anode, creating an inverted-U shaped dense and hot plasma profile that is not symmetric in the pictures. The arms of the inverted $U$ then appear to link up enclosing a toroidal region that appears "empty" in both the XUV and interferometry images.

b. This kind of low density toroidal structure external to the dense plasma column was observed also at the time of plasma constriction and in correlation with the second neutron pulse.

c. Interpretation of this data is discussed in Section 4.3.1.3.

(10) The process of plasma column break-up coinciding with the second neutron pulse is clearly documented [106] in Figure 87 below, $\left(D_{2}\right.$ at $170 \mathrm{~Pa}, 16 \mathrm{kV}$, standard electrodes) 

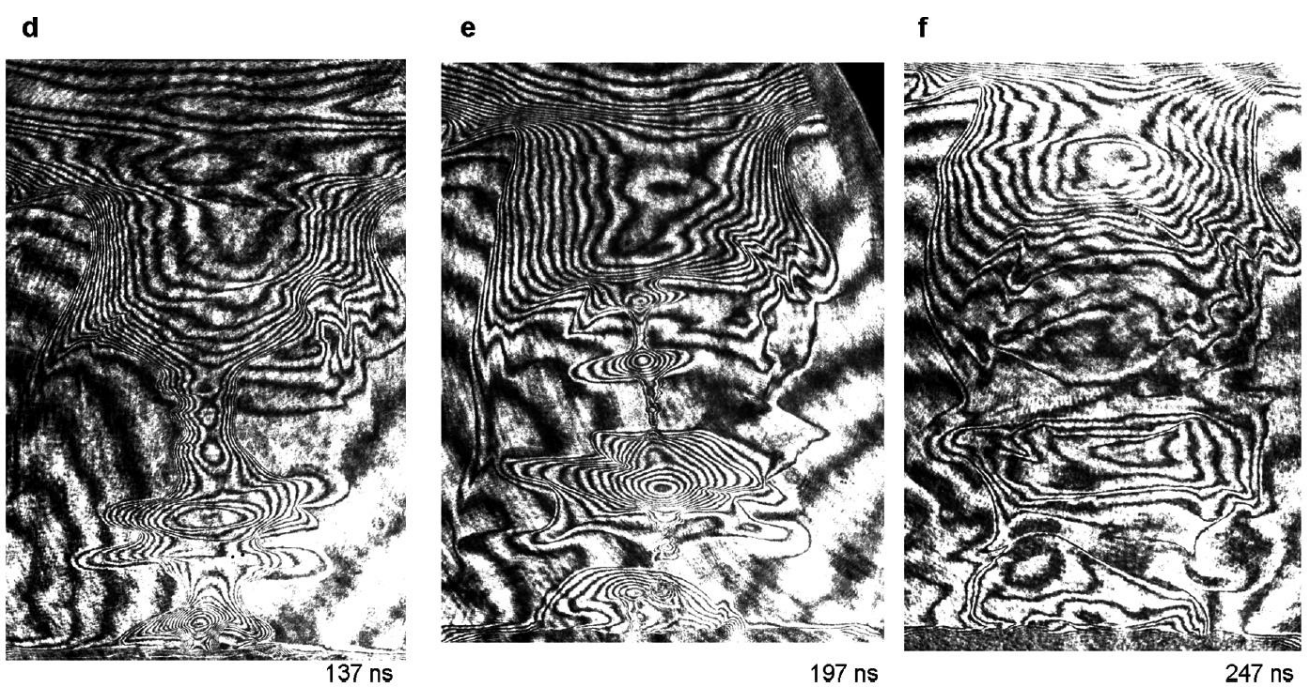

Figure 87. Adapted from Figure 2 of [106]. (Matter Radiat. Extremes 2020, 5, 046401), with the permission of AIP Publishing. Note the narrow dense necks connecting small plasmoids in the middle frame, which ultimately disappear leaving behind large distorted plasmoids.

(11) A clearer image of plasma column break-up is shown [105] in Figure 88 below, $\left(\mathrm{D}_{2}\right.$ at 80-100 Pa, $16 \mathrm{kV}$, conical tips at the anode and anti-anode). However, caution must be exercised when comparing results of an experiment with anode inserts with the standard electrode geometry of PF-1000 which has a cavity in the anode $[44,45]$ (see Section 2.3.1).

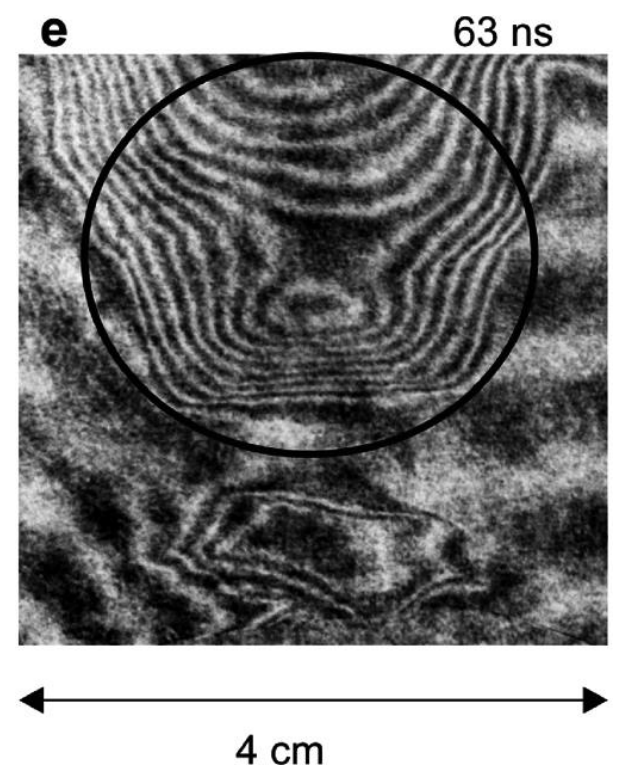

Figure 88. Adapted from Figure 2e of [105], (Phys. Plasmas 2019, 26, 032702) with the permission of AIP Publishing. Note the plasmoid at the top and the low density region below separating it from a smaller plasmoid. This coincides with the second HXR pulse.

B. XUV spectroscopy and imaging

This section discusses utilization of extreme ultra violet (XUV) radiation in the 10-124 eV range for diagnostics purposes in PF-1000 facility.

(1) Time and space integrated XUV spectrum of $\mathrm{H}$ and He like ions of $\mathrm{O}$, C, and $\mathrm{N}$ (present as impurities) was interpreted [397] as indicating an average temperature of $\sim 70 \mathrm{eV}$ "at the final phase of the stagnation". 
(2) A 4-frame micro-channel-plate (MCP) camera equipped with 4 pinholes without any filters was used to obtain XUV images [107]. The lower limit of photon energy $\sim 30 \mathrm{eV}$ was set by absorption in the working gas and the upper energy was a few $\mathrm{keV}$ set by the MCP spectral response. The XUV frames compared with interferometry pictures show details of a lobule structure that forms an inverted $U$ profile pointing towards the anode (Section 4.3.1.1 A(8)).

(3) A segmented 4-frame gated MCP camera with $5.2 \mu$ m polyester $\left(\mathrm{C}_{8} \mathrm{H}_{8}\right)$ filter, $2 \mathrm{~ns}$ gating time and inter-frame separation of 10-50 ns was used [398] to obtain XUV images in a window $200-300 \mathrm{eV}$ and above $600 \mathrm{eV}$. Its response to radiation below $10 \mathrm{eV}$ was negligible as compared to XUV response. A p-i-n silicon diode with $3 \mu \mathrm{m}$ thick aluminized mylar, having spectral response similar to the MCP camera was used to obtain temporal variation of XUV radiation. The origin of time was defined not with respect to the current derivative minimum as in later publications but with respect to the maximum of the p-i-n diode signal. (See Section 4.3.1.1 C below for signal waveforms).

a. The p-i-n diode records a peak 0 to $50 \mathrm{~ns}$ before the minimum of the current derivative. It usually has two peaks. The first peak has a rise time of 20 to $30 \mathrm{~ns}$ and FWHM of 30-60 ns. The second peak of considerably lower intensity has its maximum 120 to $170 \mathrm{~ns}$ after the first peak with FWHM 50-100 ns. In later publications with interferograms, this peak corresponds with the compression of the constriction.

b. $\quad$ During the rise of the p-i-n diode signal, which occurs during the onset of sheath stagnation but before maximum compression, an intensely radiating region is seen in the XUV framing pictures at -20 and -10 ns with a corresponding region in the visible framing pictures, which indicates its position with respect to the umbrella shape.

c. XUV frames coinciding with the two neutron peaks also show intensely radiating regions in the plasma column coinciding with 1-2 cm diameter spherical structures seen in visible frames (See Section 4.3.1.1 C below).

(4) Comparison of interferometric and XUV images shows [106] striking evidence of closed loop currents outside the dense column. An example is shown in Figure 89. Helical deformations of the plasma boundary are clearly seen in the XUV image.
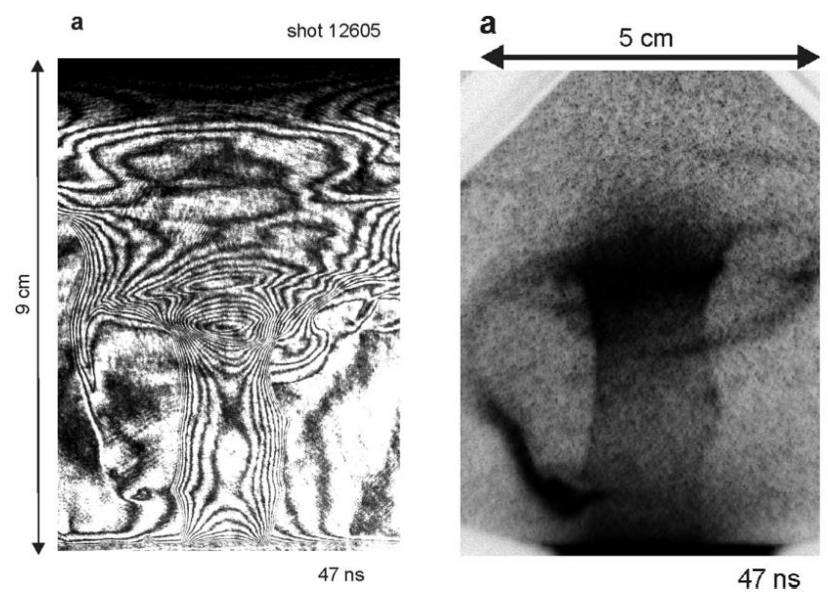

Figure 89. Adapted from Figures 2a and 3a of [106] (Matter Radiat. Extrem. 2020, 5, 046401), with the permission of AIP Publishing. Comparison of interferometric (left) and XUV (right) images The lateral lobular extension of the plasma loops back and joins the plasma column near its bottom, effectively short-circuiting the pinch. 
C. Visible framing and Streak

(1) Visible radiation in a $10 \mathrm{~nm}$ spectral band centered on $589 \mathrm{~nm}$ (spectral region free of intense line radiation) was imaged [398] with a 4-frame camera with a gating time of $1 \mathrm{~ns}$ and inter-frame separation of 10-20 ns. A visible streak camera looking perpendicular to the axis of the plasma with a slit imaged at $3 \mathrm{~cm}$ above anode recorded the radial motion of the plasma. A sequence of 16 frames from 4 shots covering the time interval -60 to $170 \mathrm{~ns}$ was shown. The origin of time was defined not with respect to the current derivative minimum as in later publications but with respect to the maximum of the p-i-n diode signal.

a. The minimum radius of the visible column occurs at the $t=0$. The minimum of current derivative occurs 0 to $50 \mathrm{~ns}$ after the first XUV peak.

b. At $-30 \mathrm{~ns}$, an intensely radiating region is seen in the middle of the imploding plasma stem, that is also seen in XUV pictures. The p-i-n diode records a pulse that peaks at this time.

c. Frames coinciding with the two neutron emission peaks show a spherical structure of 1-2 cm diameter located on the axis at 6-8 $\mathrm{cm}$ from the anode at the junction of the umbrella-like shape and the top of the plasma column. Streak picture showed evidence of a second pinching which is also seen in visible framing pictures.

(2) Streak pictures of PF-1000 discharges [399] for low and high neutron shots show significant differences in the implosion velocity.

\subsubsection{Emissions of Various Kinds and Their Inter-Correlation}

A. Neutrons and Hard X-rays (HXR)

Investigations [105,106,399-402] primarily concerned with characterizing neutron emission (as opposed to providing a context for other measurements, such as interferometry (see Section 4.3.1.1 A), are discussed here. Fast scintillator-photomultiplier detectors, with $5 \mathrm{~cm}$ thick NE102A scintillators, were used [398] to record HXR above a few hundreds of $\mathrm{keV}$ (penetrating 3 to $5 \mathrm{~cm}$ of steel or lead) and fusion neutrons.

(1) First results [400] of neutron emission on PF-1000 were obtained from a configuration different from that described in Section 3.1.2-anode diameter $244 \mathrm{~mm}$, anode length $447 \mathrm{~mm}$, insulator $113 \mathrm{~mm}$ long of $244 \mathrm{~mm}$ diameter, inner diameter of cathode $368 \mathrm{~mm}$.

a. $\quad$ Two groups of $X$-ray and neutron pulses separated by $\sim 2 \mu$ s were observed. Neutron signals measured at $15 \mathrm{~m}$ downstream (along the direction of current flow) and at $40 \mathrm{~m}$ and $85 \mathrm{~m}$ upstream indicated maximum neutron energy of first pulse to be $2.70 \mathrm{MeV}$ upstream and $2.68 \mathrm{MeV}$ downstream whereas the second pulse showed $2.49 \mathrm{MeV}$ upstream and $2.77 \mathrm{MeV}$ downstream. The fact that the maximum downstream neutron energy exceeded the $2.45 \mathrm{MeV}$ d-d reaction energy in the second pulse suggested that an appreciable number of deuterons moved away from the anode with a high velocity during the second pulse.

b. Upstream maximum neutron energy in the range $2.95 \pm 0.15 \mathrm{MeV}$ was reported.

(2) In order to reconstruct the energy spectrum of neutrons by the time of flight method, fast scintillator-photomultiplier detectors were set up [398] along the axis both in upstream and downstream directions in two configurations: a) 7.0 and $16.3 \mathrm{~m}$ downstream from the center of the neutron source (assumed to be $6 \mathrm{~cm}$ in front of the anode) and 7.0, 17.0, 30.3, 44.3, and $58.3 \mathrm{~m}$ upstream. b) 7.0,16.3 and $58.3 \mathrm{~m}$ downstream and 7.0, 17.0, and $58.3 \mathrm{~m}$ upstream. Two HXR and neutron peaks were obtained in most shots, with the second neutron peak dominating. The report was compiled from data from 200 shots. Temporal uncertainty in each shot was 5 ns. "The presented values of time, full-width at half maximum (FWHM) of pulses, and energies of neutrons are mean values taken from all the recorded shots. The shot-to-shot variability of time increases with the value of this time, and it could be estimated as $10 \%$ to 
$15 \%$. The variability of the FWHM values of pulses is higher. in average, it is $20 \%$. The inaccuracy of the determination of the neutron-energy values is estimated to be 0.1 to $0.2 \mathrm{MeV}^{\prime \prime}$.

a. Energy of neutrons corresponding to the first and second peaks (corresponding to the dominant portion of deuteron velocity distribution) was $2.7 \mathrm{MeV}$ and 3.0 $\mathrm{MeV}$ in the downstream direction and $2.3 \mathrm{MeV}$ and $2.0 \mathrm{MeV}$ in the upstream direction. (The numbers mentioned in Section 4.3.1.2 A(1)a refer to the beginning of the neutron signal, the maximum neutron energy).

b. "A sharp onset of the pulses downstream and a sharp decrease of those upstream have been observed. On the contrary, the decrease in the pulses observed downstream is slower, and the increase is slower in the upstream ones". This asymmetric feature has been "... explained with a production of a smaller portion of neutrons with energies between 2.2 and $2.4 \mathrm{MeV}$ in the downstream directions". Such neutrons, with energy less than the reaction energy of $2.45 \mathrm{MeV}$, would be produced by deuterons moving in the upstream direction.

c. HXR emission (recorded with upstream detector at $30.3 \mathrm{~m}$ ) has two peaks and usually, the second peak is more intense than the first in contrast to the soft $X$-rays whose first peak is the more intense one. The first HXR peak begins $40 \mathrm{~ns}$ before the peak of the p-i-n diode XUV signal, has FWHM of $45 \mathrm{~ns}$ and reaches a peak shortly after the peak of the XUV signal (see Section 4.3.1.2 D). The second peak occurs $170 \mathrm{~ns}$ after the first peak and has an FWHM of $70 \mathrm{~ns}$.

d. For the first neutron pulse, the FWHM is $50-70 \mathrm{~ns}$. In its initial portion, the downstream neutron energy is $2.6 \mathrm{MeV}$ to $3.0 \mathrm{MeV}$. Towards its end, it becomes $2.2 \mathrm{MeV}$ to $2.4 \mathrm{MeV}$. This conclusion was a result of a Monte Carlo simulation. e. $\quad$ For the second neutron pulse, the FWHM is 70-100 ns. In its initial part, the downstream neutron energy is 3.0 to $3.2 \mathrm{MeV}$. Towards its end, it becomes 2.2 to $2.4 \mathrm{MeV}$.

f. The second pulse has 3 to 10 times the number of neutrons as the first pulse.

(3) Neutron time of flight spectroscopy was used [86] to determine the energy distribution of neutron-producing deuterons in the operating parameter regime $27 \mathrm{kV}$ charging voltage (500 kJ stored energy), $470 \mathrm{~Pa}$ deuterium pressure, $2 \mathrm{MA}$ maximum current. Fast scintillator-photomultiplier probes with $5 \mathrm{~cm}$ thick BC408 scintillators were placed at 7.0, 16.3, 58.3, and $84 \mathrm{~m}$ downstream, 7.0, 16.3, 30.3, 44.2, 58.3, and $84 \mathrm{~m}$ upstream and $7 \mathrm{~m}$ in the radial direction.

a. Energy distribution of neutrons detected downstream ranged from 2.2 MeV to 3.2 MeV with a peak at $2.75 \mathrm{MeV}$, which is $0.3 \mathrm{MeV}$ above the $2.45 \mathrm{MeV}$ reaction energy of $d-d$ neutrons in the center of mass frame of reference. This indicates that the dominant population of neutron-producing deuterons is moving downstream. The downstream neutrons of energy less than $2.45 \mathrm{MeV}$ are produced by deuterons moving upstream. The scattered neutrons were excluded using the intersection of signals from detectors placed in the same distance in opposite directions. Energy distribution for deuterons moving axially was calculated for both upstream and downstream directions (see Figure 2 of [86] reproduced as Figure 9 in Section 2.3.1).

b. Signals from detectors placed at $7 \mathrm{~m}$ in downstream, upstream, and radial directions have practically the same FWHM. This suggests that the energy distribution of reacting deuterons is approximately isotropic.

(4) Correlations between neutron and HXR emission and interferometry images [397] show that the first neutron pulse begins before the current derivative minimum when the front portion of dense plasma sheath has just reached the axis on the surface of the anode (see Figure 90a). The beginning of a closed fringe system at this time indicated the start of formation of the first plasmoid in coincidence with the start 
of first neutron pulse $30 \mathrm{~ns}$ before the current derivative minimum. This plasmoid continued to evolve during the 200 ns duration of the neutron pulse.

a. The first neutron pulse had similar profiles in the downstream, upstream, and side-on detectors indicating that energy anisotropy is very small.

b. The side-on neutron spectrum had dominant energy of $2.8 \mathrm{MeV}$ (determined from the time difference between the first HXR and neutron pulse peaks) indicating a deuteron side-on motion with kinetic energy $\sim 70 \mathrm{keV}$ (velocity $\sim 2.6 \times 10^{6} \mathrm{~m} / \mathrm{sec}$ ). The transit time of a deuteron with this velocity across $1 \mathrm{~cm}$ is $3.86 \mathrm{~ns}$. The duration of the neutron pulse was $200 \mathrm{~ns}$. This indicates that the deuterons that produced neutrons were radially confined. Continued radial outflow of deuterons would have depleted the plasma fast enough to be noticed in interferograms.

c. The second neutron pulse was correlated in time with the disruption of the plasma column. Figure 91 shows a sequence of interferograms overlapping with the second neutron pulse.
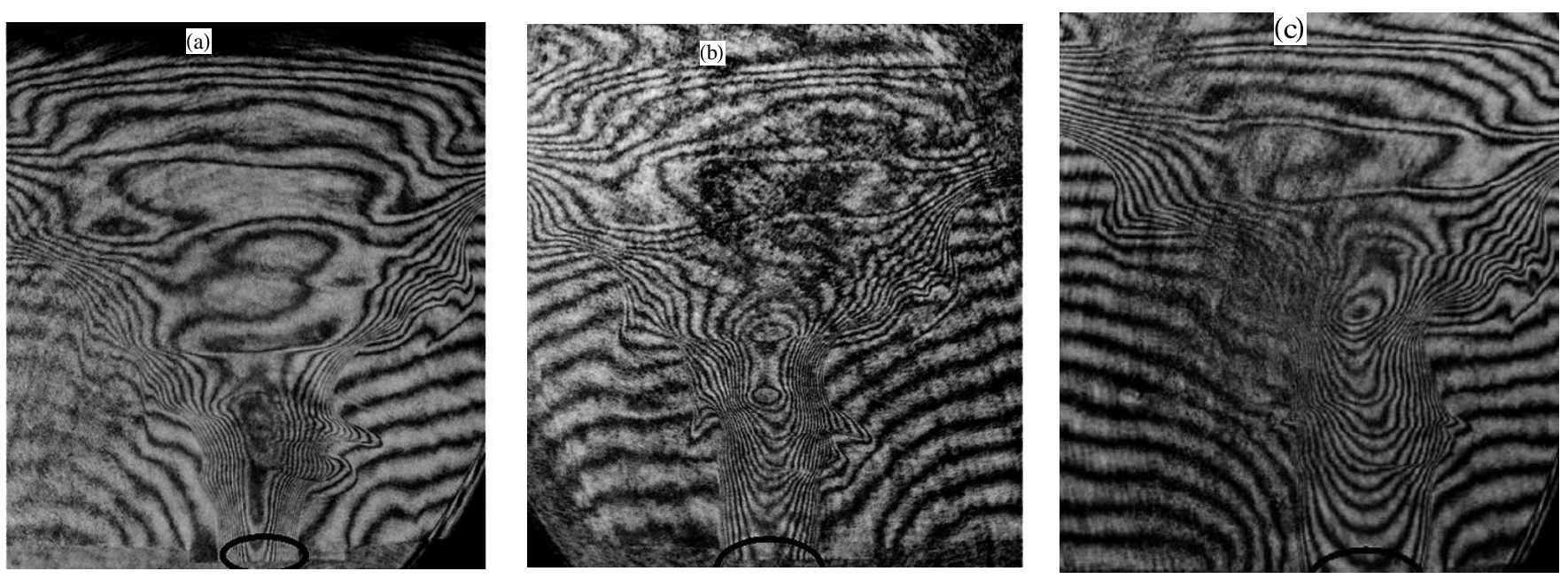

Figure 90. (a): Adapted from Figure 1c of [397] (Phys. Plasmas 2017, 24, 072706) with permission from AIP Publishing. This image corresponds to a time $29 \mathrm{~ns}$ before current derivative minimum. The low density front portion of the dense plasma sheath has just collapsed on itself at the anode surface. This instant also marks the beginning of the first neutron pulse. Closed fringes indicate the beginning of the first plasmoid. (b) Adapted from Figure 1d of [397]. This corresponds to a time $1 \mathrm{~ns}$ after the current derivative minimum. The first neutron pulse has its maximum at this time. (c) Adapted from Figure 1e of [397]. This corresponds to a time $21 \mathrm{~ns}$ after current derivative minimum. The first neutron pulse is still at its first peak.
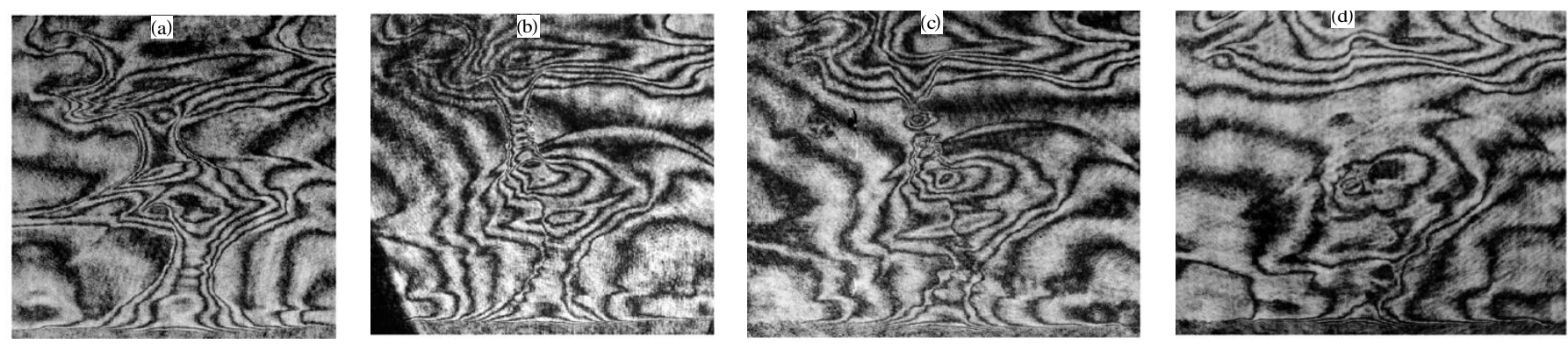

Figure 91. Adapted from Figure 2c-f of [397] (Phys. Plasmas 2017, 24, 072706) with permission from AIP Publishing), corresponding to times 271,301, 311 and $331 \mathrm{~ns}$ after the current derivative minimum. Note the decrease in plasma radius eventually leading to apparent breakup of the plasma column, with density below the detection limit.

(5) The early part of first phase of neutron emission was shown to coincide [103] with axial movement of the plasmoid formed near the bottom of the plasma column (see Figure 92). 


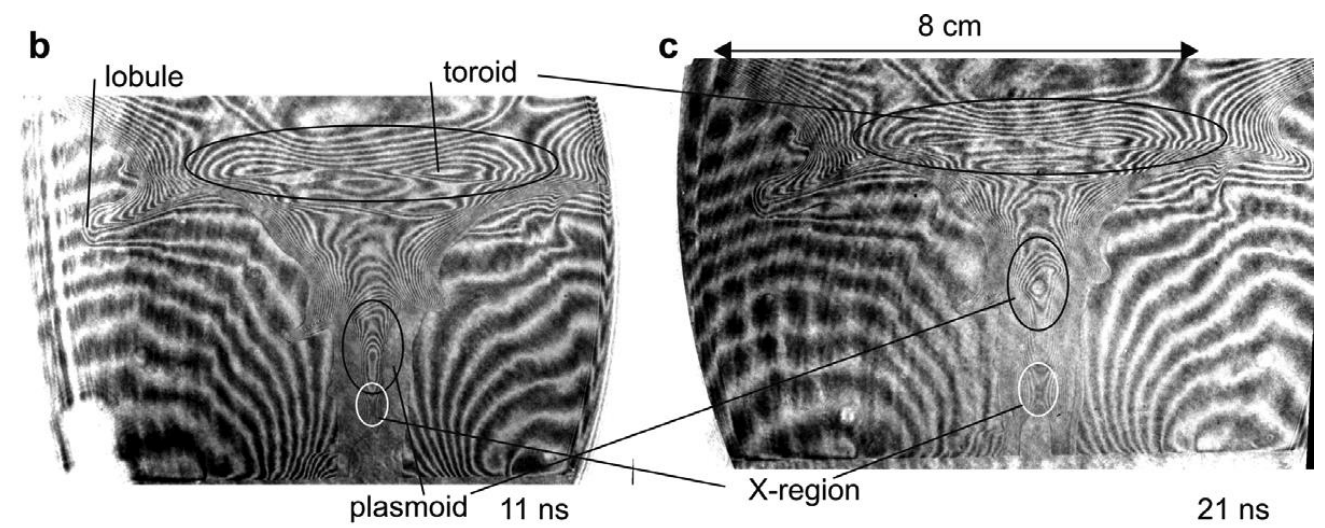

Figure 92. Adapted from Figure 2 of [103]. A system of nested closed fringes marked by black ellipses, indicating a plasmoid structure, is separated from another system of open axially oriented fringes by $X$-regions, marked by white ellipses. The figure illustrates axial movement of the plasma axially displacing the center of the plasmoid, as well as the X-region. Growing size of the plasmoid, elongation of the plasma column and plasma flow into the toroid can be discerned. (C) 2021 IEEE. Reprinted, with permission, from IEEE Trans. Plasma Sci. 2019, 47, 339-345.

A toroidal structure seen at the top separating the plasma column from the umbrellalike structure was formed at an early stage of stagnation and persisted until the breakup of the plasma column. A lobule surrounding the toroid transported mass from the dense plasma into the surrounding low density plasma. Later in the neutron pulse, the plasma column seems to undergo both $\mathrm{m}=0$ and $\mathrm{m}=1$ instabilities (see Figure 93).

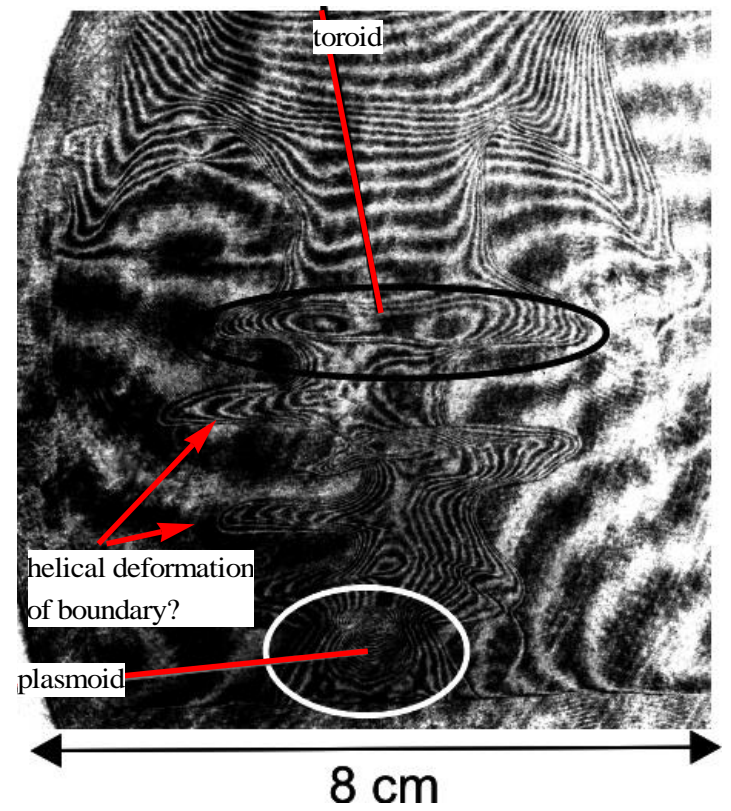

Figure 93. Adapted from Figure 3b of [103]. The boundary of the dense column looks like the projection of a helix. Plasmoid and toroid structures are seen at different elevations. (C) 2021 IEEE. Reprinted, with permission, from IEEE Trans. Plasma Sci. 2019, 47, 339-345.

B. Fusion protons

(1) Fusion protons from PF-1000 were imaged [401] using ion pinhole cameras using calibrated CR-39 and PM 351 solid state nuclear track detectors (SSNTD), along with aluminium foil filters which discriminated against fast deuterons and provided protection against plasma damage. 
a. Preliminary experiments were conducted on PF-1000 configuration with $230 \mathrm{~mm}$ diameter anode, $28 \mathrm{kV}$ charging voltage, and $4 \mathrm{hPa}$ deuterium pressure with a single $0.5 \mathrm{~mm}$ diameter pinhole camera using a CR-39 detector covered with 80 $\mu \mathrm{m} \mathrm{Al}$ foil placed at $68^{\circ}$ to the axis with 3 to 4 shots superimposed for getting sufficient number of tracks.

b. More detailed experiments were performed with anode of $244 \mathrm{~mm}$ diameter and $447 \mathrm{~mm}$ length, insulator of $244 \mathrm{~mm}$ diameter, and $114 \mathrm{~mm}$ length and 20 to $40 \mathrm{kV}$ charging voltage. Three pinhole cameras placed at $0^{\circ}, 68^{\circ}$, and $90^{\circ}$ to the axis were deployed at distances of 500,610, and $1000 \mathrm{~mm}$ from the center of the inner electrode. PM 355 detectors covered with $80 \mu \mathrm{m}$ Al foil were used and exposed to 5 shots. The developed foils at $90^{\circ}$ show 5 to $9 \mathrm{~mm}$ diameter spots (or clusters of tracks). The images were also shifted from the geometric center of the detector. Histograms of number of proton tracks versus track diameter along with previous calibration revealed 3 energy groups of fusion protons at $3.01 \mathrm{MeV}, 3.30 \mathrm{MeV}$ and $3.83 \mathrm{MeV}$ with different intensity at the three detectors. Highest track density was observed at $0^{\circ}$.

(2) Comparison of a time-integrated fusion proton image with time resolved images from interferometry and a 4-frame filtered gated MCP camera [402] show a spherical structure in all three images at the same axial location. The fusion proton image appears to be larger in size than the X-ray image.

(3) Fusion protons were recorded with 7 ion pinhole cameras having $3 \mathrm{~mm}$ diameter pinholes with SSNTDs covered with $80 \mu \mathrm{m}$ Al foil. The PF operating parameters were $31 \mathrm{kV}$ charging voltage, $5.32 \mathrm{hPa} \mathrm{D}_{2}$ producing $6.7 \times 10^{11}$ neutrons/shot. In order to improve statistics, the SSNTDs were exposed to 5 consecutive shots.

a. Fusion protons were found to be emitted anisotropically.

b. They had energy ranging from $3.2 \mathrm{MeV}$ to $4.5 \mathrm{MeV}$, with a peak at $3.5 \mathrm{MeV}$.

C. Soft X-rays (SXR)

(1) Temporal evolution of soft X-rays with photon energy below $1 \mathrm{keV}$ was studied [398] using a thin NE102 scintillator-photomultiplier detector covered with $10 \mu \mathrm{m}$ Al foil. Soft X-ray emission had 3 peaks of decreasing amplitude (see Figure 94 below). First emission started before maximum compression and reached a peak before the current derivative minimum. Comparison with other signals is discussed in Section 4.3.1.1 B(3), Section 4.3.1.2 A(2), and Section 4.3.1.2 D. Note the absence of a strong SXR pulse corresponding to the second HXR and neutron pulses in Figure 94. 


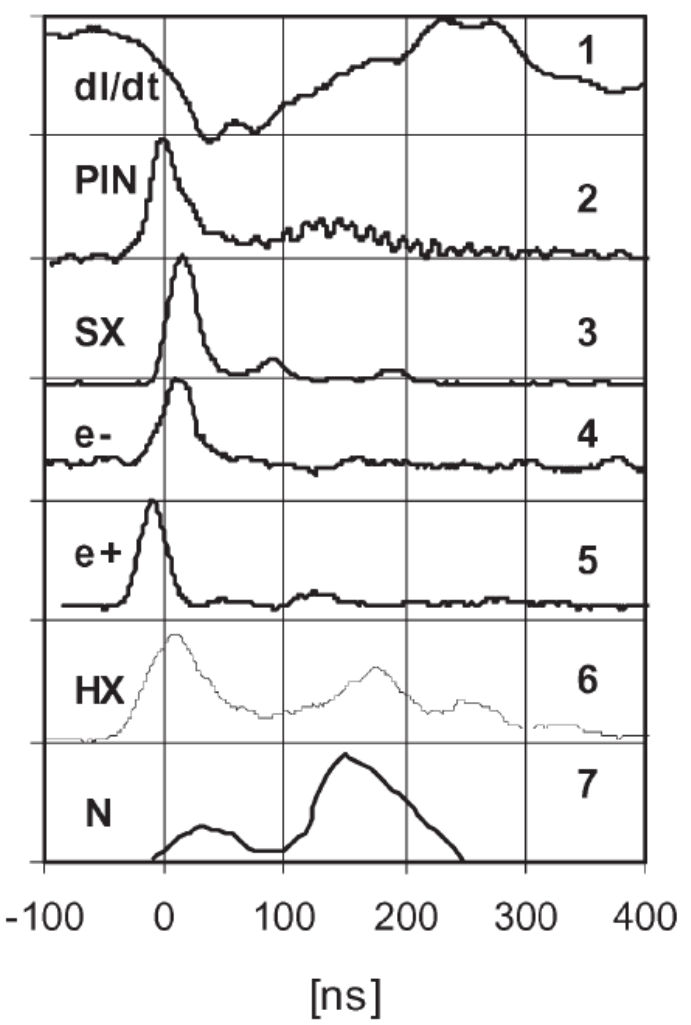

Figure 94. Shot 4618. Records of current derivative (trace 1), p-i-n-detector signal (trace 2), soft X-rays above $1 \mathrm{keV}$ (trace 3), electrons of energy above $70 \mathrm{keV}$, as measured upstream (trace 4), electrons above $40 \mathrm{keV}$, as measured downstream (trace 5), hard X-rays above hundreds of kilo-electron-volts (trace 6), and estimation of neutrons shifted by the time-of-flight method to the source, which is assumed to be in the center of the pinch $6 \mathrm{~cm}$ in front of the anode outlet (trace 7). Temporal uncertainty in each shot was 5 ns [398] (C) 2021 IEEE. Reprinted, with permission, from Figure 3, IEEE Trans. Plasma Sci. 2006, 34, 2349-2351.

(2) An MCP camera divided into 4 quadrants shielded with $5 \mu \mathrm{m} \mathrm{C}_{8} \mathrm{H}_{8}, 3 \mu \mathrm{m} \mathrm{Al}$, and $10 \mu \mathrm{m}$ Be foils was used [402] to compare regions emitting above and below $1500 \mathrm{eV}$. Two silicon p-i-n diodes with $3 \mu \mathrm{m} \mathrm{Al}$ and $10 \mu \mathrm{m}$ Be filters also looked at temporal revolution. A dense spherical region located $7 \mathrm{~cm}$ along the axis in the interferogram was also shown to have an electron temperature of roughly $750 \mathrm{eV}$. (There is a possibility that this might be incorrectly evaluated: $750 \mathrm{eV}$ may be the average photon energy and temperature may be $\sim 4$ times less in line with other measurements).

(3) Time integrated soft X-ray pinhole camera images [127] with the PF-1000 facility were compared for two modes of operation: normal mode and with gas puffing. The helical deviation of the boundary was discernible.

D. Electrons

Three Cerenkov detectors (made of diamond or rutile crystals and shielded with 20- $\mu$ m-thick Al filters) were deployed [398] to detect fast electrons of energies in the range of $50 \mathrm{keV}$ to $400 \mathrm{keV}$ downstream, upstream (behind a 5-cm-diameter central hole in the anode), and side on. Temporal correlation between these signals was accurate to 3 ns.

The upstream electron emission was 3 times more intense than the downstream electron emission. The downstream electron pulse started (see Figure 94), along with the p-i-n diode XUV signal (see Section 4.3.1.1 B) and had a peak before the XUV pulse. The upstream electron peak had an FWHM of 10-30 ns, occurred after a delay of 0 to $30 \mathrm{~ns}$ with respect to the downstream electron peak and was coincident with the first SXR peak (Section 4.3.1.2 C). Note the near absence of electron signals corresponding to the second HXR and neutron pulses. 


\section{E. Ions}

Fast deuteron emission recorded by ion pinhole cameras is reviewed in detail in Section 2.3.1. Two recent papers on PF-1000 [101,105] are summarized in this section, because they report certain conclusions derived with inputs from interferometric diagnostics. Both reports have used ion pinhole cameras with PM 355 SSNTDs filtered with Al foils of different thicknesses.

(1) In the first of these [101], one pinhole camera with $500 \mu \mathrm{m}$ pinhole was at polar angle $-60^{\circ}$ with the axis placed at $77 \mathrm{~cm}$ from anode center. It had a magnification of 0.091 with a blur of $3 \mathrm{~mm}$. A similar camera was placed at $0^{\circ}$ at a distance of $160 \mathrm{~cm}$ from the anode. That had a magnification of 0.044 with a blur of $5 \mathrm{~mm}$. Both had $3 \mu \mathrm{m}$ $\mathrm{Al}$ filters so they detected deuterons above $360 \mathrm{keV}$. For comparison, the dominant energy for deuterons deduced from neutron time of flight was $130-150 \mathrm{keV}$ during the first neutron pulse and $300 \mathrm{keV}$ in the second pulse. Estimates of deuteron trajectories using a model for magnetic field based on interferometer images indicated that the deuteron trajectories would be deflected by not more than 1-2 $\mathrm{mm}$.

a. In one of the reported shots, the $0^{\circ}$ camera recorded an ion image composed of two patterns: first a nearly circular image of $2 \mathrm{~cm}$ diameter with a lower intensity central spot of $8 \mathrm{~mm}$ diameter and second a circular arc of 3-5 cm radius. The $60^{\circ}$ detector had no signal. In another shot, the $0^{\circ}$ camera recorded "a small central spot of $8 \mathrm{~mm}$ in diameter and two ring-shaped regions of diameters equal to about $6-8$ and $11-13 \mathrm{~cm}$, respectively". The larger ring diameter was larger than the field of view of the interferometer and could not be ascribed to any particular plasma region. The smaller ring could be associated with a $3 \mathrm{~cm}$ diameter lobule seen in the interferometer images. The smaller central spot could be ascribed to a plasmoid formed on the axis.

b. Six cameras with $200 \mu \mathrm{m}$ pinholes were placed on an arc of $32 \mathrm{~cm}$ radius centered on the anode center at polar angles $-42^{\circ},-23^{\circ},-4^{\circ},+4^{\circ},+23^{\circ}$, and $+42^{\circ}$ in addition to the ones at $0^{\circ}$ and $-60^{\circ}$. The SSNTDs were placed $4 \mathrm{~cm}$ behind the pinholes, i.e., on an arc of $36 \mathrm{~cm}$ radius looking at the anode center. Three cameras were unfiltered, recording deuterons above $30 \mathrm{keV}$, and others had $3 \mu \mathrm{m} \mathrm{Al} \mathrm{filters.} \mathrm{The} \mathrm{results} \mathrm{showed} \mathrm{that} \mathrm{there} \mathrm{were} \mathrm{two} \mathrm{sources} \mathrm{of} \mathrm{fast}$ deuterons. The one within the central pinch region was recorded in all detectors. The other had a radius of about $3 \mathrm{~cm}$ and produced images in the shape of circular arcs only in the detectors near the axis. Their probable location was in the vicinity of the extreme edges of the lobule structures.

(2) The second paper [105] interpreted earlier ion pinhole camera images obtained in [79] in terms of deflection of ions by magnetic field created by local closed-loop currents inferred from interferometric pictures, and possibly filaments which were not directly observed in this experiment. Additional data were obtained with a modified geometry having a conical tip on the anode and another $6 \mathrm{~cm}$ away on the axis connected to an "anti-anode" connected to the cathode rods with 12 metallic radial ribbons. The ion pinhole images detected at $0^{\circ}$ beyond the anti-anode structure exhibited a number of azimuthally distributed spots.

\subsubsection{Interpretation of Results}

This section reviews the interpretation of the experimental results discussed above. Interpretation aims to find logical causal linkages between observations from many experiments and the existing accumulated knowledge of the subject. The motivation for discussing interpretation separately from experimental results is twofold: Firstly, all the results can be considered together facilitating attempts at a unified interpretation of all data, rather than attempting partial explanations of each experiment separately. Secondly, assumptions, postulates, analogies, models, scenarios, and other similar considerations that are not directly supported by experimental data but are an inevitable part of interpretation 
of experimental results can be stated explicitly, creating opportunities for further experimentation to confirm them. This review is organized by the central theme of interpretation. Some aspects are unique to the experiments done at PF-1000 based on correlation between interferometry and other diagnostics. They are discussed separately in "Section 4.3.1.3 A. Internal currents and magnetic fields" and "Section 4.3.1.3 B Evolution of structure". The topic of "Section 4.3.1.3 C Generation of fusion neutrons, fast electrons, XUV, and X-rays" involves interpretation of energy dissipation mechanisms. The knowledge base relevant to this interpretation is still evolving on many fronts. It would be prudent to keep open the possibility of multiple interpretations. For this purpose, Section 4.3.1.3 C is structured in a question-answer format, where the questions are formulated from the PF-1000 data presented above and the possibility of multiple answers is kept open.

A. Internal currents and magnetic fields

Results of magnetic probe measurements, interferometry, and XUV imaging on PF-1000 have been considered together to construct the following interpretation concerning existence of internal currents and magnetic fields.

- Magnetic probe data and correlated interferometry pictures (Section 4.3.1.1 A) demonstrate that axial magnetic field $\mathrm{B}_{\mathrm{z}}$ and associated azimuthal currents exist in close vicinity of toroidal structures formed ahead of the imploding dense plasma sheath. In a few frames after stagnation and before breakup, the movement of fringes is negligible suggesting that a quasi-stationary state exists. Estimates of rotational drift velocity lead to associated kinetic energy density an order of magnitude less than the estimated pressure. This allows local dynamics to be approximated by the relation $\vec{\nabla} \mathrm{p} \approx \overrightarrow{\mathrm{J}} \times \overrightarrow{\mathrm{B}}$. The average temperature of the plasma has been estimated to lie in the rather broad interval 50-150 eV from intensities of spectral lines of $\mathrm{H}$ - and He-like ions of ambient impurities $(\mathrm{O}, \mathrm{N}$, and $\mathrm{C})$ using VUV emission spectroscopy. This is assumed to be spatially uniform. This allows density gradients to be associated with pressure gradients and, hence, with magnetic force density within the limitations imposed by the assumptions and approximations.

- Regions of low density surrounded by high density have some boundaries with positive radial pressure gradient and hence positive radial (directed away from the axis) magnetic force density $(\vec{J} \times \vec{B})_{r}=J_{\theta} B_{Z}-J_{z} B_{\theta}$. These regions are, therefore, identified as locations of internal closed currents with both toroidal and poloidal components. Positive force density can result either from $\mathrm{J}_{\theta} \mathrm{B}_{\mathrm{Z}}$ from toroidal currents $\mathrm{J}_{\theta}$ dominating the negative force density $-\mathrm{J}_{z} \mathrm{~B}_{\theta}$ from poloidal currents $\mathrm{J}_{z}$ moving downstream or from poloidal currents directed towards the anode in a returning current loop.

- Using simultaneous XUV and interferometry images (Section 4.3.1.1 B(2)), such regions are found on the external surface of the dense plasma column where the radial lobule structures bend towards the anode forming an inverted-U profile, with the arms of the $\mathrm{U}$ at higher density than the space enclosed by them (see Figures 89 and 95). Similarly, negative pressure gradients on the outside boundary of these structures correspond to the negative force density (directed towards the axis) associated with external currents. This implies that the discharge current and the dense plasma column are separated by a "plasma cushion" that contains both toroidal and poloidal currents and associated magnetic fields. 


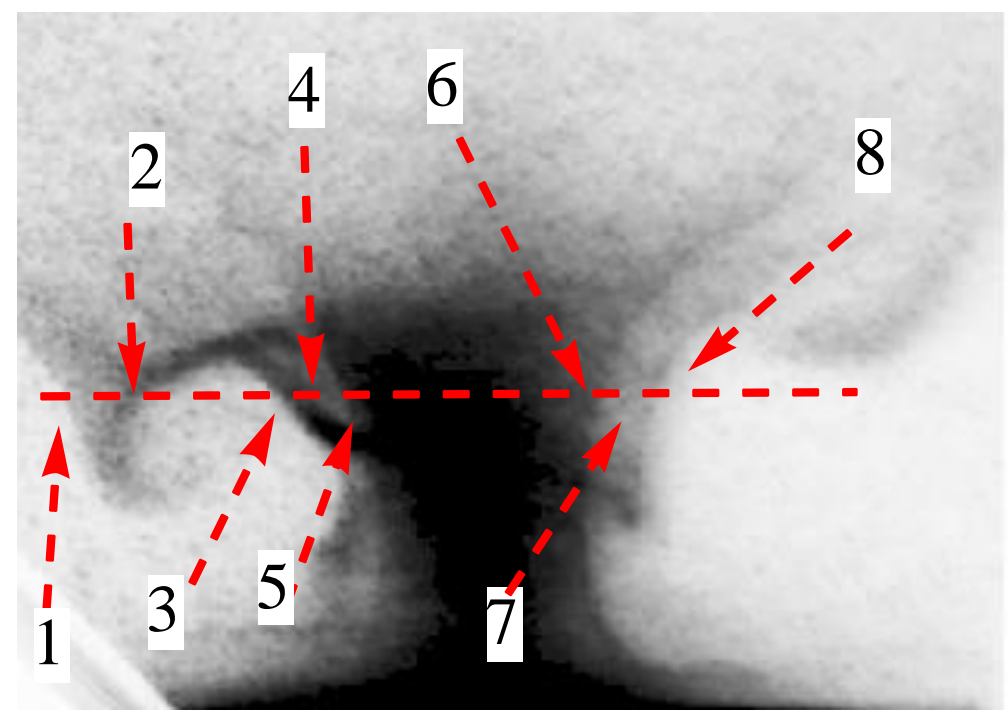

Figure 95. XUV image adapted from original data presented in Figure 2b from [107] (Phys. Plasmas 2020, 27, 092702), reproduced with permission from AIP Publishing. The time is $-10 \mathrm{~ns}$. As one traverses the image from left to right along the red dashed line, the sign of the pressure gradient changes as (1) - ve, (2) +ve, (3) - ve, (4) + ve (5) - ve (6) - ve (7) +ve (8) - ve. The interpretation described above would have a compressive force density at points (1) and (8) arising from the main discharge current. The region between (2) and (3), (4) and (5), and (6) and (7) are interpreted as locations of internal current loops that exert a positive force density on their external boundary and negative force density on the internal boundary.

- During the radial implosion phase, axial magnetic field has been detected by magnetic probes on the downstream side of the plasma shell (Section 4.3.1.1 A(4)c). A toroidal structure has also been detected by interferometry in the low density plasma region on the downstream side of the dense plasma sheath (Section 4.3.1.1 A(4)c). Since the shock is yet to reach this structure, the kinetic energy can be neglected allowing interpretation of positive pressure gradient with positive magnetic force density that is associated with toroidal currents $\mathrm{J}_{\theta}$. This region is, therefore, identified as the location of a toroidal current distribution (see Figure 84).

- Specific plasmoidal structures, with a single density peak, observed within the dense plasma column, have been associated with numerical estimates of toroidal and poloidal magnetic field distributions using density profiles obtained by Abel inversion assuming cylindrical symmetry [397]. They are interpreted as being similar to spheromaks in magnetic topology.

- Helical perturbations of the dense plasma column boundary are seen in soft X-ray, XUV and interferometry images $[107,127,392]$. They are potentially helical current flow paths that have both axial and azimuthal current components. They can generate the axial flux occupying the entire dense plasma column. This axial flux is an essential component of magnetic helicity and is necessary for operation of dynamo mechanisms that alter topology of the magneto-plasma.

- The hypothesis of formation of the plasma cushion spatially separating the main discharge current from the dense plasma column explains the observed delay between the voltage peak and the current derivative minimum measured at the base of the plasma focus seen in Figure 80 that results in the double peaked voltage across the plasma column seen in Figure 82. The current derivative minimum occurs when the current reaches the axis, causing a sharp increase in inductance. Figure 89 shows a short circuit occurring across the dense column before this time. This prevents the voltage spike associated with the sharply increasing inductance from being detected by the voltage probe. The occurrence of this short circuit is reflected in the valley between the two voltage peaks in Figure 82. 


\section{B. Evolution of structure}

The evolution of three structural forms-toroidal, plasmoidal, and lobular-is interpreted in terms of magnetic force density in the following manner:

- The toroidal current density in the toroidal structures formed in front of the imploding dense plasma sheath (Section 4.3.1.1 A(4)c) is amplified by a dynamo mechanism involving radial transport of the axial magnetic field by the imploding sheath, resulting in toroidal discs at one or more axial locations.

- The toroidal discs are harder to compress than the adjacent plasma column (see Figure 84) because of their internal magnetic stresses. The pinching force compresses this adjacent plasma region to a lower radius than the toroidal discs, forming a shape with one or more lobular structures of larger radius distributed axially along a plasma column of lesser radius.

- The magnetic pressure is much larger at the lower radius of the plasma column than at the radius of the toroidal discs. This leads to continuous decrease in the radius of the plasma column creating constrictions. The plasma contained in the constricted zone is pushed axially into the higher radius toroidal discs, eventually leading to plasma erosion in the constricted parts of the plasma column and transformation of the toroidal discs into plasmoids.

- This cross-field axial plasma transport drives a magnetic dynamo that creates and amplifies both toroidal and poloidal current distributions by converting plasma kinetic energy into magnetic energy, that results in a spheromak-like plasmoidal magnetoplasma structure.

- The toroidal discs increase in diameter, creating a lobular structure that bends towards the anode enclosing a part of the azimuthal flux creating a magnetic bubble. The poloidal current inside this bubble is directed towards the anode near its exterior boundary and away from the anode near the interior boundary (see Figure 89 above and Figure 2 of [106]).

- Erosion of the constricted plasma between the lobules (or between a single lobule and the anode) results from the axial transport of plasma into the toroidal discs that get transformed into plasmoids (see Figures 88 and 91).

- The resulting phenomenology differs from the traditional description of $\mathrm{m}=0 \mathrm{MHD}$ instability, which does not take into account any poloidal flux and, thus, does not include dynamo mechanisms powered by 3D cross-field plasma flows. Note that no interpretation has been associated with the origin of the axial magnetic field detected in the radial collapse phase. This is just taken as an experimental observation. Subsequent interpretation is based on the validity of this experimental observation. Possible mechanisms for the origin of this axial magnetic field are discussed in a subsequent section (Section 8.2.1).

C. Generation of fusion neutrons, fast electrons, XUV, and X-rays

Rapid transfer of energy from the plasma to fast ions and electrons in time correlation with evolution of structure (Section 4.3.1.3 B) and internal currents (Section 4.3.1.3 A) has been postulated in terms of magnetic reconnection, possibly associated with annihilation of current filaments $[103,105,106,403]$. Under these considerations, fast displacement of magnetic flux leads to induction of electric field perpendicular to the magnetic field, which is expected to accelerate ions [103,105,106,403].

Magnetic reconnection is a general term applied to the topological rearrangement of the magnetic field lines in a plasma. Recent reviews of this field [168,404,405] clearly bring out the point that this is still 'work in progress'. The traditional view $[168,404,405]$ that magnetic reconnection necessarily involves two-dimensional current sheets with Xtype neutral points has been shown to be erroneous in a recent paper by Boozer [406]. He demonstrates existence of "an effective parallel electric field $E_{\|}$which can accelerate particles despite the particle acceleration due to the true parallel electric field $\mathrm{E}_{\|}$being negligible". His analysis distinguishes between the circular motion of the charged particles 
perpendicular to the magnetic field about its guiding center and motion of the guiding center along the magnetic field. Electric field perpendicular to the magnetic field transfers energy to the motion of the charged particle around its guiding center, resulting in increase in the instantaneous local gyroradius. When the associated gyroradius becomes comparable to the scale length of the magnetic field, the perpendicular electric field starts getting decoupled, limiting the transfer of energy. In contrast, the effective parallel electric field accelerates the guiding center of the charged particles along the magnetic field. Unlike the case of the perpendicular electric field, the parallel electric field remains coupled with the guiding center, increasing its effectiveness in accelerating the charged particle. Following Boozer [406], if one considers the trajectories of the guiding centers rather than ions, then it is clear that the trajectories of the accelerated guiding centers share the topology of the magnetic flux surfaces. Boozer's expression for the effective parallel electric field (Equation (19) of [406]) includes a term related to the rate of change of magnetic field suggesting that the direction of the electric field could be different for the rising and falling parts of the magnetic field. This term is also proportional to the mass-to-charge ratio of the charged particle indicating that it could be different for ions and electrons.

The interpretation of internal currents and magnetic fields (Section 4.3.1.3 A) can then be used to relate the direction of the effective accelerating electric field to the direction of the local magnetic field (both being parallel). This can in turn be used to interpret observations of fast electrons, ions, and neutrons at various stages of plasma evolution. Such interpretation needs to take into account certain reservations regarding the use of current derivative minimum as a time marker (see Section 4.3.1.1 A).

Since the experimental knowledgebase is still evolving (both at PF-1000 and other laboratories), multiple interpretations are possible (and also desirable). In order to streamline this process, the following section paraphrases the experimental knowledge base for PF-1000 discussed above into a set of specific questions that must be comprehensively answered by any candidate interpretation. For each question $(\mathrm{Q})$, one likely answer (LA) is provided with the understanding that other such likely answers may also exist and searching for them may provide fruitful directions for further research.

I. Energy dissipation mechanism before maximum compression/current derivative minimum:

Q: The results of [398] shown in Figure 94 and discussed in Section 4.3.1.1 B(3) indicate that XUV, SXR, and fast electrons above $40 \mathrm{keV}$ in the downstream direction are all emitted much before the current derivative minimum, in a time zone where the plasma is still seen to be imploding in visible framing pictures (Figure 6 of [398]). XUV images in this time zone (Figure 7a,b of [398]) show intense emission over the entire length of the yet-to-be formed plasma column. Since the shock has not reached the axis by this time and the rapid rise of inductance associated with current diffusion to the axis has not yet taken place (see Figure 86), the energy dissipation cannot be because of shock reflection, adiabatic compression, phenomena associated with a pinch instability, or anomalous resistivity. What then is the mechanism of rapid energy transfer to the plasma at this time?

LA-1: Boozer's effective parallel electric field [406], expected to be much larger than the real electric field, could provide a likely explanation as follows: Radial flow of the imploding plasma powers a dynamo mechanism that continuously amplifies the poloidal and toroidal magnetic field components present ahead of the imploding dense sheath seen as precursor pulses in probe signals (Figures 76 and 79). An effective parallel electric field is generated with both poloidal and toroidal components. The poloidal component of this electric field would be directed away from the anode close to the axis (at small radii) and towards the anode away from the axis (at large radii) in the growing magnetic field. Since in the implosion phase the plasma density is concentrated in the imploding shell, the plasma ions and electrons experience the portion of the poloidal electric field that is pointed towards the anode. This electric field generates an electron beam downstream and an ion beam upstream [92] which partially dissipate their energy in the plasma to produce the intense radiating region in the XUV frames. Associated toroidal electric field also may generate circulating ion and electron beams which can also dissipate energy in 
the imploding plasma shell. The existence of an electric field directed towards the anode within the imploding plasma is corroborated by other evidence. (A) Emission of fast ions upstream [92]. (B) Start of neutron emission well before current derivative minimum (Section 4.3.1.2 B(3)). (C) The observation of neutrons with energy less than $2.45 \mathrm{MeV}$ moving downstream indicating a significant number of accelerated deuterons moving towards the anode. (D) Observation of maximum neutron energy of $2.95 \pm 0.15 \mathrm{MeV}$ in the upstream direction (Section 4.3.1.2 A(1)).

The detection of $40 \mathrm{keV}$ electrons in the downstream direction while the plasma is still imploding is a key observation that must be explained by any alternative interpretation.

Note that this answer is incomplete. To complete it, Boozer's effective parallel electric field needs to be evaluated and shown to account for the time, magnitude, and direction of the proposed electric field. This requires a model for the precursor axial and azimuthal magnetic fields in the implosion phase as a prerequisite.

II. Neutron emission mechanism associated with the first pulse:

Q: When the first HXR and neutron pulses are generated, the axially oriented open fringes transform into a system of nested closed fringes indicating the formation of a plasmoid (Section 4.3.1.1 A(3)a). In the initial portion of the first neutron pulse, the interferogram shows (see Figure $90 \mathrm{a}$ ) the dense column to consist of axially oriented open fringes at the bottom and a toroidal structure above it. The center of the system of closed fringes moves along the axis and the number of nested closed fringes increases during the first neutron pulse indicating increase in the number of particles in the plasmoid (see Figure 90). In the final portion of the first neutron pulse, the bottom stagnated portion of the column is again without closed fringes, with increase in column radius and decrease in its density (see Figure 85, Figure 86, and Figure 96 from the same shot). The initial upper toroid is transformed into a plasmoid due to injection of plasma from the column (see Figure 96b).
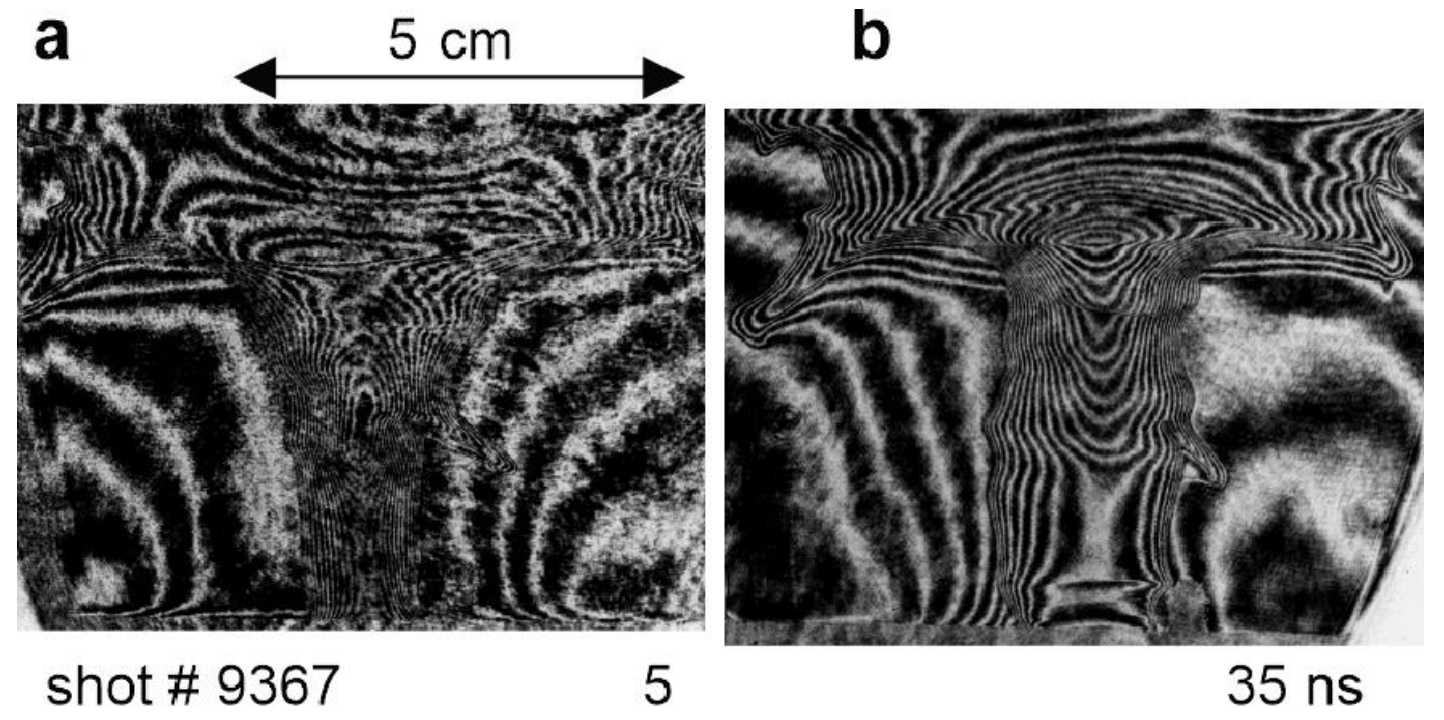

Figure 96. (Unpublished data of Shot \#9367). This is the same shot as Figures 84-86. Image (a) shows formation of the plasmoid and (b) shows the formation of the stagnation plasma configuration between the dense structures near the end of the first neutron pulse.

The first pulse of neutron emission is found to have the following properties:

- It begins before plasma stagnation and ends when the plasma column re-expands before becoming unstable.

- The neutron time-of-flight spectra observed along downstream, upstream, and side-on directions indicate energy distribution for the first neutron pulse is more isotropic than the second pulse (Section 4.3.1.2 A(1)a), Section 4.3.1.2 A(3)b, Section 4.3.1.2 A(4)a). 
Deuterons that produce neutrons have a side-on kinetic energy $70 \mathrm{keV}$ (Section 4.3.1.2 A(4)a). Their transit time across $1 \mathrm{~cm}$ is $\sim 3.86 \mathrm{~ns}$ while the duration of the first neutron pulse is $200 \mathrm{~ns}$. A continuous radial outflow of $70 \mathrm{keV}$ ions for $200 \mathrm{~ns}$ is ruled out as that would have been detected in a rapid depletion of particle content in interferometric pictures. This indicates that the deuterons that produced neutrons were radially confined.

- $\quad$ For the first neutron pulse, the FWHM is 50-70 ns. In its initial portion, the downstream neutron energy is $2.6 \mathrm{MeV}$ to $3.0 \mathrm{MeV}$ and towards its end, it becomes 2.2 to $2.4 \mathrm{MeV}$ according to a Monte Carlo simulation of the experimental results (Section 4.3.1.2 A(2)d).

- The neutron energy corresponding to the first peak is $2.7 \mathrm{MeV}$ in the downstream direction and 2.3 MeV in the upstream direction (Section 4.3.1.2 A(2)a).

- According to Section 4.3.1.2 A(3(a): “Energy distribution of neutrons detected downstream ranged from $2.2 \mathrm{MeV}$ to $3.2 \mathrm{MeV}$ with a peak at $2.75 \mathrm{MeV}$, which is $0.3 \mathrm{MeV}$ above the $2.45 \mathrm{MeV}$ reaction energy of d-d neutrons in the center of mass frame of reference"

What mechanism of ion acceleration can explain these properties of the first pulse of neutron emission and their relation with the observed plasma dynamics consistently?

LA1: The simultaneous presence of both poloidal and toroidal components of magnetic field during the period before and after stagnation indicates that cross-field plasma flows always occur and amplify both of them by a dynamo effect. This converts kinetic energy of plasma flow into energy of magnetic and electric fields. The resulting magnetoplasma consists of nested flux surfaces topologically similar to a spheromak. Boozer's effective parallel electric field [406], expected to be much stronger than the real parallel electric field, should then also have the topology of nested toroidal surfaces (with toroidal and poloidal field components) and the guiding centers of accelerated ions should share this topology of the magnetic flux surfaces. This acceleration begins when the cross-field plasma flow (both radial and axial) starts amplifying the poloidal and toroidal magnetic fields (Section 4.3.1.3 C I). It ends when the plasma column density decreases both because of radial expansion and axial flow into the plasmoid at the top ending the dynamo action. The magnetic field of the plasmoid is increasing during the rise of the neutron pulse and decreasing during its fall. The sign of the electric field is therefore different in the rising and falling part of the neutron pulse.

The 3D trajectories of such accelerated ions (with all 3 velocity components) have turning points that also lie on toroidal surfaces [407]. The ratio of axial and radial velocities would then be related to the axial and radial scale lengths of the plasmoid, which is of the order of unity, explaining the observed near-isotropy of energy spectrum. Radial component of kinetic energy $\sim 70 \mathrm{keV}$ would correspond to the kinetic energy of ions at the turning points of its trajectory where the radial and axial components of velocity change their sign. These ions would remain confined in the radial direction [407] as a consequence of approximate conservation of their canonical angular momentum because of the approximate azimuthal symmetry of the plasma seen during the first neutron pulse (see Figures 84-86 and 96 from Shot \#9367).

The rise time of the neutron pulse would result from the duration of the magnetic dynamo powered by cross-field flow. Taking ballpark figures of plasma column height $\sim 6-8 \mathrm{~cm}$, axial flow velocity $\sim 10^{6} \mathrm{~m} / \mathrm{sec}$ [398] provides estimates of $60-80 \mathrm{~ns}$ in reasonable agreement with the observations.

The inference of accelerated ions both downstream and upstream from the fusion neutron spectrum observed downstream is consistent with trajectories of ions accelerated by poloidal and toroidal electric fields. In the portions of the poloidal component of ion trajectory closer to the axis, the ions move in the downstream direction (away from the anode) while away from the axis the ions move upstream (towards the anode). Since the density of the plasmoid is higher closer to the axis, more neutrons are generated by ions moving in the downstream direction closer to the axis than those moving in the upstream direction. The dominant energy of the neutrons downstream is, thus, more than the reaction energy of 2.45 in the center of mass frame while the lowest neutron energy is less than 
2.45 MeV from the ions moving towards the anode in the periphery of the plasma. In the falling part of the neutron pulse, the sign of the Boozer electric field is reversed. This could explain the result of the Monte Carlo simulation (Section 4.3.1.2 A(2)d) revealing that the downstream neutron energy is less than the reaction energy in the falling part of the neutron pulse.

Corroboration of this mechanism is found in the fact that the reaction proton image is much larger in radial size than the $X$-ray pinhole image in the same shot (Section 4.3.1.2 B(2)), which would happen if the ions that move through the dense plasma column in the downstream direction make a return trip outside the dense column. This interpretation also explains the increased visible luminosity in a spherical region at the end of the dense plasma column in Figure 6g,h of [398] which coincide with the peak of the first neutron pulse.

This mechanism of first phase neutron production could be concisely described by the phrase "hot ions circulating inside plasmoid (HICIP)" mechanism.

III. Neutron emission mechanism associated with the second pulse:

Q: Before the second pulse begins, the toroidal structure at the junction of the dense plasma column and the umbrella like structure has already transformed into a plasmoid because of axial inflow from the dense plasma column (see Figure 96). The second neutron pulse is known to coincide with the constriction of the plasma column and its breakup by the $\mathrm{m}=0$ instability (see Section 4.3.1.2 A(4)c). Interferometric visualization of this phase (see Figures 88 and 91) shows that the radius of the plasma column varies sharply along its length. Interpretation of the dynamics that leads to this situation (Section 4.3.1.3 B) shows internal transformations, whose physics is similar to the $\mathrm{m}=0$ MHD instability with one important difference.

The traditional MHD conception of $\mathrm{m}=0$ instability depends on the fact that the magnetic pressure that compresses the plasma varies inversely with radius. So a smallamplitude axial modulation of the plasma column radius leads to a higher pressure on the lower radius portion leading to plasma getting axially squeezed out into the neighboring regions of higher radius. The lower radius portion loses plasma and the higher radius portion gains plasma. As a consequence, the lower radius portion further shrinks in radius and the higher radius portion swells further, ultimately leading to the lower radius portions losing all their plasma to the surrounding higher radius portions, forming a low density region (often below the detection limit of diagnostics) between isolated regions of high density.

What actually occurs in the plasma focus differs from this traditional description of $\mathrm{m}=0 \mathrm{MHD}$ instability because of the presence of pre-existing poloidal and toroidal magnetic fields generated during the implosion phase (see Figure 84, Section 4.3.1.3 A, Section 4.3.1.3 C II). The axial flow associated with the instability together with the poloidal and toroidal magnetic fields leads to a dynamo that converts the kinetic energy of the forced axial outflow of plasma into magnetic energy, amplifying both the toroidal and poloidal magnetic field components, leading to growth of plasmoids (Figure 88 shows many plasmoids along the plasma column joined together by thin plasma necks just about to vanish in a short time). The experimental evidence in support of this scenario is quite clear. The prominent properties of the second neutron pulse (Section 4.3.1.2 A(3)a) are:

- The neutron energy corresponding to the second peak is $3.0 \mathrm{MeV}$ in the downstream direction and 2.0 MeV in the upstream direction (Section 4.3.1.2 A(2)a).

- The strong second HXR and neutron pulses are not accompanied with strong SXR and fast electron signals as in the first pulse (see Fix 63, Section 4.3.1.2 C, Section 4.3.1.2 D).

- For the second pulse, the FWHM is 70-100 ns. In its initial portion, the downstream neutron energy is $3.0 \mathrm{MeV}$ to $3.2 \mathrm{MeV}$. Towards its end, it becomes 2.2 to $2.4 \mathrm{MeV}$ (Section 4.3.1.2 A(2)e) according to a Monte Carlo simulation of the experimental results.

What mechanism of ion acceleration can explain these properties of the second pulse of neutron emission and their relation with the observed plasma dynamics consistently? 
LA1: The interferometer images demonstrate axial transport of plasma from the constricted zone into the plasmoids. This results in two effects: (1) Decrease in plasma density in the constricted zone to a value below the detection limit of the interferometer ("breakup zone") (see Figures 87 and 88). (2) Increase in the plasma density of the plasmoids. The absence of strong SXR and fast electron signals corresponding to the strong second HXR and neutron pulses (see Figure 83, Section 4.3.1.2 C, Section 4.3.1.2 D) can be interpreted as a consequence of plasma erosion, which decreases the electron density in the breakup region, so that bremsstrahlung soft $\mathrm{X}$-rays and accelerated electrons are in short supply. The breakup zone is effectively a relatively high impedance plasma-filled diode with virtual electrodes [10] that holds off the pinch voltage. A significant portion of current in the breakup zone must then be carried by ions because of relative shortage of electrons. This ion current is directed away from the anode. The plasmoid downstream of the breakup zone acts as a magnetic trap for this ion current. Ions make trajectories that have a large velocity component directed downstream but the combination of poloidal and toroidal magnetic fields associated with the plasmoid serve to bend the ion trajectories, such that the ions are returned to the plasmoid after traversing its axial dimension by moving radially out, down towards the anode, radially inwards and again at the breakup zone to begin a new travel along the plasmoid, much like water in a fountain that is pumped up as a strong jet in the center that falls back into the surrounding pool as a shower to be pumped up again as a jet. In each such circuit, the ion would gain additional energy while traversing the virtual diode. More neutrons would be produced by the accelerated deuterons moving downstream through the plasmoid than during their return trip outside the plasmoid, giving the impression of a linear axially directed beam. During this transit, ion trajectories would also undergo azimuthal displacement on account of deflection by the 3D magnetic field structure. This could be concisely described by the phrase "rotating-fountain-beam, plasmoid-target fusion (RFBPTF)" mechanism.

Interestingly, this seems to have been anticipated by Gribkov et al. [10] in their discussion of the virtual plasma-filled diode model of neutron production in the following quote "To 'fill' the whole pinch volume by these medium-energy ions, to confine them there and to give them the possibility of going away eventually from the pinch we have to suppose the existence of a longitudinal component of magnetic field (along the Z-axis)...". The additional element in the present case is the experimentally identified presence of a plasmoid at the end of the plasma column as an ion trap. The numerical estimates of neutron yield in the Lee model (Section 3.3.1.1), which are based on the diode model of [10] may, therefore, hold as well for the RFBPTF mechanism discussed above, with the difference that PF-1000 observations listed above in the question are additionally accounted for.

This interpretation explains the axially directed dominant deuteron energy of several hundred $\mathrm{keV}$ (Section 4.3.1.1 A(2), Section 4.3.1.2 E(1)), as well as the long duration of the second neutron pulse as compared with the axial transit time for ions of this energy. If one assumes that the second neutron pulse to be produced in a single axial transit of the accelerated ions, there would be rapid depletion of the plasma density. The fact that the plasmoid is seen in interferometry pictures for the duration of neutron emission supports the idea of multiple ion passes through the plasmoid. The occurrence of high intensity spherical structure in the visible frames in Figure 6n-p of [398] supports this interpretation. The return journey of the ions would occur through the low density plasma external to the dense plasma column (that has transformed into the plasmoid) accounting for a small number of downstream neutrons having energy less than the reaction energy of $2.45 \mathrm{MeV}$. The duration of neutron emission would depend on the lifetime of the plasmoid. There might be a variable number of plasmoids in different shots, each making its contribution to the neutron emission, accounting for variability of the neutron emission. These plasmoids are likely to be misaligned with respect to the device axis as a result of $\mathrm{m}=0$ and $\mathrm{m}=1$ instabilities in some shots. Fast deuterons escaping confinement in these plasmoids are then likely to be emitted along their local magnetic null axis [407] causing azimuthally 
asymmetric ion track clusters in time-integrated ion pinhole camera pictures (Section 2.3.1, Figure 1, Figure 8, and Figure 13).

The high HXR pulse at this time must then correspond to the portion of current in the breakup region carried by electrons. These nearly relativistic electrons would pinch in their self-magnetic field unless their motion has an azimuthal component that also produces an axial magnetic field that prevents such pinching. The result is likely to be a sub-millimeter region where hot electrons are confined in their self magnetic field consisting of both toroidal and poloidal components. Physical axial movement of this confined electron bunch in the breakup region would carry part of the current. This would be seen in X-ray pinhole cameras as occurrence of hotspots that are correlated with plasma constrictions. Such hotspots are known to be moving axially and have a short life time (Section 2.3.2 (3)e). This could also account for the occurrence high density plasma domains that cause relatively high secondary triton burnup (Section 2.3.2 (4)b).

\subsubsection{Other PF Devices}

This section discusses some experimental results from PF devices other than PF-1000 in a standard operating mode with deuterium that are relevant for a discussion of the pinch phase phenomenology. Since cross-correlated data from many diagnostics on a single plasma focus facility are rather rare, this section has opted to select a small set of results that exhibit some level of overlap with results from PF-1000. This is justified by the following argument.

The credibility of the interpretation of PF-1000 results (Section 4.3.1.3) suffers from a fundamental defect, that is not related to the quality of experiments or of their evaluation. Rather, it refers to the doubt that these results may be inherent to the device or its particular sub-optimized mode of operation, and not indicative of a general phenomenon that occurs in all plasma focus devices. The purpose of this section is to mitigate that doubt by pointing out that similar conclusions have been reached by other researchers on other devices using other diagnostics. For this reason, these interpretations of correlated data from PF-1000 must be regarded as a generic part of plasma focus physics and not specific to the PF-1000 device.

Serving this purpose does not require an exhaustive reportage of all plasma focus research nor of all experiments that report such findings, which would be an overkill. The selected experiments are compared in Section 4.3.3 with PF-1000 data and interpretation to demonstrate a certain degree of convergence in the physical understanding.

(1) Roshan et al. [408] used activation of a graphite target of $20 \mathrm{~mm}$ diameter inserted into the anode tip of the NX2 plasma focus working at 8 mbar deuterium and $12 \mathrm{kV}$ charging voltage to demonstrate presence of accelerated deuterons in the upstream direction (towards the anode). The relevant reaction ${ }^{12} \mathrm{C}(\mathrm{d}, \mathrm{n}){ }^{13} \mathrm{~N}$ has a deuteron threshold energy of $328 \mathrm{keV}$. The reaction product ${ }^{13} \mathrm{~N}$ decays with a half-life of 9.96 min producing a positron. This is supporting evidence for the inference of an electric field directed towards the anode (see Section 4.3.1.3 C(1)) from PF-1000 data.

(2) Lerner et al. $[95,140]$ have used neutron time of flight method to obtain the average ion energy $E_{i}$ of a plasma undergoing fusion using the following formula:

$$
E_{i}=E\left(W^{2}-\tau^{2}\right) / t^{2}
$$

where $t$ is the time required for a neutron of energy $\mathrm{E}$ ( $2.45 \mathrm{MeV}$ for $\mathrm{d}-\mathrm{d}$ fusion) to travel to the detector, $\mathrm{W}$ is the FWHM of the pulse at the detector, and $\tau$ is the duration of the neutron pulse at the origin of the pulse. This formula assumes a Maxwellian velocity distribution for ions. Using two scintillator-photomultiplier detectors with $25 \mathrm{~mm}$ thick BC404 scintillator (rise time $\sim 2 \mathrm{~ns}$ ) kept at $11 \mathrm{~m}$ and $17 \mathrm{~m}$ at $90^{\circ}$ to the axis, this relation was used to solve for $E_{\mathrm{i}}$ and $\tau$ for each of 44 shots. In another series of 23 shots, an additional detector was placed at $1.2 \mathrm{~m}$ from the axis at a polar angle of $4^{\circ}$ with the axis. Using the time difference between the neutron peaks at $11 \mathrm{~m}$ and $17 \mathrm{~m}$ 
detectors, an average perpendicular neutron energy $E_{p}$ was estimated. This was used to estimate the time of generation of neutrons (see Section 4.3.2 (4)d below). From this and the signal from the axial detector at $1.2 \mathrm{~m}$, the average axial neutron energy $E_{a}$ was calculated. The report mentions that the detector at $17 \mathrm{~m}$ is larger than the one at $11 \mathrm{~m}$. However, there is no mention of correction for possible photomultiplier electron transit time differences between the two detectors. Neutron fluence anisotropy was measured using bubble detectors at $0,4,12.5$, and 90 degrees with the axis. Ion beams were measured using Rogowski coils placed at $31 \mathrm{~cm}$ from the end of the anode inside a drift tube. A gated intensified charge-coupled device camera with exposure time $\sim 0.2 \mathrm{~ns}$ was used to take visible and near UV framing pictures. The following major conclusions were reported:

a. There were 4 shots with $\mathrm{E}_{\mathrm{i}}>150 \mathrm{keV}$ in the first series. A table showing frequency distribution of $E_{i}$ has been reported. In another series of experiments [140], the maximum $\mathrm{E}_{\mathrm{i}}$ is reported as $240 \mathrm{keV}$.

b. In the second series, mean values $\mathrm{E}_{\mathrm{p}} \approx 2.25 \pm 0.03 \mathrm{MeV}$ and $\mathrm{E}_{\mathrm{a}} \approx 2.65 \pm 0.45 \mathrm{MeV}$ have been reported. This has been interpreted as having no statistically significant anisotropy. According to the authors, the difference in mean $\mathrm{E}_{\mathrm{p}}$ and the reaction energy of $2.45 \mathrm{MeV}$ "...implies a mean bulk velocity of the neutron source of only $0.087 \mathrm{~cm} / \mathrm{ns}$. This would give the deuterons only $8 \mathrm{keV}$ of energy, which is small compared to the up to $160 \mathrm{keV}$ energy inferred from the TOF data".

c. The deuteron beam energy was estimated from ion time of flight. Mean deuteron kinetic energy of $\sim 320 \mathrm{keV}$ and maximum $\sim 500 \mathrm{keV}$ were reported.

d. The fluence anisotropy data were interpreted to imply that “...at most $30 \%$ of the neutrons observed could have come from an unconfined beam and that thus at least $70 \%$ of the neutrons come from confined high-energy ions".

e. ICCD image taken within 2 ns of the X-ray emission "...appears to show the plasmoid core consisting of a coil of plasma filaments. The bright filament is $\sim 60 \mu \mathrm{m}$ in diameter and is wrapped in a coil that is $400 \mu \mathrm{m}$ in diameter and $1.5 \mathrm{~mm}$ long. There is also a $500 \mu \mathrm{m}$ radius halo of less dense filaments surrounding the inner core". Assuming that this visible light image shows the volume from which the neutrons are emitted, the authors conclude that the density of this structure is $\sim 3 \times 10^{19} \mathrm{~cm}^{-3}$.

f. The above data have been interpreted by the authors as follows: "...our results show that $>70 \%$ of the neutrons observed in these shots originate in a plasmoid with $\mathrm{n}$ in the range of $3-4 \times 10^{19} \mathrm{~cm}^{-3}$, with radii of about $500 \mu \mathrm{m}$ and lengths of about $1.5 \mathrm{~mm}$, confined ion average $\mathrm{E}_{\mathrm{i}}$ up to $160 \mathrm{keV}$ and lifetimes of 30-60 ns".

(3) Experiments on the PACO device in Argentina [409] used a Bicron BC 400 scintillator of diameter $180 \mathrm{~mm}$ coupled with the high current photomultilier Philips 150 AVP kept at a distance of $2.3 \mathrm{~m}$ perpendicular to the axis. Out of the neutron yield of $2 \times 10^{8}$ neutrons measured using a silver activation detector, $7.6 \times 10^{4}$ neutrons were expected to reach the scintillator. The investigations report the following conclusions:

a. "The time differences between the maximum neutron emission and that of X-ray emission... puts into evidence that both events are related but not simultaneous".

b. "The time correlation between voltage peaks and X-ray peaks ... is in reasonable agreement with the $X$-rays being generated by an electron beam produced in turn by a voltage drop on the forming plasma column...".

c. "The fact that the neutrons are emitted on average $\approx 20 \mathrm{~ns}$ before the formation of an electron beam is hardly compatible with a beam-target model for the neutron production in this device [a similar conclusion arises from the essential lack of correlation between the amplitude of the X pulse and the neutron yield...]".

d. "The correlation between the dip in $\mathrm{dI} / \mathrm{dt}$ and voltage divider signals shows that both events are closely time related but not simultaneous". 
(4) Experiments on a plasma focus operated at $320 \mathrm{~J}$ energy at $27 \mathrm{kV}, 9$ mbar $\mathrm{D}_{2}, 12 \mathrm{~mm}$ anode radius [410] are described below in some detail in view of some unexpected conclusions. Apart from current and voltage monitors, the diagnostics consisted of two moderated ${ }^{3} \mathrm{He}$ counters, calibrated with an AmBe neutron source, kept radially and axially and 6 scintillator-photomultiplier detectors based on BC-408 BICRON scintillator optically coupled to a $51 \mathrm{~mm}$ diameter R1828-01 Hamamatsu photomultiplier tubes (PMT). Two spatial configurations of detectors were utilized. In the first, all 6 detectors were placed radially, separated by $50 \mathrm{~cm}$, the closest $1 \mathrm{~m}$ from the $\mathrm{PF}$, which allowed placement of the ${ }^{3} \mathrm{He}$ counters for total yield measurements. In the second case, 3 detectors were placed radially at $1.5 \mathrm{~m}, 2 \mathrm{~m}$, and $3.05 \mathrm{~m}$ and axially at $2 \mathrm{~m}, 2.46 \mathrm{~m}$, and $3.05 \mathrm{~m}$. At a distance of $1 \mathrm{~m}$, the PMT receives a fraction $1.56 \times 10^{-4}$ of all neutrons emitted in $4 \pi$. With a total neutron yield of $\sim 5 \times 10^{5}$ neutrons, the farthest detector at $3 \mathrm{~m}$ would, thus, receive $\sim 26$ neutrons. The detector signals were recorded on $500 \mathrm{MHz}$ oscilloscope channels. The initial rise of X-ray and neutron signals were taken as time references. Differences in PMT delay times were eliminated by adjusting them such that the leading edge of X-ray pulse arrived at each PMT at the expected $X$-ray flight time. Linear regression on the detector position and rise of the $\mathrm{X}$-ray and neutron signals led to determination of the time of origin of the X-ray $\left(t_{x}\right)$ and neutron pulses $\left(t_{N}\right)$ at the plasma focus, as well as "the mean energy of the fastest neutrons" in each shot for axial and radial directions separately. The data show the following:

a. Times of production of X-rays and neutrons can be different showing that they are produced by independent mechanisms.

b. Correlation exists between the mean energy of the fastest neutrons and the neutron emission delay with respect to $\mathrm{X}$-ray emission.

c. This correlation is different along the axial and radial directions.

d. The time of production of neutrons can be different in axial and radial directions in the same shot (see Section 4.3.2 (2)). In the authors" words “...the neutron pulse detected radially does not necessarily correspond to the same neutron pulse detected axially since ( $\mathrm{tN} \_a x i a l-t N \_$radial) is not always zero".

e. "...the neutron emission on the different directions (radial or axial) present differences in their energy and yield, showing a clear anisotropy with higher values and broader statistical distributions in the axial direction. Furthermore, the results of the observed neutron energies on the different directions and the temporal differences of the radiation emission suggest the existence of two temporally separated neutron pulses, which agrees with observations made in larger energy devices.".

(5) Experiments on a $400 \mathrm{~J}$ plasma focus [411] using a time history analysis technique show that in some shots the neutrons are emitted before the hard X-rays and the pinch. The experiment used 2 PMTs axially and 2 PMTs radially separated by $20 \mathrm{~cm}$ distance kept at $1.57 \mathrm{~m}$ and $1.77 \mathrm{~m}$ from the center of the anode. The PMTs could intercept a fraction $5 \times 10^{-5}$ of neutrons emitted by the PF in $4 \pi$. The current derivative signal from a Rogowski coil was split and recorded on two fast oscilloscopes, both as time marker and trigger. Signals from two axial PMTs and two radial PMTs were recorded along with the current derivative signal. The time of appearance of the neutron (X-ray) signal on the oscilloscope was determined as the sum of the time of origin of neutrons (X-rays), time of flight from the source to the PMT, electron transit time of the PMT and the cable transit time from the PMT to the oscilloscope. This analysis enabled determination of the time difference $\Delta t \equiv t_{N, O}-t_{x r y, O}$ in the time of origin of neutrons and X-rays. Making the assumptions that: (1) X-rays are produced at the time of the pinch and (2) the pinch duration was given by the scaling law of Lee and Serban (Section 3.3.3.1) $\mathrm{t}_{\mathrm{pf}}(\mathrm{ns}) \approx 2 \mathrm{a}(\mathrm{mm})$ to be $12 \mathrm{~ns}$, the report concluded that:

a. In many shots some neutrons are produced with $\Delta t<0$ (before the pinch phase). Similar observations are reported from larger installations (see Section 2.4.2, 
Section 4.3.1.1 A(3)a, Section 4.3.1.2 A(3), Section 4.3.1.3 C(1)). This is more marked in the axial direction than in the radial direction.

b. Some neutrons are produced with $\Delta \mathrm{t}>\mathrm{t}_{\mathrm{pf}} \sim 12 \mathrm{~ns}$ (post-pinch phase). This is more marked in the radial direction than in axial direction.

c. Mean energy of fastest neutrons, determined from the time difference between the start of the neutron signals in the detectors (see Figure 4 of [411]), increases monotonically over the range from $1 \mathrm{MeV}$ to $6 \mathrm{MeV}$ with $\Delta \mathrm{t}$ but the variation is markedly different in the axial and radial directions.

d. Neutrons were produced $40 \mathrm{~ns}$ before $\mathrm{X}$-rays in the axial direction and $20 \mathrm{~ns}$ in the radial direction.

(6) Zakaullah et al. [412] used a $0.5 \mathrm{~mm}$ diameter pinhole to image the reaction zone of a $2.5 \mathrm{~kJ}$ plasma focus on a CR-39 SSNTD using emission of fast charged particles. The pinhole camera had a magnification of 1 and the distance between the pinhole and the source was $8 \mathrm{~cm}$. Ion pinhole images (see Figure 97) showed significant variation over the pressure range 0.25 to 3.0 mbar $\mathrm{D}_{2}$ pressure. At each pressure, 3-5 images were recorded and good reproducibility was observed.

The authors state their conclusions in the following words "It seems that the neutron production mechanism at play strongly depends upon the filling pressure. The data suggest beam-target mechanism at low pressure, trapped gyrating particles in some anomalous magnetic field at optimum pressure, and a jet-like moving boiler at high pressure".

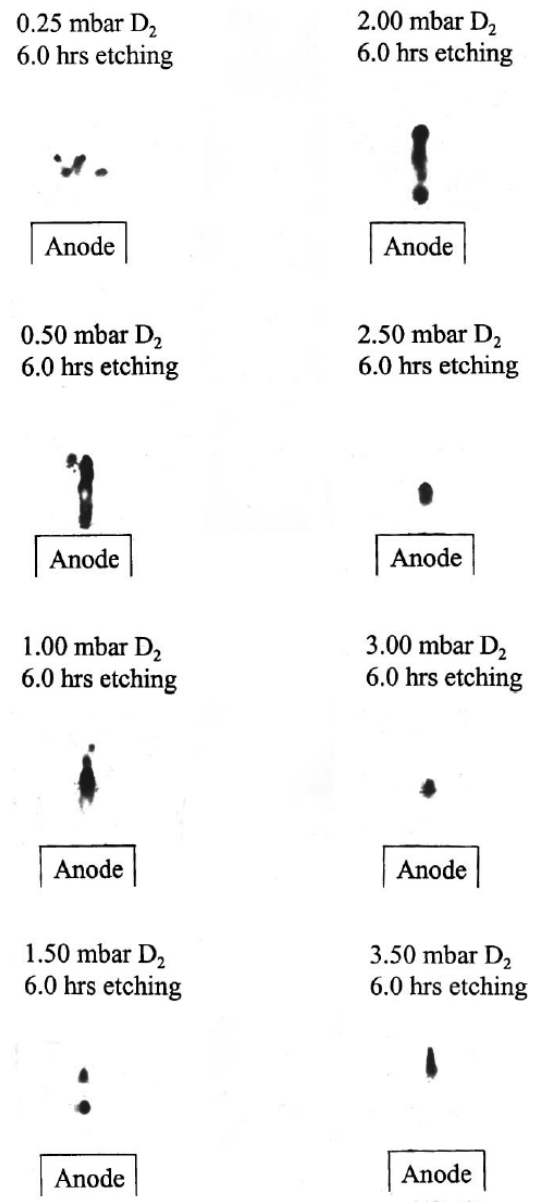

Figure 97. Reproduced from Figure 5 of [412] (Phys. Plasmas 1999, 6, 3188-3193) with the permission of AIP Publishing. The authors state: "At 0.25 mbar, a dotted region spread over a large volume is 
observed. On increasing the pressure to $0.5 \mathrm{mbar}$, an elongated beaded structure, starting just from the anode tip, is observed. At $1.0 \mathrm{mbar}$, about $8 \mathrm{~mm}$ long a rocket-type plasma column, which appears moving away from the anode tip is observed. At $1.5 \mathrm{mbar}$, this rocket-type plasma column breaks into two whereas, at 2.0 mbar, about $12 \mathrm{~mm}$ long plasma column which is broken at many locations, starting $3 \mathrm{~mm}$ away from the anode tip is seen. At $2.5 \mathrm{mbar}$, an almost stable dense plasma filament is seen, which is about $3 \mathrm{~mm}$ in length, $2 \mathrm{~mm}$ in diameter, and $5 \mathrm{~mm}$ away from the anode tip. With a further increase in pressure, dense plasma region squeezes in size and shifts away from the anode. It is $8 \mathrm{~mm}$ away at $3.0 \mathrm{mbar}, 15 \mathrm{~mm}$ away at $3.5 \mathrm{mbar}$, and not visible at higher pressure, probably formed outside the field view of the imaging camera.".

(7) The time-integrated spatial distribution of $\mathrm{D}(\mathrm{d}, \mathrm{p}) \mathrm{T}$ fusion reactions for pure $\mathrm{D}_{2}$ gas, and $\mathrm{D}_{2}-\mathrm{Kr}$ gas admixture, was studied by using the proton-CAI technique to image individual NX2 plasma focus shots $[219,221,222]$ as described in Section 3.2.5. CAI permits a high proton throughput to be obtained, counterbalancing the NX2's modest proton yield per shot. For a typical NX2 shot with $Y_{n}=3 \times 10^{8}$ neutron yield, the number of recognized and processed proton tracks on the CR-39 detector was about $2 \times 10^{5}$. In addition, sub-millimeter image resolution was obtained.

Figure 98 shows a typical result which compares an X-ray pinhole CCD camera image with the fusion proton image. The shape of the fusion source resembles a tapered cigar of length $\sim 10 \mathrm{~mm}$, with diameters of $\sim 2 \mathrm{~mm}$ near the anode (upstream end) and about $4 \mathrm{~mm}$ at the downstream end. The maximum proton density occurs about 4 $\mathrm{mm}$ above the anode. The X-ray image shows a hot dense pinch region of $\sim 4 \mathrm{~mm}$ length and $\sim 0.5 \mathrm{~mm}$ diameter. Comparison of the $\mathrm{X}$-ray and fusion proton images indicates that the zone with the highest concentration of $\mathrm{DD}$ fusion occurs close to the downstream end of the hot dense plasma column, seen in the X-ray image [221]. The size of the proton image was larger than the size of the pinch seen in X-ray pinhole pictures [219], a feature also found in PF-1000 (see Section 4.3.1.2 B(2)).

Figure 99 shows de-convoluted CAI images of a single NX2 PF shot with pure $\mathrm{D}_{2}$ gas operation viewed by the two cameras with Mask-1365 and Mask-4680. There is a $60^{\circ}$ difference in azimuthal viewing angle, but the fusion images show very similar spatial distributions confirming the genuineness of the images and the validity of the applied deconvolution procedures. The leftmost pane shows an X-ray image of the hot plasma pinch (obtained by CCD camera) with $20 \mu \mathrm{m}$ beryllium foil which cuts off all visible light and X-rays with energy less than $700 \mathrm{eV}$. The horizontal red dashed line indicates the level of the hollow anode tip for each set of images. The maximum intensity of the proton image is at the same height as the downstream end of the X-ray image.

Figure 100 shows de-convoluted proton CAI images from a single shot of NX2 PF for $\mathrm{D}_{2}-\mathrm{Kr}$ gas admixture operation ( $2 \% \mathrm{Kr}$ by volume). Due to the high intensity of $\mathrm{x}$-ray radiation for $\mathrm{D}_{2}-\mathrm{Kr}$ operation, the $20 \mu \mathrm{m}$ Be filter used for pure $\mathrm{D}_{2}$ was replaced by $50 \mu \mathrm{m} \mathrm{Be}+12.5 \mu \mathrm{m}$ Ti filters to attenuate incident $X$-rays and prevent CCD saturation. This filter combination transmits a suitable fraction of $\mathrm{X}$-rays in the energy range $1.0 \mathrm{keV}$ to $4.9 \mathrm{keV}$.

The X-ray image in Figure 100 contains evident plasma hot spots and $\mathrm{m}=0$ instabilities in final pinch compression. Despite this, there are no corresponding dense zones in the fusion source image, and this finding is characteristic of the $\mathrm{D}_{2}-\mathrm{Kr}$ data. This finding is significant in the context of hypotheses concerning dense hot spots being significant sites for the occurrence of fusion reactions (see Section 2.3.2 (3)), [95,140,158,159,200]. 


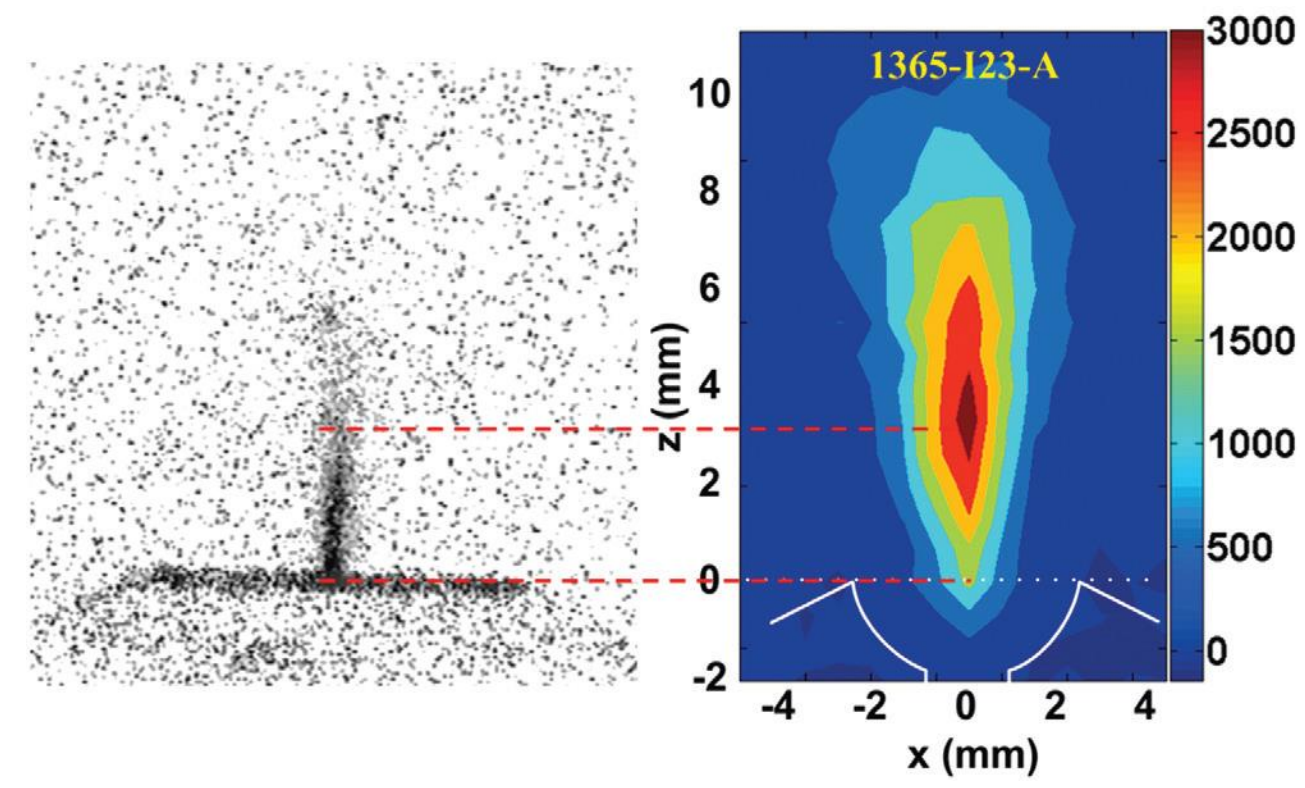

Figure 98. Reproduced from Figure 2 of [221]. (Appl. Phys. Lett. 2012, 101, 114104) with the permission of AIP Publishing. Left side image is the X-ray pinhole CCD camera image and the right side image is the de-convoluted coded aperture reaction proton image.

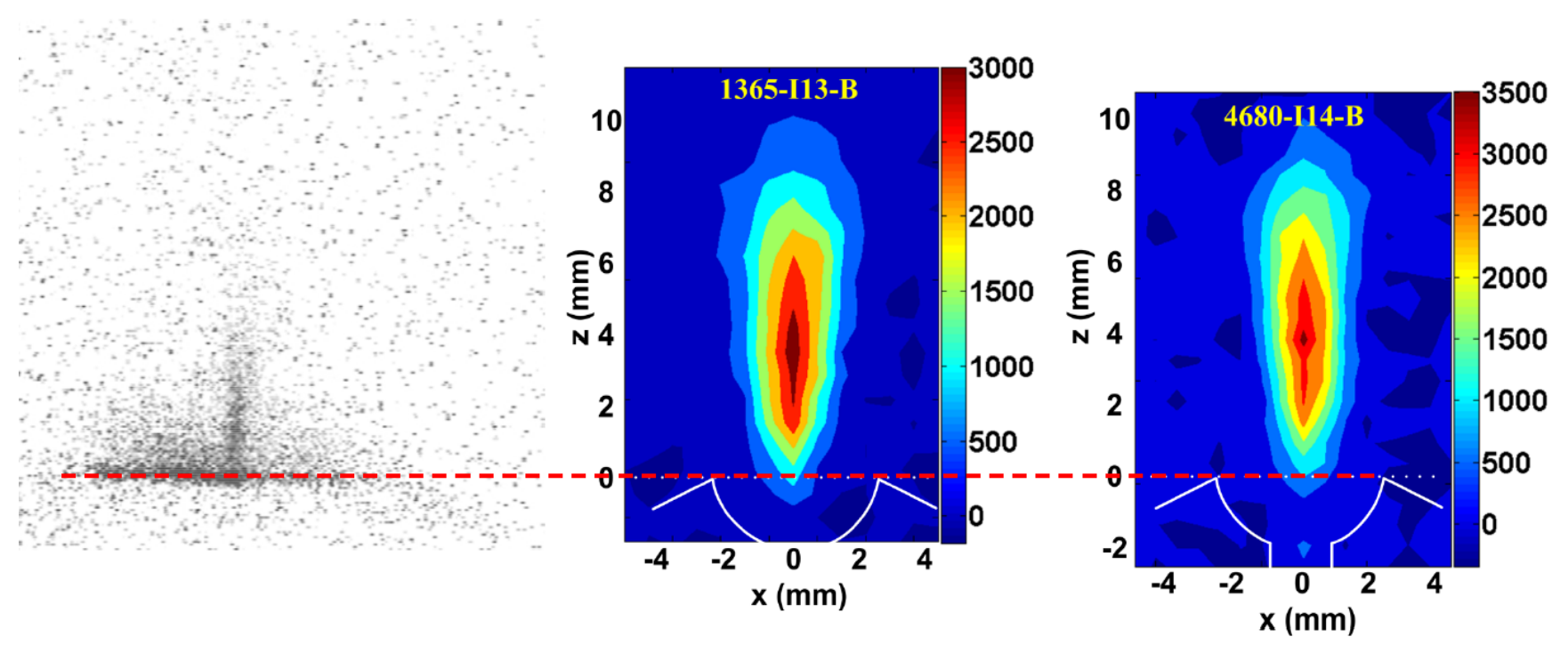

Figure 99. (middle and right panes) De-convoluted proton CAI images for a single NX2 PF shot with pure $\mathrm{D}_{2}$ gas at 13 mbar. The neutron (proton) yield for this shot was $2.38 \times 10^{8}$. (left pane) CCD X-ray image with $20 \mu \mathrm{m}$ Be filter. Reproduced from A. Talebitaher, Ph.D. thesis, NTU, Singapore (2012) [223]. 


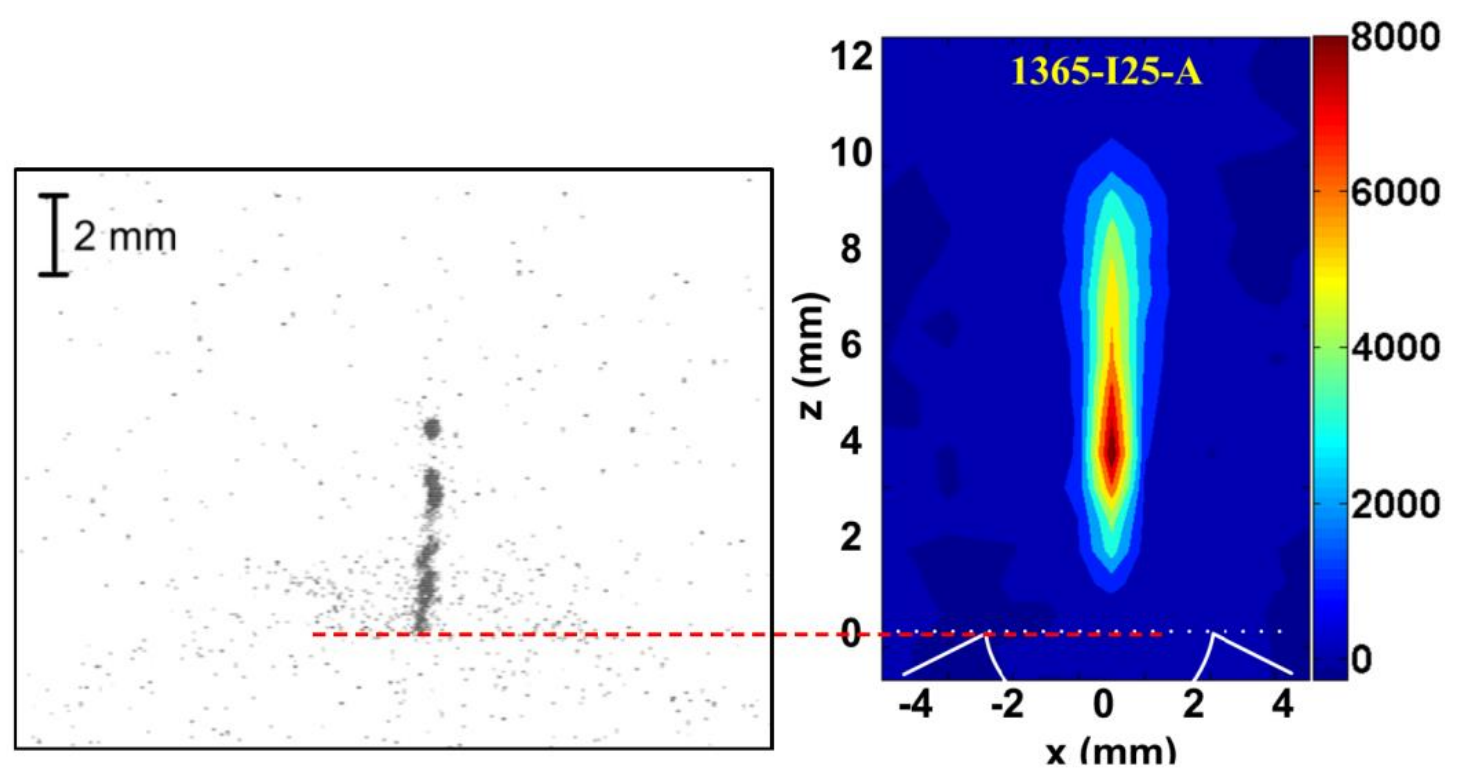

Figure 100. (Right): de-convoluted proton CAI image from a single shot of NX2 PF for $\mathrm{D}_{2}-\mathrm{Kr}$ gas admixture operation ( $2 \% \mathrm{Kr}$ by volume). (Left): X-ray pinhole CCD camera image with a $50 \mu \mathrm{m}$ Be $+12.5 \mu \mathrm{m}$ Ti filter. Reproduced from A. Talebitaher, Ph.D. thesis, NTU, Singapore (2012) [223].

(8) The time-integrated quasi-average energy of fusion neutrons emitted at $0^{\circ}$ and $90^{\circ}$ in each shot of NX3 plasma focus at Singapore was measured by using the Zirconium Beryllium Pair detector technique described in Section 3.2.6.

Figure 101 shows the shot-to-shot characteristic neutron energy, at $0^{\circ}$ and $90^{\circ}$, for 2 series $\left(16+16\right.$ shots) at 5.0 mbar $D_{2}$ gas pressure. The neutron energies at $90^{\circ}$ to the $\mathrm{PF}$ axis are predominantly in the $2.45 \mathrm{MeV}$ to $2.55 \mathrm{MeV}$ range. with a mean of $2.50 \mathrm{MeV}$. At $0^{\circ}$, most shots produce neutrons with energies above $2.8 \mathrm{MeV}$. The variance in energy is larger than at $90^{\circ}$, and the mean energy is $2.81 \mathrm{MeV}$. In terms of the $\mathrm{d}$ - $\mathrm{d}$ fusion reaction kinematics, neutron energies of $2.81 \mathrm{MeV}$ at $0^{\circ}$ to the PF axis is associated with a beam-target mechanism in which a forward-directed $\left(0^{\circ}\right) \mathrm{D}+$ ions of kinetic energy about $80 \mathrm{keV}$ interact with stationary deuterons. The marked difference in neutron energies between $0^{\circ}$ and $90^{\circ}$ indicates predominance of neutrons created by an axially directed beam.

The inference of an axially directed beam from this large difference in the effective neutron energy between end-on and side-on neutrons was cross-checked by placing an aluminium obstacle plate (OP) $6 \mathrm{~cm}$ from the anode top to interrupt the propagation of the beam in the neutral gas above the pinch. Collecting the data for all shots fired at a given deuterium gas pressure, the mean neutron energy was calculated for the cases with and without the plate. These results from a total of 596 shots are plotted in Figure 102.

It is seen that the average neutron energy depends only weakly on $\mathrm{D}_{2}$ gas pressure. The results and discussion presented in [225] conclude that their experimental data are consistent with a beam-target mechanism (with an axial beam interacting with a plasma and neutral gas target). 


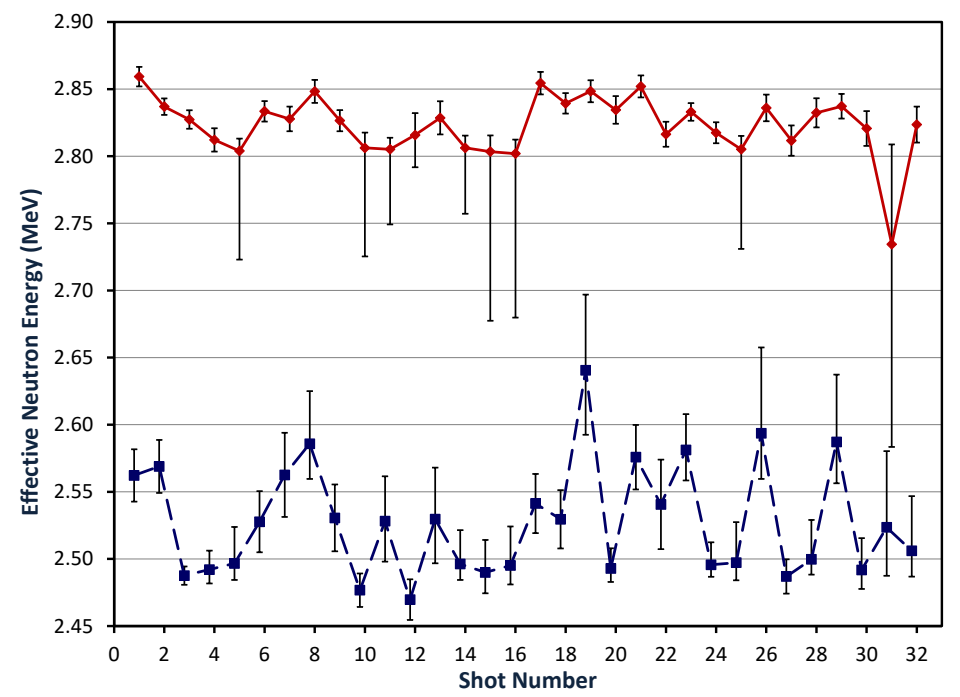

Figure 101. Shot-to-shot neutron energy at the $0^{\circ}$ orientation (red solid line) and the $90^{\circ}$ orientation (blue dashed line) for 2 consecutive series (of 16 shots) at $\mathrm{D}_{2}$ gas pressure of 2.0 mbar.

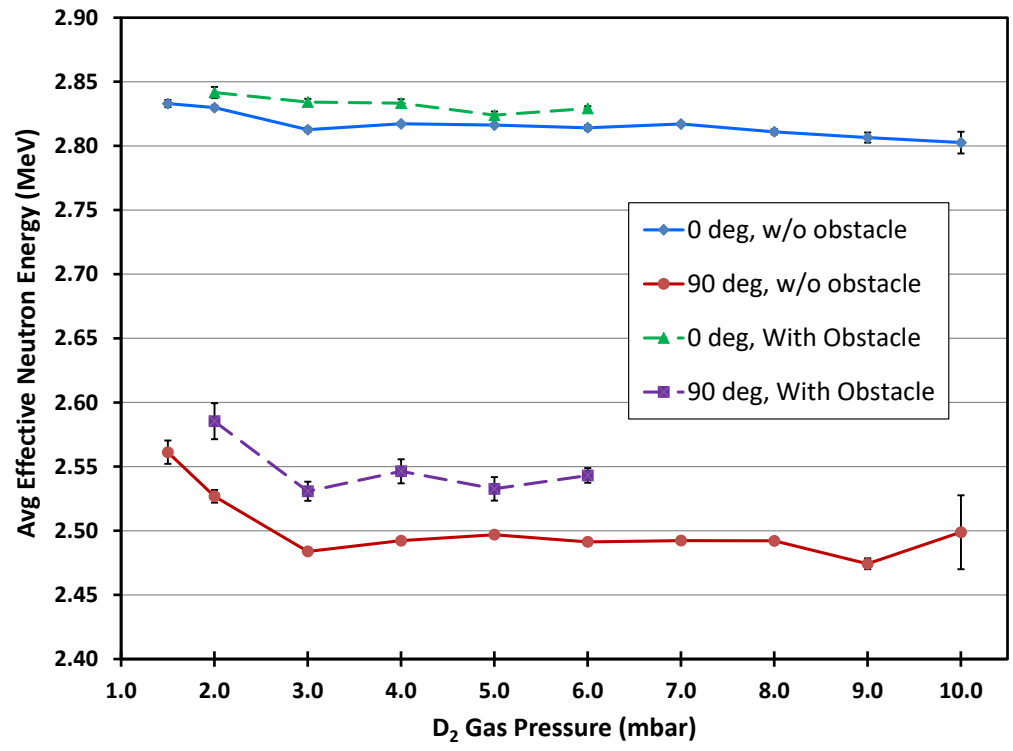

Figure 102. Variation of mean neutron energies over the range of deuterium gas pressures experimentally investigated.

(9) The UNU/ICTP PFF at the University of Malaya was optimized for neutron yield at about 4 mbar of deuterium, producing $\sim 10^{8}$ neutrons [188]. Using a deuterated titanium target and a plain copper obstacle at the different distances in front of the anode, it could be shown that less than $15 \%$ of the neutrons were produced within the pinch, and the remaining $85 \%$ of the neutrons arose from the neutral deuterium gas 20-60 $\mathrm{mm}$ ahead of the anode end [248].

(10) Using a Maxwellian curve fitting technique [413], it was shown that the neutron signals in the UNU/ICTP PFF at the University of Malaya could be fitted to two pulses that possibly correspond with two phases of neutron emission found in POSEIDON [173] and PF-1000 (see Section 4.3.1). The neutron detectors were "...cylindrical NE102A scintillator (diameter $5 \mathrm{~cm}$, length $10 \mathrm{~cm}$ ) wrapped with a fluorescent fibre and optically coupled to a normal plastic optical fibre". The optical signals were carried by this optical fiber to the photomultiplier tube, which was placed inside a screened room to diminish electrical interference, as well as spurious signals 
from the photoelectrons ejected by hard-X-rays from photomultiplier dynodes [414]. Four detectors were placed as follows: at distances of $50 \mathrm{~cm}$ and $80 \mathrm{~cm}$ at $0^{\circ}$ and at $50 \mathrm{~cm}$ and $100 \mathrm{~cm}$ at $90^{\circ}$. The energies of the two phases of neutrons were estimated from the time of flight using the peaks of the corresponding curves. These fitted curves were also integrated to give the magnitudes of the neutron yields and for the calculation of the end-on to side-on anisotropy.

The results obtained show that the first pulse was dominant (contributing $65 \%$ of yield) and had neutron energy $2.6 \pm 0.4 \mathrm{MeV}$ at $0^{\circ}$ and $2.6 \pm 0.3 \mathrm{MeV}$ at $90^{\circ}$. The end-on to side-on yield anisotropy factor was about 1 . The second pulse of neutrons contributing less then $35 \%$ of the total neutron yield had energy $3.6 \pm 0.5 \mathrm{MeV}$ at $0^{\circ}$ and $2.6 \pm 0.3 \mathrm{MeV}$ at $90^{\circ}$. The end-on to side-on yield anisotropy factor was about 1.5. The results of a similar analysis of argon-doped shots in the same paper is discussed in Section 5.3 (6).

\subsubsection{Convergence between Results from Various Laboratories}

This section demonstrates that the results on the standard operating mode of PF-1000 with deuterium are not a unique feature of the PF-1000 device and that similar results have been reported from other devices by other researchers using different diagnostics. These must, therefore, be regarded as generic features of the plasma focus phenomenon and not peculiarities specific to PF-1000. This convergence between conclusions from different laboratories using different methodologies is an important aspect of due diligence while drawing significant inferences that could be translated into theories and models.

\subsubsection{Existence of Axial Component of Magnetic Field in the Radial Implosion Phase}

This is a crucial corner-stone of the interpretation of the formation of toroids and plasmoids (Section 4.3.1.3 A, Section 4.3.1.3 B) as well as of the associated dynamo mechanism and the rapid transfer of energy to the plasma much before stagnation (Section 4.3.1.3 C I). It is also involved in the interpretation of the properties of the first pulse of neutron emission (Section 4.3.1.3 C II). Although the existence of $B_{z}$ in the pinch phase can be ascribed to helical deformation of the plasma boundary (see Figure 89), this is not possible for the radial implosion phase where very symmetric plasma shells are routinely seen. The evidence from PF-1000 is based on magnetic probe measurements [212] and is supported by the observations on contactless compression of a laser-plasma plume by an imploding plasma focus sheath [169-171] and magnetic probe measurements [174] in POSEIDON (see Section 2.4.1).

\subsubsection{Existence of Accelerated Deuterons Moving towards the Anode}

Plasma focus literature has used the phrase "beam-target mechanism" for a long time as short-form for 'a unidirectional beam of ions accelerated by an electric field pointed in the direction of current flow caused by current interruption because of the $\mathrm{m}=0 \mathrm{MHD}$ instability resulting in neutron emission during its single transit through the plasma and neutral gas beyond'. The principal evidence in favor of this description is the fact that a large fraction of neutrons detected downstream have energy much higher than the reaction energy 2.45 MeV. Confirmation of this fact is obtained on PF-1000 (see Section 4.3.1.2 A(2)a) by neutron time of flight technique and on NX3 by Zirconium Beryllium Pair activation detector technique in recent times but has been a part of plasma focus literature from its early days. This commonly held view of neutron emission mechanism, however, is inconsistent with other data.

Existence of fast deuterons moving towards the anode in PF-1000 is revealed by the presence of downstream neutrons with less than $2.45 \mathrm{MeV}$ energy (Section 4.3.1.2 A(2)b,d,e, (3)a), presence of upstream neutrons with maximum energy $2.95 \pm 0.15 \mathrm{MeV}$ (Section 4.3.1.2 A(1)b) and few hundred keV deuteron tracks in the upstream direction [92]. This reveals the existence of either an electric field that is pointing towards the anode at least for some time or of a magnetic field that bends the path of ions moving along the axis through $180^{\circ}$ or 
both. The detection of a fast electron peak in the downstream direction (Section 4.3.1.2 D) while the plasma is still imploding clearly shows that such electric field exists.

The detection of graphite activation by the ${ }^{12} \mathrm{C}(\mathrm{d}, \mathrm{n}){ }^{13} \mathrm{~N}$ reaction with a deuteron energy threshold of $328 \mathrm{keV}$ by Roshan et al. [408] (see Section 4.3.2 (1)) shows that acceleration of ions towards the anode is not a phenomenon unique to PF-1000. The PF1000 data on downstream electron emission before plasma stagnation, therefore, becomes very significant because it associates a time-stamp with the electric field responsible for acceleration of ions towards the anode.

Note that the presence of fast ions directed towards the anode does not invalidate the experimental fact that the major fraction of neutrons are caused by ions moving along the axis. It forms part of the evidence for an alternate narrative that suggests that the axially moving ions make a return journey because of the presence of a 3D magnetic field that bends their path. This is discussed in Section 4.3.3.3.

\subsubsection{Fusion Proton Images Larger Than X-ray Pinhole Images}

The time-integrated proton-pinhole image from PF-1000 (Section 4.3.1.2 B(2), Figure 3 of [16]) appears larger than the X-ray image from the same shot. This has also been observed in POSEIDON $[171,182]$ and in NX2 [219,221]. This is a property of the ion-trapping mechanism suggested in Section 4.3.1.3 C III.

In this proposed mechanism, a plasmoid near the junction of the straight plasma column and the umbrella-like structure serves as a magnetic trap for fast ions injected into it by a virtual, plasma-filled diode created by plasma erosion in the neck of the $\mathrm{m}=0$ instability. These ions make trajectories that have a large velocity component directed downstream but the combination of poloidal and toroidal magnetic fields associated with the plasmoid serve to bend the ion trajectories, such that the ions are returned to the plasmoid after traversing its axial dimension by moving radially out, down towards the anode, radially inwards and again at the breakup zone to begin a new travel along the plasmoid, possibly accompanied with an azimuthal displacement as well. In each such circuit, the ions gain additional energy while traversing the virtual diode. During their return journey, the ions cause fusion reactions outside the dense plasma column, making the reaction proton image larger than the soft X-ray image of the dense plasma column.

This proposed mechanism (Section 4.3.1.3.C III) would predict a high concentration of fusion reactions in a localized region situated at the end of the dense plasma column away from the anode. This can be clearly seen in the reaction proton images in NX2 [220] (see Figure 98, Section 4.3.2 (7)) and in fast ion pinhole images reported by Zakaullah et al. (see Figure 97, Section 4.3.2 (6)). Visible and soft X-ray framing pictures at the time of maximum neutron emission in PF-1000 (Figures 6g,h,o,p and 7g,h of [398]) also show bright regions located at the far end of the dense plasma column.

The POSEIDON time-integrated reaction proton image dataset was evaluated [182] for the energy distribution of reaction protons at 8 points selected on the time-integrated soft X-ray image from the same shot at coordinates: points 1 and 2: $\mathrm{x}= \pm 2 \mathrm{~cm}, \mathrm{z}=8 \mathrm{~cm}$, 3 and 4: $x= \pm 0.5 \mathrm{~cm}, z=4.5 \mathrm{~cm}, 5$ and $6: x= \pm 2 \mathrm{~cm}, z=3 \mathrm{~cm}$, and 7 and $8: x= \pm 0.5 \mathrm{~cm}$, $\mathrm{z}=1.5 \mathrm{~cm}$. This evaluation showed that the peak of the energy distribution function was shifted to energy higher and lower than the reaction energy on pairs of points 3 and 4,5 and 6 . Points 5 and 6 clearly lie outside the pinch zone, while 3 and 4 are well within the pinch zone. This is a clear indication of the existence of an azimuthal component of the velocity of the deuterons that caused the proton emission. This is consistent with the proposed mechanism (Section 4.3.1.3 C III) where poloidal magnetic field would deflect the ions in the azimuthal direction without loss of energy. The shift at points 5 and 6 could also be because of neutron emission before the stagnation that could be caused by azimuthally circulating fast deuterons (see Section 8.2.1).

The features of reaction proton images in PF-1000 discussed above are thus also observed on at least two other devices and are, therefore, not a feature specific to PF-1000. 


\subsubsection{Observation of Lobular Protrusion Reaching the Anode}

The observation of lobular protrusions that bend towards the anode (Section 4.3.1.1 A(9)a,b, Figure 95) was also reported in the Frascati 1 MJ plasma focus (see Section 2.4.3). It was recognized $[117,184]$ that they might reconnect to form "long-lasting plasma blobs whose decay time (100-150 ns) corresponds to that of the local neutron emission".

\subsubsection{Non-Simultaneity of Current Derivative and Voltage Extrema}

The observation that characteristic extrema of voltage and current waveforms on PF-1000 are not simultaneous (Section 4.2.3, Figure 80) leads to the conclusion that the voltage drop across the plasma column has a double peak separated by a valley (Figure 82). The observation mentioned in Section 4.3.3.3 above provides the following interpretation of the valley between this double peak. The rising voltage drop across the plasma column is short-circuited by the reconnecting lobular protrusions so that the circuit external to the short-circuiting plasma (which includes the voltage monitor) is decoupled from the plasma column. The conclusion [409] that "The correlation between the dip in $\mathrm{dI} / \mathrm{dt}$ and voltage divider signals shows that both events are closely time related but not simultaneous" (Section 4.3.2 (3)d) on a smaller device shows that the conclusions on PF-1000 are not an artifact of measurement or a unique property of the device.

\subsubsection{Near-Isotropy of Neutron Emission Spectrum in the First Pulse}

Nearly identical FWHM of the first pulse of neutron emission in downstream, upstream and side-on detectors placed at $7 \mathrm{~m}$ (Section 4.3.1.2 A(3)b, Section 4.3.1.2 A(4)a) show that the neutron energy spectrum is nearly isotropic. Such isotropy has also been reported on the FF-1 device [95] (see Section 4.3.2 (2)) and in the UNU/ICTP FF at University of Malaya [413] (see Section 4.3.2 (9)). However, the results on FF-1 need to be reviewed in the light of the results mentioned in Section 4.3.2 (4), Section 4.3.2 (5).

\subsubsection{Deuteron Acceleration, Electron Acceleration, and Hard X-ray Emission Are Separate Phenomena}

It is sometimes tacitly assumed (see, for example, [95]) that the hard X-rays and the neutrons are emitted at the same time. The difference in their flight times is then used to evaluate the neutron energy. The underlying logic is that hard X-rays are produced by fast electrons, neutrons are produced by fast ions, and the same electric field must be responsible for acceleration of both ions and electrons.

PF-1000 data raises doubts on this simple logic. Figure 94 shows that fast electron signal detected by Cerenkov detectors in downstream and upstream directions, soft $\mathrm{X}$ ray, hard X-ray, and neutron signals (time-translated for neutron time of flight at energy $2.54 \mathrm{MeV}$ ) occur at times and with amplitudes which do not have the correlation expected if the soft X-rays were to be generated by heating of the plasma by electron beams, hard $X$-rays were to be emitted by bremsstrahlung of runaway fast electrons and ions were to be produced by beam target interactions between the accelerated ions and the plasma or the neutral gas. Although a clearer identification of all the mechanisms at play is not available at present, the data are sufficient to cast doubt on the commonly held view that electric fields generated by an instability cause acceleration of both ions and electrons at the same time so the time of origin of X-rays and neutrons must be identical.

This is corroborated by three experiments done on small machines (Section 4.3.2 (3), (4), and (5)), which can, in principle, be replicated in many other laboratories. Some of the conclusions are repeated here:

- "The fact that the neutrons are emitted on average $\approx 20 \mathrm{~ns}$ before the formation of an electron beam is hardly compatible with a beam-target model for the neutron production in this device" [409].

- "...the neutron emission on the different directions (radial or axial) present differences in their energy and yield, showing a clear anisotropy with higher values and broader statistical distributions in the axial direction. Furthermore, the results of the observed 
neutron energies on the different directions and the temporal differences of the radiation emission suggest the existence of two temporally separated neutron pulses, which agrees with observations made in larger energy devices." [410].

- "In most of the discharges, it was found that neutrons are originated before HXRs in the axial direction and after HXRs in the radial direction" [411].

Although the conclusions from [410,411] could be questioned on account of poor particle statistics at the detector, that does not apply to the conclusions from [409]. This difference in the time of origin of HXRs and neutrons indicates that electron and deuteron acceleration are caused by different mechanisms. It is worth noting that Boozer's effective parallel electric field (Equation (19) of [406]) contains a term proportional to the mass-tocharge ratio of the charged particle and the rate of change of the direction of magnetic field caused by non-ideal phenomena. This provides a likely scenario where ions and electrons are accelerated by related but different effective electric fields.

The above discussion of experimental results obtained on PF-1000 using multiple correlated diagnostics and convergence between those results and data from other experiments using very different methodologies provides sufficient reason to conclude that the standard narrative, outlined in Section 2.2, is in need of revision. Although the outlines of a new emerging narrative are not quite well-defined at this moment, it is clear the phenomena discussed in this section need to be integral to its structure. This is discussed further in Section 8.

\section{Experimental Results: Special Modes of PF Operation-Wires, Gas Puffing, and Other Gases}

This section summarizes experimental results of special modes of PF operation that include operation with gases other than pure deuterium, wires placed on the axis, special modifications of electrode geometry (conical tips, anti-anode, etc.) and gas puffing, and where the interest lies in the modified phenomenology. This is distinguished from experiments where the plasma focus is used as a means of rapidly transferring current to another plasma load, which is the principal object of interest, rather than the plasma focus itself. Such experiments are discussed in Section 6. There are also experiments which explore electrode geometries other than the classical Mather and Filippov type. These are discussed in Section 7.

The reason for discussing these results separately from the standard mode of PF operation using pure deuterium has to do with uncertainty regarding extrapolation of conclusions derived from such investigations to the standard mode. Several physical phenomena contribute to this uncertainty:

- Formation and propagation of the plasma sheath both depend on material properties of the gas such as various atomic cross-sections and specific ionization energy (Section 3.3.2.3, Section 3.3.3.6). Admixtures of gases with differences in these properties can affect both these aspects.

- High atomic number gases added to deuterium or used as fill gases by themselves have higher resistivity as compared to a pure deuterium discharge. This can contribute to faster development of resistive "tearing mode" instability [132] that causes a uniform current distribution to break up into a non-uniform one that may become manifested as current filaments.

- Higher effective atomic number also implies higher rates of radiation loss that could cause radiative collapse [164] of local magnetic equilibria that might be the mechanism responsible for micropinches.

- Higher average mass of the gaseous species can affect characteristic timescales associated with macroscopic motion of the plasma and the growth rates of some instabilities. They might lead to apparently higher lifetime of structures as compared to those in a pure deuterium plasma.

- In an annular gas puff pinch experiment, a mixture of $90 \%$ deuterium and $10 \%$ argon was found to physically separate into distinct concentric cylinders [365]. This kind 
of mass separation may be present to some extent in plasma focus experiments with heavy gas admixtures with deuterium.

- Gas puffs are likely to introduce turbulent eddies into the initial state of the gas which is different from the stationary uniform density of the standard mode of operation. This turbulence could get amplified while interacting with a supersonic plasma shock.

- Gas puffs may involve condensation of trace amounts of moisture (inevitably present in gases or adsorbed on the surface of the gas puffing apparatus) into water droplets resulting from rapid expansion, causing an unwitting fine scale non-uniformity that might trigger gradient driven instabilities or droplet micro-explosions when the supersonic plasma sheath diffuses through it.

- Electrode modifications, such as conical tips placed on the anode or "anti-anode" structures erected above the cathode, influence boundary conditions of the radial collapse phase and the stagnation phase. They are likely to modify the details of axial shock wave propagation during stagnation. Cross-field axial plasma flows are suspected to be involved in evolution of plasmoidal and toroidal structures and associated dynamo processes. Consequences of modifications of axial flows for emergence and growth of self-organized structures are not fully understood.

- Electrode material from the bare anode $[68,69,146]$ or from conical or other inserts placed at the anode center may enter into the high electric field zone of the pinch phase. This is already demonstrated [44,45] by detection of multiply-ionized electrode material ions of MeV-level kinetic energy in a Filippov device. Influence of high atomic number impurities on the fine structure of plasma focus pinch phase has been demonstrated (Section 2.3.2). Extrapolation of conclusions from experiments with solid anode centers to those with cavities at the anode centers may not always be correct.

Faced with such multiple possibilities, unambiguous and reliable conclusions seem to be out of reach at the present scale of experimental efforts. It would, therefore, be prudent to summarize the state of experimental knowledge of these special modes separately. Extrapolation of conclusions from this research to the standard mode of operation with pure deuterium needs to exercise extreme caution.

On the other hand, these special modes of operation afford a unique glimpse into aspects of plasma dynamics that are not accessible from the standard mode of operation. A consistent understanding of plasma dynamics must be able to account for peculiarities of the special modes of operation using the framework developed from interpretation of the standard mode of operation which is more extensively studied. The purpose of this section is to review the state of experimental knowledge on special modes of plasma focus operation and bring out their noteworthy features.

This section is organized by the facility from which experimental results have been reported. PF-1000 results are discussed first and more extensively because it has more experimental data correlated across multiple diagnostics than any other experiment.

\section{1. $P F-1000$}

In PF-1000 plasma focus facility, operating modes with static filling of deuterium, helium, and neon, and puffing of deuterium, neon, and nitrogen through annular nozzles have been investigated. In some shots, an aluminium wire was placed on the axis. These special modes were used as diagnostic tools for the study of the behavior of internal structures in different gases, of the influence of the conducting material placed on the $z$-axis above the anode on the formation and evolution of internal structures, and of their influence on the production of high energy particles.

This section reviews the experimental results and their interpretations. The interpretations that are not directly supported by experimental data are significant for conceptualizing a unified picture of the behavior of the plasma. They are also expected to provide a stimulus for further experimentation to confirm or refute them. 
5.1.1. Deuterium and Neon Gas Puffs Impacted by Deuterium and Neon Plasma Focus Sheaths

For studying compression of an annular cloud of deuterium by a plasma focus sheath launched in a static filling of either deuterium or neon, a gas puff was injected above the anode $[415,416]$ through an annular nozzle of inner and outer diameters $2.4 \mathrm{~cm}$ and $3.6 \mathrm{~cm}$ which was placed $1.5 \mathrm{~cm}$ below the face of the anode inside a cavity (see Figure 103).

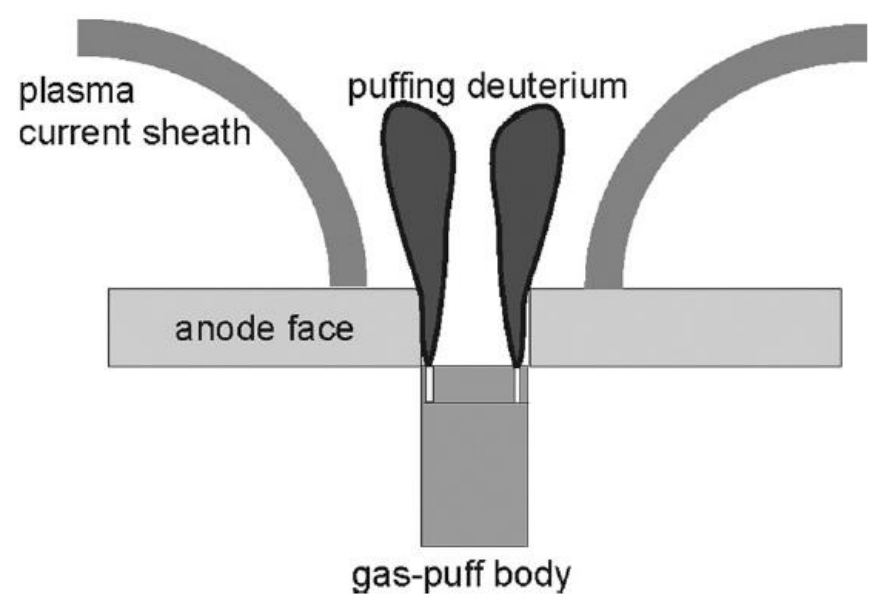

Figure 103. Schematic of the gas puff compressed by the plasma focus current sheath. Reproduced from [415] (Phys. Plasmas 2014, 21, 122706) with the permission of AIP Publishing.

The gas puff valve was operated 2 ms before the capacitor bank ( $350 \mathrm{~kJ}, 1.7-1.9 \mathrm{MA}$ ) with a pressure of $1.5 \times 10^{5} \mathrm{~Pa}$ in a plenum of volume $1 \mathrm{~cm}^{3}$. Diagnostics included interferometry, current and voltage monitoring, time variation of soft and hard X-rays, time integrated soft $X$-ray pinhole camera, neutron emission side on, downstream, and upstream at $7 \mathrm{~m}$ from the anode center.

(1) Inter-comparison of measured parameters of (A) 13 shots with pure deuterium, (B) 8 shots with deuterium plasma focus on deuterium gas puff, (C) 18 shots with pure neon, and (D) 27 shots of neon plasma focus on deuterium gas puff revealed the following notable observations [416] where the uncertainties refer to shot to shot variations in the series.

a. Neutron emission in (A), (B), and (D) was nearly the same $(4.5 \pm 3,5.9 \pm 3,4.0 \pm 3) \times 10^{10}$. Average energy of neutrons measured downstream was nearly the same $\sim 2.85 \mathrm{MeV}$.

b. The implosion velocity was (A) $(1.7 \pm 0.4) \times 10^{5} \mathrm{~m} / \mathrm{sec}$, (B) $(1.5 \pm 0.2) \times 10^{5} \mathrm{~m} / \mathrm{sec}$, (C) $(2.1 \pm 0.2) \times 10^{5} \mathrm{~m} / \mathrm{sec}$, (D) $(1.7 \pm 0.4) \times 10^{5} \mathrm{~m} / \mathrm{sec}$. The corresponding ion energy works out to (A) $0.3 \pm 0.1 \mathrm{keV}$, (B) $0.25 \pm 0.1 \mathrm{keV}$, (C) $4.6 \pm 0.1 \mathrm{keV}$, and (D) $3.6 \pm 0.1 \mathrm{keV}$.

c. There was a marked difference in:

i. $\quad$ pinch length (A) $6-8 \mathrm{~cm},(B) 6-8 \mathrm{~cm}$, (C) $1-2 \mathrm{~cm}$, (D) $1.5-2 \mathrm{~cm}$.

ii. voltage peak (A) $28 \pm 5 \mathrm{kV}$ (B) $28 \pm 5 \mathrm{kV}$ (C) $32 \pm 6 \mathrm{kV}$ (D) $35 \pm 6 \mathrm{kV}$.

iii. minimum pinch diameter (A) $1.4 \pm 0.2 \mathrm{~cm}$, (B) $1.5 \pm 0.2 \mathrm{~cm},(\mathrm{C}) 0.5 \pm 0.1 \mathrm{~cm}$, (D) $0.7 \pm 0.1 \mathrm{~cm}$.

iv. $\quad$ SXR FWHM (A) $44 \pm 10 \mathrm{~ns}$, (B) $65 \pm 10 \mathrm{~ns}$, (C) $40 \pm 20 \mathrm{ns,} \mathrm{(D)} 55 \pm 20 \mathrm{~ns}$.

v. SXR total emission (A) $2.3 \pm 0.3 \mathrm{~V}$-ns, (B) $4.6 \pm 1.0 \mathrm{~V}$-ns, (C) $22 \pm 5 \mathrm{~V}$-ns, (D) $1.0 \pm 0.3 \mathrm{~V}$-ns.

The increase in SXR yield and duration and neutron yield between deuterium PF and deuterium PF on puff show the stabilizing effect of the plasma focus sheath colliding with the deuterium gas puff. Reduction in pinch height has been reported by Ming Fang Lu as a result of an unstable mode of operation (see Section 4.2.4). 
d. The neon plasma focus shot described in the paper has an interesting feature (see Figure 104).

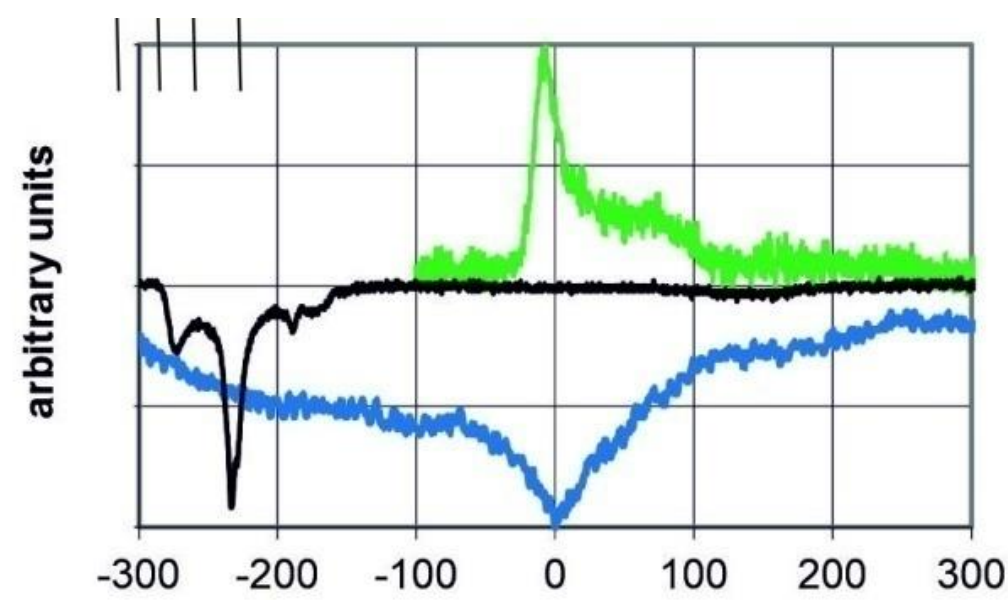

Figure 104. Adapted from Figure 7a of [416] (Phys. Plasmas 2014, 21, 082706). The black trace is the soft X-ray signal from a PIN diode covered with $10 \mu \mathrm{m}$ Be. The green signal is the hard X-ray signal from a scintillator photomultiplier detector in energy range above $100 \mathrm{keV}$ limited by the steel walls in the discharge chamber. The blue signal is the current derivative. The marks in the upper left corner show the time of registered interferograms: $-322,-282,-262,-232$ ns before the current dip minimum.

The soft X-ray signal has three well-marked peaks. Interferograms at $-282 \mathrm{~ns}$ and $-262 \mathrm{~ns}$ show a well formed pinch which is much shorter than the pinch in the standard deuterium operation (see Section 4.2.4). The interferogram at $-232 \mathrm{~ns}$ shows a welldeveloped instability. It would normally be expected that after the instability, the wellformed pinch configuration is permanently destroyed. The occurrence of a sharp hard X-ray peak (which is generally taken to indicate energetic electrons) a short time before the current-derivative minimum is suggestive of significant magneto-plasma activity occurring much after the pinch breakup. There is insufficient information to speculate about the nature of this activity.

The occurrence of the current derivative minimum more than $200 \mathrm{~ns}$ after the destruction of the plasma column (seen in interferometry) and after the soft X-ray signal is difficult to reconcile with the phenomenology inferred from pure deuterium shots in Section 4.3.1.1 A(4)d. The interferogram in Figure 86, taken at the maximum of the azimuthal magnetic field, shows that all the current is located within the already expanding plasma column at $22 \mathrm{~ns}$ past the current derivative minimum. This indicates that in a deuterium plasma focus, the arrival of the current at the axis and the current derivative minimum are simultaneous to within experimental errors, even taking into account possible delays in the occurrence of current derivative minimum at the axis and its detection nearly $2 \mathrm{~m}$ away outside the chamber. This has led to a justifiable conjecture concerning a causal relation between the two events. This conjecture is put in doubt by the observed delay of more than $200 \mathrm{~ns}$ between the destruction of plasma column and the current derivative minimum. Recently, this issue has been discussed in a first-principles framework [364] but the question remains open. This aspect is discussed further in Section 8.2.4.

(2) Observations of filamentation and other structures in the deuterium gas puff impacted by deuterium or neon plasma sheath are reported in $[100,416]$. The principal diagnostics was a 4-quadrant micro-channel plate (MCP) camera, with $80 \mu \mathrm{m}$ pinholes covered with $5 \mu \mathrm{m}$ of polystyrene, which imaged photons above $10 \mathrm{eV}$ with a $2 \mathrm{~ns}$ exposure time, $10 \mathrm{~ns}$ inter-frame separation, and $800 \mu \mathrm{m}$ spatial resolution. One of the four quadrants had much higher sensitivity compared to the other three. Supporting diagnostics was interferometry and soft X-ray PIN diode signals filtered by $10 \mu \mathrm{m}$ Be foils sensitive to photons in the $0.7 \mathrm{keV}$ to $1.5 \mathrm{keV}$ range. 
a. The following pictures in Figure 105 show the XUV frames recorded in different shots at different times with deuterium gas puff impacted with deuterium sheath. Origin of time $t=0$ corresponds to the current derivative minimum and timing errors are mentioned as 2-3 ns. Four kinds of structures are seen in the reported pictures." (i) short filaments with the width about $1 \mathrm{~mm}$ and length up to $1 \mathrm{~cm}$, (ii) spots with the dimension of about $1 \mathrm{~mm}$, [(iii)] two sorts of the long filaments in pictures $(\mathrm{g})$ and $(\mathrm{h})$ with the length up to $(2-3) \mathrm{cm}$ and $1 \mathrm{~mm}$ diameter, and (iv) small balls of a few mm diameter with a regular spherical shape existing usually outside the dense region of the dense column". In Figure 105, the image at -66 ns shows " ... traces of the short filaments passing through the imploding dense plasma sheath, mainly in the direction perpendicular to the column surface... ". The image at $6 \mathrm{~ns}$ was recorded at the "... the maximum of the first SXR, HXR, and neutron pulse ... "for a good shot with neutron yield $(11.1 \pm 0.3) \times 10^{10}$. The image at $29 \mathrm{~ns}$ was also recorded at the peak of X-ray and neutron pulses but for a bad shot with neutron yield $(1.9 \pm 0.3) \times 10^{10}$. This image shows an asymmetric compression, with a larger diameter of column, higher XUV emission and larger number of short filaments. The authors particularly mention:" (In general, we observe higher energetic SXR pulses in shots with the lower neutron yield.)"

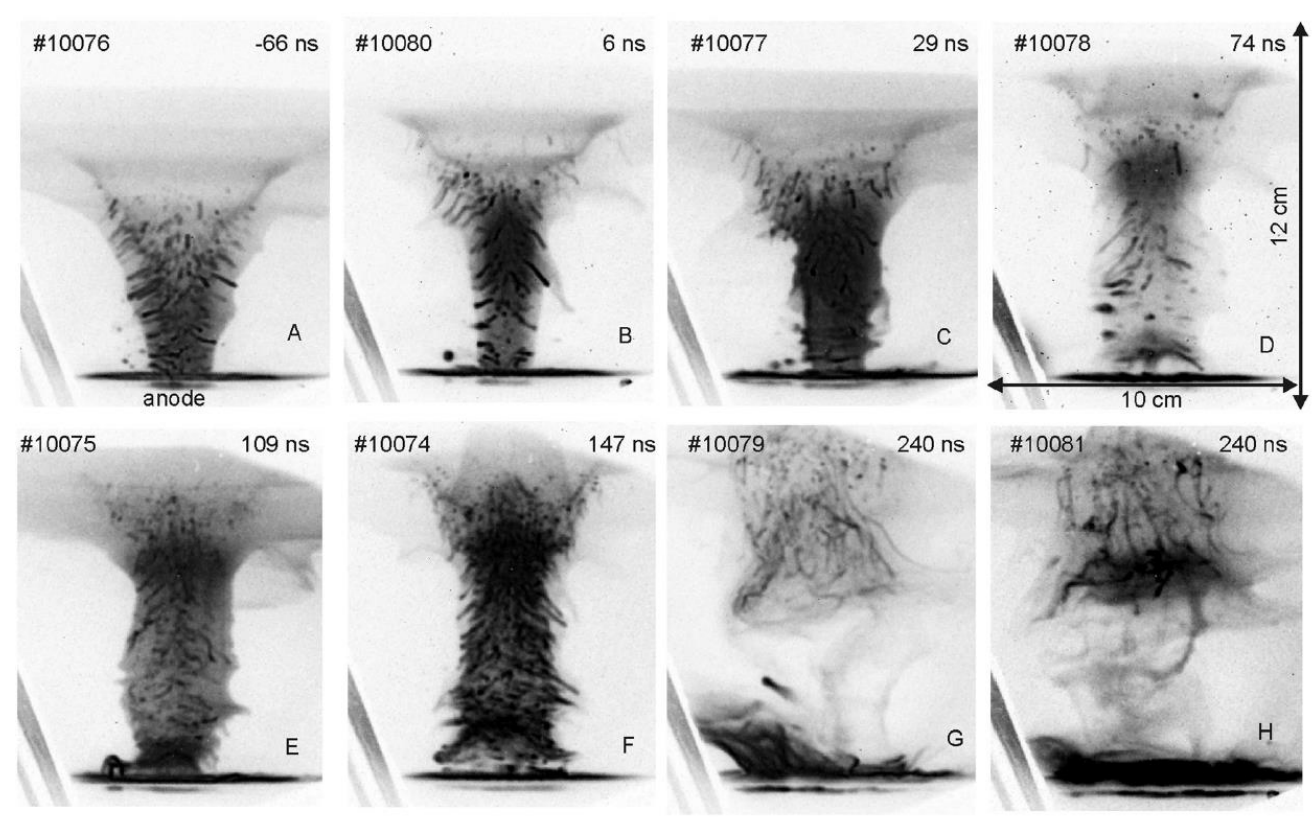

Figure 105. Adapted from Figure 2 of [415] (Phys. Plasmas 2014, 21, 122706) with the permission of AIP Publishing. For details of the experiment, see Figure 103 and text preceding it.

The authors' conclusions for deuterium sheath collapsing on deuterium puff are as follows:

(1) The filaments penetrate the external, probably colder, imploding layer of the pinch, and they can connect the external low-density region with the denser internal region of the pinch.

(2) The short filaments accompany the transformation of internal plasmoidal structures and their number varies proportionally with the magnitude of SXR and neutron production.

(3) The direction of the short filaments has radial (dominant near the anode) and axial (dominant in the region of the first plasmoid) components. 
(4) The fine radiated structure is registered only in shots with deuterium puffing, probably at the lower temperature of the plasma in the external layers of the column. It is absent in shots without deuterium puffing.

(5) The higher intensity of the radiated fine structures can be caused by their higher plasma density and/or temperature in comparison with the surrounding plasma."

These filaments appear to be of a different kind than the filaments described by Bostick and others (see Section 2.3.2). The difference lies in the orientation of filaments with respect to the sheath. The Bostick filaments lie along the sheath, while those described in this report are perpendicular to the sheath.

b. With neon sheath collapsing on deuterium puff, a much smaller number of fainter filaments or spots were observed, at the time of neutron production.

c. The report mentions "In some shots with deuterium puffing, one or more small symmetrical and spherical balls a few $\mathrm{mm}$ in diameter are observed in the interferometry and XUV pictures with both deuterium and neon filling and with deuterium puffing". An interesting example of the evolution of these ball-like structures is shown in Figure 106.
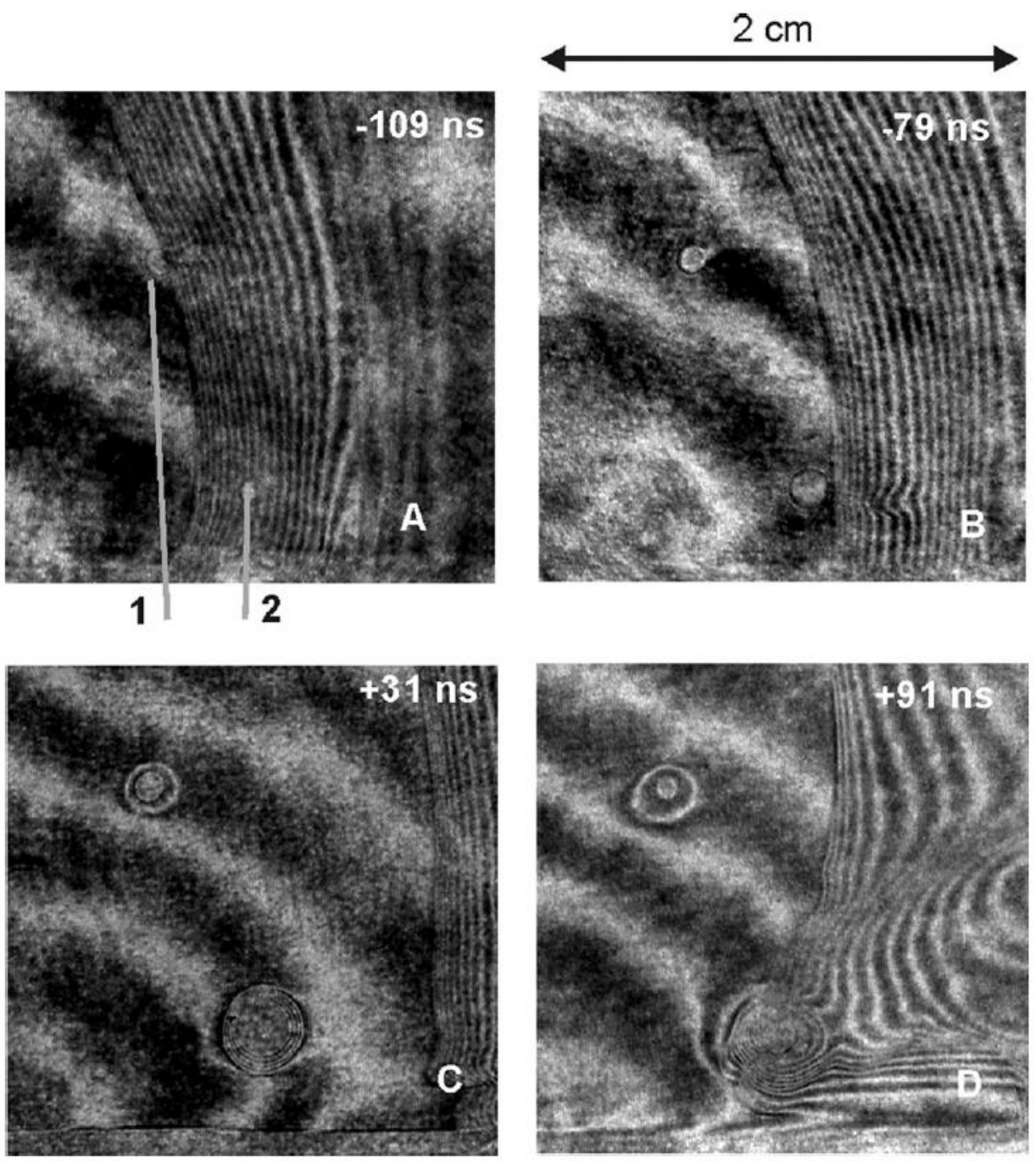

Figure 106. Reproduced from Figure 9 of [415] (Phys. Plasmas 2014, 21, 122706) with the permission of AIP Publishing. Shot \#10080. Evolution of small balls 1 and 2 registered in interferograms. The anode is at the bottom of the pictures and the axis of symmetry of the interferograms is $18 \mathrm{~mm}$ right from the center of ball 2 . The balls appear at the same location in the interferogram while the plasma moves to the right towards the device axis and comes back during expansion. 
The balls appear at the same location in the interferogram while the plasma moves to the right towards the device axis and comes back during expansion. The balls have a clear, surprisingly symmetric internal density distribution that grows both in size from 1 to 5-6 mm and in the number of internal fringes (either too dense to be resolved or blurred by rapid turbulent motion in some portions). The maximum internal electron density is about $1 \times 10^{25} \mathrm{~m}^{-3}$ and average density about $(3-4) \times 10^{24} \mathrm{~m}^{-3}$. Regarding the nature of the balls the authors' write:

"In several interferograms, a small shift of fringes in the ball's surroundings gives an indication of the connection of the ball to the anode or to the dense plasma column. The intense emission of X-rays also shows its high energy density. As a result, the pressure inside of the ball should be higher than in the surrounding plasma and we can speculate that this higher pressure can be caused by the pinched pressure of the current filament flowing in the low density region around the ball body out of the dense column".

Similar investigations of filaments and plasma balls are also reported in [100], already referred in Section 2.3.2 and Figure 19.

While the interpretation of these observations in terms of plasma phenomena $[100,107]$ are certainly an interesting subject of further research, a simpler explanation in terms of an experimental artifact cannot be ignored. The nearly stationary and quite symmetric spherical balls could also be conjectured to be water droplets formed during condensation of trace amounts of moisture (inevitably present in gases or adsorbed on the surface of the gas puffing apparatus) because of Joule-Thomson effect during rapid expansion of deuterium gas (initially at 1.5 bar pressure) through the nozzle. The droplets can persist for the $2 \mathrm{~ms}$ period until the arrival of the sheath and later expand and evaporate. This is supported by the observations [100] "In the experiments described in this paper, the filaments and small plasma-balls appeared only when the gas-puffing was applied".

(3) Addition of nitrogen to the deuterium gas puff impacted by a deuterium sheath revealed [403] axial and radial filaments in the XUV images and sometimes, also in interferometry images (see Figure 107).

B

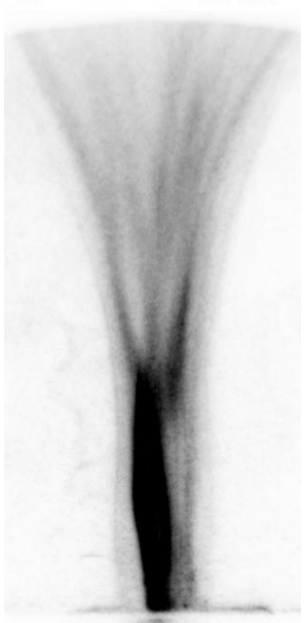

(a)

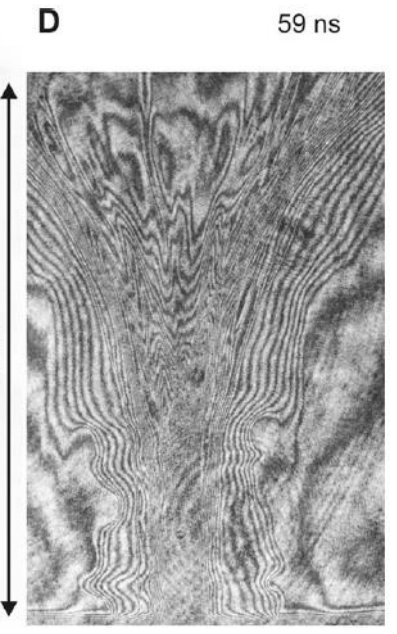

(b)

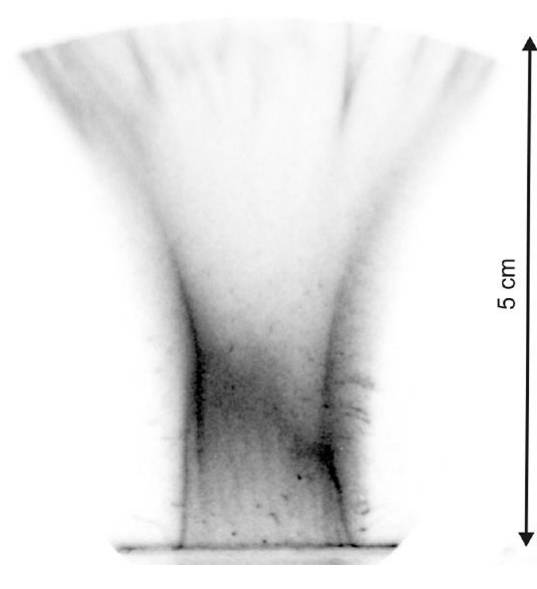

(c)

Figure 107. (a,b) Reproduced from Figure 2B,D of [403]. "The interferometric image (D), which was recorded at the same instant as image (B), seems to show the same current filaments in the upper half of the picture". (c) is reproduced from Figure 3 of [403]. All figures are reproduced or adapted from (Phys. Plasmas 2016, 23, 082704) with permission from AIP Publishing.

(4) An apparently surprising result was observed when the experimental configuration consisted of neon in the gas puff compressed by a deuterium plasma focus sheath [417]—it produced almost the same level of neutron yield as a pure deuterium 
plasma focus. This is explained by the authors as a result of penetration of the deuterium into the neon gas puff. The report contains many details of the interaction between the deuterium sheath and the neon gas puff, of which, Figure 108 provides a good example. 


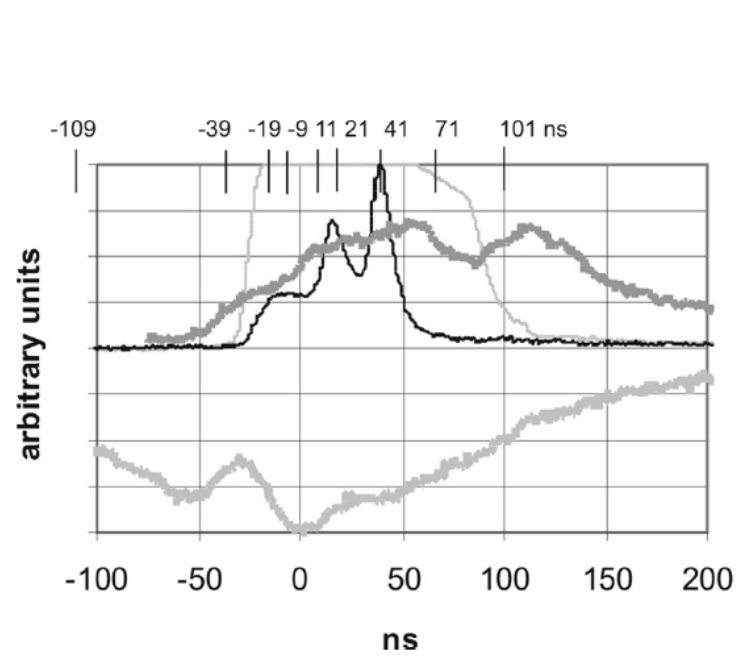

(a)

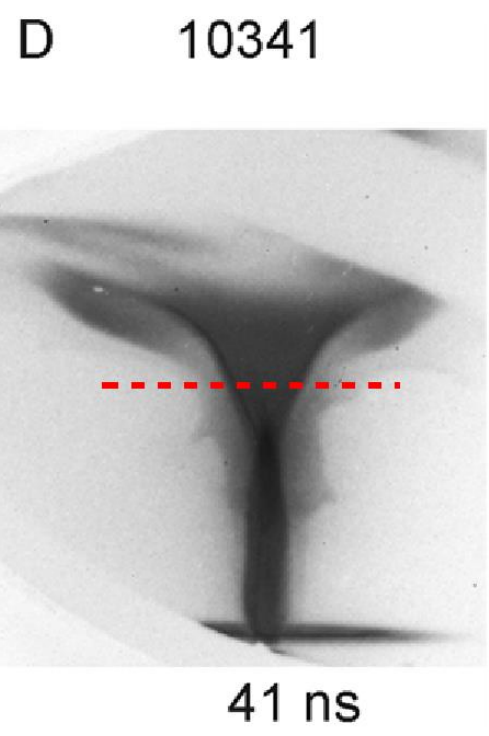

(b)

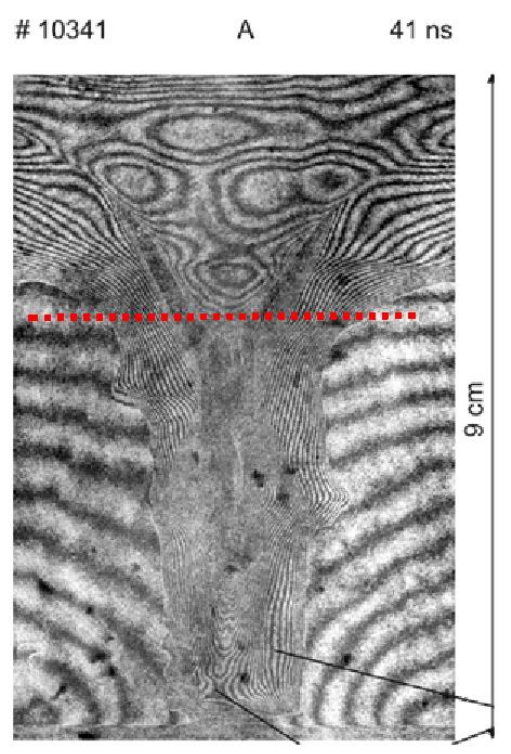

(c)

Figure 108. (a) Shot \#10341.Signals of PIN (with the peak out of oscilloscope screen, thin gray): HXRs (thin black), neutrons (thick dark gray), and current temporal derivative (thick light gray). The side-on neutron signal temporally shifted by the time-of-flight of $2.45 \mathrm{MeV}$ neutrons. Reproduced from Figure 1 of [418], ((b) XUV gated MCP pinhole camera picture at the 3rd peak of HXR emission at $41 \mathrm{~ns}$. Note the dense $\mathrm{Y}$ shaped top with a less dense outer zone surrounding it. Adapted from Figure $4 \mathrm{~d}$ of [418]. (c) Interferogram corresponding to the same instant. Adapted from Figure 6a of [418]. All figures are from Phys. Plasmas 2015, 22, 062705) reproduced or adapted with the permission from AIP Publishing.

The XUV and interferometer images correspond to the 3rd peak of the HXR emission at $41 \mathrm{~ns}$. Neutron and SXR emissions are into their first peak. Both the images show a distinct funnel-shaped structure. The XUV image shows a dark Y-shaped core surrounded by a lighter layer. In the interferometer image, the lighter region has well-resolved fringes while the dark core does not have discernible fringes. Both images show a well-marked boundary between the two regions near the fork of the Y-shape, which persists, without any deviations from its regular shape, in images from -39 ns to $101 \mathrm{~ns}$ in the same shot. The stem of the Y-shape starts showing fringes near the anode (see Figure 108c) at a time that coincides with the peak of the neutron emission.

The authors conclude that the first part of the neutron emission in this configuration coincides with the formation of plasmoids and the second part with the growth of instability in the stem of the funnel shape. During the second part of the neutron emission, several systems of nested closed fringes are seen to form and merge. The dark upper portion of the Y-shape is explained as a region opaque to the laser because of the presence of neon (possibly leading to electron density above the critical density for the laser wavelength) and possibly because of turbulent density fluctuations.

The authors infer the presence of deuterium inside a pure neon gas puff compressed by deuterium sheath from the emission of fusion neutrons. The diagnostic data are interpreted to indicate penetration of the deuterium sheath into the neon gas puff region. However, since the neon puff is emitted into stationary deuterium gas filling the chamber $\sim 2 \mathrm{~ms}$ before the arrival of the sheath, it is reasonable to expect the lighter deuterium gas to have already diffused into the neon gas puff well before the arrival of the deuterium sheath. Therefore, no special mechanism is really required to explain the presence of deuterium inside a "pure neon" gas puff as inferred from fusion neutron emission.

The persistence of a regular boundary between the dense neon-rich upper portion of the Y-shape and the lighter deuterium sheath appears to be contrary to the expectations of gravitational instability theory, where the interface between a lighter fluid pushing a denser fluid is supposed to become unstable to modes propagating along the interface. As one follows the dashed line drawn across the fork of the Y shape in Figure $108 \mathrm{~b}, \mathrm{c}$, it is clear 
that the radial gradient of pressure has large values at the outer and inner boundaries of the Y. The considerations of Section 4.3.1.3 A should then apply. This would imply existence of surface currents and magnetic field at the boundaries of the Y shape. Such surface currents can be visualized in terms of Chandrasekhar-Kendal (CK) modes which have streamlines spanning a toroidal surface [407]. The funnel shape could just be a portion of the toroidal CK mode of the magnetic flux surface that is populated by the plasma. This is also an appropriate place to recall (see Section 2.4.1) the radial confinement of the laser-ablated anode plasma plume observed at the Lebedev Institute.

\subsubsection{Wire Placed on the Axis}

A $280 \mu \mathrm{m}$ diameter $\mathrm{Al}$ wire placed at the anode axis was used as a probe for testing of the possibility of the formation of the organized structures in the pinch column [417]. It was shown that it had no influence on the formation and evolution of the dense plasma structures and also on the neutron production. It increased the plasma density, and the estimated currents around the surface of the wire corona inside the plasmoids. It seems that the currents in plasmoids and wire corona flow independently. The dominant portion of fusion d-d neutrons was produced during the evolution of instabilities, when the uninterrupted wire corona prevented the total interruption of the dense column.

A similar series of shots was carried with helium instead of deuterium and $270 \mu \mathrm{m}$ diameter Al-wire [419]. The velocity of plasma transformations was observed to be decreased. The toroidal structures were found to persist for longer time as compared with a deuterium plasma. The plasma corona around the Al-wire (formed in the axis of the internal structures) impeded the formation of a small diameter of the pinch constriction. This configuration did not prevent the production of hard X-rays with energies above $100 \mathrm{keV}$, similar to those observed with the deuterium filling. In the helium shots, the formation of the plasmoids seems to be more difficult, and their ordering was not as good as in deuterium.

\section{2. $P F-3$}

PF-3 is a Filippov PF device, that currently holds the record for the largest stored energy of nearly $3 \mathrm{MJ}$ (see Section 3.1.6) for an operating plasma focus facility. It does not work well with pure deuterium for reasons not quite well understood-experiments [211] that study "discharges in deuterium" in reality contain a small admixture of a noble gas (e.g., 3 torr $\mathrm{D}_{2}+3 \%$ Xe in Figure 3 of [211]). It is mostly operated with neon and argon. That is the reason why experimental results from PF-3 are discussed under "Section 5 Special modes of PF operation".

In a Filippov plasma focus, the rundown phase of Mather geometry is replaced by a rather long radial compression phase, with a good diagnostic access from the return current conductor facing the anode just $10 \mathrm{~cm}$ away. This has been utilized to study the structure and propagation of the neon or argon [205,206] and deuterium (with Xe admixture [211]) plasma current sheath (PCS) in this phase, using the magnetic and magneto-optical probes described in Section 3.2.2, with the schematic similar to one shown in Figure 109. A significant aspect of these investigations concerns the effect of secondary breakdowns on the efficiency of current transport from the insulator region to the axis, which is the main theme of this section. It also cites relevant results from some other facilities. 


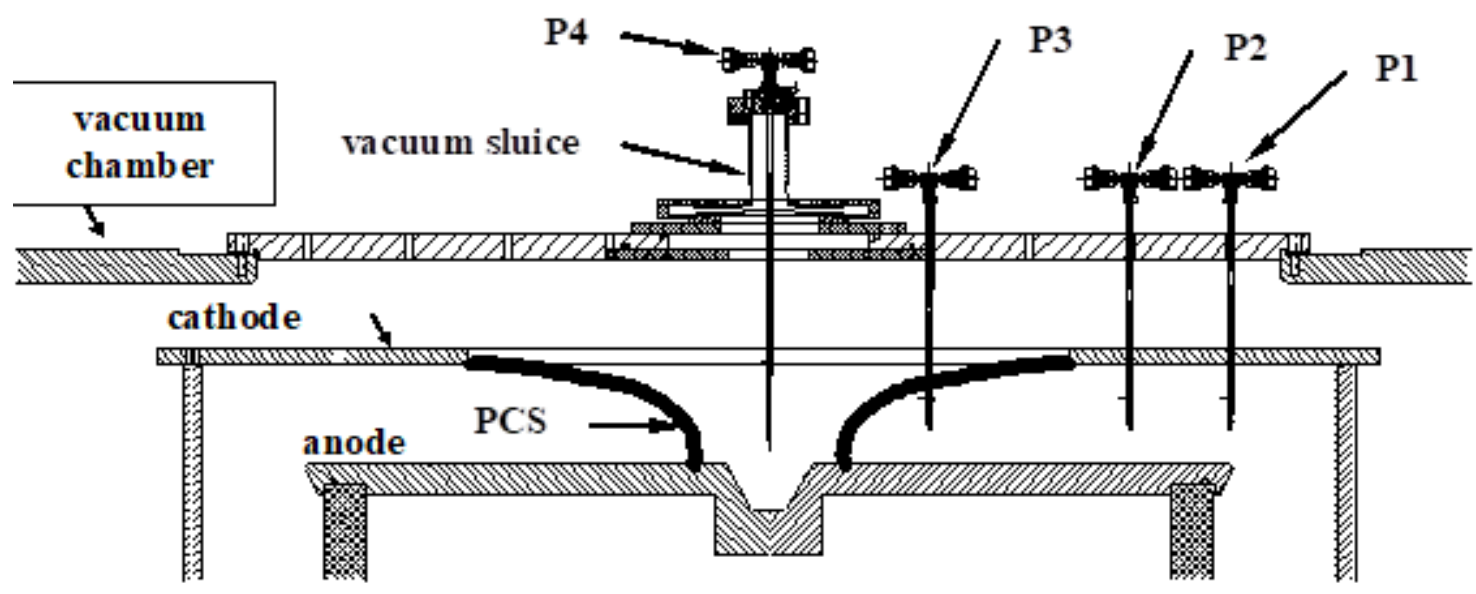

Figure 109. Arrangement of probes in the chamber of the PF-3 facility: (P1-P4) probes for measuring the azimuthal magnetic field at the radii 46, 36, 16, and $2 \mathrm{~cm}$, respectively. Reproduced with permission from Figure 2 of [206], Plasma Physics Reports, 2010, 36, 937-952. (c) 2010. Pleiades Publishing, Ltd.

The following is a summary of salient results:

(1) Analysis of signals from magnetic probes shows that the current growth rate increases from $\approx 10^{12} \mathrm{~A} / \mathrm{s}$ at $\mathrm{r}=46 \mathrm{~cm}$ (anode radius) to $\approx 3 \times 10^{12} \mathrm{~A} / \mathrm{s}$ at $\mathrm{r}=16 \mathrm{~cm}$ and to $\approx 1.5 \times 10^{13} \mathrm{~A} / \mathrm{s}$ at $\mathrm{r}=2 \mathrm{~cm}$. The rate of rise of current in the final stages of the radial collapse is nearly 75 times what the power source is capable of providing. This circumstance makes it possible to consider the plasma focus as a driver for novel condensed plasma loads discussed in Section 6. The rise time of the front of the current pulse decreases from $\approx 5.5 \mu \mathrm{s}$ at $\mathrm{r}=46 \mathrm{~cm}$ to $\approx 1.5 \mu \mathrm{s}$ at $\mathrm{r}=16 \mathrm{~cm}$ and to $\approx 120$ ns near the facility axis. The radial component of the sheath velocity increases from $(1-3) \times 10^{6} \mathrm{~cm} / \mathrm{s}$ at $\mathrm{r}=46 \mathrm{~cm}$ to $\sim 10^{7} \mathrm{~cm} / \mathrm{s}$ in the final stage of the discharge.

(2) The signals from the magnetic and optical channels overlap during the initial stages of radial collapse phase indicating absence of a plasma shock layer distinct from a magnetic piston layer. As the sheath approaches the axis, the dense plasma layer moves ahead of the current carrying layer forming a distinct shock wave followed by a well-separated magnetic piston [211] which carries the major portion of the current.

(3) Estimates show that the thickness of the current carrying layer decreases from $6 \pm 1 \mathrm{~cm}$ at $46 \mathrm{~cm}$ to $3 \pm 1 \mathrm{~cm}$ inside the region of radius $16 \mathrm{~cm}$. In the final stage of implosion inside the region of radius $\mathrm{r}=2 \mathrm{~cm}$, this can be less than $1 \mathrm{~cm}$.

(4) The thickness of the current carrying layer in the near-axis region is numerically equal to the width of the radial distribution of the plasma electron density obtained by laser interferometry in the final stage of implosion (Figure 110) [420,421].

(5) The measurements lead to two important conclusions:

a. At currents of up to $\sim 2 \mathrm{MA}$, almost the entire discharge current can be transferred to the near-axis region of the system (see Figure 111). Within measurement errors, the signals from the probes 1,2 , and 3 coincide with the total current at the corresponding instants. i.e., the current is efficiently transported toward the axis: as the PCS passes near the probes, the current measured by each probe is equal to the total discharge current. The current amplitude at $\mathrm{R}=2 \mathrm{~cm}$ (probe 4 ) is only $0.75 \mathrm{MA}$, which amounts to $\sim 30 \%$ of the maximum current. It should be noted that both the amplitude of this signal and the character of its subsequent decay agree well with the signal from the probe located at the radius $160 \mathrm{~mm}$. Due to its axially diverging shape, the PCS reaches the probe located at a height of $10-20 \mathrm{~mm}$ above the anode plane after the formation of the pinch in the cavity of the conical anode (the signal of the probe 4 reaches the maximum after the peak in the total current derivative). At this instant, the 
current begins to be switched to the insulator region. As a result, after pinching, the signals from all the probes are less than the total current.

b. Taking into account the similarity of the shapes and amplitudes of the decaying signals from the probes installed at the radii $2 \mathrm{~cm}$ and $16 \mathrm{~cm}$, the current at the instant of pinch formation can be estimated with high reliability from the current value provided by the probe installed at $16 \mathrm{~cm}$. The significance of this conclusion lies in its role in estimating the prospects and capabilities of future high-current PF facilities. This result is not trivial, because the efficiency of current transfer depends strongly on the operating mode of the discharge. An increase in the voltage across the discharge gap due to an increase in the load impedance and a sharp increase in the pinch resistance at the instant of maximum compression, as well as other factors, can cause a shunting breakdown of the discharge gap near the insulator after the singularity or even earlier, as was observed in the example presented in Figure 112.

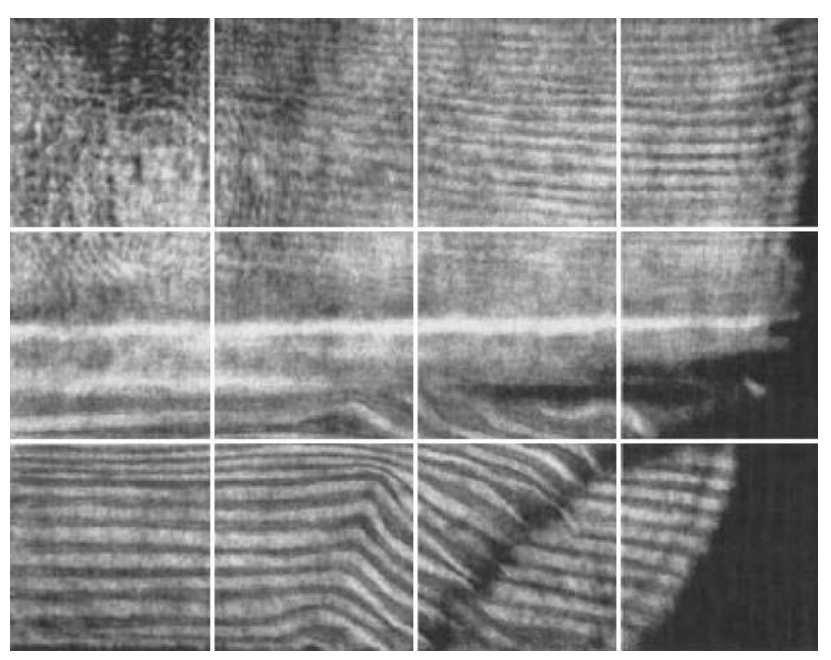

(a)

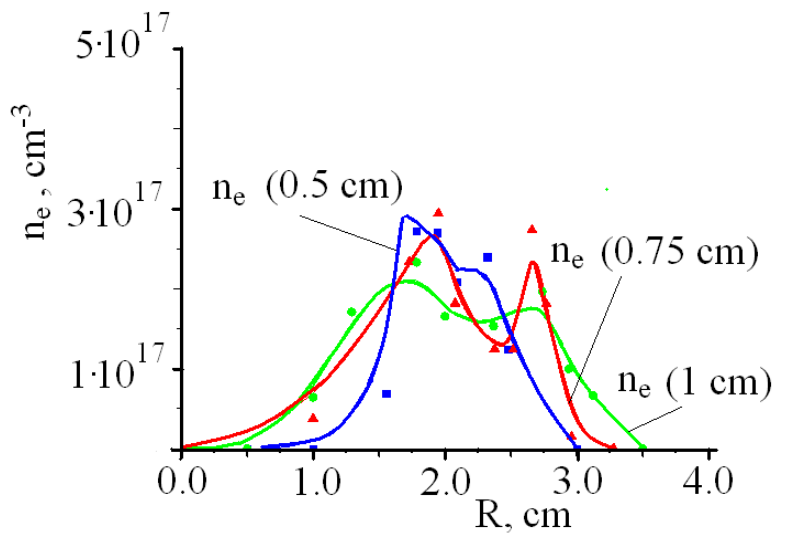

(b)

Figure 110. (a) PCS interferogram obtained in a PF-3 discharge $20 \mathrm{~ns}$ before the arrival of the sheath front at the axis for a neon pressure of 1 Torr and stored energy of $\mathrm{W}=360 \mathrm{~kJ}$. Scale $1 \mathrm{~cm}$. (b) Electron density profiles at distances of $0.5 \mathrm{~cm}$, $0.75 \mathrm{~cm}$, and $1 \mathrm{~cm}$ from the anode plane. Adapted from authors' original data also presented in Figure 3a,c of [420].

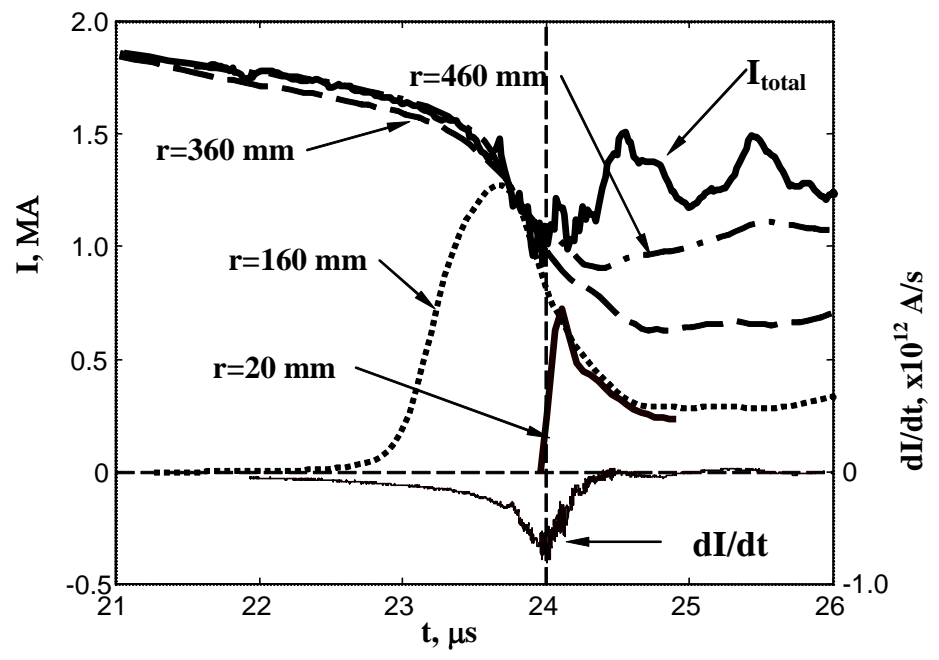

Figure 111. Current measured with magnetic probes in different positions and total current derivative dI/dt. Reproduced with permission from Figure 6 of [208] (IEEE Trans. Plasma Sci. 2010, 38, 92-99, (c) 2021 IEEE). 


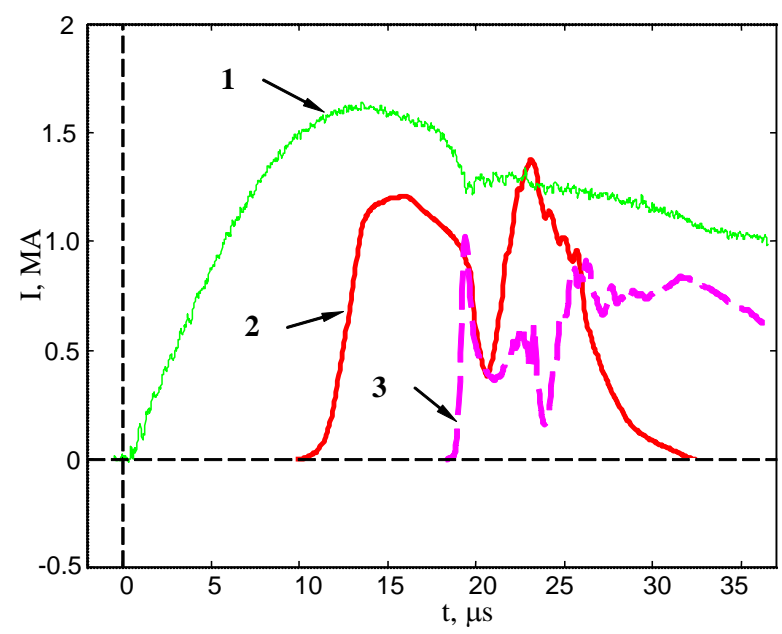

Figure 112. Discharge in $\mathrm{Ne}, \mathrm{P}_{0}=1.5$ Torr, $\mathrm{W}=290 \mathrm{~kJ}$ : Total discharge current measured by the integrating Rogowski coil (curve 1) and currents measured by the probes installed at the radii 46 and $16 \mathrm{~cm}$ (curves 2 and 3, respectively). The probes are installed $2.6 \mathrm{~cm}$ above the anode [422].

The character of signals from magnetic probes indicates the formation of autonomous closed current loops disconnected from the main discharge circuit as shown in Figure 113.

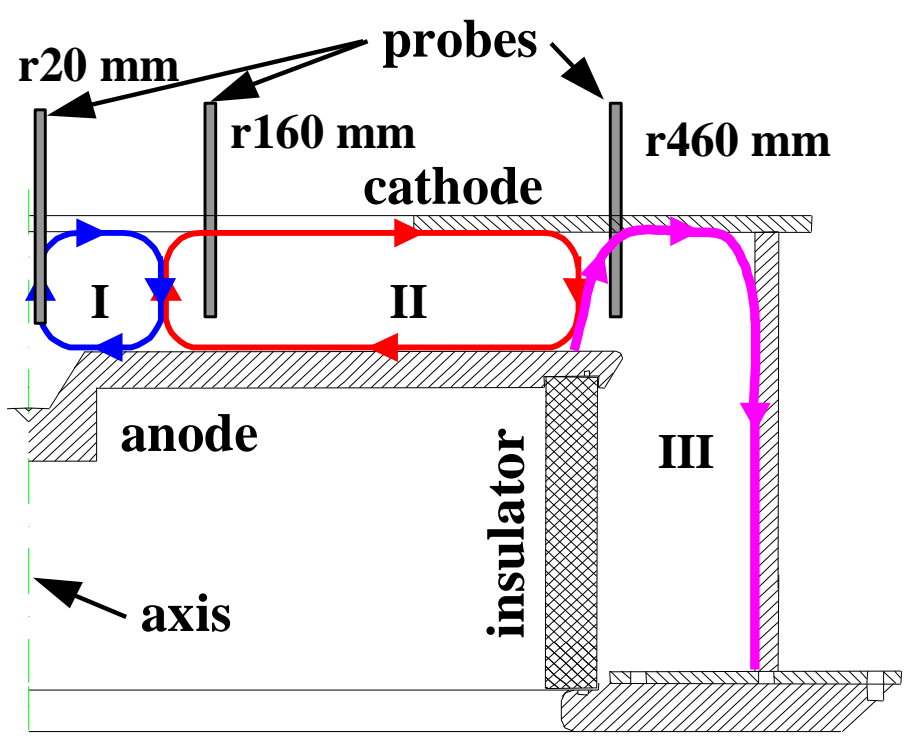

Figure 113. Scheme illustrating the formation of closed current loops in the electrode gap. Reproduced with permission from Figure 11 of [206] Plasma Physics Reports, 2010, 36, 937-952. (C) 2021 Pleiades Publishing Ltd.

The data confirming this conclusion were also obtained on the PF-3 facility with the use of small-size Rogowski coils mounted in the return current conductors [423], as well as on some other facilities with the use of magnetic probes [424,425] (see Section 4.2.2 (1)).

The effect of the formation of closed current formations was observed in experiments on a small installation ( $\mathrm{W}=540-600 \mathrm{~J}$, Imax $=75 \mathrm{kA}$ ) [426] (see Figure 114). 


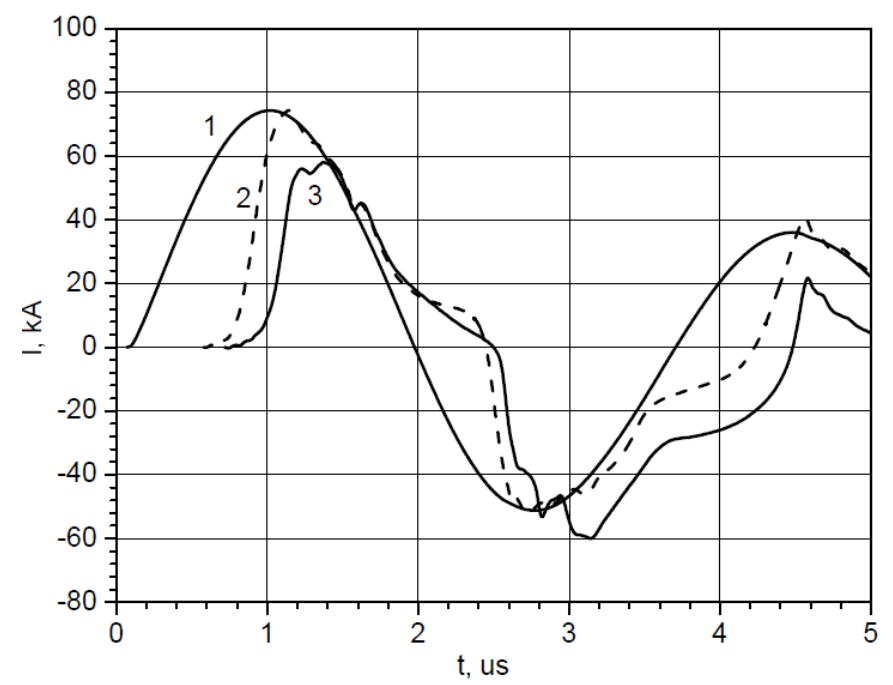

Figure 114. Waveforms of the total current measured by the Rogowski coil (1) and currents obtained by integration of magnetic probe signals $(2,3)$. The positions of the probes with respect to the anode top are $-5 \mathrm{~mm}$ (curve 2-dashed) and $0 \mathrm{~mm}$ (curve 3). Facility PFV-600 (W= $540 \mathrm{~J}$ ). Adapted from Figure 5b of [426] (Plasma Physics Reports, 2014, 40, 146-159) (c 2021 Pleiades Publishing Ltd.

Sometime after PCS compression on the axis (the dip in the current time derivative), the current measured by the probes becomes oppositely directed to the total current. This indicates the development of a secondary breakdown, most probably near the insulator. In this case, a new PCS forms near the insulator and the probe detects the magnetic field inside a certain closed current formation arising near the anode end. After the arrival of the second PCS, the direction of the current detected by the probe changes abruptly and coincides again with the direction of the total current. The shunting of the discharge current by the residual plasma is directly related to the efficiency of snowplowing of the working gas by the PCS as it propagates from the insulator. Strong magnetic field of the discharge current behind the PCS is one of the key properties of the PF discharge, which provides its operation in the PF mode. Shunting breakdowns probably occur through the ionized plasma remaining after the passage of the CS, provided that magnetic isolation is disturbed. In $[208,427]$, the density of the residual plasma at which it can be considered un-magnetized was estimated. Taking into account that plasma can be assumed un-magnetized when $\omega_{\mathrm{ce}} \tau_{\mathrm{ei}}<1$ (where $\omega_{\mathrm{ce}}$ is the electron cyclotron frequency and $\tau_{\mathrm{ei}}$ is the electron-ion collision time), it was shown in both those papers that, for typical discharge parameters, current shunting can occur when the snowplowing efficiency becomes lower than $80 \%-90 \%$. The second CS formed in this case snowplows the residual plasma toward the axis, thereby reducing its density. If the residual plasma density decreases below a certain threshold value, no subsequent shunting breakdowns occur.

\subsection{Other Devices}

This section discusses special mode investigations from other devices which were not as extensively pursued as from PF-1000 and PF-3.

\section{POSEIDON at Stuttgart, Germany}

The motivation of exploring gas puff operation of the $500 \mathrm{~kJ}$ POSEIDON facility [428] was "to control and possibly optimize the interaction of a well-formed current sheath with a gas-puffed target formed in front of the anode upper surface at higher bank energy $(>100 \mathrm{~kJ})$ than in experiments performed so far". "The working gas (pure deuterium, deuterium with admixtures, or pure noble gas)..." was injected "... through 16 specially shaped $5 \mathrm{~mm}$ diameter holes arranged symmetrically at $\mathrm{r}=28.5 \mathrm{~mm}$ in the front plate of the inner electrode. Depending on the initial pressure... of the working gas and the time delay... of the PF discharge with respect to the triggering of the valve, the injected 
gas forms a tubular gaseous curtain or a quasi-spherical gas cloud in front of the inner electrode plate". The following results were reported:

a. Schlieren pictures showed that "the puffing of the Ar gas target in front of the inner electrode plate induces a distinct rise of microturbulence within the collapsing plasma sheath".

b. "For shots with $\mathrm{D}_{2}$ filling and Ar puffing considerable changes in the radial, compression phase were observed. Argon streams, as injected by the valve, form a turbulent gas target, which can hardly be compressed by the current sheath. Framing pictures taken with the pulsed planar diode prove, however, that the pinch column can be formed above the gas cloud...".

c. "...schlieren pictures show that the puffing of an Ar admixture induces stronger turbulence and changes the pinch formation. With a relatively short discharge delay time $\left(t_{d}<450 \mu \mathrm{s}\right)$ the Ar target is formed only in the close proximity of the inner electrode front plate...Even in that case the radial compression of the heavy gas target is not very efficient, and the pinch column exhibits strong MHD instabilities.".

d. "...by appropriate gas puffing one can obtain PF discharges with numerous 'hot spots' inside the pinch column, ...".

e. Using $\mathrm{D}_{2}$ puffing, with optimized valve pressure and delay, “...the neutron yield could be increased by about $30 \%$ in comparison with operation without gas puffing. For non-optimum gas target properties the neutron yield was usually reduced, whereas its reproducibility from shot to shot was generally increased".

f. Schlieren pictures taken during the radial compression phase show "distinct streams of the gas injected" and the resulting turbulence.

g. "...soft X-ray observations have also confirmed the turbulent character of the current sheath collapse in the cases when a relatively dense gas target was introduced...".

2. SPEED-II at Düsseldorf, Germany:

The SPEED-II plasma focus was used to reproducibly generate and study micropinches $[117,154,155]$ using a deuterium plasma focus discharge to compress an argon cloud that was injected above the anode through a fast electrically operated valve opened a few milliseconds before the plasma focus discharge. Too short an interval between the gas valve and plasma focus trigger led to pure deuterium pinches without any micropinches. Too large a delay caused failure to launch a proper plasma focus sheath "Optimum deuterium filling pressure (5 mbar) and valve-discharge delay $(6.5 \mathrm{~ms})$ result in bright deuterium-argon pinch columns within which micropinches are actuated in an orderly manner along the axis of symmetry". Although filament-like features are apparent in both time integrated and time resolved soft $\mathrm{X}$-ray pictures, the authors have not claimed to have observed filaments. Micropinches have been observed in these experiments and discussed in Section 2.3.2.

\section{PACO device in Tandil, Argentina}

Effect of non-uniform deuterium gas distribution on neutron emission was investigated on the PACO device [429] by creating a "gas cloud" and a "gas jet" using a fast electric valve operated with an auxiliary capacitor bank. The gas cloud was created with gas injected in the device through the back of the anode via holes located circumferentially halfway along the anode length. The gas jet was created by using a nozzle to inject gas at the center of the anode. In the static, gas cloud and gas jet modes, the best neutron yield was $5 \times 10^{8}, 6 \times 10^{8}$, and $1.7 \times 10^{9}$ while the fluence anisotropy was 2.1 (static $1 \mathrm{mbar}$ ), 1.9 (static 2 mbar), 3.25 and 3.38 .

\section{DPF-78 in Stuttgart, Germany}

Dynamics of DPF 78 operated at $60 \mathrm{kV}, 28 \mathrm{~kJ}$ in either pure deuterium or in admixtures (up to $60 \%$ by mass) of different combinations of neon, argon, and krypton was studied using multiple diagnostics [430]. The fill pressure of the discharge ranged from below 1 mbar to over 5 mbar of deuterium. When different admixtures were used, a near constant 
equivalent fill mass was adopted to minimize the effect on the collapse time so that valid comparisons could be made between the different impurity types and concentrations. The salient observations were:

a. In pure deuterium discharges, "[t]he classical formation of the dense pinch column, some $3 \mathrm{~mm}$ in diameter, at the end of the radial collapse, is observed to begin first near the anode and then extending to a length of about $12 \mathrm{~mm}$ in front of the anode. After the compression stage, the expanded plasma maintains the cylindrical structure whose border is detected in the Schlieren photographs. At late time, the expanded plasma column appears to be disrupted and no longer confined. However, with the smaller aperture in the Schlieren system, the photographs clearly show that a large plasma column, some 15 to $20 \mathrm{~mm}$ in diameter, still remains at this time."

b. "In most discharges with pure deuterium, the electron density gradient, and hence the refractive index gradient is much stronger on the outside of the sheath compared with that from the inner surface, closer to the pinch axis."

c. "In admixtures of deuterium doped with neon up to $60 \%$ by mass, it is found that the stable plasma column extended to about $15 \mathrm{~mm}$ in length, longer than in discharges with pure deuterium at an equivalent mass. The sheath structure is clearly defined on both sides of the collapsing plasma shell, with a thickness similar to that observed in pure discharges. There is a smaller variation in thickness along the length of the collapsing column. In general, the plasma column remains grossly stable for over $30 \mathrm{~ns}, \ldots$ This is longer than that in discharges with pure deuterium.".

d. "The rapid expansion observed in pure discharges is absent at high doping level, but strong surface perturbations can be found throughout the evolution of the pinch. These surface perturbations could be traced back to that appearing early on in the sheath during the radial compression. Measurements from the double frame system indicate that they are formed as the plasma sheath emerges from the axial run down phase and turns around the edge of the anode. These perturbation remains more or less frozen in until pinch time and tends not to destroy the integrity of the collapsing column. The upper part of the column appears to be less sensitive to disturbances and hence more stable. These perturbations either have a ring like shape around the surface of the plasma column... or are helical around the plasma column...".

e. " "...for low $\mathrm{Z}$ doping (neon) the plasma column is grossly stable for more than $30 \mathrm{~ns}$ but multiple necking begins to occur some 15-20 ns after first pinch formation in high $Z$ doping (krypton). The plasma column is observed to expand after first compression at low level of low $\mathrm{Z}$ doping, with a larger expansion for pure discharges. Rayleigh Taylor type instabilities are observed to grow in the expanding plasma column. With pure discharge, a re-pinching is often observed at high z position and is correlated to the onset of a second period of neutron emission. In the case of high $\mathrm{Z}$ doping, no global expansion of the plasma column is observed...".

Another study on DPF 78 [431] investigated the effect of high Z admixtures of neon, argon, and krypton to deuterium on the polar distribution of the neutron emission, as well as on the total yield. The pure deuterium pressure required to match the arrival of the plasma at the axis with the peak of the current was 5 mbar and the mass density of the admixtures was always kept equal to the mass density of deuterium at 5 mbar. Five leadshielded plastic scintillators [414] wrapped with fluoresecent optical fibers coupled with a normal plastic optical fiber (see Section 4.3.2 (10)) to remote, lead-shielded photomultiplier tubes were deployed in a polar array just $50 \mathrm{~cm}$ away from the focus. They were crosscalibrated against each other by placing them together from time to time. The key findings of this report are given below:

i. $\quad$ For each admixture studied, the shot-averaged total neutron yield decreased monotonically with the mass fraction of the high $\mathrm{Z}$ dopant.

ii. Time resolved polar distribution of neutron emission shows that the $90^{\circ}$ neutron signal in pure $\mathrm{D}_{2}$ has FWHM of $146 \mathrm{~ns}$, while at $0^{\circ}$ it is $170 \mathrm{~ns}$. With admixtures, 
the FWHM at $0^{\circ}$ increases and progressively displays evidence of a double pulse structure, while that at $90^{\circ}$ decreases.

iii. " "...the polar pattern of the neutron emission becomes more and more peaked in the forward direction in the second period with increasing Z.".

iv. "...in the forward direction (end-on) the width of the neutron pulse does not change substantially, but the amplitude increases by a factor of two for the krypton doped discharge compared with the argon doped. On the other hand, in the side-on direction, the neutron pulse is much narrower in the krypton-doped discharge.".

v. The neutron and HXR emission begins before the tight pinch formation in the final stages of stagnation.

vi. " .... there is no direct correlation between the neutron yield and the appearance of the hard X-ray emission.".

vii. "The results obtained in this experiment indicate that there is no correlation between the presence or the number of hot spots and the total neutron yield".

\section{5. "FMPF-1" in Singapore}

This fast miniature plasma focus ( $200 \mathrm{~J}, 2.4 \mu \mathrm{F}, 27 \mathrm{nH}, \mathrm{T}_{1 / 4} \sim 400 \mathrm{~ns}$ ) was operated [432] with varied concentrations of $\mathrm{D}_{2}-\mathrm{Kr}$ admixtures. The maximum neutron yield with admixture operation increased by 30,20 , and 1.2 times for $\mathrm{D}_{2}+2 \% \mathrm{Kr}$ (volumetric concentration), $\mathrm{D}_{2}+5 \% \mathrm{Kr}$, and $\mathrm{D} 2+10 \% \mathrm{Kr}$, respectively, as compared to pure deuterium operation at 3 mbars. Neutron and HXR signals recorded by 3 detectors comprising NE102A plastic scintillator and EMI 9813B photomultiplier tube showed FWHM of $7 \pm 1$ and $9 \pm 1$ ns for pure deuterium discharges and 25-35 ns and 20-25 ns for various admixtures with $\mathrm{Kr}$. The signals with admixtures appeared to consist of multiple pulses. The FWHM of the current derivative dip also increased with the increase in $\mathrm{Kr}$ concentration.

Another study on the same device [433], on the other hand, concludes "...for an optimized plasma focus device $\mathrm{D}_{2}-\mathrm{Kr}$ admixtures may give little or no neutron yield enhancement relative to pure $\mathrm{D}_{2}$ operation". They suggest that "...the admixture operation is an evaluation methodology to determine the level of optimization of device geometry".

\section{UNU/ICTP PFF $(15 \mathrm{kV}, 3.3 \mathrm{~kJ})$ at the University of Malaya}

The neutron yield, as well as the width of the neutron time profile [413,434], was found to change with the percentage of argon admixture to the deuterium gas. The neutron yield measured with indium activation was highest (about $80 \%$ higher than pure deuterium) at $30 \%-40 \%$ of argon by mass. The detectors (described in Section $4.3 .2(9)$ ) were placed [434] as follows: $\mathrm{CH} 1$ at a distance of $41 \mathrm{~cm}$ at $15^{\circ}$ to the axis, $\mathrm{CH} 2$ at a distance of $50 \mathrm{~cm}$ at $50^{\circ}$ to the axis, $\mathrm{CH} 3$ at a distance of $101 \mathrm{~cm}$ at $90^{\circ}$ to the axis, $\mathrm{CH} 4$ at a distance of $50 \mathrm{~cm}$ at $90^{\circ}$ to the axis, $\mathrm{CH} 5$ at a distance of $80 \mathrm{~cm}$ at $0^{\circ}$ to the axis, $\mathrm{CH} 6$ at a distance of $47 \mathrm{~cm}$ at $25^{\circ}$ to the axis. The resulting neutron pulse shapes were interpreted as being a superpostion of two neutron pulses (see Section 4.3.2 (9)). For argon doped discharges [413], the second pulse was dominant ( $60 \%$ of yield) and had energy $4.2 \pm 0.8 \mathrm{MeV}$ at $0^{\circ}$ and $2.8 \pm 0.3 \mathrm{MeV}$ at $180^{\circ}$. The weaker first pulse had energy $2.8 \pm 0.4 \mathrm{MeV}$ at $0^{\circ}$ and $2.8 \pm 0.3 \mathrm{MeV}$ at $180^{\circ}$. The dominant second pulse anisotropy was 1.5 and the weaker first pulse anisotropy was 1 .

The Maxwellian curve fitting technique enabled the authors to translate the two pulses back in time to the time of the voltage maximum which is defined as $t=0$. "The calculated first phase of neutron emission at time zero has a FWHM of about $20 \mathrm{~ns}$. The pulse starts right after the peak of the voltage signal... However, the voltage probe may not show the true peak of the signal as it rises rapidly to a much higher value, and is partially shunted by the crowbar effect due to a secondary breakdown at the insulator. In this case, the start of the first neutron pulse will take place during the final pinching of the plasma, before the stagnation on axis". "The time of occurrence of the second phase, which is 20 ns after the first phase is in agreement with the disruption phase as shown in the shadowgraphy observation of a similar $3 \mathrm{~kJ}$ plasma focus device." 


\section{7. $500 \mathrm{~J} \mathrm{PF}$ at Alameda Applied Sciences Corporation, San Leandro, USA}

This device [435] " ... ha[d] an alumina insulator sleeve extending $4.8 \mathrm{~cm}$ above the cathode plane. Two anodes were studied. Both [we]re composed of W/Cu (20\%). A cylindrical anode with a 20-mm length and a 20-mm rundown with a bore hole of $17 \mathrm{~mm}$ in the center [was] the baseline anode. A second anode with a conical profile (20-mm base diameter converged to a 5-mm diameter over the rundown) also had a 20-mm active length, and a bore hole of $17 \mathrm{~mm}$ diameter [??]. The cathode ha[d] 16 3-mm-diameter SS304 rods that [we]re of the same length as those of the anodes.". The bank capacitance and inductance were $6 \mu \mathrm{F}$ and $17 \mathrm{nH}$. Its normal operation at $13.2 \mathrm{kV}$ produced peak currents from 120 to $140 \mathrm{kA}$, depending on the gas fill. The conical anode was found to have a 5 times higher neutron yield that was optimized at a 2.3 times higher pressure of pure $\mathrm{D}_{2}$. as compared to the cylindrical anode. The addition of $2 \%, 4 \%$, and $8 \%$ (by mass) of argon improved the neutron yield in the cylindrical anode case by a maximum of 3.6 times but not in the conical anode case.

\section{Gemini device at Las Vegas, USA}

An investigation regarding the effect of krypton admixture in deuterium on neutron yield in the Gemini plasma focus (see Section 3.1.4) reports [436] the following observations:

a. There is a 1.7 times increase in neutron yield operating at 2.66 MA peak current, 11 torr deuterium with a rundown time of $6.56 \mu$ s caused by addition of $0.1 \% \mathrm{Kr}$ by volume. A similarly high level of yield enhancement was not observed when operating at 2.26 MA.

b. Both side-on and end-on neutron time of flight signals show two pulses for pure deuterium but "... addition of krypton at both $0.1 \%$ and $1 \%$ alters the double peak structure to a predominately single peak".

c. Addition of krypton reduces the neutron signal FWHM. "Increasing the voltage/current also reduces the FWHM, for example, from $240 \mathrm{~ns}$ at $0.1 \% \mathrm{Kr}$ for $35 \mathrm{kV}$ to $160 \mathrm{~ns}$ at $0.1 \%$ $\mathrm{Kr}$ at $40 \mathrm{kV}$.

d. "The observed flux asymmetry (end/side) for most shots is less than 1".

e. The end-on neutron time of flight signal begins earlier than the side-on signal, indicating a higher neutron energy end-on.

9. Sahand Filippov type facility at Tabriz, Iran

The Sahand, Filippov type, dense plasma focus device $(288 \mu \mathrm{F}, 25 \mathrm{kV}, 90 \mathrm{~kJ}, 1.1 \mathrm{MA}$, has a $50 \mathrm{~cm}$ diameter copper disc anode, a $48 \mathrm{~cm}$ diameter, $11 \mathrm{~cm}$ height, cylindrical ceramic insulator. The discharge chamber, $76 \mathrm{~cm}$ in diameter and $26 \mathrm{~cm}$ in height, acts as a cathode for current return. A report [437] of its operation with a mixture of deuterium and krypton finds:

"The highest average neutron yield of $2.2 \times 10^{9}$ neutrons per shot was achieved at 1.00 Torr deuterium with $3 \%$ krypton at charging voltage of $18 \mathrm{kV}$ which was about an order of magnitude higher than the highest neutron yield for any combination of gas pressure and operating voltage for pure deuterium operation. The krypton seeding of deuterium not only enhanced the neutron yield but also made the neutron emission more reliable over wider operating pressure range from 0.25 to 1.00 Torr. The estimation of average neutron energy using time-of-flight method showed that the maximum and minimum average energy are $2.98 \pm 0.6 \mathrm{MeV}$ at $16 \mathrm{kV}$ in $0.25 \mathrm{Torr}+3 \% \mathrm{Kr}$ and $2.09 \pm 0.2 \mathrm{MeV}$ at $18 \mathrm{kV}$ with 0.5 Torr $+3 \% \mathrm{Kr}$, respectively"

\section{DENA Filippov type facility at Teheran, Iran}

The DENA Filippov type plasma focus $(25 \mathrm{kV}, 288 \mu \mathrm{F})$, was also operated [438] with a deuterium-krypton mixture. One of the significant findings is: "The dip of negative spike in the current derivative signal for the case of krypton admixture is four times more than deuterium only gas discharges". 


\section{Plasma Focus as Driver for Condensed Targets}

One of the interesting directions in PF research is experiments with diverse plasmaproducing media. This direction was originally initiated by studies of the possibility of using PF as a driver for magnetic compression of liners [439,440]. The basic idea is that $\mathrm{PF}$ is a magnetic energy storage with power amplification in the final stage of discharge (see Section 5.2). The energy of the power source (regardless of its type) is transformed into the kinetic and magnetic energy of the plasma-current sheath (PCS) at the stage of its movement to the axis of the discharge system. In the classical PF discharge scenario, due to the outflow of a significant part of the raked plasma, in the final part of the compression, the energy of the sheath is transferred to a small number of particles, which leads to the formation of a dense hot pinch. However, if a conductive liner is placed on the system axis, the sheath current can be transferred to this liner. Current rise time on the load will be determined in the first approximation by the current layer thickness, $\delta$, and by the radial sheath velocity, $\mathrm{V}_{\mathrm{r}}$. At the real accessible parameters $\mathrm{I} 33 \mathrm{MA}, \delta \sim 1 \mathrm{~cm}$ and $\mathrm{V}_{\mathrm{r}} \sim 10^{7} \mathrm{~cm} / \mathrm{s}$, it is possible to achieve a rate of a current rise on a load

$$
\mathrm{I} \sim \mathrm{I}\left(\mathrm{V}_{\mathrm{r}} / \delta\right) \geq 3 \times 10^{13} \mathrm{~A} / \mathrm{s}
$$

One of the key issues in such experiments is the efficiency of current switching-over.

The first experiments on the interaction between the mega-ampere PCS of microsecond plasma focus and an agar-agar foam load at the PF-1000 facility demonstrated promising results [439]. The formation of homogeneous plasma of the foam load during its interaction with the lighter PCS was observed. Later, similar experiments were also carried out with other types of loads. A thin-walled foam liner with a diameter of 10-20 mm [421,440], tungsten wire arrays [440-443], fine aluminium wires and deuterated polyethylene fibers [441,444], fine-dispersed submicron alumina dust powder $[421,440,441,445]$, and other loads were employed. The possibility of load compression after the current is switched over from a non-cylindrical PCS to different types of loads was demonstrated in experiments carried out at the PF-3 facility. Scheme of the experiments is shown in Figure 115.

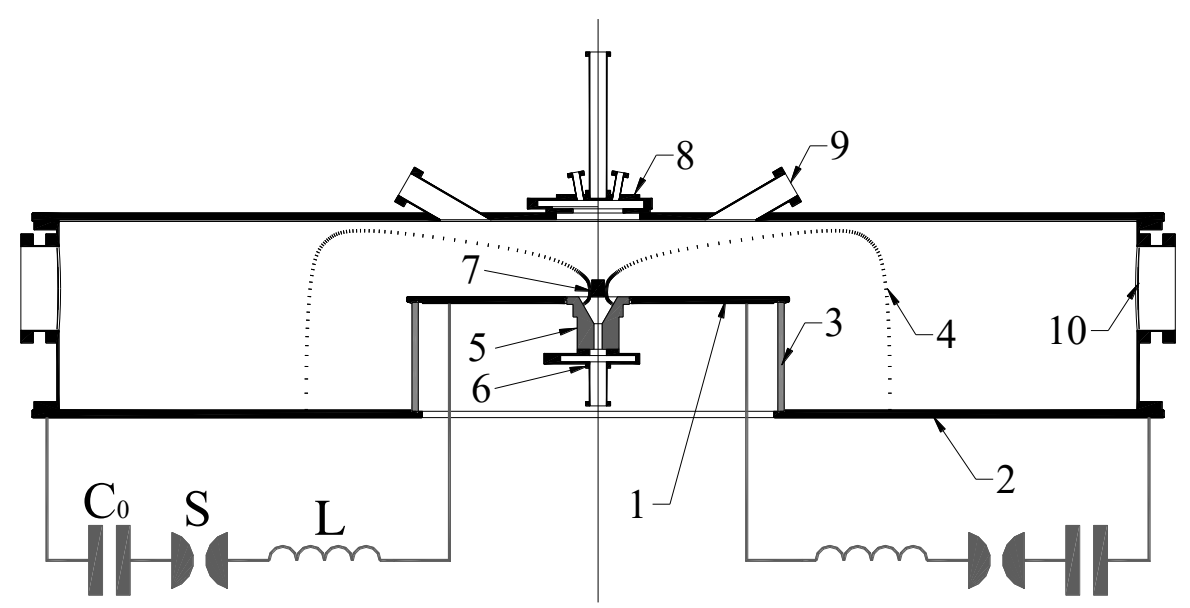

Figure 115. Diagram of the liner experiments at the PF-3-facility: 1-anode. 2-cathode. 3-insulator. 4-plasma current sheath. 5-anode insert. 6-suspension ware. 7-liner. 8-loading unit with a vacuum lock. 9, 10-diagnostics ports. $\mathrm{C}_{0}$ - capacitor bank. S-low pressure spark gap switch. L-external inductance. Reproduced from Figure 1 in [440] (AIP Conf. Proc. 2002, 651, 37-42.) with the permission from AIP Publishing.

A special load installation device with vacuum lock was developed (Figure 115, item 8). The device is located on the upper cover (cathode) of the discharge chamber. All the construction elements are located outside the discharge volume and they do not introduce heterogeneity affecting the PCS-dynamics. The liner (7) is suspended from a thin 
$(\sim 60 \mu \mathrm{m})$ metallic filament (6) and lowered to the necessary position at the system axis with the mobile rod located in the cylindrical column sluice.

Thin-walled hollow liners with an average density of $10 \mathrm{mg} / \mathrm{cm}^{3}$ and a linear mass of $0.2-1.0 \mathrm{mg} / \mathrm{cm}$ are manufactured in TRINITI (Troitsk) by vacuum drying using their own unique technology from agar-agar gel. The technology of manufacturing wire arrays, which are assemblies of 40-60 tungsten wires with a diameter of 6-8 microns and linear mass $220-660 \mu \mathrm{g} / \mathrm{cm}$, was also developed at TRINITI. Wires $15 \mathrm{~mm}$ long are stretched between two metal discs on a diameter of $20 \mathrm{~mm}$ with a step of $\sim 1 \mathrm{~mm}$. The design of the wire array with a magnetic probe for detecting the current entering the assembly is shown in Figure 116.

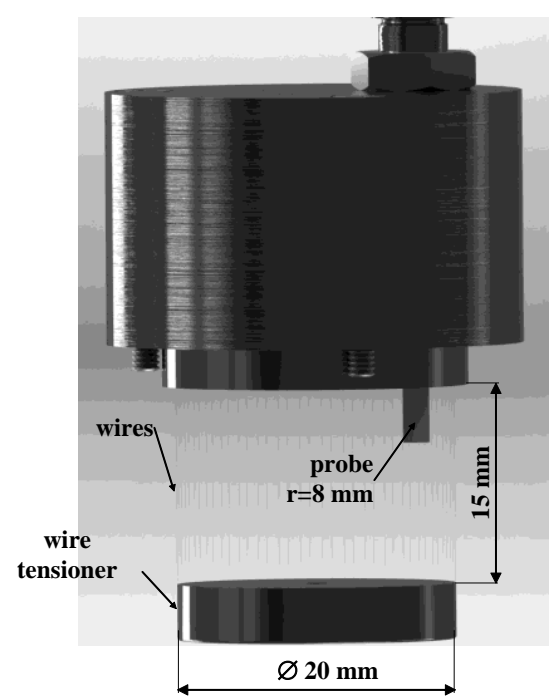

Figure 116. Design of wire array with magnetic probe.Reproduced from Figure 1 of [442] (Plasma Physics Reports, 2014, 40, 110-134.). () 2021 Pleiades Publishing Ltd.

Experiments with foam have shown effective compression of the foam load (Figure 117). The compression rate depends on the mass of the liner and on the value of the current in the sheath at the time of its contact with the liner. In these experiments, a two-fold increase in the velocity of the liner's compression with linear mass $0.3 \mathrm{mg} / \mathrm{cm}$ was obtained at increasing the current from 1.2 MA to 2.5 MA. Moreover, the compression of the liner with linear mass up to $1.0 \mathrm{mg} / \mathrm{cm}$ was demonstrated at I $=2.5 \mathrm{MA}$, which was impossible with a lower current value [201]. 

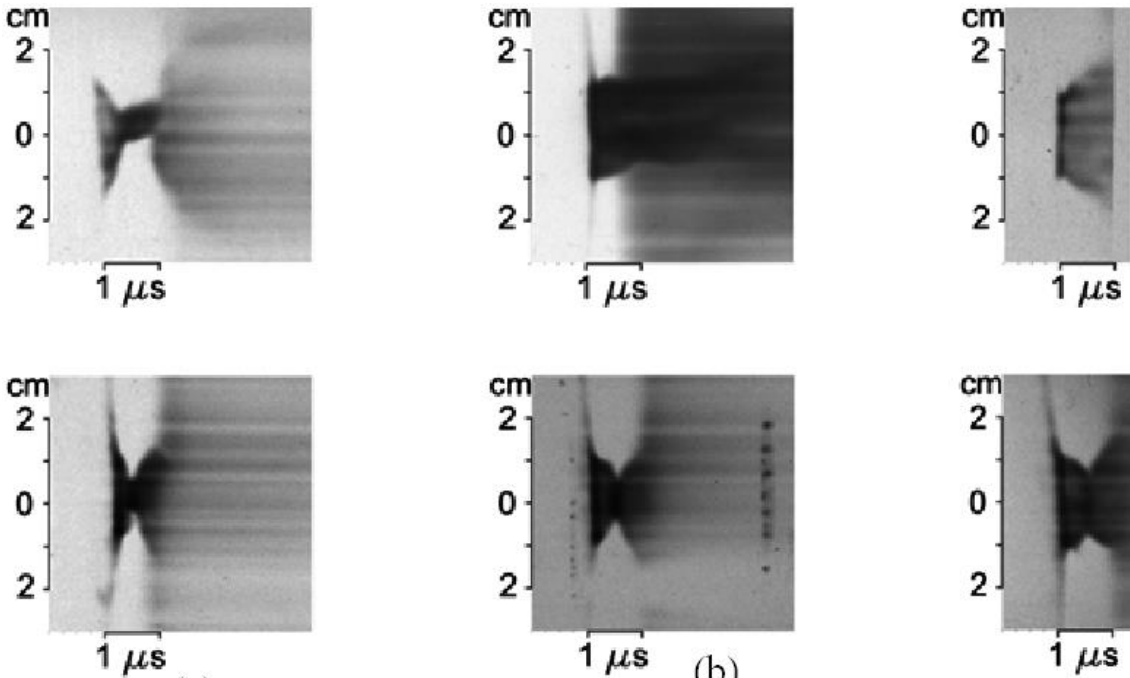

(a)
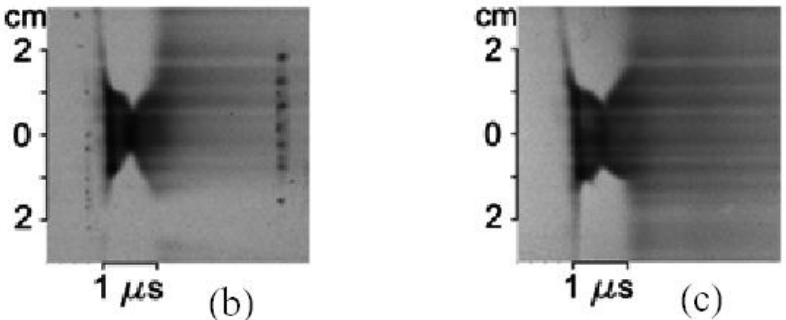

Figure 117. Streak-camera images of foam liner compression at $\mathrm{I}_{\text {pinch }} \approx 1.2 \mathrm{MA}$ (upper line,) and at $\mathrm{I}_{\text {pinch }} \approx 2.5 \mathrm{MA}$ (lower line). Linear mass: (a) $0.33 \mathrm{mg} / \mathrm{cm}$. (b) $0.6 \mathrm{mg} / \mathrm{cm}$. (c) $1.0 \mathrm{mg} / \mathrm{cm}$. Reproduced from authors' original data, also presented as Figure 3 of [201].

Similar results were obtained with wire arrays. It has been shown [440] that, after current sheath contacts with the liner, a major portion of the current continues to flow in the vicinity of the wires during the period necessary for transferring the current to the liner. However, some portion of the current is "dropped" down to the axis, producing a pinch (Figure 118).

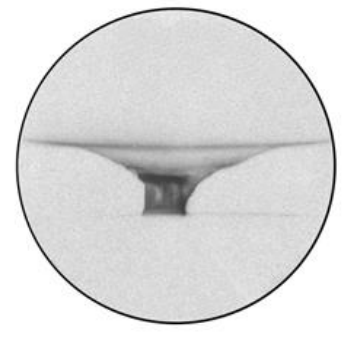

a)

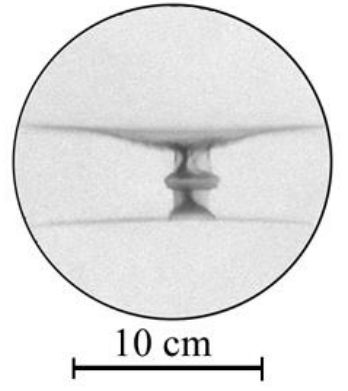

b)

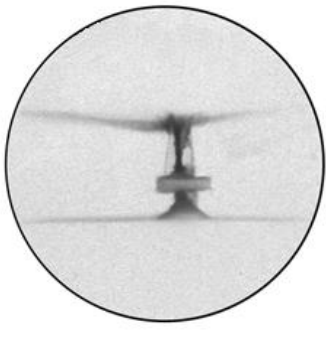

c)

Figure 118. Frame camera pictures of the discharge with the wire array, $\mathrm{m}=330 \mu \mathrm{g} / \mathrm{cm}$. Frame exposure is $10 \mathrm{~ns}$. $(\mathbf{a}-\mathbf{c})$ different instants of the pinch formation with time delay of $150 \mathrm{~ns}$. Reproduced from the original images also published in Figure 2 of [440] (AIP Conf. Proc. 2002, 651, 37-42).

The wires remain immobile during $\sim 150 \mathrm{~ns}$ for an assembly with $\mathrm{m}=330 \mu \mathrm{g} / \mathrm{cm}$, then a rather fast compression $\left(\sim 3 \times 10^{6} \mathrm{~cm} / \mathrm{s}\right.$ for $\left.\mathrm{I}=1.2 \mathrm{MA}\right)$ of the liner to the axis occurs. Later on, the pinch expands at a velocity slower than the compression velocity by an order of magnitude.

The time of the wires "standing" at initial radius and the velocity of their subsequent compression depends on the linear mass of the liner: the greater the mass, the longer it takes to transition to the plasma state and the lower the compression velocity (Figure 119). 


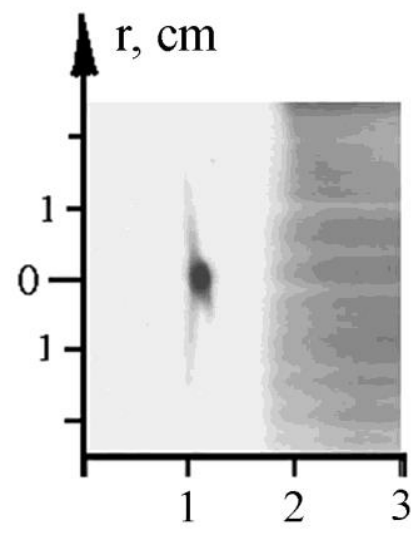

a)

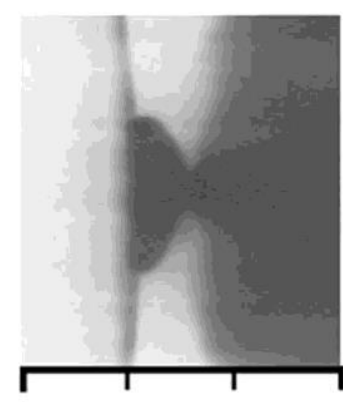

b)

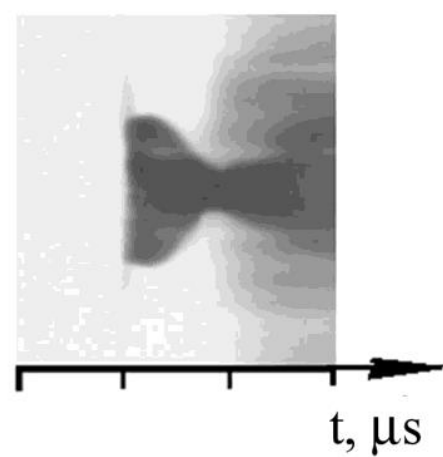

c)

Figure 119. Streak camera pictures: (a) discharge in neon without liner. (b) wire array, $330 \mu \mathrm{g} / \mathrm{cm}$. (c) wire array, $600 \mu \mathrm{g} / \mathrm{cm}$. Reproduced from the original images also published in Figure 3 of [440] (AIP Conf. Proc. 2002, 651, 37-42).

In the beginning of the 1960s, Ch. Maisonnier, J. G. Linhart, and M. Haegi $[446,447]$ proposed to use a microparticle diode for the production of a plasma sheath in the experiments with a hollow dynamical pinch by means of a thin layer of microparticles.

The implementation of this technique seems to be rather promising for producing the target as a dust cloud in the experiments by the liner program [440]. This approach has a number of the advantages related, first of all, with an opportunity to vary-on a wide scale - the mass, configuration and the element composition of such liner. In fact, the formation of predetermined density distributions is equivalent to an additional injection (gas puff) of a material into a given region of the discharge.

In the experiments performed on the PF-3 facility the dust target was produced at the system axis as a freely-falling flow of the finely-dispersed $(2-10 \mu \mathrm{m})$ powder of $\mathrm{Al}_{2} \mathrm{O}_{3}$. The scheme of the experiment is shown on Figure 120. This scheme is similar to the scheme in experiments with foam liners and wire arrays.

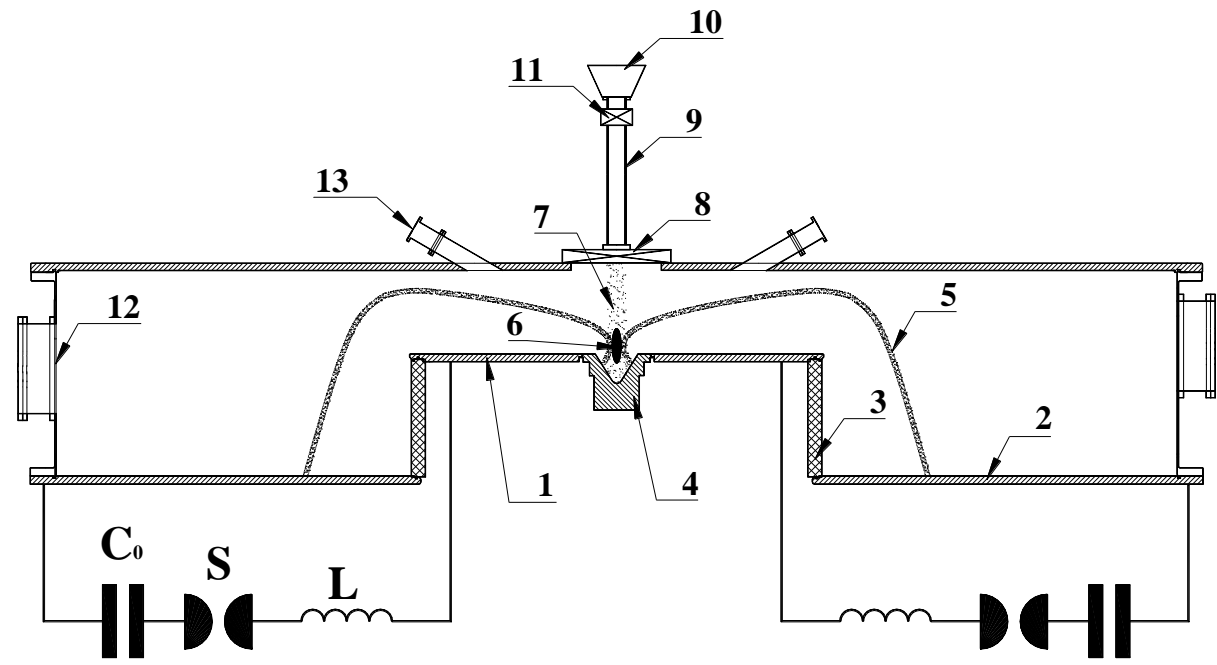

Figure 120. Experimental layout with dust target: (1) anode, (2) cathode, (3) insulator, (4) anode insert, (5) PCS, (6) pinch, (7) dust column, (8) vacuum gate, (9) transit tube, (10) dust container, (11) electromagnetic gate, $(12,13)$ diagnostic windows, $\left(\mathrm{C}_{0}\right)$ capacitor bank, $(\mathrm{S})$ low-pressure spark gap, and (L) external inductance. Reproduced from Figure 2 of [445] (Plasma Phys. Rep. 2006, 32, 642-655). (C) 2021 Pleiades Publishing Ltd. 
A unit for injecting dust is mounted on the upper cover (cathode) of the discharge chamber and consists of a dust container, an electromagnet, a transit tube, and a vacuum gate. All of the components of the injection unit are located outside of the discharge volume and do not introduce perturbations that might affect the PCS dynamics. When the vacuum gate is closed, the volume of the dust container can be evacuated to a pressure of $\sim 10^{-2}$ Torr or can be filled with air without deteriorating vacuum conditions in the discharge chamber. This permits preconditioning of the discharge chamber for improving the PCS parameters. The dust target was produced from a fine dust falling freely along the system axis. After filling with dust, the container was sealed, evacuated, and then was connected through a vacuum gate to the discharge volume filled with a working gas $(\mathrm{Ne}$ at a pressure of 1-3 Torr). The dust consisted of $\mathrm{Al}_{2} \mathrm{O}_{3}$ powder with grain sizes of 2-6 or $10-50 \mu \mathrm{m}$. Such a powder is characterized by a fairly low resistance to shear deformation and exhausts uniformly under the action of gravity through a narrow annular slot $\sim 1 \mathrm{~mm}$ in width and $6 \mathrm{~mm}$ in diameter when the electromagnetic gate is opened. The design of powder source was such that parameters of dust targets (the diameter and the linear mass) were close to those of foam liners and wire arrays used in PF experiments [443]. A distinctive feature of a dust target is that its mass is distributed over the entire target volume, so its average mass density is fairly low (in this sense, such a target is similar to a porous target).

One of the main results of experiments with dust is a significant increase in the lifetime of a stable pinch (see Figures 121 and 122).
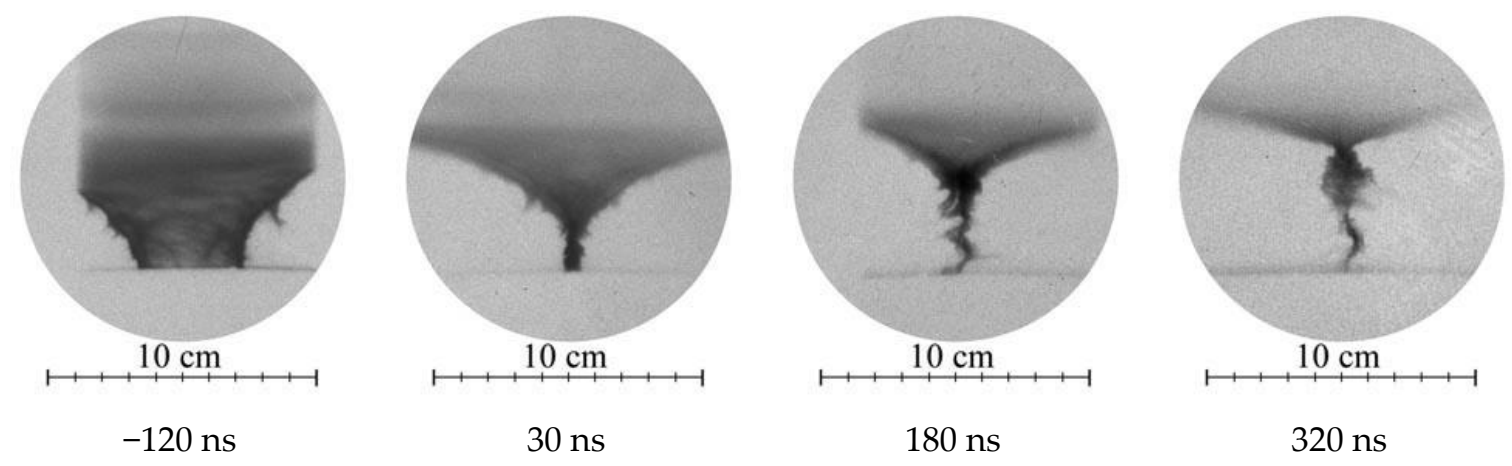

Figure 121. Frame camera pictures of a discharge in pure neon. The time is counted from the dip in the current derivative. Adapted from Figure 10 of [445] (Plasma Phys. Rep. 2006, 32, 642-655) (c 2021 Pleiades Publishing Ltd.

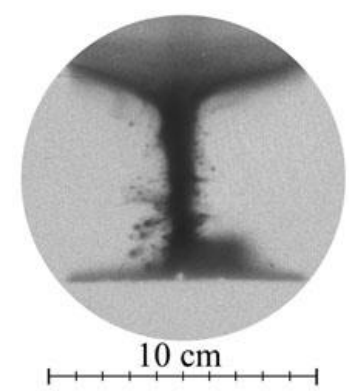

$500 \mathrm{~ns}$

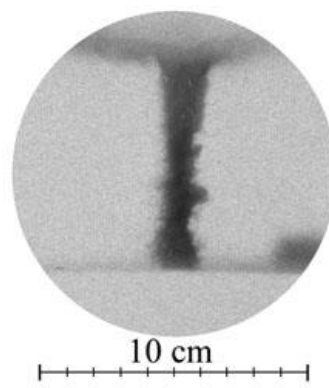

$650 \mathrm{~ns}$

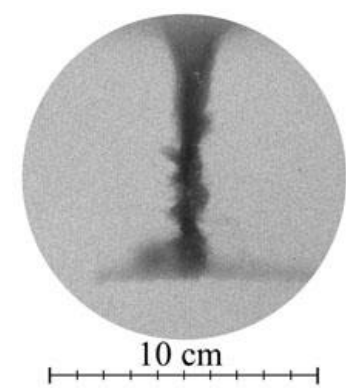

800 ns

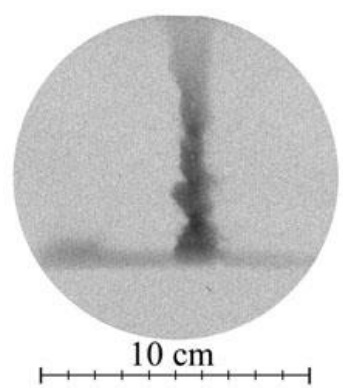

$950 \mathrm{~ns}$

Figure 122. Frame camera pictures of a discharge with a dust target. The time is counted from the dip in the current derivative. Adapted from Figure 13 of [445] (Plasma Phys. Rep. 2006, 32, 642-655) @ 2021 Pleiades Publishing Ltd.

In PF experiments with foam or dust liners, the preliminary stages of the discharge can substantially affect the phase state of the target before the current sheath comes into contact with the load. The type of working gas can significantly influence both the initial stages of the discharge and the efficiency of the sheath-load interaction. In the first experiments 
with foam liners [439], the influence of the sheath radiation was not taken into account, because the discharge was produced in the Mather-type electrode geometry, in which the duration of the radial implosion stage $(\sim 0.5 \mu \mathrm{s})$ was much shorter than the total discharge duration. In contrast, in Filippov's electrode configuration, in which the anode diameter is much larger than the length of the system, the effect of the preheating of the target by the sheath radiation can be very strong, especially when operating with high-Z working gases. In particular, in experiments carried out in the PF-3 Filippov-type facility with an anode diameter of $0.92 \mathrm{~m}$, the stage of the radial implosion of the current sheath is fairly long and the target is exposed to the sheath radiation for $\sim 10 \mu \mathrm{s}$. At sufficiently high radiation intensities, this can lead to a substantial preheating of the target located at the axis and a faster transition of the condensed target material into a plasma state. Radiative losses from the current sheath of a plasma focus in neon, argon, and hydrogen with a $2 \%$ admixture of $\mathrm{Xe}$ are calculated with allowance for the line, bremsstrahlung, and recombination radiation [421]. It is shown that the temperature of the neon plasma $(10-15 \mathrm{eV})$ is quite sufficient to evaporate $\mathrm{Al}_{2} \mathrm{O}_{3}$ grains of radii $10-20 \mu \mathrm{m}$. In the final stage of a discharge, when the PCS meets the dust load, the system under study becomes multiphase. The dispersed medium in our case is a neon plasma with a dispersed phase consisting of incompletely sublimated dust grains and their agglomerates, as well as of the neutral and ionized vapor of the dust material. The volume character of dust interaction with the plasma and radiation allows the dust component to be efficiently sublimated and ionized. This additional plasma source in the pinch region suppresses the onset of MHD instabilities and results in more stable pinch. The stability improves with increasing target linear mass.

The effect of radiation on the state of the liner load was also found in experiments with a foam liner. In Figure 123a, it can be noted that the diameter of the target at the moment of interaction with the sheath exceeds the initial size of the liner. In this case, compression begins immediately after contact with the shell, without the "standing" time characteristic of a wire array. In the case of a wire array, there is no pre-evaporation of the liner and no increase in its initial size (Figure 123b). This is due to the small irradiated surface of the wires and the high melting point of tungsten. For the transition of the substance of the wires to the plasma state, a sufficiently long duration of current flow in the "standing" phase is necessary, which is observed in the experiment.

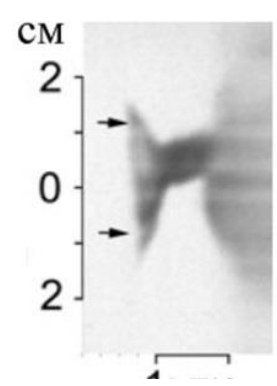

(a)

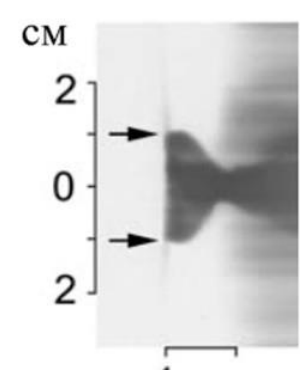

(b)

Figure 123. Streak images of PF discharges produced with a (a) $0.3-\mathrm{mg} / \mathrm{cm}$ foam liner (Ip = 1.2 MA), (b) $0.66-\mathrm{mg} / \mathrm{cm}$ wire array (Ip = 1.2 MA). The arrows show the initial liner diameter $(20 \mathrm{~mm})$. Time scale is 1 microsecond. Adapted from Figure 9 of [421] (Plasma Phys. Rep. 2008, 34, 43-51) (C) 2021 Pleiades Publishing Ltd.

In principle, a similar approach was applied in many experiments with fast z-pinches, in which liner loads of complicated configuration (nested liners of different composition, implosion of a wire array onto a deuterated fiber, pulsed gas puffing, etc.) were used to optimize the process of implosion. A substantial difference, however, is that, in fast z-pinches, the energy is supplied to an "unprepared" cold load. as a result, the implosion begins with a so-called "cold-start" phase. 
This experimental scheme allows one not only to study the influence of the cold-start conditions on the implosion dynamics, but also many other phenomena inherent in highcurrent facilities. The possibility of using the PCS as a current driver for the compression of various liners in a wide range of linear masses has been demonstrated. In experiments with wire arrays, almost the entire PCS current was switched over to the wire array. No less than $80 \%$ of the initial wire mass is compressed onto the array axis [442]. It was shown that the phenomena occurring during the implosion of wire arrays in the plasma focus device (such as the formation of the current precursor on the array axis before the compression of the main plasma mass and the plasma rainstorm in the final stage of the implosion) have much in common with those observed in experiments with wire arrays carried out at the Angara-5-1, ZR, MAGPIE, and others.

It has been demonstrated that, in the presence of a tungsten wire array in a PF discharge, the power and energy of X-ray emission in the spectral range from $100 \mathrm{eV}$ to $1 \mathrm{keV}$ increases at least three to four times as compared to the compression of a conventional neon PCS [443]. Estimates show that, at a discharge current of $\sim 1 \mathrm{MA}$, the linear SXR $(h v<1 \mathrm{keV})$ power and energy generated by the wire array z-pinch amount to 6-20 GW/ cm and $4-13 \mathrm{~kJ} / \mathrm{cm}$, respectively.

In dust-target experiments, the duration of the X-ray signals did not increase, though the time during which the pinch emitted visible light increased significantly. Moreover, experiments with dust targets are characterized by a reduced integral radiation yield in the range $>100 \mathrm{eV}$ (the detection threshold). Provided that the dust grains are completely evaporated, the gas density in the pinch formation region can be two orders of magnitude higher than the initial density of neon in the chamber. This naturally affects the PCS dynamics and the pinch parameters. A considerable increase in the number of particles per unit length leads to a decrease in the plasma temperature under the Bennett equilibrium conditions and, consequently, to a decrease in the energy of the radiated photons as compared to that in discharges in pure neon. As a result, the measured radiation intensity in the photon energy range $\geq 100 \mathrm{eV}$ decreases. A PF discharge with a dust component shows promise as an efficient source of VUV radiation.

\section{Exploration of Un-Conventional Plasma Focus Geometries}

Standard plasma focus research deals with the Mather and Filippov configurations [1,2]. Mather geometry has flourished because of the ease of its construction that is constrained by the commercial availability of insulators in the required size and the possibility of making a small, easy-to-use configuration popularized by the UNU-ICTP design (see Section 3.1.1). Filippov geometry required a large diameter insulator that was difficult to source commercially for most laboratories and had no counterpart like the UNU-ICTP design and thus, did not become so widely popular. It also had problems with scaling and with deuterium operation (see Section 3.1.6). Other geometries have been explored that differ considerably from both but have not been found to have major advantages over the standard geometries.

This section reviews the following non-conventional configurations: (1) Two-guns PF, (2) Hypocycloidal PF, (3) Staged PF, (4) Spherical PF, (5) "mushroom shaped" PF, (6) Interrupted PF, (7) Nanofocus.

(1) Two-guns PF-In this geometry, pioneered by Mather [143], two similar PF devices are placed in the same vacuum chamber in a counter-facing position so that the two imploding plasma sheaths are brought into collision.

The connection of the two PF to the same capacitor bank and trigger allows a simultaneous start of the discharges in the two devices and, the two circuits being in parallel, if one sheath advances more rapidly than the other, the impedance of that branch grows more rapidly than in the second branch, causing a relative decrease in the current and a relative slowing of the velocity. This mechanism allows the simultaneity of the arrival of the sheaths to the end of the inner electrode and a simultaneous collapse. 
The hope was that the collision of two foci moving one against the other with a velocity of $2 \times 10^{7} \mathrm{~cm} / \mathrm{s}$ would cause a more powerful neutron emission compared with the standard configuration, since the axial beam of each plasma focus would treat the other plasma focus as an additional target. The results did not agree with these expectations. Measurements done with capacitor banks up to $140 \mathrm{~kJ}, 1,9$ MA [448] showed a neutron production one order of magnitude lower than in the standard configuration.

Three modifications of the two gun configuration as shown in Figure 124 were studied in [449]: (a) a normal standard configuration used as a reference for the other configurations, (b) a two-gun configuration with a deep hole on the top of the central electrodes, (c) a two-gun configuration with hemi-spherically shaped top of the central electrodes, $(\mathrm{d})$ the two facing central electrodes connected with a metallic rod. No neutrons are detected when the central electrodes have negative polarity and the experimental results refer to positive polarity of central electrodes.

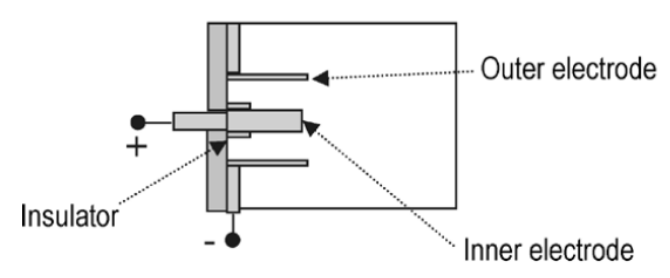

a) PF SINGLE

(conventional arrangement)

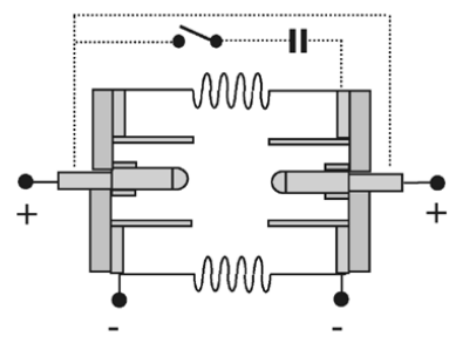

c) PF TWIN PHAL

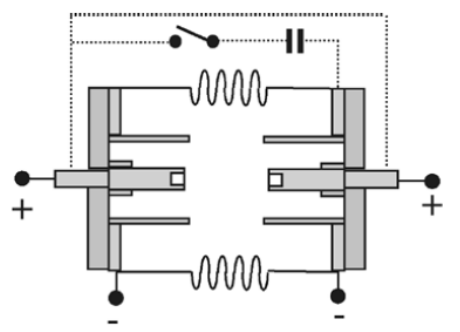

b) PF TWIN GYN

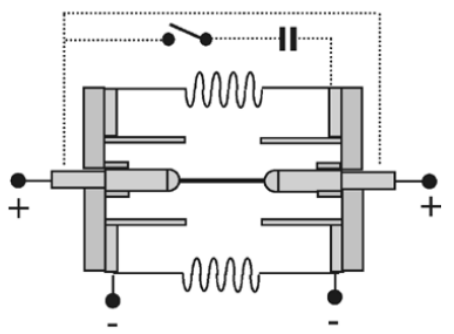

d) PF COLL

Figure 124. Experimentally tested configurations: (a) standard single anode PF (used as reference), (b) two opposing anodes with a deep hole at the tip, (c) two opposing anodes with hemispherical shape at the tip, (d) two opposing anodes connected with a metal rod. (C) 2021 IEEE. Reprinted, with permission, from Figure 1 of [449], IEEE Trans. Plasma Sci. 2006, 34, 36-54.

The anodes had a diameter of $3.45 \mathrm{~cm}$ and the electrode separation could be adjusted between $1 \mathrm{~cm}$ and $16 \mathrm{~cm}$. The capacitor bank energy was about $10 \mathrm{~kJ}$ and the working gas was deuterium. The experiments showed that the hollow shaped electrodes produced twice the neutron yield than the hemispherical shaped (also in the reference single electrode configuration). For distances between the two anodes lower than twice the anode radius the neutron production was progressively reduced with the gap reduction but not totally eliminated being present also at a distance of $1 \mathrm{~cm}$ (the minimum available). For the inter-anode distance greater than twice the electrodes radius, the neutron production corresponded to the sum of two independent PF not interacting (each with half the bank energy). As the scaling of the production fit the proportionality to the square of the bank energy, the final neutron production is about one half of that generated by the reference single configuration with the same total bank energy. This result gives some indication of the fact that the neutron production mechanism is not a beam-target (at a convenient small distance between the two colliding PF the reciprocal beams hitting the incoming targets would cause an increase in the neutron production with respect to the production of the simple sum of the two separate PF as experimentally seen). A mechanism dealing with long time trapping of the ions in local poloidal and toroidal magnetic configurations, 
as mentioned in this review paper, would also predict that there would be no effect of interaction between the two PF. The study of the configuration with a metallic rod (stainless steel or tungsten) connecting the two anodes was performed changing several parameters such as the rod diameter or length, the gas pressure, the current. The results agreed with a simple model of ballistic collision of two plasma sheets. The length of the rods was varied from $3 \mathrm{~cm}$ to $10 \mathrm{~cm}$ and the diameter from $3 \mathrm{~mm}$ to $13 \mathrm{~mm}$. The neutron release was of the order of that measured in previously mentioned cases of two disconnected electrodes when the inter-electrode gap is smaller than the electrode diameter. As the velocity in the run-down phase is inversely proportional to the electrode radius for a rod diameter of $3 \mathrm{~mm}$ the velocity was about $10^{8} \mathrm{~cm} / \mathrm{s}$ and the impact energies were about $35 \mathrm{keV}$.

Results on other emissions from the two-gun PF were more encouraging than the neutron emission. Indeed the life of the hot plasma was more than that of the standard PF. A duration of about $2 \mu \mathrm{sec}$ of the radiation emission would make it a better source for some applications. In particular the emission in the extreme ultraviolet light (EUV) is useful for photo-lithography as reported in [450] where a two-gun $\mathrm{PF}$ of $1 \mathrm{kHz}$ repetition rate has been studied.

(2) Hypocycloidal PF-The structure of this kind of PF is a sandwich with two metallic discs electrically connected (cathode) and a third metallic disc (anode) placed between and isolated from the cathode discs by two circular insulators placed at the external radius of the discs. The discs have the same radius and have an hole in the central zone (see Figure 125).

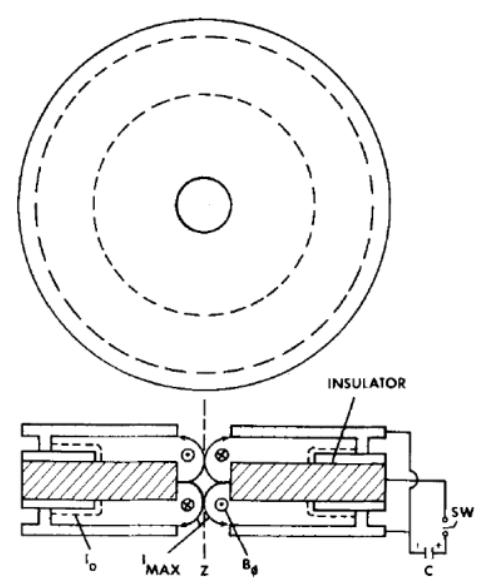

Figure 125. Reproduced from Figure 1 of [451] (Phys. Fluids 1977, 20, 313-321), with permission of AIP Publishing. The figure shows the cross-section of the disc-shaped electrodes and insulators that is revolved around the axis of symmetry marked $\mathrm{Z}$.

When high voltage is applied, two discharges start simultaneously in the external zone on the insulators up and down the anode, and move symmetrically toward the axis as in a rundown phase in standard PF, and eventually collapse on itself in the central hole zone generating two focus points along the device axis, with emission of radiations and, with deuterium filling, emission of neutrons. Experimental results [451] show that the neutron production is poor but there is a powerful emission of soft X-rays and extreme ultraviolet (EUV) radiation. The most important characteristic of the hypocycloidal PF is the long duration of the radiation emission. This indeed has been measured to last at least $5 \mu \mathrm{sec}$ (the limit was put from the duration of the current). This result is interpreted to show that after the implosion the plasma reaches a stable pinch configuration along the axis of the central hollow zone of the anode. With deuterium the temperature of the plasma has been measured, being about $1 \mathrm{keV}$ with a density greater than $10^{18} \mathrm{~cm}^{-3}$. The radiation emission characteristics are useful for some applications, mainly for EUV lithography. To that goal, hypocycloidal PF are developed with argon gas [452] and small capacitor bank (4.5 kV-1.53 $\mu \mathrm{F})$. 
(3) Staged PF-The configuration of this PF is a combination of the two-gun and hypocycloidal PF. In a two-gun configuration a metallic disc with a central hole is placed in the mid-plane between the two facing central electrodes. The two plasma sheets of the opposite two-gun sides, after the run-down along the cylindrical zone, reach opposite surfaces of the conducting disc and implode like in the hypocycloidal configuration forming two focus points along the axis, just as in the hypocycloidal PF. The outputs of the staged PF are more or less the same as in the hypocycloidal apparatus. Additionally, in this configuration the very positive point is the long duration of the soft X-ray and EUV radiations [453], limited only by the half period of the current. The neutron production lasts about $100 \mathrm{~ns}$ as in a normal PF. The neutron emission is in perfect agreement with the production of a two-gun device and with the square of the bank energy dependence (for instance $5 \times 10^{8}$ for $17 \mathrm{~kJ}$ bank versus $2.2 \times 10^{8}$ for a two-gun bank of $11.25 \mathrm{~kJ}$ ). The fields of possible applications are clearly the same of the hypocycloidal PF.

(4) Spherical PF-This is part of a truck-transportable high intensity DT-neutron source, producing $\sim 10^{13}$ DT neutrons per burst-apparently, the most intense publicly reported transportable high intensity neutron source. In this PF, the cylindrical configuration of the standard PF is changed into a spherical configuration. Two metallic spheres of different diameter, placed one inside the other, are the anode and the cathode of the PF, (Figure 126), with the outer stainless steel sphere also acting as a hermetically sealed, UHV compatible vacuum chamber.

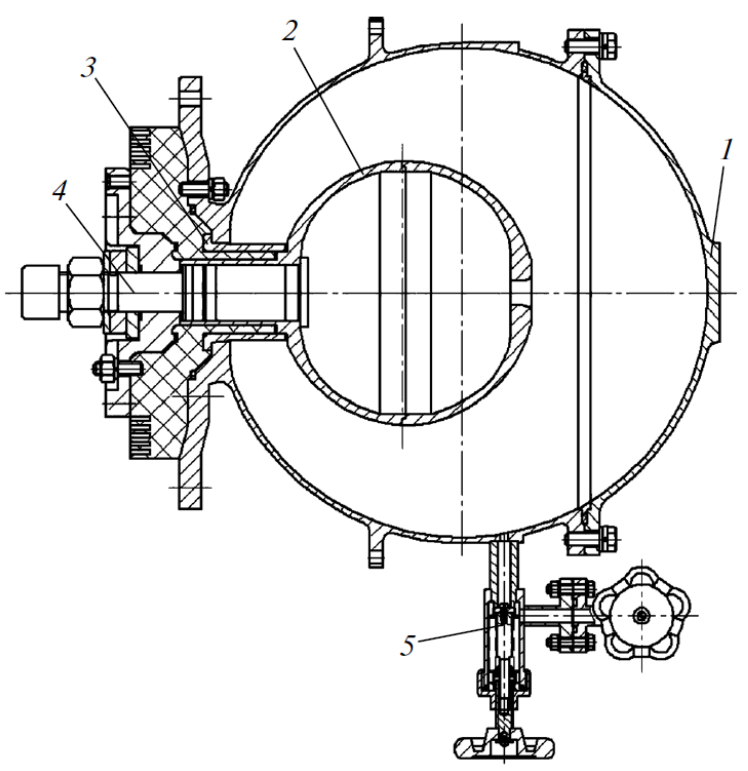

Figure 126. Construction of the spherical plasma focus "PNK-13 plasma neutron chamber": (1) cathode-housing, (2) anode, (3) insulator, (4) anode leadin, and (5) valves for pumping and filling the chamber with gas. Reproduced with permission from Figure 3 of [289], (Instruments and Experimental Techniques. 2014, 57, 131-134) ( ) (2014) Pleiades Publishing Ltd.

The internal sphere is supported by a metallic rod, passing through a hole in the external sphere, connected to the HV generator and surrounded by an insulator allowing the electrical insulation of the two spheres. When the high voltage is applied, the discharge develops on the insulator and travels between the surfaces of the two spheres starting from the north pole (first antipodal), passing through the equator and collapsing in the south pole (second antipodal). Practically the trip from the north pole to the equator coincides with the run-down phase of the Mather PF, and from the equator to the south pole to the radial implosion. The physics of the sheath do not change with respect to the Mather models, as shown in successful simulations utilizing the same methods (snow-plow, shock wave, slug configuration) with spherical coordinates instead of cylindrical coordinates, as 
in the Mather geometry $[247,454]$. The spherical PF is very compact with respect to the standard geometries (useful in some applications) and has a neutron production of the same level. For instance $[289,290]$ a spherical PF with $16 \mathrm{~cm}$ diameter of the internal sphere and $29 \mathrm{~cm}$ of the external sphere (which also serves as the vacuum chamber), operating at 1.4 MA produces $8 \times 10^{10}$ neutrons in DD and operating at $1.5 \mathrm{MA}$ produces $1.3 \times 10^{13}$ neutrons in DT. Additionally, the proportionality of the neutron production to the fourth power of the current is preserved. The neutron and hard X-ray emissions last about $100 \mathrm{ns,}$ like in the standard PF. Regarding the emissions, the spherical PF is, therefore, analogous to the standard PF.

(5) "Mushroom" plasma focus: Ivanov et al. [146] mention a plasma focus referred as the LV2 device $(180 \mu \mathrm{F}, 25 \mathrm{kV})$ whose copper anode resembles a mushroom shape with a top portion that is $110 \mathrm{~mm}$ in diameter and a stem that is inserted into the chamber via a ceramic insulator of $55 \mathrm{~mm}$ diameter (see Figure 127). This has two interesting properties. One is the increased reproducibility of micropinches in deuterium filling at pressures of 1 to 6 mbar. The second is the occurrence of several pinches "at intervals of fractions of a microsecond". The authors state "At the time of the first pinching, as a result of contact with the hot and dense plasma, the central part of the anode surface explodes and the partially ionized copper plasma spreads along the pinch axis with a velocity of $2-3 \mathrm{~cm} \cdot \mu \mathrm{s}^{-1}$.

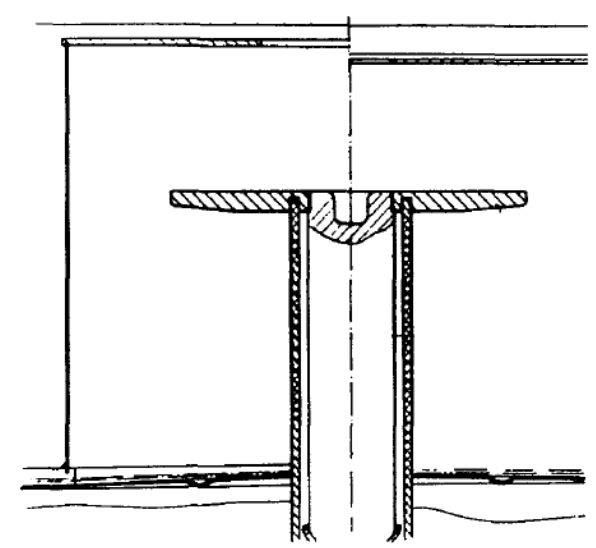

Figure 127. Reproduced with permission from Figure 3 of [146].

During the second (and subsequent) pinching, a part of the discharge current flows through the region occupied by the metal plasma, which undergoes compression under radiative cooling conditions."

(6) "Interrupted" plasma focus as an ion beam generator.

In this modification [455] of the UNU/ICTP FF, the plasma is prevented from reaching the axis by the arrangement shown in Figure 128. Instead, the plasma is diverted, sharply increasing its resistivity and generating a high local electric field between the anode and an auxiliary cathode. A high energy ion beam is extracted through a hole in the latter. 


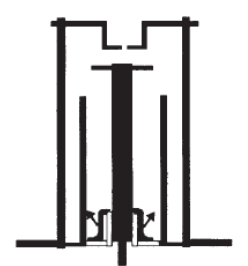

Breakdown

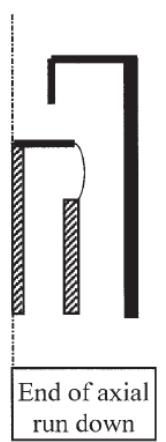

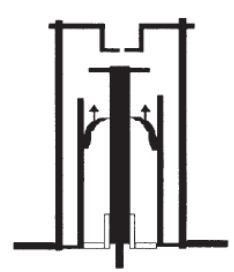

Axial run down

(a)

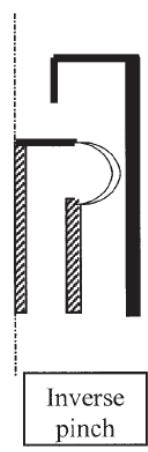

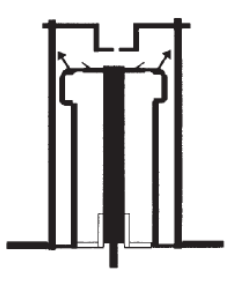

Current diversion

Figure 128. Reproduced with permission from Figure 1 of [455]. (a) The three stages in the operation of the modified plasma focus. (b) The final stage of the dynamics of the modified plasma focus, when the plasma resistivity is raised sharply due to the diversion of the current sheet. Copyright (2002) The Japan Society of Applied Physics.

This arrangement has been found to generate ion beams of argon ( $285 \pm 23 \mathrm{keV})$, nitrogen (225 $\pm 66 \mathrm{keV})$ and hydrogen $(96 \pm 12 \mathrm{keV})$. Further studies show:

- $\quad$ Longer electrodes and lower pressures of deuterium produced more energetic ions [456].

- $\quad$ Faraday cups were used to determine deuteron-beam energy and deuteron-beam fluence per shot while CR-39 solid-state nuclear track detectors were employed in studying the angular distribution of deuteron emission [457]. In the most optimum deuterium filling of 0.2 mbar, the ion beam measured was $52 \pm 7 \mathrm{keV}$, with fluence of $10^{15}$ ions $/ \mathrm{m}^{2}$. The ion beam emission was anisotropic and predominately in a forward cone of $60^{\circ}$.

- $\quad$ The time profile of the ion beam emission was simultaneously measured with soft and hard X-ray signals using BPX 65 silicon PIN diode and a set of scintillatorphotomultiplier detectors [458]. Two components of the ion beam were identified. The first component is of lower energy but higher intensity compared to the later. The emission of the first component of the ion beam and the hard X-ray due to electron beam bombardment of the anode are correlated. It is believed they are emitted by the induced electric field in the pinch.

- $\quad$ The X-ray line spectra correspond to $\mathrm{Cu}-\mathrm{K} \beta 1$ at $8.80 \mathrm{keV}$ [459] were measured using the XR100CR spectrometer.

(2) Nanofocus "NF" at the Chilean Nuclear Energy Commission

This creation of L. Soto's group [460] holds the record for the smallest plasma focus in the world $[6,339,358]$. It operates from a $5 \mathrm{nF}$ capacitor charged to $5-10 \mathrm{kV}$ storing 60-250 mJ of energy, producing 5-10 kA of peak current in $16 \mathrm{~ns}$. Its anode has submillimeter radius ( 0.8 and $0.21 \mathrm{~mm}$ in two versions). It has no cathode-one terminal of the capacitor serves its purpose.

Evidence of pinch was observed in electrical signals in discharges operating at $3 \mathrm{mbar}$ of hydrogen and $\sim 100 \mathrm{~mJ}$. Visible plasma images obtained with image converter camera (4 ns exposure) showed photographs consistent with (a) the formation of a plasma sheath close to the insulator surface, (b) the plasma covering the anode, (c) radial compression 
over the anode, and, finally, (d) the plasma is detached from the anode in the axial direction. The total time from stages (a) to (d) is $\sim 30 \mathrm{~ns}$ [460].

$\mathrm{X}$-ray emission with effective mean energy $4.3 \pm 0.3 \mathrm{keV}$ was measured [244]. Evidence of neutron emission from the Nanofocus operating in deuterium at only $0.1 \mathrm{~J}$ was obtained using the technique described in Section 3.2.8 in collaboration with the Balseiro Atomic Center of Argentina [231,234].

\section{Outlines of the "Emerging Narrative", Open Questions, and Potential Implications}

The discussion up to now was centered around reviewing the research developments in the last 20 years. This section is about exploring their implications regarding plasma focus physics-how the new results relate with the "Standard Narrative" discussed in Section 2.2 and how they define the outlines of an "Emerging Narrative" that is not quite 'established science' as yet but can no longer be dismissed as 'mere conjecture'. Several issues are flagged as open questions-the information available collectively is still not sufficient for a clear answer. These must continue to receive attention in the future. Finally, the big picture that emerges out of these deliberations is summarized under the heading 'implications'.

This discussion begins with a short conceptual summary of the new developments.

\section{1. "What has Changed?"-A Brief Overview}

Experimental results on the standard mode of PF operation discussed in Section 4 need to be distilled for their conceptual essence before their implications for PF physics can be discussed on a conceptual level. This also applies to some theoretical results. This section attempts to perform that task.

(a) Improved visualization of plasma phenomena using interferometry and imaging with visible, SXR, XUV, and reaction proton emissions and their correlation with time resolved measurements of neutron, electron, ion, SXR, and HXR emissions indicates that the formation, and decay of bounded 3D plasma structures appears to be correlated with neutron emission. Some aspects of SXR, HXR, electron, ion, and XUV emission support that view (see Section 4.3.1.3 and Section 4.3.3). Internal pressure gradients associated with these bounded structures can be sustained over the observation time windows only if they are accompanied with internal current distributions that can provide requisite counter-balancing magnetic force density. This is possible only if the current density and associated magnetic field have all the three components. Quite difficult and sophisticated magnetic probe measurements (Sections 3.2.2 and 4.2.2) provide crucial support for such interpretation.

(b) There is a better appreciation of the topology of 3D magnetic fields. Most researchers still believe that a current carrying straight cylindrical conductor produces an azimuthal magnetic field symmetric about the conductor. This is clearly an impossibility anywhere in the universe which is permeated by a small but non-zero magnetic field that may be locally uniform and unidirectional in the vicinity of the conductor. The magnetic field of the current carrying conductor will clearly have all three components in the cylindrical frame of reference with $z$-axis along the conductor: the azimuthal component given by Ampere's Law and partly by the ambient magnetic field, and the axial and radial components related to the ambient magnetic field and its inclination with respect to the conductor. The resulting magnetic lines of force are helical rather than circular with their axis of symmetry shifted. The difference in physics in the two topologies can be significant. A charged particle gyrating around the magnetic field lines can drift over larger and different regions of space in a helical magnetic field than in a purely azimuthal magnetic field. The simple motional dynamo process described by the electric field $-\overrightarrow{\mathrm{v}} \times \overrightarrow{\mathrm{B}}$ acts on all three components, allowing the directed plasma motion to amplify all three magnetic field components, and increasing magnetic energy at the expense of plasma kinetic energy. Since the plasma motion is itself 
driven by the magnetic force density $\overrightarrow{\mathrm{J}} \times \overrightarrow{\mathrm{B}}$, initially unidirectional plasma motion can transform into a 3D plasma flow after a sufficiently long time (see Section 8.1 (c)). This can, in turn, affect the dynamo process. The observation of 3D plasma structures and axial magnetic field by multiple techniques thus exposes the error in making the simplifying assumption [166] that axial and radial magnetic fields are initially zero and remain zero throughout the plasma evolution under the assumption of strict azimuthal symmetry and, thus, can be safely omitted from plasma focus research. There is a profound physical difference between the mathematical number zero and a very small number "tending to zero". This is explained below.

(c) The origin of this error, and of the general neglect of geomagnetic field in plasma focus research literature, lies in non-appreciation of the mathematics of multiple-scale analysis of non-linear systems of equations (Chapter 6 of [461]). A small perturbation of any system of dynamical equations is wrongly neglected by putting it identically equal to zero. The proper mathematical procedure would be to define a small parameter proportional to the perturbation for the non-dimensionalized system of equations, define the solution as a power series in the small parameter and obtain each term in the power series by a successive approximation procedure. A crucial step in this process is defining a hierarchy of timescales [461] which are related to the real time by inverse powers of the small parameter. The small term is then seen to influence the dynamics at longer times- the smaller the term, the longer it takes to manifest its effect. By putting this term identically equal to zero, one is forever dismissing the possibility that its effect may be manifested as an unexplained phenomenon at late times within the observational time window. In the plasma focus example, the dynamics of the rundown phase would be relatively unaffected by the small but non-zero geomagnetic field but that could provide the seed for the nonlinear dynamo processes that can create spontaneously self-organized structures much later in the discharge, lasting well after the apparent breakup of the plasma column. This is manifested in the experimental observations of monotonic increase in plasma inductance (see Section 3.2.3) that continues well past the current derivative singularity. Observations of the current derivative singularity and HXR emission $200 \mathrm{~ns}$ after the formation of the pinch in neon (Section 5.1.1 (1)d) and formation of dense structures well after the pinch break up observed using $\mathrm{CO}_{2}$ laser scattering [163] also indicate occurrence of significant plasma evolution after the so-called breakup of the plasma column. These could very well correspond to the higher order terms in the perturbation series solution of plasma dynamical equations with the ambient geomagnetic field defining a small parameter.

(d) A related conceptual error has been analyzed by Boozer [406]. This refers to the process of acceleration of charged particles in a magnetic field whose topology is changing in time. Although Boozer' analysis is mathematically quite complex, its physical essence can be heuristically described in the context of the plasma focus in the following manner. In textbook examples, a changing magnetic field is expected to create an electric field that is perpendicular to the magnetic field. So the expectation would be that charged particles would be accelerated perpendicular to the magnetic field. By tracking the particle energy perpendicular and parallel to the magnetic field whose local direction and magnitude are both changing in time, Boozer shows that it is the parallel kinetic energy that increases. The charged particle motion is effectively resolved into a gyration around the particle's guiding center that lies on the magnetic field line and acceleration of the guiding center along the magnetic field line. The transfer of kinetic energy into the gyration merely increases its local instantaneous gyroradius until it approaches a scale length of the magnetic field distribution, after which the perpendicular motion is effectively decoupled from the influence of the local electric field. The increase in the kinetic energy associated with the motion of the guiding center along the magnetic field has no such constraint. Boozer's analysis leads to an expression for the effective parallel electric field that would accelerate the 
guiding center along the magnetic field lines, that is much larger than the Maxwellian parallel electric field, and which contains a term proportional to the rate of change of the unit vector along the magnetic field multiplied by the mass-to-charge ratio of the charged particle. In other words, the effective accelerating electric field is much larger for ions than for electrons. It is also different in different directions. This challenges one of the common assumptions in plasma focus research-that ions and electrons are accelerated at the same time by the same local electric field. This assumption is used to calculate the neutron energy in time of flight experiments taking the HXR signal for a time marker for the start of neutron emission. Experiments discussed in Section 4.3.2 (3)-(5) indicate that HXR and neutrons may not be generated at the same time. They also show that neutron emission has measurably different characteristics in the axial and radial directions.

(e) Convergence of certain experimentally validated conclusions across multiple plasma focus installations (discussed in Section 4.3.3) provides a set of phenomena that must form part of any narrative concerning plasma focus physics, which are listed below.

- Existence of axial component of magnetic field in the radial implosion phase.

- Existence of accelerated deuterons moving towards the anode.

- Fusion proton images larger than X-ray pinhole images.

- Observation of lobular protrusion reaching the anode.

- Non-simultaneity of current derivative and voltage extrema.

- Near-isotropy of neutron emission spectrum in the first pulse.

- Deuteron acceleration, electron acceleration, and hard X-ray emission are separate but related phenomena.

The "Standard Narrative" formulated in Section 2.2 obviously continues to be accurate within its literal meaning. However, it no longer serves as a reliable basis for understanding the above mentioned experimental features of plasma focus physics. Its limitations are discussed in the next section along with the contours of an "Emerging Narrative".

\subsection{Limitations of the "Standard Narrative"}

This section attempts an examination of limitations of the "Standard Narrative" in conceptual terms. Along the way, features that might overcome such perceived limitations are flagged as possible ingredients of an "Emerging Narrative". Where such limitations cannot be addressed at the present time, an "Open Question" is tagged for later discussion.

\subsubsection{Ab Initio Neglect of the Ambient (Geomagnetic) Field}

Although the ambient magnetic field is certainly negligible as compared to the magnetic field in the current-carrying layer of the plasma, there are two situations where it is not negligible. One is during the formation phase (see Section 3.3.2.3), when the magnetic field due to the discharge current begins to rise from its zero initial value. The second is at the interface between the neutral gas and partially ionized plasma shock front (see Section 4.2.1 (1) and Figure 68), where it is the only component of magnetic field. In both cases, basic plasma parameters, such as the electron density, temperature, and magnetic field vary over many orders of magnitude over gradient scale-lengths of physical quantities and over time scales of plasma evolution. Corresponding characteristic plasma scalelengths, such as Debye length, various collision mean free paths, collisionless electron and ion skin-depths, Larmor radii of ions and electrons also, therefore, vary over orders of magnitude. The range of variation of each of these spans values both larger and smaller than the physical scale-length of the transition zone. Similarly, characteristic time scales, such as electron and ion plasma periods, various collision times, ion and electron Larmor periods also change significantly over plasma evolution time scales. Usual simplifying assumptions concerning applicability of fluid models, neglect of electron momentum convection and Hall effect are not applicable in this situation. All arguments that directly or indirectly invoke such assumptions for justifying the ab initio neglect of the ambient magnetic field are, therefore, fundamentally and irreparably invalid [462]. 
On the other hand, several physical arguments can be put forth against the ab initio neglect of the ambient magnetic field. These are discussed below.

Azimuthal currents can arise in the situations described above even when the plasma has good azimuthal symmetry [462] as a joint consequence of the constraint of quasineutrality and momentum conservation, which can be heuristically understood as follows.

In situations where fluid approximation is admissible, a force on a volume element containing both ions and electrons would produce a larger acceleration in the direction of force for electrons than for ions on account of their smaller mass. However, this difference in accelerations cannot lead to unlimited charge separation because of quasi-neutrality. This is possible only if the electron acceleration is predominantly convective (i.e., determined by $\left(\vec{v}_{\mathrm{e}} \cdot \vec{\nabla}\right) \overrightarrow{\mathrm{v}}_{\mathrm{e}}$ rather than by $\left.\partial_{\mathrm{t}} \overrightarrow{\mathrm{v}}_{\mathrm{e}}\right)$. Electrons and ions would then also move in a direction perpendicular to the direction of force, leading to both a transverse current [462] and transverse mass flow.

Experimental reports on axial magnetic field at Frascati [172], Stuttgart [42,173] and PF-1000 [212] mention that the polarity of the axial magnetic field does not change from shot to shot. Since the equations of plasma dynamics do not contain a preferred direction, the existence of a fixed polarity of axial magnetic field must be explained in terms of initial conditions, or equivalently, in terms of interaction of the plasma with an external physical vector field [463]. Only four such external vector fields are identifiable: Earth's magnetic field, Earth's angular momentum, direction of current density and direction of the plasma accelerator (from breech towards muzzle of the coaxial gun) [463]. It is difficult to imagine how the last three vector fields interact with plasma dynamics in order to create an axial magnetic field with a fixed polarity, leaving the geomagnetic field as the most likely reason behind the observation of the fixed polarity of the axial magnetic field.

Compression of the geomagnetic field has been suggested as a mechanism of generation of $B_{Z}[169,170]$. However, this fails to explain the polarity in the PF-1000 case [463].

The mechanism by which the ambient magnetic field may govern the polarity of self-generated axial magnetic field in a situation where fluid approximation may or may not be valid is described below [462].

Consider an idealized description of the interface between the neutral gas and the plasma shock in the radial phase (see Section 4.2.1 (1) and Figure 68). Electrons just liberated by ionization of the neutral gas in this thin cylindrical layer would tend to move away from the ions on account of their lighter mass until they are pulled back by the electrostatic field resulting from charge separation. On their return journey, they would overshoot charge equilibrium condition because of acquired momentum and would be pushed back by the excess charge density, starting a radial electrostatic oscillation at the electron plasma frequency. This would produce a radial displacement current density oscillating at the plasma frequency. Hall effect would then produce an oscillating electric field at the plasma frequency in the azimuthal direction in the presence of a small but non-zero constant ambient axial magnetic field and the oscillating radial current density at plasma frequency. This would displace the electrons in the azimuthal direction with respect to ions creating an azimuthal current density and hence an axial magnetic field. Lenz's Law would then create an azimuthal electric field that would resist the azimuthal motion of electrons. In that process, the electrons would acquire momentum and would overshoot their starting position, starting an azimuthal electromagnetic oscillation at the electron plasma frequency. The azimuthal current density at plasma frequency, together with the constant ambient axial magnetic field, would generate a radial Hall electric field at plasma frequency that would reinforce the initial radial oscillation. Both the radial and azimuthal modes would thus grow together in mutual resonance at the plasma frequency. The Hall effect between oscillating current density of the radial electrostatic mode and oscillating axial magnetic field associated with the azimuthal electromagnetic mode, both at plasma frequency, would generate an azimuthal electric field at zero frequency proportional to the initial constant axial magnetic field, causing its exponential growth. This growth occurs at the electronion collision time scale because of phase difference between the two modes introduced 
by collisional damping [462]. This mechanism explains how initial axial magnetic field, however small in magnitude, can determine the polarity of the spontaneously generated axial magnetic field even in ideally symmetric plasma.

Since the electron density begins with zero value, the electron plasma period would change by orders of magnitude during the rise of ionization. This might represent an insurmountable difficulty in realistic numerical simulations. However, its ultimate result can be arrived at by a different argument [463] involving existence of a paramagnetic drift current in imploding plasmas [176] that is a direct consequence of electron momentum convection induced by an applied force.

An average electron at the interface between the neutral gas and the plasma shock in the radial implosion phase would be moving in the $-\hat{\mathrm{r}}$ direction and would move in the $-\hat{\theta}$ direction under the action of the Lorentz force of an ambient magnetic field in the $+\hat{z}$ direction. The resulting current would be in the $+\hat{\theta}$ direction and the magnetic field produced by it would be along $+\hat{z}$ direction. That is to say that the current would be paramagnetic. Paramagnetic currents should cooperatively strengthen the initial axial magnetic field. It can be shown [463], using a procedure that makes no assumptions concerning validity of fluid approximation to this region, that the axial magnetic field in the region between the axis and the imploding plasma shock having a pressure profile $p(r)$ is given by the following relations which contain only the sign but not the magnitude of the ambient magnetic field:

$$
\mathrm{B}_{\mathrm{z}}=\operatorname{Sign}\left(\mathrm{B}_{\mathrm{z} 0}\right) \sqrt{2 \mu_{0}\left(\mathrm{p}_{\max }-\mathrm{p}(\mathrm{r})\right)} \quad \mathrm{r}<\mathrm{r}_{\mathrm{pmax}}
$$

For the region beyond the maximum of plasma pressure, it is given by:

$$
B_{z}=-\operatorname{Sign}\left(B_{z 0}\right) \sqrt{2 \mu_{0}\left(\int_{r_{p} \max }^{r} d r\left(J_{z} B_{\theta}+\frac{\partial p}{\partial r}\right)\right)} \quad r>r_{p \max }
$$

The growth of axial magnetic field in this region must be accompanied with an azimuthal electric field during the radial collapse phase. Signatures of this azimuthal electric field are probably seen in the time integrated reaction proton spectra in POSEIDON and observation of neutrons before the pinch phase in many large and small devices (see Section 4.3.3.7).

An alternative way of looking at the same phenomena is the concept of "constrained dynamics" [464], which " ... is based on the fact that terms of the momentum conservation equation are governed by widely dissimilar and independent processes. For example, pressure gradient is governed by processes which add or subtract energy (such as ohmic heating, thermal conduction, radiation, etc.) and particles (such as ionization and recombination) to the plasma and by irrotational flow which redistributes plasma particles in space. In contrast, a part of the magnetic (Lorentz) force is governed by local skin effect from temperature dependent resistivity and current supplied by an external power source, which may impose a temporal profile on the magnetic force different from that of the pressure gradient. The resultant of these should ordinarily govern the plasma acceleration. However, in DPF and many other kinds of Z-pinches, the fully ionized current-carrying plasma is constrained to move through an initially neutral medium. The viscous drag offered by the neutral medium to the magnetically accelerated plasma should result in a nearly constant drift velocity and a negligible acceleration. Streak camera pictures of the radial collapse phase of the DPF confirm this...".

"Under this condition, momentum balance can be satisfied only if there exists a degree of freedom in the dynamics, which can instantaneously supply the shortfall in the momentum balance equations produced as a result of different phenomena influencing different terms. This degree of freedom is, in fact, related to the absence of external solenoidal forces, which ensures that the center of mass of the plasma can have only irrotational 
motion". This postulated degree of freedom is manifested through the paramagnetic drift of electrons discussed above [462,463].

Both experimental and theoretical considerations thus indicate that the plasma current sheath must have a 3D magnetic structure in the radial collapse phase. Ab inito neglect of the geomagnetic field is, therefore, a fundamental limitation of the standard narrative. This needs to form a major feature of the "Emerging Narrative". Tracking time evolution of its effects from the beginning of the discharge during the rundown phase both experimentally and theoretically is still an open question.

Numerical simulations discussed in Section 3.3.2 routinely neglect the existence of this 3D magnetic structure of the plasma by assuming azimuthal currents and electric fields to be identically zero and, therefore, are inherently handicapped in dealing with dynamo processes that amplify the 3D magnetic structure at the expense of plasma kinetic energy. They also are not equipped for taking into account ion acceleration in 3D orbits.

For the next generation of simulations to follow dynamo processes in $3 \mathrm{D}$, the influence of the ambient field on the initial state of the plasma needs to be determined. The inclusion of the effect of the ambient magnetic field in the numerical simulations of the initial phase and the transition region between the neutral gas and the plasma would probably require extension of the EMPIC approach discussed in Section 3.3.2.3.

During the growth and decay of these magnetic structures, ions and electrons would get accelerated such that their guiding centers continue to move along the magnetic field lines wrapped around flux surfaces while their gyroradii may be of the order of the scale length of the 3D magnetic field structures. This would be consistent with the inference of ion acceleration towards the anode (Section 4.3.3.2) and the larger size of reaction proton images as compared with X-ray images (Section 4.3.3.3).

\subsubsection{Is the Pinch Phase a Pressure Equilibrium State (the So-Called "Bennett Pinch")?}

Streak camera pictures $[370,398,399]$ typically show a converging plasma shell whose inner boundaries merge and outer boundaries remain stationary for about 100 ns and then diverge again. The duration over which the radius of the outer boundary remains nearly constant is usually taken to represent the formation of a z-pinch equilibrium state with zero velocity and zero acceleration. This is often justified on the grounds that the Alfven transit time across the plasma radius is much less than the time scale of observed plasma motion. The Bennett relation is used to estimate the "temperature" of this state. Assumption of the Bennett equilibrium forms the core of many models and analyses of the plasma focus.

PF-1000 data from Section 4.3.1.1 A(4) and Figure 86 can be used to examine this assumption. From the measured magnetic field of $22 \mathrm{~T}$ at $13 \mathrm{~mm}$ radius, one can infer the current as $1.43 \mathrm{MA}$. The average density of the plasma column is about $4 \times 10^{18} \mathrm{~cm}^{-3}$ and the radius is nearly $1 \mathrm{~cm}$. The Alfven velocity is $\sim 10^{5} \mathrm{~m} / \mathrm{sec}$ and the radial transit time is about $100 \mathrm{~ns}$. The sequence of interferograms on PF-1000 taken at 10-20 ns intervals show dramatic changes in the spatial structure of the plasma during one Alfven transit time. This demonstrates that the assumption of a Bennett "equilibrium" is very far from experimental reality. In fact, the radial velocity measured in POSEIDON [370], PF-360 [370], KPF4-PHONEIX [210], and PF-1000 [393] is in the range (1-2) $\times 10^{5} \mathrm{~m} / \mathrm{sec}$, not very different from estimated Alfven speeds [358].

The Bennett temperature from these data comes to $\sim 250 \mathrm{eV}$. Temperature estimates using XUV spectroscopy (see Section 4.3.1.1 B) yield a number $\sim 70 \mathrm{eV}$ "at the final phase of the stagnation" [397]. Additionally, the plasma has a highly non-uniform pattern of evolution that corresponds to the "constant radius phase" seen in the streak pictures [398]. Magnetic probe measurements [393] show the presence of axial magnetic field larger than $2 \mathrm{~T}$ on the axis during the pinch phase, an aspect that is missing from the Bennett z-pinch concept.

These experimental observations demonstrate that the common view of the plasma focus as a Bennett z-pinch equilibrium [167] is certainly not a correct representation of 
reality. Analyses of plasma properties [167,358] based on the use of Bennett z-pinch equilibrium model (with zero velocity, zero acceleration and zero axial magnetic field) as a fundamental assumption may not, therefore, be extrapolated into practical decisions without adequate additional due diligence.

The so-called radiative collapse hypothesis [164] used to explain the occurrence of plasma hotspots by the addition of high $\mathrm{z}$ impurities (see Section 2.3.2 (3) is strongly dependent on the assumption that the plasma can be described as a Bennett equilibrium. The presence of magneto-plasma structures that carry 3D currents and magnetic fields may necessitate a review of the effect of radiative cooling on the properties of plasma microstructure.

\subsubsection{The Beam-Target Mechanism of Neutron Emission}

Plasma focus literature dealing with neutron emission frequently uses statements to the effect that their data are consistent with a 'beam-target interaction model'. This section explores various aspects of such declarations in the light of the experimental data discussed in Section 4.

The reaction $\mathrm{D}(\mathrm{d}, \mathrm{n})^{3} \mathrm{He}$ is known to produce a neutron of reaction energy $\mathrm{Q}=2.45 \mathrm{MeV}$ in the center-of-mass (CM) frame of the reactants. Observation of neutrons of energy different from this value is then ascribed to the motion of the CM of an average pair of reactants. When the neutron energy is more than $Q$, the $C M$ is said to be moving towards the detector; when it is less than $\mathrm{Q}$, it is taken to be moving away. When the neutron energy spectrum is centered at $Q$ but has broad wings on both sides $[173,180,181]$, the $\mathrm{CM}$ is probably having either a random motion or a periodic motion that makes it move both towards and away from the detector at separate instants in the observation time window.

Collectively, such CM motion is interpreted as the consequence of a "beam" that is interacting with a "target", in analogy with accelerator-driven nuclear reactions, for lack of a better description. This does not imply any particular kind of directionality that is associated with the commonplace usage of the term "beam". It also does not imply existence of two physically separate entities that can be identified as a "beam" and a "target". A comprehensive discussion of the main ideas of the "beam-target interaction" is given by Bernstein and Comissar [178] and Jaeger and Herold [42]. The concept fundamentally pertains to fitting of experimental neutron emission data to a numerically defined velocity distribution of interacting ions, along with a suitably defined spatial distribution of stationary ions, which may be a function of both space and time. Gribkov et al. [10] go beyond this to attempt relating the "beam" parameters with the plasma focus parameters. The Lee model (see Section 3.3.1.1) incorporates the scheme suggested by Gribkov et al. [10] into a handy tool that functions as a virtual plasma focus laboratory.

This should be very carefully distinguished from the study of ions by diagnostic techniques other than the neutron emission data, as discussed in Section 2.3.1. Such investigations provide a lot of information about high energy ions by themselves and the plasma environment that they experience, but extrapolation of that information to interpretation of neutron emission data would have to be treated as conjecture. The difference between the two cases pertains to the "interaction" part. "Beam ions" may simply make a single transit through a stationary collection of "target ions" or may have a trajectory that repeatedly passes through that region. The probability of interaction between the "beam" and "target" ions would be very different in the two cases.

For the sake of emphasizing this distinction, this section chooses to define the following nomenclature:

- Ions whose velocity distribution including directionality is inferred from reaction product (neutrons and protons) data or is proposed to interpret such data would be collectively referred as ION BEAM, or BEAM in short. As a matter of convention, such interpretation would assume the other participant in the reaction, to be referred as 
TARGET, to be stationary with respect to the plasma. Ions whose physical parameters are determined in any other manner would be collectively referred as 'ion beam'.

- A different BEAM could be invoked for dealing with data pertaining to different times in the plasma evolution.

Obviously, both the ion beams and ION BEAMS are related to the microscopic details of the plasma environment and the main motivation for studying them would be to construct a comprehensive understanding of this relationship that is applicable to all plasma focus devices and not specific to a single device. As an example, the non-uniform pattern of ion tracks discussed in Section 2.3.1 has been compared with model magnetic field distributions within the plasma leading to the inferences concerning existence of filamentary structures in the pinch phase.

Since BEAMs are an end product of data interpretation, the nature of data plays a significant role in determination of the conclusions. This is illustrated in the following examples:

- In the NX2 plasma focus, the shot-averaged peak neutron energy (corresponding to the peak of neutron signal) at $0^{\circ}, 90^{\circ}$, and $180^{\circ}$ found by time of flight method [465] was reported as $2.89 \pm 0.25,2.49 \pm 0.20,2.11 \pm 0.12 \mathrm{MeV}$, respectively, while the shotaveraged maximum neutron energy (corresponding to the start of the neutron signal) was $3.39 \pm 0.28,3.12 \pm 0.24$, and $2.73 \pm 0.14 \mathrm{MeV}$, respectively. These results were arrived at by determining the time of flight from the time between the peaks or the beginnings of the $\mathrm{X}$-ray and neutron pulses, under the implicit assumption that the $\mathrm{X}$ rays and neutrons are produced at the same time. The conclusion of the investigation was that the neutrons were produced predominantly by a BEAM TARGET mechanism where $100 \mathrm{keV}$ deuterons produced by the $\mathrm{m}=0$ instability traveled along the axis.

- In the experiment discussed in Section 4.3.2 (4), the time of flight and the instants of origin of the X-ray and neutron pulses were independently determined using a pair of detectors placed at different known distances. The start of the X-ray and neutron pulses were taken as time markers so that the "neutron energy" was the energy of the fastest neutrons and not of the majority of neutrons. The conclusion was that the neurons and X-rays were NOT produced at the same time. In fact, in some shots neutrons were produced as much as $20 \mathrm{~ns}$ before the X-rays. Similar conclusions were reported from experiments discussed in Section 4.3.2 (3) and Section 4.3.2 (5). The energy of fastest neutrons estimated from the time-of-flight in Section 4.3.2 (5) varied over the range $1 \mathrm{MeV}$ to $6 \mathrm{MeV}$ in both the axial and radial directions as a function of the measured time difference between $\mathrm{X}$-rays and neutrons. These results are interpreted as evidence that sometimes, the (fastest) neutrons are produced before the pinch, while the plasma is still imploding.

- The comparison of neutron yield with and without an obstacle plate placed $6 \mathrm{~cm}$ above the axis in NX3 plasma focus showed [225] that up to 75\% of estimated neutron yield appeared to be produced (with some caveats of interpretation) in the neutral gas above the pinch, by a conical fast deuteron beam. Earlier results on the UNU/ICTP PFF [248] using copper and deuterated obstacles had reached similar conclusions.

- Data on imaging of the reaction zone using charged particle emission (Section 4.3.2 (6)) as a function of deuterium pressure have been interpreted as suggesting operation of various mechanisms, such as "... beam-target mechanism at low pressure, trapped gyrating particles in some anomalous magnetic field at optimum pressure, and a jet-like moving boiler at high pressure".

What these examples demonstrate is the presence of an inherent indeterminacy in the BEAM-TARGET interpretation associated with the standard narrative- the inferred BEAM for one device may differ considerably from the one in another device or even within the same device at different pressures.

The interpretation of data on PF-1000 discussed in Section 4.3.1.3, however, provides a conceptualization of BEAM-TARGET interaction that can be identified as a superset of various descriptions of BEAM-TARGET interaction proposed on other devices, including 
the gyrating particle model [42], the crossed-field acceleration model [178] and the "virtual diode model" [10]. This shall be referred to as the ICDMP BEAM-TARGET model, until another nomenclature is proposed and adopted. Its basic elements are the following:

- TARGET is a 3D bounded plasma structure kept together by 3D distributions of current and magnetic field that evolve by dynamo processes.

- $\quad$ BEAM is a collection of ion guiding centers that are accelerated by the effective parallel electric field proposed by Boozer [406] that follow the magnetic field lines that have a 3D structure.

- The magnetic field lines that guide the BEAM could be either (1) spanning a toroidal flux surface [407] partially or fully embedded within the dense plasma TARGET or (2) could have a helical geometry that takes the ions outside the dense plasma into the neutral gas region.

- They can produce significant neutron yield in the neutral gas as observed in NX3 [225] and UNU ICTP PFF [248], in case the magnetic field lines are either helical or the flux surface extends beyond the dense plasma.

- In the toroidal flux surface case, if the BEAM has a partial overlap with the dense plasma, it would resemble an axially directed beam on the inner part of the toroidal flux surface, producing appropriate signatures in energy spectrum and fluence anisotropy, as observed in NX2 [465] and other devices which describe their neutron emission in terms of an axial BEAM-TARGET mechanism.

- In such devices, the reaction zone imaged with reaction protons would appear to be radially larger than the dense plasma X-ray image because the part of the BEAM that lies outside the dense plasma would still produce neutrons in the low density exterior region of the plasma as observed in Section 2.4.2, Section 4.3.1.2 B(2) and Section 4.3.2 (7).

- The ions in this external part of the BEAM would be directed towards the anode as they follow magnetic field lines which return outside the dense plasma [407], as discussed in Section 4.3.2 (1). Section 4.3.1.2 A(1)b, Section 4.3.1.2 A(2)b.

- The production of neutrons before the pinch while the plasma is still imploding, discussed in Section 2.4.2, Section 4.3.1.1 A(3)a, Section 4.3.1.2 A(3), Section 4.3.1.3 C(1), Section 4.3.2 (5), is included in this conceptualization by the recognition that the process that generates the axial magnetic field during the implosion phase would also generate an azimuthal electric field (related to the time derivative of the azimuthal magnetic vector potential associated with the poloidal magnetic field) that would accelerate ions in the azimuthal direction. The azimuthal velocity of ions would be approximately related to the change in azimuthal magnetic vector potential by the conservation of canonical angular momentum [407] as

$$
\mathrm{E}_{\mathrm{i}} \sim \frac{1}{2} \mathrm{~m}_{\mathrm{i}} \mathrm{v}_{\theta \mathrm{i}}{ }^{2} \sim \frac{1}{2} \mathrm{~m}_{\mathrm{i}}\left(\frac{\mathrm{e} \Delta \mathrm{A}_{\theta}}{\mathrm{m}_{\mathrm{i}}}\right)^{2}
$$

where the loss of energy by collisions is neglected. This would also explain the observation of higher FWHM of neutron spectrum in the radial direction as compared to axial direction in the Limeil device, the evidence of azimuthal motion of reacting deuterons in Frascati and POSEIDON and anisotropy less than unity in the first neutron pulse of POSEIDON discussed in Section 2.4.2 and in the Gemini plasma focus [436] discussed in Section 5.3 (8)d.

- The difference in the time of production of X-rays and neutrons in Section 4.3.2 (3), (4), and (5) could be accounted for in this conceptualization of BEAM-TARGET process by the term in Equation (19) of Boozer's paper [406] for the effective parallel electric field that is proportional to mass-to-charge ratio, which is 3 orders of magnitude larger for deuterons than for electrons which generate X-rays. It may also result from a low particle count reaching the detector in experiments discussed in Section 4.3.2 (4) and (5), which could lead to an X-ray photon, even if produced 
in coincidence with the neutron, missing the detector. However, in Section 4.3.2 (3), the same conclusion is reached but the particle count on the detector is not so low.

- The observed differences in the "energy of the fastest neutrons versus time difference between X-rays and neutrons" plots in the axial and radial detectors described in Section 4.3.2 (4) and (5) would follow from the projection of ION BEAM trajectory along the magnetic field lines on to the respective direction of observation. It could also be accounted for by the fact that the start of the neutron and X-ray signals would be defined by a rather small fraction of neutrons and $x$-ray photons out of the already small fraction $\sim 10^{-4}$ of the total neutron yield $\left(\sim 5 \times 10^{5}\right)$ and X-ray emission that does reach the detector. If the individual neutron that defines the start of the neutron signal happens to be produced by a deuteron that is moving away from the detector, its energy would be less than $Q$. Because of low particle numbers reaching the detector, the probability that the $\mathrm{X}$-ray photon that results from a simultaneously generated fast electron misses the detector would be quite high.

- The energy source that powers the ion acceleration would be the same as the one that drives the changing topology of the magnetic field, i.e., a dynamo process that converts plasma kinetic energy into magnetic energy and partly into particle kinetic energy by the process described by Boozer [406]. Phenomenological evidence from PF-1000 indicates the role played by cross-field axial flow during the first neutron pulse (see Section 4.3.1.3 C II) and the resulting growth and decay of a plasmoid. The ICDMP BEAM-TARGET mechanism suggested by the evidence for the first pulse (see Section 4.3.1.3 C II) can be concisely described as "hot ions circulating inside plasmoid (HICIP)". For the second pulse, the PF-1000 evidence suggests (see Section 4.3.1.3 C III) involvement of a plasma-filled virtual diode formed by plasma erosion in the neck formed by the $\mathrm{m}=0$ instability just below a plasmoid situated at the top end of the plasma column that can be concisely described by the phrase "rotating-fountain-beam, plasmoid-target fusion (RFBPTF)" mechanism.

- This mechanism is also seen to explain certain features of other experiments discussed in Section 4.3.2.

- It resolves an apparent contradiction between two obsevations on the University of Malaya, UNU/ICTP FF device: (1) Almost $85 \%$ of neutron emission arose from the neutral gas outside the pinch zone [188]. (2) Almost 65\%of the neutron emission which had anisotropy factor $\sim 1$, was produced [413] near the time of the voltage maximum and had energy $2.6 \mathrm{MeV}$ both at $0^{\circ}$ and at $90^{\circ}$. The remaining $35 \%$ had anisotropy factor 1.5 , was produced nearer the $\mathrm{m}=0$ instability and had energy $3.6 \pm 0.5 \mathrm{MeV}$ at $0^{\circ}$ and $2.6 \pm 0.3 \mathrm{MeV}$ at $90^{\circ}$. The first observation suggests a beam escaping the pinch. The second suggests confined ions in the first phase. The HICIP mechanism would have the ions trapped in a plasmoid that surrounds the dense plasma [407], which would be intercepted by a solid obstacle. The RFBPTF mechanism would be a good fit to the second part of the neutron emission.

- It explains the size difference in the reaction proton images and soft X-ray images in the NX2 plasma focus and the position of the maximum of the reaction proton density coinciding with the far end of the soft $x$-ray pinch image (see Section 4.3.2 (7), Figure 98). The RFBPTF mechanism provides a good match to this observation.

- It explains the lumpy appearance of the ion pinhole images reported by Zakaullah et al. [412] (see Section 4.3.2 (6), Figure 97). The RFBPTF mechanism again provides a good match to this observation. It is interesting to note the observation of Zakaullah et al. [412] in 1999: "One may explain the observed data by considering the notion of some anomalous magnetic field in the pinch region" — an astute anticipation of future developments indeed!

- $\quad$ Two plasma foci facing each other (see Section 7 (1)) should have produced more neutrons than the sum expected from the two acting individually at half the 
current because each would have acted as a target for an ion beam produced by the other one. That it fails in that respect is an indirect refutation of the single-pass axial beam + plasma-target model, which cannot account for any of these features and must, therefore, be treated as a conjecture not supported by experiments.

\subsubsection{Plasma Focus as a Circuit Element}

The plasma focus is usually treated as a time-varying inductance that increases during the rundown phase as the volume occupied by the magnetic field increases. It is believed to vary as the logarithm of the pinch radius [1,3] accounting for its sharp increase during the pinch phase. However, the apparent pinch impedance calculated from the current and voltage waveforms is more than what can be accounted for by the time-varying inductance calculated from the time variation of the pinch radius [1] leading to the hypothesis of an anomalous impedance that might arise as a result of micro-instabilities.

The experimental evidence presented in Sections 4.2.3 and 5.1.1 (1)d raises some question marks on this aspect of the standard narrative. The inductance variation calculated from the current and voltage waveforms (see Figure 83) is seen to increase monotonically. If the inductance were to vary as the logarithm of the pinch radius, it should have decreased as the plasma column expands. Its continuous increase even after the disruption of the plasma column presents conceptual difficulties, since a defined geometry of plasma is no longer visible in the interferometry pictures and a traditional definition of inductance in terms of a defined geometry of current flow is apparently not feasible. In a neon plasma focus, the plasma column forms and breaks up $200 \mathrm{~ns}$ before the current derivative singularity and associated HXR emission. If the current derivative singularity is really because of the rise in inductance as the plasma radius decreases, then this delay cannot be explained. This question has been examined from a first-principles point of view [364]. Its gist is given below.

At the interface between the capacitor bank and the plasma focus, the voltage $V(t)$ and current $I(t)$ define the power that passes from the capacitor bank into the plasma focus chamber. Although the power output from the capacitor bank is well-represented in terms of constant circuit parameters, such as capacitance, inductance, and resistance, the power input into the plasma focus can be represented as a volume integral over the power density $\overrightarrow{\mathrm{J}} \cdot \overrightarrow{\mathrm{E}}$ expressed in terms of current density $\overrightarrow{\mathrm{J}}$ and electric field $\overrightarrow{\mathrm{E}}$. The following expression is derived [364] from application of Poynting's theorem:

$$
\begin{aligned}
& \mathrm{I}(\mathrm{t}) \mathrm{V}(\mathrm{t})=\underbrace{\frac{\mathrm{d}}{\mathrm{dt}} \int_{\Omega} \mathrm{d}^{3} \overrightarrow{\mathrm{r}}\left(\frac{1}{2} \mu_{0}^{-1} \mathrm{~B}^{2}\right)}_{\text {I }}+\underbrace{\int_{\Sigma_{\mathrm{p}}} \mathrm{d} \overrightarrow{\mathrm{S}} \cdot \overrightarrow{\mathrm{v}}\left(\frac{1}{2} \mu_{0}^{-1} \mathrm{~B}^{2}\right)}_{\text {II }} \underbrace{+\frac{\mathrm{d}}{\mathrm{dt}} \int_{\Omega} \mathrm{d}^{3} \overrightarrow{\mathrm{r}}\left(\frac{1}{2} \varepsilon_{0} \mathrm{E}^{2}\right)}_{\text {III }}-\underbrace{-\int_{\Sigma_{\mathrm{p}}} \mathrm{d} \overrightarrow{\mathrm{S}} \cdot \overrightarrow{\mathrm{v}}\left(\frac{1}{2} \varepsilon_{0} \mathrm{E}^{2}\right)}_{\text {IV }} \\
& \underbrace{+\mu_{0}^{-1} \oint_{\Sigma_{\mathrm{p}}} \mathrm{d} \overrightarrow{\mathrm{S}} \cdot \eta \overrightarrow{\mathrm{J}} \times \overrightarrow{\mathrm{B}}}_{\mathrm{V}}-\underbrace{\mu_{0}{ }^{-1} \oint_{\Sigma_{\mathrm{p}}} \mathrm{d} \overrightarrow{\mathrm{S}} \cdot \overrightarrow{\mathrm{B}}(\overrightarrow{\mathrm{B}} \cdot \overrightarrow{\mathrm{v}})}_{\mathrm{VI}}
\end{aligned}
$$

The domain of integration $\Omega$ includes the volume enclosed by the current carrying parts of the electrodes and the plasma and $\Sigma_{\mathrm{p}}$ represents the current carrying skin layer of the moving plasma.

Comparing terms on the right hand side with expressions for electric power through standard electric circuit elements, it can be shown [364] that while terms labeled I and III are proper analogs of rate of change of energy in a suitably defined inductance and capacitance, there is no exact analog of the power associated with the motional impedance represented by the rate of change of that inductance. There is also no exact circuit theory analog of the last term that would be non-zero in case there are 3D magnetic and velocity fields, e.g., in the post pinch plasma. These terms in the expression for power would have to be accounted for in terms of an anomalous impedance which would be essentially a correction for an imperfect circuit element representation of the plasma focus. 
In the light of this discussion, the validity of the method of calculating dynamic inductance of the discharge (see Section 3.2.3) and its conclusions (Section 4.2.3) both need a closer scrutiny. Clearly, this is a major departure from the standard narrative that should form part of the emerging narrative. This subject also needs considerable additional investigation and is to be flagged as an open question.

\subsection{Contours of the "Emerging Narrative"}

The experimental evidence and its interpretation discussed in Section 4 and the above discussion clearly demonstrate that the plasma focus:

- $\quad$ is not a pure z-pinch with zero poloidal magnetic field;

- cannot be represented as a Bennett z-pinch equilibrium;

- cannot be represented as a manifestation of a fusion process involving a single-pass, axial beam interacting with a combined plasma + neutral-gas target with the beam generated by the $m=0$ instability and

- cannot be consistently described as a time-varying inductance in a circuit element representation.

The discussion above suggests the broad outline of an "Emerging Narrative" that is not quite 'established science' but can no longer be dismissed as 'mere conjecture'. It certainly has many gaps or uncertainties, which are open questions quite ripe for further attention from researchers. This section attempts to formulate this emerging narrative in the light of all the discussion so far in the following paragraphs.

(a) A plasma sheath is initially formed as a surface discharge over the insulator, where the just-liberated electrons interact with the magnetic field that results from (1) their own drift in the electric field that builds up in the breakdown process and (2) the drift that results from their interaction with the ambient magnetic field. The result is an initial distribution of current that is not purely axial but has a helical character.

(b) The initial plasma sheath gets detached from the insulator and gets accelerated by magnetic pressure. The magnetic field associated with the current flowing through the plasma sheath has all three components, with the dominant component being the azimuthal component.

(c) The accelerated plasma turns around the anode shoulder and moves towards the axis in a characteristic curved shape, while retaining and amplifying a 3D magnetic field structure associated with its current carrying layer. In particular, it is associated with a poloidal magnetic field that is present also in the neutral gas between its dense plasma front and the axis.

(d) The current flows behind the dense plasma sheath until it approaches the axis. A characteristic sharp dip in the current derivative signal and a spike in the voltage across the electrodes occur as the plasma reaches the axis. The moving current layer partially mimics a time-varying inductance that increases as the plasma approaches the axis and keeps increasing even after the apparent disassembly of the plasma column.

(e) One or more bounded toroidal plasma structures are spontaneously formed during the radial implosion phase of the plasma sheath in the region between the dense plasma and the axis, near the interface between the neutral gas and the dense plasma, at different elevations above the anode. These structures carry all three components of current density.

(f) The sheath stagnates at the axis in a funnel-shaped formation. The bounded toroidal structures are embedded in this funnel shaped formation at different axial locations. The stem of the funnel-shaped formation is compressed by the magnetic pressure of the discharge current. The axial regions of the stem between the axially separated embedded toroidal structures are compressed more than those at the axial locations of the toroids. The resulting axially non-uniform radial compression leads to a poloidal plasma flow that removes plasma from the region between the toroidal structures, leading to decrease in their radius, and adds it into the region associated 
with the toroidal structures. This leads to formation of one or more constrictions in the plasma column whose density gradually decreases below the detection limit of existing diagnostics.

(g) The part of the stem near the axial locations of the toroidal structures bulges out into a lobular structure because of plasma that flows in. These structures bend towards the anode, creating a low density magnetized plasma bubble bounded by 3D surface currents that maintain its density gradients. This magnetized plasma bubble acts as a pressure transmitting medium that transfers the compressive magnetic pressure from the discharge current that flows at larger radial distances to the magneto-plasma formations that arise within the plasma stem. It also electrically shorts the pinch column so that the pinch voltage spike detected outside the chamber is not exactly coincident with the current derivative minimum.

(h) The poloidal plasma flow pattern drives a dynamo process amplifying all the 3 components of magnetic field associated with the toroidal structures resulting in conversion of plasma kinetic energy into magnetic energy. The toroidal structures evolve into one or more plasmoidal structures with a single centrally-located density maximum. The plasmoidal and toroidal structures have internal magnetic fields which can be described as magnetic flux surfaces. Since electron motion and thermal conduction are faster along the magnetic field rather than normal to the magnetic field, the magnetic flux surfaces are also surfaces of equal electron density and electron temperature. They are, therefore, visible in soft X-ray, schlieren, and interferometry pictures (see Section 2.4.3).

(i) The $3 \mathrm{D}$ boundaries of the magneto-plasma structures are maintained by the magnetic force density $\overrightarrow{\mathrm{J}} \times \overrightarrow{\mathrm{B}}$ involving internal current distributions driven by the dynamo processes.

(j) The evolution of magneto-plasma structures is accompanied by ion acceleration into trajectories, which can be resolved into motion perpendicular and parallel to the instantaneous local magnetic field. The perpendicular motion is a gyration about a guiding center that lies on a local magnetic field line and the parallel motion is the motion of the guiding center along that magnetic field line. The energy associated with the perpendicular motion can increase only until the local instantaneous Larmor radius becomes comparable with the scale length of the magneto-plasma, after which it is not affected by the local electric field. The energy associated with parallel motion increases without this restriction.

(k) When the magnetic field line is fully embedded inside a magnetic flux surface, that also serves as a surface of constant electron density and temperature, the guiding centers of accelerated ions also appear to be moving on the flux surface. The resulting fusion reaction products have a kinetic energy different from the reaction energy in the center of mass frame. If these ions are collectively referred as a BEAM, their directionality reflects the toroidal directionality of the magnetic field lines which span a flux surface.

(1) When the magnetic field lines do not span a magnetic flux surface but rather have an open helical character, the guiding centers of accelerated ions can escape outside the dense plasma. They can cause fusion reactions in the neutral gas and be detected by ion diagnostics.

(m) The constricted regions of the plasma column have too few electrons to carry the existing current leading to an increase in the impedance of a virtual plasma-filled diode [10] and an increase in the local electric field. Ions accelerated in this virtual diode region move along the axis through the remains of the plasma stem, encounter a bounded magneto-plasma structure having all 3 components of embedded magnetic field, are magnetically deflected radially outward and axially towards the anode with an azimuthal displacement, and radially inward again. In this manner the ions pass through the virtual diode region repeatedly, gaining energy in each cycle. The fusion reactions between these ions and the bounded plasma structure at the far end of 
the plasma column have the spectral character of reactions produced by an axial ion beam interacting with a stationary target when viewed axially. The fusion neutrons or protons have the character of an azimuthally circulating ion beam when viewed perpendicular to the axis if there is a spatially resolved spectrum measurement. An enhanced spectral width is observed when they are viewed perpendicular to the axis without spatial resolution.

This "Emerging Narrative" consistently explains results from many laboratories collected over several decades of research and listed in Sections 2.4 and 4. Of particular interest is the recent (2020) observation of neutron yield anisotropy (end/side) less than 1 (see Section 5.3 (8)d) on the Gemini device [436], the only MA-level Mather type current operating device other than PF-1000. This is the opposite of what is expected from an axial beam-target mechanism, but is understandable in terms of the ICDMP BEAM-TARGET model.

Several key aspects, however, need to be more critically examined and more extensively validated before it can be accepted with full confidence as 'established science'. These are discussed below along with many other open questions of plasma focus research. Known phenomenological deviations from (or additions to) the standard narrative reviewed in Section 2.3 continue to remain outside the purview of the emerging narrative.

\subsection{Open Questions of Plasma Focus Research}

This section describes some open questions of plasma focus research that do not have a clear answer in sight in spite of considerable amount of data and conjecture. These are divided into two categories. The first category refers to old open questions that have been recognized over the past few decades of plasma focus research. The second category consists of open questions that have arisen from more recent developments. Where possible, promising pathways to potential answers are suggested.

\subsubsection{Classical Open Questions}

8.4.1.1. Why Does the Neutron Yield Scale as the Fourth Power of Current?

The experimental observation of neutron yield scaling as the 4 th power of current flowing in the pinch for well-optimized plasma focus devices was one of the main driving forces behind the growth of plasma focus research in its early days and is still frequently cited as one of the main attractions of doing plasma focus research. Early discussions of this scaling law are found in [466-468] which do not address the question "why".

A more recent attempt to "derive" this scaling law [319] assumes that the neutrons are produced by a thermonuclear mechanism, disregarding experimental evidence to the contrary. A more generic likely answer would be along the following lines:

'Neutron optimized PF devices operate at similar intensive plasma parameters, such as temperature, density, and magnetic field-this is a consequence of the optimization process. In that case, the neutron yield would depend on the size of the reaction zone and the time for which it exists. Since the Alfven speed would be the same for all such devices, the neutron yield would depend on the 4 th power of linear dimensions. The constancy of the drive parameter (see Sections 3.3.3.1 and 3.3.3.6, [467]) $I_{p} / a \sqrt{p}$ would then imply the 4th-power-of-current scaling law for the neutron yield since most PF devices operate over a limited range of deuterium pressures...'

This or similar 'answers' simply exchange one 'why' question for another. Why do PF devices operate at similar temperature, density and magnetic field? What are the underlying physical mechanisms that place upper or lower bounds on the density and magnetic field? Is it because nobody has explored a plasma focus design that might operate at a much higher density [318] or magnetic field or because of a real phenomenon nobody has thought of before?

It would seem that a well-explored experimentally-supported understanding of the neutron emission process should be a prerequisite for answering this question properly. 


\subsubsection{2. "Optimization" of PF Devices}

The scaling law discussed above is supposed to apply to "well-optimized" PF devices. Clearly, a plasma focus experiment that does not manage to transfer a large fraction of stored energy into the pinch phase would not be expected to perform as well as one which does. It is noteworthy that in PF-1000 experiments, the operating parameters were often deliberately chosen to be different from those which gave maximum neutron yield for diagnostic convenience (see Section 4.3.1). It remains an open question whether conclusions derived from the observed phenomenology in sub-optimized modes of operation apply to PF devices that operate in a "truly optimized" mode.

The significance of this point can be seen from the fact that the Limeil and Frascati devices were optimized to produce only one neutron pulse, while POSEIDON and PF-1000 had two neutron pulses. The second one, correlated with the instability, was the stronger one. Frascati had neutron optimized modes where the plasma column expanded without an instability. In PLAMYA device, the instability was a rare occurrence but the neutron yield was good. Would it then be correct to say that the main portion of the neutron yield in all plasma focus devices is because of the instability as inferred from POSEIDON and PF-1000?

It would seem that an objective definition of "optimization" [318] should involve the fraction of energy remaining in the capacitor bank at the time of the pinch. The lower this fraction, the better optimized the device would be. Other figures of merit could be the ratios of the maximum current and the pinch current to the ideal shortcircuit current $V_{0} \sqrt{C_{0} / L_{0}}$. This would give a basis of comparison between results from different experiments, particularly with respect to the degree to which it is 'well optimized'. However, this question is still open as no informed consensus has emerged on this point. It has been suggested [433] that enhancement of neutron yield by addition of krypton is an indication that the plasma focus electrode geometry is not optimized.

\subsubsection{Why Does the Neutron Yield Scaling Fail at High Bank Energies?}

This question needs to be refined into three cases that have different implications:

(1) A larger capacitor bank does not lead to a larger pinch current because of progressively more imperfect matching between the plasma load and its power source.

(2) A pinch is formed and is driven at a high current but fails to produce expected neutron yield.

(3) A larger capacitor bank or operation at a higher voltage fails to form a pinch-no current derivative singularity or voltage spike results anywhere during the discharge.

An example of the first condition is found in PF-3, see Section 3.1.6. Related theoretical considerations are discussed $[253,254,269,469]$ under various assumptions in the context of scaling up plasma focus to multi-mega-joule energy.

The best current example of the second condition is shown in Figure 129 from NSTec (see Section 3.1.4). 


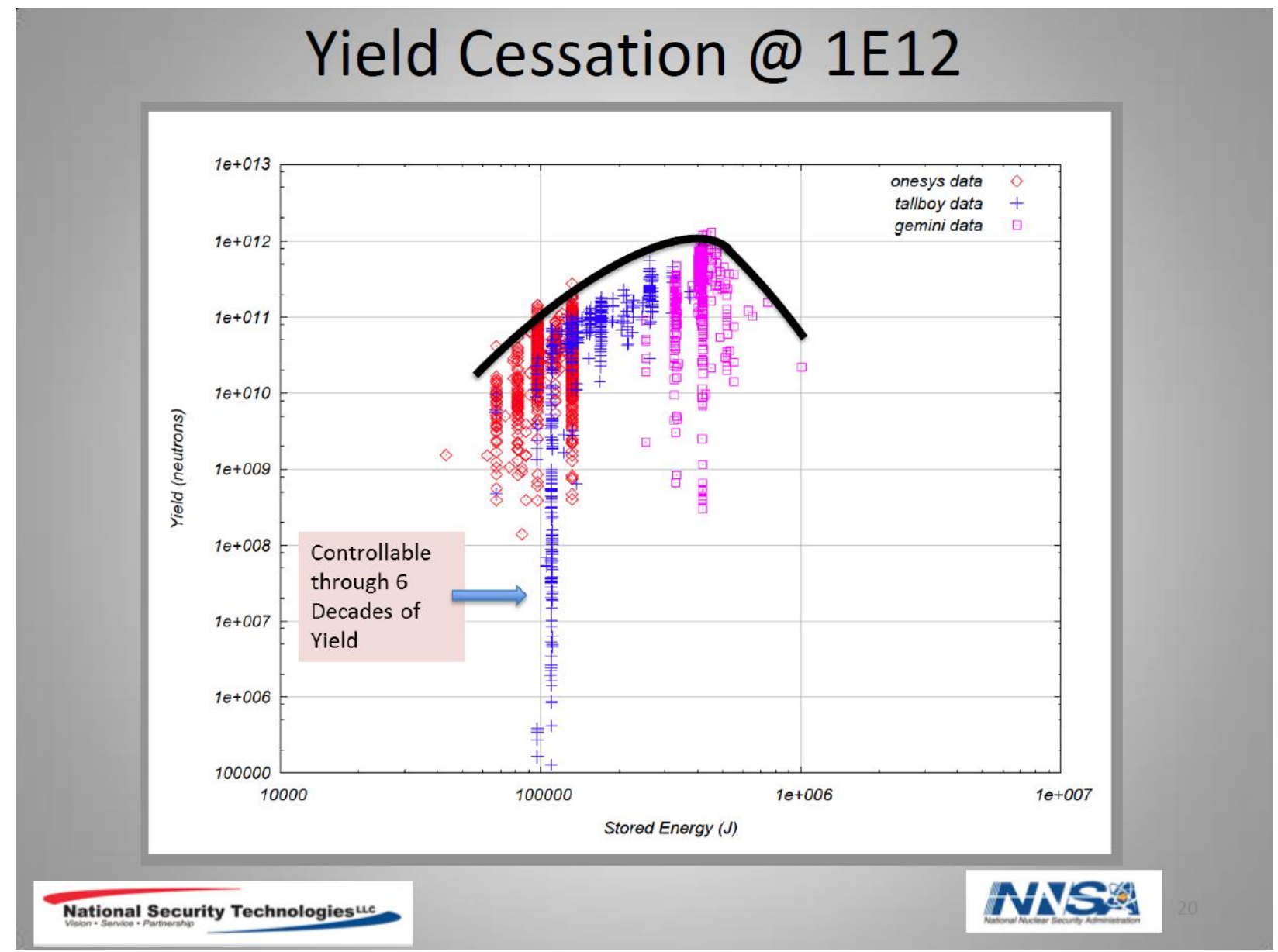

Figure 129. The abrupt reduction in neutron yield in Gemini. The last two data points for Gemini are at $61 \mathrm{kV}(0.8 \mathrm{MJ}) 4 \mathrm{MA}$ and $70 \mathrm{kV}$ (1 MJ) 6 MA. Reproduced from slide 14 of [199] with permission.

An early example of the second condition is found in POSEIDON and PF-360 [370]. In POSEIDON, the replacement of a Pyrex insulator with an alumina insulator overcame the neutron yield saturation [470]. Measurements of the rundown velocity using magnetic probes inserted through the anode showed that shots with low neutron yield generally had lower rundown velociy than those with good neutron yield and the shots with Pyrex insulator had lower rundown velocity than with alumina insulator. Streak pictures also show lower radial velocity for bad shots than for good shots, a fact also observed in PF-1000 (see Section 4.3.1 AIII(2)).

Magnetic probe measurements revealed no current loss across the alumina insulator at $60 \mathrm{kV}$, while for the Pyrex insulator there was a 15\% loss of current. Very significcantly, with an alumina insulator, the first neutron pulse was the dominant one [470], similar to the finding from UNU/ICTP FF at University of Malaya (Section 4.3.2 (10)) and the the second one correlated with the $\mathrm{m}=0$ instability was relatively small. With a Pyrex insulator, the first pulse was smaller.

The third condition is probably encountered in all large devices which defines the upper limit of device operation but is seldom reported because there is hardly any data to report. The only known exception is a conference poster paper [471] where the schlieren photographs of the radial phase were compared for good shots, shots with deliberately added impurities and a shot at the limit of neutron yield (shot \#12056 see Figure 130). The memorable aspect of this shot was that the previous shot \#12055 was at $87 \mathrm{kV}$ and had the highest neutron yield recorded on POSEIDON but was not reported because the delay setting for the laser pulse was improper so the schlieren photograph did not capture the image of the sheath. When this was corrected in the next shot at slightly higher voltage, 
the pinch was not formed and the neutron yield was zero. This was not repeated for the fear of damaging the insulator.

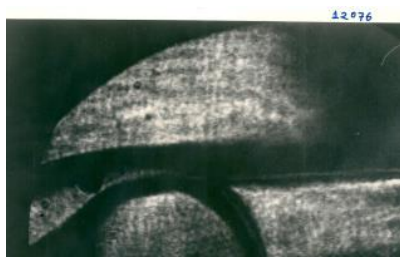

(a) Shot\#12076: $60 \mathrm{kV}, 4$

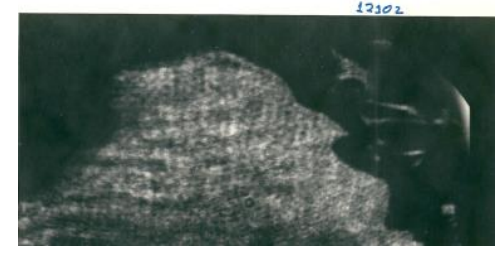

(b) Shot\#12102: $60 \mathrm{kV}, 3.8 \mathrm{mbar}$ $\mathrm{D}_{2}+0.2 \mathrm{mbar} \mathrm{O}_{2}, \mathrm{Y}_{\mathrm{n}} \sim 2.8 \times 10^{8}$

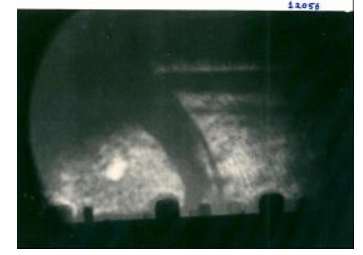

(c) Shot \#12056: $88 \mathrm{kV}$, 6.5 mbar $\mathrm{D}_{2}, \mathrm{Y}_{\mathrm{n}} \sim 0$

Figure 130. Adapted from [471].

The good, bad, and failed shots differed in the thickness of the dense sheath in the schlieren photographs seen in Figure 130a-c, respectively. This appears to be consistent with the physical requirement that the transverse electric field in the neutral gas just ahead of the dense plasma sheath be less than the dielectric strength of the gas (see Section 3.3.3.6). This is met in the good shot in Figure 130a, forming a relatively thin sheath with sharp boundaries. With oxygen admixture, the dielectric breakdown strength decreases as the ionization potential of oxygen is less than that of hydrogen so electron impact ionization is more probable at lower electron energy. This can cause the ionization wave to move ahead of the snowplow shock. In the case of the high voltage shot in Figure 130c, the ionization wave moves ahead of the sheath because of violation of the upper bound on the snowplow velocity (see Section 3.3.3.6) leading to apparent thickening of the sheath.

From the state of knowledge on this topic, it is clear that there is a lot of scope to further investigate this question, which has remained open for several decades.

8.4.1.4. What Is the Efficiency with Which the Sheath Snowplows the Gas Ahead of It? How Can It Be Measured? How Does It Affect Plasma Focus Operation? What Factors Govern It?

The snowplow concept is based on the idea that the magnetic piston driven shock sweeps all the gas that it encounters during its propagation. There are indications that it may be sweeping only a fraction of the encountered gas. The Lee model defines a mass fraction to account for this aspect. The same number, however, may also be correcting for the sheath curvature effect to some unknown extent.

The density of plasma left behind the sheath during its axial transit has been estimated by Mather [2] by the following logic. "The propagation speed of an electromagnetic wave down the coaxial tube was obtained by determining the time delay between a dI/dt signal near the focus and its appearance at the breech of the accelerator. This time difference corresponds to a wave speed $\mathrm{v}_{\mathrm{w}} \sim 10^{9} \mathrm{~cm} / \mathrm{sec}^{\prime \prime}$ The magnetized residual plasma in the coaxial gun has a dielectric constant given by $\mathrm{k}=1+4 \pi \rho \mathrm{c}^{2} / \mathrm{B}^{2}$. Equating the measured wave speed with $\mathrm{c} / \sqrt{\mathrm{k}}$, Mather estimated that the residual plasma density was about $10^{-4}$ times the initial fill density, demonstrating excellent snowplowing efficiency.

Formation of multiple sheaths in the PF-3 (see Section 5.2, Figure 113) is believed to be because of incomplete sweeping of the neutral gas by the sheath. Incomplete transfer of current from the capacitor bank to the current sheath has also been blamed on the inefficient snowplow (see Section 4.2.2 (1), Section 4.2.2 (2)). On the other hand, nearly 100\% current transfer from the capacitor bank to the sheath has been reported (see Section 4.2.2 (3)b), indicating that such efficient current transfer is feasible.

Filamentation of the current sheath has been suggested [140] as one possible reason for neutral gas to slip through the plasma sheath. 
8.4.1.5. To What Extent Is the Plasma Focus Neutron Yield Determined by the Micro-Structure of the Plasma Such as Filaments and Hot Spots?

The experimental observations show that the ion emission is strongly influenced by filamentation phenomena, because magneto-hydro-dynamic instabilities of individual filaments (macroscopic and miniature ones) lead to the formation of local plasma micro-diodes accelerating ions and electrons, while the local magnetic fields deflect trajectories of the accelerated charged particles (see Section 2.3.1). This effect is particularly connected with macroscopic (quasi-axial and spiral) filaments [102], but it can be neglected for numerous tiny filaments [100]. It should be noted that plasma-current filaments can be formed inside two or three cylindrical plasma layers, as well as at the discharge axis.

Although microstructures, such as filaments and hot spots, are certainly present in the plasma focus to a greater or lesser degree, a quantification of their effect on the neutron emissions is not available in any experiment. Detection of ion micro-beams of high energy lends support to conjectures regarding their role in acceleration of ions (see Section 2.3.1). Triton burnup data from Frascati, analyzed years later (see Section 2.3.2 (4)b), and the correlation between neutron signals and framing pictures of filaments apparently undergoing annihilation (see Figure 15) are the only experimental data that directly relate the presence of microstructure with nuclear reactions. Research $[95,200]$ based on the assumption that a major portion of the fusion yield from the plasma focus can arise from such microstructure has so far not yielded unambiguous data that confirms this assumption. On the other hand, there are explicit mentions to the contrary:

- "Hot spots are not a primary source of neutron emission" [470].

- "The results obtained in this experiment indicate that there is no correlation between the presence or the number of hot spots and the total neutron yield." [431].

- $\quad$ There are no correlations between the number of hot-spots and the neutron yields [152].

This must, therefore, be treated as an open question. A diagnostics concept [407] that can measure neutron fluence distribution with a sub-millimeter spatial resolution is discussed later (see Section 8.4.2.2). If microstructure really plays a significant role in neutron emission, its signature should be revealed by this diagnostic.

8.4.1.6. Why Does the Addition of High z Gases Increase the Neutron Yield in Some but Not All Devices?

The increase in neutron yield by doping of deuterium with high $\mathrm{z}$ gases is observed in Filippov devices PF-3 (see Section 3.1.6), Sahand [437], a 1 MJ Mather device Gemini [436], and in smaller Mather type devices in Singapore [221,432] and University of Malaya [413,434]. It is not observed in DPF78 [431]. It has been suggested [433] that in “... an optimized plasma focus device $\mathrm{D}_{2}-\mathrm{Kr}$ admixtures may give little or no neutron yield enhancement relative to pure $\mathrm{D}_{2}$ operation". This issue appears to be not quite well understood at the present moment.

\subsubsection{Open Questions Related to the Emerging Narrative}

This section looks at some questions pertaining to the emerging narrative discussed above.

\subsubsection{Does There Exist an Axial Magnetic Field in Every Plasma Focus?}

The main point of departure between the standard narrative and the emerging narrative lies in the role of the axial magnetic field. Although there are reports about the detection of axial magnetic field from 3 large PF facilities (Frascati, POSEIDON and PF-1000), it is not known with any degree of certainty that it exists also in other PF devices. This is because of technical difficulties of the diagnostics. It is very important to distinguish between "measurement" of the axial magnetic field, which is a space and time dependent quantity with a magnitude that needs to be determined with a specified error bar with certain space and time resolution, and its "detection", which involves just a yes or no answer without specifying its magnitude and accuracy of measurement. Recently, a technique for detection of the azimuthal component of the vector potential has been proposed [407] and 
demonstrated [472]. It is only after improvement and replication of this experiment on multiple PF facilities that a definite answer can be found. It would of course be very nice if a technique for determination of azimuthal current density distribution from measurements made outside the plasma could be developed.

\subsubsection{Does the ICDMP BEAM-TARGET Model Explain the Neutron Yield from Every Plasma Focus?}

This is a very important open question. There are some very distinctive signatures of the neutron emission according to the ICDMP BEAM-TARGET model.

The neutron emission from the post-instability phase is suggested as being due to accelerated ions that move like water in a rotating fountain that shoots like a tight jet in the middle but falls back into its pool as a scattered shower, from which it is re-pressurized by the pump and sent up. In this analogy, the virtual plasma-filled diode, formed as a result of the $\mathrm{m}=0$ instability, plays the role of the pump. The ions playing the role of the central jet of the fountain would produce reactions whose center of mass would appear to be moving along the axis exactly like an axial beam-target mechanism "confirmed" as being the major neutron production mechanism in many papers. The reaction protons produced by the ions playing the role of the scattered shower falling into the pool would show up as an image larger in radial size than the corresponding soft $X$-ray images. This has been reported from 3 facilities: POSEIDON, PF-1000, and NX2. Space resolved time-integrated neutron and reaction proton images from Frascati and POSEIDON, respectively, show the rotational features of opposite spectral shifts at laterally mirror symmetric points.

A diagnostic has been proposed [407] to verify the spectral signatures of the ICDMP BEAM-TARGET model that can be fielded on every plasma focus producing more than $10^{8}$ neutrons. This consists of a $\sim 50 \mathrm{~mm}$ thick stack of $75 \mu \mathrm{m}$ thick polyimide films drilled with a $5 \mathrm{~cm} \times 10 \mathrm{~cm}$ matrix of $0.1 \mathrm{~mm}$ diameter holes on $0.2 \mathrm{~mm}$ pitch which are carefully aligned to generate a fast-neutron micro-collimator array. An acrylic sheet placed in contact with this stack converts neutrons, that travel along the $0.1 \mathrm{~mm}$ diameter tunnel formed by aligned holes without collisions, into recoil protons, whose energy bears a mathematical relationship with the energy of incident neutrons. A similar $5 \mathrm{~mm}$ thick stack of foils acts as an array of recoil proton collimators. A CR-39 SSNTD film kept in contact with the second stack records a time-integrated image of recoil protons, whose energy and fluence distribution can be related with the energy and fluence distribution of fast neutrons incident on the stack. Such stacks can be placed at $0^{\circ}, 90^{\circ}$, and $180^{\circ}$ to the plasma focus axis at distances as close as physically possible to the plasma focus. Two stacks can be placed at $90^{\circ}$ to the axis facing the plasma from opposite sides. The CR-39 tracks can be developed using protocols which can relate their diameter to the energy of the incident recoil protons. The micro-collimators provide natural discrimination against neutrons that do not travel along the collimator because they lose nearly half their energy in the first collision they make in the hydrogenous material that the collimator is made of. According to the ICDMP BEAM TARGET model, the neutron energy spectra measured at $0^{\circ}$ and $180^{\circ}$ should have complementary spatial dependence. The central regions of the recoil proton image at $0^{\circ}$ and $180^{\circ}$ should have spectral peaks shifted above and below $Q$, while the peripheral regions should have the shifts below and above $Q$, respectively. The same complementary signature should be seen in the two images measured perpendicular to the axis with $180^{\circ}$ azimuthal separation. Smaller neutron yield devices may need to expose the detector to multiple shots while on large machines, a single shot measurement should be sufficient. Using gated bare MCP detectors for recording the recoil protons can perhaps also provide simultaneous sub-millimeter space resolution and nanosecond time resolution for neutron source localization in different phases of PF evolution.

This diagnostic can also resolve the question raised in Section 8.4.1.4 regarding the role of microstructure in the neutron emission. Super dense "plasma domains of enhanced nuclear reactivity" discussed in Section 2.3.2 (4) should be revealed as statistically significant small scale non-uniformity in the neutron fluence distribution in the track images. 


\subsubsection{Are Hard X-rays and Neutrons Generated at the Same Time in All PF Devices?}

This question has been raised by experiments discussed in Section 4.3.2 (3), (4), and (5). Clearly, the importance of this question in the overall scheme of PF physics requires that these experiments be repeated on other devices perhaps with better particle statistics at the detector and if possible using multiple diagnostics. A related question is whether HXRs and neutrons are generated at the same location inside the plasma. It should be possible to construct a micro-collimator array for hard X-rays in a manner similar to the one discussed above for neutrons. Instead of using polyimide films, one can use a $25 \mathrm{~mm}$ thick stack of stainless steel sheets of $50 \mu \mathrm{m}$ thickness where one can use photochemical milling to create a $5 \mathrm{~cm} \times 10 \mathrm{~cm}$ matrix of $0.1 \mathrm{~mm}$ square holes on $0.2 \mathrm{~mm}$ pitch. Standard medical $\mathrm{X}$-ray films can be used to record the X-rays or MCPs with gold photo-cathodes can be used for time resolved measurements. Comparison of X-ray and neutron images with sub-millimeter spatial resolution should go a long way towards answering most of the unresolved questions related to PF physics.

\subsubsection{Does Neutron Emission Begin before the Current Derivative Singularity in All PF Devices?}

Although neutron emission is known to begin before the current derivative singularity in POSEIDON, SPEED-II, Frascati, and PF-1000 installations, the experiments discussed in Section 4.3.2 (3) and (5) suggest that this might be the case also in smaller devices. This has a bearing on acceleration of ions in the azimuthal direction either by the mechanism suggested by Deutsch and Kies [177], where the question of a preferred direction of azimuthal acceleration (clockwise or counterclockwise) is not addressed, or by the mechanism suggested by Auluck $[174,335,463]$ where it is. There is, therefore, a very strong case for replication of these experiments on all neutron producing PF devices. If this feature is found in many PF devices, a major revision of the current understanding of PF phenomena would be required. The diagnostics discussed in Section 8.4.2.2 would also help in resolving this question.

\subsubsection{Are Plasmoids Generated in All PF Devices?}

The observation of spherical clusters in ion pinhole images in Figure 97 is strongly suggestive of occurrence of plasmoids in at least one kJ-level PF device. Perhaps such measurements can be repeated on other devices. In principle, digital interferometry has the spatial and temporal resolution to look for existence of plasmoids in smaller PF devices. Detection of the axially symmetric part of azimuthal component of current density [472] may be another way of looking for the existence of dynamo processes and possibly also plasmoids in smaller plasma focus devices.

\subsection{Implications: Question Mark over the Fundamental Premise of Controlled Fusion Research}

In the early days of controlled fusion research (CFR), the scientific community was aware of three methods of generating fusion reactions: (1) bombardment of a solid target with a beam of accelerated ions as in a Cockcroft-Walton machine, (2) generation and confinement of a hot, fusion-reactive plasma as in the stars and (3) explosive release of fusion energy as in a hydrogen bomb. Only the last two methods were developed into mainline CFR programs known as magnetic confinement fusion and inertial confinement fusion, because the first was dismissed with the following argument:

The fusion reaction cross-sections are many orders of magnitude smaller than the Coulomb scattering cross-section. Hence a Coulomb collision is much more probable event than a fusion reaction in a beam-target fusion. The accelerated ions that undergo collisions lose energy to electrons and may be additionally scattered out of the reaction zone so that they do not have another chance of a fusion reaction. Therefore, the energy loss rate would always be much higher than the energy production rate. Hence this is fundamentally unviable. However, if the scattered ions can be provided with many more chances of undergoing fusion reaction by making them part of a confined thermal plasma at a high enough temperature, this restriction would not apply. 
The conclusion that any kind of beam-target fusion process would always be inefficient as compared with a thermonuclear process became rather an article of faith, but no longer a fit topic for reasoned scientific discussion. This is the Fundamental Premise of Controlled Fusion Research (FPCFR) [473].

The realization that fusion reactions in various z-pinches and plasma focus installations were caused by accelerated ions was a sufficient reason to discontinue funding support for the Frascati and Limeil installations. It was no longer necessary to look into the merits of the beam-target fusion where the target was not a solid target but a warm plasma, the beam was not produced by an expensive machine but was created gratis by the plasma itself and the ion beam was kept confined in self-organized magneto-plasma structures (as revealed in the last experimental reports from Frascati). The leadership of the Frascati plasma focus project migrated to the MCF research program because, by then, the Fundamental Premise of Controlled Nuclear Fusion (FPCFR) was already taken as an established fact not subject to any serious re-examination

This situation has changed, as seen from the experimental results and their interpretation reviewed in this article. The ions that produce fusion reactions seem to repeatedly cross the reaction zone. They do not spend their energy to heat the target since the target is already hot enough so they do not slow down significantly. The target ions remain in their spatial position for times much longer the ion transit times so the beam-target interaction has much higher probability as compared to the numbers originally advanced while banishing the beam-target process from CFR. This seems to be occurring in multiple devices, although more extensive experimentation is necessary to establish it conclusively.

The emerging narrative may quite possibly demonstrate the FPCFR to be based on an incorrect understanding of the underlying physics in the early days of CFR as it evolves towards becoming established science. In that case, the possibility of a net energy producing machine based on the ICDMP BEAM TARGET mechanism of the plasma focus may need to be seriously re-examined. This is potentially a very big deal for controlled fusion research in general and for plasma focus research community in particular.

However, much preparatory work would be needed before such re-examination becomes meaningful. This is discussed in Section 9.

\section{Future Course of Action}

The potential invalidation of the Fundamental Premise of Controlled Fusion Research (FPCFR) by the emerging narrative of plasma focus physics presents opportunities for the plasma focus community to influence the trajectory of its own scientific progress by carefully planning its future course of action. This section looks at various promising ideas for such planning, which may be voluntarily adopted by various plasma focus groups and further tailored to suit their particular circumstances. The discussion is divided into short term ( $0-3$ years), medium term (1-7 years), and longer term (2-12 years) activities.

The inclusion of discussion of such planning in this review needs some explanation. The historical review presented in Section 2 reveals that the course of plasma focus research in this period was governed by factors other than the science involved in the plasma focus phenomenon. It was the coordinated activity by the plasma focus community culminating in the establishment of the International Center for Dense Magnetized Plasmas, with a clear mandate for carrying out fundamental research, that led to most of the research reported in Section 4.3. In hindsight, it is clear that many of the scientific advances reported in this review would not have taken place in the absence of such coordination. Large MJ facilities being planned in the USA for the future are clearly seen to have a more limited mandate related to priorities set by other US government agencies and not by requirements of science. The opportunity presented by the potential invalidation of the Fundamental Premise of Controlled Fusion Research (FPCFR) by the emerging narrative of plasma focus physics may be squandered unless the plasma focus community decides to coordinate its future course of action in the manner it did in the 1990s. 


\subsection{Short Term (0-3 Years) Activities}

The short term activities described below can be carried out without significant additional expenses using existing budgets and capabilities of most plasma focus laboratories. In the short term, plasma focus laboratories would do well to begin to position themselves as nodes of a networked community in addition to following their institutional mandate. This would be relevant for the long term activities as discussed later. The following suggestions might help.

\subsubsection{Voluntary Adoption of Certain Reporting Standards}

It would be a good scientific practice for experimental plasma focus publications to mention a few shot quality measures (SQM). For example, those who measure the current waveform could report the ratio of the maximum current $\left(\mathrm{I}_{\max }\right)$ and pinch current $\left(\mathrm{I}_{\text {pinch }}\right)$ to the ideal short circuit current

$$
\mathrm{SQM}_{1}=\mathrm{I}_{\max } / \mathrm{I}_{0} ; \mathrm{SQM}_{2}=\mathrm{I}_{\text {pinch }} / \mathrm{I}_{0} ; \mathrm{I}_{0} \equiv \mathrm{V}_{0} \sqrt{\mathrm{C}_{0} / \mathrm{L}_{0}}
$$

The fraction of energy remaining in the capacitor bank at the time of the pinch is another useful SQM. This could perhaps be found by fitting the Lee model or the RGV model to the experimental waveform. Reporting SQMs would allow a basis for comparison between results from different devices or from the same device under different operating conditions. For example, variation of X-ray or ion yield with pressure or voltage could be correlated with variation of the corresponding SQM to better understand the factors underlying the observed trend and allow similar measurements from different devices to be plotted together.

\subsubsection{Replication of Unusual Observations}

One of the most useful goals of short term activities of plasma focus laboratories should be to replicate unusual observations of their peers in order to establish that these phenomena are not artifacts of one device or one diagnostic but are real phenomena of more general validity. Some examples of such phenomena are:

(a) Non-simultaneity of characteristic voltage and current-derivative extrema.

(b) Monotonic growth of inductance calculated from current and voltage waveforms.

(c) Different times of origin of X-ray and neutron pulses.

(d) In heavy gases, time of emission of SXR and HXR in relation with current derivative waveform.

(e) Ion time of flight measurements in different angular directions.

In case of successful replication, the phenomenon becomes a validated feature that must be included in any scientific discourse regarding the plasma focus. In case of confirmed non-replication in many devices, it gets tagged as a possible artifact that should not be included in such deliberations.

\subsubsection{Detection of Azimuthal Electric Field outside the Plasma}

One of the key differences between the standard narrative and the emerging narrative is the role played by the axial magnetic field which is generated by an azimuthal component of current density. This should create an azimuthal component of electric field outside the plasma focus cathode. An open circular metallic ring placed outside the cathode, centered with the axis, should then develop a voltage difference across its ends, which should change its sign if the ends are interchanged. In addition, the entire ring should acquire an electric potential because of the capacitance between the plasma and itself. Two such rings, connected in clockwise and counterclockwise sense to two oscilloscope channels, should then show different signals. From their difference, the part that represents the time variation of the azimuthal electric field can be determined [472]. Exploration of such signals under different operating conditions and in different devices should create a database that can be used to examine whether azimuthal current density components exist in all plasma 
focus devices and, if so, their general behavior in relation to other diagnostic signals can be inferred.

\subsection{Medium Term (1-7 Years) Activities}

The objective of the medium term activities should be to build up critical infrastructure and capabilities in individual laboratories for carrying out more ambitious diagnostics that can potentially settle most questions in plasma focus physics. This is likely to require additional budget and may need formal cooperation agreements between different laboratories for sharing of diagnostics facilities, expertise, and expenses.

\subsubsection{Digital Interferometry on kJ Level Plasma Focus Devices with Nanosecond Laser}

This powerful technique can look for evidence of plasmoid formation and their correlation with neutron emission in small plasma focus devices. Although demonstrated with a $50 \mathrm{~J}$ plasma focus, it would be very useful on a $\mathrm{kJ}$ level machine producing more than $10^{7}$ neutrons. It can also be correlated with the azimuthal electric field measurements (Section 9.1.3) which is difficult to do with a large device, such as PF-1000. Variations on the basic principle can be explored as potential Ph.D. level projects. For example, an interferometer scheme where the interfering beams traverse the plasma from opposite directions has been suggested [361]. Interferograms produced by a double pulse with a separation less than the pulse width may be used to look at the flow patterns.

\subsubsection{Neutron and HXR Source Imaging with Sub-Millimeter Spatial Resolution}

This idea has been explained in Sections 8.4.2.2 and 8.4.2.3. On a small plasma focus, the micro-collimator array can be of a size just $1 \mathrm{~cm} \times 2 \mathrm{~cm}$ which should cost about 25 times less than the $5 \mathrm{~cm} \times 10 \mathrm{~cm}$ array required for a large device, such as PF-1000 or FF-1. Multiple laboratories could share equipment so that multi-device validation of essential physics can be achieved at a lesser overall cost for each laboratory, perhaps under a coordinated research project funded through IAEA.

\subsubsection{Pilot Projects for Exploration of Alternate Plasma Initiation Methods}

Simulations of the initial phase of the plasma focus (Section 3.3.2.3) show the critical role of photon emission and re-absorption in the propagation of the ionization front. The processes involved depend crucially on the density of the fill gas and place limits on the plasma focus operation that are not related to the propagation and pinch phases. Development of alternative methods of plasma initiation that result in plasma focus action would thus remove some of the limits on plasma focus operation.

The effect of deliberate electric field intensification using a cathodic knife edge has been quantified [474] in terms of the measured voltage surge. A scheme employing the principle of field intensification to form the initial plasma in a plasma focus utilizes an inside-out washer gun [382].

In this configuration (see Figure 131), the anode passes through a stack of $50 \mu \mathrm{m}$ thick stainless steel washers, separated by $200 \mu \mathrm{m}$ thick mylar washers, via a thick insulating sleeve. The metallic washers and the insulating washers have outer diameter that matches the anode diameter. Application of voltage leads to a sequential self-breakdown of the gaps between metallic washers. The characteristic current derivative singularity appears after a few tens of training shots which probably burn off non-uniformities in the edges of the washers. 

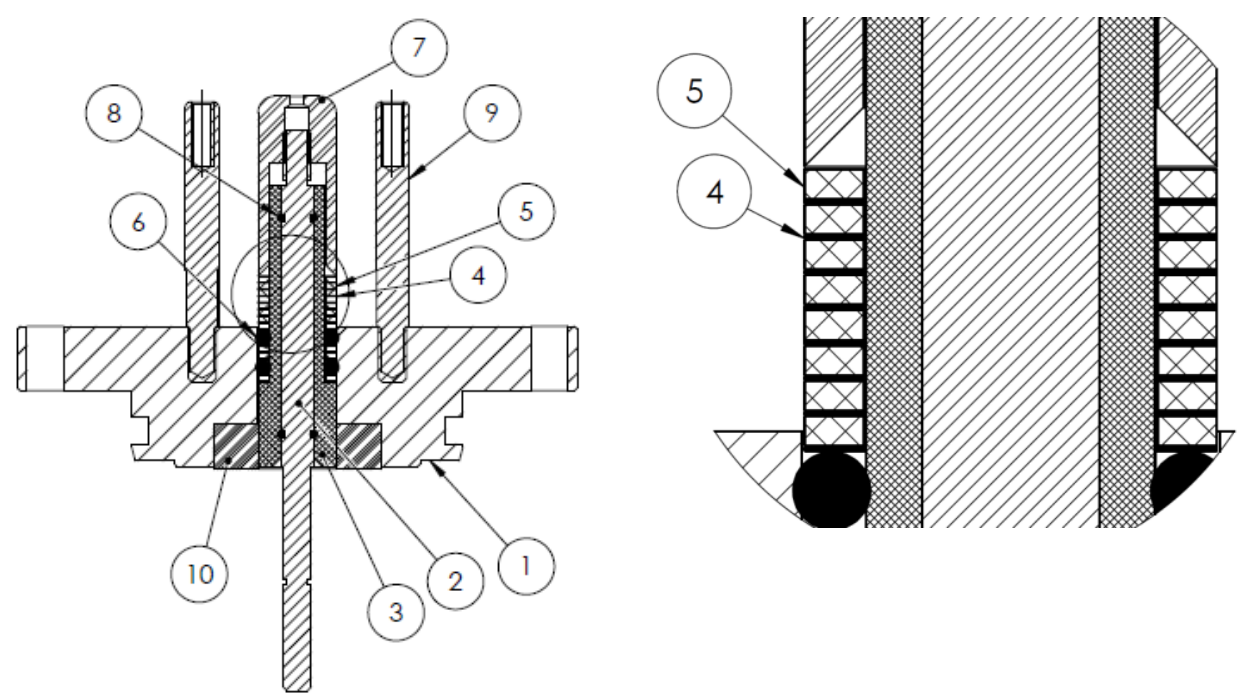

Figure 131. (1) PF support, (2) anode core, (3) insulating sleeve, (4) metallic washers, (5) mylar washers, (6) outer $\mathrm{O}$ rings, (7) anode cap, (8) inner $\mathrm{O}$ rings, (9) cathode rods $6 \mathrm{~mm}$ diameter on $34 \mathrm{~mm}$ centers (8 Nos), (10) silicone rubber gasket (Adapted from [382]).

This and similar configurations could be explored for their operating properties under small scale pilot projects. These are expected to break new ground and enable discovery of plasma focus physics in new regions of parameter space. One possibility is the optimized operation of plasma focus at deuterium pressures of 0.1 to $1 \mathrm{bar}$ [318].

\subsection{Longer Term Activities (2-10 Years)}

Before the establishment of ICDMP, there was no research program with a clear mandate for answering fundamental questions related to plasma focus physics (see Section 2.1). As a result, most reports from large plasma focus facilities in the 1970s and 1980s were conference papers that mainly reported first impressions which were not followed up with a detailed series of invstigations leading to well-researched scientific conclusions. The work on PF-1000, reviewed in Sections 4.3.1 and 5.1, shows a marked contrast to published research from other large plasma focus facilities, both past and present. This illustrates the significant role played by a large facility with a clear mandate for fundamental research, and also provides the context for identifying an appropriate long term goal for the plasma focus community.

The goal of the longer term activities of plasma focus community should be to conceptualize a worthy successor institution to the ICDMP, which is based on the PF-1000 device at the IPPLM. ICDMP is the result of coordinated initiatives between many scientists active in plasma focus research in the 1990s (see Appendix B). This goal becomes necessary because of many reasons. Firstly, the PF-1000 facility would be approaching the end of its technical hardware life sooner or later. Secondly, most of the plasma focus experiments that can be done on that device would have already been done over the next few years and many that need to be done may not be feasible with the existing installation for various reasons. Thirdly, the emerging narrative or one of its variants may become established science in not too distant future. It may indicate a path towards an energy producing machine based on the plasma focus or its modifications. That may generate new ideas for experimentation that need to be tried out at the scale of $\sim 100 \mathrm{~kJ}$ as a proof-of-concept for an ITER-like international project based on the developments in plasma focus research. This would be beyond the resources available to the ICDMP.

This goal requires the formation of a working group of scientists active in plasma focus research for carrying out deliberations similar to those that led to the establishment of ICDMP. The working group should aim to elaborate the long term vision of a successor institution to the ICDMP, conceptually explore different pathways of achieving that vision, 
identify potential avenues of continued financial support and the essential elements of its organization structure necessary for realization of its vision. This initiative would be a fitting tribute to the memory of Vladimir Gribkov, one of the founders of ICDMP.

\section{Tribute to Vladimir Gribkov}

On 30 April 2020, one of the founders and ideological inspirers of ICDMP, Professor Vladimir Alekseevich Gribkov, passed away in a tragic accident. A world-renowned expert in the fields of plasma focus, laser technology, pulsed high current discharges and charged particle beams, plasma diagnostics, X-ray technology, powerful pulsed sources of penetrating radiation, and their applications in radiation physics, chemistry, biology, and medicine, he was a prime mover of international cooperation in plasma focus research. A friend and mentor to many of the co-authors of this article, he was a warm human being with an ever present lively smile on his face, always willing to bring laughter and gaiety to any gathering he was part of.

Prof. Vladimir Alekseevich Gribkov

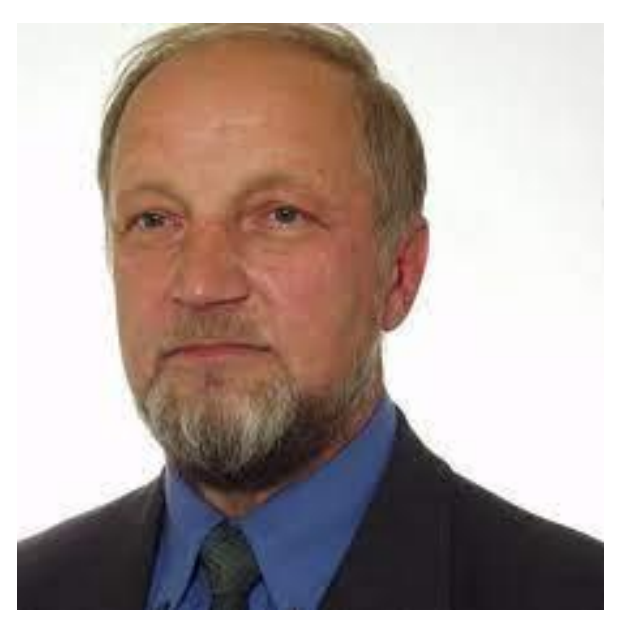

Professor Vladimir Alekseevich Gribkov graduated from one of the leading universities of the Soviet Union, the Moscow Institute of Physics and Technology (MIPT) in 1967 and immediately found himself in the thick of scientific research in the field of quantum radiophysics and electronics, since he joined the Lebedev Physical Institute of the USSR Academy of Sciences (FIAN), in the laboratory of Prof. N. G. Basov, the Nobel laureate in physics. A significant part of his career path was connected with the P. N. Lebedev Physical Institute.

In the early 1970s, in collaboration with Nikolai and Tatiana Filippov, he built the FLORA installation, where pioneering studies of the plasma focus were conducted on a wide range of issues, including the study of plasma dynamics, PF parameters at various stages of discharge development, neutron, hard and soft X-rays, charged particle beams, IR radiation, etc. In particular, he proposed a mechanism for isolating the discharge gap by turbulent plasma generated by leakage currents, a mechanism for autostabilizing the Rayleigh-Taylor instability, a mechanism for accelerating particles based on a plasma diode, and a number of others. In 1974, V. A. Gribkov defended his Ph.D. thesis "Experimental studies of cumulative phenomena in laser plasma and plasma focus", and —in 1990_the full (doctoral) dissertation on "Physical processes in high-current discharges of the "plasma focus "type". Since 1991, Vladimir Gribkov was a professor at the Moscow Institute of Engineering and Physics (MIPhI).

The FLORA installation was originally created as a complex "Plasma Focus + power laser". This allowed him to perform a number of new interferometric studies of the plasma focus. Using laser action on the plasma—the so-called "combined laser-beam heating" —as well as additional injection of material into the pinch using a laser, an improvement in the emission parameters was achieved. In 1986, Vladimir Gribkov, as a member of the team 
of scientists, was awarded the USSR State Prize for the series of works "Creation of laser diagnostic methods and research of high-temperature plasma in a physical experiment". In 1987, he was awarded the 1st degree Prize of the Polish Ministry of Science and Education for his research on Plasma Focus. In the 1990s, he was the winner of the Presidential Award for Outstanding Scientist of the Russian Federation.

Vladimir Gribkov was directly involved in the creation of a number of PF installations: PF-60 (FIAN), PLAMYA (FIAN-Experimental Design Bureau Horizon), PF-1000 and PF-6 (IPPILM, Warsaw), PF-0.2 (together with Dukhov Automatics Research Institute (VNIIA)), etc. Of particular note is the NX1 installation, the creation of which contributed to the spread of the plasma focus in many universities and research centers in different countries. The most important contribution was his participation in the creation of the PF-1000 installation, which became the main experimental base of ICDMP.

For the last 12 years, Vladimir Gribkov worked as a chief researcher at the Baikov Institute of Metallurgy and Materials Science of the Russian Academy of Sciences (IMET RAS). V. A. Gribkov was one of the pioneers of the use of high-power plasma installations in radiation materials science. For many years, his professional interests were focused on the use of dense magnetized plasma produced in the "Plasma Focus" type installations for pulsed irradiation exposure to metals, alloys, ceramics, organics and biomaterials for various purposes. Among these works are such studies as radiation testing of promising materials for nuclear and thermonuclear energy, modification of the structural and phase state of materials (including in hard-to-reach cavities), non-destructive dynamic flaw detection of machine parts and mechanisms during their operation (for example, tires or blades of jet aircraft engines).

V. A. Gribkov conducted scientific research focused on the possibility of using PF installations to detect hidden illegal materials as well as in the field of radiobiology. He also conducted exploratory research in the field of application of PF installations as pulsed sources of neutron radiation in medicine, in particular, in oncology-for solving problems of boron-neutron capture therapy of cancer formations.

Vladimir Gribkov co-authored 6 monographs, 1 textbook for universities, more than 300 scientific articles. He was the guide of 10 successfully defended Ph.D. theses. He was the President of the public organization "Moscow Physical Society" and a member of the Board of the United Physical Society of the Russian Federation, Chairman of the Physics Section of the Central House of Scientists of the Russian Academy of Sciences.

V. A. Gribkov was a member of the editorial board of the journal "Perspective Materials" and the Encyclopedia of Low Temperature Plasma. He was a visiting professor and an expert of the IAEA in Canada, Poland, the United States, Singapore, Malaysia, and Argentina. He was also an associate member of the Abdus Salam International Center for Theoretical Physics (Trieste, Italy) and a member of the Institute of Physics (UK).

In the 1990s, together with Horacio Bruzzone, he floated the idea of an International Center for Dense Magnetized Plasmas, with a mandate for fundamental research dedicated to understanding the mysteries of the plasma focus and similar phenomena. His drive, persistence, and charm helped rally the international plasma focus community around a proposal that was ultimately supported by the UNESCO. A large portion of the research described in this review became possible only because of this initiative.

He will be missed by many and remembered by many more. Had he not been a victim of this accident, he would have been an active co-author of this article. This article is therefore dedicated to the memory of Prof. Vladimir Alekseevich Gribkov.

\section{Summary and Conclusions}

This paper reviews the developments in the field of plasma focus research in the last two decades on the occasion of the 20th anniversary of establishment of the International Center for Dense Magnetized Plasmas under the auspices of the UNESCO in the year 2000. This was a landmark event, which mandated the utilization of the 1 MJ PF-1000 facility for fundamental scientific research with the involvement of the international scientific 
community. The review demonstrates that (1) this event had a positive impact on the elucidation of significant aspects of plasma focus physics, and (2) the reported progress could not have been achieved without the establishment of ICDMP.

The principal outcomes of this review are:

- A coherent presentation of major experimental results and their interpretations.

- Demonstration that certain commonly held beliefs about the plasma focus phenomenon are not supported by experimental data.

- Delineation of the outlines of an emerging narrative concerning the plasma focus phenomenon that is supported by experimental data but does require more validation.

- An appreciation of several open questions, some known for many decades and yet to be satisfactorily resolved and some new ones that could be answers by newly suggested experiments.

- A recommendation for a future course of action for the plasma focus community.

One of the significant conclusions of the review is that the described developments potentially invalidate the Fundamental Premise of Controlled Fusion Research, which takes it as an already settled matter that any fusion reaction having the character of a beam-target process must necessarily be more inefficient than a thermonuclear process with a confined thermal plasma at a suitably high temperature. The plasma focus is seen to provide a counter-example where a warm magnetically confined plasma target and an energetic ion beam that repeatedly passes through it are naturally created.

This creates a unique opportunity for the scientific community to explore a new pathway towards realization of a net fusion energy producing system.

Funding: This review paper was prepared by the voluntary contributions of its coauthors. No direct funding was received from any institution specifically for the preparation of this review paper.

Data Availability Statement: Data sharing not applicable. No new data were created or analyzed in this study. Data sharing is not applicable to this article.

Acknowledgments: S. Auluck would like to thank Alain Bernard and Horacio Bruzzone for many useful discussions and their consistent support and encouragement. He would also like to thank Valery Nikulin for access to his work on the Frascati plasma focus and Ming Fang Lu for making available unpublished material from his research.

P. Kubes would like to acknowledge financial support for his work under grants MSMT LTT17015, LTAUSA17084, CZ.02.1.01/0.0/0.0/16_019/0000778, and GACR 19-02545S.

Vyacheslav Krauz would like to acknowledge PF-3 team and Konstantin Mitrofanov (TRINITI, Troitsk, Moscow) for their help and financial support under Project 18-29-21006 from the Russian Foundation for Basic Research.

Cristian Pavez and Leopoldo Sotowould like to thank theNational Research and Development Agencyfor the ANID PIA ACT172101, ANID FONDECYT1151471, and ANID FONDECYT1211885 grants.

Ryszard Miklaszewski would like to thank International Atomic Energy Agency for support through the grant Coordinated Research Projects (CRP) no. F13019 for the project titled "Plasma Focus Devices and Diagnostics for Plasma and Fusion-relevant Education, Training and Research". The research reported in this paper has also been co-financed from the IAEA CRP grants 23071 and 23225 as well as by the Polish Ministry of Science and Higher Education within a framework of the international co-financed projects in 2021.

Alexander Blagoev would like to acknowledge Dr. Vasil Yordanov for his help and the financial support for his work under contract 1827 of Scientific Research Center of the University of Sofia.

Karel Kolacek would like to thank Ministry of Education, Youth, and Sports (MEYS) of the Czech Republic for its auspice and financial support of this work under grants LTT17015, and LTC20061.

Stuart V Springham is grateful to the National Institute of Education, Nanyang Technological University, Singapore, for AcRF grant numbers RI 7/16 SSV and RI 8/07 SVS.

M. Akel would like to express his thanks to the Director General of AECS for his encouragement and permanent support, as well as to PF-24 Lab. (IFJ) and PF-1000 Lab. (IPPLM) and ISCDMP and AAAPT for some financial support. 
Rishi Verma expresses his sincere gratitude to Archana Sharma, Associate Director (BTDG) and Head (PP\&EMD), BARC, Mumbai for her encouragement, support and keen interest in promoting applications and research on plasma devices of scientific, industrial, and academic interests.

Tõnu Laas would like to thank the International Atomic Energy Agency Coordinated Research Project F13020 in the frame of the CRP grant RC-24179.

Conflicts of Interest: Authors declare no conflict of interest.

\section{Appendix A}

A Brief Summary of Activities of ICDMP over Its 20 Year Span

The International Center for Dense Magnetized Plasmas (ICDMP) has been set up at the Institute of Plasma Physics and Laser Microfusion (IPPLM), 23 Hery St. Warsaw under the following agreements:

(1) AGREEMENT between the United Nations Educational, Scientific and Cultural Organizaion and the National Atomic Energy Agency of the Republic of Poland concerning establishment and operation of the International Center for Dense Magnetized Plasmas dated 24 August 1999 (http: / / www.icdmp.pl/2012-07-03-16-49-16/unesco-agreement, accessed on 15 July 2021).

(2) AGREEMENT between the International Scientific Committee for Dense Magnetized Plasmas and the Institute of Plasma Physics and Laser Microfusion on the establishment and operation of the "International Centre for Dense Magnetised Plasmas" dated 17 October 2000 (http: / / www.icdmp.pl/icdmp-charter, accessed on 15 July 2021).

Article II of the second agreement states the objectives of ICDMP as follows: Article II "Objectives of Activities of the Centre"

1. The main tasks of the centre are:

- Contribution - through research, training and education - to the progress of knowledge in dense magnetized plasma physics, as well as related technology fields.

- Promotion and implementation of research findings into practical applications.

- Support and maintenance of international scientific co-operation, exchange, and communication.

- Dissemination of information on scientific achievements.

2. To this effect, the centre will carry out the following activities:

- Experimental and theoretical research.

- Education activity and specialized training.

- Provision of a platform for contacts and exchange of information among scientists from all the countries.

- Co-operation with industrial enterprises and institutions in order to implement scientific findings into practical use.

- Dissemination of the knowledge, mainly through publications and organization of scientific meetings.

3. The centre will arrange agreements on bilateral or multilateral collaboration with other laboratories and institutions. The agreements have to be accepted by partners according to their national rules. Joint research programmes of the centre and the partners will be approved by the International Scientific Committee for Dense Magnetized Plasmas on a regular basis.

4. The centre shall not be considered as constituting a part of, and agent for, UNESCO, and shall not carry out any activities contrary to the principles of UNESCO.

In order to facilitate these objectives, the International Scientific Committee for Dense Magnetized Plasmas organizes its annual meeting at Warsaw along with an International Workshop and Expert Meeting and a meeting of the Council of the ICDMP Foundation. The workshop is aimed at an exchange of scientific insights, examination of various viewpoints, and dissemination of recent results beyond the ambit of more formal conventional meetings. The presentations are available at the website http: / / www.icdmp.pl/2012-07-03-16-46-37/2000, 
accessed on 15 July 2021. Many ideas informally discussed at the blackboard have eventually blossomed into significant experimental campaigns which would not have materialized without such exchange.

Many young scientists have spent some time at the PF-1000 installation and participated in experiments organized by more experienced scientists. This exposure has had a significant impact on the research at their home institutions.

\section{Appendix B}

Group photos from the first meeting in Moscow (organized by the Lebedev Institute) where the idea of ICDMP was first discussed.

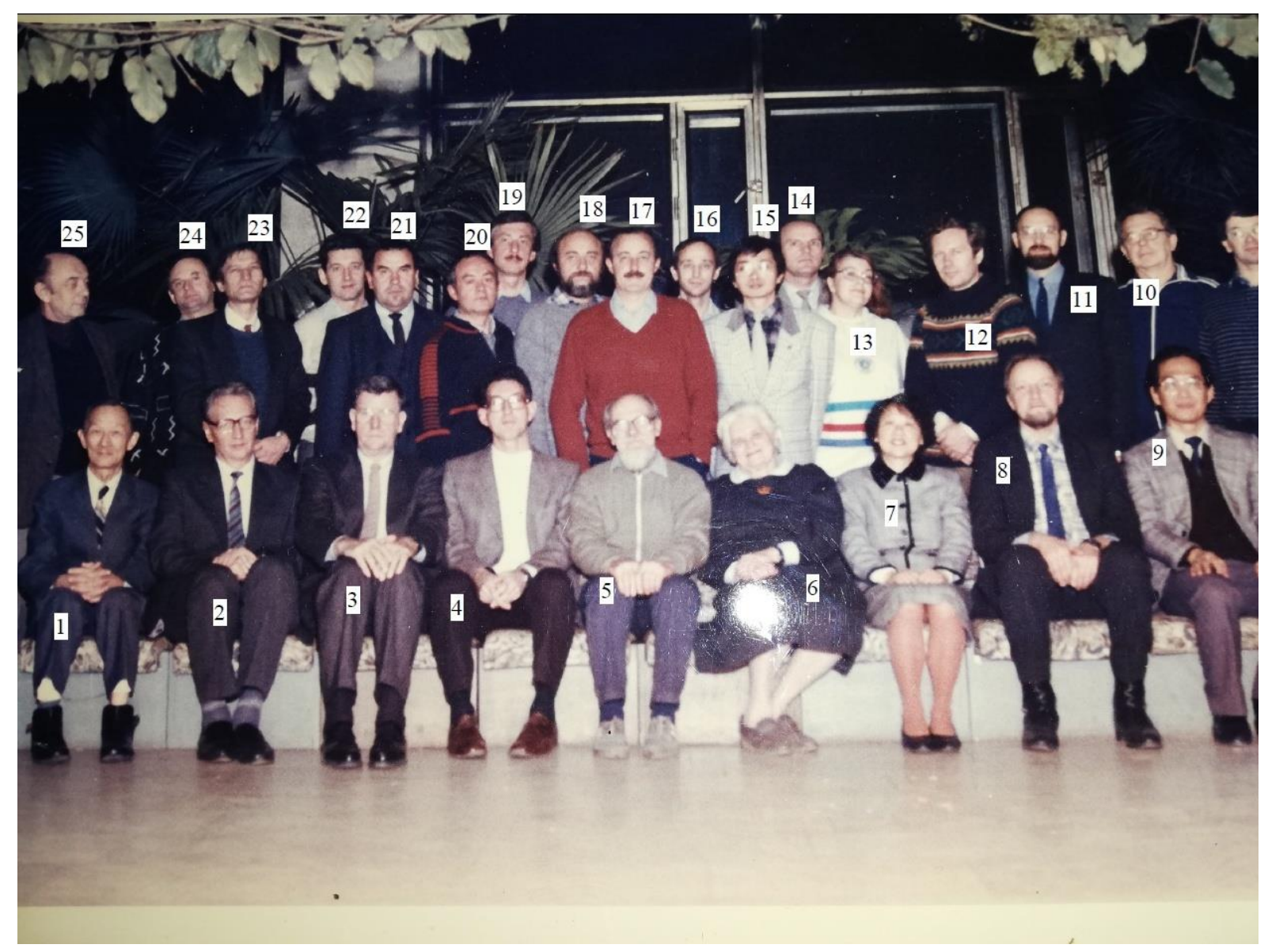

(1) Prof. T C Yang (Dept of Electrical Engineering, Tsinghua University, China). (2) Prof. M. J. Sadowski (Poland). (3) Dr. Hans Conrads (Germany). (4) Dr A. Bernard (France). (5) Dr N. V. Filippov (Russia). (6) Dr T. I. Filippova (Russia). (7) Dr. Min Han (Dept of Electrical Engineering Tsinghua Univ, China). (8) Prof. V. A. Gribkov (Russia). (9) Prof Sing Lee (University of Malaya, Malaysia) (10) Prof. Yu.S. Sigov (Russia, Keldysh Institute). (11) Dr. N. Kalachev (Russia, Lebedev Institute). (12) Dr. V.V. Yan'kov (Russia). (13) Dr. G. I. Zmievskaya (Keldysh Institute). (14) (?). (15) Dr P. Choi (UK). (16) Dr. Luis Bilbao (Argentina). (17) Prof. H. Bruzzone (Argentina). (18) Dr V. I. Krauz (Russia). (19) Dr R. Miklaszewski (Poland). (20) Dr. E.Yu. Khautiev (Sukhumi Institute of Physics and Technology). (21). Dr. N.G. Reshetnyak (Sukhumi Institute of Physics and Technolgy, now at Kharkov Institute of Physics and Technology, Ukraine). (22) (?). (23) Dr. Igonin (Facility "Plamay"). (24) L. Kociński (Poland). (25) Dr. A.A. Filukov (Keldysh Institute). 


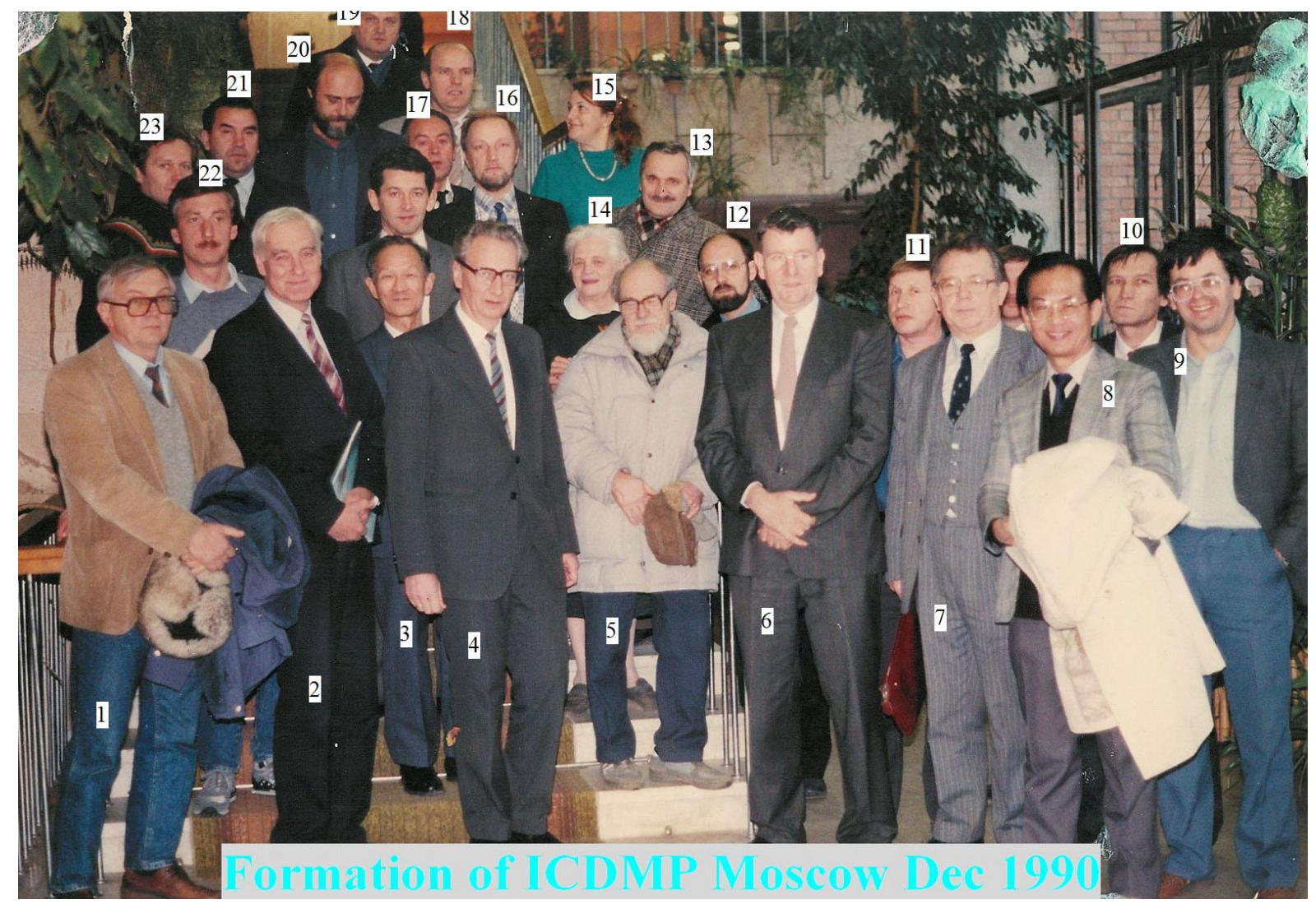

(1) (?). (2) Prof. O. N. Krokhin (Russia). (3) Prof. T C Yang (China). (4) Prof. M. J. Sadowski (Poland). (5) Dr N. V. Filippov (Russia). (6) Dr. Hans Conrad (Germany). (7) Yu. S. Sigov (Keldysh Institute). (8) Prof Sing Lee (University of Malaya, Malaysia). (9) Dr. A.V. Gerusov (Russia). (10) Dr. Igonin (Facility „Plamay”). (11) Dr V. Ya. Nikulin (Russia, Lebedev Iustitute). (12) Dr. N. Kalachev (Russia, Lebedev Iustitute). (13) Dr. V.P. Vinogradov (Kurchatov Institute). (14) Dr T. I. Filippova (Russia). (15) Dr. G. I. Zmievskaya (Keldysh Institute). (16) Prof. V. A. Gribkov (Russia). (17) Dr. E.Yu. Khautiev (Sukhumi Institute of Physics and Technology). (18) (?). (19) (?). (20) Dr V. I. Krauz (Sukhumi Institute of Physics and Technology, now Kurchatov Institute, Russia). (21) Dr. N.G. Reshetnyak (Sukhumi Institute of Physics and Technolgy, now Kharkov Institute of Physics and Technology, Ukraine). (22) (?). (23) Dr. V.V. Yan'kov (Russia).

\section{References}

1. Bernard, A.; Bruzzone, H.; Choi, P.; Chuaqui, H.; Gribkov, V.; Herrera, J.; Hirano, K.; Lee, S.; Luo, C.; Mezzetti, F.; et al. Scientific status of the dense plasma focus. J. Mosc. Phys. Soc. 1998, 8, 93-170.

2. Mather, J.W. Dense Plasma Focus. Methods Exp. Phys. 1971, 9, 187-249.

3. Bernard, A. Plasma Focus at Applications, LE VIDE. Soc. Fr. Vide 2002, 306, 836.

4. Gribkov, V.A.; Banaszak, A.; Jakubowski, L.; Sadowski, M.J.; Szydlowski, A.; Bienkowska, B.; Borowiecki, M.; Ivanova-Stanik, I.; Karpinski, L.; Miklaszewski, R.A.; et al. Transient Phenomena in the Final Implosion Stage at the PF-1000 Facility; Preprint IC/2006/United Nations: 2006. Abdus Salam International Center for Theoretical Physics: Trieste, Italy, 2006; Available online: https://inis.iaea.org/collection/NCLCollectionStore/_Public/39/016/39016690.pdf (accessed on 15 July 2021).

5. Brzosko, J.S.; Degnan, J.H.; Filippov, N.V.; Freeman, B.L.; Kiutlu, G.F.; Mather, J.W. Comments on the Feasibility of Achieving Scientific Break-Even with a Plasma Focus Machine. In Current Trends in International Fusion Research; Springer Science and Business Media LLC: Berlin/Heidelberg, Germany, 1997; pp. 11-32.

6. Soto, L. New trends and future perspectives on plasma focus research. Plasma Phys. Control Fusion 2005, 47, A361-A381. [CrossRef]

7. Krauz, V. Progress in plasma focus research and applications. Plasma Phys. Control Fusion 2006, 48, B221-B229. [CrossRef]

8. Vikhrev, V.V.; Korolev, V.D. Neutron generation from Z-pinches. Plasma Phys. Rep. 2007, 33, 356-380. [CrossRef]

9. Gribkov, V.; Bienkowska, B.; Borowiecki, M.; Dubrovsky, A.V.; Ivanova-Stanik, I.; Karpinski, L.; Miklaszewski, R.; Paduch, M.; Scholz, M.; Tomaszewski, K. Plasma dynamics in PF-1000 device under full-scale energy storage: I. Pinch dynamics, shock-wave diffraction, and inertial electrode. J. Phys. D Appl. Phys. 2007, 40, 1977-1989. [CrossRef] 
10. Gribkov, V.; Banaszak, A.; Bienkowska, B.; Dubrovsky, A.V.; Ivanova-Stanik, I.; Jakubowski, L.; Karpinski, L.; Miklaszewski, R.; Paduch, M.; Sadowski, M.J.; et al. Plasma dynamics in the PF-1000 device under full-scale energy storage: II. Fast electron and ion characteristics versus neutron emission parameters and gun optimization perspectives. J. Phys. D Appl. Phys. 2007, 40, 3592-3607. [CrossRef]

11. Krishnan, M. The Dense Plasma Focus: A Versatile Dense Pinch for Diverse Applications. IEEE Trans. Plasma Sci. 2012, 40, 3189-3221. [CrossRef]

12. Rawat, R.S. Dense Plasma Focus-From Alternative Fusion Source to Versatile High Energy Density Plasma Source for Plasma Nanotechnology. J. Phys. Conf. Ser. 2015, 591, 012021. [CrossRef]

13. Dubinov, A.E.; Fomicheva, E.I.; Senilov, L.A. Research with plasma foci in countries of Asia, Africa, and Latin America. Rev. Mod. Plasma Phys. 2020, 4, 1-173. [CrossRef]

14. Filipov, N.V.; Filipova, T.I.; Vinogradov, V.P. Dense high temperature plasma in a non-cylindrical Z-pinch compression (in Russian). Nucl. Fusion Suppl. 1962, 2, 577-587.

15. Mather, J.W. Investigation of the High-Energy Acceleration Mode in the Coaxial Gun. Phys. Fluids 1964, 7, S28. [CrossRef]

16. Zucker, O.; Bostick, W.; Long, J.; Luce, J.; Sahlin, H. The plasma focus as a large fluence neutron source. Nucl. Instrum. Methods 1977, 145, 185-190. [CrossRef]

17. Post, R.F. Controlled Fusion Research-An Application of the Physics of High Temperature Plasmas. Rev. Mod. Phys. 1956, 28, 338-362. [CrossRef]

18. Bostick, W.H. The pinch effect revisited. Int. J. Fus. Energy 1977, 1, 1-55.

19. Bennett, W.H. Magnetically Self-Focussing Streams. Phys. Rev. 1934, 45, 890-897. [CrossRef]

20. Ware, K.D.; Williams, A.H.; Clark, R.W. Operation of a 720 kJ, 60 kV, Dense Plasma Focus. Bull. Amer. Phys. Soc. $1973,18,1363$.

21. Nuckolls, J.; Wood, L.; Thiessen, A.; Zimmermann, G. Laser Compression of Matter to super high densities: Thermonuclear (CTR) applications. Nature 1972, 239, 139-142. [CrossRef]

22. Brueckner, K.A.; Jorna, S. Laser-driven fusion. Rev. Mod. Phys. 1974, 46, 325-367. [CrossRef]

23. Conrads, H.; Cloth, P.; Demmeler, M.; Hecker, R. Velocity distribution of the ions producing neutrons in a plasma focus. Phys. Fluids 1972, 15, 209-210. [CrossRef]

24. Belyaeva, I.; Filippov, N. Location of fast deuterons in a plasma focus. Nucl. Fusion 1973, 13, 881-882. [CrossRef]

25. Krompholz, H.; Michel, L.; Schönbach, K.H.; Fischer, H. Neutron-, ion-, and electron-energy spectra in a 1 kJ plasma focus. Appl. Phys. A 1977, 13, 29-35. [CrossRef]

26. Gullikson, R.L.; Sahlin, H.L. Measurements of high-energy deuterons in the plasma-focus device. J. Appl. Phys. 1978, 49, 1099-1105. [CrossRef]

27. Bernard, A.; Garçonnet, J.P.; Jolas, A.; Le Breton, J.P.; De Mascureau, J.; Romary, P. Plasma focus research at the Centre d'Etudes de Limeil (Invited paper). In Proceedings of the Plasma Sci. Montreal, Vancouver, BC, Canada, 7-9 June 1993.

28. Maisonnier, C.; Rager, J.P. Associazione Euratom-CNEN Sulla Fusione Report 79.31/cc June; Associazione Euratom-CNEN Sulla Fusione: Frascati, Italy, 1979.

29. Filippov, N.V.; Filippova, T.I. Subterrawatt ion beams in a plasma focus. Sov. JETP Lett. 1979, 29, 689-692.

30. Bertalot, L.; Herold, H.; Jäger, U.; Mozer, A.; Oppenländer, T.; Sadowski, M.J.; Schmidt, H. Mass and energy analysis and space-resolved measurements of ions from plasma focus devices. Phys. Lett. A 1980, 79, 389-392. [CrossRef]

31. Sadowski, M.; Schmidt, H.; Herold, H. Time-resolved studies of deuteron beams emitted from a plasma focus. Phys. Lett. 1981, 83, 435-439. [CrossRef]

32. Cebanu, A.; Chera, T.; Dinu, L.; Ionescu, G.; Ionescu-Bujor, T.; Iordanescu, A.; Mandache, N.; Tsois, N.; Vlad, M.; Zaharescu, M.; et al. Generation and diagnostics of energetic particles and plasmas in focus devices. In Proceedings of the 8th International Conference Plasma Physics \& Controlled Nuclear Fusion Research, Brussels, Belgium, 1-10 July 1980; IAEA: Vienna, Austria, 1981; Volume II, p. 197. Available online: http:/ / www-naweb.iaea.org/napc/physics/FEC/old_fus_en_conf.htm (accessed on 15 July 2021).

33. Stygar, W.; Gerdin, G.; Venneri, F.; Mandrekas, J. Particle beams generated by a 6-12.5 kJ dense plasma focus. Nucl. Fusion 1982, 22, 1161-1172. [CrossRef]

34. Mozer, A.; Sadowski, M.J.; Herold, H.; Schmidt, H. Experimental studies of fast deuterons, impurity- and admixture-ions emitted from a plasma focus. J. Appl. Phys. 1982, 53, 2959-2964. [CrossRef]

35. Bocancea, A.; Mandache, N. Analyzer system of the plasma-focus ionic component. Rev. Roum. Phys. 1983, $28,413$.

36. Sadowski, M.; Chyrczakowski, S.; Komar, W.; Rydygier, W.; Żebrowski, J. Studies on ion beams pro-duced in a plasma focus, Part II. In Proceedings of the 3rd International Workshop on Plasma Focus Research, Stuttgart, Germany, 12-13 September 1983; Report IPF-83-6. pp. 39-42.

37. Khautiev, E.Y.; Krauz, V.I.; Salukvadze, R.G. Some features of ion beam formation in a plasma focus. In Proceedings of the 11th Europ. Conf. Controlled Fusion \& Plasma Physics, Aachen, Germany, 5-9 September 1983; ECA 7D, Part I. p. 505.

38. Sadowski, M.J.; Zebrowski, J.; Rydygier, E.; Herold, H.; Jager, U.; Schmidt, H. Multi-spike structure of ion pulses generated by plasma focus discharges. Phys. Lett. A 1985, 113, 25-31. [CrossRef]

39. Schneider, R.F.; Rhee, M.J. Proceedings of the 4th International Workshop on Plasma Focus and Z-Pinch Research, Warsaw, Poland, 9-11 September 1985; p. 108. 
40. Yamamoto, T.; Shimoda, K.; Hirano, K. Neutrons, X-rays and Charged Particle Beams Emission in a 65 kV Plasma Focus. Jpn. J. Appl. Phys. 1985, 24, 324-327. [CrossRef]

41. Nardi, V. Megagauss Technology and Power Applications; Plenum: New York, NY, USA; London, UK, $1987 ;$ p. 269.

42. Jäger, U.; Herold, H. Fast ion kinetics and fusion reaction mechanism in the plasma focus. Nucl. Fusion 1987, 27, 407-423. [CrossRef]

43. Sadowski, M.J.; Zebrowski, J.; Rydygier, E.; Kucinski, J. Ion emission from plasma-focus facilities. Plasma Phys. Control Fusion 1988, 30, 763-769. [CrossRef]

44. Filippov, N.V. Acceleration of Aluminium ions in a plasma focus. Sov. Phys. JETP 1979, 49, 785-786.

45. Filippov, N.V.; Filippov, A.N.; Brzosko, J.R. Generation and Acceleration of Multicharged Heavy Ions in the Anode Contact Zone of the Plasma Focus. In Proceedings of the XXII International Conference on Phenomena in Ionized Gases, Hoboken, NJ, USA, 31 July-4 August 1995; Contributed Papers 4. pp. 9-10.

46. Kelly, H.; Lepone, A.; Sadowski, M.J.; Baranowski, J.; Skladnik-Sadowska, E.; Marquez, A. Analysis of the nitrogen ion beam generated in a low-energy plasma focus device by a Faraday cup operating in the secondary electron emission mode. IEEE Trans. Plasma Sci. 1998, 26, 113-117. [CrossRef]

47. Pasternak, A.; Sadowski, M. Analysis of ion trajectories within a pinch column of a PF-type discharge. In Proceedings of the 1998 ICPP \& 25th EPS Conf. CF\&PP, Prague, Czech Repulic, 29 June-3 July 1998; ECA Volume 22C. pp. 2161-2164.

48. Scholz, M.; Karpinski, L.; Pisarczyk, T.; Sadowski, M.; Jakubowski, L.; Szydlowski, A.; Faenov, A.Y.; Pikuz, S.A.; Romanova, V.M. Study of current sheath dynamics and charged particle emission from PF-1000. In Proceedings of the 1998 ICPP \& 25th EPS Conf. CF\&PP, Prague, Czech Repulic, 29 June-3 July 1998; ECA Volume 22C. pp. 2868-2871.

49. Sadowski, M.; Zebrowski, J.; Jakubowski, L. Research on emission of charged particle beams from PF-360 facility. In Proceedings of the 1998 ICPP \& 25th EPS Conf. CF\&PP, Prague, Czech Repulic, 29 June-3 July 1998; ECA Volume 22C. pp. $2876-2879$.

50. Pasternak, A.; Sadowski, M. Theoretical study of ion motion within a plasma-focus region. J. Techn. Phys. 1998, $39,45$.

51. Scholz, M.; Karpiński, L.; Kasperczuk, A.; Pisarczyk, T.; Sadowski, M.; Jakubowski, L.; Szydlowski, A.; Faenov, A.Y.; Romanova, V.M. Studies of noncylindrical pinch dynamics and charged particle emis-sion within PF-1000 facility. J. Techn. Phys. 1998, 39, 67.

52. Szydlowski, A.; Sadowski, M.; Scholz, M. Measurements of ion beams on the PF-1000 facility by means of nuclear track detectors. J. Techn. Phys. 1998, 39, 73.

53. Kelly, H.; Lepone, A.; Marquez, A.; Sadowski, M.; Baranowski, J.; Skladnik-Sadowska, E. Faraday-cup measurements of deuteron beams generated by a small PF device. J. Techn. Phys. 1998, 39, 103.

54. Sadowski, M.; Skladnik-Sadowska, E.; Baranowski, J.; Milanese, M.; Moroso, R.; Pouzo, J. Structure of ion beams emitted from a small PACO PF-device. J. Techn. Phys. 1998, 39, 109.

55. Sadowski, M.; Zebrowski, J. Diagnostic methods of experimental studies on emission of pulsed deuteron and electron beams from the PF-360 facility. J. Techn. Phys. 1998, 39, 115.

56. Sadowski, M.J.; Szydlowski, A.; Scholz, M.; Kelly, H.; Marquez, A.; Lepone, A. Application of solid-state nuclear track detectors for studies of fast ion beams within PF-1000 and other plasma-focus facilities. Radiat. Meas. 1999, 31, 185-190. [CrossRef]

57. Składnik-Sadowska, E.; Baranowski, J.; Milanese, M.M.; Moroso, R.; Pouzo, J.; Sadowski, M.; Żebrowski, J. Research on structure of ion beams from the PACO-PF device. J. Tech. Phys. 1999, 40, 129.

58. Jakubowski, L.; Baranowski, J.; Sadowski, M.; Żebrowski, J. Ion beam measurements within MA-JA-Plasma-Focus facility. J. Tech. Phys. 1999, 40, 137.

59. Pasternak, A.; Sadowski, M. Modeling of ions kinetics in dynamic PF pinch column. J. Tech. Phys. 1999, 40, 141.

60. Pasternak, A.; Sadowski, M.J.; Galkowski, A. 3D modelling of ion motion within dynamic filamentary PF-pinch column. Czechoslov. J. Phys. 2000, 50, 159-163. [CrossRef]

61. Sadowski, M.; Skladnik-Sadowska, E.; Baranowski, J.; Zebrowski, J.; Kelly, H.; Lepone, A.; Marquez, A.; Milanese, M.; Moroso, R.; Pouzo, J. Comparison of characteristics of pulsed ion beams emitted from dif-ferent small PF devices. Nukleonika 2000, 45, 179-184.

62. Jakubowski, L.; Sadowski, M.; Zebrowski, J. Measurements of charged particle beams from plas-ma-focus discharges. Nucl. Fusion 2001, 41, 755-760. [CrossRef]

63. Pasternak, A.; Sadowski, M. Study of deuteron motion in a filamentary PF pinch column for different configurations of filaments. Nukleonika 2001, 46, S29-S32.

64. Szydłowski, A.; Scholz, M.; Karpiński, L.; Sadowski, M.; Tomaszewski, K.; Paduch, M. Neutron and fast ion emission from PF-1000 facility equipped with new large electrodes. Nukleonika 2001, 46, S61-S64.

65. Skladnik-Sadowska, E.; Baranowski, J.; Milanese, M.; Moroso, R.; Pouzo, J.; Sadowski, M.J.; Zebrowski, J. Spatial structure and energy spectrum of ion beams studied with CN detectors within a small PF device. Radiat. Meas. 2001, 34, 315-318. [CrossRef]

66. Jakubowski, L.; Sadowski, M.J. Hot-spots in plasma-focus discharges as intense sources of different ra-diation pulses. Brazil. J. Phys. 2002, 32, 187-192. [CrossRef]

67. Pasternak, A.; Sadowski, M.J.; Galkowski, A. Progress in modeling of ion kinetics in plasma-focus dis-charges. Czech. J. Phys. 2002, 52, D177-D181.

68. Moreno, J.; Pedreros, J.; Soto, L. Measurements of ions emission using Tof method and CR39 SSNTD in a Small Plasma Focus device of Hundreds of Joules. J. Phys. Conf. Ser. 2014, 511, 012025. [CrossRef]

69. Moreno, J.; Morales, D.; Avaria, G.; Cuadrado, O.; Soto, L. Ion emission study using visible spectroscopy and Tof method in a plasma focus device of two kilojoules. J. Phys. Conf. Ser. 2015, 591, 012023. [CrossRef] 
70. Jain, J.; Moreno, J.; Davis, S.; Bora, B.; Pavez, C.; Avaria, G.; Soto, L. Experimental evidence of more than one ions acceleration mechanisms in plasma focus device: Observations and interpretations. Phys. Plasmas 2019, 26, 103105. [CrossRef]

71. Banaszak, A.; Szydlowski, A.; Sadowski, M.J.; Paduch, M.; Schmidt, H.; Scholz, M.; Wolowski, J.; Bonheure, G.; Van Wassenhove, G. Application of pm-351 solid-state nuclear track detectors for ion diag-nostics in high-temperature plasma experiments. Czech. J. Phys. 2004, 54, C223-C227. [CrossRef]

72. Szydlowski, A.; Banaszak, A.; Bienkowska, B.; Ivanova-Stanik, I.; Scholz, M.; Sadowski, M.J. Measure-ments of fast ions and neutrons emitted from PF-1000 plasma focus device. Vacuum 2004, 76, 357-360. [CrossRef]

73. Gribkov, V.A.; Dubrovsky, A.V.; Miklaszewski, R.; Paduch, M.; Tomaszewski, K.; Scholz, M.; Pimenov, V.N.; Ugaste, Y.E.; Sadowski, M.J.; Skladnik-Sadowska, E.; et al. Experimental studies of the interaction of ion- and plasma-streams with carbon-based targets placed near a cathode of plasma-focus facility. Plasma Phys. 2005, 10, 92-94.

74. Szydlowski, A.; Banaszak, A.; Sadowski, M.J.; Scholz, M.; Wolowski, J. Advantages of the use of solidstate nuclear track detectors in high-temperature plasma experiments. Radiat. Meas. 2005, 40, 479-482. [CrossRef]

75. Zebrowski, J.; Sadowski, M.J.; Jakubowski, L. Correlation of Neutron Emission with Other Corpuscular and X-ray Pulses in Different Plasma-Focus Experiments. AIP Conf. Proc. 2006, 812, 229-232. [CrossRef]

76. Malinowska, A.; Szydlowski, A.; Zebrowski, J.; Sadowski, M.J.; Scholz, M.; Schmidt, H.; Karpinski, P.; Jaskola, M.; Korman, A. Time-integrated measurements of fusion-produced protons emitted from PF-facilities. AIP Conf. Proc. 2006, 812, 237-240. [CrossRef]

77. Malinowski, K.; Skladnik-Sadowska, E.; Sadowski, M.J.; Scholz, M.; Schmidt, H.; Czaus, K. Analysis Of The Structure Of Ion Micro-Beams Emitted From RPI- And PF-Type Facilities. AIP Conf. Proc. 2006, 812, 256-259. [CrossRef]

78. Gribkov, V.A.; Malinowska, A.; Bieńkowska, B.; Dubrovsky, A.V.; Ivanova-Stanik, I.; Jakubowski, L.; Karpiński, L.; Miklaszewski, R.; Paduch, M.; Sadowski, M.J.; et al. Plasma and beams in PF-1000 device under the full-scale energy storage. AIP Conf. Proc. 2006, 875, 5-10.

79. Malinowska, A.; Szydlowski, A.; Malinowski, K.; Sadowski, M.J.; Scholz, M.; Paduch, M.; Ivanova-Stanik, I.; Kubes, P. Investigation of fusion-reaction protons from PF-discharges. Czechoslov. J. Phys. 2006, 56, B303-B308. [CrossRef]

80. Sadowski, M.J.; Gribkov, V.; Kubes, P.; Malinowski, K.; Skladnik-Sadowska, E.; Scholz, M.; Tsarenko, A.; Zebrowski, J. Application of intense plasma-ion streams emitted from powerful PF-type discharges for material engineering. Phys. Scr. 2006, T123, 66-78. [CrossRef]

81. Scholz, M.; Bienkowska, B.; Ivanova-Stanik, I.M.; Karpinski, L.; Paduch, M.; Stepniewski, W.; Zielinska, E.; Kravarik, J.; Kubes, P.; Malinowska, A.; et al. Studies of pinch dynamics and fusion-products emission within megajoule plasma-focus facility. Rus. Phys. J. 2006, 49, 161-164.

82. Żebrowski, J.; Sadowski, M.J.; Jakubowski, L.; Scholz, M.; Kubes, P. Research on correlation of x-ray pulses with electron-, ionand neutron-emission from high-current PF-discharges. Rus. Phys. J. 2006, 49, 165-168.

83. Skladnik-Sadowska, E.; Malinowski, K.; Marchenko, A.; Sadowski, M.J.; Scholz, M.; Karpinski, L.; Paduch, M.; Zielinska, B.; Gribkov, V.A.; Hartfuss, H.-J.; et al. Studies of Pulsed Plasma-Ion Streams During Their Free Propagation And Interaction with Carbon-Tungsten Targets in PF-1000 Facility. AIP Conf. Proc. 2008, 993, 365-368. [CrossRef]

84. Malinowska, A.; Malinowski, K.; Sadowski, M.J.; Zebrowski, J.; Szydlowski, A. Experimental Studies of Fast Protons Originated from Fusion Reactions in Plasma-Focus Discharges. AIP Conf. Proc. 2008, 993, 354-356. [CrossRef]

85. Malinowska, A.; Szydlowski, A.; Sadowski, M.J.; Zebrowski, J.; Scholz, M.; Paduch, M.; Jaskola, M.; Korman, A. Measurements of fusion-produced protons by means of SSNTDs. Radiat. Meas. 2008, 43, S295-S298. [CrossRef]

86. Kubes, P.; Kravarik, J.; Klir, D.; Rezac, K.; Bohata, M.; Scholz, M.; Paduch, M.; Tomaszewski, K.; Ivanova-Stanik, I.; Karpinski, L.; et al. Determination of Deuteron Energy Distribution From Neutron Diagnostics in a Plasma-Focus Device. IEEE Trans. Plasma Sci. 2008, 37, 83-87. [CrossRef]

87. Sadowski, M.J.; Scholz, M.; Stepniewski, W.; Malinowski, K. Modeling of motions of fast protons in fila-mentary pinches. In Proceedings of the 29th ICPIG, Cancun, Mexico, 12-17 July 2009; pp. 5-6.

88. Malinowska, A.; Szydlowski, A.; Czaus, K.; Sadowski, M.J.; Scholz, M.; Zebrowski, J.; Scholz, M.; Paduch, M.; Zielinska, E.; Jaskola, M.; et al. Application of SSNTD for measurements of fusion prod-ucts in high-temperature plasma experiments. Radiat. Meas. 2009, 44, 878-880. [CrossRef]

89. Springham, S.V.; Roshan, M.V.; Malik, F.; Sim, T.H.; Mahmood, S.; Hassan, S.M.; Shutler, P.M.E.; Tan, T.L.; Rawat, R.S.; Lee, P. Plasma Focus Fusion Research in Singapore. In Dense Magnetized Plasmas; IAEA-TECDOC-16992013; IAEA: Vienna, Austria, 2007; pp. $115-130$.

90. Sadowski, M.J.; Czaus, K.; Malinowski, K.; Skladnik-Sadowska, E.; Zebrowski, J. Mass- and energy-analysis of ions from plasma by means of a miniature Thomson spectrometer. Rev. Sci. Instrum. 2009, 80, 053504. [CrossRef] [PubMed]

91. Składnik-Sadowska, E.; Czaus, K.; Kwiatkowski, R.; Malinowski, K.; Sadowski, M.J.; Zebrowski, J.; Karpinski, L.; Paduch, M.; Scholz, M. Recent studies of the ion emission from high-current PF-1000 experiments. Probl. Atom. Sci. Technol.Ser. Plasma Phys. 2010, 16, 199-201.

92. Kwiatkowski, R.; Skladnik-Sadowska, E.; Sadowski, M.J.; Malinowski, K.; Czaus, K.; Żebrowski, J.; Karpinski, L.; Paduch, M.; Scholz, M.; Garkusha, I.E.; et al. Measurements of electron and ion beams emitted from PF-1000 device in the upstream and downstream direction. Nukleonika 2011, 56, 119-123. 
93. Kwiatkowski, R.; Sadowski, M.J.; Składnik-Sadowska, E.; Żebrowski, J.; Malinowski, K.; Czaus, K.; Garkusha, I.E. Research on spatial- and energetic-characteristics of ion beams emitted from PF-360 discharges. Nukleonika 2012, 57, 67-74.

94. Kwiatkowski, R.; Sadowski, M.J.; Składnik-Sadowska, E.; Żebrowski, J.; Malinowski, K.; Czaus, K.; Garkusha, I.E. Time- and space-resolved measurements of high-energy ion beams emitted from PF-type discharges. Nukleonika 2012, 57, 211-214.

95. Lerner, E.J.; Murali, S.K.; Shannon, D.; Blake, A.M.; Van Roessel, F. Fusion reactions from $>150 \mathrm{keV}$ ions in a dense plasma focus plasmoid. Phys. Plasmas 2012, 19, 032704. [CrossRef]

96. Kwiatkowski, R.; Czaus, K.; Skladnik-Sadowska, E.; Sadowski, M.J.; Zaloga, D.R.; Paduch, M.; Zielinska, E. Recent ion measurements within the modified DPF-1000U facility. Nukleonika 2015, 60, 297-302. [CrossRef]

97. Kwiatkowski, R.; Czaus, K.; Paduch, M.; Sadowski, M.J.; Skladnik-Sadowska, E.; Zielinska, E. Time- And Energy-Resolved Measurements Of Ion Beams Emitted From Plasma-Focus Type Discharges. In Proceedings of the 1st EPS conference on Plasma Diagnostics, Frascati, Italy, 14-17 April 2015; Volume 240, p. 121. Available online: https://pos.sissa.it/240/121/ (accessed on 15 July 2021).

98. Kwiatkowski, R.; Czaus, K.; Paduch, M.; Sadowski, M.J.; Skladnik-Sadowska, E.; Zaloga, D.R.; Zielińska, E.; Zebrowski, J. Energyand Time-Resolved Measurements of Fast Ions Emitted from Plasma-Focus Discharges by Means of a Thomson Spectrometer; SPIE: Wilga, Poland, 2015. [CrossRef]

99. Malinowski, K.; Skladnik-Sadowska, E.; Sadowski, M.J.; Szydlowski, A.; Czaus, K.; Kwiatkowski, R.; Zaloga, D.; Paduch, M.; Zielińska, E. Research on anisotropy of fusion-produced protons and neutrons emission from high-current plasma-focus discharges. Rev. Sci. Instrum. 2015, 86, 13502. [CrossRef]

100. Kubes, P.; Paduch, M.; Cikhardt, J.; Cikhardtova, B.; Klir, D.; Kravarik, J.; Rezac, K.; Zielinska, E.; Sadowski, M.J.; Szymaszek, A.; et al. Filamentation in the pinched column of the dense plasma focus. Phys. Plasmas 2017, 24, 32706. [CrossRef]

101. Kubes, P.; Paduch, M.; Sadowski, M.J.; Cikhardt, J.; Cikhardtova, B.; Klir, D.; Kravarik, J.; Munzar, V.; Rezac, K.; Zielinska, E.; et al. Charac-terization of fast deuterons involved in the production of fusion neutrons in a dense plasma focus. Phys. Plasmas 2018, 25, 012712. [CrossRef]

102. Sadowski, M.J. Comments on recent achievements of research on dense magnetized plasmas in Poland. Probl. Atom. Sci. Technol. Ser. Plasma Phys. 2018, 6, 121-126.

103. Kubes, P.; Paduch, M.; Sadowski, M.J.; Cikhardt, J.; Cikhardtova, B.; Klir, D.; Kravarik, J.; Munzar, V.; Rezac, K.; SkladnikSadowska, E.; et al. Evolution of a Pinch Column During the Acceleration of Fast Electrons and Deuterons in a Plasma-Focus Discharge. IEEE Trans. Plasma Sci. 2019, 47, 339-345. [CrossRef]

104. Damideh, V.; Chin, O.; Saw, S.; Lee, P.; Rawat, R.S.; Lee, S. Characteristics of Fast ion beam in Neon and Argon filled plasma focus correlated with Lee Model Code. Vacuum 2019, 169, 108916. [CrossRef]

105. Kubes, P.; Paduch, M.; Sadowski, M.J.; Cikhardt, J.; Cikhardtova, B.; Klir, D.; Kravarik, J.; Kwiat-kowski, R.; Munzar, V.; Rezac, K.; et al. Features of fast dfeuterons emitted from plasma focus discharges. Phys. Plasmas 2019, 26, 032702. [CrossRef]

106. Kubes, P.; Paduch, M.; Sadowski, M.J.; Cikhardt, J.; Klir, D.; Kravarik, J.; Kwiatkowski, R.; Munzar, V.; Rezac, K.; Szymaszek, A.; et al. Scenario of a magnetic dynamo and magnetic reconnection in a plasma focus discharge. Matter Radiat. Extrem. 2020, 5, 046401. [CrossRef]

107. Kubes, P.; Paduch, M.; Sadowski, M.J.; Cikhardtova, B.; Cikhardt, J.; Klir, D.; Kravarik, J.; Kwiatkowski, R.; Munzar, V.; Rezac, K.; et al. Characteristics of closed currents and magnetic fields outside the dense pinch column in a plasma focus discharge. Phys. Plasmas 2020, 27, 092702. [CrossRef]

108. Sadowski, M.; Paduch, M.; Kubes, P.; Krauz, V.; Gribkov, V. Recent Achievements of Plasma Studies within PF-1000U Facility. Acta Phys. Pol. A 2020, 138, 613-621. [CrossRef]

109. Kubes, P.; Paduch, M.; Sadowski, M.J.; Cikhardt, J.; Cikhardtova, B.; Klir, D.; Kravarik, J.; Kwiatkowski, R.; Munzar, V.; Rezac, K.; et al. Characteristics of fast deuteron sources generated in a dense plasma focus. Eur. Phys. J. Plus 2021, 136, 810. [CrossRef]

110. Bostick, W.H.; Nardi, V.; Prior, W. Production and Confinement of High-Density Plasmas. Ann. N. Y. Acad. Sci. 1975, 251, 2-29. [CrossRef]

111. Bostick, W.H.; Grunberger, L.; Prior, W. Neutron Production by Vortex Annihilation in the Plasma Focus. Proc. of 3rd EPS conf. on Cont. Fusion and Plasma Physics, Utrecht, Netherlands. 1969, p. 125. Available online: http:/ /dx.doi.org/10.1007/BF01118673 (accessed on 13 July 2021).

112. Bostick, W.H.; Grunberger, L.; Prior, W.; Nardi, N. Neutron Production by Vortex Annihilation in the Plasma Focus. Proc. 4th Eur. Conf. Contr. Fus. Plas. Phys. Rome, Italy. 1970, p. 108. Available online: http:/ / dx.doi.org/10.1007/BF01164689 (accessed on 13 July 2021).

113. Bostick, W.H. Pair Production of Plasma Vortices. Phys. Fluids 1966, 9, 2078. [CrossRef]

114. Herold, H.; Bertalot, L.; Jaeger, U.; Schmidt, H.; Schmidt, R.; Shakhatre, M. Plasma Dynamics, Neutron and Ion Emission of the POSEIDON Plasma Focus. Proc. 11th Europ. Conf. Control Fusion Plasma Phys, Aachen, Germany. 1983, p. 477. Available online: http:/ / iopscience.iop.org/issue/0741-3335/26/1A (accessed on 13 July 2021).

115. Sadowski, M.J.; Herold, H.; Schmidt, H.; Shakhatre, M. Filamentary structure of the pinch column in plasma focus discharges. Phys. Lett. A 1984, 105, 117-123. [CrossRef]

116. Bilbao, L.; Bruzzone, H.; Nikulin, V.Y.; Rager, J.P. Plasma Dynamics during Neutron Production in the Frasacti 1 MJ plasma Focus; Laboratory Preprint from Centro di Frascati, ENEA: Frascati, Italy, 1980.

117. Rager, J.P.; Bilbao, L.E.; Bruzzone, H.A.; Gourlan, C.; Guidoni, U.; Kroegler, H.; Podda, S.; Ro-bouch, B.V.; Steinmetz, K. Experiments on neutron production phase of Frascati 1-MJ plasma focus (IAEA-CN-38/G-3). In Proceedings of the 8th International 
Conference on Plasma Physics and Controlled Fusion, Brussels, Belguim, 1-10 July 1980; Volume II, p. 209. Available online: http://www-naweb.iaea.org/napc/physics/FEC/STIPUB563_VOL2.pdf (accessed on 13 July 2021).

118. Gribkov, V.A.; Krokhin, O.N.; Nikulin, V.Y.; Fadeev, V.M. Self-organizing current-Plasma structures and their effect on plasma dynamics in a plasma focus. In Proceedings of the 1998 ICPP \& 25th EPS Conf. CF\&PP, Prague, Czech Repulic, 29 June-3 July 1998; ECA Volume 22C. p. 2272.

119. Decker, G.; Kies, W.; Nadolny, R.; Röwekamp, P.; Schmitz, F.; Ziethen, G.; Koshelev, K.N.; Sidelnikov, Y.V.; Sopkin, Y.V. Micropinch actuation in the SPEED 2 plasma focus. Plasma Sources Sci. Technol. 1996, 5, 112-118. [CrossRef]

120. Soto, L.; Pavez, C.; Castillo, F.; Veloso, F.; Moreno, J.; Auluck, S.K.H. Filamentary structures in dense plasma focus: Current filaments or vortex filaments? Phys. Plasmas 2014, 21, 72702. [CrossRef]

121. Milanese, M.M.; Niedbalski, J.J.; Moroso, R.L. Filaments in the Sheath Evolution of the Dense Plasma Focus as Applied to Intense Auroral Observations. IEEE Trans. Plasma Sci. 2007, 35, 808-812. [CrossRef]

122. Filaments in FF-1. Available online: https://lppfusion.com/images-catch-ff-2b-in-action (accessed on 24 April 2021).

123. Khautiev, E.Y.; Krauz, V.I.; Vikhrev, V.V.; Fadeev, V.M. On the Mechanism of the Current Sheath Structure Formation in the Plasma Focus Discharge. In Proceedings of the XXIV ICPIG, Warsaw, Poland, 11-16 July 1999; pp. 89-90.

124. Lu, M.-F. Evolution of the filamentary current sheath in a plasma focus device. In Proceedings of the the Fourth International Conference on Dense z-Pinches, Vancouver, BC, Canada, 28-31 May 1997; Volume 409, pp. 397-400.

125. Sadowski, M.J. Plasma self-organization effects in high-current discharges of axial symmetry. In Proceedings of the 11th Kudowa Summer School “Towards Fusion Energy," (IPPLM), Kudowa, Poland, 11-15 June 2012; pp. 53-57.

126. Kubes, P.; Paduch, M.; Pisarczyk, T.; Scholz, M.; Chodukowski, T.; Klir, D.; Kravarik, J.; Rezac, K.; Ivanova-Stanik, I.; Karpinski, L.; et al. Interferometric Study of Pinch Phase in Plasma-Focus Discharge at the Time of Neutron Production. IEEE Trans. Plasma Sci. 2009, 37, 2191-2196. [CrossRef]

127. Sadowski, M.J.; Paduch, M.; Skladnik-Sadowska, E.; Surala, W.; Zaloga, D.; Miklaszewski, R.; Zielinska, E.; Tomaszewski, K. Soft x-ray studies of plasma-focus pinch structures in PF-1000U experiments. Plasma Sources Sci. Technol. 2015, 24, 55003. [CrossRef]

128. Alfven, H. Cosmology in the plasma universe: An introductory exposition. IEEE Trans. Plasma Sci. 1990, 18, 5-10. [CrossRef]

129. Peratt, A.L. Guest Editorial Special Issue on Space and Cosmic Plasma. IEEE Tran. Plasma Sci. 2007, 35, 741-744. [CrossRef]

130. Hayashi, T.; Sato, T.; the Complexity Simulation Group. Self-organizing plasmas. Plasma Phys. Control Fusion 1999, 41, A229-A238. [CrossRef]

131. Alfven, A. Filamentary Structure Produced by an Electric Current in a Plasma. Arkiv Fysik 1960, 19, 25.

132. Furth, H.P.; Killeen, J.; Rosenbluth, M.N. Finite-Resistivity Instabilities of a Sheet Pinch. Phys. Fluids 1963, 6, 459. [CrossRef]

133. Nardi, N. Magnetic bundles in reacting flowing plasmas. Phys Rev. Lett. 1970, 25, 718-721. [CrossRef]

134. Marklund, G. Steady State Models for Filamentary Plasma Structures Associated with Force-Free Magnetic Fields. Report TRITAEPP-78-09. 1978. Available online: https://www.iaea.org/inis/collection/NCLCollection-Store/_Public/09/403/9403234.pdf (accessed on 26 April 2021).

135. Auluck, S.K.H. A possible mechanism for the generation of filamentary plasma structures in the plasma focus. Atomkernenerg. Kerntech. 1980, 36, 173-176.

136. Ferro Fontan, C.; Sicardi Schifino, A. Generation of kilogauss radial magnetic fields in the plasma fo-cus current sheath. In Energy Storage, Compression and Switching; Nardi, V., Sahlin, H., Bostick, W.H., Eds.; Plenum: New York, NY, USA, 1983 ; Volume 2, p. 607.

137. Haines, M.G.; Marsh, F. A steady-state model of current filamentation caused by the electrothermal instability in a fully ionized magnetized plasma. J. Plasma Phys. 1982, 27, 427-435. [CrossRef]

138. Trubnikov, B.A. Current filaments in plasmas. Plasma Phys. Rep. 2002, 28, 312-326. [CrossRef]

139. Di Vita, A. Hot spots and filaments in the pinch of a plasma focus: A unified approach. Eur. Phys. J. D 2009, 54, 451-461. [CrossRef]

140. Lerner, E.J.; Hassan, S.M.; Karamitsos, I.; Von Roessel, F. Confined ion energy $>200 \mathrm{keV}$ and increased fusion yield in a DPF with monolithic tungsten electrodes and pre-ionization. Phys. Plasmas 2017, 24, 102708. [CrossRef]

141. Nikulin, V.Y.; Startsev, S.; Tsybenko, S.P. Supersonic, subsonic and stationary filaments in the plasma focus. J. Phys. Conf. Ser. 2017, 907, 12024. [CrossRef]

142. Fadeev, V.M.; Kvartskhava, I.F.; Komarov, N.N. Self-focusing of local plasma currents. Nucl. Fusion 1965, 5, 202-209. (In Russian) [CrossRef]

143. Mather, J.W. Formation of a High-Density Deuterium Plasma Focus. Phys. Fluids 1965, 8, 366. [CrossRef]

144. Bostick, W.H.; Nardi, V.; Prior, W. X-ray fine structure of dense plasma in a co-axial accelerator. J. Plasma Phys. 1972, 8, 7-20. [CrossRef]

145. Schönbach, K.H.; Michel, L.; Fischer, H. Correlation of soft x-ray spots with hard radiation and neutron emission in a 1-kJ plasma focus. Appl. Phys. Lett. 1974, 25, 547. [CrossRef]

146. Ivanov, V.D.; Kochetov, V.A.; Moiseeva, M.P.; Palkin, A.A.; Svirskij, E.B.; Terent'ev, A.R.; Filippova, T.I.; Filippov, N.V.; Veretennikov, V.A.; Vyskubov, V.P.; et al. Experimental Studies on the Plasma Focus. IAEA-CN-38/G-1-1. In Proceedings of the 8th Int. Conf. on Plasma Phys. and Contr. Nucl. Fus. Res.; Brussels, Belgium, 1-10 July 1980, IAEA: Vienna, Australia, 1981; Volume 2, pp. 161-176. Available online: http:/ / www-naweb.iaea.org/napc/physics/FEC/STIPUB563_VOL1.pdf (accessed on 15 July 2021).

147. Koshelev, K.N.; Krauz, V.I.; Reshetniak, N.G.; Salukvadze, R.G.; Sidelnikov, Y.V.; Khautiev, E.Y. The formation of micropinch structures in plasma focus with addition of heavy impurities. J. Phys. D 1988, 21, 1827. [CrossRef]

148. Koshelev, K.N.; Krauz, V.I.; Reshetnyak, N.G.; Salukvadze, R.G.; Sidel'nikov, Y.V.; Khautiev, E.Y. Formation of micropinch structures in plasma-focus discharges with admixtures of heavy atoms. Sov. J. Plasma Phys. 1989, 15, 619. 
149. Krauz, V.I. Plasma Focus. In Encyclopedia of Low-Temperature Plasma; Fortov, V.E., Ed.; Ser. B.; Yanus-K: Moscow, Russia, 2007; Volume IX-2, pp. 152-195. (In Russian)

150. Antsiferov, P.S.; Koshelev, K.N.; Krauz, V.I.; Sidelnikov, Y.V.; Khautiev, E.Y.; Yartseva, O.N. Use of dielectronic satellites for measuring the electron density in micropinches in a plasma focus. Sov. J. Plasma Phys. 1990, 16, 754.

151. Antsiferov, P.; Franz, D.; Herold, H.; Jakubowski, L.; Jonas, A.; Sadowski, M.; Schmidt, H. Hot spot formation and emission characteristics of the plasma focus. In Proceedings of the 18th EPS Conf. Control Fusion and Plasma Phys., Berlin, Germany, 3-7 June 1991; Volume 15C, Part II. p. 221.

152. Choi, P.; Aliaga-Rossel, R.; Dumitrescu-Zoita, C.; Deeney, C.; Haines, M. Dynamics of Hot Spots Formation in a Dense Plasma Focus Optical and X-ray Observations. AIP Conf. Proc. 1994, 299, 288-298. [CrossRef]

153. Schmidt, H.; Schulz, D.; Antsiferov, P. X-ray Emission from Micropinches in the DPF78 Plasma Focus. AIP Conf. Proc. 1994, 299, 348-355. [CrossRef]

154. Rowekamp, P.; Decker, G.; Kies, W.; Schmitz, F.; Ziethen, G.; Bayley, J.M.; Koshelev, K.N.; Sidelnikov, Y.V.; Rosmej, F.B.; Schulz, A.; et al. Spectral Investigations of Micropinches in the Speed 2 Plasma Focus. AIP Conf. Proc. 1994, 299, 332. [CrossRef]

155. Bayley, J.M.; Decker, G.; Kies, W.; Malzig, M.; Muller, F.; Rowekamp, P.; Westheide, J.; Sidelnikov, Y.V. Observations of soft x-ray production in the speed 2 plasma focus. J. Appl. Phys. 1991, 69, 613-617. [CrossRef]

156. Favre, M.; Silva, P.; Choi, P.; Chuaqui, H.; Dumitrescu-Zoita, C.; Wyndham, E.S. Experimental in-vestigations of hotspots in a low energy plasma focus operating in hydrogen-argon mixtures. IEEE Trans. Plasma Sci. 1998, 26, 1154-1161. [CrossRef]

157. Silva, P.; Favre, M. Properties of hotspots in plasma focus discharges operating in hydrogen argon mixtures. J. Phys. D Appl. Phys. 2002, 35, 2543-2550. [CrossRef]

158. Brzosko, J.; Nardi, V.; Goldstein, D.; Brzosko, J. Observation of plasma domains with fast ions and enhanced fusion in plasma-focus discharges. Phys. Lett. A 1994, 192, 250-257. [CrossRef]

159. Brzosko, J.S.; Brzosko, J.R.; Robouch, B.V.; Ingrosso, L. Triton burnup in plasma focus plasmas. Phys. Plasmas 1995, 2, 1259-1269. [CrossRef]

160. Jakubowski, L.; Sadowski, M. Studies of hot-spots and their correlation with other phenomena in PF-type discharges. In Proceedings of the 22nd EPS Conf. on CFPP, Bournemouth, UK, 3-7 July 1995; Part II. p. 161.

161. Surała, W.; Sadowski, M.J.; Paduch, M.; Zieliñska, E.; Tomaszewski, K. Recent measurements of soft X-ray emission from the DPF-1000U facility. Nukleonika 2015, 60, 303-308. [CrossRef]

162. Zaloga, D.R.; Sadowski, M.J.; Skladnik-Sadowska, E.; Paduch, M.; Zielinska, E.; Tomaszewski, K. In-fluence of gas conditions on electron temperature inside a pinch column of plasma-focus discharges. J. Phys. Conf. Ser. 2018, 959, 012003. [CrossRef]

163. Neil, G.R.; Post, R. Observation of overdense infrared scattering from a post-pinch plasma focus. Plasma Phys. 1981, 23, 425-434. [CrossRef]

164. Shearer, J.W. Contraction of $Z$ pinches actuated by radiation losses. Phys. Fluids 1976, 19, 1426. [CrossRef]

165. Meierovich, B. Toward the realization of electromagnetic collapse. Sov. Phys. Uspekhi 1986, 29, 506-529. [CrossRef]

166. D'yachenko, V.F.; Imshennik, V.S. Magnetohydrodynamic theory of the poinch effect in a dense high-temperature plasma (dense plasma focus). In Reviews of Plasma Physics; Leontovich, M.A., Ed.; Consultants Bureau: New York, NY, USA, 1970; pp. 447-495. [CrossRef]

167. Haines, M.G. A review of the dense Z-pinch. Plasma Phys. Control Fusion 2011, 53, 093001. [CrossRef]

168. Zweibel, E.G.; Yamada, M. Perspectives on magnetic reconnection. Proc. R. Soc. A Math. Phys. Eng. Sci. 2016, 472, 20160479. [CrossRef]

169. Gribkov, V.; Dubrovsky, A.; Kalachev, N.; Kozlova, T.; Nikulin, V. Dynamics of plasma phenomena in "plasma focus" under the action of powerful laser radiation. J. Phys. Colloq. 1979, 40, C7-763-C7-764. [CrossRef]

170. Gribkov, V.A.; Dubrovsky, A.V.; Isakov, A.I.; Kalachev, N.V.; Kozlova, T.A.; Korzavin, V.M.; Nikulin, V.Y. Dynamics of Plasma Phenomena in "Plasma Focus" under the Action of Powerful Laser Radiation. In Proceedings of the Lebedev Physical Institute; 1980; Volume 127, pp. 32-61. (In Russian). Available online: http://proceedings.lebedev.ru/wordpress/wp-content/uploads/ 2013/03/1980-127.djvu (accessed on 15 July 2021).

171. Auluck, S.K.H. Axial magnetic field and toroidally streaming fast ions in the dense plasma focus are natural consequences of conservation laws in the curved axisymmetric geometry of the current sheath. Phys. Plasmas 2014, 21, 102515. [CrossRef]

172. Rager, J.P. Progresses on Plasma Focus Research at Frascati, Invited Paper at the 10th European Conference on Controlled Fusion and Plasma Physics, Moscow, Russia. 1981, pp. 243-267. Available online: http://iopscience.iop.org/article/10.1088/0029-5515/ 22/2/014 (accessed on 15 July 2021).

173. Herold, H.; Bertalot, L.; Hirano, K.; Jager, U.; Kaeppeler, H.J.; Sadowskj, M.; Schmidt, H.; Schmidt, R.; Shakhatre, M.; Shyam, A.; et al. Two phases of neutron production in the POSEIDON plasma focus. IAEA-CN-44/D-III-6-3. In Proceedings of the Inter. Conf. on Plasma Physics and Controlled Nuclear Fusion Research, London, UK, 1984; Volume 2, p. 579. Available online: http:/ / www-naweb.iaea.org/napc/physics/FEC/STIPUB670_VOL2.pdf (accessed on 15 July 2021).

174. Auluck, S.K.H. An apparent momentum balance anomaly in the plasma focus. IEEE Trans. Plasma Sci. 1997, 25, 37-41. [CrossRef]

175. Mather, J.; Ahluwalia, H. The geomagnetic field-an explanation for the microturbulence in coaxial gun plasmas. IEEE Trans. Plasma Sci. 1988, 16, 56-57. [CrossRef]

176. Auluck, S.K.H. Spontaneous generation of magnetic field in an imploding plasma. J. Plasma Phys. 1984, 32, 349-357. [CrossRef]

177. Deutsch, R.; Kies, W. Ion acceleration and runaway in dynamical pinches. Plasma Phys. Control Fusion 1988, 30, 263-276. [CrossRef]

178. Bernstein, M.J.; Comisar, G.G. Neutron Energy and Flux Distributions from a crossed-field accelera-tion model of plasma focus and z-pinch discharges. Phys. Fluids 1972, 15, 700-707. [CrossRef] 
179. Bernard, A.; Coudeville, A.; Jolas, A.; Launspach, J.; De Mascureau, J. Experimental studies of the plasma focus and evidence for nonthermal processes. Phys. Fluids 1975, 18, 180. [CrossRef]

180. Milanese, M.M.; Pouzo, J.O. Evidence of non-thermal processes in a 1-MJ plasma focus device by ana-lyzing the neutron spectra. Nucl. Fusion 1978, 18, 533-536. [CrossRef]

181. Hübner, K.; Rager, J.P.; Steinmetz, K. Space-resolved investigation on the plasma focus neutron emission. In Proceedings of the 10th European Conference on Controlled Fusion and Plasma Physics, Moscow, Russia, 14-19 September 1981; Volume 1, p. D2. Available online: http://fusionwiki.ciemat.es/wiki/European_Physical_Society_Conference_on_Plasma_Physics (accessed on 15 July 2021).

182. Jaeger, U. Über die Reaktionsmechanismen im Plasmafokus-Untersuchungen an Beschleungiten Deuteronen und Reaktionsprotonen. Report-IPF-86-1. Ph.D. Thesis, Institut Fuer Plasmaforschung, University of Stuttgart, Stuttgart, Germay, 1986. (In German).

183. Schmidt, H.; Kubes, P.; Sadowski, M.; Scholz, M. Neutron Emission Characteristics of Pinched Dense Magnetized Plasmas. IEEE Trans. Plasma Sci. 2006, 34, 2363-2367. [CrossRef]

184. Steinmetz, K.; Hubner, K.; Rager, J.P.; Robouch, B.V. Neutron pinhole camera investigations on tem-poral and spatial structures of plasma focus neutron source. Nucl. Fusion 1982, 22, 30-32. [CrossRef]

185. Thong, S.P.; Lee, S. A simplified method of switching a 2 mega-ampere capacitor bank using a volt-age-division technique. Malays. J. Sci. 1973, 2, 157-169.

186. Lee, S.; Chen, Y.H. Measurements of neutrons from a focussed plasma. Malays. J. Sci. 1975, 3, $159-163$.

187. Lee, S.; Wong, C.S. Initiating and Strengthening Plasma Research in Developing Countries. Phys. Today 2006, 59, 31-36. [CrossRef]

188. Lee, S. Plasma focus model yielding trajectory and structure. Radiations in Plasmas. In Proceedings of the Spring College in Plasma Physics, Trieste, Italy, 24 May-17 June 1983; World Scientific Pub Co.: Singapore, 1984; pp. $978-987$.

189. Lee, S.; Tan, B.C.; Wong, C.S.; Chew, A.C. Laser and plasma technology. In Proceedings of the First Tropical College on Applied Physics, Kuala Lumpur, Malaysia, 26 December 1983-14 January 1984; World Scientific Publishing Co.: Singapore, 1985; pp. 38-62.

190. Lee, S.; Tou, T.Y.; Moo, S.P.; Eissa, M.A.; Gholap, A.V.; Kwek, K.H.; Mulyodrono, S.; Smith, A.J.; Suryadi; Usada, W.; et al. A simple facility for the teaching of plasma dynamics and plasma nuclear fusion. Am. J. Phys. 1988, 56, 62-68. [CrossRef]

191. Lee, S.; Moo, S.P.; Wong, C.S.; Chew, A.C. Twelve Years of UNU/ICTP PFF-A Review.IC/98/231. In Proceedings of the Satellite Meeting of the International Meeting on Frontiers of Physics, Kuala Lumpur, Malaysia, 30 October 1998; ICTP Preprint, ICTP: Trieste, Italy, 1998; p. 101.

192. Bernard, A.; Bruzzone, H.; Choi, P.; Chuaqui, H.; Gribkov, V.; Herrera, J.; Hirano, K.; Krejei, A.; Lee, S.; Luo, C.; et al. International Centre for Dense Magnetised Plasmas: A Proposal prepared on the request of UNESCO, 1994; Unpublished.

193. Moo, S.P.; Chakrabarty, C.K.; Lee, S. An Investigation of the Ion Beam of a Plasma Focus Using a Metal Obstacle and a Deuter-ated Target. IEEE Trans. Plasma Sci. 1991, 19, 515-519. [CrossRef]

194. Scholz, M.; Miklaszewski, R.; Gribkov, V.; Mezzetti, F. PF-1000 device. Nukleonika 2000, 45, 155-158.

195. Hagen, E.C. Dense Plasma Focus Fusion Neutron Sources. International Workshop and Expert Meeting of ICDMP. 2009. Available online: http:/ / www.icdmp.pl/images/2009/hagen_2009.pdf (accessed on 15 July 2021).

196. Goldin, F.J.; Meehan, B.T.; Hagen, E.C.; Wilkins, P.R. Time-resolved spectra of dense plasma focus using spectrometer, streak camera, and CCD combination. Rev. Sci. Instrum. 2010, 81, 10. [CrossRef]

197. Bennett, N.; Blasco, M.; Breeding, K.; Constantino, D.; DeYoung, A.; DiPuccio, V.; Friedman, J.; Gall, B.; Gardner, S.; Gatling, J.; et al. Development of the dense plasma focus for short-pulse applications. Phys. Plasmas 2017, 24, 012702. [CrossRef]

198. Savage, M.E.; Johns, O.; García, M.; Lake, P.; Moore, J.K.; Ormond, E.; Webb, T.J.; Bennett, N.; Gall, B.; Gardner, S.; et al. Electrical and X-ray diagnostics on the NSTec 2-MA dense plasma focus system. In Proceedings of the 2017 IEEE 21st International Conference on Pulsed Power (PPC), Brighton, UK, 18-22 June 2017; pp. 1-7.

199. Lowe, D. The NSTec Dense Plasma Focus Laboratories-An Overview. Available online: https://www.osti.gov/servlets/purl/ 1245474 (accessed on 24 April 2021).

200. Lerner, E.J.; Murali, S.K.; Haboub, A. Theory and Experimental Program for p-B11 Fusion with the Dense Plasma Focus. J. Fusion Energy 2011, 30, 367-376. [CrossRef]

201. Karakin, M.A.; Khautiev, E.Y.; Krauz, V.I.; Mokeev, A.N.; Mourenas, D.; Myalton, V.V.; Simonet, F.; Smirnov, V.P.; Tykshaev, V.P.; Vierne, J.; et al. Studies on the Liner Compres-sion in the PF-3-Facility Modernized Discharge System.BEAMS'04. In Proceedings of the 15th Int. Conf. on High-Power Particle Beams, Saint-Petersburg, Russia, 18-23 July 2004; pp. 738-741.

202. Filippov, N.V.; Filippova, T.I.; Krauz, V.I.; Azizov, E.A.; Golubchikov, L.G.; Lototsky, A.P.; Nastoyashchy, A.N.; Makeev, N.G.; Rumyantsev, V.G. Recent Progress in Plasma Focus Development: From an Existing 3 MJ to Projected 100 MJ Plasma Focus Installation. In Current Trends in International Fusion Research-Proc. of the Second Symposium, Washington; Panarella, E., Ed.; NRC Research Press: Ottawa, ON, Canada, 1999; pp. 27-40.

203. Filippov, N.V.; Filippova, T.I.; Khutoretskaia, I.V.; Mialton, V.V.; Vinogradov, V.P. Mega-joule Scale Plasma Focus as Efficient X-ray Source. Phys. Lett. A 1996, 211, 168-171. [CrossRef]

204. Zielinska, E.; Paduch, M.; Scholz, M. Sixteen-Frame Interferometer for a Study of a Pinch Dynamics in PF-1000 Device. Contrib. Plasma Phys. 2011, 51, 279-283. [CrossRef] 
205. Mitrofanov, K.N.; Krauz, V.I.; Grabovski, E.V.; Myalton, V.V.; Paduch, M.; Gritsuk, A.N. Features of the Application of the Magnetic-probe Method for Diagnostics of High-temperature Plasma. Instrum. Exp. Tech. 2018, 61, 239-259. [CrossRef]

206. Krauz, V.I.; Mitrofanov, K.N.; Myalton, V.V.; Vinogradov, V.P.; Vinogradova, Y.V.; Grabovski, E.V.; Zukakishvili, G.G.; Koidan, V.S.; Mokeev, A.N. Magnetic probe measurements of the current sheath on the PF-3 facility. Plasma Phys. Rep. 2010, 36, 937-952. [CrossRef]

207. Krauz, V.; Mitrofanov, K.; Scholz, M.; Paduch, M.; Karpinski, L.; Zielinska, E.; Kubes, P. Experimental study of the structure of the plasma-current sheath on the PF-1000 facility. Plasma Phys. Control Fusion 2012, 54, 025010. [CrossRef]

208. Krauz, V.I.; Mitrofanov, K.N.; Myalton, V.V.; Grabovski, E.V.; Koidan, V.S.; Vinogradov, V.P.; Vinogradova, Y.V.; Zukakishvili, G.G. Dynamics of the Current Distribution in a Discharge of the PF-3 Plasma Focus Facility. IEEE Trans. Plasma Sci. 2010, 38, 92-99. [CrossRef]

209. Mitrofanov, K.N.; Krauz, V.I.; Kubes, P.; Scholz, M.; Paduch, M.; Zielinska, E. Study of the fine structure of the plasma current sheath and magnetic fields in the axial region of the PF-1000 facility. Plasma Phys. Rep. 2014, 40, 623-639. [CrossRef]

210. Krauz, V.I.; Mitrofanov, K.N.; Voitenko, D.A.; Mateev, Yu.V.; Astapenko, G.I. Study of the azimuthal magnetic fields and scaling laws at the KPF-4-Phoenix facility. Plasma Phys. Rep. 2013, 39, 888-899. [CrossRef]

211. Krauz, V.I.; Mitrofanov, K.N.; Myalton, V.V.; Vinogradov, V.P.; Vinogradova, Y.V.; Grabovski, E.V.; Koidan, V.S.; Kor̆dan, V.S. Dynamics of the structure of the plasma current sheath in a plasma focus discharge. Plasma Phys. Rep. 2011, 37, 742-754. [CrossRef]

212. Krauz, V.I.; Mitrofanov, K.N.; Scholz, M.; Paduch, M.; Kubes, P.; Karpinski, L.; Zielinska, E. Experimental evidence of existence of the axial magnetic field in a plasma focus. EPL Europhys. Lett. 2012, 98, 45001. [CrossRef]

213. Mather, J.W.; Bottoms, P.J. Characteristics of the Dense Plasma Focus Discharge. Phys. Fluids 1967, 11, 611-618. [CrossRef]

214. Bruzzone, H.; Acuña, H.; Barbaglia, M.O.; Clausse, A. A simple plasma diagnostic based on processing the electrical signals from coaxial discharges. Plasma Phys. Control Fusion 2006, 48, 609-620. [CrossRef]

215. Bruzzone, H.; Acuña, H.; Clausse, A. Neutron correlations with electrical measurements in a Plasma Focus device. Braz. J. Phys. 2008, 38, 117-122. [CrossRef]

216. Veloso, F.; Pavez, C.; Moreno, J.; Galaz, V.; Zambra, M.; Soto, L. Correlations among neutron yield and dynamical discharge characteristics obtained from electrical signals in a 400 joules plasma focus. J. Fusion Energy 2012, 31, 30-37. [CrossRef]

217. Lu, M.F. Use of a double-Wollaston prism laser differential interferometer in a plasma focus. Rev. Sci. Instrum. 1997, 68, 1149-1151. [CrossRef]

218. Jager, U.; Bertalot, L.; Herold, H. Energy spectra and space resolved measurements of fusion reaction protons from plasma focus devices. Rev. Sci. Instrum. 1985, 56, 77-80. [CrossRef]

219. Talebitaher, A.; Springham, S.V.; Shutler, P.M.E.; Lee, P.; Rawat, R.S. Imaging of Plasma Focus Fusion by Proton Coded Aperture Technique. J. Fusion Energy 2011, 31, 234-241. [CrossRef]

220. Talebitaher, A.; Shutler, P.M.E.; Springham, S.V.; Rawat, R.S.; Lee, P. Coded aperture imag-ing of alpha source spatial distribution. Radiat. Meas. 2012, 47, 992-999. [CrossRef]

221. Springham, S.V.; Talebitaher, A.; Shutler, P.M.E.; Lee, S.; Rawat, R.S.; Lee, P. Coded aperture imaging of fusion source in a plasma focus operated with pure $\mathrm{D}_{2}$ and a $\mathrm{D}_{2}-\mathrm{Kr}$ gas admixture. Appl. Phys. Lett. 2012, 101, 114104. [CrossRef]

222. Talebitaher, A.; Springham, S.V. Neutron and Proton diagnostics for Pulsed Plasma Fusion Devices. In Plasma Science and Technology for Emerging Economies; Rawat, R.S., Ed.; Springer Nature: Singapore, 2017; pp. 293-354.

223. Talebitaher, A. Coded Aperture Imaging of Nuclear Fusion in the Plasma Focus Device. Ph.D. Thesis, NTU, Singapore, 2012.

224. Shutler, P.M.E.; Talebitaher, A.; Springham, S.V. Signal-to-noise ratio in coded aperture imaging. NIMA 2012, 669, 22-31. [CrossRef]

225. Springham, S.V.; Verma, R.; Zaw, M.; Rawat, R.; Lee, P.; Talebitaher, A.; Ang, J. Plasma focus neutron energy and anisotropy measurements using zirconium-beryllium pair activation detectors. NIMA 2021, 988, 164830. [CrossRef]

226. Talebitaher, A.; Springham, S.V.; Rawat, R.S.; Lee, P. Beryllium neutron activation detector for pulsed DD fusion sources. NIMA 2011, 659, 361-367. [CrossRef]

227. Pavez, C.; Pedreros, J.; Curín, C.; Muñoz, G.; Soto, L. Digital interferometry applied to transient dense plasmas. IEEE Trans. Plasma Sci. 2012, 40, 3384-3389. [CrossRef]

228. Soto, L.; Chuaqui, H.; Saavedra, R. Interferometry of phase micro-inhomogeneities within macroscopic objects. Meas. Sci. Technol. 1997, 8, 875-879. [CrossRef]

229. Pavez, C.; Pedreros, J.; Tarifeño-Saldivia, A.; Soto, L. Observation of plasma jets in a table top plasma focus discharge. Phys. Plasmas 2015, 22, 040705. [CrossRef]

230. Pavez, C.; Pedreros, J.; Avaria, G.; Bora, B.; Moreno, J.; Soto, L. A methodology for the digital reconstruction of an interferogram, a schlieren image, or a shadowgram from a single digital holographic recording. Rev. Sci. Instrum. 2018, 89, 123103. [CrossRef]

231. Moreno, J.; Birstein, L.; Mayer, R.; Silva, P.; Soto, L. System for measurement of low yield neutron pulses from D-D fusion reactions based upon a3He proportional counter. Meas. Sci. Technol. 2008, 19, 087002. [CrossRef]

232. Soto, L.; Silva, P.; Moreno, J.; Zambra, M.; Kies, W.; Mayer, R.; Clausse, A.; Altamirano, L.; Pavez, C.; Huerta, L. Demonstration of neutron production in a table-top pinch plasma focus device operating at only tens of joules. J. Phys. D Appl. Phys. 2008, 41, 205215. [CrossRef]

233. Tarifeño-Saldivia, A.; Soto, L. Statistical characterization of the reproducibility of neutron emission of small plasma focus devices. Phys. Plasmas 2012, 19, 092512. [CrossRef] 
234. Soto, L.; Pavéz, C.; Moreno, J.; Altamirano, L.; Huerta, L.; Barbaglia, M.O.; Clausse, A.; Mayer, R.E. Evidence of nuclear fusion neutrons in an extremely small plasma focus device operating at 0.1 Joules. Phys. Plasmas 2017, 24, 082703. [CrossRef]

235. Tarifeño-Saldivia, A.; Mayer, R.E.; Pavez, C.; Soto, L. Calibration methodology for propor-tional counters applied to yield measurements of a neutron burst. Rev. Sci. Instrum. 2014, 85, 013502. [CrossRef]

236. Veloso, F.; Moreno, J.; Tarifeño-Saldivia, A.; Pavez, C.; Zambra, M.; Soto, L. Non-intrusive plasma diagnostics for measuring sheath kinematics in plasma focus discharges. Meas. Sci. Technol. 2012, 23, 087002. [CrossRef]

237. Veloso, F.; Tarifeño-Saldivia, A.; Pavez, C.; Moreno, J.; Zambra, M.; Soto, L. Plasma sheath kinematics and some implications on the modeling of very low energy plasma focus devices. Plasma Phys. Control Fusion 2012, 54, 095007. [CrossRef]

238. Avaria, G.; Ardila-Rey, J.; Davis, S.; Orellana, L.; Cevallos, B.; Pavez, C.; Soto, L.; Cevallos, B.-N. Hard X-ray Emission Detection Using Deep Learning Analysis of the Radiated UHF Electromagnetic Signal From a Plasma Focus Discharge. IEEE Access 2019, 7, 74899-74908. [CrossRef]

239. Orellana, L.; Ardila-Rey, J.; Avaria, G.; Diaz, M.A.; Pavez, C.; Schurch, R.; Soto, L. On the Relationship between the Electromagnetic Burst and Inductive Sensor Measurement of a Pulsed Plasma Accelerator. IEEE Access 2019, 7, 133043-133057. [CrossRef]

240. Orellana, L.; Avaria, G.; Ardila-Rey, J.; Davis, S.; Schurch, R.; Pavez, C. Inference of X-ray Emission From a Plasma Focus Discharge: Comparison Between Characteristic Parameters and Neural Network Analyses. IEEE Access 2020, 8 , 79273-79286. [CrossRef]

241. Da Re, A.; Mezzetti, F.; Tartari, A.; Verri, G.; Rapezzi, L.; Gribkov, V.A. Preliminary study on X-ray source from Plasma Focus device for fast radiography. Nukleonika 2001, 46, S123-S125.

242. Raspa, V.; Moreno, C.; Sigaut, L.; Clausse, A. Effective hard x-ray spectrum of a tabletop Mather-type plasma focus optimized for flash radiography of metallic objects. J. Appl. Phys. 2007, 102, 123303. [CrossRef]

243. Zambra, M.; Silva, P.; Moreno, M.; Pavez, C.; Soto, L. Experimental results on hard x-ray en-ergy emitted by a low energy plasma focus device: A radiographic image analysis. Plasma Phys. Control Fusion 2009, 51, 125003. [CrossRef]

244. Pavez, C.; Soto, L. Demonstration of X-ray Emission from an Ultraminiature Pinch Plasma Focus Discharge Operating at $0.1 \mathrm{~J}$ Nanofocus. IEEE Trans. Plasma Sci. 2010, 38, 1132-1135. [CrossRef]

245. Siahpoush, V.; Tafreshi, M.A.; Sobhanian, S.; Khorram, S. Adaptation of Sing Lee's model to the Filippov type plasma focus geometry. Plasma Phys. Control. Fusion 2005, 47, 1065-1076. [CrossRef]

246. Lee, S. Radiative Dense Plasma Focus Computation Package: RADPF. Available online: http://www.plasmafocus.net/IPFS/ modelpackage/File1RADPF.htm (accessed on 24 April 2021).

247. Lee, S. Plasma Focus Radiative Model: Review of the Lee Model Code. J. Fusion Energy 2014, 33, 319-335. [CrossRef]

248. Lee, S.; Saw, S.H. Plasma Science and Technology for Emerging Economies; Springer Science and Business Media LLC: Singapore, 2017; p. 113.

249. Potter, D. The formation of high-density z-pinches. Nucl. Fusion 1978, 18, 813-823. [CrossRef]

250. Lee, S.; Serban, A. Dimensions and lifetime of the plasma focus pinch. IEEE Trans. Plasma Sci. 1996, 24, 1101-1105. [CrossRef]

251. Lee, S.; Saw, S.H.; Rawat, R.S.; Lee, P.; Verma, R.; Talebitaher, A.; Hassan, S.M.; Abdou, A.E.; Ismail, M.; Mohamed, A.; et al. Measurement and Processing of Fast Pulsed Discharge Current in Plasma Focus Machines. J. Fusion Energy 2011, 31, 198-204. [CrossRef]

252. Auluck, S.K.H. Resistive Gratton-Vargas Model. Available online: http:/ / www.icdmp.pl/rgv (accessed on 24 April 2021).

253. Lee, S.; Saw, S.H. Pinch current limitation effect in plasma focus. Appl. Phys. Lett. 2008, 92, 021503. [CrossRef]

254. Lee, S.; Lee, P.; Saw, S.H.; Rawat, R.S. Numerical experiments on plasma focus pinch cur-rentlimitation. Plasma Phys. Control Fusion 2008, 50, 065012. [CrossRef]

255. Karami, F.; Roshan, M.V.; Habibi, M.; Lee, P.; Saw, S.H.; Lee, S. Neutron yield scaling withinductance in plasma focus. IEEE Trans. Plasma Sci. 2015, 43, 2155-2159. [CrossRef]

256. Akel, M.; Al-Hawat, S.; Lee, S. Pinch current and soft X-ray yield limitation by numerica-lexperiments on nitrogen plasma focus. J. Fusion Energy 2010, 29, 94-99. [CrossRef]

257. Lee, S. Current and neutron scaling for megajoule plasma focus machines. Plasma Phys. Control Fusion 2008, 50, 105005. [CrossRef]

258. Lee, S.; Saw, S.H. Neutron Scaling Laws from Numerical Experiments. J. Fusion Energy 2008, 27, 292-295. [CrossRef]

259. Saw, S.H.; Lee, S. Scaling Laws for Plasma Focus Machines from Numerical Experiments. Energy Power Eng. 2010, 02, 65-72. [CrossRef]

260. Lee, S.; Saw, S.H.; Lee, P.; Rawat, R.S. Numerical experiments on plasma focus neon soft x-ray scaling. Plasma Phys. Control Fusion 2009, 51, 105013. [CrossRef]

261. Akel, M.; Al-Hawat, S.; Saw, S.H.; Lee, S. Numerical experiments on oxygen Soft X-rayemissions from low energy plasma focus using Lee model. J. Fusion Energy 2010, 29, 223-231. [CrossRef]

262. Akel, M.; Lee, S.; Saw, S.H. Numerical Experiments in Plasma Focus Operated in Various Gases. IEEE Trans. Plasma Sci. 2012, 40, 3290-3297. [CrossRef]

263. Saw, S.H.; Lee, S. Multi-scaling of the dense plasma focus. J. Phys. Conf. Ser. 2015, 591, 012022. [CrossRef]

264. Lee, S.; Saw, S.H. Plasma focus ion beam fluence and flux-Scaling with stored energy. Phys. Plasmas 2012, 19, 112703. [CrossRef]

265. Lee, S.; Saw, S.H. Plasma focus ion beam fluence and flux-For various gases. Phys. Plasmas 2013, 20, 062702. [CrossRef]

266. Akel, M.; Lee, S. Dependence of Plasma Focus Argon Soft X-ray Yield on Storage Energy, Total and Pinch Currents. J. Fusion Energy 2011, 31, 143-150. [CrossRef]

267. Akel, M.; Lee, S. Scaling Laws of Nitrogen Soft X-ray Yields from 1 to 200 kJ Plasma Focus. J. Fusion Energy 2012, 32, 107-110. [CrossRef] 
268. Saw, S.H.; Lee, S. The plasma focus scaled for neutrons, soft X-rays, fast ion beams and fast-plasma streams. J. Sci. Eng. Technol. 2014, 10, 42-49.

269. Lee, S. Neutron yield saturation in plasma focus: A fundamental cause. Appl. Phys. Lett. 2009, 95, 151503. [CrossRef]

270. Lee, S.; Saw, S.H.; Ali, J. Numerical experiments on radiative cooling and collapse in plasmafocus operated in krypton. J. Fusion Energy 2012, 32, 42-49. [CrossRef]

271. Akel, M.; Lee, S. Radiative Collapse in Plasma Focus Operated with Heavy Noble Gases. J. Fusion Energy 2012, 32, 111-116. [CrossRef]

272. Lee, S.; Saw, S.H.; Akel, M.; Ali, J.; Kunze, H.-J.; Kubes, P.; Paduch, M. Conditions for Radiative Cooling and Collapse in the Plasma Focus Illustrated with Numerical Experiments on PF. IEEE Trans. Plasma Sci. 2015, 44, 165-173. [CrossRef]

273. Akel, M.; Ismael, S.; Lee, S.; Saw, S.H.; Kunze, H.J. Effects of Power Terms and Thermodynamics on the Contraction of Pinch Radius in Plasma Focus Devices Using the Lee Model. J. Fusion Energy 2016, 35, 807-815. [CrossRef]

274. Lee, S.; Saw, S.H.; Abdou, A.; Torreblanca, H. Characterizing Plasma Focus Devices-Role of the Static Inductance-Instability Phase Fitted by Anomalous Resistances. J. Fusion Energy 2010, 30, 277-282. [CrossRef]

275. Singh, A. Comparative Study of Plasma Focus Machines. Ph.D. Thesis, INTI International University, Nilai, Malaysia, 2015.

276. Lim, L.H.; Yap, S.L.; Lee, M.C.; Poh, H.S.; Ma, J.; Lee, S.; Lim, L.K.; Yap, S.S.; Lim, L.H. Comparison of measured and computed radial trajectories of plasma focus devices UMDPF1 and UMDPF0. Phys. Plasmas 2015, 22, 092702. [CrossRef]

277. Lee, S.; Saw, S.H.; Rawat, R.S.; Lee, P.; Talebitaher, A.; Abdou, A.E.; Chong, P.L.; Roy, F.A.; Singh, A.; Wong, D.; et al. Correlation of Measured Soft X-ray Pulses with Modeled Dynamics of the Plasma Focus. IEEE Trans. Plasma Sci. 2011, 39, 3196-3202. [CrossRef]

278. Saw, S.H.; Rawat, R.S.; Lee, P.; Talebitaher, A.; Abdou, A.E.; Chong, P.L.; Roy, F.; Ali, J.; Lee, S. SXR Measurements in INTI PF Operated in Neon to Identify Typical (Normal N) Profile for Shots with Good Yield. IEEE Trans. Plasma Sci. 2013, 41, 3166-3172. [CrossRef]

279. Silva, P.; Moreno, J.; Soto, L.; Birstein, L.; Mayer, R.E.; Kies, W. Neutron emission from a fast plasma focus of 400 Joules. Appl. Phys. Lett. 2003, 83, 3269-3271. [CrossRef]

280. Lee, S.; Saw, S.H.; Soto, L.; Springham, S.V.; Moo, S.P. Numerical experiments on plasma fo-cus neutron yield versus pressure compared with laboratory experiments. Plasma Phys. Control Fusion 2009, 51, 075006.

281. Saw, S.H.; Subedi, D.P.; Khanal, R.; Shrestha, R.; Dugu, S.; Lee, S. Numerical Experiments on PF1000 Neutron Yield. J. Fusion Energy 2014, 33, 684-688. [CrossRef]

282. Saw, S.H.; Lee, P.; Rawat, R.S.; Verma, R.; Subedi, D.; Khanal, R.; Gautam, P.; Shrestha, R.; Singh, A.; Lee, S. Comparison of Measured Neutron Yield Versus Pressure Curves for FMPF-3, NX2 and NX3 Plasma Focus Machines Against Computed Results Using the Lee Model Code. J. Fusion Energy 2014, 34, 474-479. [CrossRef]

283. Castillo, F.; Herrera, J.E.; Rangel, J.; Alfaro, A.; Maza, M.A.; Sakaguchi, V. Neutron Anisotropy and X-ray Production of the FN-II Dense Plasma Focus Device. Braz. J. Phys. 2002, 32, 3-12.

284. Singh, A.; Lee, S.; Saw, S.H. Numerical experimentation on focusing time and neutron yield in GN1 plasma focus machine. Int. J. Mod. Phys. Conf. Ser. 2014, 32, 1460325. [CrossRef]

285. Singh, A.; Sing, L.; Saw, S.H. Effect of the Variation of Pressure on the Dynamics and Neutron Yield of Plasma Focus Machines. IEEE Trans. Plasma Sci. 2017, 45, 2286-2291. [CrossRef]

286. Singh, A.; Heoh, S.S.; Sing, L. Numerical study on the variation of pressure on India Bhabha Atomic Research Center (BARC) and Imperial College plasma focus machines. Int. Conf. PLASMA Sci. Appl. 2017, 1824, 30002.

287. Saw, S.H.; Lee, S. Numerical Experiments on Bora- Invited Workshop/Lecture. In Proceedings of the School and Training Course on Dense Magnetized Plasma as a Source of Ionizing Radiations, Their Diagnostics and Applications, Trieste, Italy, 8-12 October 2012.

288. Akel, M.; Marciniak, L.; Ismael, S.; Gannom, D.; Kulinska, A.; Lee, S.; Scholz, M.; Kunze, H.-J.; Saw, S.H. Investigation of the Measured and Computed Neutron Yield From the PF-24 Device Operated with D2-xx \%Ar Admixture. IEEE Trans. Plasma Sci. 2019, 47, 4301-4311. [CrossRef]

289. Maslov, V.V.; Rumyantsev, V.G.; Basmanov, V.F.; Budnikov, D.V.; Garin, A.V.; Drozdov, I.Y.; Ershov, D.A.; Korkin, D.S.; Makeev, N.G.; Molodtsev, D.A.; et al. A KPU-200 movable capacitor installation. Instrum. Exp. Tech. 2014, 57, 131-134. [CrossRef]

290. Zavyalov, N.; Maslov, V.V.; Rumyantsev, V.G.; Drozdov, I.Y.; Ershov, D.A.; Korkin, D.S.; Molodtsev, D.A.; Smerdov, V.I.; Falin, A.P.; Yukhimchuk, A.A. A source with a 1013 DT neutron yield on the basis of a spherical plasma focus chamber. Plasma Phys. Rep. 2013, 39, 243-247. [CrossRef]

291. Lee, S.; Lee, P.; Zhang, G.; Feng, X.; Gribkov, V.; Liu, M.; Serban, A.; Wong, T.K.S. High rep rate high performance plasma focus as a powerful radiation source. IEEE Trans. Plasma Sci. 1998, 26, 1119-1126. [CrossRef]

292. Zhang, G.X. Plasma Soft X-ray Source for Microelectronic Lithography. Ph.D. Thesis, NTU, Singapore, 1999.

293. Shan, B. Comparative Study: Dynamics and SXR Emission of Several Plasma Focus Devices. Ph.D. Thesis, NTU, Singapore, 2000.

294. Lee, S.; Rawat, R.S.; Lee, P.; Saw, S.H. Soft x-ray yield from NX2 plasma focus. J. Appl. Phys. 2009, 106, 023309. [CrossRef]

295. Gautam, P.; Khanal, R.; Saw, S.H.; Lee, S. Comparison of Measured Soft X-ray Yield Versus Pressure for NX1 and NX2 Plasma Focus Devices Against Computed Values Using Lee Model Code. J. Fusion Energy 2015, 34, 686-693. [CrossRef]

296. Liu, M.H.; Feng, X.P.; Springham, S.V.; Lee, S. Soft X-ray yield Measurement in a smallplas-ma focus operated in neon. IEEE Trans. Plasma Sci. 1998, 26, 135-140.

297. Liu, M.H. Soft X-rays from Compact Plasma Focus. Ph.D. Thesis, NTU, Singapore, 1997.

298. Roy, F., Jr. Investigation and Optimisation of Neon Soft X-ray of the INTI Plasma Focus at 12 kV. Ph.D. Thesis, INTI International University, Nilai, Malaysia, 2015. 
299. Roy, F.A., Jr.; Saw, S.H.; Lee, P.C.K.; Rawat, R.A.; Lee, S. Correlation of Characteristic Ne SXR Signal Pulse with Computed Plasma Focus Dynamics in the Ne (97.5\%)-Kr (2.5\%) Admixtures of the INTI PF Machine at $12 \mathrm{kV}$. IEEE Trans. Plasma Sci. 2019, 47, 1297-1301. [CrossRef]

300. Akel, M.; Salo, S.A.; Ismael, S.; Saw, S.; Lee, S. Comparison of measured and computed beam ion current densities emitted from two $2 \mathrm{~kJ}$ plasma focus machines. Vacuum 2017, 136, 163-167. [CrossRef]

301. Mohanty, S.R.; Neog, N.K.; Bhuyan, H.; Rout, R.K.; Rawat, R.S.; Lee, P. Effect of Anode Designs on Ion Emission Characteristics of a Plasma Focus Device. Jpn. J. Appl. Phys. 2007, 46, 3039-3044. [CrossRef]

302. Hassan, M.; Qayyum, A.; Ahmad, R.; Murtaza, G.; Zakaullah, M. Nitriding of titanium by using an ion beam delivered by a plasma focus. J. Phys. D: Appl. Phys. 2007, 40, 769-777. [CrossRef]

303. Akel, M.; Salo, S.A.; Ismael, S.; Saw, S.H.; Lee, S. Deuterium Plasma Focus as a Tool for Testing Materials of Plasma Facing Walls in Thermonuclear Fusion Reactors. J. Fusion Energy 2016, 35, 694-701. [CrossRef]

304. Soto, L.; Pavez, C.; Moreno, J.; Inestrosa-Izurieta, M.J.; Veloso, F.; Gutierrez, G.; Vergara, J.; Clausse, A.; Bruzzone, H.; Castillo, F.; et al. Characterization of the axial plas-ma shock in a table top plasma focus after the pinch and its possible application to testing mate-rials for fusion reactors. Phys. Plasmas 2014, 21, 122703. [CrossRef]

305. Damideh, V.; Ali, J.; Saw, S.H.; Rawat, R.S.; Lee, P.; Chaudhary, K.T.; Rizvi, Z.H.; Dabagh, S.; Ismail, F.D.; Sing, L. Fast Faraday cup for fast ion beam TOF measurements in deuterium filled plasma focus device and correlation with Lee model. Phys. Plasmas 2017, 24, 063302. [CrossRef]

306. Akel, M.; Cikhardt, J.; Kubes, P.; Kunze, H.-J.; Lee, S.; Paduch, M.; Saw, S.H. Experiments and simulations on the possibility of radiative contraction/collapse in the PF-1000 plasma focus. Nukleonika 2016, 61, 145-148. [CrossRef]

307. Saw, S.H.; Lee, S. Measurement of Radiative Collapse in 2.2 kJ PF: Achieving High Energy Density (HED) Conditions in a Small Plasma Focus. J. Fusion Energy 2016, 35, 702-708. [CrossRef]

308. Marciniak, Ł. Investigation of Plasma Compression in the PF-24 Device with the Use of Different Z Working Gases. Ph.D. Thesis, Institute of Nuclear Physics, Polish Academy of Sciences, Krakow, Polish, 2019.

309. Marciniak, Ł.; Akel, M.; Kulińska, A.; Lee, S.; Kunze, H.-J.; Scholz, M.; Saw, S.H. Results of plasma radiative compression investigation in the PF-24 device operated with D2, Ar and (100\%-x)D2+x\%Ar mixtures obtained using the 5-phase Lee model code. Appl. Radiat. Isot. (Under Review).

310. Piriaei, D.; Mahabadi, T.D.; Javadi, S.; Ghoranneviss, M.; Saw, S.H.; Lee, S. The study of pinch regimes based on radiationenhanced compression and anomalous resistivity phenomena and their effects on hard x-ray emission in a Mather type dense plasma focus device (SABALAN2). Phys. Plasmas 2015, 22, 123507. [CrossRef]

311. Auluck, S.K.H. Re-appraisal and extension of the Gratton-Vargas two-dimensional analytical snowplow model of plasma focus evolution in the context of contemporary research. Phys. Plasmas 2013, 20, 112501. [CrossRef]

312. Auluck, S.K.H. Re-appraisal and extension of the Gratton-Vargas two-dimensional analytical snowplow model of plasma focus. II. Looking at the singularity. Phys. Plasmas 2015, 22, 112509. [CrossRef]

313. Auluck, S.K.H. Re-appraisal and extension of the Gratton-Vargas two-dimensional analytical snowplow model of plasma focus. III. Scaling theory for high pressure operation and its implications. Phys. Plasmas 2016, 23, 122508. [CrossRef]

314. Gratton, F.; Vargas, J.M. Two dimensional electromechanical model of the plasma focus. In Energy Storage, Compression and Switching; Nardi, V., Sahlin, H., Bostick, W.H., Eds.; Plenum: New York, NY, USA, 1983; pp. 354-386.

315. Vargas, J.M.; Gratton, F.; Gratton, J.; Bruzzone, H.; Kelly, H. Experimental Verification of a Theory of the Current Sheath in the Plasma Focus. In Proceedings of the 6th Int. Conf. on Plasma Phys. and Controlled Fusion Res., Berchtesgaden, Germany, 6-13 October 1976; IAEA-CN-35/E18.5(a). p. 483. Available online: http:/ / www-naweb.iaea.org/napc/physics/FEC/STIPUB439_VOL1.pdf (accessed on 15 July 2021).

316. Auluck, S.K.H. Axial magnetic field and toroidally streaming fast ions in the dense plasma focus are natural consequences of conservation laws in the curved axisymmetric geometry of the current sheath. II. Towards a first principles theory. Phys. Plasmas 2017, 24, 112502. [CrossRef]

317. Auluck, S.K.H. Global parameter optimization of a Mather-type plasma focus in the framework of the Gratton-Vargas twodimensional snowplow model. Plasma Sources Sci. Technol. 2014, 23, 065015. [CrossRef]

318. Auluck, S.K.H. Design Parameter Space for a High Pressure Optimized Dense Plasma Focus Operating with Deuterium. J. Fusion Energy 2017, 36, 218-229. [CrossRef]

319. Moreno, C.; Bruzzone, H.; Martinez, J.; Clausse, A. Conceptual engineering of plasma-focus thermonuclear pulsors. IEEE Trans. Plasma Sci. 2000, 28, 1735-1741. [CrossRef]

320. Casanova, F.; Moreno, C.; Clausse, A. Finite-elements numerical model of the current-sheet movement and shaping in coaxial discharges. Plasma Phys. Control Fusion 2005, 47, 1239-1250. [CrossRef]

321. Casanova, F.; Tarifeño-Saldivia, A.; Veloso, F.; Pavez, C.; Clausse, A.; Soto, L. Toroidal High-Density Singularity in a Small Plasma Focus. J. Fusion Energy 2011, 31, 279-283. [CrossRef]

322. Dyachenko, V.P.; Imshennik, V.S. Plasma Focus and the Neutron Emission Mechanism in A Z-Pinch. Zh. Eksp. Teor. Fiz. 1969, 56, 1766-1777.

323. Potter, D.E. Numerical Studies of the Plasma Focus. Phys. Fluids 1971, 14, 1911. [CrossRef]

324. Maxon, S.; Eddleman, J. Two-dimensional magnetohydrodynamic calculations of the plasma focus. Phys. Fluids 1978, 21, 1856. [CrossRef]

325. Behler, K.; Bruhns, H. Three-fluid magnetohydrodynamical simulation of plasma focus discharges. Phys. Fluids 1987, 30, 3767. [CrossRef] 
326. Stepniewski, W. MHD numerical modelling of the plasma focus phenomena. Vaccum 2004, 76, 51-55. [CrossRef]

327. Garanin, S.F.; Mamyshev, V.I. Two-Dimensional MHD Simulations of a Plasma Focus with Allowance for the Acceleration Mechanism for Neutron Generation. Plasma Phys. Rep. 2008, 34, 639-649. [CrossRef]

328. Kueny, C.S.; Flicker, D.G.; Rose, D.V. ALEGRA-HEDP Simulations of the Dense Plasma Focus; Report SAND2009-6373; SANDIA Laboratory: Albuquerque, NM, USA, 2009. [CrossRef]

329. Meehan, B.T.; Niederhaus, J.H.J. Fully three-dimensional simulation and modeling of a dense plasma focus. J. Déf. Model. Simulation: Appl. Methodol. Technol. 2016, 13, 153-160. [CrossRef]

330. Liu, J.X.; Sears, J.; McMahon, M.; Tummel, K.; Cooper, C.; Higginson, D.; Shaw, B.; Povilus, A.; Link, A.; Schmidt, A. Seeding the $\mathrm{m}=0$ Instability in Dense Plasma Focus Z-Pinches with a Hollow Anode. 2016. Available online: https://arxiv.org/abs/1610.09092 (accessed on 15 July 2021).

331. Li, H.; Li, S.; Jungman, G.; Hayes-Sterbenz, A.C. Dense Plasma Focus Modeling; LA-UR-17-25546, Los Alamos Report, USA. 2017. Available online: https:/ / doi.org/10.2172/1369162 (accessed on 24 July 2021). [CrossRef]

332. Schmidt, A.; Tang, V.; Welch, D. Fully Kinetic Simulations of Dense Plasma Focus Z-Pinch Devices. Phys. Rev. Lett. 2012, 109, 205003. [CrossRef] [PubMed]

333. Schmidt, A.; Link, A.; Welch, D.; Ellsworth, J.; Falabella, S.; Tang, V. Comparisons of dense-plasma-focus kinetic simulations with experimental measurements. Phys. Rev. E 2014, 89, 061101. [CrossRef] [PubMed]

334. Schmidt, A.; Link, A.; Welch, D.; Meehan, B.T.; Tang, V.; Halvorson, C.; May, M.; Hagen, E.C. Fully kinetic simulations of megajoule-scale dense plasma focus. Phys. Plasmas 2014, 21, 102703. [CrossRef]

335. Bazdenkov, S.V.; Vikhrev, V.V. Three-fluid MHD-model of a current shell in Z-pinch. Sov. J. Plasma. Phys. 1975, 1, $250-253$.

336. Scholz, M.; Ivanova-Stanik, I. Initial phase in plasma focus device-model and computer simulation. Vacuum 2000, 58, 287-293. [CrossRef]

337. Yordanov, V.; Genov, D.; Ivanova-Stanik, I.; Blagoev, A. Ionization growth in the breakdown of plasma focus discharge. Vac. 2004, 76, 365-368. [CrossRef]

338. Yordanov, V.; Ivanova-Stanik, I.; Blagoev, A. PIC-MCC method with finite element solver for Poisson equation used in simulation of the breakdown phase in dense plasma focus devices. J. Phys. Conf. Ser. 2006, 44, 215-220. [CrossRef]

339. Yordanov, V.; Ivanova-Stanik, I.; Blagoev, A. The role of photo electrical effect in sustaining the preionization process in Plasma Focus device. J. Phys. Conf. Ser. 2007, 63, 012022. [CrossRef]

340. Yordanov, V.; Ivanova-Stanik, I.; Blagoev, A. Development of the ionization wave in the breakdown of the plasma focus device. J. Phys. D: Appl. Phys. 2007, 40, 2522-2525. [CrossRef]

341. Grasso, G.; Rocchi, F.; Sumini, M. Development of a Particle-In-Cell code with Structured Adaptive Mesh Refinement for Plasma Focus devices breakdown simulation. Nuovo Cim. C 2009, 32, 147-151.

342. Seng, Y.S.; Lee, P.; Rawat, R.S. Electromagnetic particle in cell modeling of the plasma focus: Current sheath formation and lift off. Phys. Plasmas 2014, 21, 023509. [CrossRef]

343. Blagoev, A.; Yordanov, V. A modelling of the breakdown phase in the plasma focus dis-charge. Plasma Phys. Contr. Fusion 2009, 51, 124022. [CrossRef]

344. Dobretsov, L.N.; Gomoyunova, M.V. Emissionaya Electronica; Nauka: Moscow, Russia, 1966. (In Russian)

345. Tawara, H.; Itikawa, Y.; Nishimura, H.; Yoshino, M. Cross Sections and Related Data for Electron Collisions with Hydrogen Molecules and Molecular Ions. J. Phys. Chem. Ref. Data 1990, 19, 617-636. [CrossRef]

346. The Siglo Data Base, CPAT and Kinema Software. Available online: http:/ / www.siglo-kinema.com (accessed on 15 July 2021$).$

347. Seng, Y.S.; Lee, P.; Rawat, R.S. Current sheath formation dynamics and structure for different insulator lengths of plasma focus device. Phys. Plasmas 2014, 21, 113508. [CrossRef]

348. Seng, Y.S. The Sliding Discharge Phase of a Plasma Focus. IEEE Trans. Plasma Sci. 2019, 47, 4883-4890. [CrossRef]

349. Seng, Y.S. Electrical Oscillations of the UNU-ICTP Plasma Focus Device in the Early Break-down Phase. IEEE Trans. Plasma Sci. 2018, 46, 943-946. [CrossRef]

350. Seng, Y.S.; Lee, P.; Rawat, R.S. External circuit integration with electromagnetic particle in cell modeling of plasma focus devices. Phys. Plasmas 2015, 22, 033524. [CrossRef]

351. Ohsawa, Y. Strong ion acceleration by a collisionless magnetosonic shock wave propagating perpendicularly to a magnetic field. Phys. Fluids 1985, 28, 2130. [CrossRef]

352. Davidson, R.C.; Gladd, N.T. Anomalous Transport Properties Associated with Lower-Hybrid-Drift Instability. Phys.Fluids 1975, 18, 1327-1335. [CrossRef]

353. Bruzzone, H.; Bernal, L. The need of using anomalous resistivity due to Lower Hybrid In-stabilities in plasma-magnetic field interfaces. Nukleonika 2001, 46, 59-61.

354. Gary, S.P.; Hohl, F. Ion kinematics in Plasma Focus. Phys.Fluids 1973, 16, 997-1002. [CrossRef]

355. Jiang, S.; Higginson, D.P.; Link, A.; Holod, I.; Schmidt, A. Effect of polarity on beam and plasma target formation in a dense plasma focus. Phys. Plasmas 2019, 26, 042702. [CrossRef]

356. Saito, S.; Sakai, J.I. Surfatron acceleration of ions by fast magnetosonic shocks generated dur-ing two current loops' coalescence. Astrophys. J. 2004, 604, L133-L136. [CrossRef]

357. Yousefi, R.; Sakai, J.I.; Ito, H.; Masugata, K. A New Ion Acceleration Mechanism in Z-Pinch Discharges of a Plasma Focus Device. Iranian Phys. J. 2007, 1, 31-34. 
358. Soto, L.; Pavez, C.; Tarifeño, A.; Moreno, J.; Veloso, F.; Tarifeño-Saldivia, A. Studies on scalability and scaling laws for the plasma focus: Similarities and differences in devices from $1 \mathrm{MJ}$ to $0.1 \mathrm{~J}$. Plasma Sources Sci. Technol. 2010, 19, 055017. [CrossRef]

359. Klir, D.; Soto, L. Drive Parameter of Neutron-Optimized Dense Plasma Foci. IEEE Trans. Plasma Sci. 2012, 40, 3273-3279. [CrossRef]

360. Turner, L. Hall Effects on Magnetic Relaxation. IEEE Trans. Plasma Sci. 1986, 14, 849-857. [CrossRef]

361. Auluck, S.K.H. Description of plasma focus current sheath as the Turner relaxed state of a Hall magnetofluid. Phys. Plasmas 2009, 16, 122504. [CrossRef]

362. Auluck, S.K.H. Evaluation of Turner relaxed state as a model of long-lived ion-trapping structures in plasma focus and Z-pinches. Phys. Plasmas 2011, 18, 32508. [CrossRef]

363. Auluck, S.K.H. Bounds imposed on the sheath velocity of a dense plasma focus by conservation laws and ionization stability condition. Phys. Plasmas 2014, 21, 090703. [CrossRef]

364. Auluck, S.K.H. On the representation of dense plasma focus as a circuit element. Phys. Plasmas 2021, 28, 030703. [CrossRef]

365. Bailey, J.; Ettinger, Y.; Fisher, A.; Rostoker, N. Gas-puff Z pinches with D2and D2-Ar mixtures. Appl. Phys. Lett. 1982, 40, 460-462. [CrossRef]

366. Zhang, T.; Lin, X.; Chandra, K.; Tan, T.L.; Springham, S.; Patran, A.; Lee, P.; Lee, S.; Rawat, R.S. Current sheath curvature correlation with the neon soft x-ray emission from plasma focus device. Plasma Sources Sci. Technol. 2005, 14, 368-374. [CrossRef]

367. Zakaullah, M.; Baig, T.; Beg, S.; Murtaza, G. Effect of insulator sleeve length on neutron emission in a plasma focus. Phys. Lett. A 1989, 137, 39-43. [CrossRef]

368. Koohestani, S.; Habibi, M.; Amrollahi, R.; Baghdadi, R.; Roomi, A. Effect of Quartz and Pyrex Insulators Length on Hard-X ray Signals in APF Plasma Focus Device. J. Fusion Energy 2010, 30, 68-71. [CrossRef]

369. Dönges, A.; Herziger, G.; Krompholz, H.; Rühl, F.; Schönbach, K. The breakdown phase in a coaxial plasma gun. Phys. Lett. A 1980, 76, 391-392. [CrossRef]

370. Herold, H.; Jerzykiewicz, A.; Sadowski, M.; Schmidt, H. Comparative analysis of large plas-ma focus experiments performed at IPF, Stuttgart, and IPJ, Świerk. Nucl. Fusion 1989, 29, 1255-1270. [CrossRef]

371. Beg, F.N.; Zakaullah, M.; Murtaza, G.; Beg, M.M. Effect of insulator sleeve material on neu-tron emission from a plasma focus. Phys. Scr. 1992, 46, 152-154. [CrossRef]

372. Hussain, S.; Shafiq, M.; Badar, M.A.; Zakaullah, M. Effect of insulator sleeve material on the x-ray emission from a plasma focus device. Phys. Plasmas 2010, 17, 092705. [CrossRef]

373. Decker, G.; Kies, W.; Pross, G. Experiments solving the polarity riddle of the plasma focus. Phys. Lett. A 1982, 89, 393-396. [CrossRef]

374. Mathuthu, M.; Zengeni, T.; Gholap, A. Variation of focus action with electrode length for an inner electrode of negative polarity. IEEE Trans. Plasma Sci. 1998, 26, 14-18. [CrossRef]

375. Soto, L.; Tarifeño-Saldivia, A.; Pavez, C.; Avaria, G.; Moreno, J.; Cabrini, N.; Zambra, M. Experimental Study in a Plasma Focus with Modified Electrodes. In Proceedings of the 39th EPS Conference \& 16th Int. Congress on Plasma Physics, Stockholm, Sweden, 2-6 July 2012; P5.103. Available online: http:/ / ocs.ciemat.es/epsicpp2012pap/pdf/P5.103.pdf (accessed on 15 July 2021).

376. Barbaglia, M.O.; Bruzzone, H.; Rios, I.; Acuña, H.; González, J.; Clausse, A. Multiple pinch formations in small plasma-focus devices. Plasma Phys. Control Fusion 2010, 52, 032001. [CrossRef]

377. Barbaglia, M.O.; Soto, L.; Clausse, A. Multiple pinching in the miniature plasma focus nanoPLADEMA. J. Physics: Conf. Ser. 2012, 370, 012058. [CrossRef]

378. Bruzzone, H.; Vieytes, R. The initial phase in plasma focus devices. Plasma Phys. Control Fusion 1993, 35, 1745-1754. [CrossRef]

379. Gribkov, V.A.; Tyagunov, M.G. USSR Patent No. 1012707, 26 January 1981.

380. Gribkov, V.A.; Tyagunov, M.G. USSR Patent No. 1026439, 1 March 1981.

381. Gribkov, V.A.; Tyagunov, M.G. USSR Patent No. 1101838, 20 December 1982.

382. Auluck, S.K.H.; Parhar, S.; Kulkarni, M.G.; Magtum, A.; Ghatge, S.; Suryavanshi, N.; Mirajkar, P.U. Demonstration of Plasma Focus Operation without Using Sliding Discharge on a Glass or Ceramic Insulator for Plasma Initiation. 2021. Available online: https: / / arxiv.org/abs/2104.00958 (accessed on 15 July 2021).

383. Bruzzone, H.; Fischfeld, G. On The Structure of Plasma Focus Imploding Current Sheaths. Phys. Lett. A 1989, 134, 484-487. [CrossRef]

384. Bruzzone, H. The role of anomalous resistivities in Plasma Focus discharges. Nukleonika 2001, 46, S3-S7.

385. Volobuev, I.V.; Gurei, A.E.; Nikulin, V.Y.; Polukhin, S.N. Magnetic and Neutron Measurements on the PF-400 Plasma Focus Facility. Plasma Phys. Rep. 2010, 36, 1013-1022. [CrossRef]

386. Bruzzone, H.; Acuña, H.N.; Barbaglia, M.O.; Milanese, M.M.; Miklaszewski, R.; Paduch, M.; Zieliñska, E.; Clausse, A. TimeVarying Inductance of the Plasma Sheet in the PF1000 Plasma-Focus Device. IEEE Trans. Plasma Sci. 2016, 44, 968-972. [CrossRef]

387. Mitrofanov, K.N.; Krauz, V.I.; Grabovski, E.V.; Myalton, V.V.; Vinogradov, V.P.; Paduch, M.; Scholz, M.; Karpiński, L. Study of the interrelation between the electrotechnical parameters of the plasma focus discharge circuit and the plasma compression dynamics on the PF-3 and PF-1000 facilities. Plasma Phys. Rep. 2015, 41, 379-398. [CrossRef]

388. Barbaglia, M.O.; Milanese, M.; Soto, L.; Clausse, A.; Moreno, J.; Pavez, C.; Moreno, C. Temporal Variation of the Current Sheet Inductance from PACO Plasma Focus Device. J. Fusion Energy 2016, 35, 561-566. [CrossRef]

389. Lu, M.F. Instability of the current sheath implosion in an 18-kJ Mather-type plasma focus. Phys. Rev. E 1996, 54, R1074-R1077. [CrossRef]

390. Lu, M.F. Different modes of plasma sheath motion in the radial compression and pinch phases in plasma focus. J. Phys. D Appl. Phys. 1996, 29, 660-680. [CrossRef] 
391. Kubes, P.; Paduch, M.; Pisarczyk, T.; Scholz, M.; Klir, D.; Kravarik, J.; Rezac, K.; Chodukow-ski, T.; Ivanova-Stanik, I.; Karpinski, L.; et al. Transformation of the Pinched Column at aPeriod of the Neutron Production. IEEE Trans. Plasma Sci. 2010, 38, 672-679. [CrossRef]

392. Kubes, P.; Paduch, P.; Pisarczyk, T.; Scholz, M.; Chodukowski, T.; Klir, D.; Kravarik, J.; Rezac, K.; Ivanova-Stanik, I.; Karpinski, L.; et al. Spontaneous Transformation in the Pinched Column of the Plasma Focus. IEEE Trans. Plasma Sci. 2011, 39, 562-568. [CrossRef]

393. Kubes, P.; Krauz, V.; Mitrofanov, K.; Paduch, M.; Scholz, M.; Piszarzcyk, T.; Chodukowski, T.; Kalinowska, Z.; Karpinski, L.; Klir, D.; et al. Correlation of magnetic probe and neutron signals with interferometry figures on the plasma focus discharge. Plasma Phys. Control Fusion 2012, 54, 105023. [CrossRef]

394. Kubes, P.; Klir, D.; Kravarik, J.; Rezac, K.; Kortanek, J.; Krauz, V.; Mitrofanov, K.; Paduch, M.; Scholz, M.; Pisarczyk, T.; et al. Scenario of pinch evolution in a plasma focus discharge. Plasma Phys. Control Fusion 2013, 55, 035011. [CrossRef]

395. Kubes, P.; Paduch, M.; Cikhardt, J.; Klir, D.; Kravarik, J.; Rezac, K.; Kortanek, J.; Cikhardtova, B.; Zielinska, E. Existence of a return direction for plasma escaping from a pinched column in a plasma focus discharge. Phys. Plasmas 2015, 22, 052706. [CrossRef]

396. Kubes, P.; Paduch, M.; Cikhardt, J.; Klir, D.; Kravarik, J.; Rezac, K.; Cikhardtova, B.; Kortanek, J.; Zielinska, E. The evolution of the plasmoidal structure in the pinched column in plasma focus discharge. Plasma Phys. Control Fusion 2016, 58, 045005. [CrossRef]

397. Kubes, P.; Paduch, M.; Cikhardt, J.; Cikhardtova, B.; Klir, D.; Kravarik, J.; Rezac, K.; Zielinska, E.; Sadowski, M.J.; Szymaszek, A.; et al. Transformation of the ordered internal structures during the acceleration of fast charged particles in a dense plasma focus. Phys. Plasmas 2017, 24, 072706. [CrossRef]

398. Kubes, P.; Kravarik, J.; Klir, D.; Rezac, K.; Scholz, M.; Paduch, M.; Tomaszewski, K.; Ivanova-Stanik, I.; Bienkowska, B.; Karpinski, L.; et al. Correlation of Radiation with Electron and Neutron Signals Taken in a Plasma-Focus Device. IEEE Trans. Plasma Sci. 2006, 34, 2349-2351. [CrossRef]

399. Scholz, M.; Bieńkowska, B.; Borowiecki, M.; Ivanova-Stanik, I.; Karpiński, L.; Stępniewski, W.; Paduch, M.; Tomaszewski, K.; Sadowski, M.; Szydłowski, A.; et al. Status of a mega-joule scale Plasma-Focus experiments. Nukleonika 2006, 51, 79-84.

400. Schmidt, H.; Kasperczuk, A.; Paduch, M.; Pisarczyk, T.; Scholz, M.; Tomaszewski, K.; Szydłowski, A. Review of Recent Experiments with the Megajoule PF-1000 Plasma Focus Device. Phys. Scr. 2002, 66, 168-172. [CrossRef]

401. Malinowska, A.; Malinowski, K.; Skladnik-Sadowska, E.; Sadowski, M.J.; Scholz, M.; Szydlowski, A.; Czaus, K.; Jaskóla, M.; Korman, A.; Schmidt, H. Measurements of ion micro-beams in RPI-type discharges and fusion protons in PF-1000 experiments. Phys. Scr. 2006, T123, 104-111. [CrossRef]

402. Kubes, P.; Kravarik, J.; Klir, D.; Rezac, K.; Scholz, M.; Paduch, M.; Ivanova-Stanik, I.; Karpinski, L.; Tomaszewski, K.; Kusse, B. R.; et al. Neutron Production and Fast Deuteron Characteristics at the Plasma Focus Discharge. AIP Conf. Proc. 2009, 1088, 207. [CrossRef]

403. Kubes, P.; Paduch, M.; Cikhardtova, B.; Cikhardt, J.; Klir, D.; Kravarik, J.; Rezac, K.; Kortanek, J.; Zielinska, E.; Sadowski, M.J.; et al. The influence of the nitrogen admixture on the evolution of a deuterium pinch column. Phys. Plasmas 2016, 23, 082704. [CrossRef]

404. Yamada, M.; Kulsrud, R.; Ji, H. Magnetic reconnection. Rev. Mod. Phys. 2010, 82, 603-664. [CrossRef]

405. Loureiro, N.F.; Uzdensky, D. Magnetic reconnection: From the Sweet-Parker model to stochastic plasmoid chains. Plasma Phys. Control Fusion 2016, 58, 014021. [CrossRef]

406. Boozer, A.H. Particle acceleration and fast magnetic reconnection. Phys. Plasmas 2019, 26, 082112. [CrossRef]

407. Auluck, S.K.H. Acceleration and trapping of fast ions in self-organized magneto-plasma structures in the dense plasma focus. Phys. Plasmas 2020, 27, 022308. [CrossRef]

408. Roshan, M.V.; Lee, P.; Lee, S.; Talebitaher, A.; Rawat, R.S.; Springham, S.V. Backward high energy ion beams from plasma focus. Phys. Plasmas 2009, 16, 074506. [CrossRef]

409. Bruzzone, H.; Milanese, M.M.; Niedbalski, J.J.; Acuna, H.N.; Moroso, R.L.; Supán, J.L.; Guichón, S.; Malamud, F. Temporal Correlations Between Hard X-ray and Neutron Pulses in the PACO Plasma Focus Device. IEEE Trans. Plasma Sci. 2010, 38, 1592-1597. [CrossRef]

410. Moreno, J.; Veloso, F.; Pavez, C.; Tarifeño-Saldivia, A.; Klir, D.; Soto, L. Neutron energy distribution and temporal correlations with hard x-ray emission from a hundreds of joules plasma focus device. Plasma Phys. Control Fusion 2015, 57, 035008. [CrossRef]

411. Jain, J.; Moreno, J.; Morales, D.; Davis, S.; Bora, B.; Avaria, G.; Inestrosa-Izurieta, M.J.; Soto, L. Observation and interpretation of neutron origin prior to hard X rays and pinch in a hundred joules plasma focus device. Laser Part. Beams 2017, 35, 656-662. [CrossRef]

412. Zakaullah, M.; Akhtar, I.; Murtaza, G.; Waheed, A. Imaging of fusion reaction zone in plasma focus. Phys. Plasmas 1999, 6, 3188-3193. [CrossRef]

413. Yap, S.L.; Wong, C.S.; Choi, P.; Dumitrescu, C.; Moo, S.P. Observation of Two Phases of Neutron Emission in a Low Energy Plasma Focus. Jpn. J. Appl. Phys. 2005, 44, 8125-8132. [CrossRef]

414. Aliaga-Rossel, R.; Choi, P. A novel technique for scintillator light collection in neutron meas-urements. Rev. Sci. Instrum. 1994, 65, 3034.

415. Kubes, P.; Paduch, M.; Cikhardt, J.; Kortanek, J.; Cikhardtova, B.; Rezac, K.; Klir, D.; Kravarik, J.; Zielińska, E. Filamentary structure of plasma produced by compression of puffing deuterium by deuterium or neon plasma sheath on plasma-focus discharge. Phys. Plasmas 2014, 21, 122706. [CrossRef] 
416. Kubes, P.; Paduch, M.; Cikhardt, J.; Kortanek, J.; Batobolotova, B.; Rezac, K.; Klir, D.; Kravarik, J.; Surala, W.; Zielinska, E.; et al. Neutron production from puffing deuterium in plasma focus device. Phys. Plasmas 2014, 21, 082706. [CrossRef]

417. Kubes, P.; Paduch, M.; Cikhardtova, B.; Cikhardt, J.; Klir, D.; Kravarik, J.; Rezac, K.; Zielinska, E.; Zaloga, D.; Sadowski, M.J.; et al. Influence of the $\mathrm{Al}$ wire placed in the anode axis on the transformation of the deuterium plasma column in the plasma focus discharge. Phys. Plasmas 2016, 23, 062702. [CrossRef]

418. Kubes, P.; Paduch, M.; Cikhardt, J.; Cikhardtova, B.; Rezac, K.; Klir, D.; Kravarik, J.; Kortanek, J.; Zielińska, E. Investigation of compression of puffing neon by deuterium current and plasma sheath in plasma focus discharge. Phys. Plasmas 2015, 22, 062705. [CrossRef]

419. Kubes, P.; Paduch, M.; Cikhardtova, B.; Cikhardt, J.; Klir, D.; Kravarik, J.; Rezac, K.; Kortanek, J.; Zielinska, E.; Sadowski, M.J.; et al. Interferometry and X-ray diagnostics of pinched helium plasma in a dense plasma focus with an Al-wire on the axis. Phys. Plasmas 2016, 23, 112708. [CrossRef]

420. Oginov, A.V.; Karakin, M.A.; Krauz, V.I.; Myalton, V.V.; Vinogradov, V.P.; Gurey, A.E.; Nikulin, V.Y.; Tikhomirov, A.A. Study of the Dynamics and Structure of Plasma-Current Sheath of Plasma Focus Discharge. BEAMS'04. In Proceedings of the 15th Int. Conf. on High-Power Particle Beams, Saint-Petersburg, Russia, 18-23 July 2005; pp. 746-749.

421. Krauz, V.I.; Levashova, M.G.; Karakin, M.A.; Krokhin, O.N.; Lisitsa, V.S.; Mokeev, A.N.; Myalton, V.V.; Nikulin, V.Y.; Oginov, A.V.; Smirnov, V.P.; et al. Influence of the radiation of the plasma-focus current sheath on the implosion dynamics of condensed targets. Plasma Phys. Rep. 2008, 34, 43-51. [CrossRef]

422. Krauz, V.; Mitrofanov, K.; Scholz, M.; Myalton, V.; Paduch, M.; Grabovski, E.; Karpinski, L.; Koidan, V.; Vinogradov, V.; Vinogradova, Y.; et al. Magnetic field measurements on PF-1000 and PF-3 facilities: Current sheath structure and neutron scaling. Nukleonika 2012, 57, 201-204.

423. Krauz, V.I.; Mitrofanov, K.N.; Myalton, V.V.; Khautiev, E.Y.; Mokeev, A.N.; Vinogradov, V.P.; Vinogradova, Y.V.; Grabovsky, E.V.; Zukakishvili, G.G. Studies of Dynamics and Structure of Current Sheath on Plasma Focus Facility PF-3. In Proceedings of the 34th EPS Conference on Plasma Phys., Warsaw, Poland, 2-6 July 2007. ECA Vol.31F, P-1.018.

424. Gurey, G.; Nikulin, V.; Polukhin, S.; Volobuev, I. Current sheath studies by magnetic probes on plasma focus PF-400.Problems of atomic science and technology. Plasma Phys. 2009, 15, 98-100.

425. Andreeshchev, E.A.; Voŭtenko, D.A.; Krauz, V.I.; Markoliya, A.I.; Matveev, Yu.V.; Reshetnyak, N.G.; Khautiev, É.Y. Study of the dynamics of a plasma current sheath in the KPF-4 Phoenix plasma-focus facility. Plasma Phys. Rep. 2007, 33, 218-226. [CrossRef]

426. Vinogradov, V.P.; Nashilevskii, A.V.; Krauz, V.I.; Remnev, G.E.; Vinogradova, Y.V.; Kanaev, G.G.; Mitrofanov, K.N.; Myalton, V.V. Development and study of a portable plasma focus neutron source. Plasma Phys. Rep. 2014, 40, 146-159. [CrossRef]

427. Nikulin, V.Y.; Polukhin, S.N.; Tikhomirov, A.A. A simple criterion for the snowplowing efficiency of the working gas in a kJ plasma focus. Plasma Phys. Rep. 2005, 31, 591-595. [CrossRef]

428. Schmidt, H.; Sadowski, M.J.; Jakubowski, L.; Skladnik-Sadowska, E.; Stanislawski, J. Gas-puff target experiments with the Poseidon plasma focus facility. Plasma Phys. Control Fusion 1994, 36, 13-24. [CrossRef]

429. Milanese, M.; Moroso, R.; Pouzo, J. A study of the production of neutrons in a dense plasma focus source operated with a non-uniform density of deuterium neutral gas. J. Phys. D: Appl. Phys. 1998, 31, 85-92. [CrossRef]

430. Choi, P.; Aliaga-Rossel, R. Dynamics of a Medium Energy Plasma Focus. In DENSE Z-PINCHES: Third International Conference; AIP Publishing: College Park, MD, USA, 1994; Volume 299, pp. 299-307.

431. Aliaga-Rossel, R.; Choi, P. Experimental Observations of the Spatial Anisotropy of the Neu-tron Emission in a Medium Energy Plasma Focus. IEEE Trans. Plasma Sci. 1998, 26, 1138-1145. [CrossRef]

432. Verma, R.; Lee, P.; Lee, S.; Springham, S.V.; Tan, T.L.; Rawat, R.S.; Krishnan, M. Order of magnitude enhancement in neutron emission with deuterium-krypton admixture operation in miniature plasma focus device. Appl. Phys. Lett. 2008, 93, 101501. [CrossRef]

433. Talebitaher, A.; Lee, S.; Kalaiselvi, S.M.P.; Verma, R.; Lee, P.; Springham, S.V.; Tan, T.L.; Rawat, R.S. Influence of Krypton Seeding on DD Fusion Neutron Production: Evaluation Methodology for Plasma Focus Optimization. J. Fusion Energy 2015, 35, 370-377. [CrossRef]

434. Yap, S.L.; Moo, S.P.; Wong, C.S.; Choi, P.S.; Dumitrescuzoita, C. Spatial and temporal evolution of neutron emission from a small plasma focus. AIP Conf. Proc. 1997, 409, 75-78. [CrossRef]

435. Bures, B.L.; Krishnan, M.; Madden, R.; Blobner, F. Enhancing Neutron Emission From a 500-J Plasma Focus by Altering the Anode Geometry and Gas Composition. IEEE Trans. Plasma Sci. 2010, 38, 667-671. [CrossRef]

436. Hahn, E.N.; Housley, D.; Narkis, J.; Conti, F.; Lowe, D.R.; Beg, F.N. Effect of krypton admixture in deuterium on neutron yield in a megaampere dense plasma focus. J. Appl. Phys. 2020, 128, 143302. [CrossRef]

437. Mohammadi, M.A.; Sobhanian, S.; Rawat, R.S. Neutron production with mixture of deuter-ium and krypton in Sahand Filippov type plasma focus facility. Phys. Lett. A 2011, 375, 3002-3006. [CrossRef]

438. Babazadeh, A.R.; Sadat Kiai, S.M. Study of Gas Admixture Influences on the Pinch Dynam-ics in a 90 kJ Filippov Type Plasma Focus. In Proceedings of the 32nd EPS Conference on Plasma Phys., Tarragona, Spain, 27 June-1 July 2005; ECA Volume 29C. p. 1.128.

439. Scholz, M.; Karpinski, L.; Stepniewski, W.; Branitski, A.; Fedulov, M.; Medovschikov, S.; Nedoseev, S.; Smirnov, V.P.; Zurin, M.; Szydlowski, A. Foam liner driven by a plasma focus current sheath. Phys. Lett. A 1999, 262, 453-456. [CrossRef] 
440. Fortov, V.E.; Karakin, M.A.; Khautiev, E.Y.; Krauz, V.I.; Medovschikov, S.F.; Mokeev, A.N.; Myalton, V.V.; Nedoseev, S.L.; Smirnov, V.P.; Vinogradov, V.P.; et al. Study of the Plasma Focus as a Driver for the Magnetic Compression of Liners. In Proceedings of the 5th Int. Conf, on Z-pinches, Albuquerque, NM, USA, 23-28 June 2002; Davids, J., Deeney, C., Pereira, N.R., Eds.; Melville: New York, NY, USA, 2002; Volume 651, pp. 37-42.

441. Krauz, V.I.; Karakin, M.A.; Khautiev, E.Y.; Mokeev, A.N.; Myalton, V.V.; Smirnov, V.P.; Vinogradov, V.P.; Fortov, V.E.; Nikulin, V.; Oginov, A.V.; et al. Recent results of the Filippov-type PF experiments at Kurchatov Institute. AIP Conf. Proc. 2006, 812, 43. [CrossRef]

442. Mitrofanov, K.N.; Krauz, V.I.; Myalton, V.V.; Vinogradov, V.P.; Vinogradova, Y.V.; Grabovski, E.V.; Dan'ko, S.A.; Zelenin, A.A.; Medovshchikov, S.F.; Mokeev, A.N. Study of the Implosion of Wire Arrays at the PF-3 Facility. Plasma Phys. Rep. 2014, 40, 110-134. [CrossRef]

443. Dan'ko, S.A.; Krauz, V.I.; Myalton, V.V.; Zhuzhunashvili, A.I.; Vinogradov, V.P.; Kharrasov, A.M.; Ananev, S.S.; Vinogradova, Y.V.; Kalinin, Y.G. Study of Soft X-ray Emission during Wire Array Implosion under Plasma Focus Conditions at the PF-3 Facility. Plasma Phys. Rep. 2015, 41, 882-894. [CrossRef]

444. Kubes, P.; Kravarik, J.; Klir, D. Influence of CD2 fiber on the compression in the PF-1000 facility. Czechoslov. J. Phys. 2004, 54, 285-290. [CrossRef]

445. Vinogradova, V.P.; Karakin, M.A.; Krauz, V.I.; Mokeev, A.N.; Myalton, V.V.; Smirnov, V.P.; Fortov, V.E.; Khautiev, É.Y. Dynamics of a high-temperature pinch in the presence of dust. Plasma Phys. Rep. 2006, 32, 642-655. [CrossRef]

446. Maisonnier, C.; Linhart, J.G.; Haegi, M. Generation of Shocks. In A Collapsing Cylindrical Plasma Shell Nuclear Fusion Supplement. 1962. Part II. pp. 727-732. Available online: http://www-naweb.iaea.org/napc/physics/FEC/1961.pdf (accessed on 15 July 2021).

447. Maisonnier, C.; Haegi, M.; Linhart, J.G. Hollow Dynamic Pinch Proc. of Conf. on Plasma Phys and Contr. Fus. Res., Culham, 6-10September 1965, V.II; IAEA: Vienna, Australia, 1966; pp. 345-365. Available online: http://www-naweb.iaea.org/napc/ physics/FEC/STIPUB111_VOL2.pdf. (accessed on 15 July 2021).

448. Yeh, T.R.; Wen, M.; Tzeng, C.C.; Shang, D.J.; Yeh, C.K.; Wu, K.S.; Kuo, Y.Y.; Hou, W.S. Neutron localization measurements from a two-gun plasma focus device. Laser Part. Beams 1989, 7, 773-779. [CrossRef]

449. Samuelli, M.; Rapezzi, L.; Angelone, M.; Pillon, M.; Rapisarda, M.; Vitulli, S. Unconventional plasma focus devices. IEEE Trans. Plasma Sci. 2006, 34, 36-54. [CrossRef]

450. Sodekoda, T.; Kuwabara, H.; Masuda, M.; Horioka, K. Demonstration of rapid electrical re-covery in repetitive operation of a counter-facing plasma-focus device. Plasma Fusion Res-arch: Rapid Commun. 2017, 12, 1206021. [CrossRef]

451. Lee, J.H.; McFarland, D.R.; Hohl, F. Production of dense plasmas in a hypocycloidal pinch apparatus. Phys. Fluids 1977, 20, 313. [CrossRef]

452. Hong, Y.J.; Lee, S.H.; Uhm, H.S.; Choi, E.H. Measurement of extreme ultraviolet light and electron temperature for a dense plasmas from hypocycloidal pinch device. Curr. Appl. Phys. 2011, 11, S177-S181. [CrossRef]

453. Lee, J.H.; McFarland, D.R.; Harries, W.L. Investigation of a staged plasma-focus apparatus. Plasma Phys. 1978, 20, 1025-1038. [CrossRef]

454. Ay, Y.; Al-Halim, M.A.; Bourham, M.A. Simulation of the plasma sheath dynamics in a spherical plasma focus. Eur. Phys. J. D 2015, 69, 1-9. [CrossRef]

455. Wong, C.S.; Choi, P.; Leong, W.S.; Singh, J. Generation of High Energy Ion Beams from a Plasma Focus Modified for Low Pressure Operation. Jpn. J. Appl. Phys. 2002, 41, 3943-3946. [CrossRef]

456. Wong, C.S.; Yap, S.L. Generation of Deuteron Beam from the Plasma Focus. Solid State Phenom. 2005, 107, 151. [CrossRef]

457. Lim, L.K.; Yap, S.L.; Bradley, D.A. Time-resolved characteristics of deuteron-beam generated by plasma focus discharge. PLoS ONE 2018, 13, e0188009. [CrossRef]

458. Lim, L.K.; Yap, S.L.; Nee, C.H. Dynamics of ion beam emission in a low pressure plasma focus device. Plasma Phys. Control Fusion 2021, 63, 035012. [CrossRef]

459. Khan, M.Z.; Yap, S.L.; Yaqoob, I.; Kumar, N.N.; Kuang, L.L.; San, W.C. Low-Energy Plasma Focus Device as an Electron Beam Source. Sci. World, J. 2014, 2014, 1-9. [CrossRef] [PubMed]

460. Soto, L.; Pavez, C.; Moreno, J.; Barbaglia, M.; Clausse, A. Nanofocus: An ultra-miniature dense pinch plasma focus device with submillimetric anode operating at 0.1 J. Plasma Sources Sci. Technol. 2008, 18, 015007. [CrossRef]

461. Nayfeh, A.H. Perturbation Methods; WILEY-VCH Verlag GmbH \& CO. KGaA: Weinheim, Germany, 2004.

462. Auluck, S.K.H. Role of Electron-inertia-linked Current Source Terms in the Physics of Cylin-drically Symmetric Imploding Snow-Plow Shocks. Phys. Plasmas 2002, 9, 4488-4494. [CrossRef]

463. Auluck, S.K.H. Dense Plasma Focus: A question in search of answers, a technology in search of applications. Int. J. Mod. Physics: Conf. Ser. 2014, 32, 1460315. [CrossRef]

464. Auluck, S.K.H. Manifestation of Constrained Dynamics in a Low-Pressure Spark. IEEE Trans. Plasma Sci. 2013, 41, 437-446. [CrossRef]

465. Koh, J.M.; Rawat, R.S.; Patran, A.; Zhang, T.; Wong, D.; Springham, S.V.; Tan, T.L.; Lee, S.; Lee, P. Optimization of the high pressure operation regime for enhanced neutron yield in a plasma focus device. Plasma Sources Sci. Technol. 2004, 14, 12-18. [CrossRef]

466. Decker, G.; Flemming, L.; Kaeppeler, H.J.; Oppenlander, T.; Pross, G.; Schilling, P.; Schmidt, H.; Shakhatre, M.; Trunk, M. Current and neutron yield scaling of fast high voltage plasma focus. Plasma Phys. 1980, 22, 245-260. [CrossRef]

467. Krompholz, H.; Rühl, F.; Schneider, W.; Schönbach, K.; Herziger, G. A scaling law for plasma focus devices. Phys. Lett. A 1981, 82, 82-84. [CrossRef] 
468. Milanese, M.; Pouzo, J. Neutron yield scaling laws for plasma focus devices. Small Plasma Physics Experiments. In Proceedings of the Symposium on small scale laboratory plasma experiments, Spring College on Plasma Physics, Trieste, Italy, 25 May-19 June 1987; p. 66.

469. Nikulin, V.Y.; Polukhin, S.N. Saturation of the Neutron Yield from Megajoule Plasma Focus Facilities. Plasma Phys. Rep. 2007, 33, 271-277. [CrossRef]

470. Herold, H.; Kaeppeler, H.J.; Schmidt, H.; Shakhatre, M.; Wong, C.S.; Deeney, C.; Choi, P. Progress in plasma focus operation up to $500 \mathrm{~kJ}$ bank energy. IAEA-CN-S0/C-IV-S-2, 1988. In Proceedings of the 12th International Conference on Plasma Physics and Controlled Nuclear Fusion Research, Nice, France, 12-19 October 1988; Volume 2, p. 587. Available online: http:/ / www-naweb.iaea.org/napc/physics/FEC/STIPUB787_VOL2.pdf (accessed on 15 July 2021).

471. Shakhatre, M.; Auluck, S. Saettigung der Neutronenausbeute am Plasmafokus Poster. In Proceedings of the Spring meeting of the German Physical Society, Kiel, Germany, 11-16 March 1991.

472. Mitov, M.; Blagoev, A.; Zapryanov, S.; Auluck, S.K.H. First dedicated experiment on the az-imuthal electric field as a new diagnostic tool for Dense Plasma Focus. In Proceedings of the 9th International Workshop and Summer School on Plasma Physics, Sofia, Bulgaria, 30 November-4 December 2020.

473. Auluck, S.K.H. Energetic Ions in Dense Magnetized Plasma and the Fundamental Premise of Thermonuclear Fusion-Significance of Energetic Ions, the Fundamental Premise of Thermonucle-AR Fusion and Present State of Understanding in DMP. School and Training Course on Dense Magnetized Plasma, Abdus Salam International Center for Theoretical Physics (ICTP), Trieste, Italy, 8-12 October 2012. Available online: http://indico.ictp.it/event/a11188/session/11/contribution/7 (accessed on 15 July 2021).

474. Mannucci, S.; Mostacci, D.; Rocchi, F.; Sumini, M.; Angeli, E.; Tartari, A. Effect of a cathodic knife edge on the PFMA-1 pinch performance. Plasma Sources Sci. Technol. 2009, 18, 436-449. [CrossRef] 